\title{
Selected Hydrologic and Water-Quality Data, 1997 through 1999, for the Lake Traverse Reservation/Roberts County Water-Resources Investigation in South Dakota
}

By Ryan F. Thompson

Open-File Report 00-445

Prepared in cooperation with the South Dakota Geological Survey, Sisseton-Wahpeton Sioux Tribe, and Roberts County 


\section{U.S. Department of the Interior}

Bruce Babbitt, Secretary

\section{U.S. Geological Survey}

Charles G. Groat, Director

The use of firm, trade, and brand names in this report is for identification purposes only and does not constitute endorsement by the U.S. Geological Survey.

Rapid City, South Dakota: 2000

For additional information write to:

District Chief

U.S. Geological Survey

1608 Mt. View Road

Rapid City, SD 57702

Copies of this report can be purchased from:

U.S. Geological Survey

Branch of Information Services

Box 25286

Denver, C0 80225-0286 


\section{CONTENTS}

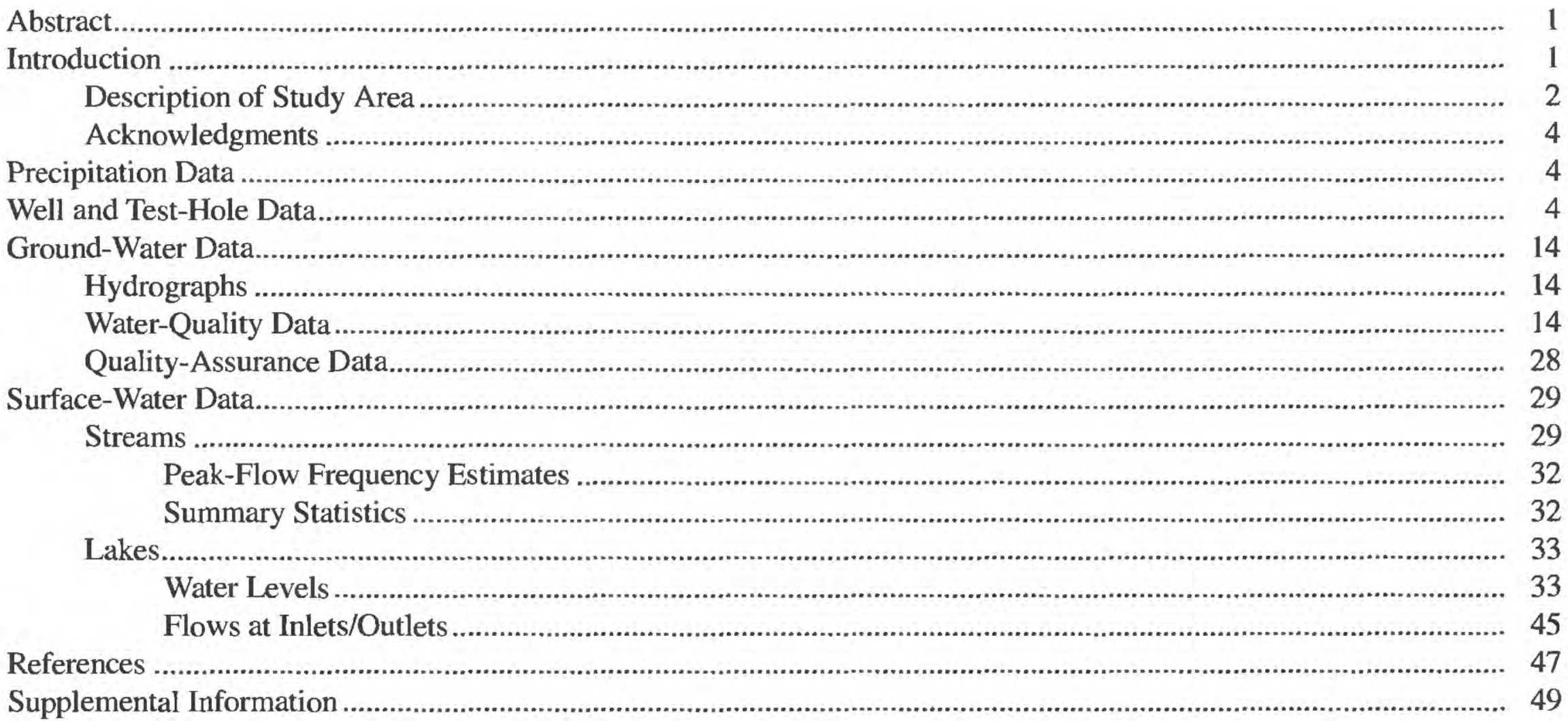

\section{FIGURES}

1-7. Maps showing:

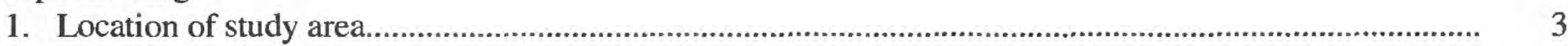

2. Location of precipitation stations within and near study area ...............................................................

3. Location of observation wells installed between 1997 and 1999 for the study .......................................... 8

4. Location of test holes drilled between 1997 and 1999 for the study ........................................................ 9

5. Location of selected observation wells in and near study area, which were part of the water-level monitoring program .................................................................................................... 22

6. Location of ground-water sites sampled during 1997-99 ….................................................................. 23

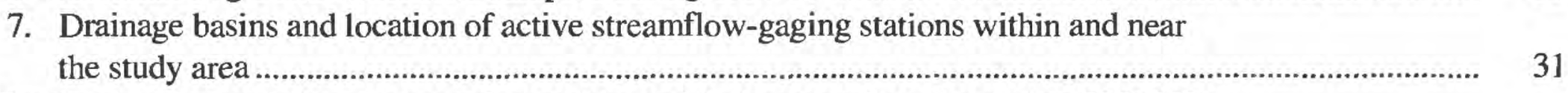

8. Graphs showing variations in annual, monthly, and daily mean flow for station 5051650 ,

LaBelle Creek near Veblen, S. Dak., water years 1988-99 ............................................................................ 34

9. Graphs showing variations in annual, monthly, and daily mean flow for station 05289985 ,

Big Coulee Creek near Peever, S. Dak., water years 1988-99 ........................................................................ 35

10. Map showing location of lakes with recorded water-level elevations................................................................. 36

11-20. Hydrographs for lakes:

11. Bitter Lake in Day County ................................................................................................................. 37

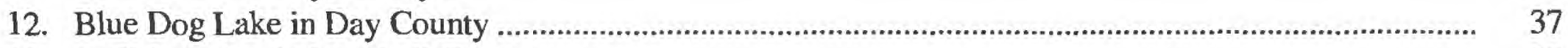

13. Buffalo Lakes in Marshall County ......................................................................................................... 38

14. Clear Lake in Marshall County ........................................................................................................... 38

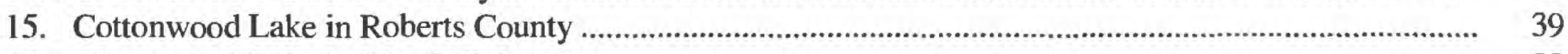

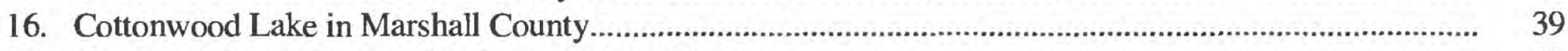

17. North and South Drywood Lakes in Roberts County .........................................................................

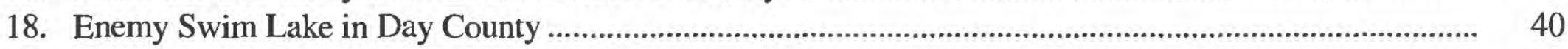

19. Hillebrands Lake in Day County .................................................................................................

20. Hurricane Lake in Roberts County .................................................................................................. 
FIGURES-Continued

21-25. Hydrographs for lakes:

21. Pickerel Lake in Day County .

22. Piyas Lake in Marshall County .

23. North and South Red Iron Lakes in Marshall County

24. Spring Lake in Day County.

25. North and South Whitestone Lakes in Roberts County

26. Map showing location of miscellaneous streamflow measurements at lake inlets or outlets.

\section{TABLES}

1-8. Precipitation, in inches, for the:

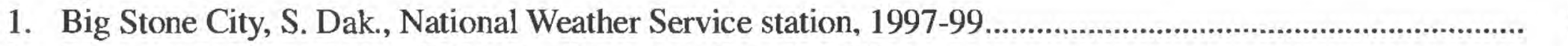

2. Sisseton, S. Dak., National Weather Service station, 1997-99 ......................................................................... 6

3. Summit, S. Dak., National Weather Service station, 1997-99 ……................................................................... 6

4. Victor, S. Dak., National Weather Service station, 1997-99 ........................................................................... 6

5. Watertown, S. Dak., National Weather Service station, 1997-99................................................................. 6

6. Waubay National Wildlife Refuge, S. Dak., National Weather Service station, 1997-99.............................. 7

7. Wilmot, S. Dak., National Weather Service station, 1997-99 ……................................................................. 7

8. Forman, N. Dak., National Weather Service station, 1997-99 ….............................................................. 7

9. Selected site information for observation wells and test holes completed from 1997 through $1999 \ldots \ldots \ldots \ldots \ldots \ldots \ldots . . . . .10$

10. Selected site information for observation wells in water-level network ........................................................ 15

11. Water-quality data from observation wells sampled by the U.S. Geological Survey from 1997-99.................... 24

12. Constituent concentrations for quality-assurance laboratory and field blanks ............................................... 29

13. Peak-flow estimates for selected recurrence intervals for active gaging stations within and near the study area

14. Miscellaneous streamflow measurements at lake inlets or outlets .....

\section{CONTENTS OF SUPPLEMENTAL INFORMATION SECTIONS}

A. Geologic logs for:

Test Holes Drilled and Observation Wells Installed, 1997-99

Selected Existing Test Holes and Observation Wells in Which the Bedrock was Reached ...................................... 119

B. Hydrographs for South Dakota Department of Environment and Natural Resources

North Dakota State Water Commission observation wells:

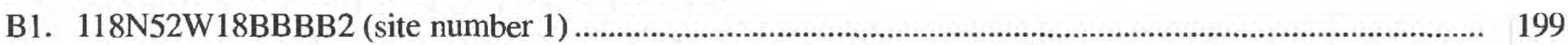

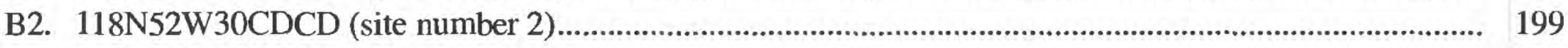

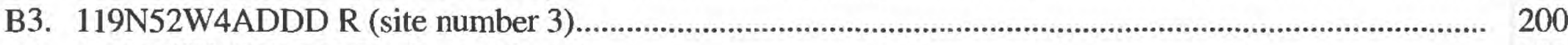

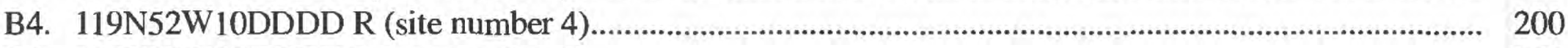

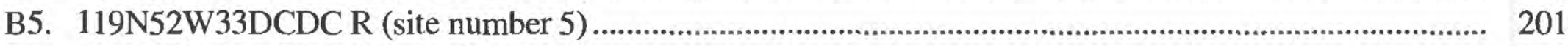

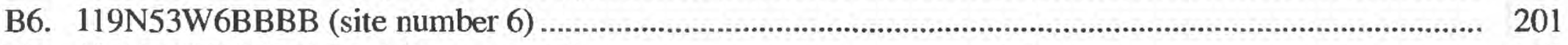

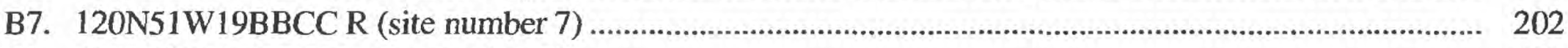

B8. 120N52W9BBBB R (site number 8) ….................................................................................. 202

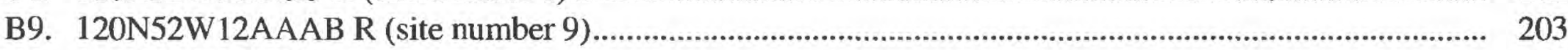

B10. 120N52W23BBBB R (site number 10) …............................................................................... 203

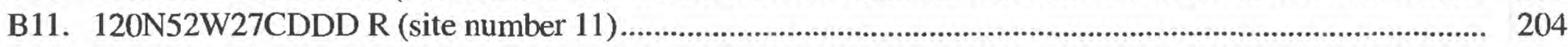

B12. 120N52W28DDDD R (site number 12) .................................................................................. 204

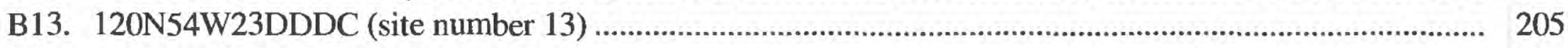

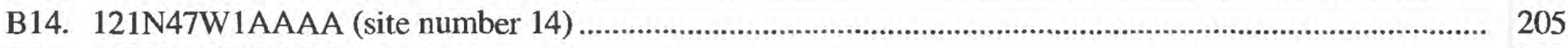

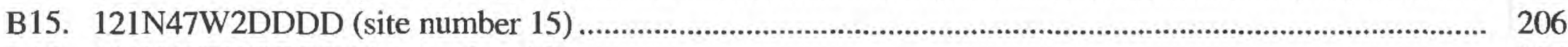

B16. 121N47W6CCCC (site number 16) ............................................................................................... 206

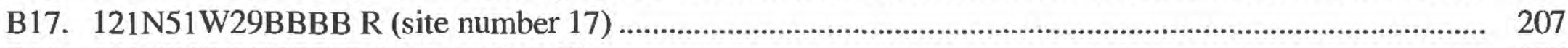

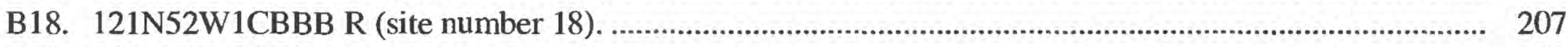

B19. 121N52W2ADDD R (site number 19) ..................................................................................... 208

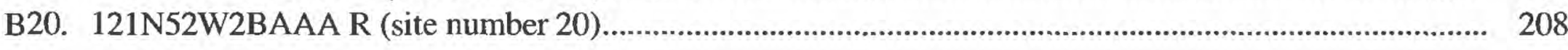




\section{CONTENTS OF SUPPLEMENTAL INFORMATION SECTIONS-Continued}

B. Hydrographs for South Dakota Department of Environment and Natural Resources

North Dakota State Water Commission observation wells-Continued:

B21. 121N52W2BBBA R (site number 21) ...................................................................................... 209

B22. 121N52W2CCCC R (site number 22) ................................................................................................. 209

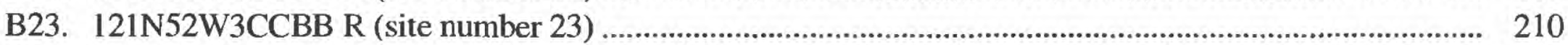

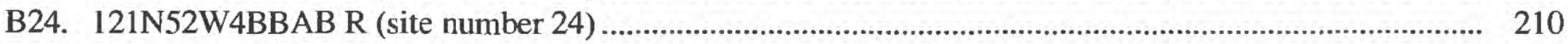

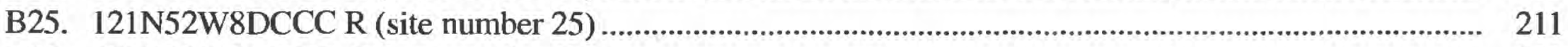

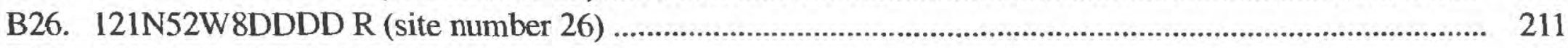

B27. 121N52W13BBBA R (site number 27) ….......................................................................... 212

B28. 121N52W22CCCC R (site number 28) ....................................................................................... 212

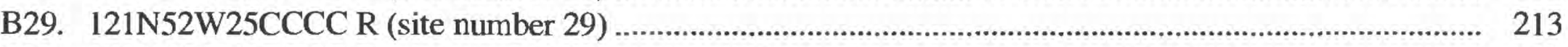

B30. 121N52W29CCBB R (site number 30) ……............................................................................. 213

B31. 121N54W34AADA (site number 31) .............................................................................................. 214

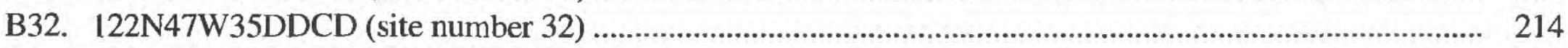

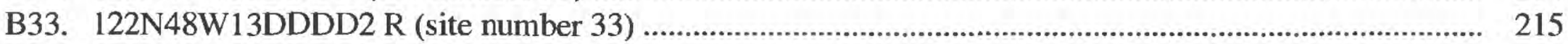

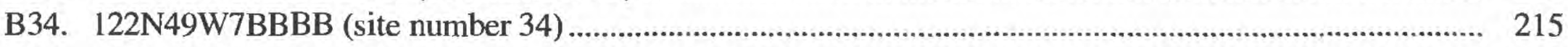

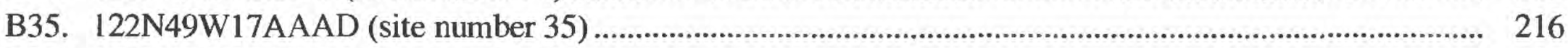

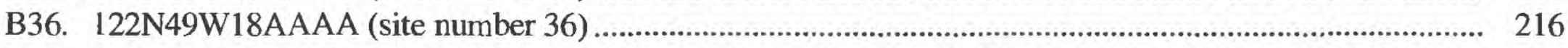

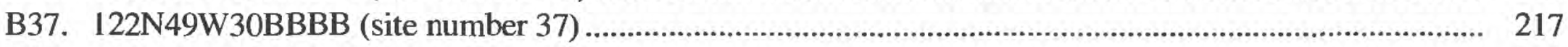

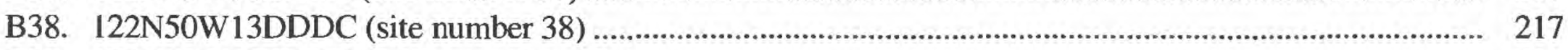

B39. I22N51W4DDDD2 R (site number 39) ....................................................................................... 218

B40. 122N51W27BBBB2 R (site number 40) ..................................................................................... 218

B41. 122N52W1DDCC R (site number 41) ....................................................................................... 219

B42. 122N52W2ABBB R (site number 42) ……................................................................................ 219

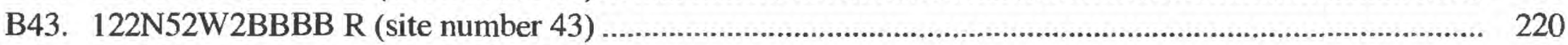

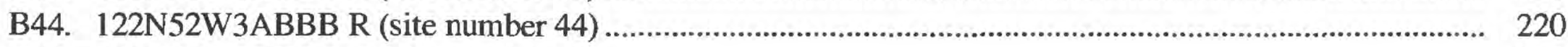

B45. 122N52W3CCDD R (site number 45) ........................................................................................... 221

B46. 122N52W3DAAA R (site number 46) …….............................................................................. 221

B47. 122N52W5CCCC R (site number 47) ……................................................................................ 222

B48. 122N52W9AAAA2 R (site number 48) ………....................................................................... 222

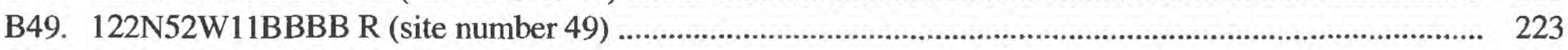

B50. 122N52W12CCCC R (site number 50) ……............................................................................ 223

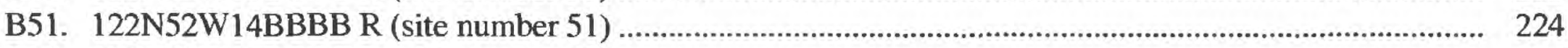

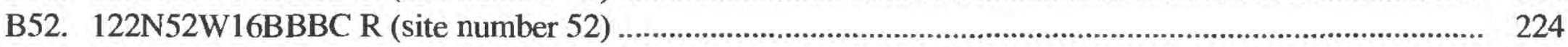

B53. 122N52W27BBBB2 R (site number 53) …................................................................................... 225

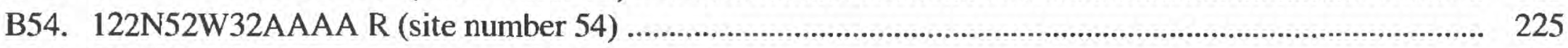

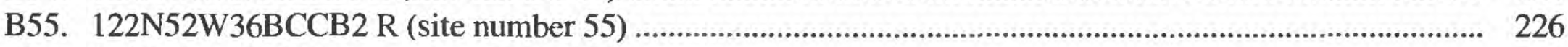

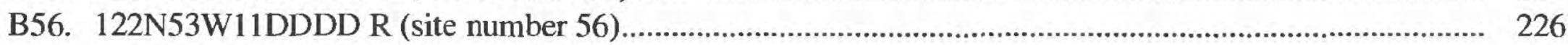



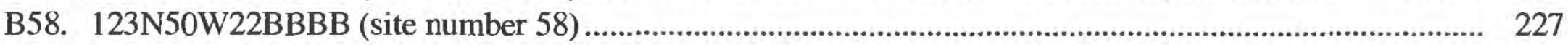

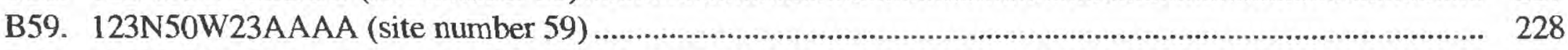

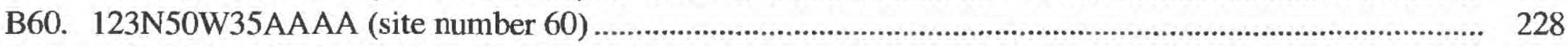

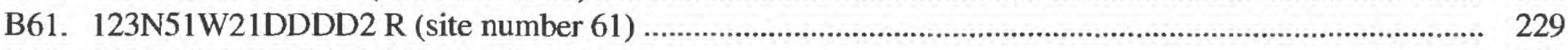

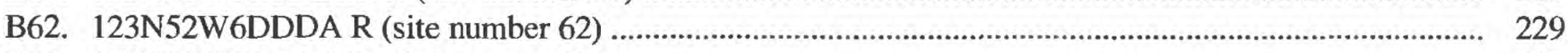

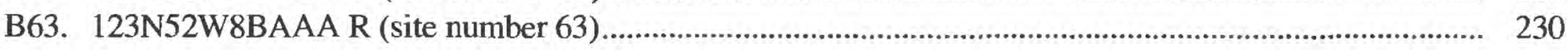

B64. 123N52W19BABA R (site number 64) ….................................................................................... 230

B65. 123N52W25CCCC R (site number 65). ........................................................................................ 231

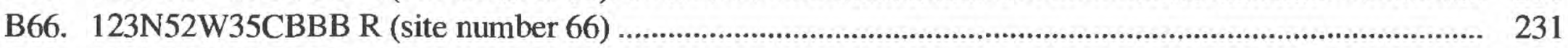

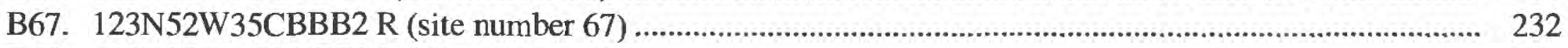

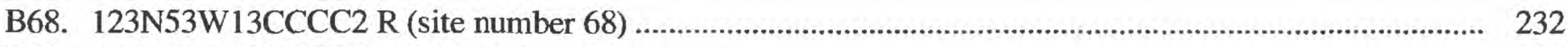

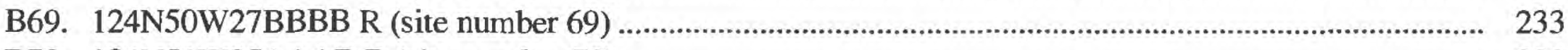

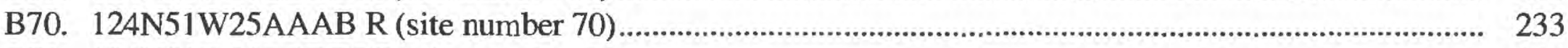

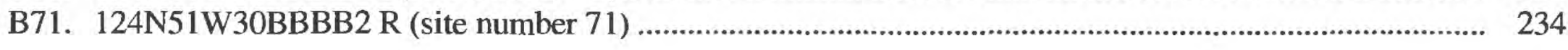




\section{CONTENTS OF SUPPLEMENTAL INFORMATION SECTIONS-Continued}

B. Hydrographs for South Dakota Department of Environment and Natural Resources

North Dakota State Water Commission observation wells-Continued:

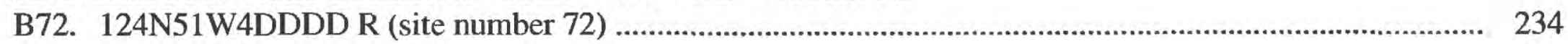

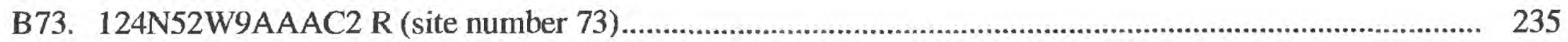

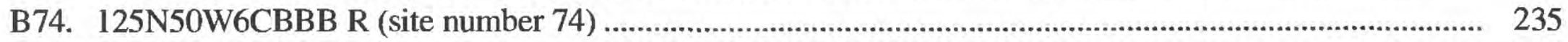

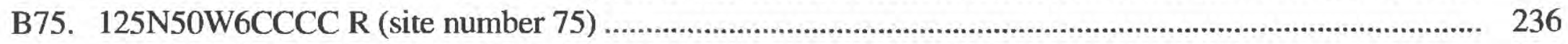

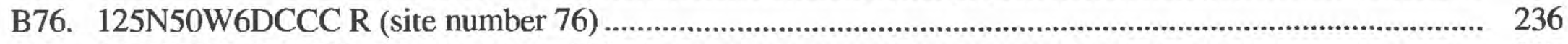

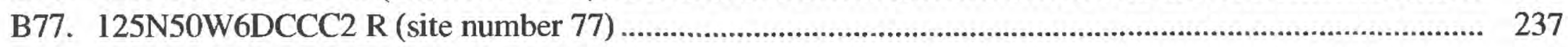

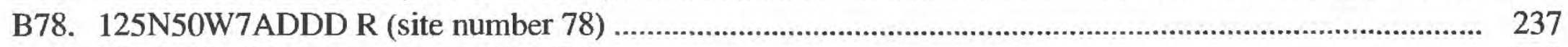

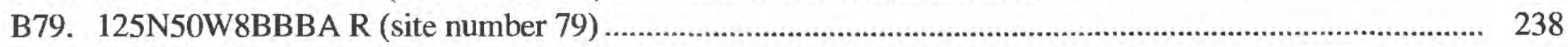

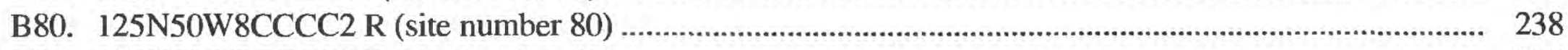

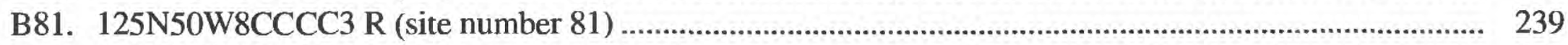

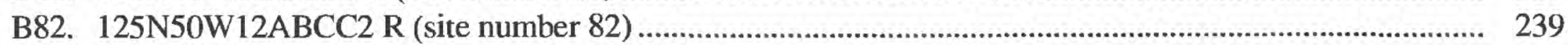

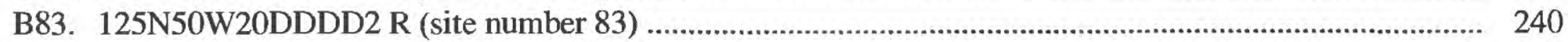

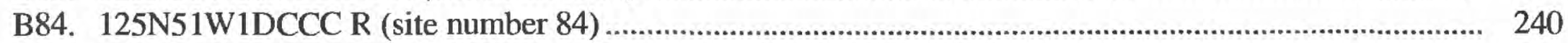

B85. 125N51W1DCCC2 R (site number 85) …............................................................................. 241

B86. 125N51W13ABBB2 R (site number 86) .................................................................................... 241

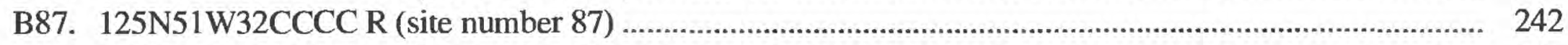

B88. 125N51W7BBBB2 R (site number 88) ................................................................................... 242

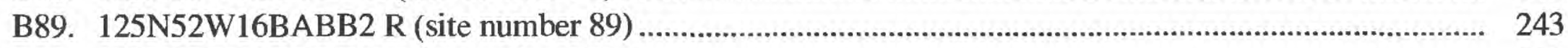

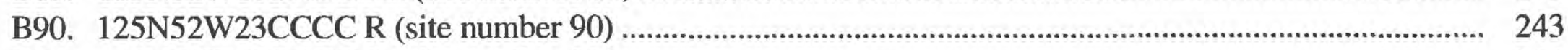

B91. 125N53W12CCCC R (site number 91) ……................................................................................... 244

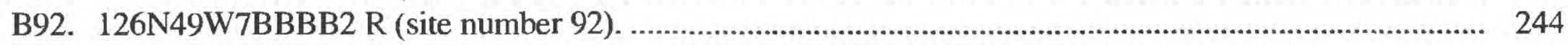

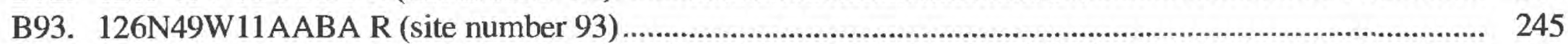

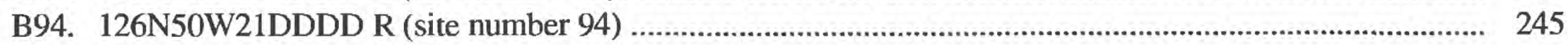

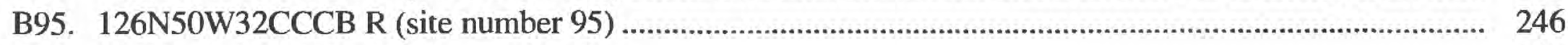

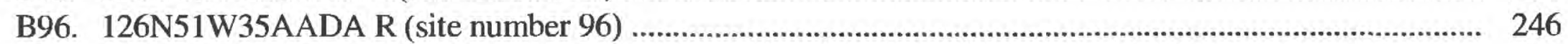

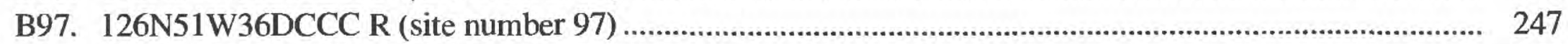

B98. 127N48W2BBBB R (site number 98) ........................................................................................ 247

B99. 127N48W7BBBB R (site number 99) ..................................................................................... 248

B100. 127N48W7BBBB2 R (site number 100) …........................................................................... 248

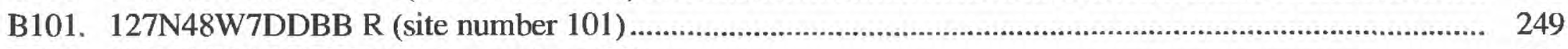

B102. 127N48W18DDDD R (site number 102) ...................................................................................... 249

B103. 127N48W28AAAA R (site number 103) ............................................................................... 250

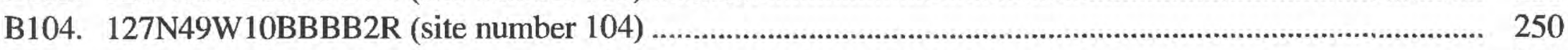

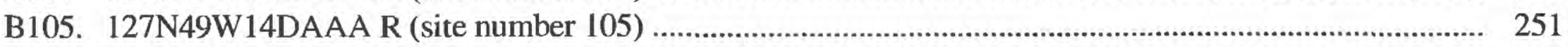

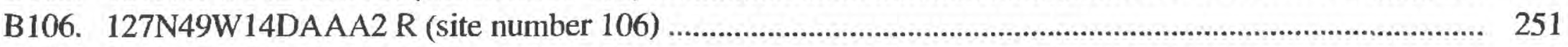

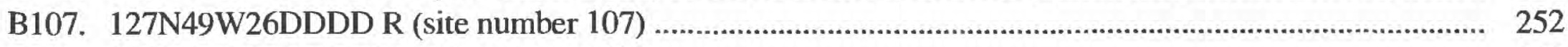

B108. 127N49W29DCDC2 R (site number 108) ……............................................................................... 252

B109. 127N49W36BBBB R (site number 109) …................................................................................ 253

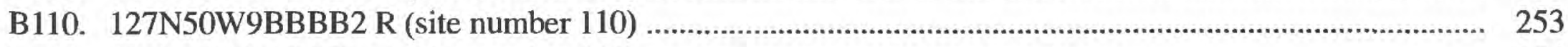

B111. 127N50W19CCCB R (site number 111) ....................................................................................... 254

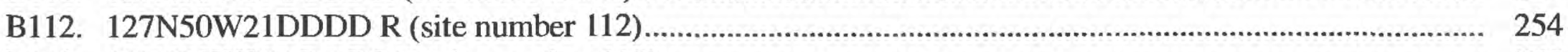

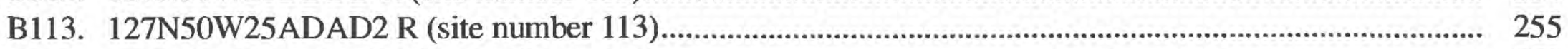



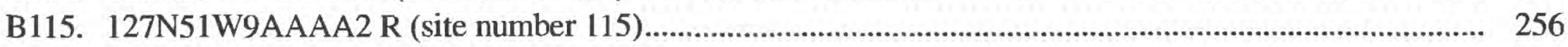

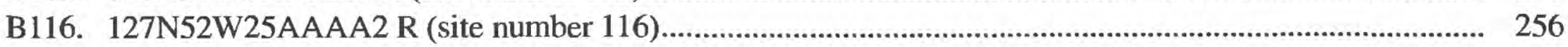

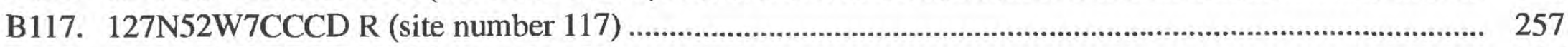

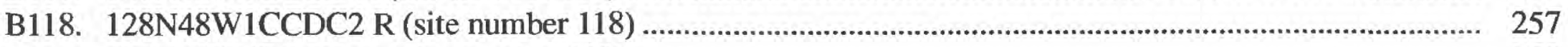



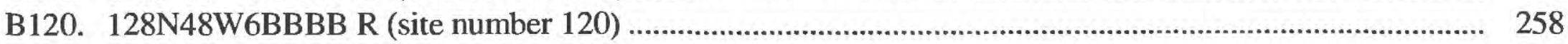

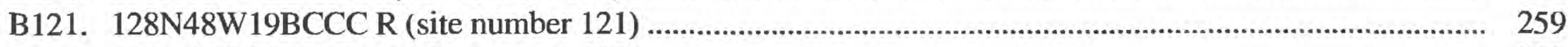

B122. 128N48W20BBBB R (site number 122) .................................................................................... 259 


\section{CONTENTS OF SUPPLEMENTAL INFORMATION SECTIONS-Continued}

B. Hydrographs for South Dakota Department of Environment and Natural Resources North Dakota State Water Commission observation wells - Continued:

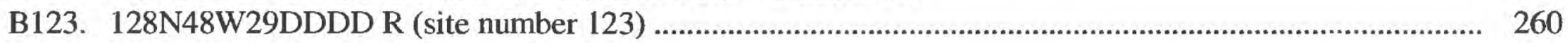

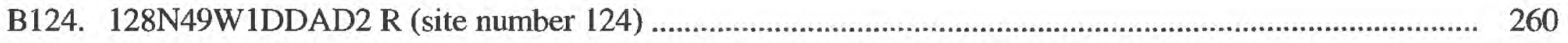

B125. 128N49W20BBBB R (site number 125) ................................................................................. 261

B126. 128N49W36AAAA R (site number 126) ……......................................................................... 261

B127. 128N50W10BBBB2 R (site number 127) ……................................................................................. 262

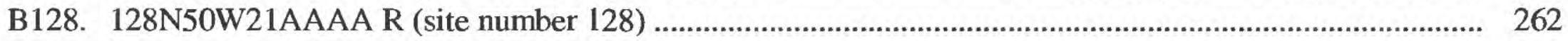

B129. 128N50W21AABA R (site number 129)....................................................................................... 263

B130. 128N50W21CCCC R (site number 130) ................................................................................ 263

B131. 128N50W25AAAB2 R (site number 131) ……............................................................................... 264

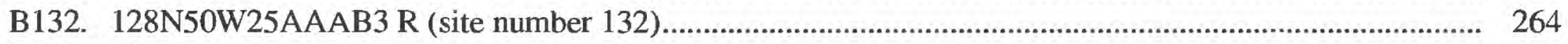

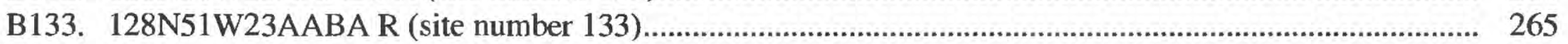

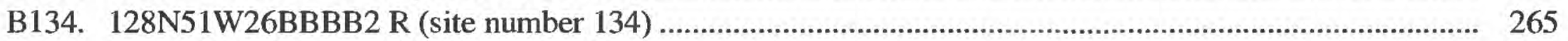

B135. 128N52W1DDDD2 R (site number 135) ……......................................................................... 266

B136. 128N52W3CCCB2 R (site number 136) …........................................................................... 266

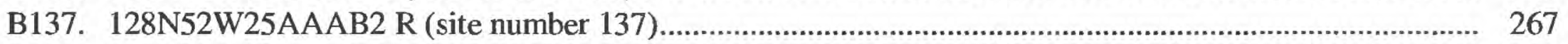

B138. 128N52W25AAAB R (site number 138) ……................................................................................. 267

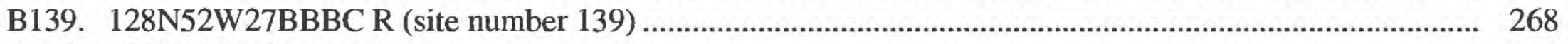

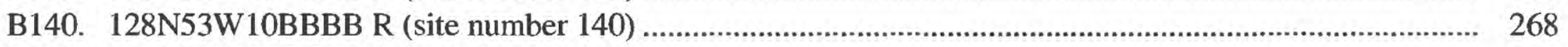

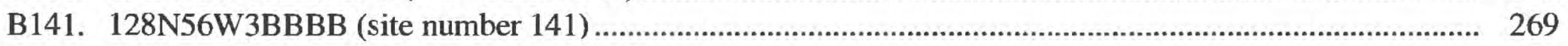

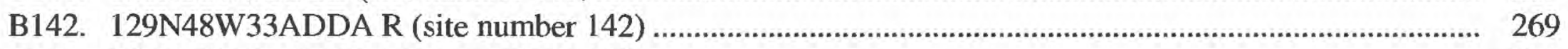

B143. 129N50W24CCD1 R (site number 143) .................................................................................. 270

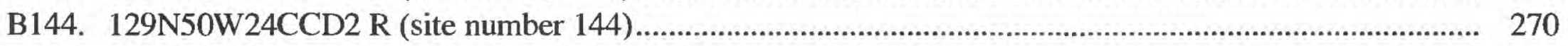

B145. 129N50W24CCD3 R (site number 145) ................................................................................ 271

B146. 129N50W27BBBB2 R (site number 146) …................................................................................ 271

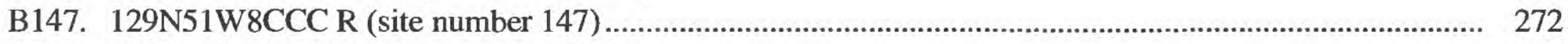

B148. 129N51W19ABA R (site number 148)................................................................................ 272

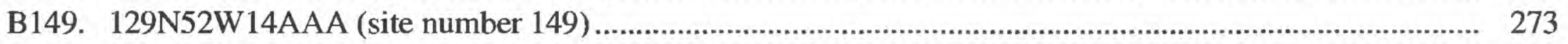

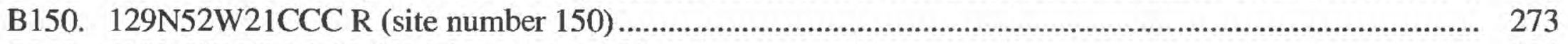

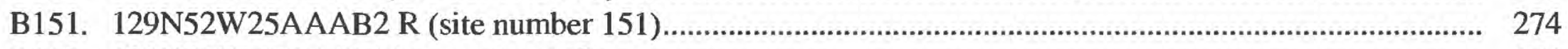

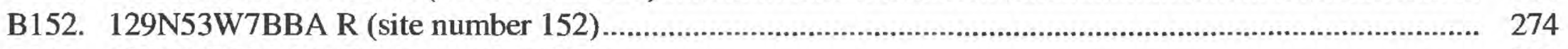

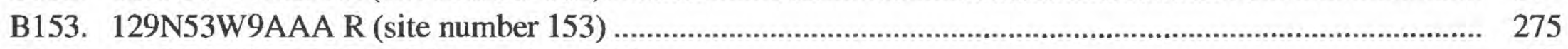

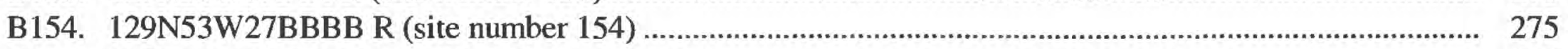

B155. 129N54W1AAA (site number 155) …………......................................................................... 276

B156. 129N54W1AAA2 (site number 156) .................................................................................. 276

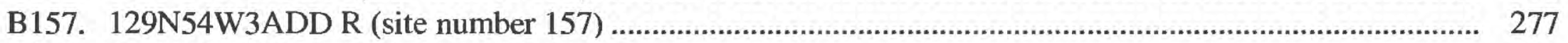

B158. 129N54W25AAAA R (site number 158) …................................................................................. 277

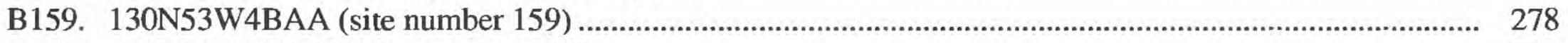

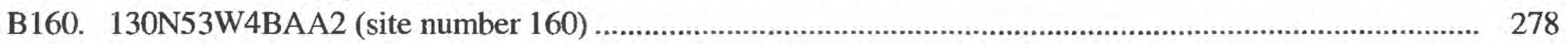

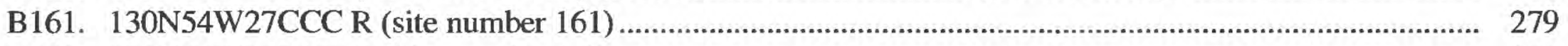

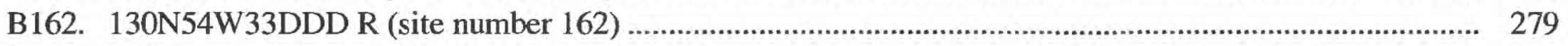

B163. 130N54W35CCC R (site number 163) ..................................................................................... 280

C. Annual peak-flow information:

C1. Station 05050000, Bois de Sioux River near White Rock, S. Dak. ................................................... 283

C2. Station 05051650, La Belle Creek near Veblen, S. Dak. .................................................................. 283

C3. Station 05289985, Big Coulee Creek near Peever, S. Dak. .................................................................. 283

C4. Station 05290000, Little Minnesota River near Peever, S. Dak................................................................ 284

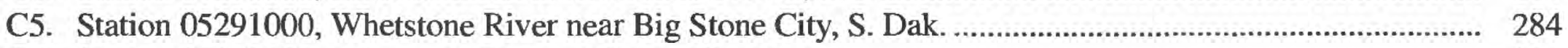

C6. Station 05292000, Minnesota River at Ortonville, Minn....................................................................... 284

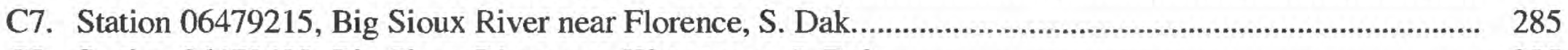

C8. Station 06479438, Big Sioux River near Watertown, S. Dak. …............................................................. 285 


\section{CONTENTS OF SUPPLEMENTAL INFORMATION SECTIONS-Continued}

D. Mean flow and summary statistics

D1. Station 05051650, LaBelle Creek near Veblen, S. Dak.

1. Mean flow, in cubic feet per second

2. Statistics on mean flow, in cubic feet per second (October 1998 through September 1999)

3. Serial correlation for 1-year lag for monthly and annual mean flows (October 1998 through September 1999)

4. Correlation matrix for monthly mean flow (October 1998 through September 1999).

5. Lowest mean flow, in cubic feet per second, and ranking for the following number of consecutive days

6. Highest mean flow, in cubic feet per second, and ranking for the following number of consecutive days

D2. Station 05289985, Big Coulee Creek near Peever, S. Dak.

1. Mean flow, in cubic feet per second.

2. Statistics on mean flow, in cubic feet per second (October 1988 through September 1999)

3. Serial correlation for 1-year lag for monthly and annual mean flows (October 1988 through September 1999).

4. Correlation matrix for monthly mean flow (October 1988 through September 1999)

5. Lowest mean flow, in cubic feet per second, and ranking for the following number of consecutive days

6. Highest mean flow, in cubic feet per second, and ranking for the following number of consecutive days

E. Water-level measurements for:

E1. Bitter Lake in Day County .

E2. Blue Dog Lake in Day County

E3. Buffalo Lake North in Marshall County

E4. Buffalo Lake South in Marshall County

E5. Clear Lake in Marshall County

E6. Clubhouse Lake in Roberts County

E7. Cottonwood Lake (North) in Roberts County.

E8. Cottonwood Lake in Marshall County.

E9. Drywood Lakes in Roberts County.

E10. Enemy Swim Lake in Day County

E11. Hillebrands Lake in Day County

E12. Hurricane Lake in Roberts County

E13. Pickerel Lake in Day County

E14. Piyas Lake in Marshall County

E15. Red Iron Lake North in Marshall County ........

E16. Spring Lake in Day County.

E17. Whitestone Lake North in Roberts County

E18. Whitestone Lake South in Roberts County 


\section{WELL-NUMBERING SYSTEM}

The Federal land-survey system is one method used to number observation wells and test holes. This system uses township, range, and section number. Number 126N52W20CCCB indicates a well in Township 126 North, Range 52 West, section 20. The last four letters show location within the section as shown below. When a nest of wells is drilled, sequential numbers identify each well in the nest and appear after the four-letter code. Wells located within the former boundary of the Lake Traverse Indian Reservation are given a suffix of "R."

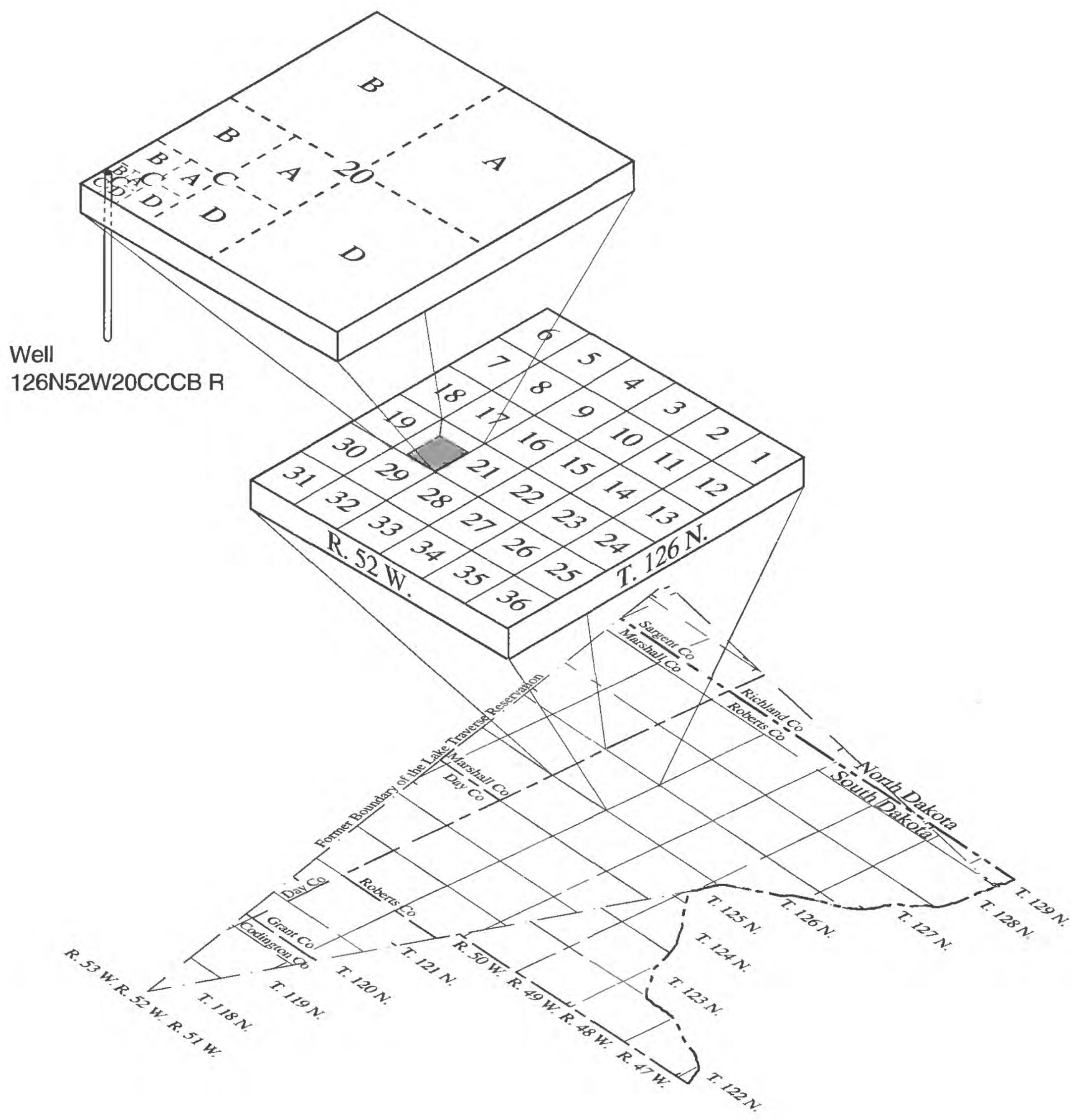




\title{
Selected Hydrologic and Water-Quality Data, 1997 through 1999, for the Lake Traverse Reservation/Roberts County Water-Resources Investigation in South Dakota
}

\author{
By Ryan F. Thompson
}

\section{ABSTRACT}

This report presents data on precipitation, geologic logs, water levels for ground water and lakes, stream discharge, and water quality for ground water that have been collected or compiled beginning in 1997 for the Lake Traverse Reservation/Roberts County Water Resources Investigation. The investigation was initiated in 1994 as a cooperative effort between the U.S. Geological Survey, the South Dakota Geological Survey, the Sisseton-Wahpeton Sioux Tribe, and Roberts County.

Prior to the water-resources investigation, a reconnaissance drilling program was accomplished from 1988 through 1990 by the U.S. Geological Survey and the Sisseton-Wahpeton Sioux Tribe. During the reconnaissance drilling, 19 test holes were drilled, and 9 observation wells were installed. After the water-resources investigation started in 1994, a comprehensive drilling program was initiated in cooperation with the South Dakota Geological Survey. This is the second of two data reports compiling data collected for the study. The first data report presented existing data within the study area, and data collected through 1996 specifically for the study. Since 1997, 59 additional test holes have been drilled and 23 observation wells have been installed. Geologic logs are presented for the test holes and wells completed during the 1997 and subsequent drilling seasons, as well as for selected existing test holes and wells in which the bedrock was reached.

Precipitation data are presented for the eight National Weather Service stations within and near the study area. Water-level data for the new observation wells are presented, as well as water-level data collected since 1996 for observation wells included in the first data report. Water-quality data are presented for the samples collected from observation wells installed between 1997 and 1999. Updated peak-flow information is provided for all gaging stations included in the first data report that are still active. Summary statistics are presented for the two active streamflow-gaging stations that had less than a 10 -year period of record when the first data report was prepared. Additional lake-level data compiled by the South Dakota Department of Environment and Natural Resources are presented to supplement the lakelevel data included in the first data report. Miscellaneous streamflow measurements taken at the outlets of two lakes in the study area also are presented.

\section{INTRODUCTION}

The Lake Traverse Reservation/Roberts County Water Resources Investigation was initiated in 1994 as a cooperative effort between the U.S. Geological Survey (USGS), the South Dakota Geological Survey (SDGS), the Sisseton-Wahpeton Sioux Tribe, and Roberts County. 
Prior to the water-resources investigation, a reconnaissance drilling program was accomplished from 1988 through 1990 by the USGS and the Sisseton-Wahpeton Sioux Tribe. During the reconnaissance drilling, 19 test holes were drilled, and 9 observation wells were installed. The reconnaissance drilling program identified the need for a more thorough knowledge of the area in order to map the thickness and extent of sand and gravel deposits and delineate aquifers. The present investigation was initiated to improve the knowledge of the area.

After the water-resources investigation was started in 1994, a comprehensive drilling program was initiated in cooperation with SDGS. Between 1994 and 1996, 41 test holes were drilled, and 19 observation wells were installed. Between 1997 and 1999, 59 additional test holes were drilled and 23 more observation wells were installed. Geologic logs for the test holes and wells completed during the reconnaissance and comprehensive drilling programs prior to 1997 are presented in Carter and Thompson (1999). Geologic logs for the test holes and wells completed during 1997-99 are presented in this report.

In addition to the observation wells installed during the reconnaissance and comprehensive drilling programs, 57 observation wells owned by the South Dakota Department of Environment and Natural Resources (SDDENR) and 18 observation wells owned by the North Dakota State Water Commission (NDSWC) were incorporated into the study for the purpose of water-level monitoring. The USGS began a water-level monitoring program in December 1996, and data collected from this effort are included in this data report.

Precipitation records from 1997 through 1999 at eight sites are compiled, as well as lake levels collected by SDDENR from 1997 through 1999 at 20 sites. In the previous data report (Carter and Thompson, 1999), two streamflow-gaging stations had only nine years of record. Both of these stations now have 12 years of record, and updated summary statistics are presented in this report for these two stations. Water-quality samples from 19 observation wells have been collected from 1997 through 1999. These samples were analyzed for field measurements, common and trace elements, nutrients, and radiometrics. The shallow wells also were tested for common pesticides and total coliform bacteria.
Specifically, this report contains (1) precipitation records for 1997-99 for eight sites; (2) geologic logs for 59 test holes and 23 observation wells completed for this study and selected other test holes and observation wells within the former boundary of the Lake Traverse Reservation that reached bedrock; (3) hydrographs for 163 wells; (4) water-quality data for 19 observation wells; (5) updated peak-flow frequency estimates for all active stations and updated summary statistics for the two active streamflow-gaging stations that had less than a 10 -year period of record in Carter and Thompson (1999); (6) water-level elevation data for 1997-99 for 20 lakes; and (7) miscellaneous streamflow measurements at lake inlets or outlets.

\section{Description of Study Area}

Most of the Lake Traverse Reservation/Roberts County study area is located in northeastern South Dakota; a small part of the former boundary of the Lake Traverse Reservation extends into southeast North Dakota (fig. 1). Roberts County has an area of 1,135 square miles. The former boundary of the Lake Traverse Reservation included 1,595 square miles in South Dakota and North Dakota.

The study area, which is included in the Central Lowlands physiographic province, occupies the Coteau des Prairies and the Minnesota River-Red River lowland physiographic divisions (Flint, 1955). Present-day topography was formed by the advancement and recession of glaciers during the Wisconsin age of the Pleistocene epic (Koch, 1975). The Coteau des Prairies, which is drained by the Big Sioux River, the James River, and the Minnesota River, is a massive plateau with rugged morainal topography (Koch, 1975). Much of the upper coteau consists of noncontributing, internal drainage except in times of high precipitation and low evaporation. The Minnesota RiverRed River lowland is a broad depression that consists primarily of ground moraine (till) and isolated outwash and lake deposits formed by glacial meltwaters (Koch, 1975). This southern area of the lowland is drained by the Minnesota River and subsequently to the Mississippi River (Gulf of Mexico), and the northern area is drained by the Bois de Sioux River and subsequently to the Red River (Hudson Bay). 


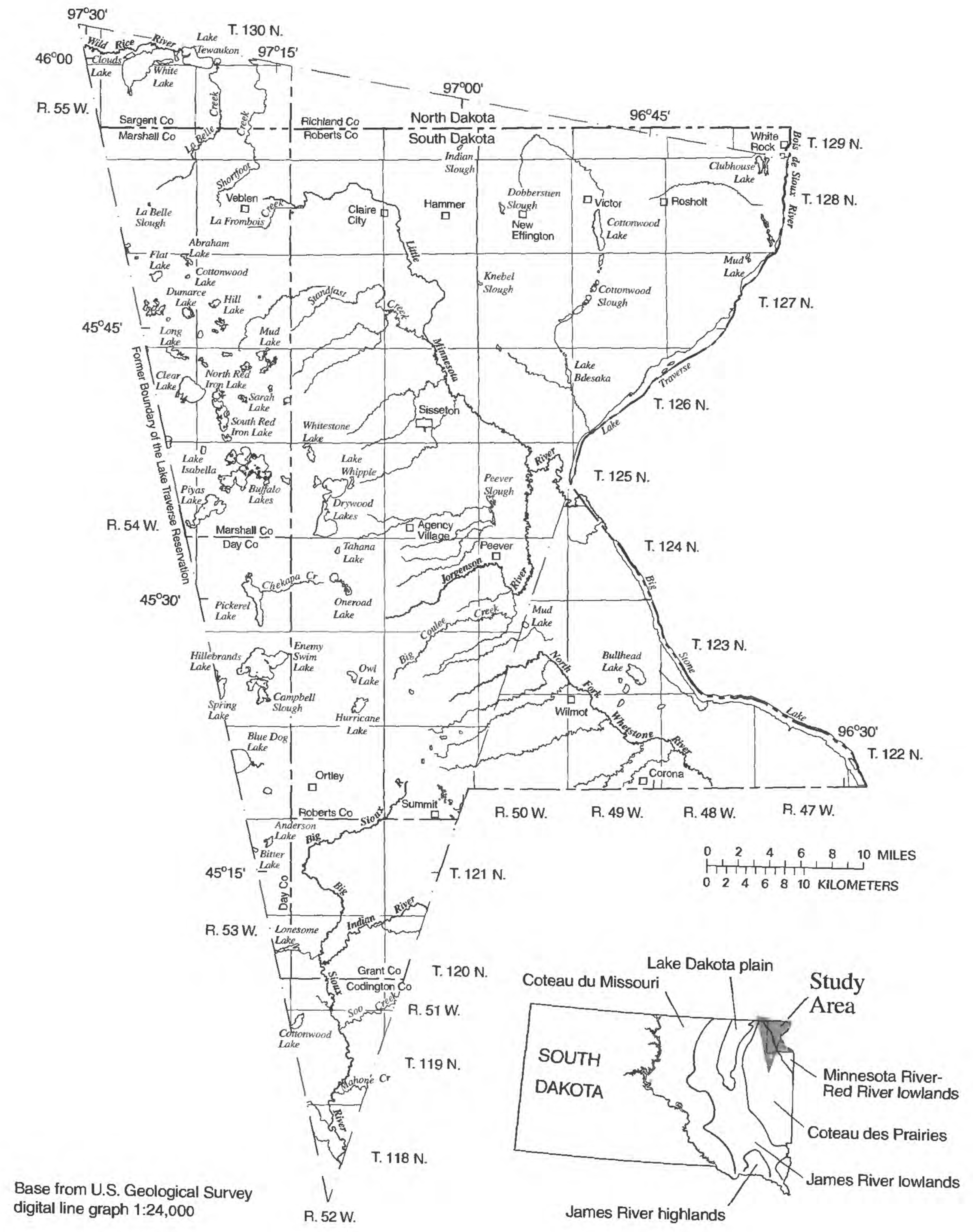

Figure 1. Location of study area. 
Glacial deposits (till and outwash) cover the majority of land surface. The drift is underlain by the Cretaceous-age Pierre Shale, Niobrara Formation, and Carlile Shale and by Precambrian-age granite (Flint, 1955). Isolated surface exposures of the Cretaceousage formations are present in the study area, although they are relatively uncommon. Quaternary-age alluvial deposits also are present in the study area.

\section{Acknowledgments}

Many people have assisted with the development and implementation of the water-resources investigation. The SDGS drilled the test holes and installed the observation wells for the project. Dennis Tomhave (SDGS) provided insight and technical guidance throughout the study and assisted extensively during the interpretation of the geologic logs. The water-level measurements for 20 lakes and many observation wells were provided by SDDENR, Water Rights Program.

\section{PRECIPITATION DATA}

Precipitation data are collected by the National Weather Service at eight sites in and near the study area (fig. 2). Monthly precipitation data were compiled from annual summaries accessed on the internet, January 22, 2000, at http://www.ncdc.noaa.gov/pub/ data/coop-precip/south-dakota.txt. Precipitation data for 1997-99 are presented for Big Stone City, S. Dak. (table 1), Sisseton, S. Dak. (table 2), Summit, S. Dak. (table 3), Victor, S. Dak. (table 4), Watertown, S. Dak. (table 5), Waubay National Wildlife Refuge, S. Dak. (table 6), Wilmot, S. Dak. (table 7), and Forman, N. Dak. (table 8).

\section{WELL AND TEST-HOLE DATA}

The Lake Traverse Reservation/Roberts County Water Resources Investigation included two drilling programs: a reconnaissance program from 1988 through 1990 and an ongoing comprehensive program that began in 1994. During the reconnaissance drilling program, 19 test holes were drilled, and 9 observation wells were installed. During the comprehensive drilling program, 41 test holes were drilled, and 19 observation wells were installed between 1994 and 1996. Another 59 test holes and 23 observation wells were installed between 1997 and 1999, and these geologic logs are presented in Section A of the
Supplemental Information section at the end of this report. All wells and test holes were drilled in Roberts County during both the reconnaissance and comprehensive drilling programs.

Three methods of station identification for the wells and test holes are used in this report. The first method is the station identification number, which is based on the international system of latitude and longitude. The number contains 15 digits. The first six digits denote the degrees, minutes, and seconds of latitude north of the equator. The next seven digits denote the degrees, minutes, and second of longitude west of the prime (Greenwich) meridian. The last two digits are sequential numbers for sites within the same latitude and longitude. The latitude and longitude were determined using Global Positioning Satellite (GPS) equipment at the time of drilling and rounded to the nearest second.

The second identification method used is the local number, which is based on the Federal landsurvey system of eastern South Dakota. The local number consists of the township number followed by "N," for north, the range number followed by "W," for west, and the section number, followed by a maximum of four uppercase letters that indicate, respectively, the $160-, 40-, 10-$, and 2.5-acre tract in which the well or test hole is located. These letters are assigned in a counterclockwise direction beginning with " $\mathrm{A}$ " in the northeast quarter. A sequential number following the last letter is used to distinguish between wells in the same 2.5-acre tract. An "R" at the end of the local number distinguishes sites that are located within the former boundary of the Lake Traverse Reservation. Thus, well $125 \mathrm{~N} 50 \mathrm{~W} 12 \mathrm{ABCC} \mathrm{R}$ is located within the former boundary of the Lake Traverse Reservation in the $S^{1} 1 / 4$ of the $S^{1} 1 / 4$ of the NW1/4 of the NE $1 / 4$ of section 12 in township 125 north and range 50 west.

The third identification method is the "other identifier," which was assigned by the SDGS. This name denotes the drilling rig number, the year, and sequence in which the well or test hole was drilled.

All observation wells have 2 -inch diameter, flush-threaded PVC casing and screen. The wells were gravel packed with washed sand (0.65- to 0.75-millimeter diameter), and were sealed with bentonite to approximately 20 feet below land surface and with neat cement from the top of the bentonite to the land surface. Well development was accomplished using compressed air. and locking metal protectors were installed over the top of each well. 


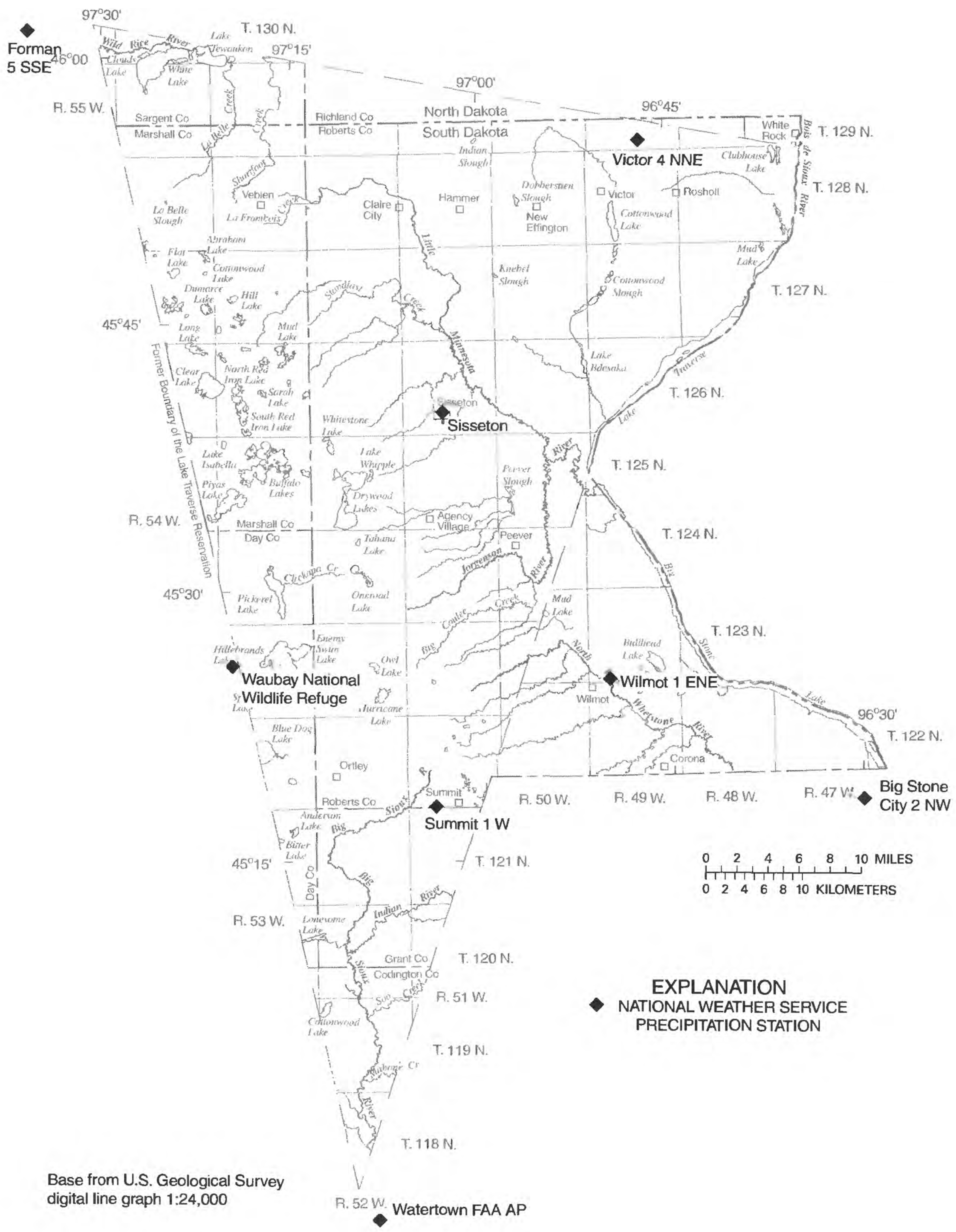

Figure 2. Location of precipitation stations within and near study area. 
Table 1. Precipitation, in inches, for the Big Stone City, S. Dak., National Weather Service station, 1997-99

\begin{tabular}{rrrrrrrrrrrrrr}
\hline Year & Jan & Feb & Mar & Apr & May & Jun & Jul & Aug & Sep & Oct & Nov & Dec & Annual \\
\hline 1997 & 1.71 & 0.43 & 1.55 & 3.07 & 1.73 & 1.16 & 3.84 & 4.89 & 1.20 & 2.13 & 0.4 & 0.21 & 22.32 \\
1998 & .96 & 1.91 & 1.14 & 2.87 & 1.99 & 4.02 & 2.40 & 3.69 & .15 & 5.59 & 1.64 & .12 & 26.48 \\
1999 & .94 & .12 & .77 & 1.45 & 3.06 & 3.63 & 5.52 & 1.92 & 3.66 & .25 & .08 & .15 & 21.55 \\
\hline
\end{tabular}

Table 2. Precipitation, in inches, for the Sisseton, S. Dak., National Weather Service station, 1997-99

\begin{tabular}{cccccccccccccc}
\hline Year & Jan & Feb & Mar & Apr & May & Jun & Jul & Aug & Sep & Oct & Nov & Dec & Annual \\
\hline 1997 & 3.13 & 0.52 & 0.91 & 2.60 & 1.55 & 0.91 & 4.41 & 2.08 & 0.41 & 2.01 & 0.75 & 0.42 & 19.70 \\
1998 & 1.30 & 2.03 & 2.06 & 2.91 & 5.51 & 3.59 & 1.88 & 2.77 & 0.00 & 7.95 & 1.81 & .07 & 31.88 \\
1999 & 1.20 & .12 & 1.28 & 1.45 & 2.41 & 4.54 & 2.20 & 4.07 & 3.65 & .57 & .00 & .20 & 21.69 \\
\hline
\end{tabular}

Table 3. Precipitation, in inches, for the Summit, S. Dak., National Weather Service station, 1997-99 [--, no data; --, not computed]

\begin{tabular}{cccccccccccccc}
\hline Year & Jan & Feb & Mar & Apr & May & Jun & Jul & Aug & Sep & Oct & Nov & Dec & Annual \\
\hline 1997 & -- & 0.61 & 1.31 & 2.23 & 1.22 & 0.59 & 5.22 & 3.99 & 1.64 & 2.88 & 0.46 & 0.51 & -- \\
1998 & 0.79 & 1.64 & 1.9 & 1.4 & 4.31 & 2.66 & 1.35 & 4.76 & .05 & 6.8 & 1.21 & .17 & 27.04 \\
1999 & 1.41 & .29 & 1.19 & 2.05 & 3.46 & 4.69 & 3.05 & 3.77 & 2.75 & .43 & .06 & .16 & 23.31 \\
\hline
\end{tabular}

Table 4. Precipitation, in inches, for the Victor, S. Dak., National Weather Service station, 1997-99

\begin{tabular}{cccccccccccccc}
\hline Year & Jan & Fab & Mar & Apr & May & Jun & Jul & Aug & Sep & Oct & Nov & Dec & Annual \\
\hline 1997 & 3.18 & 0.52 & 1.25 & 2.86 & 1.87 & 1.83 & 2.49 & 3.43 & 1.75 & 2.98 & 0.84 & 0.32 & 23.32 \\
1998 & .80 & 1.58 & 1.22 & 2.71 & 5.15 & 5.88 & 1.98 & 1.32 & .33 & 5.74 & 1.54 & .27 & 28.52 \\
1999 & 1.13 & .17 & 1.27 & 1.88 & 3.08 & 5.32 & 3.29 & 3.42 & 2.78 & .81 & .00 & .43 & 23.58 \\
\hline
\end{tabular}

Table 5. Precipitation, in inches, for the Watertown, S. Dak., National Weather Service station, 1997-99 $[--$, no data; --, not computed]

\begin{tabular}{ccccccccccccccc}
\hline Year & Jan & Feb & Mar & Apr & May & Jun & Jul & Aug & Sep & Oct & Nov & Dec & Annual \\
\hline 1997 & 0.87 & -1 & 1.02 & -- & 2.11 & 1.30 & 3.56 & 2.02 & 0.99 & 2.53 & 0.25 & 0.15 & -- \\
1998 & .71 & 0.76 & .40 & 2.17 & 2.91 & 1.62 & 2.69 & 4.06 & .13 & 7.06 & .51 & .30 & 23.32 \\
1999 & -- & .23 & .57 & 1.55 & 2.41 & 5.44 & 1.65 & 1.39 & 2.21 & .27 & .08 & .05 & -- \\
\hline
\end{tabular}


Table 6. Precipitation, in inches, for the Waubay National Wildlife Refuge, S. Dak., National Weather Service station, 1997-99

\begin{tabular}{cccccccccccccc}
\hline Year & Jan & Feb & Mar & \multicolumn{1}{c}{ Apr } & May & Jun & Jul & Aug & Sep & Oct & Nov & Dec & Annual \\
\hline 1997 & 1.60 & 0.31 & 0.65 & 1.81 & 1.49 & 1.68 & 5.71 & 2.89 & 0.84 & 2.35 & 0.59 & 0.49 & 20.41 \\
1998 & 1.24 & 1.10 & 1.10 & 4.16 & 5.42 & 3.00 & 1.97 & 2.66 & .22 & 7.78 & .80 & .14 & 29.59 \\
1999 & .69 & .07 & .74 & 1.35 & 2.98 & 7.75 & 2.37 & 3.19 & 3.64 & .54 & .00 & .17 & 23.49 \\
\hline
\end{tabular}

Table 7. Precipitation, in inches, for the Wilmot, S. Dak., National Weather Service station, 1997-99

\begin{tabular}{cccccccccccccc}
\hline Year & Jan & Feb & Mar & \multicolumn{1}{c}{ Apr } & May & Jun & Jul & Aug & \multicolumn{1}{c}{ Sep } & Oct & Nov & Dec & Annual \\
\hline 1997 & 3.15 & 0.34 & 1.38 & 2.61 & 1.43 & 0.89 & 3.69 & 3.63 & 0.94 & 1.98 & 0.49 & 0.19 & 20.72 \\
1998 & 1.03 & 1.54 & 1.18 & 3.34 & 3.54 & 3.28 & 2.50 & 3.14 & .03 & 5.65 & 1.31 & .08 & 26.62 \\
1999 & 1.25 & .10 & 1.01 & 1.39 & 1.82 & 3.40 & 2.90 & 3.22 & 2.01 & .35 & .02 & .12 & 17.59 \\
\hline
\end{tabular}

Table 8. Precipitation, in inches, for the Forman, N. Dak., National Weather Service station, 1997-99

[---, no data; --, not computed $]$

\begin{tabular}{|c|c|c|c|c|c|c|c|c|c|c|c|c|c|}
\hline Year & Jan & Feb & Mar & Apr & May & Jun & Jul & Aug & Sep & Oct & Nov & Dec & Annual \\
\hline 1997 & 1.84 & --- & --- & 1.99 & 1.23 & 1.26 & 4.55 & 2.66 & 2.54 & 2.48 & 1.03 & -- & -- \\
\hline 1998 & .54 & 1.37 & 1.46 & 2.72 & 5.87 & 8.42 & 2.15 & 1.93 & .69 & -- & 2.03 & 0.18 & -- \\
\hline 1999 & 1.54 & .06 & 1.20 & .78 & 2.61 & 4.64 & 3.70 & 3.98 & 5.34 & .79 & --- & .10 & -. \\
\hline
\end{tabular}

Selected information is presented in table 9 for the 59 test holes and 23 observation wells installed between 1997 and 1999 for the study. The last water level measured in the observation wells in calendar year 1999 is included in the table. The altitudes of all the wells installed for this study were determined using GPS software, and have vertical accuracies of approximately 0.10 foot. The location of the observation wells installed for the study are shown in figure 3 , and the test holes are shown in figure 4 .

The geologic logs in the first part of Supplemental Information Section A are presented in the order listed in table 9. Information regarding special construction details, site characteristics, and electric geophysical $\log$ (electric $\log$ ) availability are indicated in the notes following the geologic logs. The electric logs may be obtained from the USGS office in Huron, South Dakota.
In addition to the wells specifically installed for this investigation, other observation wells have been incorporated into the study. Studies similar to the Lake Traverse Reservation/Roberts County Water Resources Investigation have been completed for Codington and Grant Counties (Hansen, 1990), Marshall County (Koch, 1975), and Day County (Leap, 1988) in South Dakota by the USGS and SDGS, and for Sargent County (Armstrong, 1979, 1982) and Richland County (Baker, 1966; Baker and Paulson, 1967) in North Dakota by the USGS and North Dakota Geological Survey. Comprehensive drilling programs were conducted as part of all of these investigations. The observation wells that were installed during these other county studies that are within or near the boundary of the former Lake Traverse Reservation are included in the second part of Supplemental Section A entitled "Selected Existing Test Holes and Observation Wells in Which the Bedrock was Reached." 


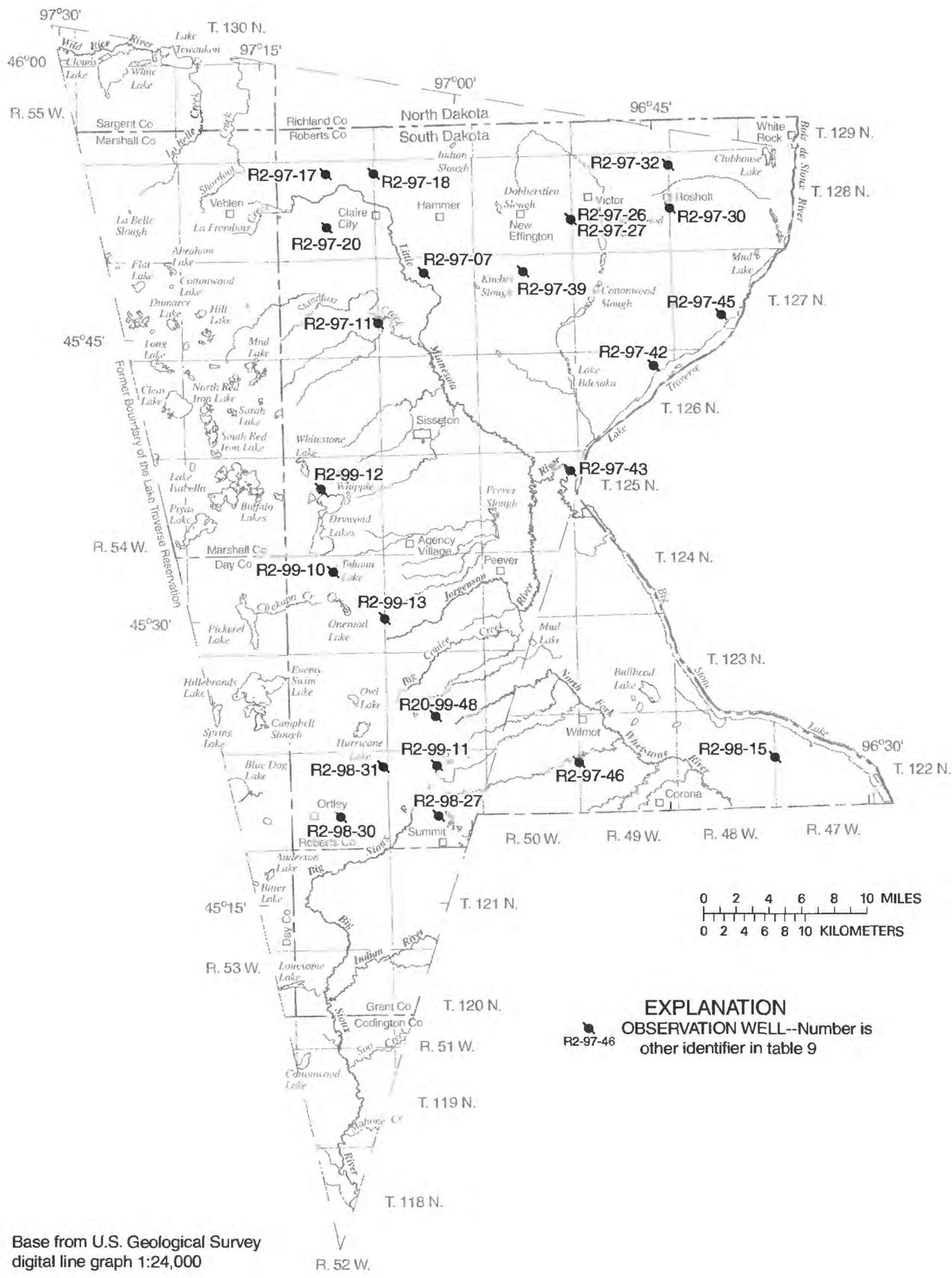

Figure 3. Location of observation wells installed between 1997 and 1999 for the study. 


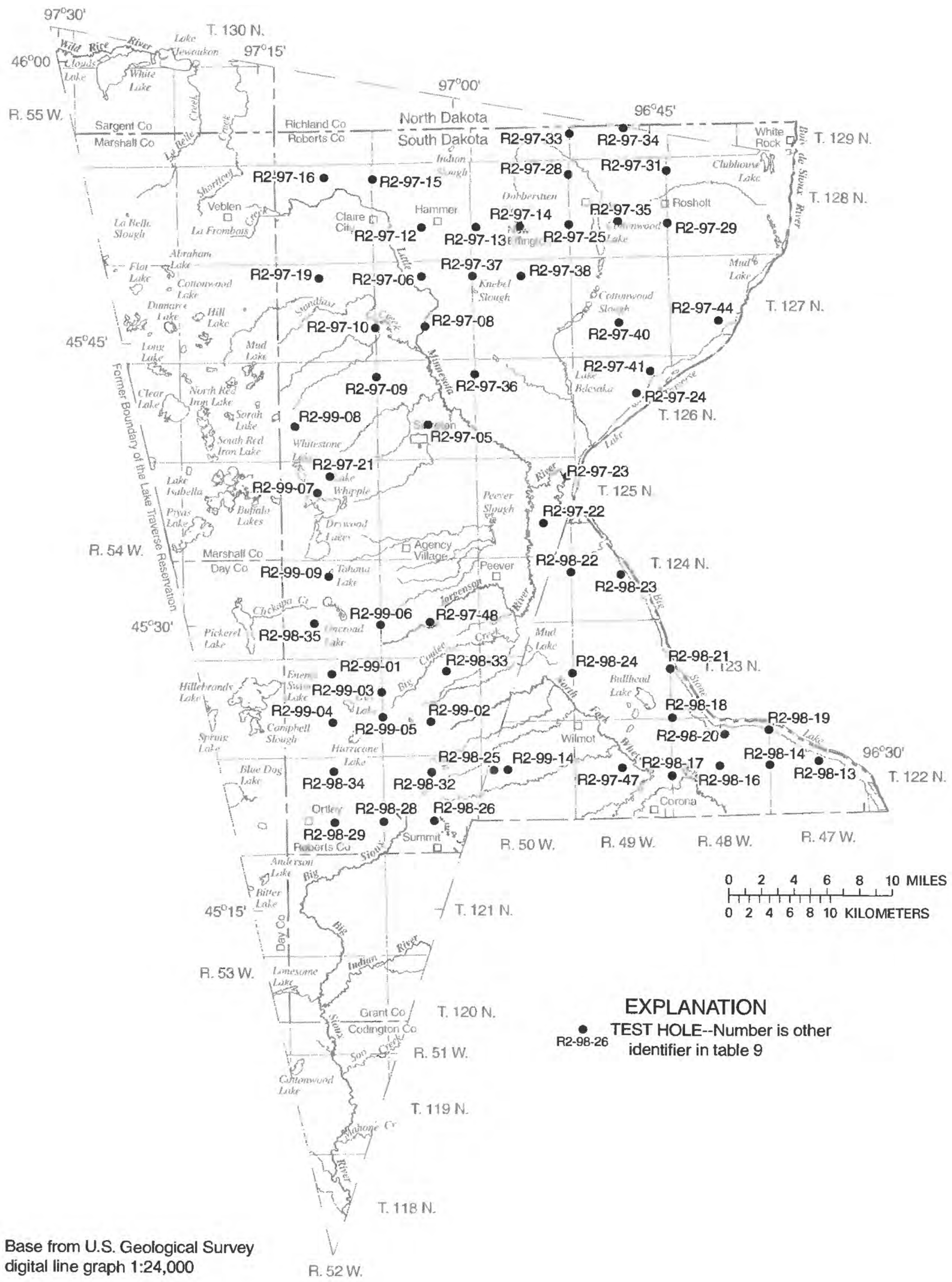

Figure 4. Location of test holes drilled between 1997 and 1999 for the study. 


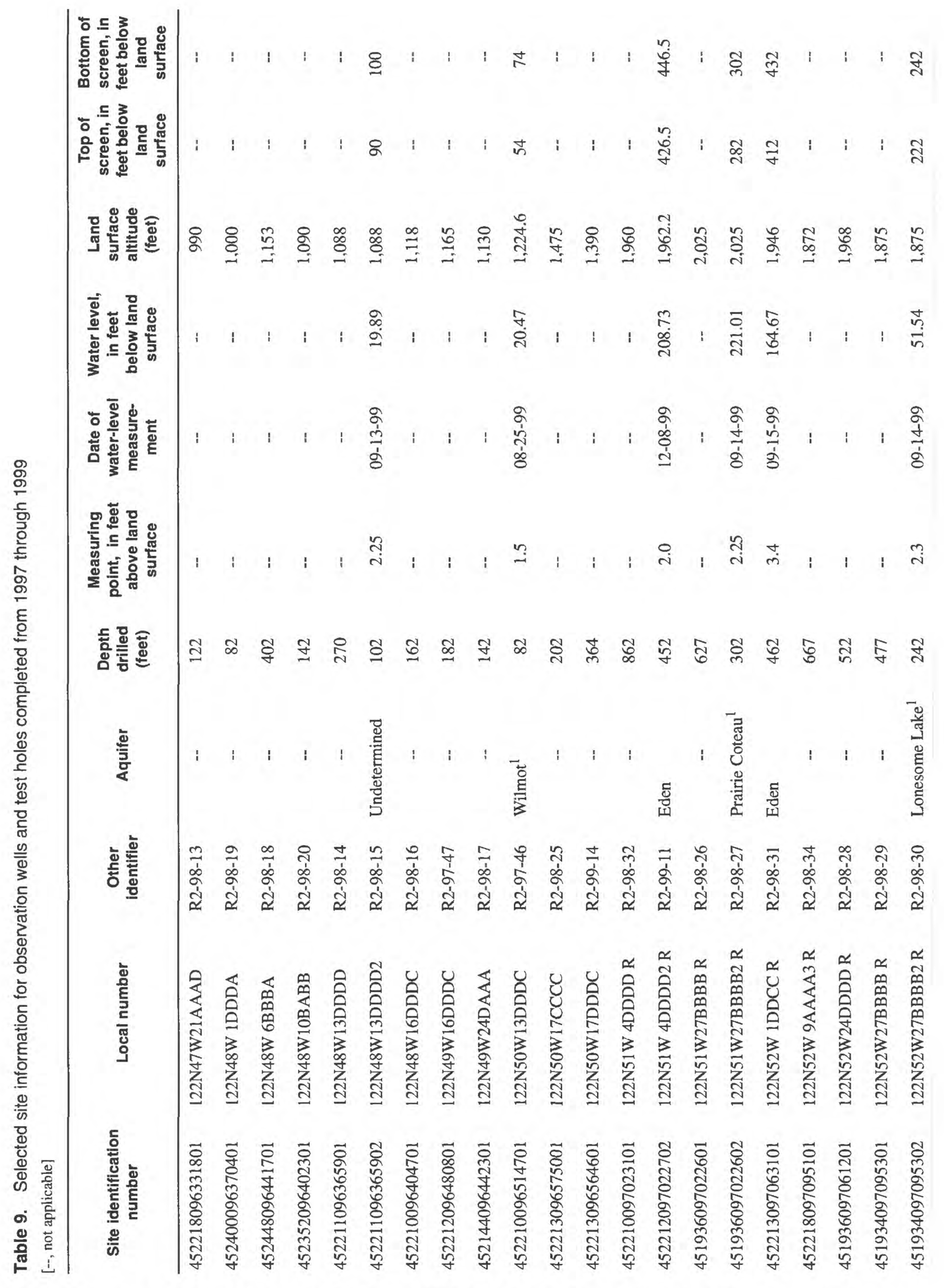




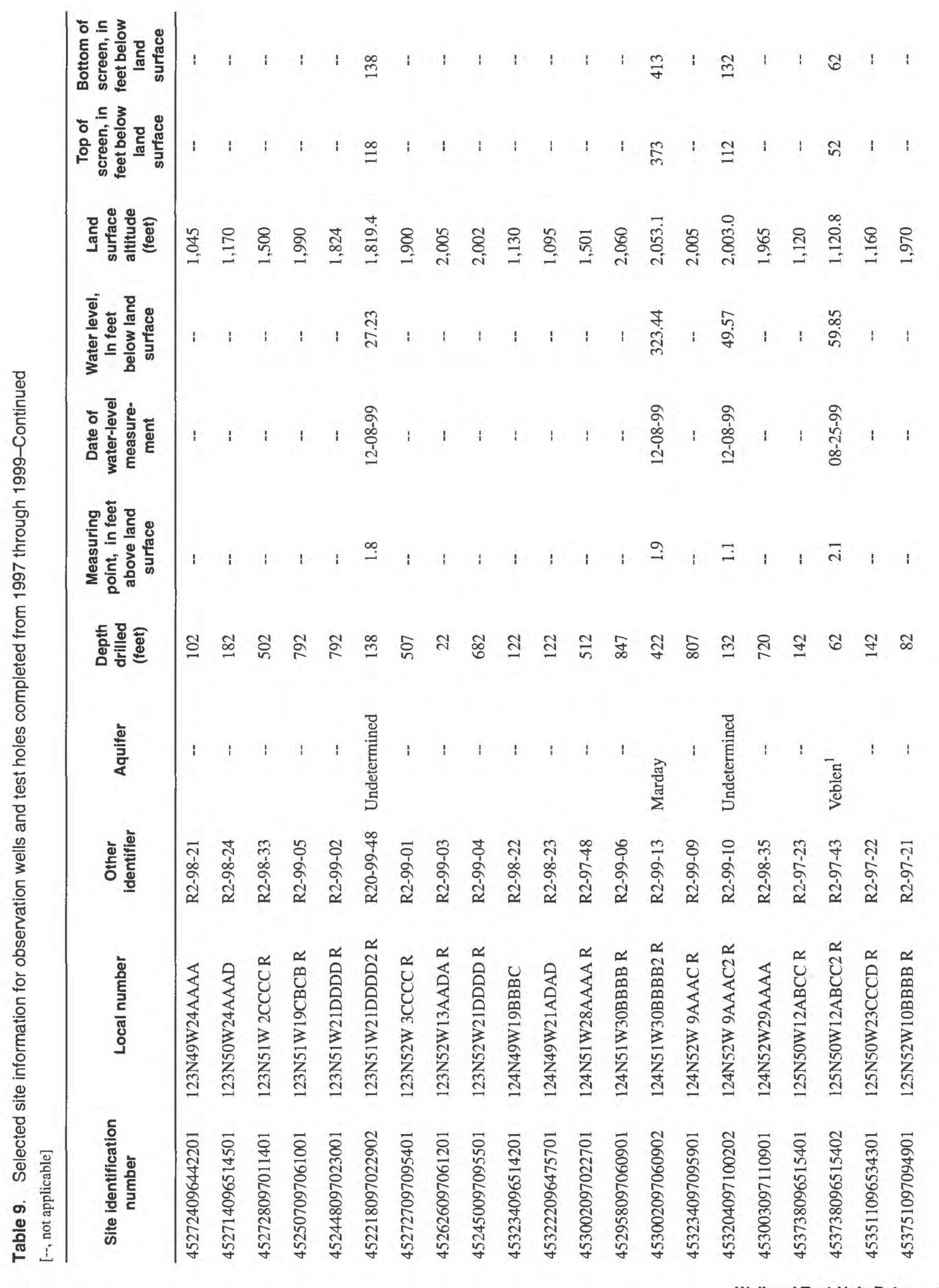




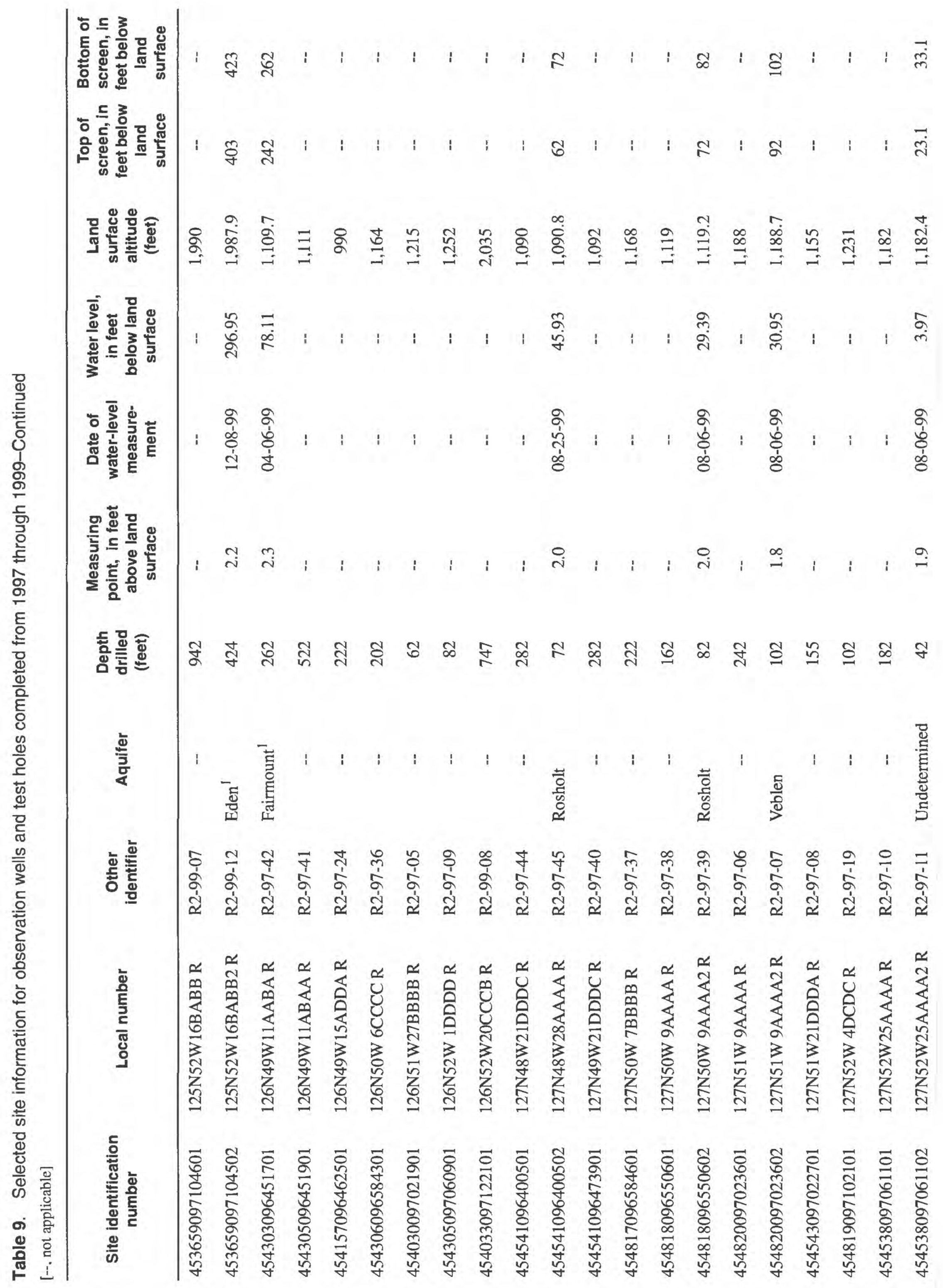

12 Hydrologic and Water-Quality Data, 1997 through 1999, Lake Traverse Reservation/Roberts County Investigation 


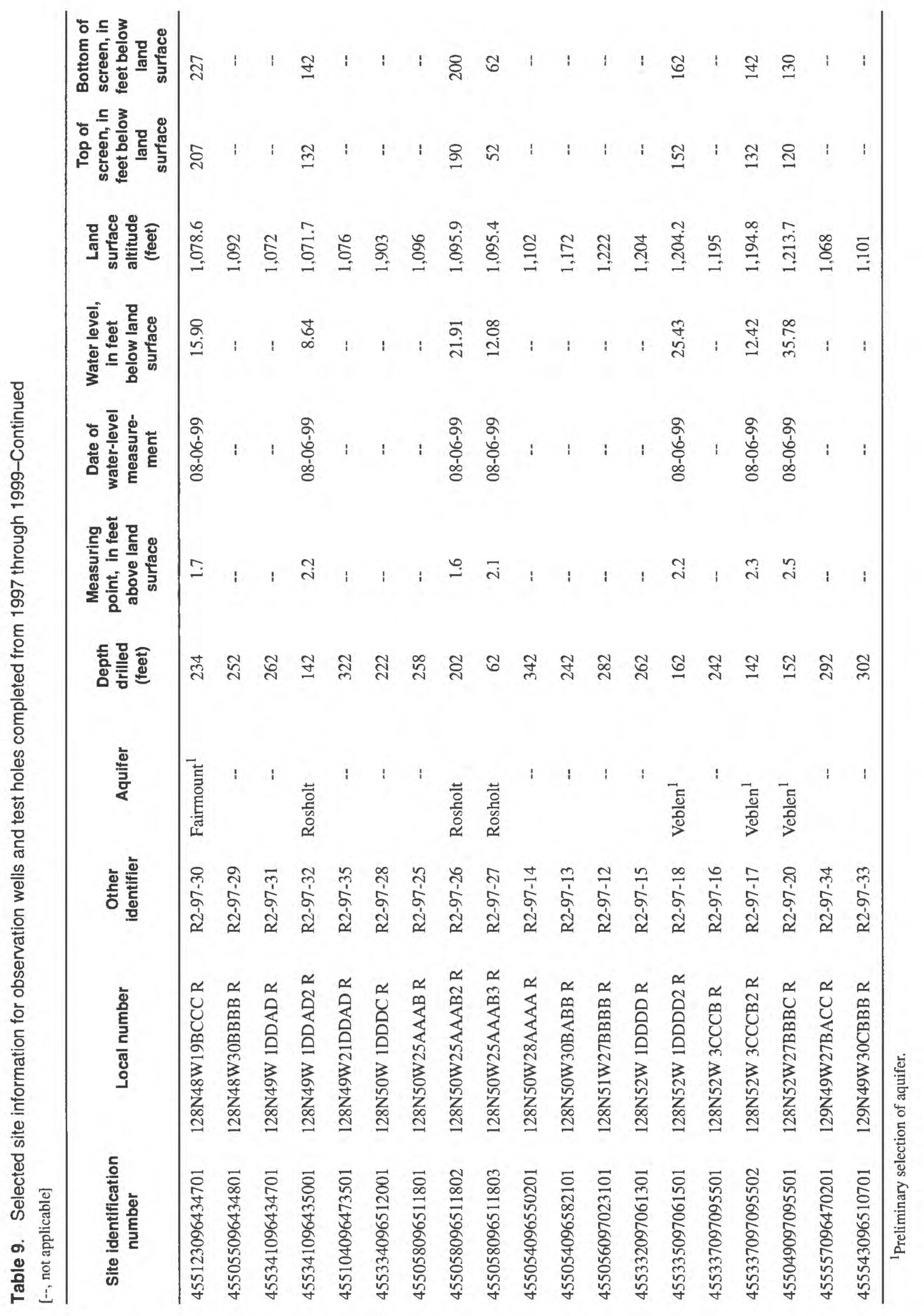


The SDDENR has installed observation wells throughout South Dakota and frequently measures the water levels in these wells. Fifty-seven SDDENR observation wells that are located within or near either Roberts County or the former boundary of the Lake Traverse Reservation also have been incorporated into the study. Selected information for the SDDENR observation wells is presented in table 10 , and the locations are shown in figure 5. Hydrographs through 1996 are presented in Carter and Thompson (1999), and hydrographs for 1997 through 1999 are presented in Section B of the Supplemental Information section. The altitudes of most of these wells that are located in Roberts County have been determined using GPS software.

The NDSWC has installed observation wells throughout North Dakota. Eighteen of these NDSWC observation wells are located within or near the former boundary of the Lake Traverse Reservation. Selected site information for the 18 NDSWC observation wells is presented in table 10, and the locations are shown in figure 5. Water-level data collected through 1996 are published in Carter and Thompson (1999). Water-level data collected after 1996 are presented Section B.

The wells in table 10 are identified by the 15-digit station identification number based on their latitudes and longitudes, the USGS local number based on the Federal land-survey system, both assigned by the same methods previously described. The other identifier (table 10) for the South Dakota wells was designated by the SDDENR or SDGS. The SDDENR identifier denotes the county (CD, Codington; GT, Grant; DA, Day; ML, Marshall; RB, Roberts) followed by the year in which it was drilled and a sequence number or letter. For example, RB-77D denotes the fourth observation well installed in 1977 in Roberts County. The SDGS identifier denotes a well name from a previous study, or the rig number, followed by the year drilled, followed by a sequence number. For example R2-97-28 denotes the 28th hole drilled by rig 2 in 1997. The other identifier (table 10) for the North Dakota wells was designated by the NDSWC.

\section{GROUND-WATER DATA}

The major aquifers in the study area consist of unconsolidated glacial drift and alluvial deposits. The major glacial aquifers that are present in the study area in South Dakota include the Big Sioux, Coteau Lakes, Fairmount, Prairie Coteau, Revillo, Veblen, Rosholt, Roslyn, Lonesome Lake, Eden, Marday, Altamont, and
Wilmot aquifers. The major glacial aquifer present in the study area in North Dakota is the Spiritwood aquifer. Because several different studies have been conducted in the area and the aquifers in these studies were named for a localized area, it is possible that some of these aquifers are interconnected but named differently based on location. This issue will be addressed during the interpretive part of this study.

\section{Hydrographs}

Water levels have been compiled for a waterlevel network consisting of 57 SDDENR observation wells, 38 existing SDGS and SDDENR observation wells from miscellaneous local studies, 18 NDSWC observation wells, and 50 observation wells completed for this study. Data collected from 1997 through 1999, and data from some wells that were measured for the first time in December 1996, are presented in this report.

Site descriptions and hydrographs for observation wells that were part of the water-level network are presented in Section B. The water levels are given in feet above $(+)$ or below $(-)$ land surface. A dashed line is shown on the hydrographs when the interval between water-level measurements exceeds 1 year. The data used to generate the hydrographs are available in the USGS Ground Water Site Inventory (GWSI) data base.

\section{Water-Quality Data}

Water-quality data from samples collected during 1997-99 are published in this report. All waterquality samples were collected by USGS personnel. Field measurements (and total coliform bacteria and immunoassay for pesticides, where applicable) were completed by South Dakota District or North Dakota District USGS staff. All other parameters were analyzed by the USGS National Water Quality Laboratory (NWQL) in Denver, Colorado. Analysis for bacteria and pesticides is generally only completed on wells with depths less than 100 feet. However, deeper wells were included when the 1997 observation wells were sampled. The locations of the sampling sites are shown in figure 6, and the analytical results are presented in table 11. Two additional wells (R2-96-56 and R2-96-58) are included in table 11 that do not appear in figure 6. These two wells were re-sampled for bacteria when the 1997 wells were sampled because they had not yet been sealed when sampled the first time. 


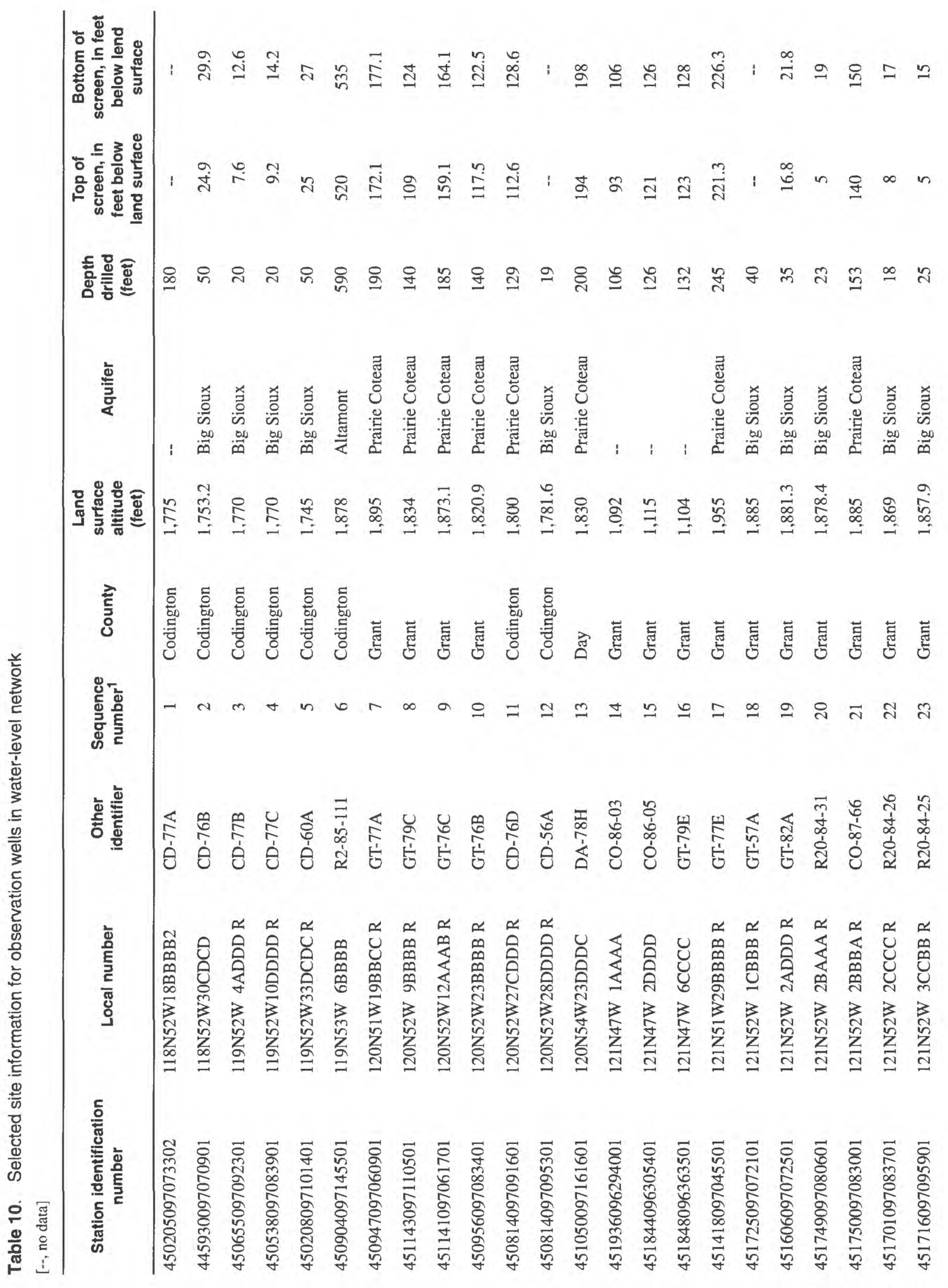




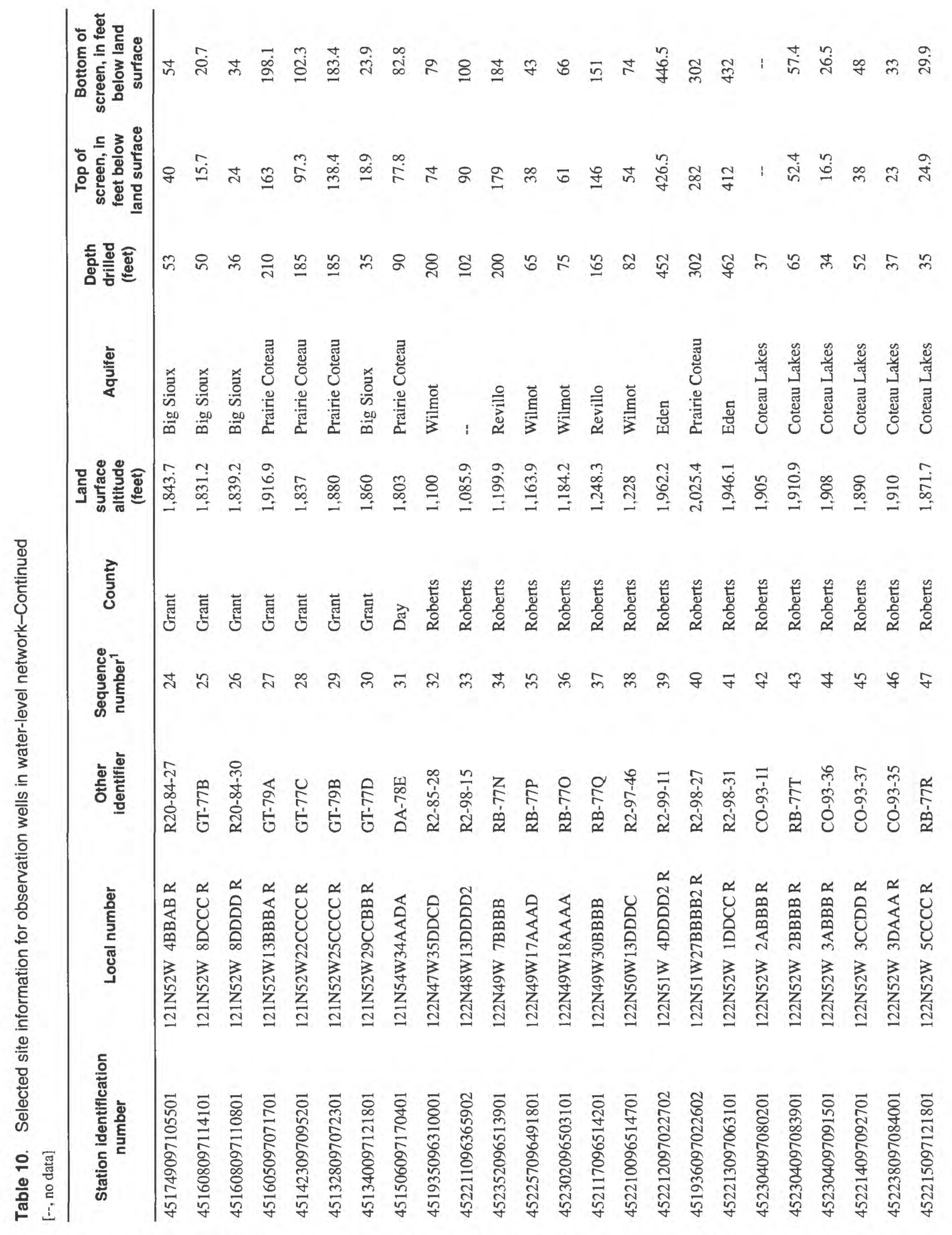

16 Hydrologic and Water-Quality Data, 1997 through 1999, Lake Traverse Reservation/Roberts County Investigation 


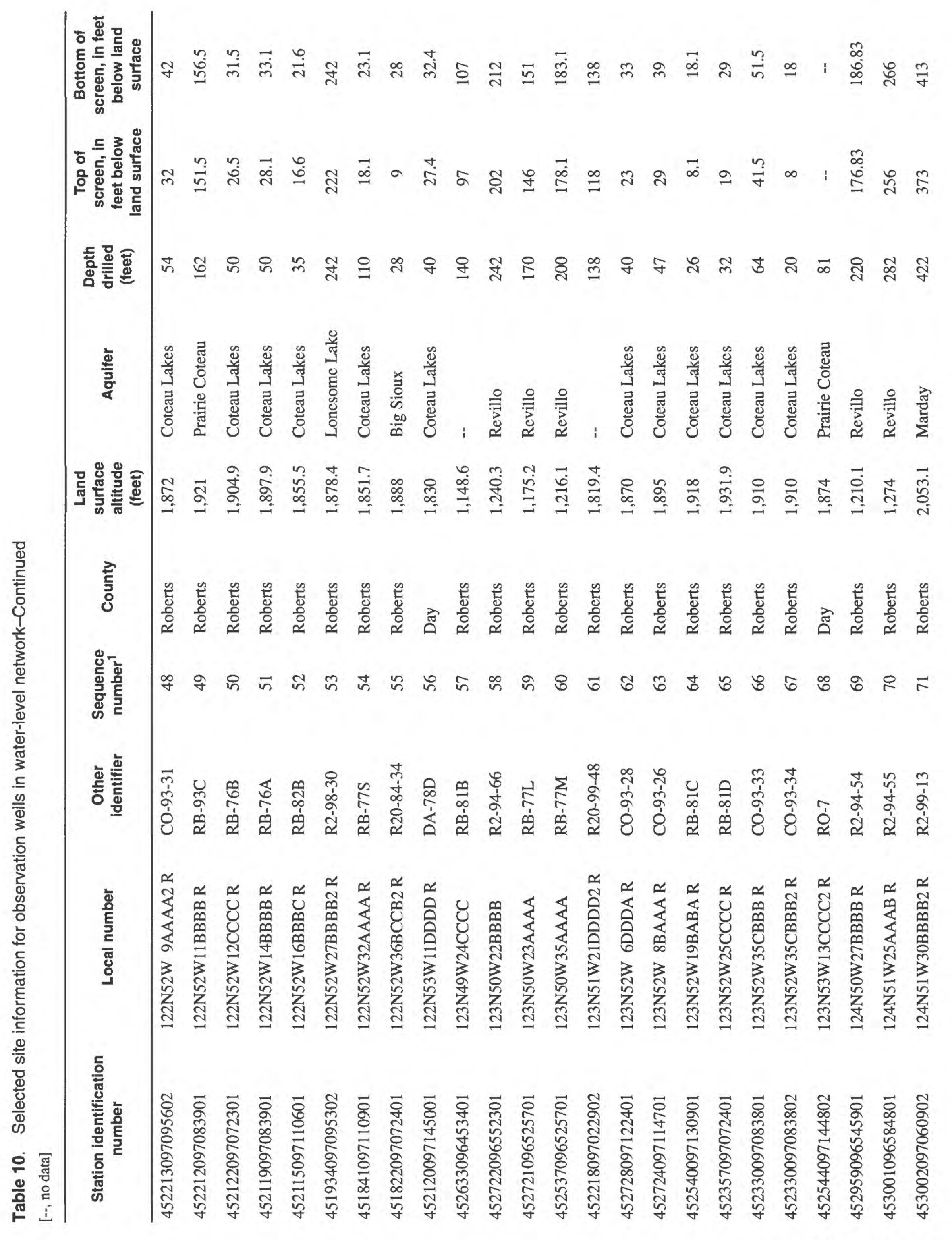

Ground-Water Data $\quad 17$ 


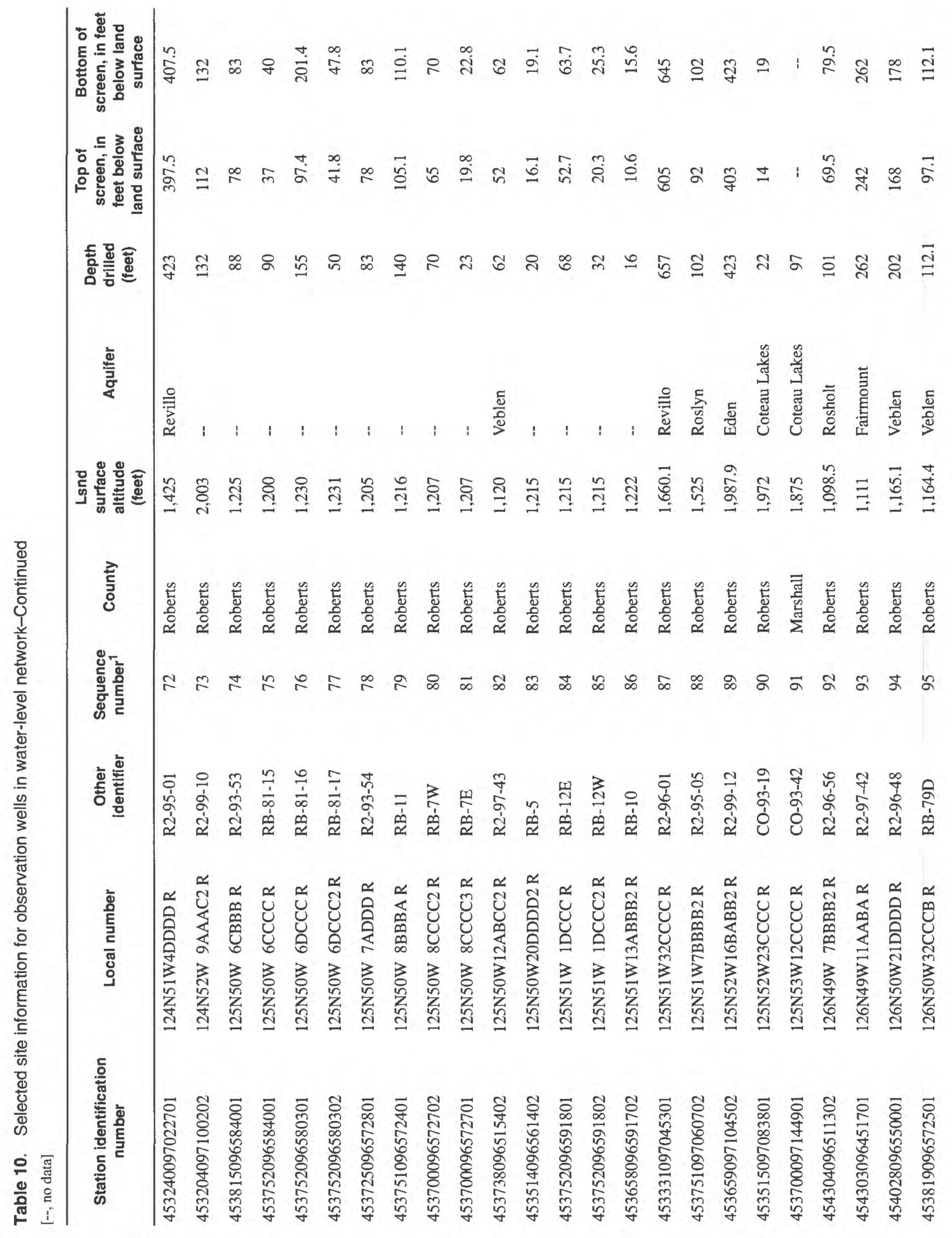

18 Hydrologic and Water-Quality Data, 1997 through 1999, Lake Traverse Reservation/Roberts County Investigation 


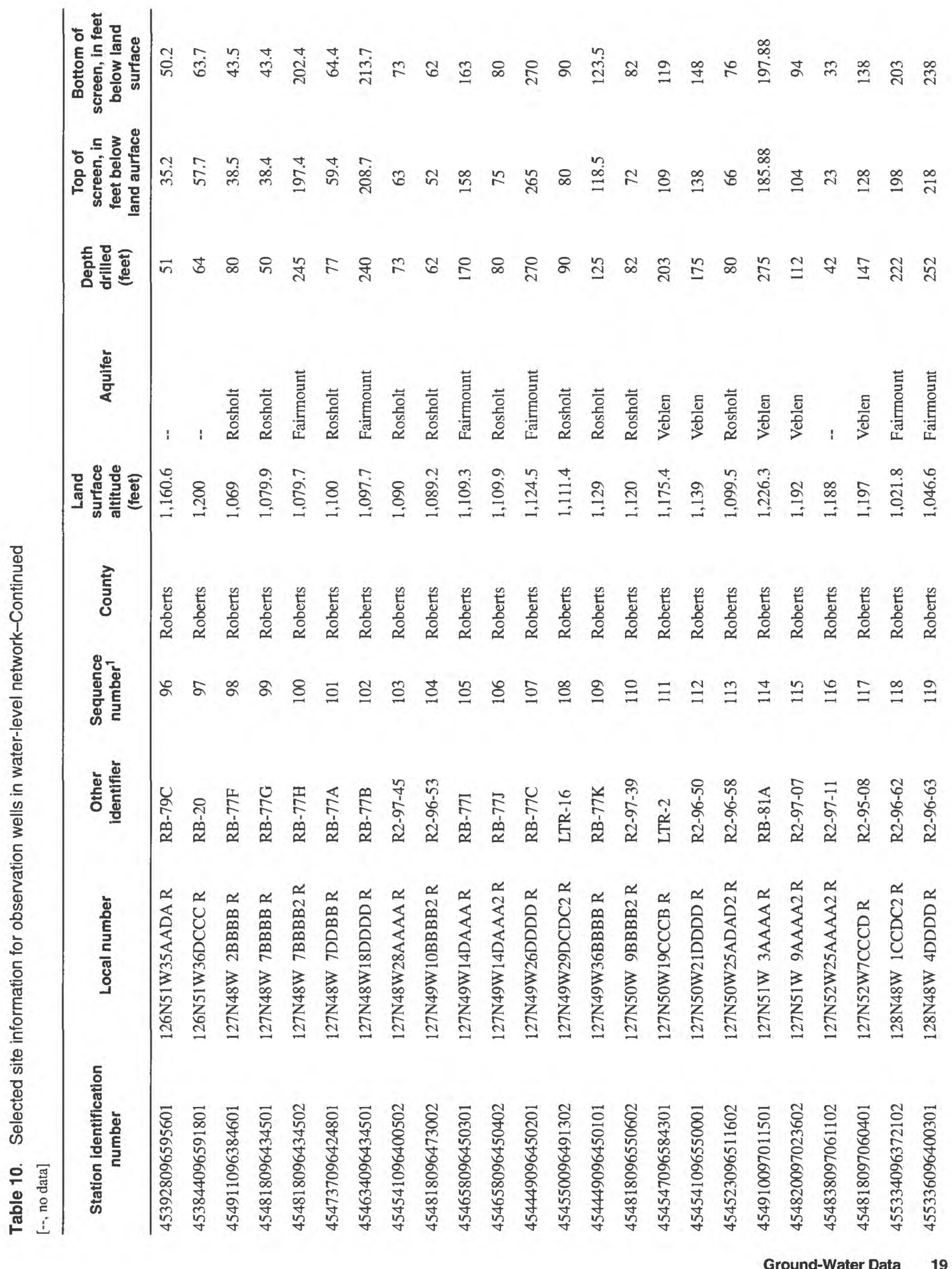




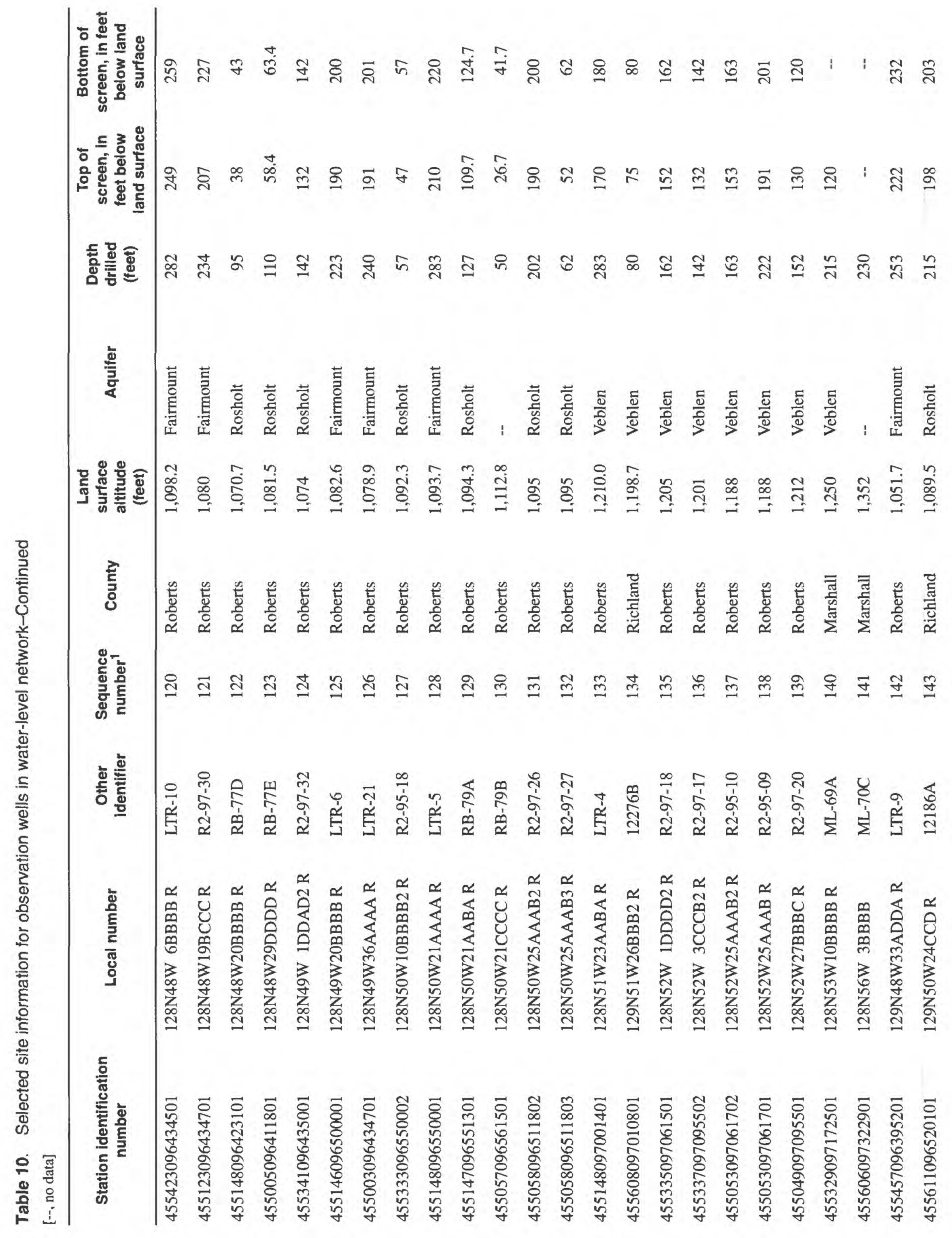

20 Hydrologic and Water-Quality Data, 1997 through 1999, Lake Traverse Reservation/Roberts County Investigation 







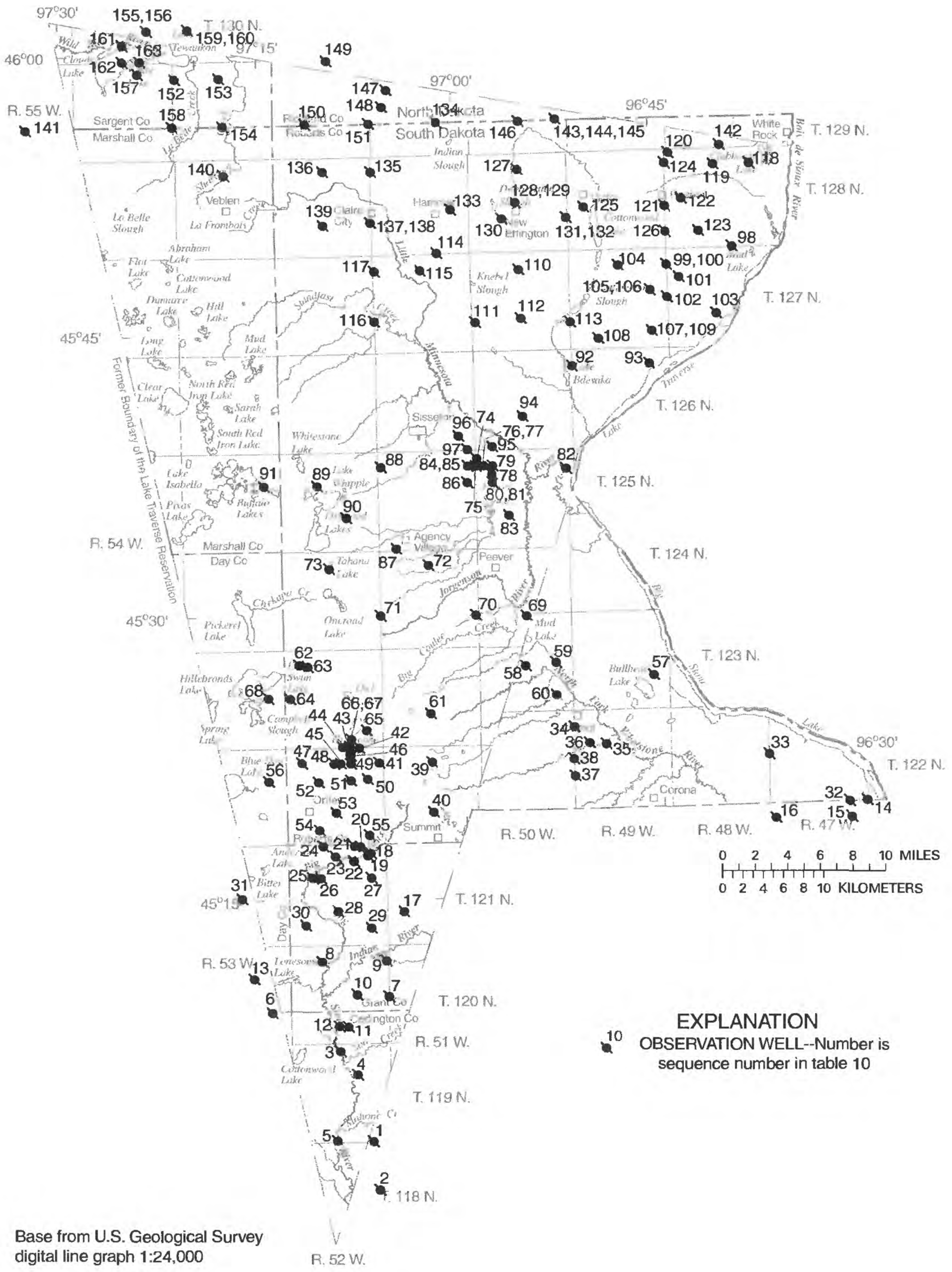

Figure 5. Location of selected observation wells in and near study area, which were part of the water-level monitoring program. 


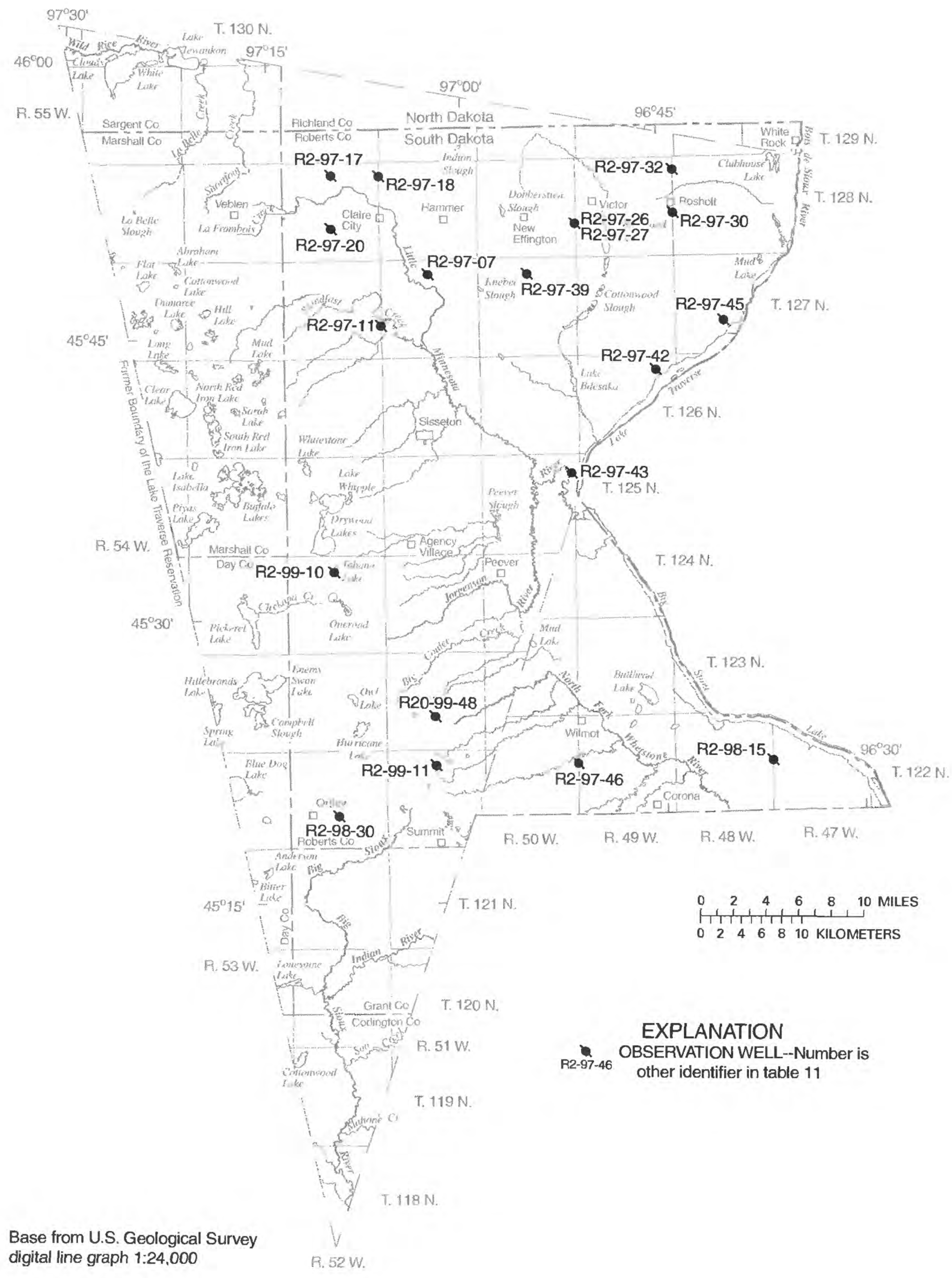

Figure 6. Location of ground-water sites sampled during 1997-99. 


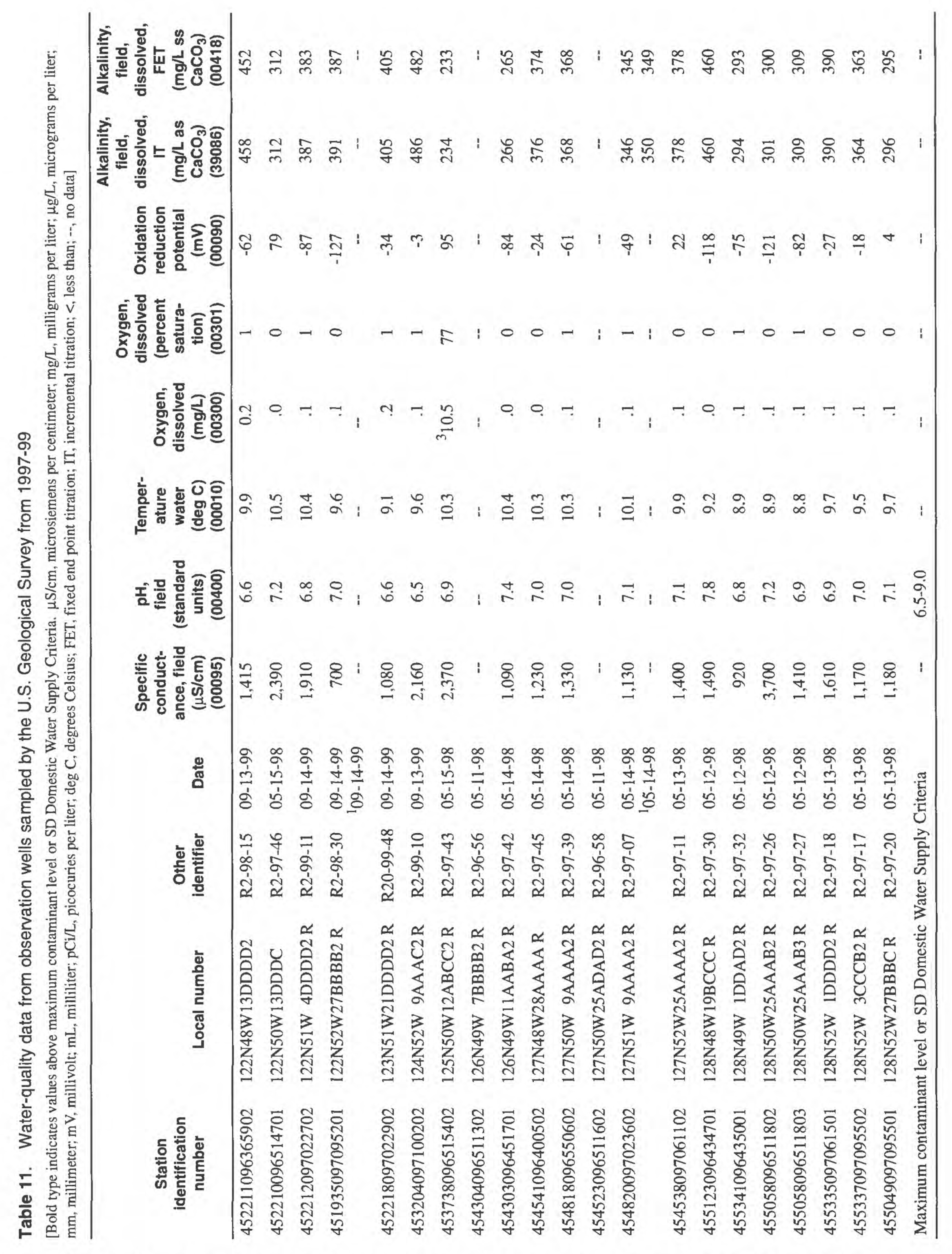




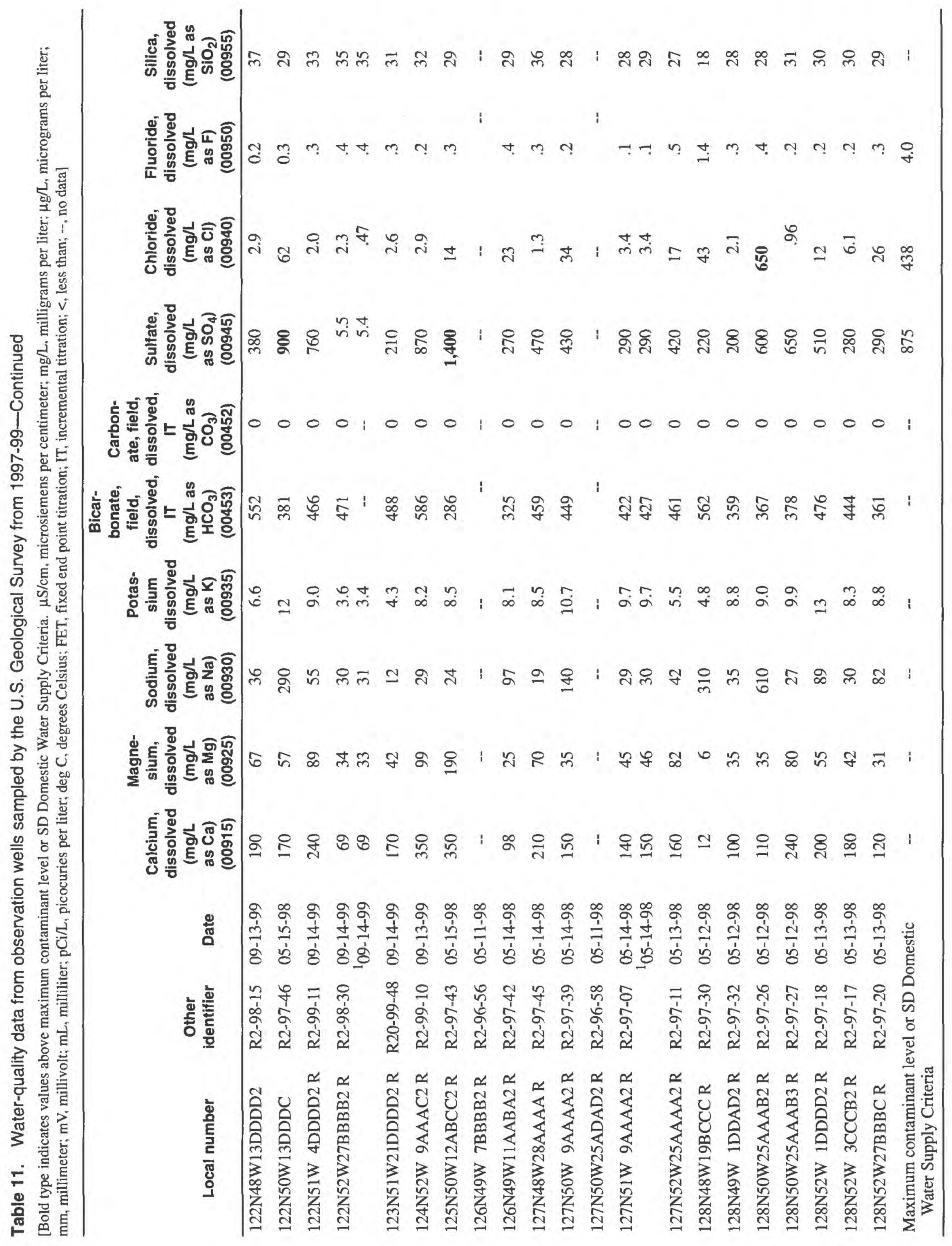




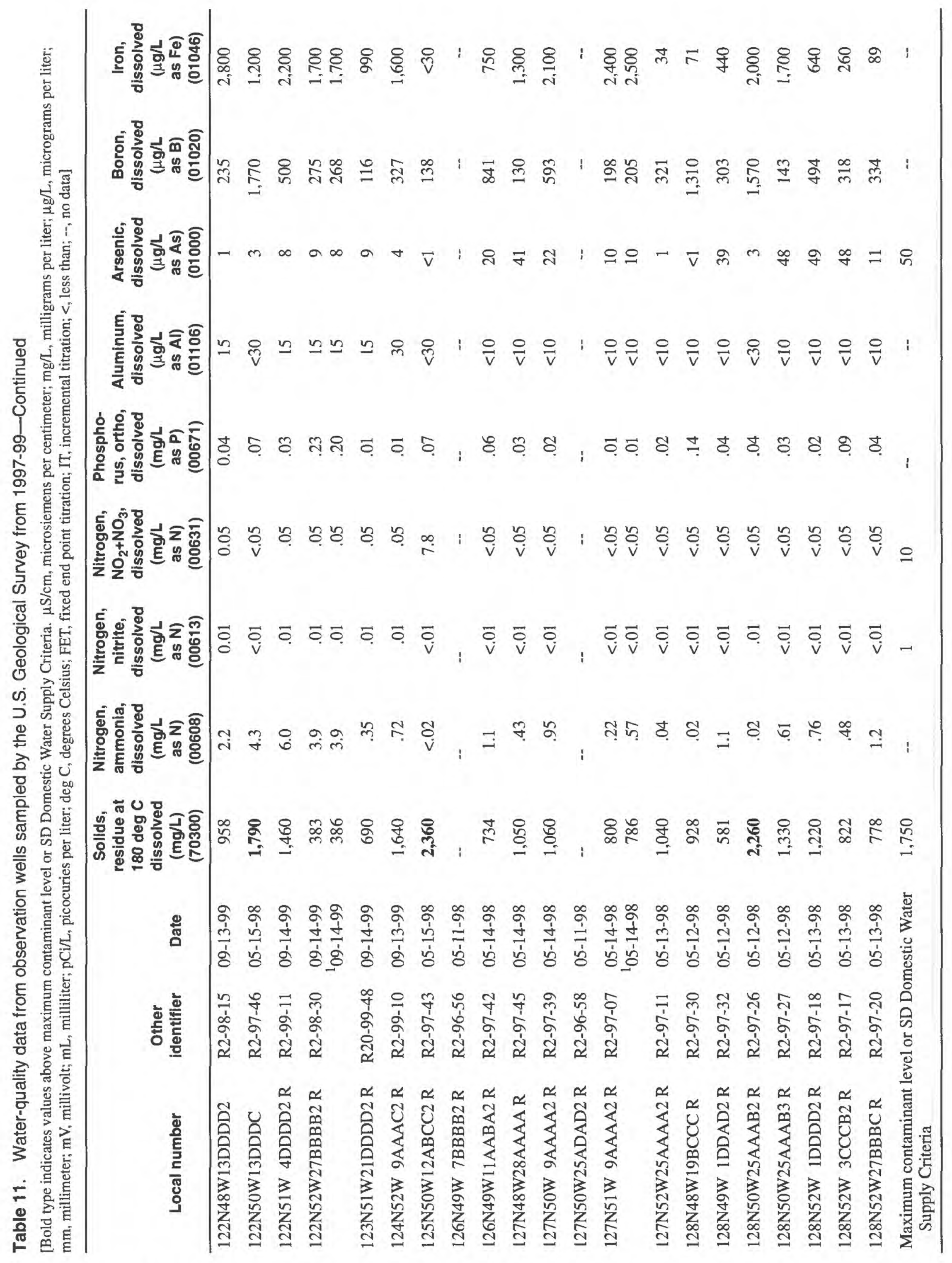




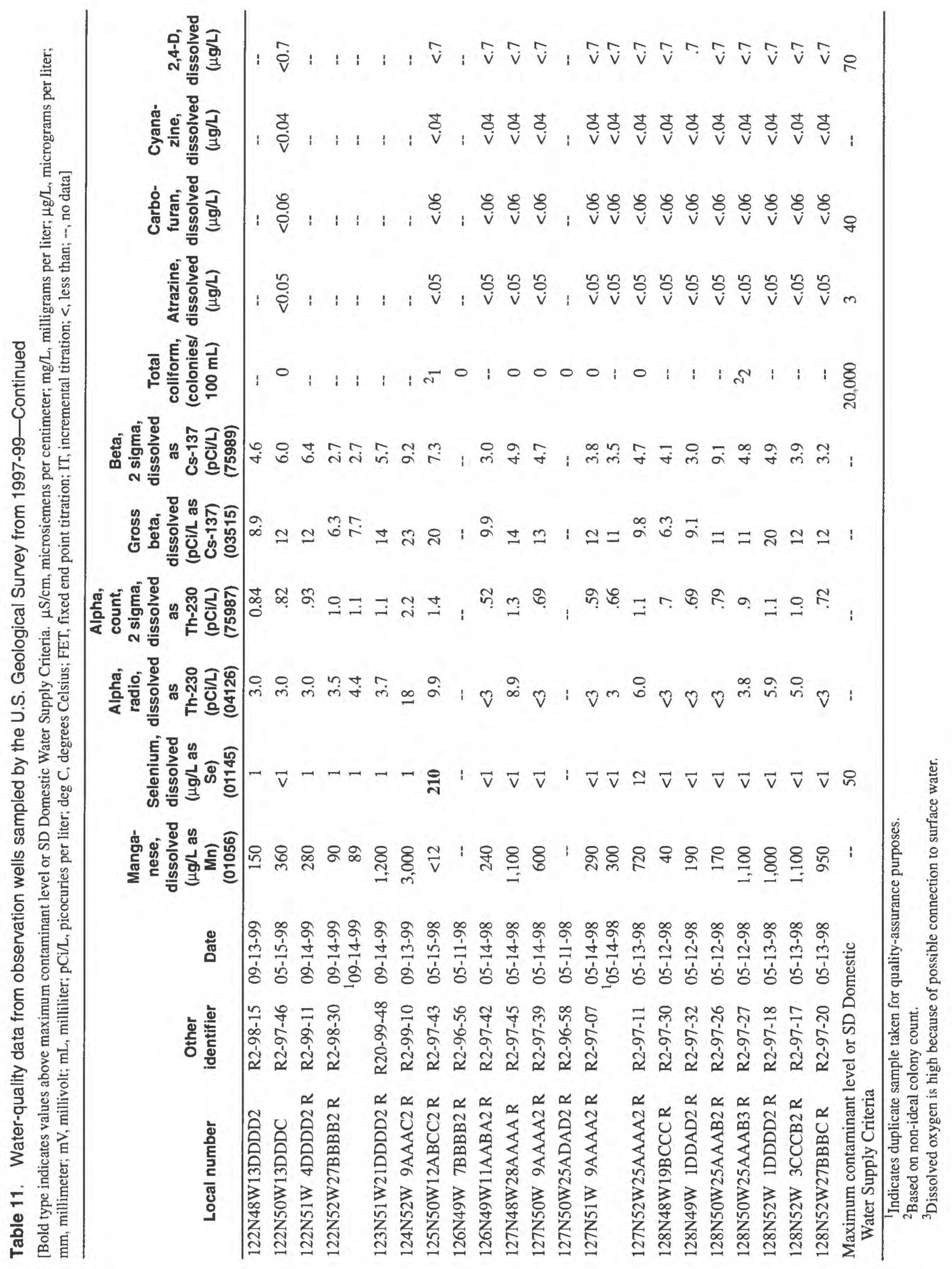


The collection of the ground-water samples followed methods presented in Wood (1976). All ground-water sampling equipment that came in contact with the sample water (submersible pump and tubing) was cleaned prior to the sampling trip with a phosphate-free detergent, dilute hydrochloric acid, and deionized water by procedures described by Horowitz and others (1994). After samples were collected from a well, the pump and tubing were rinsed with deionized water before sampling the next well.

In order to assure that a representative sample was collected from the aquifer at each well, the groundwater samples were collected after purging a minimum of three casing volumes from the well and after field measurements of specific conductance, $\mathrm{pH}$, temperature, and dissolved oxygen had stabilized (Wood, 1976). Stability was reached when specific conductance differed less than 5 percent between readings, $\mathrm{pH}$ differed less than 0.1 standard unit between readings, and water temperature differed less than $0.2^{\circ} \mathrm{C}$ between readings. Samples were collected from the observation wells using a positive-displacement, stainless-steel submersible pump. A Keck pump was used for sampling the 1997 observation wells, and a Grundflos Rediflo2 was used for sampling the 1998 and 1999 wells. Water from the observation wells passed through a flow-through chamber without coming into contact with the atmosphere so that field measurements were measured in a closed system. The water-quality samples were collected in appropriate bottles directly from a Teflon collection tube.

\section{Quality-Assurance Data}

Quality-assurance procedures were performed to evaluate the precision and accuracy of the reported analytical results. The procedures were designed to verify that no significant biases were introduced to the data during either field sampling and processing, or during laboratory analysis of the samples. Quality-assurance procedures for the USGS NWQL included analyses of reference and calibration materials and internal analyses of blanks, replicates, and spiked samples. Quality-assurance procedures for the USGS NWQL are presented in Friedman and Erdmann (1982) and Pritt and Raese (1992). Field meters were calibrated daily for $\mathrm{pH}$, dissolved oxygen, oxidation reduction potential, and specific conductance during sampling.

Quality-assurance samples collected during this study included duplicates, laboratory blanks, and field blanks. Duplicate samples are two samples collected as close in time as possible and are considered to be essentially identical in composition. They are collected to evaluate variability in the data that may be attributed to either field-collection and processing procedures, or laboratory and analytical procedures.

Two duplicate sample sets were collected for this study during 1997-99. The two duplicates were for ground-water quality samples, the only water-quality samples taken after 1996. The data indicated that the analytical results for the duplicate pairs generally compared very well and were not substantially different (table 11).

Blank samples, which included both laboratory and field blanks, were collected and analyzed to identify the presence and magnitude of contamination that potentially could bias analytical results. Laboratory blanks are samples of ultrapure deionized water that are processed through the sampling equipment used in this study within a laboratory or other controlled environment; laboratory blanks are used to identify sample contamination introduced from the sampling equipment. Field blanks are samples of ultrapure deionized water that are processed through sampling equipment at the field collection site; field blanks are used to identify contamination introduced from the sampling equipment, and contamination introduced during collection and processing of samples in the field. Two laboratory blanks and two field blanks were collected for the ground-water-quality sampling during 1997-99.

Although some of the constituents in table 12 are at or near the concentrations found in table 11 , this is due to the concentrations being at or near the reporting level for a particular constituent. One constituent does show an anomalous result that is unexpected for a blank sample. The analysis for lab alkalinity (parameter 90410) collected in a field blank on September 15, 1999, is higher than that of the other blanks. A possible source of contamination lies in the method used to collect field blanks. The volume of ultrapure deionized water required to fill all sample bottles is calculated, and an additional volume is used as a safety factor. Because the length of the tube on the Grundflos pump is great, the tube itself holds a substantial amount of water and is difficult to purge completely. The ultrapure water is run through the pump, and after a period of time has elapsed, the discharge from the sample collection tube is assumed to have purged out any water that may have remained in the tube. Bubbles can sometimes be seen at the interface between the ultrapure 
water and the previous contents of the tube. After the required volume of ultrapure water is fed into the pump, additional deionized water is needed to "push" the ultrapure water through the tube and into the remaining sample bottles. Because of the length of the tube, it would be possible for the ultrapure water to become mixed with the "push" water, or the water present in the tube before the blank sample was collected. If the first or last sample bottle was filled with mixed water, it could cause contamination, which may result in an erroneous value such as noted for lab alkalinity in the 1999 field blank. Because none of the other constituents for the 1999 field blank appear to have been impacted, any possible contamination is apparently limited to the alkalinity sample.

\section{SURFACE-WATER DATA}

Numerous streams and lakes are present within the study area. Portions of four major basins drain the study area: the Upper Red River, the Minnesota River, the Big Sioux River, and the James River (fig. 7). The portion of the study area that contains the majority of the lakes is drained by the James and Big Sioux River Basins, although much of the area is noncontributing under normal conditions. Drainage from the Minnesota, Big Sioux, and James River Basins is to the Gulf of Mexico, and drainage from the Upper Red River Basin is to the Hudson Bay.

\section{Streams}

The major streams in the study area include the Big Sioux River, Bois de Sioux River, Little Minnesota River, North Fork Whetstone River, LaBelle Creek, and Big Coulee Creek. Eight streamflow-gaging stations currently operated by the USGS are located within or near the study area (fig. 7).

Table 12. Constituent concentrations for quality-assurance laboratory and field blanks

$[\mu \mathrm{S} / \mathrm{cm}$, microsiemens per centimeter; $\mathrm{mg} / \mathrm{L}$, milligrams per liter; $\mu \mathrm{g} / \mathrm{L}$, micrograms per liter; $\mathrm{mV}$, millivolt; $\mathrm{pCi} / \mathrm{L}$, picocuries per liter; deg $\mathrm{C}$, degrees Celsius; FET, fixed end point titration; IT, incremental titration; -, no data]

\begin{tabular}{|c|c|c|c|c|c|c|c|}
\hline Station number & Local number & Date & $\begin{array}{c}\text { Calcium, } \\
\text { dissolved } \\
\text { (mg/L } \\
\text { as Ca) } \\
(00915)\end{array}$ & $\begin{array}{l}\text { Magne- } \\
\text { sium, } \\
\text { dissolved } \\
(\mathrm{mg} / \mathrm{L} \\
\text { as Mg) } \\
(00925)\end{array}$ & $\begin{array}{c}\text { Sodium, } \\
\text { dissolved } \\
\text { (mg/L } \\
\text { as } \mathrm{Na}) \\
(00930)\end{array}$ & $\begin{array}{c}\text { Potassium, } \\
\text { dissolved } \\
\text { (mg/L } \\
\text { as K) } \\
(00935)\end{array}$ & $\begin{array}{c}\text { Alkalinity, } \\
\text { lab } \\
(\mathrm{mg} / \mathrm{L} \text { as } \\
\left.\mathrm{CaCO}_{3}\right) \\
(90410)\end{array}$ \\
\hline \multirow[t]{5}{*}{442124098130700} & Equipment blank & $09-13-99$ & 0.078 & 0.12 & 0.146 & 0.24 & 2.096 \\
\hline & Equipment blank & $05-11-98$ & .021 & .004 & .1 & .1 & 1.899 \\
\hline & Field blank & $05-15-98$ & - & - & -- & -- & -- \\
\hline & Field blank & $05-15-98$ & .323 & .061 & .1 & .1 & 2.9 \\
\hline & Field blank & $09-15-99$ & .534 & .164 & .085 & .24 & 382.83 \\
\hline
\end{tabular}

\begin{tabular}{|c|c|c|c|c|c|c|c|}
\hline Local number & Date & $\begin{array}{c}\text { Sulfate, } \\
\text { dissolved } \\
\left(\mathrm{mg} / \mathrm{L} \text { as } \mathrm{SO}_{4}\right) \\
(00945)\end{array}$ & $\begin{array}{c}\text { Chloride, } \\
\text { dissolved } \\
\text { (mg/L as } \mathrm{Cl}) \\
(00940)\end{array}$ & $\begin{array}{c}\text { Fluoride, } \\
\text { dissolved } \\
\text { (mg/L as F) } \\
(00950)\end{array}$ & $\begin{array}{c}\text { Silica, } \\
\text { dissoived } \\
\text { (mg/L as } \\
\left.\mathrm{SiO}_{2}\right) \\
(00955)\end{array}$ & $\begin{array}{l}\text { Solids, } \\
\text { residue at } \\
180 \text { deg } C \text {, } \\
\text { dissoived } \\
(\mathrm{mg} / \mathrm{L}) \\
(70300)\end{array}$ & $\begin{array}{c}\text { Nitrogen, } \\
\text { ammonia, } \\
\text { dissolved } \\
\text { (mg/L as N) } \\
(00608)\end{array}$ \\
\hline Equipment blank & 09-13-99 & 0.21 & 0.29 & 0.1 & 0.09 & 10 & 0.02 \\
\hline Equipment blank & $05-11-98$ & .432 & .163 & .1 & .1 & 10 & .057 \\
\hline Field blank & $05-15-98$ & -- & - & -- & - & -- & -- \\
\hline Field blank & $05-15-98$ & .285 & .12 & .1 & .1 & 10 & .06 \\
\hline Field blank & $09-15-99$ & .8 & .29 & .1 & .089 & 10 & .02 \\
\hline
\end{tabular}


Table 12. Constituent concentrations for quality-assurance laboratory and field blanks—Continued

$[\mu \mathrm{S} / \mathrm{cm}$, microsiemens per centimeter; $\mathrm{mg} / \mathrm{L}$, milligrams per liter; $\mu \mathrm{g} / \mathrm{L}$, micrograms per liter; $\mathrm{mV}$, millivolt; $\mathrm{pCi} / \mathrm{L}$, picocuries per liter; deg $\mathrm{C}$, degrees Celsius; FET, fixed end point titration; IT, incremental titration; --, no data]

\begin{tabular}{|c|c|c|c|c|c|c|c|}
\hline Local number & Date & $\begin{array}{c}\text { Nitrogen, } \\
\text { nitrite, } \\
\text { dissolved } \\
\text { (mg/L as N) } \\
\text { (00613) }\end{array}$ & $\begin{array}{c}\text { Nitrogen, } \\
\mathrm{NO}_{2}+\mathrm{NO}_{3}, \\
\text { dissolved } \\
\text { (mg/L as } \mathrm{N} \text { ) } \\
(00631)\end{array}$ & $\begin{array}{c}\text { Phosphorus, } \\
\text { ortho, } \\
\text { dissolved } \\
\text { (mg/L as P) } \\
(00671)\end{array}$ & $\begin{array}{c}\text { Aluminum, } \\
\text { dissolved } \\
(\mu \mathrm{g} / \mathrm{L} \text { as Al) } \\
(01106)\end{array}$ & $\begin{array}{c}\text { Arsenic, } \\
\text { dissolved } \\
(\mu \mathrm{g} / \mathrm{L} \text { as As) } \\
(01000)\end{array}$ & $\begin{array}{c}\text { Boron, } \\
\text { dissolved } \\
\text { ( } \mu \mathrm{g} / \mathrm{L} \text { as B) } \\
(01020)\end{array}$ \\
\hline Equipment blank & $09-13-99$ & 0.01 & 0.05 & 0.01 & 15 & 1 & 16 \\
\hline Equipment blank & 09-13-99 & .01 & .05 & .013 & 10 & 1 & 16 \\
\hline Field blank & $05-15-98$ & - & -- & -- & -- & - & -- \\
\hline Field blank & $05-15-98$ & .01 & .05 & .043 & 10 & 1 & 16 \\
\hline Field blank & $09-15-99$ & .01 & .05 & .01 & 15 & 1 & 8.718 \\
\hline
\end{tabular}

\begin{tabular}{|c|c|c|c|c|c|c|c|}
\hline Local number & Date & $\begin{array}{c}\text { Iron, } \\
\text { dissolved } \\
(\mu \mathrm{g} / \mathrm{L} \text { as Fe) } \\
(01046)\end{array}$ & $\begin{array}{c}\text { Manganese, } \\
\text { dissolved } \\
(\mu \mathrm{g} / \mathrm{L} \text { as } \mathrm{Mn}) \\
(01056)\end{array}$ & $\begin{array}{c}\text { Selenium, } \\
\text { dissolved } \\
\text { ( } \mu \mathrm{g} / \mathrm{L} \text { as } \mathrm{Se}) \\
(01145)\end{array}$ & $\begin{array}{l}\text { Alpha, radio, } \\
\text { dissolved } \\
\text { as Th-230 } \\
(p C i / L) \\
(04126)\end{array}$ & $\begin{array}{l}\text { Alpha, count, } \\
2 \text { sigma, } \\
\text { dissolved } \\
\text { as Th-230 } \\
(\mathrm{pCi} / \mathrm{L}) \\
(75987)\end{array}$ & $\begin{array}{c}\text { Gross beta, } \\
\text { dissolved } \\
\text { (pCi/L as } \\
\text { Cs-137) } \\
(03515)\end{array}$ \\
\hline Equipment blank & 09-13-99 & 10 & 4.262 & 1 & 3 & 0.51 & 4 \\
\hline Equipment blank & $09-13-99$ & 10 & 4 & 1 & 3 & .35 & 4 \\
\hline Field blank & $05-15-98$ & -- & - & - & -- & -- & - \\
\hline Field blank & $05-15-98$ & 10 & 4 & 1 & 3 & .38 & 4 \\
\hline Field blank & $09-15-99$ & 10 & 1.672 & 1 & 3 & .51 & 4 \\
\hline
\end{tabular}

\begin{tabular}{|c|c|c|c|c|c|c|c|}
\hline Local number & Date & $\begin{array}{c}\text { Beta, } \\
2 \text { sigma, } \\
\text { dissolved } \\
\text { as Cs-137 } \\
\text { (pCi/L) } \\
(75989)\end{array}$ & $\begin{array}{c}\text { Total } \\
\text { coliform, } \\
\text { (colonies/ } \\
100 \mathrm{~mL} \text { ) }\end{array}$ & $\begin{array}{c}\text { Atrazine, } \\
\text { dissolved } \\
(\mu g / L) \\
(34756)\end{array}$ & $\begin{array}{c}\text { Carbofuran, } \\
\text { dissolved } \\
(\mu \mathrm{g} / \mathrm{L})\end{array}$ & $\begin{array}{c}\text { Cyanazine, } \\
\text { dissolved } \\
(\mu g / L)\end{array}$ & $\begin{array}{c}\text { 2,4-D, } \\
\text { dissolved } \\
(\mu \mathrm{g} / \mathrm{L})\end{array}$ \\
\hline Equipment blank & $09-13-99$ & 0.72 & -- & - & -- & -- & -- \\
\hline Equipment blank & $09-13-98$ & 68 & -- & - & - & -- & -- \\
\hline Field blank & $05-15-98$ & -- & -- & $<0.05$ & $<0.06$ & $<0.04$ & $<0.7$ \\
\hline Field blank & $09-15-98$ & .74 & -. & -- & - & -- & -- \\
\hline Field blank & 09-15-99 & .76 & - & -- & - & -- & -- \\
\hline
\end{tabular}




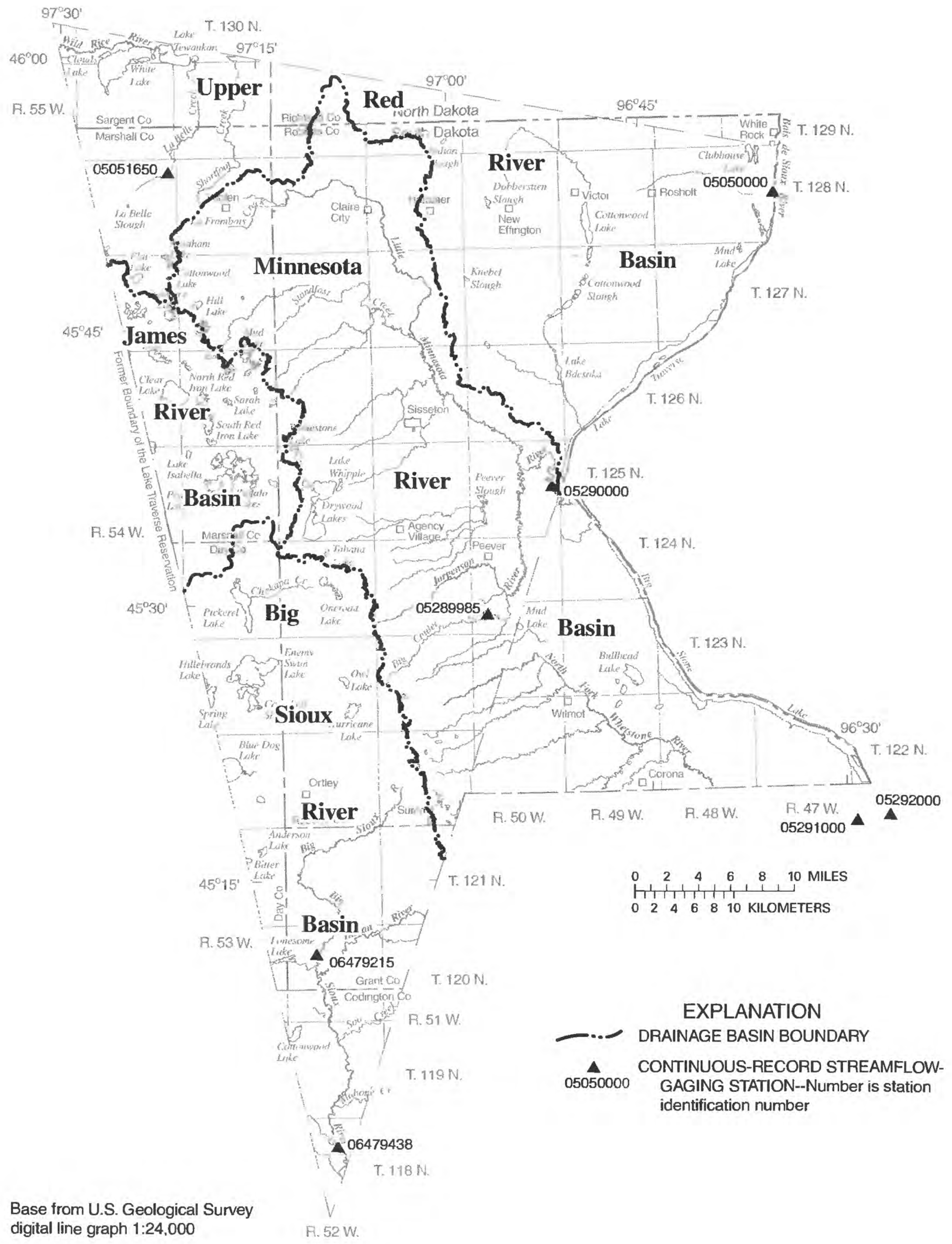

Figure 7. Drainage basins and location of active streamflow-gaging stations within and near the study area. 


\section{Peak-Flow Frequency Estimates}

Peak-flow frequency estimates were determined for the continuous-record and crest-stage gaging stations that had 10 or more years of record and were published in Carter and Thompson (1999). Of the 15 gages included in this study, two did not have 10 years of record in 1996. Also, above-normal precipitation in 1997 and 1998 may have caused significantly higher peaks than normal. For these reasons, updated peakflow frequency estimates were only determined for the eight currently operating continuous-record gaging stations. The estimates were determined using logPearson Type III procedures for recurrence intervals of $2,5,10,25,50,100$, and 500 years; the results are presented in table 13. The historic peak flows for the eight stations are presented in Section C of the Supplemental Information section.

\section{Summary Statistics}

The information presented in this section includes tabular data and graphical representation for the two continuous-record gages that had less than 10 years of record in the previous data report (Carter and Thompson, 1999). Six tables and four graphs were produced for each of the two continuous-record gages for the period of record listed in table 13. The tables list monthly and annual mean flow, statistics on monthly and annual mean flow, correlation matrices, and lowest and highest mean flow and rankings. The graphs illustrate annual mean flow, distribution of monthly mean flow, duration curves, and duration hydrographs.

The summary statistics tables are presented in Section D of the Supplemental Information section. For each site, tables D1.1 and D2.1 contain monthly and annual mean flow values for the selected statistics period.

Tables D1.2 and D2.2 present selected statistics for monthly and annual mean flow. This table includes the total number of months used in the analysis, maximum, 75 th percentile, 50 th percentile, 25 th percentile, minimum, mean, standard deviation, skewness, coefficient of variation, and the percent of annual flow.

Table 13. Peak-flow estimates for selected recurrence intervals for active gaging stations within and near the study area

\begin{tabular}{|c|c|c|c|c|c|c|c|c|c|c|}
\hline \multirow{2}{*}{$\begin{array}{l}\text { Station } \\
\text { number }\end{array}$} & \multirow{2}{*}{ Station name } & \multirow{2}{*}{$\begin{array}{c}\text { Period of } \\
\text { analysis } \\
\text { (water } \\
\text { year) }\end{array}$} & \multicolumn{8}{|c|}{$\begin{array}{l}\text { Peak flow, in cubic feet per second, for recurrence interval, in yeara, } \\
\text { and annual exceedance probability, In percent }\end{array}$} \\
\hline & & & $\begin{array}{l}\text { Years: } \\
\text { Percent: }\end{array}$ & $\begin{array}{c}2 \\
50\end{array}$ & $\begin{array}{c}5 \\
20\end{array}$ & $\begin{array}{l}10 \\
10\end{array}$ & $\begin{array}{c}25 \\
4\end{array}$ & $\begin{array}{c}50 \\
2\end{array}$ & $\begin{array}{c}100 \\
1\end{array}$ & $\begin{array}{l}500 \\
0.2\end{array}$ \\
\hline 05050000 & $\begin{array}{l}\text { Bois de Sioux River } \\
\text { near White Rock }\end{array}$ & $1942-99$ & & 1,286 & 3,438 & 5,556 & 9.038 & 12,210 & 15,860 & 26,250 \\
\hline 05051650 & $\begin{array}{l}\text { LaBelle Creek near } \\
\text { Veblen }\end{array}$ & $1988-99$ & & 83 & 298.5 & 501.9 & 789.5 & 1,006 & 1,213 & 1,636 \\
\hline 05289985 & $\begin{array}{l}\text { Big Coulee Creek } \\
\text { near Peever }\end{array}$ & $1988-99$ & & 133.8 & 380.3 & 630.8 & 1,051 & 1,438 & 1,888 & 3,180 \\
\hline 05290000 & $\begin{array}{l}\text { Little Minnesota } \\
\text { River near Peever }\end{array}$ & $\begin{array}{l}1940-81 \\
1990-99\end{array}$ & & 846.9 & 2,163 & 3,347 & 5,127 & 6,615 & 8,209 & 12,240 \\
\hline 05291000 & $\begin{array}{l}\text { Whetstone River near } \\
\text { Big Stone City }\end{array}$ & $1931-99$ & & 1,299 & 3,289 & 4,980 & 7,369 & 9,246 & 11,150 & 15,560 \\
\hline 05292000 & $\begin{array}{l}\text { Minnesota River at } \\
\text { Ortonville, Minn. }\end{array}$ & $1938-99$ & & 862.3 & 1,760 & 2,464 & 3,437 & 4,203 & 4,991 & 6,887 \\
\hline 06479215 & $\begin{array}{l}\text { Big Sioux River near } \\
\text { Florence }\end{array}$ & $1984-99$ & & 407.7 & 1,114 & 1,686 & 2,429 & 2,960 & 3,454 & 4,441 \\
\hline 06479438 & $\begin{array}{l}\text { Big Sioux River near } \\
\text { Watertown }\end{array}$ & $1973-99$ & & 856.1 & 2,235 & 3,425 & 5,120 & 6,456 & 7,815 & 10,960 \\
\hline
\end{tabular}


Tables D1.3 and D2.3 present the serial auto correlation matrix for the 1-year lag for monthly mean flow. The correlation coefficient is a measure of the strength of the linear relation between two variables (Ott, 1993). The correlation coefficient ranges from -1 to +1 ; a correlation coefficient equal to zero indicates there is no relation between the two variables. A positive correlation indicates that both variables vary in the same direction, and a negative correlation indicates that one variable increases as the other decreases. The greater the correlation coefficient, the stronger the relation is between the two variables. For example, in table D1.3, the correlation coefficient for October of each year to October of the following year is 0.099 . This indicates that streamflow in a given month is not strongly influenced by streamflow in the same month of the preceding year.

Tables D1.4 and D2.4 present a correlation matrix for monthly mean flow. This matrix contains a correlation coefficient for each month, relative to monthly flow for other months. For example, in table D1.4, the correlation coefficient between October and November is 0.596 . This indicates that high (or low) streamflow in a given month is sometimes influenced by high (or low) streamflow in the previous month.

The lowest mean flow and ranking, by year, for $1,3,7,14,30,60,90,120$, and 183 consecutive-day periods is presented in tables D1.5 and D2.5. Similarly the highest mean flow and ranking, by year, for $1,3,7$, $15,30,60,90,120$, and 183 consecutive-day periods is presented in tables D1.6 and D2.6.

The graphical representations of the variations in the annual, monthly, and daily mean flow for each of the continuous-record gages that had less than a 10-year period of record in 1996 are presented in figures 8 and 9 with four graphs on each figure labeled A, B, C, and D as described previously. Graph A shows the annual mean flow values, and graph $B$ shows a distribution of monthly mean flow. Graph $\mathrm{C}$ shows the duration curve for daily mean flows, and graph D shows the duration hydrographs for selected exceedances.

\section{Lakes}

Numerous lakes are present in the study area, and the majority of the lakes are located in the James and Big Sioux River Basins. Water-quality data from 17 lakes were presented in Carter and Thompson (1999). Lake-level data collected during 1997-99 are presented in this section.

\section{Water Levels}

Lake-level data subsequent to 1996 are presented in tables in Section E of the Supplemental Information section. The locations of the lakes within the study area with available water-level data are shown in figure 10. Water levels from 1997-99 for 19 of these lakes are shown in figures 11-25. Measurements at Clubhouse Lake in Roberts County were discontinued after May 13, 1997, so no hydrograph is presented for this lake. The extremes listed are for the period of record. Cottonwood Lake in Roberts County is referred to as Cottonwood North Lake to avoid confusion with Cottonwood Lake in Marshall County. 

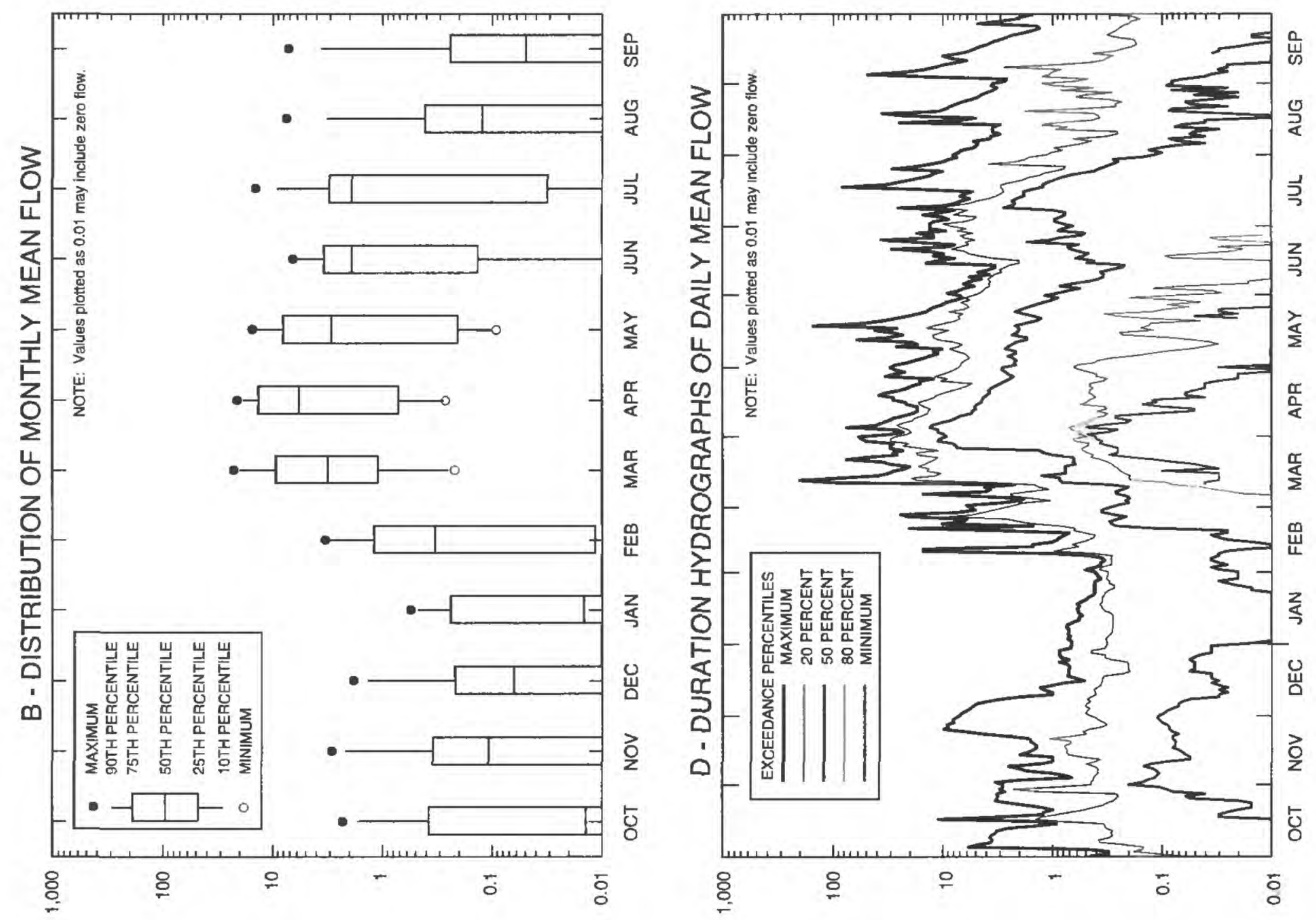

0
0
0
0
0
0
0
0
0
2
0
0
0
0
0
0
0
0
5
0
0
0
0
0
0
0
0
0
0
0
0
0
0
0
0
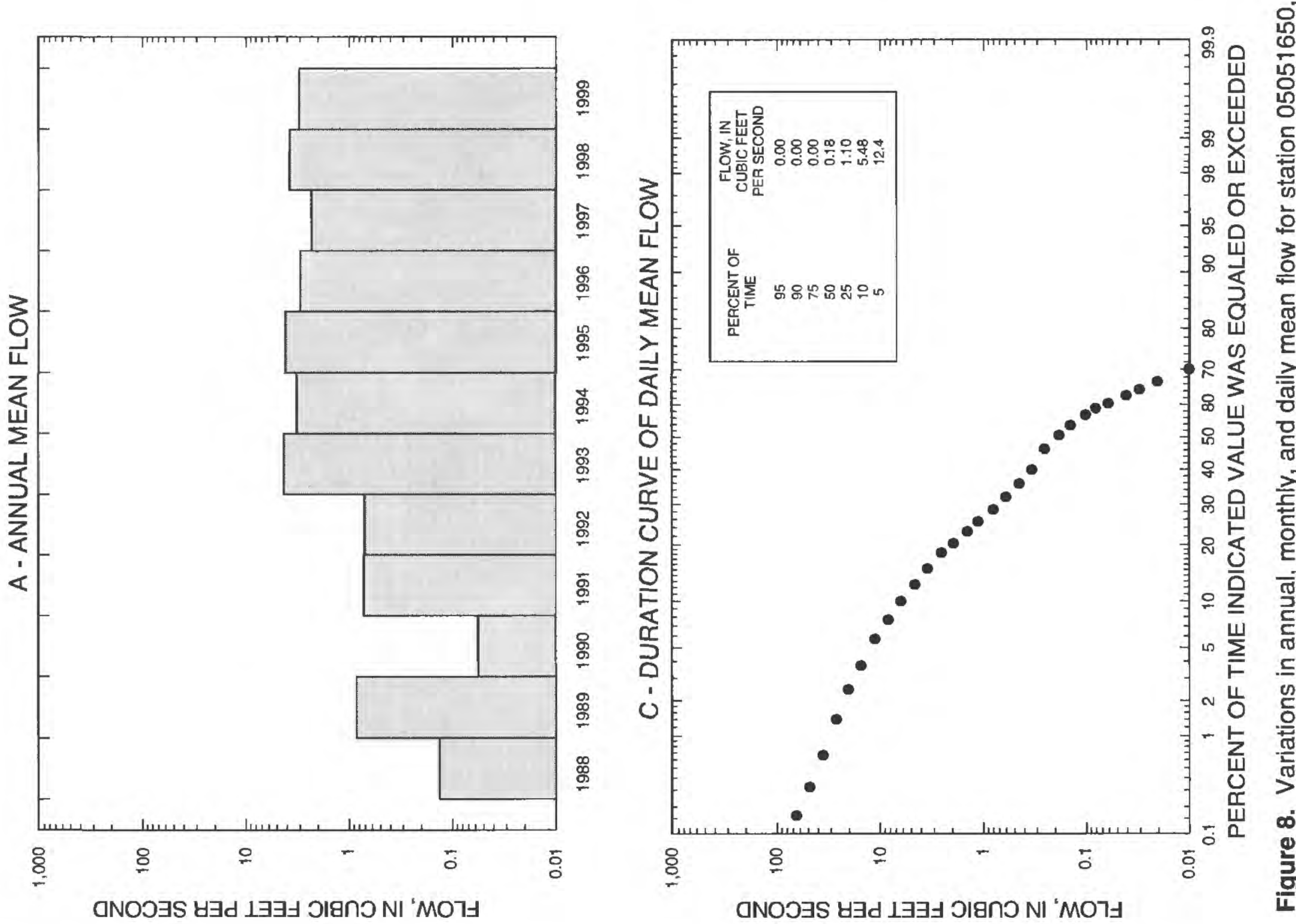

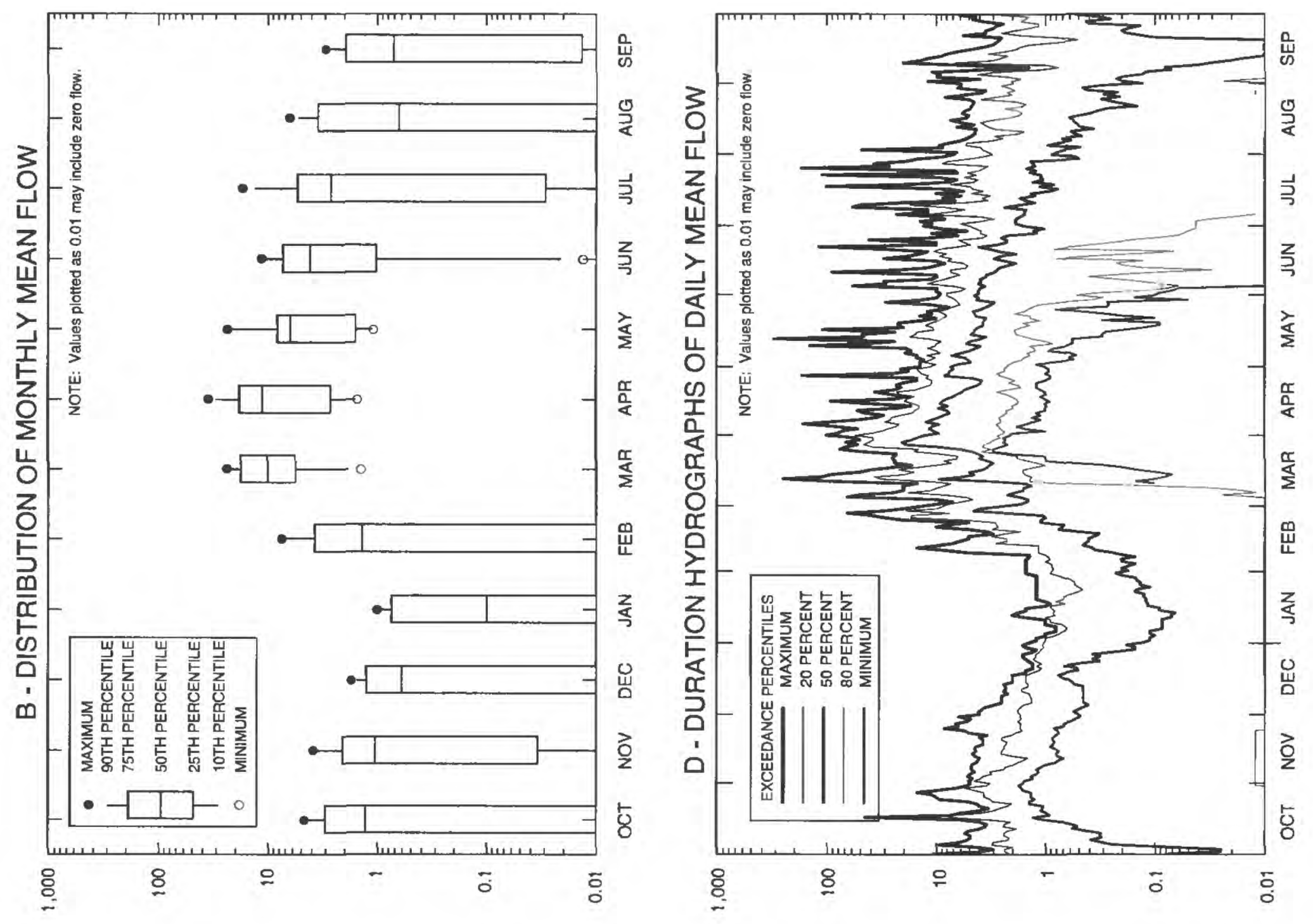

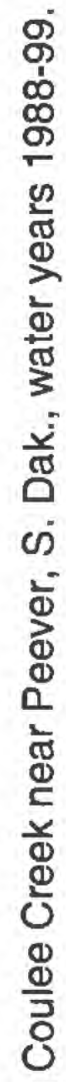
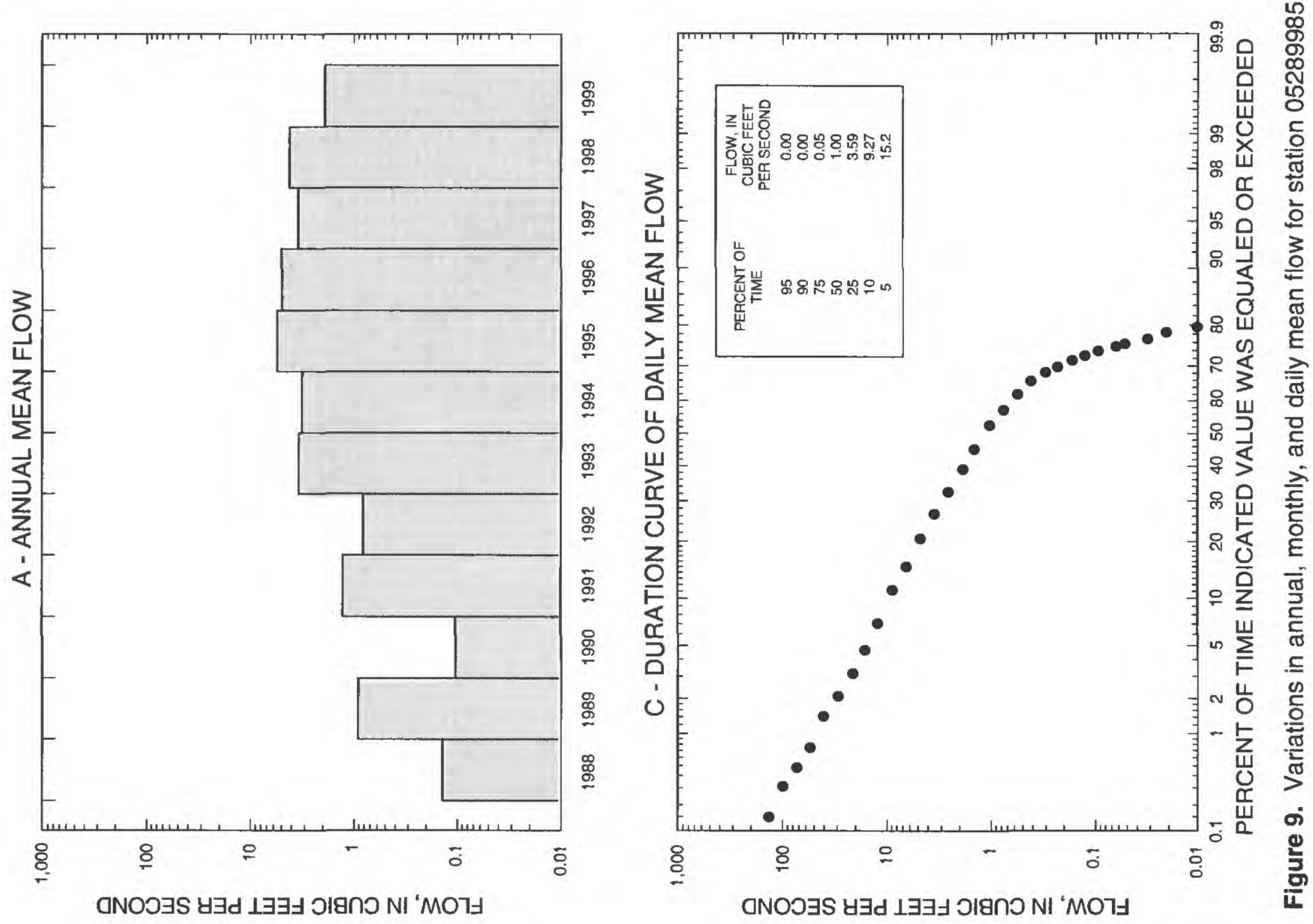


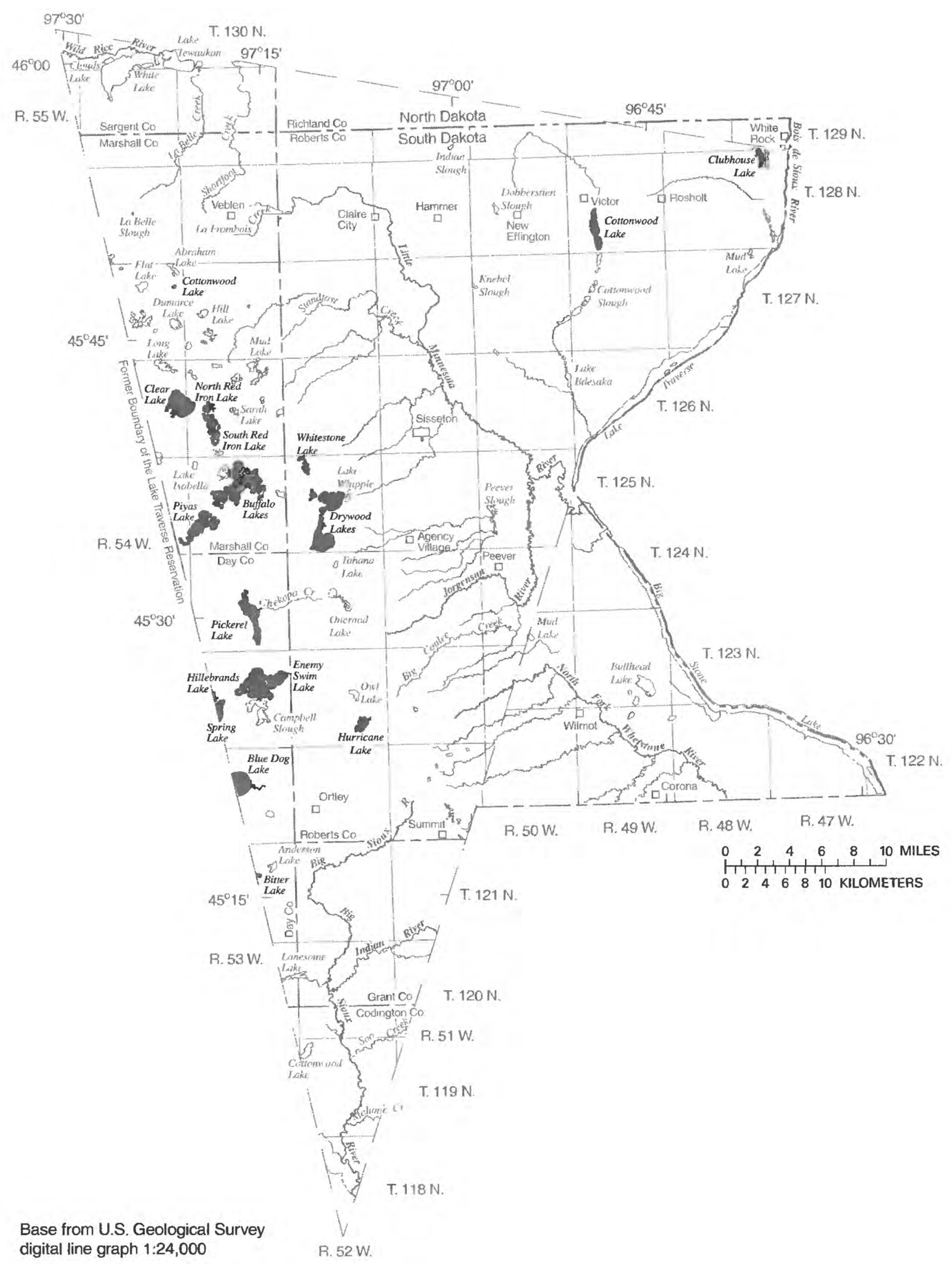

Figure 10. Location of lakes with recorded water-level elevations. 
Lake name: Bitter Lake

County: Day

Extremes: June 8, 1984, to November 2, 1999: Highest, 1,792.15 feet, November 2, 1999; lowest, 1,770.3 feet, June 8, 1984.



CALENDAR YEAR

Figure 11. Hydrograph for Bitter Lake in Day County.

Lake name: Blue Dog Lake

County: Day

Extremes: February 21, 1980, to November 2, 1999: Highest, 1,804.29 feet, June 10, 1999; lowest, 1,799.5 feet, November 4, 1980, September 21, 1988.

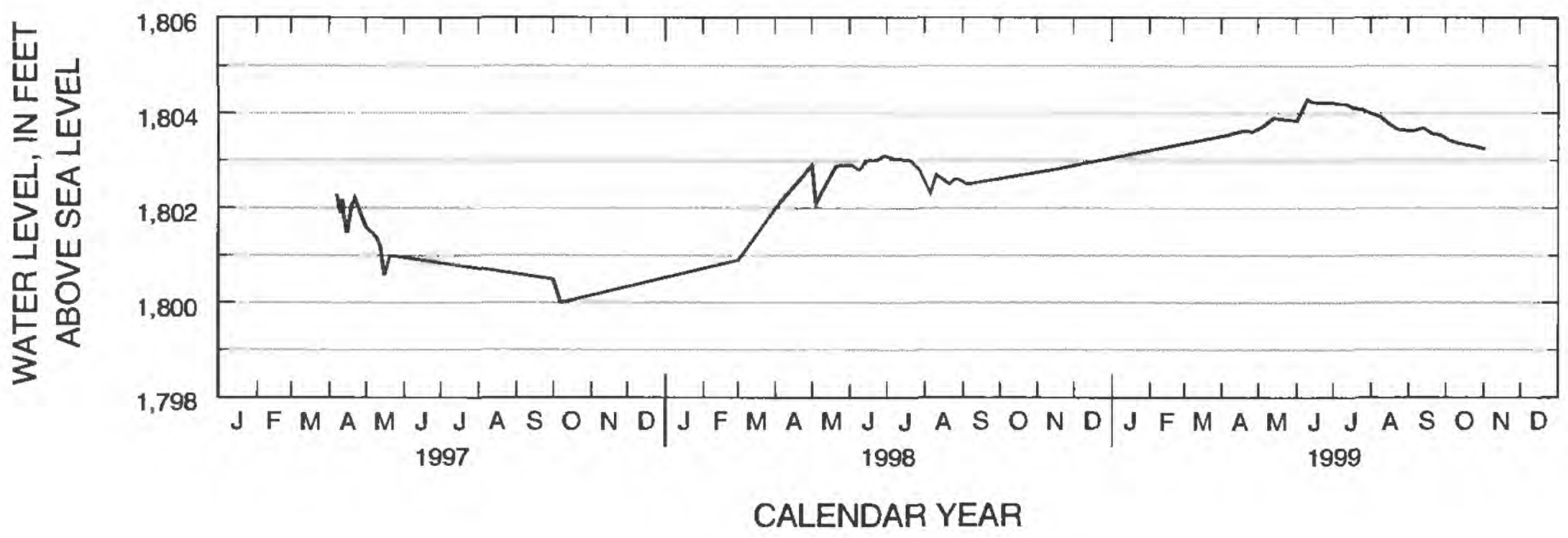

Figure 12. Hydrograph for Blue Dog Lake in Day County. 
Lake name: Buffalo Lakes

County: Marshall

Extremes (North Buffalo Lake): June 24, 1982, to September 28, 1999: Highest, 1,837.7 feet, May 19, 1997; lowest, 1,830.3 feet, October 12, 1983.

Extremes (South Buffalo Lake): June 23, 1982, to September 28, 1999: Highest, 1,838.6 feet, May 19, 1997; lowest, 1,830.7 feet, October 12, 1983.

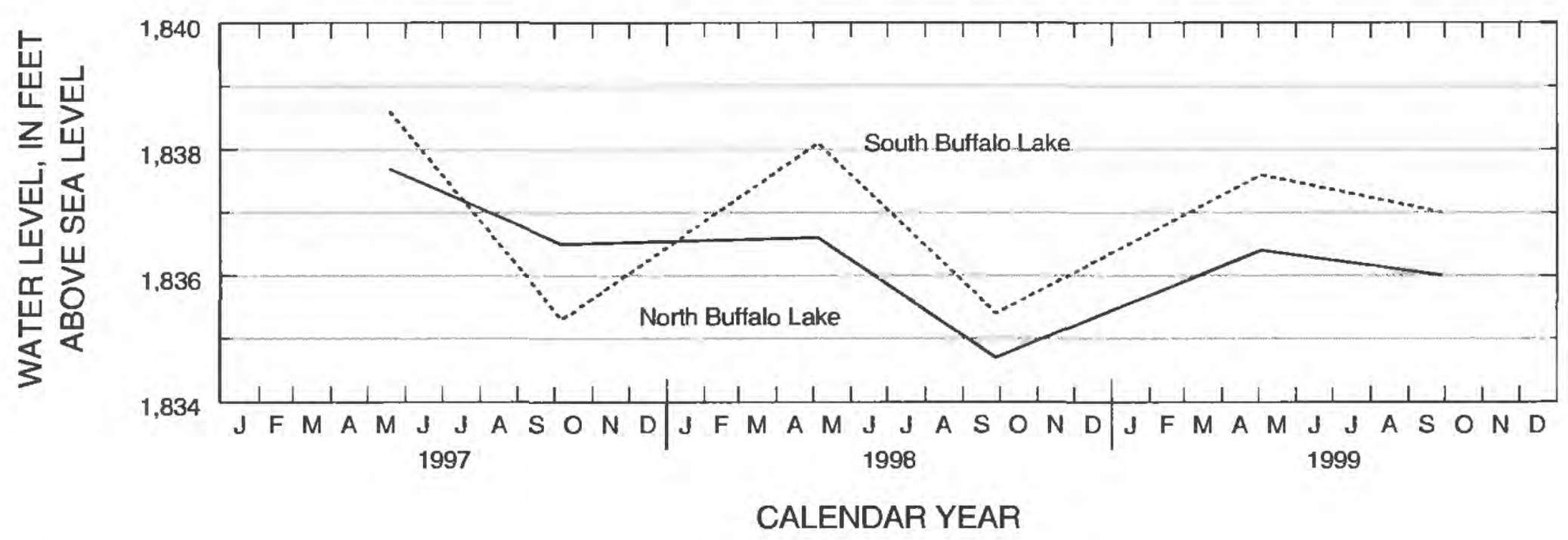

Figure 13. Hydrograph for Buffalo Lakes in Marshall County.

Lake name: Clear Lake

County: Marshall

Extremes: May 12, 1982, to September 28, 1999: Highest, 1,822.9 feet, May 5, 1994, and May 19, 1997; lowest, 1,816.4 feet, October 9, 1985.

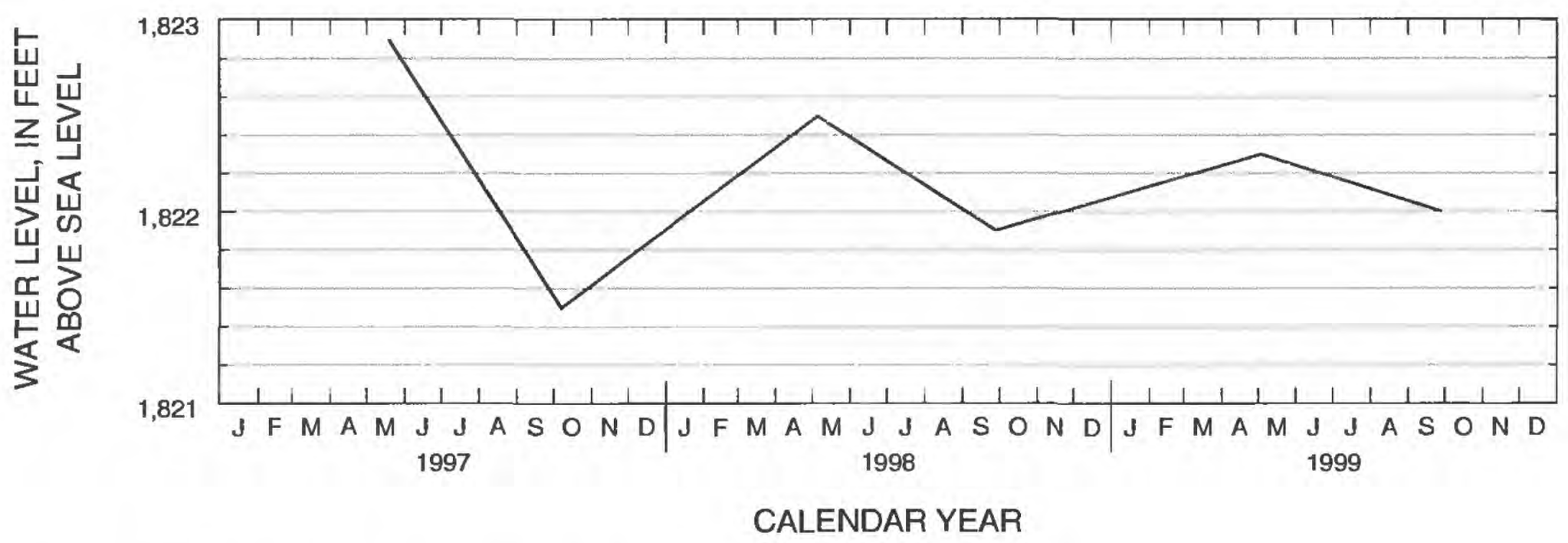

Figure 14. Hydrograph for Clear Lake in Marshall County. 
Lake name: Clubhouse Lake

County: Roberts

Extremes: July 10, 1986, to May 13, 1997: Highest, 973.1 feet, May 5, 1994; lowest, 969.5 feet, September 20, 1988. (Note: Measurements have been discontinued at this lake.)

Lake name: Cottonwood (North) Lake

County: Roberts

Extremes: July 10, 1986, to September 28, 1999: Highest, 1,030.6 feet, May 13, 1999; lowest, 1,028.3 feet, September 20, 1988.

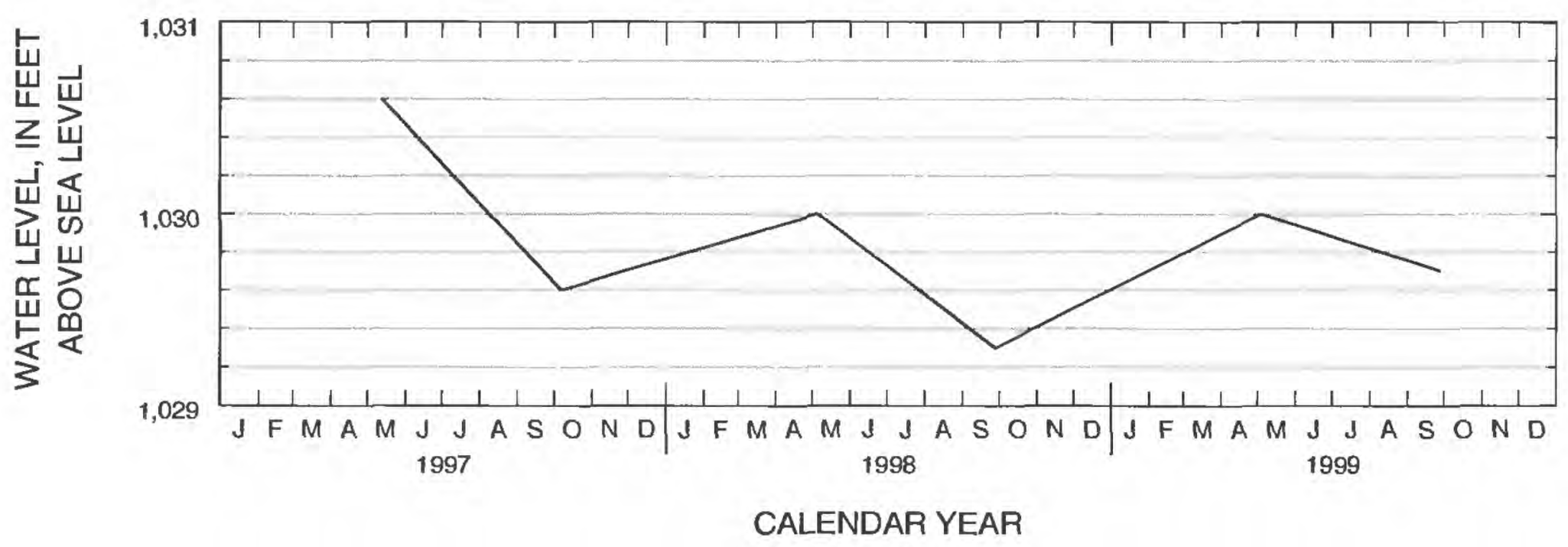

Figure 15. Hydrograph for Cottonwood Lake (North) in Roberts County.

Lake name: Cottonwood Lake

County: Marshall

Extremes: October 3, 1995, to September 28, 1999: Highest, 1,827.7 feet, May 19, 1997; lowest, 1,826.3 feet, October 7, 1997.

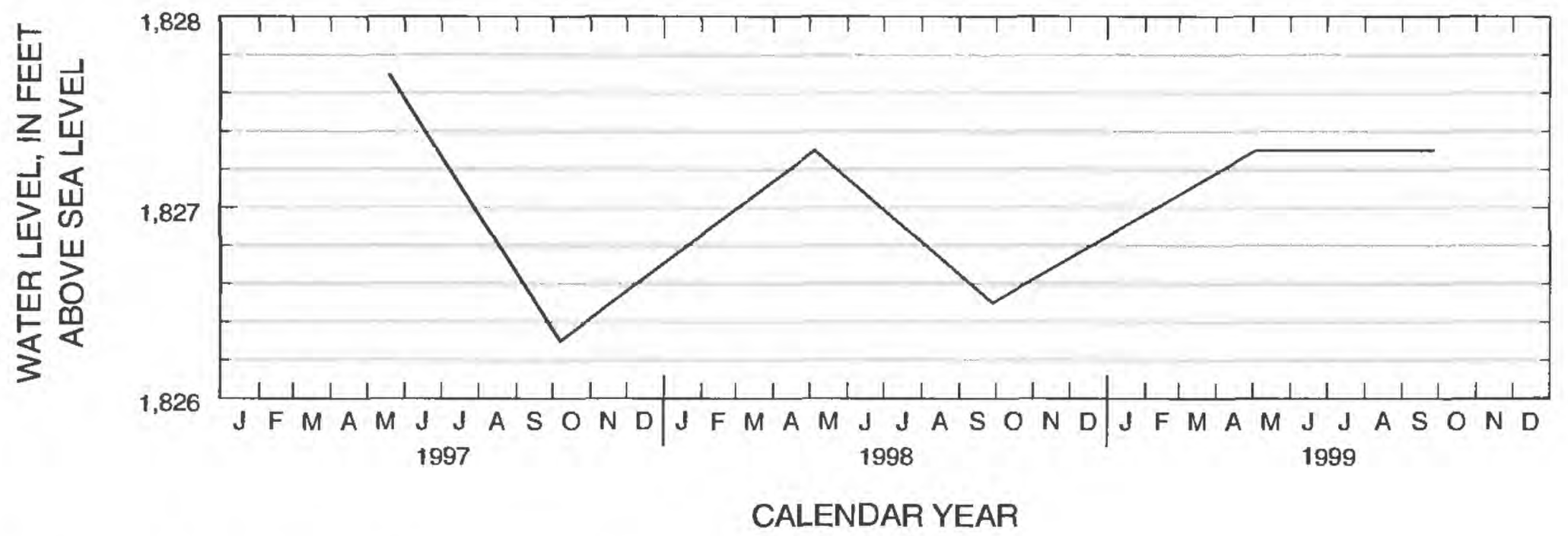

Figure 16. Hydrograph for Cottonwood Lake in Marshall County. 
Lake name: Drywood Lakes

County: Roberts

Extremes (Drywood Lake North): November 3, 1983, to September 28, 1999: Highest, 1,950.0 feet, May 4, 1999; lowest, 1,940.9 feet, September 20, 1990.

Extremes (Drywood Lake South): November 3, 1983, to September 28, 1999: Highest, 1,950.0 feet, May 4, 1999; lowest, 1,942.0 feet, May 3, 1990.



CALENDAR YEAR

Figure 17. Hydrograph for North and South Drywood Lakes in Roberts County.

Lake name: Enemy Swim Lake

County: Day

Extremes: July 7, 1982, to November 2, 1999: Highest, 1,856.3 feet, April 15, 1997; lowest, 1,850.2 feet, October 12, 1983.

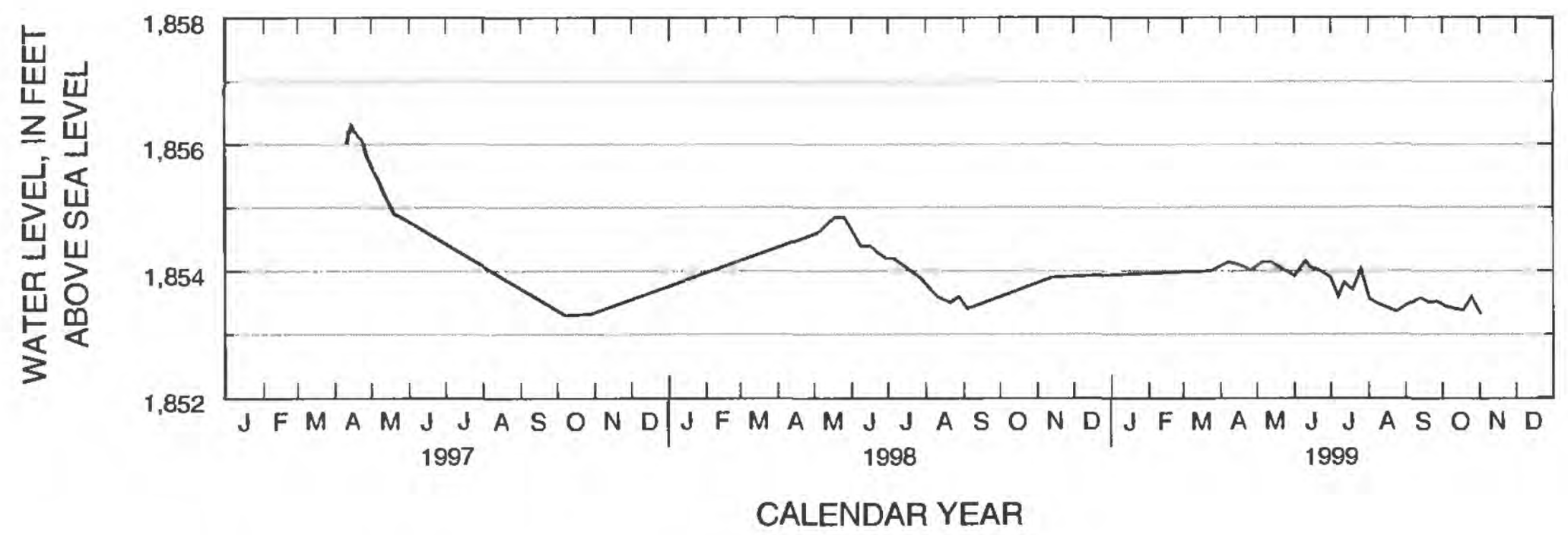

Figure 18. Hydrograph for Enemy Swim Lake in Day County. 
Lake name: Hillebrands Lake

County: Day

Extremes: October 11, 1983, to November 2, 1999: Highest, 1,804.03 feet, July 26, 1999; lowest, 1,781.3 feet, October 11, 1983.

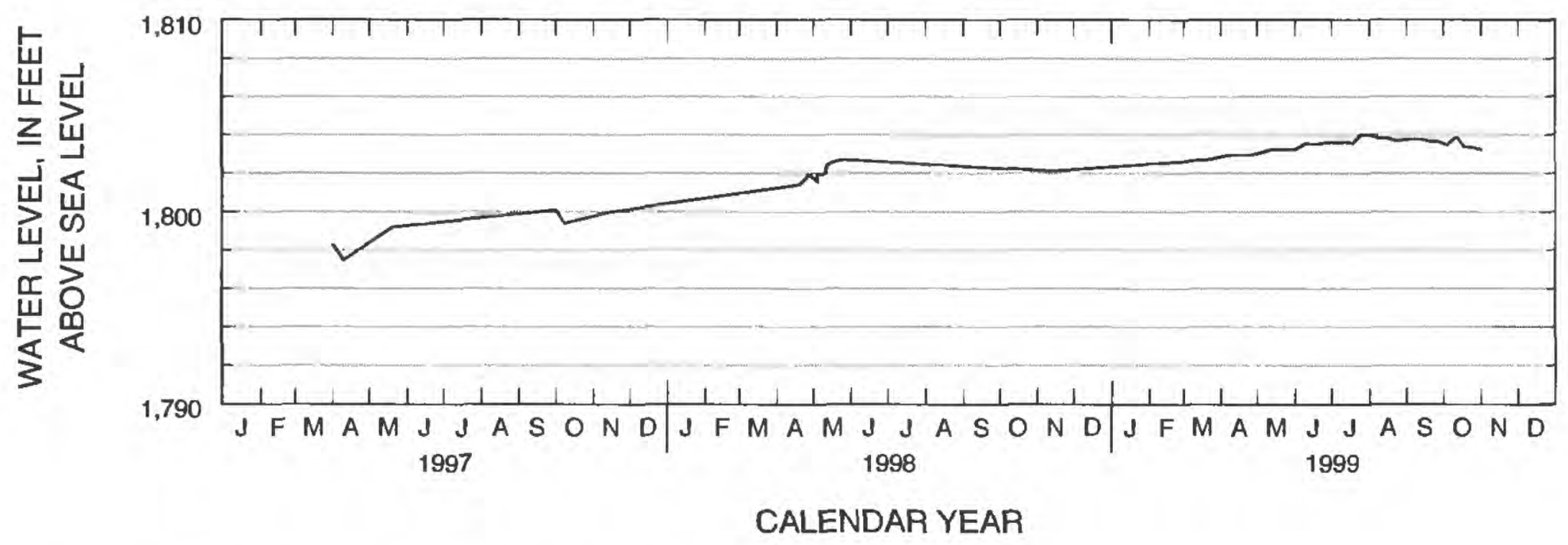

Figure 19. Hydrograph for Hillebrands Lake in Day County.

Lake name: Hurricane Lake

County: Roberts

Extremes: July 10, 1986, to September 28, 1999: Highest, 1,753.7 feet, May 10, 1995; lowest, 1,746.9 feet, May 1, 1991.



Figure 20. Hydrograph for Hurricane Lake in Roberts County. 
Lake name: Pickerel Lake

County: Day

Extremes: June 22, 1982, to November 2, 1999: Highest, 1,846.7 feet, October 8, 1997; lowest, 1,843.5 feet, September 21, 1988.

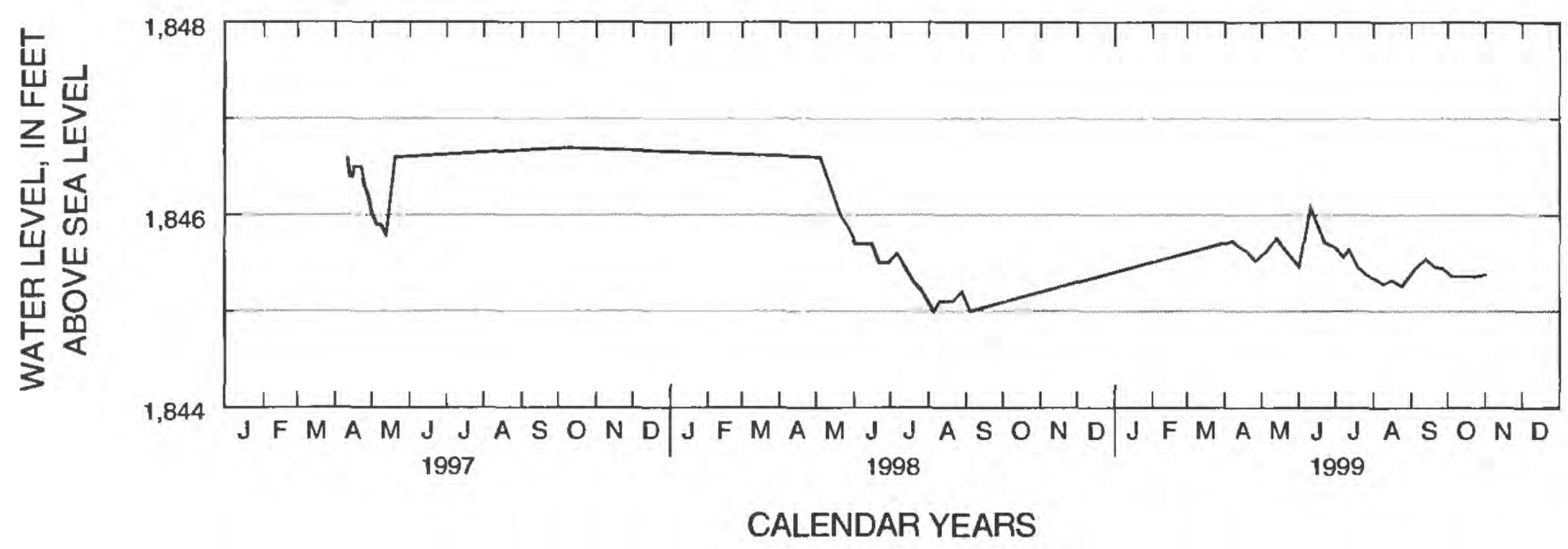

Figure 21. Hydrograph for Pickerel Lake in Day County.

Lake name: Piyas Lake

County: Marshall

Extremes: October 4, 1995, to September 28. 1999: Highest, 1,832.6 feet, September 28, 1999; lowest, 1,828.0 feet, October 4, 1995.

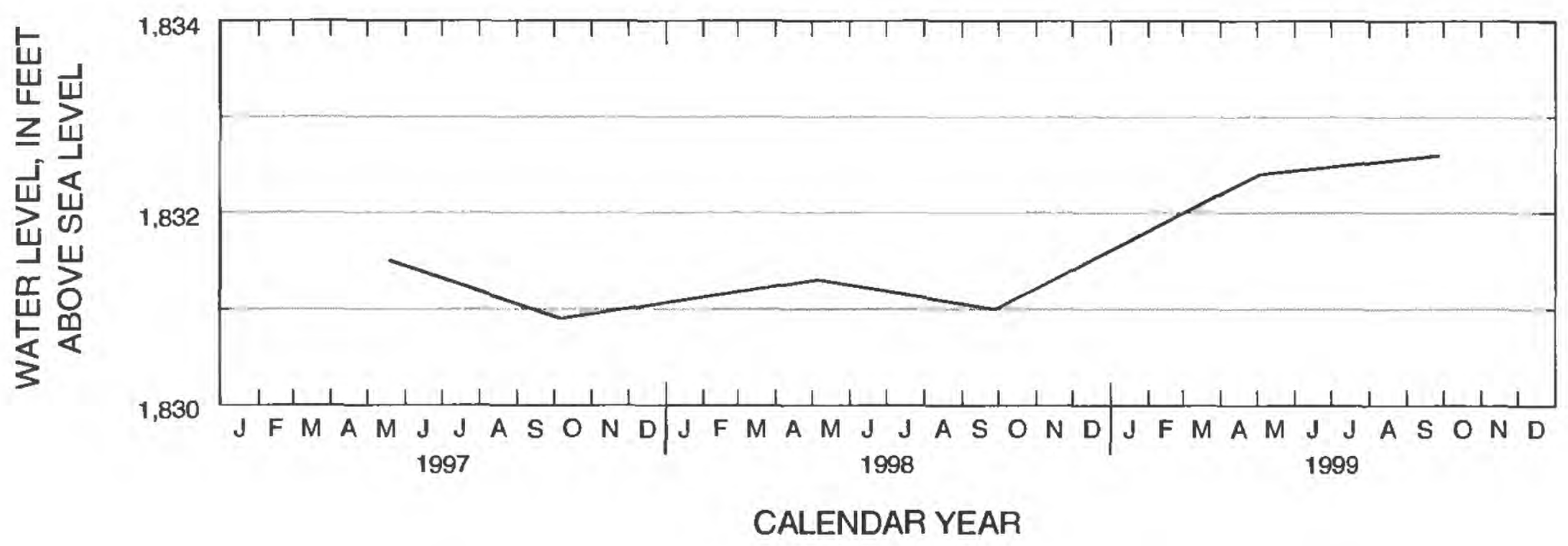

Figure 22. Hydrograph for Piyas Lake in Marshall County. 
Lake name: Red Iron Lakes

County: Marshall

Extremes (Red Iron Lake North): September 28, 1984, to September 28, 1999: Highest, 1,830.6 feet, May 19, 1997, May 11, 1995; lowest, 1,825.0 feet, September 28, 1984.

Extremes (Red Iron Lake South): September 28, 1994, to September 28, 1999: Highest, 1,832.9 feet, May 19, 1997, May 11, 1995; lowest, 1,826.8 feet, October 9, 1985, September 20, 1988.

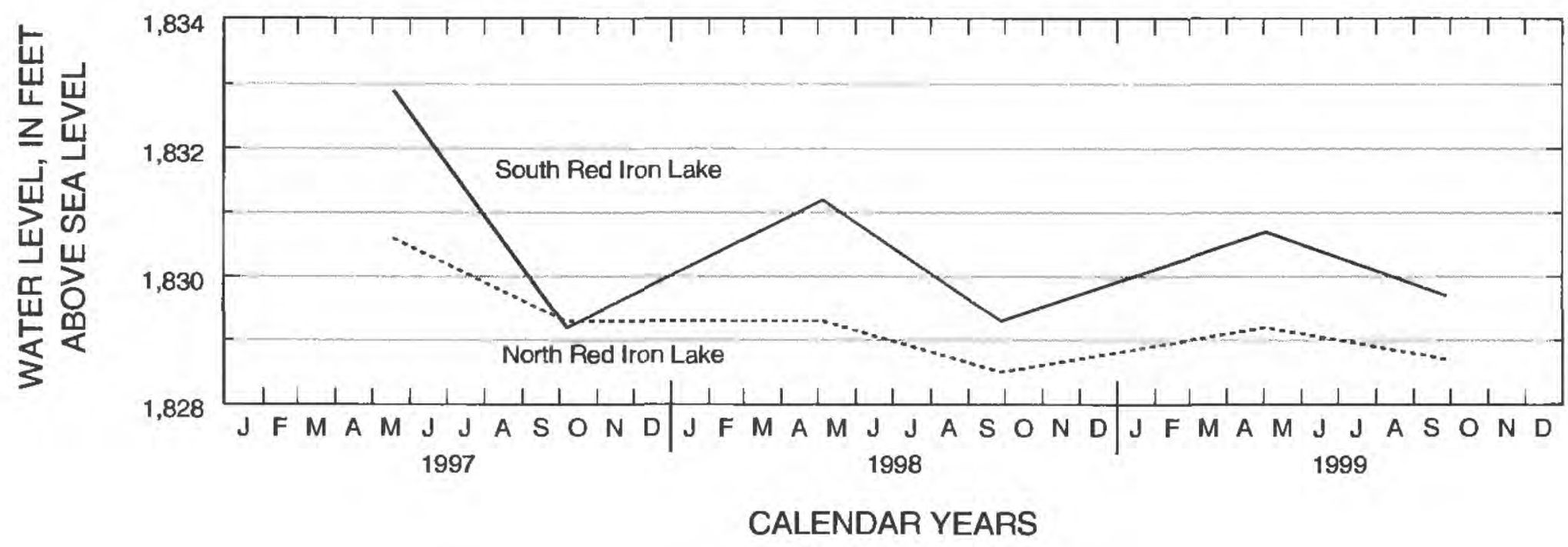

Figure 23. Hydrograph for North and South Red Iron Lakes in Marshall County.

Lake name: Spring Lake

County: Day

Extremes: October 11, 1983, to October 2, 1999: Highest, 1,804.03 feet, July 26, 1999; lowest, 1,785.0 feet, October 11, 1983.

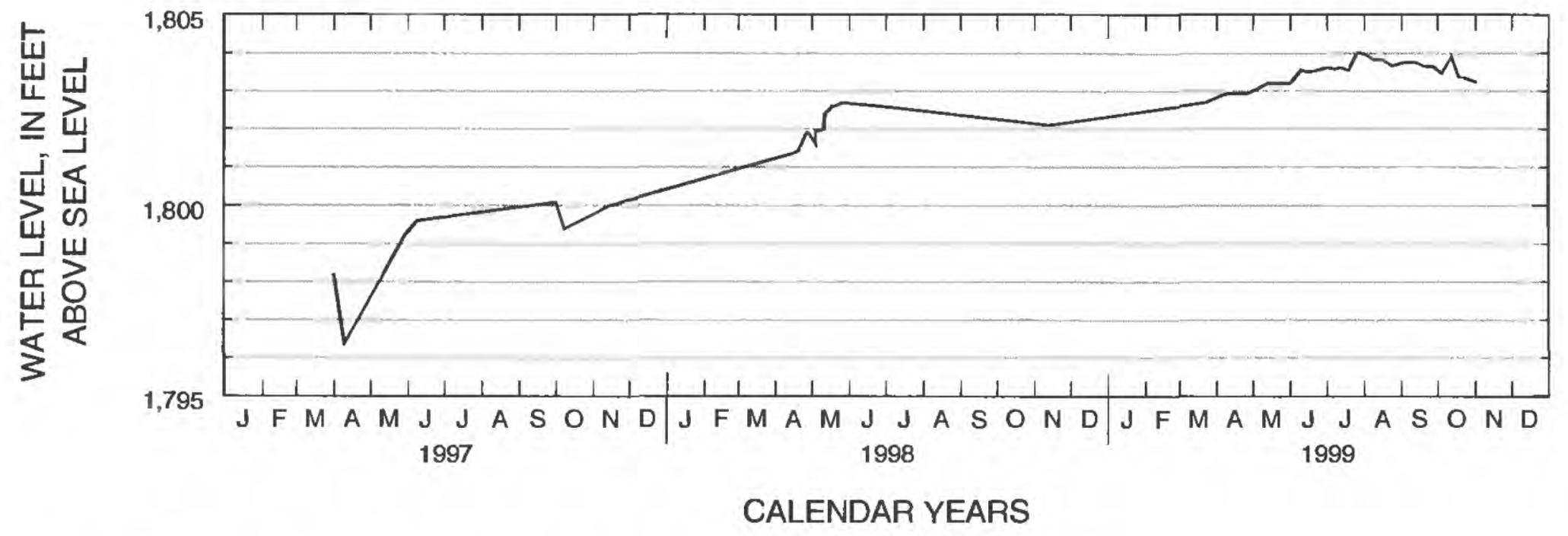

Figure 24. Hydrograph for Spring Lake in Day County. 
Lake name: Whitestone Lakes

County: Roberts

Extremes (Whitestone Lake North): June 7, 1984, to September 28, 1999: Highest, 2,015.3 feet, May 5, 1998; lowest, 2,010.4 feet, September 20, 1990.

Extremes (Whitestone Lake South): June 7, 1984, to September 28, 1999: Highest, 2,015.3 feet, May 5, 1998; lowest, 2,009.0 feet, September 17, 1992.

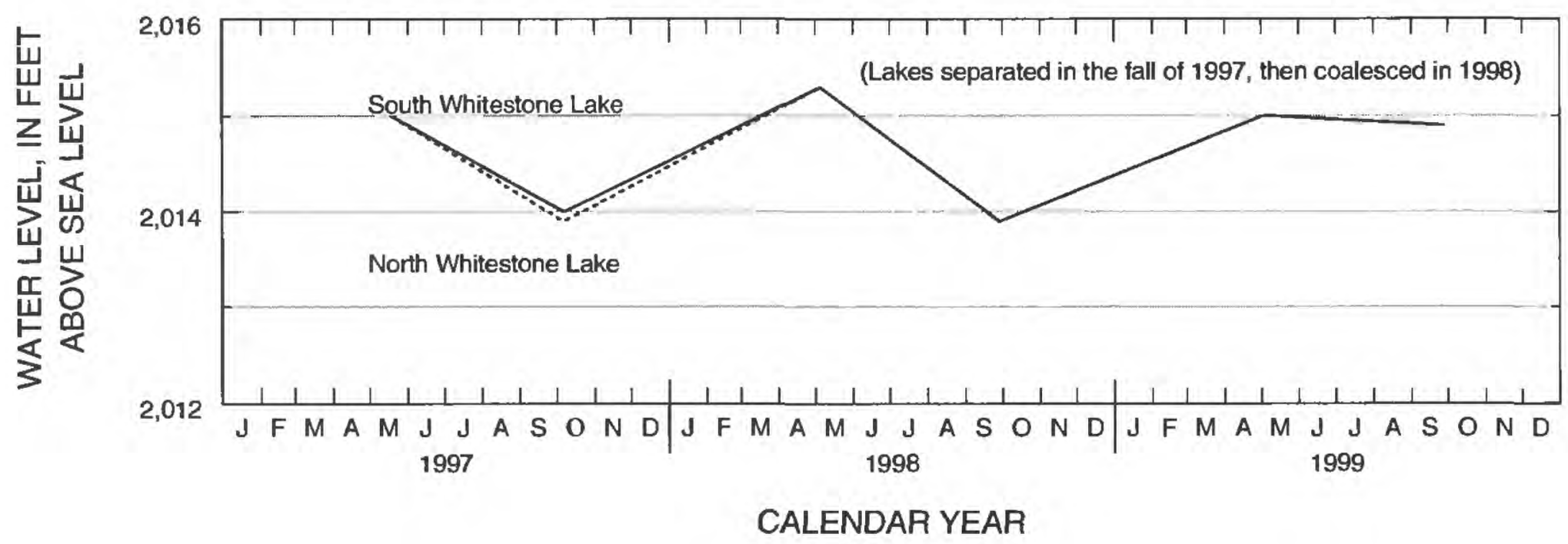

Figure 25. Hydrograph for North and South Whitestone Lakes in Roberts County. 


\section{Flows at Inlets/Outlets}

Miscellaneous flow measurements have been made by the USGS on inlets and outlets of some of the lakes within the Waubay Lakes Chain (fig. 26). Beginning in the 1990's, the lakes began to rise and coalesce. Three of the four flow measurement sites are within the study area. The fourth measurement site is outside the study area, but because of how the lakes have risen and coalesced, parts of the upstream (Rush) and downstream (Bitter) lakes are within the study area.

Table 14 summarizes the streamflow measurements and associated data at the four lake inlet/outlet locations.

Table 14. Miscellaneous streamflow measurements at lake inlets or outlets

$\left[\mathrm{ft}^{3} / \mathrm{s}\right.$, cubic feet per second; deg $\mathrm{C}$, degrees Celsius; $\mu \mathrm{S} / \mathrm{cm}$, microsiemens per centimeter; --, no data]

\begin{tabular}{|c|c|c|c|c|c|c|}
\hline Station & Date & Time & $\begin{array}{l}\text { Discharge, } \\
\text { instanta- } \\
\text { neous } \\
\left(\mathrm{ft}^{3} / \mathrm{s}\right)\end{array}$ & $\begin{array}{c}\text { Water } \\
\text { temperature } \\
(\operatorname{deg} C)\end{array}$ & $\begin{array}{c}\text { Air } \\
\text { temperature } \\
(\operatorname{deg} C)\end{array}$ & $\begin{array}{c}\text { Specific } \\
\text { conductance } \\
(\mu \mathrm{S} / \mathrm{cm})\end{array}$ \\
\hline \multirow{7}{*}{$\begin{array}{l}453014097165900 \\
\text { Pickerel Lake outfow near } \\
\text { Grenville, SD }\end{array}$} & $11-16-98$ & 1255 & 11 & 2.0 & 3.0 & 452 \\
\hline & & & & & & \\
\hline & $07-29-99$ & 1300 & 10 & 27.5 & 30.0 & 445 \\
\hline & 08-11-99 & 1605 & 3.2 & 24.0 & 27.0 & 451 \\
\hline & $08-25-99$ & 1600 & 3.2 & 25.5 & 26.0 & 440 \\
\hline & 09-08-99 & 1630 & 16 & 18.5 & 15.5 & 442 \\
\hline & 09-22-99 & 1415 & 9.4 & 16.0 & 20.0 & 447 \\
\hline \multirow{7}{*}{$\begin{array}{l}452357097165200 \\
\text { Campbell Slough outflow near } \\
\text { Waubay, SD }\end{array}$} & $11-16-98$ & 1135 & 11 & .5 & 3.0 & 429 \\
\hline & & & & & & \\
\hline & $07-29-99$ & 1150 & 7.9 & 27.0 & 29.5 & 402 \\
\hline & 08-11-99 & 1425 & 3.2 & 27.0 & 31.0 & 330 \\
\hline & $08-25-99$ & 1415 & .33 & 24.0 & 26.0 & 397 \\
\hline & 09-08-99 & 1510 & 5.7 & 18.5 & 19.0 & 380 \\
\hline & $09-22-99$ & 1215 & .02 & - & -- & -- \\
\hline \multirow{6}{*}{$\begin{array}{l}06479159 \\
\text { Blue Dog Lake inflow (Owens } \\
\text { Creek) near Waubay, SD }\end{array}$} & $07-29-99$ & 1040 & 5.8 & 25.0 & 28.0 & 566 \\
\hline & & 1100 & & & & 538 \\
\hline & $08-11-99$ & 1100 & 4.7 & 22.0 & 25.0 & 538 \\
\hline & $08-25-99$ & 1250 & 3.9 & 23.0 & 24.0 & 546 \\
\hline & 09-08-99 & 1355 & 15 & 15.5 & 14.5 & 552 \\
\hline & 09-22-99 & 1140 & 6.7 & 12.0 & 19.0 & 542 \\
\hline \multirow{7}{*}{$\begin{array}{l}451918097193700 \\
\text { Little Rush Lake outflow near } \\
\text { Waubay, SD }\end{array}$} & $07-21-99$ & 1340 & 67 & 25.0 & 28.0 & 448 \\
\hline & & & & & & \\
\hline & $07-29-99$ & 1505 & 52 & 30.0 & 36.0 & 430 \\
\hline & $08-11-99$ & 1040 & 44 & 22.5 & 25.0 & 463 \\
\hline & $08-25-99$ & 1040 & 46 & 22.0 & 22.0 & 515 \\
\hline & 09-08-99 & 1210 & 45 & 18.0 & 15.0 & 500 \\
\hline & 09-22-99 & 1030 & 39 & 15.0 & 14.0 & 559 \\
\hline
\end{tabular}




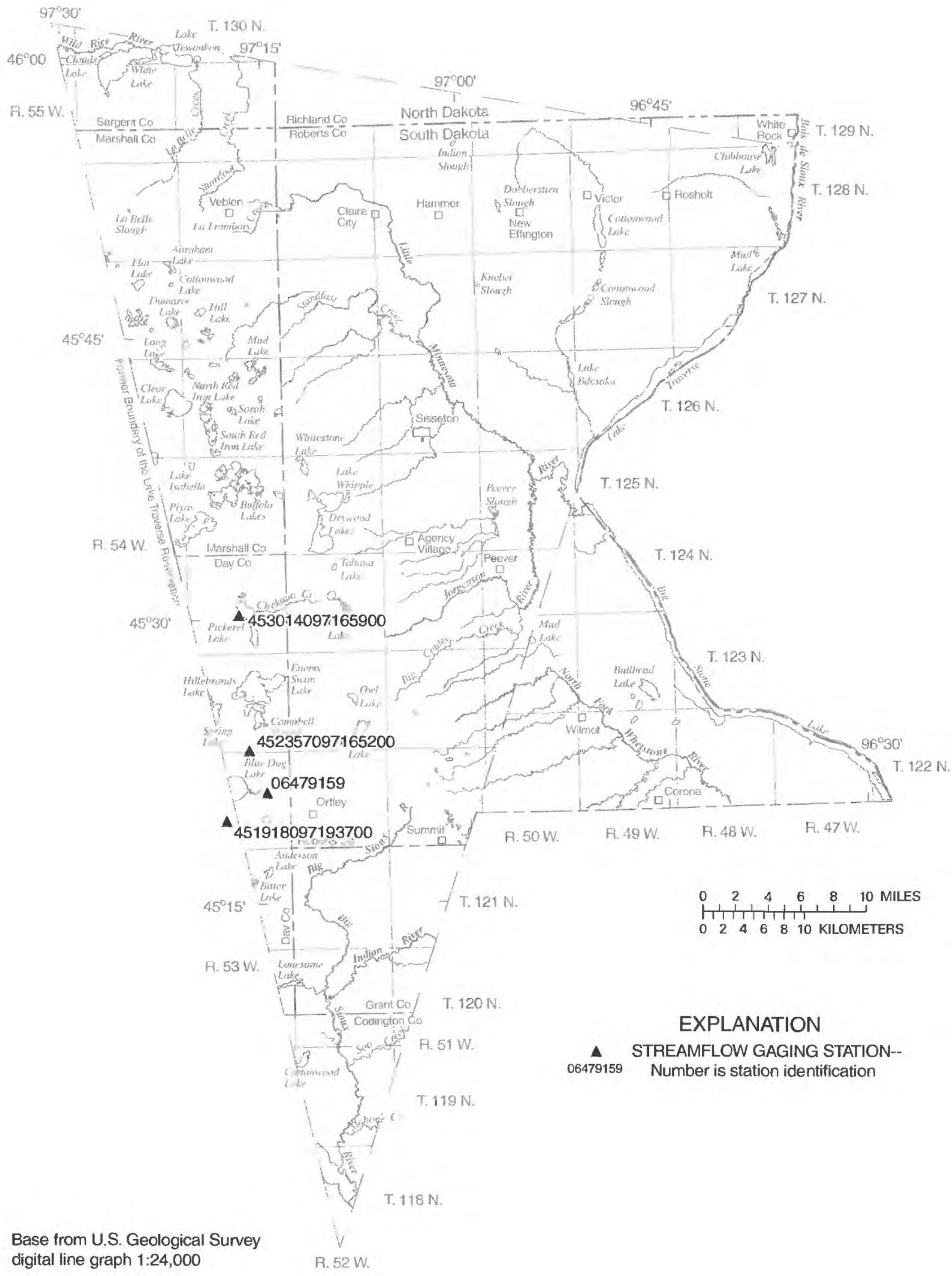

Figure 26. Location of miscellaneous streamflow measurements at lake inlets or outlets. 


\section{REFERENCES}

Armstrong, C.A., 1979, Ground-water basic data for Ransom and Sargent Counties, North Dakota, Part II-North Dakota Geological Survey Bulletin 69 and North Dakota State Water Commission County Ground Water Studies 31, 637 p.

-1982, Ground-water resources of Ransom and Sargent Counties, North Dakota, Part III-North Dakota Geological Survey Bulletin 69 and North Dakota State Water Commission County Ground Water Studies 31, $51 \mathrm{p}$.

Baker, C.H., Jr., 1966, Geology and ground water resources of Richland County, North Dakota, Part II-North Dakota Geological Survey Bulletin 46 and North Dakota State Water Commission County Ground Water Studies 7, $170 \mathrm{p}$.

Baker, C.H., Jr., and Paulson, Q.F., 1967, Geology and ground water resources of Richland County, North Dakota, Part III-North Dakota Geological Survey Bulletin 46 and North Dakota State Water Commission County Ground Water Studies 7, 48 p.

Carter, J.M., and Thompson, R.F., 1999, Selected hydrologic data through 1996 for the Lake Traverse Reservation/ Roberts County water-resources investigation in South Dakota: U.S. Geological Survey Open-File Report 98-647, 243 p.

Flint, R.F., 1955, Pleistocene geology of eastern South Dakota: U.S. Geological Survey Professional Paper 262, $173 \mathrm{p}$.
Friedman, L.C., and Erdmann, D.E., 1982, Quality assurance practices for the chemical and biological analyses of water and fluvial sediments: U.S. Geological Survey Techniques of Water-Resources Investigations, book 5 , chap. A6, $181 \mathrm{p}$.

Hansen, D.S., 1990, Water resources of Codington and Grant Counties, South Dakota: U.S. Geological Survey Water-Resources Investigations Report 89-4147, 47 p.

Horowitz, A.J., Demas, C.R., Fitzgerald, K.K., Miller, T.L., Rickert, D.A., 1994, U.S. Geological Survey protocol for the collection and processing of surface-water samples for the subsequent determination of inorganic constituents in filtered water: U.S. Geological Survey Open-File Report 94-539, 57 p.

Koch, N.C., 1975, Geology and water resources of Marshall County, South Dakota—Part I-Geology and water resources: South Dakota Geological Survey Bulletin 23, 76 p.

Leap, D.I., 1988, Geology and hydrology of Day County, South Dakota: South Dakota Geological Survey Bulletin 24, $117 \mathrm{p}$.

Ott, L.O., 1993, An introduction to statistical methods (4th ed.): Belmont, Calif., Wadsworth, Inc., 1,051 p.

Pritt, J.W., and Raese, J.W., eds., 1992, Quality assurance/ quality control manual- National Water Quality Laboratory: U.S. Geological Survey Open-File Report 92-495, $33 \mathrm{p}$.

Wood, W.W., 1976, Guidelines for collection and field analysis of ground-water samples for selected unstable constituents: U.S. Geological Survey Techniques of WaterResources Investigations, book 1, chap. D2, 24 p. 



\section{SUPPLEMENTAL INFORMATION}



Section A - Geologic logs 



\section{Test Holes Drilled and Observation Wells Installed, 1997-99}

Local number: 122N47W21AAAD

Other identifier: R2-98-13

Site identification number: 452218096331801

Date of construction: 06-15-98

Land surface altitude: 990 feet

Total depth: 122 feet

\begin{tabular}{|c|c|c|c|}
\hline Depth & $\begin{array}{l}\text { Thickness } \\
\text { (feet) }\end{array}$ & $\begin{array}{l}\text { Altitude } \\
\text { (feet) }\end{array}$ & Description \\
\hline $0-1$ & 1 & $990-989$ & Clay, black, silty; some sand, top few inches roadfill (topsoil) \\
\hline $1-10$ & 9 & $989-980$ & $\begin{array}{l}\text { Clay, tan to yellowish-brown, silty, sandy, pebbly; some light-gray mottling, oxidized } \\
\text { (till) }\end{array}$ \\
\hline $10-20$ & 10 & $980-970$ & Clay, dark-grayish-black, very shaley, silty, pebbly; unoxidized (till) \\
\hline $20-25$ & 5 & $970-965$ & Sand, grayish-brown, medium to coarse \\
\hline $25-32$ & 7 & $965-958$ & Clay, gray, silty, sandy, pebbly; unoxidzed (till) \\
\hline $32-84$ & 52 & $958-906$ & Clay, dark-gray, shaley; hard, greasy (Blue Hill Shale Member - Carlile Shale) \\
\hline $84-117$ & 33 & $906-873$ & $\begin{array}{l}\text { Clay, dark-grayish-brown; some white specks, calcareous (Fairport Shale Member - } \\
\text { Carlile Shale) }\end{array}$ \\
\hline $117-122$ & 5 & $873-868$ & Siltstone, brown with some white; hard, cherty, very calcareous (Greenhorn Limestone) \\
\hline Electric log & lable & & \\
\hline
\end{tabular}

Local number: 122N48W1DDDA

Other identifier: R2-98-19

Site identification number: 452400096370401

Date of construction: $06-23-98$

Land surface altitude: 1,000 feet

Total depth: 82 feet

\begin{tabular}{cccl}
\hline Depth & $\begin{array}{c}\text { Thicknees } \\
\text { (feet) }\end{array}$ & $\begin{array}{c}\text { Altitude } \\
\text { (feet) }\end{array}$ & \multicolumn{1}{c}{ Description } \\
\hline $0-2$ & 2 & $1,000-998$ & Clay, yellowish-brown, silty, sandy; oxidized, many cobbles (topsoil) \\
$2-10$ & 8 & $998-990$ & Gravel, medium to coarse; highly oxidized \\
$10-16$ & 6 & $990-984$ & Clay, brown, silty, sandy, pebbly; oxidized (till) \\
$16-28$ & 12 & $984-972$ & Clay, gray, silty, sandy, pebbly; unoxidized, sand lens from 22 to 24 feet (till) \\
$28-31$ & 3 & $972-969$ & Sand, gray, fine to medium \\
$31-42$ & 11 & $969-958$ & Clay, gray, silty, sandy, pebbly; harder than till interval above, unoxidized (till) \\
$42-82$ & 40 & $958-918$ & Clay, dark-gray, shaley; hard, greasy (Carlile Shale) \\
Electric $\log$ available & & \\
\hline
\end{tabular}


Local number: 122N48W6BBBA

Other identifier: R2-98-18

Site identification number: 452448096441701

Date of construction: $06-22-98$

Land surface altitude: 1,153 feet

Total depth: 402 feet

\begin{tabular}{|c|c|c|c|}
\hline Depth & $\begin{array}{l}\text { Thickness } \\
\text { (feet) }\end{array}$ & $\begin{array}{l}\text { Altitude } \\
\text { (feet) }\end{array}$ & Description \\
\hline $0-12$ & 12 & $1,153-1.141$ & Clay, tan to yellowish-brown, silty, sandy, pebbly; oxidized (till) \\
\hline $12-19$ & 7 & $1,141-1,134$ & Clay, gray, silty, sandy, pebbly; soft, unoxidized (till) \\
\hline $19-20$ & 1 & $1,134-1,133$ & Clay, tan, silty; oxidized \\
\hline $20-27$ & 7 & $1,133-1,126$ & Gravel, medium; some coarse, highly oxidized \\
\hline $27-53$ & 26 & $1,126-1,100$ & Clay, gray, silty, sandy, pebbly; soft, drills fast, unoxidized (till) \\
\hline $53-58$ & 5 & $1,100-1,095$ & Sand and gravel, coarse sand to fine gravel \\
\hline $58-100$ & 42 & $1,095-1,053$ & Clay, dark-gray, silty, sandy, pebbly; harder than till interval above, unoxidized (till) \\
\hline 100-108 & 8 & $1,053-1,045$ & Sand, gray, medium to coarse \\
\hline $108-114$ & 6 & $1,045-1,039$ & Clay, reddish-pink, silty, sandy, pebbly; oxidized (Hawk Creek Till) \\
\hline $114-136$ & 22 & $1,039-1,017$ & $\begin{array}{l}\text { Clay, dark-gray, silty, sandy, pebbly; hard, some silty, very calcareous clay, possibly } \\
\text { Niobrara Formation inclusions, unoxidized (till) }\end{array}$ \\
\hline $136-268$ & 132 & $1.017-885$ & $\begin{array}{l}\text { Clay, dark-gray, shaley: hard. greasy. concretion at } 194 \text { feet, sandy zones from } 249 \text { to } \\
255 \text { feet and } 263 \text { to } 268 \text { feet (Blue Hill Shale Member - Carlile Shale) }\end{array}$ \\
\hline 268-342 & 74 & $885-811$ & $\begin{array}{l}\text { Clay, dark-brownish-gray, shaley; gritty, hard, calcareous, few white specks (Fairport } \\
\text { Shale Member - Carlile Shale) }\end{array}$ \\
\hline $342-367$ & 25 & $811-786$ & $\begin{array}{l}\text { Clay, brown with white layers; hard, some siltstone, some chert, very calcareous } \\
\text { (Greenhorn Limestone) }\end{array}$ \\
\hline $367-402$ & 35 & 786-751 & Clay, dark-gray, shaley; hard, greasy, slightly calcareous (Graneros Shale) \\
\hline Electric log & lable & & \\
\hline
\end{tabular}


Local number: 122N48W10BABB

Other identifier: R2-98-20

Site identification number: 452352096402301

Date of construction: 6-23-98

Land surface altitude: 1,090 feet

Total depth: 142 feet

\begin{tabular}{cccl}
\hline Depth & $\begin{array}{c}\text { Thickness } \\
\text { (feet) }\end{array}$ & $\begin{array}{c}\text { Altitude } \\
\text { (feet) }\end{array}$ & \\
\hline $0-2$ & 2 & $1,090-1,088$ & Clay, black, silty (topsoil) \\
$2-20$ & 18 & $1,088-1,070$ & Clay, tan to yellowish-brown, silty, sandy, pebbly; oxidized (till) \\
$20-35$ & 15 & $1,070-1,055$ & Clay, gray, silty, sandy, pebbly; unoxidized (till) \\
$35-42$ & 7 & $1,055-1,048$ & Sand and gravel. coarse sand to fine gravel; many shale pebbles \\
$42-52$ & 10 & $1,048-1,038$ & Clay, gray, very silty; poor cuttings (lake sediment?) \\
$52-70$ & 18 & $1,038-1,020$ & Sand and gravel, brown, coarse sand to fine gravel, silty; some clay \\
$70-75$ & 5 & $1,020-1,015$ & Clay, gray, very silty; poor cuttings (lake sediment?) \\
$75-83$ & 8 & $1,015-1,007$ & Sand, fine to medium, silty; some clay \\
$83-92$ & 9 & $1,007-998$ & Clay, grayish-pink, silty, sandy, pebbly; oxidized (Hawk Creek Till) \\
$92-102$ & 10 & $998-988$ & Sand and gravel, coarse sand to fine gravel \\
$102-113$ & 11 & $988-977$ & Clay, gray, very silty, sandy, pebbly; unoxidized (till) \\
$113-142$ & 29 & $977-948$ & Clay, gray, shaley; hard, greasy (Carlile Shale) \\
Electric log available & & \\
\hline
\end{tabular}


Local number: 122N48W13DDDD

Other identifier: R2-98-14

Site identification number: 452211096365901

Date of construction: 6-16-98

Land surface altitude: 1,086 feet

Total depth: 270 feet

\begin{tabular}{|c|c|c|c|}
\hline Depth & $\begin{array}{l}\text { Thickness } \\
\text { (feet) }\end{array}$ & $\begin{array}{c}\text { Altitude } \\
\text { (feet) }\end{array}$ & Description \\
\hline $0-19$ & 19 & $1,086-1,067$ & $\begin{array}{l}\text { Clay, yellowish-brown, silty, sandy, pebbly; hard, oxidized. turns dark brown with depth } \\
\text { (till) }\end{array}$ \\
\hline $19-35$ & 16 & $1,067-1,051$ & Clay, gray, silty, sandy, pebbly; unoxidized (till) \\
\hline $35-39$ & 4 & $1,051-1,047$ & Sand, brown, medium to coarse \\
\hline $39-45$ & 6 & $1,047-1,041$ & Clay, gray, silty, sandy, pebbly; unoxidized (till) \\
\hline $45-51$ & 6 & $1,041-1,035$ & Sand, medium to coarse; some fine gravel \\
\hline $51-56$ & 5 & $1,035-1,030$ & Clay, gray, silty, sandy, pebbly; unoxidized, darker than till intervals above (till) \\
\hline $56-84$ & 28 & $1,030-1,002$ & $\begin{array}{l}\text { Clay, reddish-brown, silty, very sandy, pebbly; hard, oxidized, turning grayish-pink with } \\
\text { depth (Hawk Creek Till) }\end{array}$ \\
\hline $84-88$ & 4 & $1,002-998$ & Clay, gray, silty; unoxidized (lake sediment) \\
\hline 88-102 & 14 & 998-984 & $\begin{array}{l}\text { Sand and gravel, medium to coarse sand to fine to medium gravel; various rock types, } \\
\text { many shale pebbles }\end{array}$ \\
\hline $102-137$ & 35 & 984-949 & Clay, gray to dark-gray, silty, sandy, pebbly; unoxidized (till) \\
\hline 137-192 & 55 & $949-894$ & Clay, dark-gray, shaley; hard, greasy (Blue Hill Shale Member - Carlile Shale) \\
\hline $192-268$ & 76 & $894-818$ & $\begin{array}{l}\text { Clay, dark-brownish-gray; gritty, some white specks, calcareous (Fairport Shale } \\
\text { Member - Carlile Shale) }\end{array}$ \\
\hline $268-270$ & 2 & $818-816$ & $\begin{array}{l}\text { Siltstone, brown; with white clay beds, some chert, very calcareous (Greenhorn } \\
\text { Limestone) }\end{array}$ \\
\hline ic 1 & & & \\
\hline
\end{tabular}


Local number: 122N48W13DDDD2

Other identifier: R2-98-15

Site identification number: 452211096365902

Date of construction: $06-16-98$

Land surface altitude: 1,086 feet

Total depth: 102 feet

\begin{tabular}{cccc}
\hline Depth & $\begin{array}{c}\text { Thickness } \\
\text { (feet) }\end{array}$ & $\begin{array}{c}\text { Altitude } \\
\text { (feet) }\end{array}$ & Description \\
\hline $0-18$ & 18 & $1,086-1,068$ & $\begin{array}{c}\text { Clay, yellowish-brown, silty, sandy, pebbly; hard, oxidized, turns dark-brown with } \\
\text { depth (till) }\end{array}$ \\
$18-36$ & 18 & $1,068-1,050$ & Clay, gray, silty, sandy, pebbly; unoxidized (till) \\
$36-42$ & 6 & $1,050-1,044$ & Sand, brown, medium to coarse \\
$42-57$ & 15 & $1,044-1,029$ & Clay, gray, silty, sandy, pebbly; unoxidized (till) \\
$57-60$ & 3 & $1,029-1,026$ & Sand, reddish-brown, medium \\
$60-84$ & 24 & $1,026-1,002$ & Clay, reddish-pink, silty, very sandy, pebbly; oxidized (Hawk Creek Till) \\
$84-100$ & 16 & $1,002-986$ & Sand and gravel, medium to coarse sand to fine to medium gravel \\
$100-102$ & 2 & $986-984$ & Clay, gray, silty, sandy, pebbly; unoxidized (till) \\
Well screened from 90 to 100 feet; 2 -inch PVC & screen and casing \\
\hline
\end{tabular}


Local number: 122N48W16DDDC

Other identifier: R2-98-16

Site identification number: 452210096404701

Date of construction: $06-17-98$

Land surface altitude: 1,118 feet

Total depth: 162 feet

\begin{tabular}{|c|c|c|c|}
\hline Depth & $\begin{array}{l}\text { Thickness } \\
\text { (feet) }\end{array}$ & $\begin{array}{l}\text { Altitude } \\
\text { (feet) }\end{array}$ & Description \\
\hline $0-1$ & 1 & $1,118-1,117$ & Clay, black, silty (topsoi) \\
\hline $1-30$ & 29 & $1,117-1,088$ & $\begin{array}{l}\text { Clay, tan to yellowish-brown. silty, sandy, pebbly; oxidized, turns dark brown with } \\
\text { depth (till) }\end{array}$ \\
\hline $30-33$ & 3 & $1,088-1,085$ & Sand and gravel, coarse sand to fine gravel; some cobbles near base of interval \\
\hline $33-35$ & 2 & $1,085-1,083$ & Clay, brown, silty, sandy, pebbly; oxidized (till) \\
\hline $35-49$ & 14 & $1,083-1,069$ & Clay, gray, silty, sandy, pebbly; unoxidized (till) \\
\hline $49-76$ & 27 & $1,069-1,042$ & $\begin{array}{l}\text { Clay, gray, silty, sandy, pebbly; unoxidized, E-log signature differs from above interval } \\
\text { (till) }\end{array}$ \\
\hline $76-80$ & 14 & $1,042-1.038$ & Sand, gray, fine to medium \\
\hline $80-83$ & 3 & $1,038-1,035$ & Clay, gray, silty, sandy, pebbly; unoxidized (till) \\
\hline $83-85$ & 2 & $1,035-1,033$ & Sand, gray, fine to medium \\
\hline $85-98$ & 13 & $1,033-1,020$ & Clay, brown, silty, sandy, pebbly; oxidized, rocks at 93, and 96 to 98 feet (till) \\
\hline $98-106$ & 8 & $1,020-1,012$ & Clay, gray, shaley; hard, greasy, unoxidized (shale inclusion) \\
\hline $106-130$ & 24 & $1,012-988$ & Clay, dark-gray, silty, sandy, pebbly; unoxidized, hard, shaley (till) \\
\hline $130-133$ & 3 & $988-985$ & Sand and gravel, coarse sand to fine gravel \\
\hline $133-162$ & 30 & $985-956$ & $\begin{array}{l}\text { Clay, dark-gray, shaley; hard, greasy, noncalcareous (Blue Hill Shale Member - Carlile } \\
\text { Shale) }\end{array}$ \\
\hline Electric lo & & & \\
\hline
\end{tabular}


Local number: 122N49W16DDDC

Other identifier: R2-97-47

Site identification number: 452212096480801

Date of construction: $08-27-97$

Land surface altitude: 1,165 feet

Total depth: 182 feet

\begin{tabular}{cccl}
\hline Depth & $\begin{array}{c}\text { Thickness } \\
\text { (feet) }\end{array}$ & $\begin{array}{c}\text { Altitude } \\
\text { (feet) }\end{array}$ & Description \\
\hline $0-2$ & 2 & $1,165-1,163$ & Clay, black, silty (topsoil) \\
$2-12$ & 10 & $1,163-1,153$ & Clay, yellowish-brown, silty, sandy, pebbly; oxidized (till) \\
$12-26$ & 14 & $1,153-1,139$ & Clay, dark-gray, silty, sandy, pebbly; some shale inclusions, unoxidized (till) \\
$26-55$ & 29 & $1,139-1,110$ & $\begin{array}{c}\text { Sand, gray, fine to medium; grading to fine to medium gravel, contains abundant black } \\
\text { shale pebbles }\end{array}$ \\
$55-60$ & 5 & $1,110-1,105$ & Clay, gray, silty, sandy (till?) \\
$60-65$ & 5 & $1,105-1,100$ & Sand and gravel \\
$65-78$ & 13 & $1,100-1,087$ & Clay, light-gray, shaley; hard, greasy (Pierre Shale) \\
$78-148$ & 70 & $1,087-1,017$ & Clay, dark-gray, shaley; hard, greasy \\
$148-150$ & 2 & $1,017-1,015$ & Claystone; very hard, no sample (Niobrara Formation?) \\
$150-182$ & 32 & $1,015-983$ & Clay, dark-gray, shaley; hard, greasy (Carlile Shale) \\
\hline Electric log available & & \\
\hline
\end{tabular}


Local number: 122N49W24DAAA

Other identifier: R2-98-17

Site identification number: 452144096442301

Date of construction: 06-17-98

Land surface altitude: 1,130 feet

Total depth: 142 feet

\begin{tabular}{cccl}
\hline Depth & $\begin{array}{c}\text { Thickness } \\
\text { (feet) }\end{array}$ & $\begin{array}{c}\text { Altitude } \\
\text { (feet) }\end{array}$ & \multicolumn{1}{c}{ Description } \\
\hline $0-2$ & 2 & $1,130-1,128$ & Clay, black, silty (topsoil) \\
$2-15$ & 13 & $1,128-1,115$ & Clay, yellowish-brown, silty, sandy, pebbly; oxidized (till) \\
$15-24$ & 9 & $1,115-1,106$ & Clay, dark-gray, silty, sandy, pebbly; unoxidized (till) \\
$24-29$ & 5 & $1,106-1,101$ & Sand and gravel, coarse sand to fine gravel \\
$29-41$ & 12 & $1,101-1,089$ & Clay, reddish-brown to reddish-gray, silty, sandy, pebbly; oxidized (Hawk Creek Till) \\
$41-50$ & 9 & $1,089-1,080$ & Sand, brown, medium to coarse \\
$50-62$ & 12 & $1,080-1,068$ & Clay, dark-gray to black, shaley; some light-gray bentonite layers (Sharon Springs \\
& & Member - Pierre Shale) & Clay, light-gray to gray, shaley; greasy, noncalcareous (Carlile Shale) \\
$62-142$ & 80 & $1,068-988$ & \\
\hline
\end{tabular}

Local number: 122N50W13DDDC

Other identifier: R2-97-46

Site identification number: 452210096514701

Date of construction: $08-26-97$

Land surface altitude: $1,224.6$ feet

Total depth: 82 feet

\begin{tabular}{cccl}
\hline Depth & $\begin{array}{c}\text { Thickness } \\
\text { (feet) }\end{array}$ & $\begin{array}{c}\text { Altitude } \\
\text { (feet) }\end{array}$ & Description \\
\hline $0-1$ & 1 & $1,224.6-1,223.6$ & Clay, black, silty (topsoil) \\
$1-13$ & 12 & $1,223.6-1,211.6$ & Clay, yellowish-brown, silty, sandy, pebbly; oxidized (till) \\
$13-18$ & 5 & $1,211.6-1,206.6$ & Sand, brown, coarse; some gravel \\
$18-30$ & 12 & $1,206.6-1,194.6$ & Clay, gray, silty, sandy, pebbly; unoxidized, rock at 17 feet (till) \\
$30-82$ & 52 & $1,194.6-1,142.6$ & Sand and gravel, coarse sand to fine gravel; some coarse gravel with depth \\
Well screened from 54 to 74 feet; 2-inch PVC casing and screen used. For electric-log see R2-94-62 \\
\hline
\end{tabular}


Local number: 122N50W17CCCC

Other identifier: R2-98-25

Site identification number: 452213096575001

Date of construction: 06-30-98

Land surface altitude: 1,475 feet

Total depth: 202 feet

\begin{tabular}{|c|c|c|c|}
\hline Depth & $\begin{array}{l}\text { Thickness } \\
\text { (feet) }\end{array}$ & $\begin{array}{l}\text { Altitude } \\
\text { (feet) }\end{array}$ & Description \\
\hline $0-8$ & 8 & $1,475-1,467$ & Gravel, medium to very coarse: some large boulders, some coarse sand \\
\hline 8-13 & 5 & $1,467-1,462$ & Clay, brown, silty, sandy, pebbly; oxidized (till) \\
\hline $13-36$ & 8 & $1,462-1,439$ & Clay, gray, silty, sandy, pebbly; soft, unoxidized, rock from 28 to 30 feet (till) \\
\hline $36-58$ & 22 & $1,439-1,417$ & Gravel, fine to medium \\
\hline $58-60$ & 2 & $1,417-1,415$ & Clay, dark-brown, silty, sandy; some pebbles (soil horizon) \\
\hline $60-91$ & 31 & $1,415-1,384$ & $\begin{array}{l}\text { Clay, light-gray, silty, sandy, pebbly; unoxidized, sand lens from } 72 \text { to } 75 \text { feet, } 77 \text { to } \\
79 \text { feet, and } 89 \text { to } 91 \text { feet (till) }\end{array}$ \\
\hline $91-100$ & 9 & $1,384-1,375$ & Clay, gray, shaley; greasy (shale inclusion) \\
\hline $100-118$ & 18 & $1,375-1,357$ & $\begin{array}{l}\text { Clay, gray, silty, sandy, pebbly; unoxidized, rocks from } 100 \text { to } 101 \text { feet, gravel lens from } \\
105 \text { to } 108 \text { feet (till) }\end{array}$ \\
\hline $118-126$ & 8 & $1,357-1,349$ & Clay, dark-gray, shaley; hard, greasy, noncalcareous (shale inclusion) \\
\hline $126-130$ & 4 & $1,349-1,345$ & Sand and gravel, coarse sand to fine to medium gravel \\
\hline $130-202$ & 72 & $1,345-1273$ & Clay, dark-gray, shaley; hard, greasy, noncalcareous (Pierre Shale? or till?) \\
\hline Electric log & ble & & \\
\hline
\end{tabular}


Local number: 122N50W17DDDC

Other identifier: R2-99-14

Site identification number: 452213096564601

Date of construction: 08-30-99

Land surface altitude: 1,390 feet

Total depth: 364 feet

\begin{tabular}{|c|c|c|c|}
\hline Depth & $\begin{array}{l}\text { Thickness } \\
\text { (feet) }\end{array}$ & $\begin{array}{l}\text { Altitude } \\
\text { (feet) }\end{array}$ & Description \\
\hline $0-1$ & 1 & $1,390-1,389$ & Clay, black, silty (topsoil) \\
\hline $1-2$ & 1 & $1,389-1,388$ & Gravel, brown coarse; some sand, rock at 2 feet, oxidized \\
\hline $2-10$ & 8 & $1,388-1,380$ & Clay, yellowish-brown, silty, sandy, pebbly; oxidized (till) \\
\hline $10-52$ & 42 & $1,380-1,338$ & Clay, gray, very silty, sandy, pebbly; soft, greasy, unoxidized (till) \\
\hline $52-54$ & 2 & $1,338-1,336$ & Sand, fine to medium \\
\hline $54-63$ & 9 & $1,336-1,327$ & Clay, dark-gray, very silty; hard, calcareous (lake sediment) \\
\hline $63-68$ & 5 & $1,327-1,322$ & Sand, fine to medium \\
\hline $68-70$ & 2 & $1,322-1,320$ & Clay, gray; hard (lake sediment) \\
\hline 70-74 & 4 & $1,320-1,316$ & Gravel, fine \\
\hline $74-210$ & 136 & $1,316-1,180$ & $\begin{array}{l}\text { Clay, gray, silty, sandy, pebbly, very shaley; gravel lens from } 82 \text { to } 84 \text { feet, rock at } \\
158 \text { feet, E-log change at } 113 \text { feet. unoxidized (till) }\end{array}$ \\
\hline $210-300$ & 90 & $1,180-1,090$ & $\begin{array}{l}\text { Clay. gray. silty, sandy, pebbly; less shaley than above interval, many shale inclusions, } \\
\text { unoxidized (till) }\end{array}$ \\
\hline $300-344$ & 44 & $1,090-1,046$ & Sand, fine; hard, cemented, rig chatter, clay layer at 327 feet \\
\hline $344-356$ & 12 & $1,046-1,034$ & Clay, light-gray, silty; gritty, very calcareous (Niobrara Formation) \\
\hline $356-364$ & 8 & $1,034-1,026$ & Clay, gray, shaley; soft, greasy, noncalcareous, concretion at 363 feet (Carlile Shale) \\
\hline Electric log & lable & & \\
\hline
\end{tabular}


Local number: 122N51W4DDDD R

Other identifier: R2-98-32

Site identification number: 452210097023101

Date of construction: $08-10-98$

Land surface altitude: 1,960 feet

Total depth: 862 feet

\begin{tabular}{|c|c|c|c|}
\hline Depth & $\begin{array}{l}\text { Thickness } \\
\text { (feet) }\end{array}$ & $\begin{array}{l}\text { Altitude } \\
\text { (feet) }\end{array}$ & Description \\
\hline $0-10$ & 10 & $1,960-1,950$ & Clay, yellowish-brown, silty, sandy, pebbly; oxidized (till) \\
\hline $10-22$ & 12 & $1,950-1,938$ & Sand and gravel, oxidized \\
\hline $22-50$ & 18 & $1,938-1,910$ & Clay, gray, silty, sandy, pebbly; unoxidized (till) \\
\hline $50-65$ & 15 & $1,910-1,895$ & Sand and gravel, coarse sand to fine gravel; some coarse gravel, some clay layers \\
\hline $65-69$ & 4 & $1,895-1,891$ & Clay, tan, very silty; greasy (loess) \\
\hline $69-72$ & 3 & $1,891-1,888$ & Clay, grayish-brown to black, very silty; greasy (loess) \\
\hline $72-77$ & 5 & $1,888-1,883$ & Clay, yellowish-brown, silty, very sandy, pebbly; highly oxidized (till) \\
\hline $77-92$ & 15 & $1,883-1,868$ & $\begin{array}{l}\text { Clay, grayish-brown, silty, sandy, pebbly; rocks at } 86,89 \text {, and } 90 \text { feet, sand lens from } 82 \\
\text { to } 86 \text { feet, partially oxidized (till) }\end{array}$ \\
\hline $92-98$ & 6 & $1,868-1,862$ & Sand and gravel, coarse sand to fine gravel \\
\hline $98-110$ & 12 & $1,862-1,850$ & Clay, gray, very silty; soft. unoxidized, some sand layers (lake sediment) \\
\hline $110-162$ & 52 & $1,850-1,798$ & $\begin{array}{l}\text { Clay, gray, silty, sandy, pebbly; rocks from } 146 \text { to } 148 \text { feet and from } 161 \text { to } 162 \text { feet, } \\
\text { unoxidized (till) }\end{array}$ \\
\hline $162-196$ & 34 & $1,798-1,764$ & $\begin{array}{l}\text { Clay, yellowish-brown, silty, sandy, pebbly, cobbly; oxidized, very cobbly from } 162 \text { to } \\
178 \text { feet, rocks at } 175 \text { to } 187 \text { feet (till) }\end{array}$ \\
\hline $196-204$ & 8 & $1,764-1,756$ & Clay, grayish-brown, silty, sandy, pebbly; greasy, partially oxidized (till) \\
\hline 204-244 & 40 & $1,756-1,716$ & Clay, dark-gray, silty, sandy, pebbly; some hints of oxidation, greasy (till) \\
\hline $244-256$ & 12 & $1,716-1,704$ & Sand, coarse; cemented, rig chatter, poor sample \\
\hline $256-358$ & 102 & $1,704-1,602$ & $\begin{array}{l}\text { Clay, gray, silty, sandy, pebbly; many cobbles from } 282 \text { to } 296 \text { feet, gravel lens from } \\
351 \text { to } 354 \text { feet and } 356 \text { to } 358 \text { feet (till) }\end{array}$ \\
\hline $358-422$ & 64 & $1,602-1,538$ & $\begin{array}{l}\text { Clay, light-gray with some greenish-brown and gray, silty, sandy, pebbly, shaley; } \\
\text { oxidized and unoxidized zones, rocks at } 397 \text { and } 406 \text { feet, many shale inclusions (till) }\end{array}$ \\
\hline $422-454$ & 32 & $1,538-1.506$ & Sand and gravel, coarse sand to fine gravel; drills fast, rig chatter, cemented \\
\hline 454-506 & 12 & $1,506-1,454$ & $\begin{array}{l}\text { Clay, gray, silty, sandy, pebbly, shaley; greasy, with shale inclusions. rocks at } 472.492 \text {, } \\
\text { and } 504 \text { feet, unoxidized (till) }\end{array}$ \\
\hline $506-513$ & 7 & $1,454-1,447$ & Clay, dark-gray, shaley; greasy (shale block) \\
\hline $513-842$ & 329 & $1,447-1,118$ & $\begin{array}{l}\text { Clay, gray, silty, sandy, pebbly, shaley; greasy, with shale inclusions, rocks at } 617,759 \text {, } \\
765 \text {, and } 767 \text { feet, unoxidized, very poor cuttings (till) }\end{array}$ \\
\hline $842-862$ & 20 & $1,118-1.098$ & $\begin{array}{l}\text { Clay, dark-gray to black. shaley; very hard, greasy, very poor cuttings (Pierre Shale? or } \\
\text { till?) }\end{array}$ \\
\hline \multicolumn{4}{|c|}{ Electric log available } \\
\hline
\end{tabular}


Local number: 122N51W4DDDD2 R

Other identifier: R2-99-11

Site identification number: 452212097022702

Date of construction: $08-17-99$

Land surface altitude: $1,962.2$ feet

Total depth: 452 feet

\begin{tabular}{|c|c|c|c|}
\hline Depth & $\begin{array}{c}\text { Thickness } \\
\text { (feet) }\end{array}$ & $\begin{array}{l}\text { Altitude } \\
\text { (feet) }\end{array}$ & Description \\
\hline $0-11$ & 11 & $1,962.2-1,951.2$ & Clay, yellowish-brown, silty, sandy, pebbly; oxidized (till) \\
\hline $11-22$ & 11 & $1,951 \cdot 2-1,940.2$ & Sand and gravel; oxidized \\
\hline $22-53$ & 31 & $1,940.2-1,909.2$ & Clay, gray, silty, sandy, pebbly; rocks at 32,41 , and 46 feet, unoxidized (till) \\
\hline $53-65$ & 12 & $1,909.2-1,897.2$ & Sand and gravel, coarse sand to fine gravel; some coarse gravel, some clay layers \\
\hline $65-69$ & 4 & $1,897.2-1,893.2$ & Clay, tan, very silty; greasy (loess) \\
\hline $69-72$ & 3 & $1,893.2-1,890.2$ & Clay, grayish-brown to black, very silty; greasy (loess) \\
\hline $72-77$ & 5 & $1,890.2+1,885.2$ & Clay, yellowish-brown, silty, very sandy, pebbly; highly oxidized (till) \\
\hline $77-93$ & 26 & $1,885.2-1,869.2$ & $\begin{array}{l}\text { Clay, grayish-brown, silty, sandy, pebbly; rocks at } 86,89 \text {, and } 90 \text { feet, sand lens from } 82 \\
\text { to } 86 \text { feet, partially oxidized (till) }\end{array}$ \\
\hline 93-98 & 5 & $1,869.2-1,864.2$ & Sand and gravel, coarse sand to fine gravel \\
\hline $98-110$ & 12 & $1,864.2-1,852.2$ & Clay, gray, very silty; soft, unoxidized, some sand layers (lake sediment) \\
\hline $110-164$ & 54 & $1,852.2-1,798.2$ & $\begin{array}{l}\text { Clay, gray, silty, sandy, pebbly; rocks from } 146 \text { to } 148 \text { feet and from } 161 \text { to } 162 \text { feet, } \\
\text { unoxidized (till) }\end{array}$ \\
\hline $164-196$ & 32 & $1,798.2-1,766.2$ & $\begin{array}{l}\text { Clay, yellowish-brown, silty, sandy, pebbly, cobbly; oxidized, very cobbly from } 162 \text { to } \\
178 \text { feet, rocks at } 175 \text { and } 187 \text { feet (till) }\end{array}$ \\
\hline $196-204$ & 8 & $1,766.2-1,758.2$ & Clay, grayish-brown, silty, sandy, pebbly; greasy, partially oxidized (till) \\
\hline 204-242 & 38 & $1,758.2-1,720.2$ & Clay, dark-gray, silty, sandy, pebbly; some hints of oxidation, greasy (till) \\
\hline $242-255$ & 13 & $1,720.2-1,707.2$ & Sand, coarse; cemented, rig chatter, poor sample \\
\hline 255-349 & 16 & $1,707.2-1,613.2$ & Clay, gray, silty, sandy, pebbly; unoxidized (till) \\
\hline $349-353$ & 4 & $1,613.2-1,609.2$ & Sand; hard, cemented \\
\hline $353-419$ & 66 & $1,609.2-1,543.2$ & Clay, dark-gray, silty, sandy, pebbly, shaley; unoxidized, many shale inclusions (till) \\
\hline $419-451$ & 32 & $1,543.2-1,511.2$ & Sand and gravel, coarse sand to fine gravel; drills fast, rig chatter, cemented \\
\hline $451-452$ & 1 & $1,511.2-1,510.2$ & Clay, gray, silty, sandy, pebbly, shaley; greasy, with shale inclusions (till) \\
\hline
\end{tabular}


Local number: 122N51W27BBBB R

Other identifier: R2-98-26

Site identification number: 451936097022601

Date of construction: $07-14-98$

Land surface altitude: 2,025 feet

Total depth: 627 feet

\begin{tabular}{|c|c|c|c|}
\hline Depth & $\begin{array}{l}\text { Thickness } \\
\text { (feet) }\end{array}$ & $\begin{array}{l}\text { Altitude } \\
\text { (feet) }\end{array}$ & Description \\
\hline $0-2$ & 2 & $2,025-2,023$ & Clay, black, silty (topsoil) \\
\hline $2-8$ & 6 & $2,023-2,017$ & Clay, brown, silty, sandy, pebbly; oxidized (till) \\
\hline $8-11$ & 3 & $2,017-2,014$ & Sand and gravel, cobbly \\
\hline $11-41$ & 30 & 2,014-1,984 & Clay, gray, silty, sandy, pebbly; soft, large cuttings, unoxidized (till) \\
\hline $41-70$ & 29 & $1,984-1,955$ & $\begin{array}{l}\text { Clay, brown, silty, sandy, pebbly; hard, turns grayish-brown with depth, some cobbles, } \\
\text { rock at } 66 \text { feet, oxidized (till) }\end{array}$ \\
\hline $70-80$ & 10 & $1,955-1,945$ & $\begin{array}{l}\text { Sand and gravel, coarse sand to fine gravel, clayey; this interval is not encountered in } \\
\text { second test hole at site }\end{array}$ \\
\hline $80-159$ & 79 & $1,945-1,866$ & Clay, gray, silty, sandy, pebbly; unoxidized, rock at 107 feet (till) \\
\hline $159-162$ & 3 & $1,866-1,863$ & Gravel, fine to coarse, cobbly \\
\hline $162-243$ & 81 & $1,863-1,782$ & $\begin{array}{l}\text { Clay, gray, silty, sandy, pebbly; unoxidized, gravelly from } 168 \text { to } 177 \text { feet, gravel lens } \\
\text { from } 181 \text { to } 184 \text { feet and } 192 \text { to } 199 \text { feet (till) }\end{array}$ \\
\hline $243-278$ & 35 & $1,782-1,747$ & Clay, gray, very silty; unoxidized (lake sediment) \\
\hline $278-298$ & 20 & $1,747-1,727$ & Gravel, coarse \\
\hline $298-426$ & 128 & $1,727-1,599$ & $\begin{array}{l}\text { Clay, gray, silty, sandy, pebbly; unoxidized, sand lens from } 320 \text { to } 325 \text { feet, some shale } \\
\text { inclusions, hard spots at } 314 \text { and } 316 \text { feet (till) }\end{array}$ \\
\hline $426-470$ & 44 & $1,599-1,555$ & $\begin{array}{l}\text { Clay, gray, silty, sandy, pebbly; unoxidized, E-log signature differs from above interval, } \\
\text { gravel lens at } 442 \text { and } 454 \text { feet, rocks at } 427,447 \text {, and } 458 \text { feet, very poor cuttings } \\
\text { (till) }\end{array}$ \\
\hline $470-496$ & 26 & $1,555-1,529$ & Sand(?), very hard, cemented(?), some oxidized till noticed, very poor cuttings \\
\hline $496-528$ & 32 & $1,529-1,497$ & $\begin{array}{l}\text { Clay, gray, silty, sandy, pebbly, shaley; unoxidized, rocks from } 525 \text { to } 528 \text { feet, very } \\
\text { poor cuttings (till) }\end{array}$ \\
\hline $528-627$ & 99 & $1,497-1,398$ & Clay, dark-gray, shaley; hard, greasy, noncalcareous, poor cuttings (Pierre Shale) \\
\hline
\end{tabular}


Local number: 122N51W27BBBB2 R

Other identifier: R2-98-27

Site identification number: 451936097022602

Date of construction: $07-15-98$

Land surface altitude: 2,025 feet

Total depth: 302 feet

\begin{tabular}{|c|c|c|c|}
\hline Depth & $\begin{array}{l}\text { Thickness } \\
\text { (feet) }\end{array}$ & $\begin{array}{l}\text { Altitude } \\
\text { (feet) }\end{array}$ & Description \\
\hline $0-2$ & 2 & $2,025-2.023$ & Clay, black, silty (topsoil) \\
\hline $2-7$ & 5 & $2,023-2,018$ & Clay, yellowish-brown, silty, sandy, pebbly; oxidized (till) \\
\hline $7-11$ & 4 & $2,018-2,014$ & Sand, brown, fine to coarse \\
\hline $11-14$ & 3 & $2,014-2,011$ & Clay, yellowish-brown, silty, sandy, pebbly; oxidized (till) \\
\hline $14-40$ & 26 & $2,011-1,985$ & Clay, gray, silty, sandy, pebbly; soft, large cuttings, unoxidized (till) \\
\hline $40-79$ & 39 & $1,985-1,946$ & $\begin{array}{l}\text { Clay, brown, silty, sandy, pebbly; hard, turns grayish-brown at } 54 \text { feet, rocks at } 63,77 \text {, } \\
\text { and } 79 \text { feet, oxidized (till) }\end{array}$ \\
\hline $79-265$ & 186 & $1,946-1,760$ & $\begin{array}{l}\text { Clay, gray, silty, sandy, pebbly; unoxidized, rock at } 98 \text { feet, maybe more than one till in } \\
\text { this interval (till) }\end{array}$ \\
\hline $265-283$ & 18 & $1,760-1,742$ & Clay, gray, very silty; unoxidized (lake sediment) \\
\hline $283-298$ & 15 & $1,742-1,727$ & Sand and gravel, coarse sand to fine to medium gravel \\
\hline $298-302$ & 19 & $1,727-1,723$ & Clay, gray, silty, sandy pebbly; unoxidized (till) \\
\hline
\end{tabular}


Local number: 122N52W1DDCC R

Other identifier: R2-98-31

Site identification number: 452213097063101

Date of construction: $07-29-98$

Land surface altitude: 1,946 feet

Total depth: 462 feet

\begin{tabular}{|c|c|c|c|}
\hline Depth & $\begin{array}{l}\text { Thickness } \\
\text { (feet) }\end{array}$ & $\begin{array}{l}\text { Altitude } \\
\text { (feet) }\end{array}$ & Description \\
\hline $0-3$ & 3 & $1,946-1,943$ & Clay, black with some brown, silty (topsoil) \\
\hline $3-15$ & 12 & $1,943-1,931$ & Clay, yellowish-brown, silty, sandy, pebbly; oxidized (till) \\
\hline $15-32$ & 17 & $1,931-1,914$ & Clay, grayish-brown, silty, sandy \\
\hline $32-35$ & 3 & $1,914-1,911$ & Clay, gray, silty, sandy, pebbly; unoxidized (till) \\
\hline $35-56$ & 21 & $1,911-1,890$ & Clay, $\tan$ then brown then light-gray then tan then dark-brown, very silty; soft (loess) \\
\hline $56-85$ & 29 & $1,890-1,861$ & Clay, yellowish-brown, silty, sandy, pebbly; oxidized (till) \\
\hline $85-95$ & 10 & $1,861-1,851$ & Clay, grayish-brown, very silty; very soft, somewhat greasy, unoxidized (loess) \\
\hline $95-119$ & 24 & $1,851-1,827$ & Clay, yellowish-brown, very silty \\
\hline $119-142$ & 23 & $1,827-1,804$ & Clay, gray with brown oxidation zones, silty, sandy, pebbly; partially oxidized (till) \\
\hline $142-228$ & 86 & $1,804-1,718$ & Clay, gray, silty, sandy, pebbly; unoxidized (till) \\
\hline $228-232$ & 4 & $1,718-1,714$ & Sand and gravel, medium to coarse sand to fine gravel \\
\hline $232-316$ & 84 & $1,714-1,630$ & $\begin{array}{l}\text { Clay, gray and brown, silty, sandy, pebbly; rocks at 262,271, 274, and } 296 \text { feet, } \\
\text { oxidized and unoxidized (till) }\end{array}$ \\
\hline $316-320$ & 4 & $1,630-1,626$ & Gravel, coarse; with cobbles \\
\hline $320-333$ & 13 & $1,626-1,613$ & Clay, gray, silty, sandy, pebbly; unoxidized. very hard from 331 to 332 feet (till) \\
\hline $333-343$ & 10 & $1,613-1,603$ & Sand, medium; cemented, rig chatter, poor sample \\
\hline $343-413$ & 70 & $1,603-1,533$ & Clay, gray, silty, sandy, pebbly, shaley; unoxidized, many shale inclusions (till) \\
\hline $413-438$ & 25 & $1,533-1,508$ & Sand and gravel, medium sand to fine gravel; cemented, rig chatter, taking water \\
\hline $438-462$ & 24 & $1,508-1,484$ & Clay, dark-gray to black, shaley; very greasy (Pierre Shale) \\
\hline
\end{tabular}


Local number: 122N52W9AAAA3 R

Other identifier: R2-98-34

Site identification number: 452218097095101

Date of construction: $08-25-98$

Land surface altitude: 1,872 feet

Total depth: 667 feet

\begin{tabular}{|c|c|c|c|}
\hline Depth & $\begin{array}{c}\text { Thickness } \\
\text { (feet) }\end{array}$ & $\begin{array}{c}\text { Altitude } \\
\text { (feet) }\end{array}$ & Description \\
\hline $0-49$ & 49 & $1,872-1,823$ & Sand and gravel, coarse sand to medium to coarse gravel; oxidized \\
\hline $49-58$ & 9 & $1,823-1,814$ & Clay, gray, silty, sandy, pebbly; unoxidized (till) \\
\hline $58-65$ & 7 & $1.814-1,807$ & Gravel, medium to coarse \\
\hline $65-74$ & 9 & $1,807-1,798$ & Clay, brown to dark-brown. silty, very sandy, pebbly; oxidized (till) \\
\hline $74-85$ & 11 & $1,798-1,787$ & Clay, gray, very silty, sandy, pebbly; unoxidized (till) \\
\hline $85-90$ & 5 & $1,787-1,782$ & Sand and gravel, coarse sand to fine to medium gravel \\
\hline $90-102$ & 12 & $1,782-1,770$ & Clay, dark-gray, silty, sandy, pebbly; unoxidized, harder than till interval above (till) \\
\hline $102-106$ & 4 & $1,770-1,766$ & Gravel \\
\hline $106-160$ & 54 & $1,766-1,712$ & $\begin{array}{l}\text { Clay, light-gray, silty, sandy, pebbly; partially oxidized, turns gray by } 115 \text { feet, gravel } \\
\text { lens at } 156 \text { and } 159 \text { feet (till) }\end{array}$ \\
\hline $160-170$ & 10 & $1,712-1,702$ & Sand, medium; cemented, poor sample \\
\hline $170-177$ & 7 & $1,702-1,695$ & Clay, brown, very silty; oxidized (loess) \\
\hline $177-207$ & 30 & $1,695-1,665$ & $\begin{array}{l}\text { Clay, yellowish-brown, silty, sandy, pebbly; some gray noticed by } 190 \text { feet, oxidized } \\
\text { (till) }\end{array}$ \\
\hline $207-267$ & 60 & $1,665-1,605$ & Clay, gray, silty, sandy, pebbly; rock at 259 feet, unoxidized (till) \\
\hline $267-282$ & 15 & $1,605-1,590$ & Sand and gravel, coarse sand to fine to medium gravel; rock at 282 feet \\
\hline $282-447$ & 165 & $1,590-1,425$ & Clay, gray, silty, sandy, pebbly, shaley; unoxidized (till) \\
\hline $447-492$ & 45 & $1,425-1,380$ & $\begin{array}{l}\text { Clay, dark-gray, silty, sandy, pebbly, shaley; rocks at } 454,485 \text {, and } 490 \text { feet, E-log } \\
\text { signature differs slightly from above interval, unoxidized (till) }\end{array}$ \\
\hline $492-555$ & 63 & $1,380-1,317$ & $\begin{array}{l}\text { Clay, gray, silty, sandy, pebbly, shaley; E-log signature differs slightly from above } \\
\text { interval (till) }\end{array}$ \\
\hline $555-633$ & 78 & $1,317-1,239$ & Sand, gray, very fine to fine, very silty; some silty clay layers \\
\hline $633-654$ & 21 & $1,239-1,218$ & $\begin{array}{l}\text { Clay, dark-gray to black, shaley; greasy, possibly just a block of shale and not actual } \\
\text { shale surface (Pierre Shale) }\end{array}$ \\
\hline $654-667$ & 13 & $1,218-1,205$ & Clay, dark-gray; poor cuttings (shale? or till?) \\
\hline
\end{tabular}


Local number: 122N52W24DDDD R

Other identifier: R2-98-28

Site identification number: 451936097061201

Date of construction: $07-20-98$

Land surface altitude: 1,968 feet

Total depth: 522

\begin{tabular}{|c|c|c|c|}
\hline Depth & $\begin{array}{l}\text { Thickness } \\
\text { (feet) }\end{array}$ & $\begin{array}{l}\text { Altitude } \\
\text { (feet) }\end{array}$ & Description \\
\hline $0-5$ & 5 & $1,968-1,963$ & Sand and gravel, medium to coarse sand to medium gravel \\
\hline $5-19$ & 14 & $1,963-1,949$ & Clay, yellowish-brown, silty, sandy, pebbly; oxidized (till) \\
\hline $19-23$ & 4 & $1,949-1,945$ & Clay, light-grayish-brown, very silty; soft (loess) \\
\hline $23-26$ & 3 & $1,945-1,942$ & Clay, yellowish-brown, silty, sandy, pebbly; oxidized (till) \\
\hline 26-105 & 79 & $1,942-1,863$ & $\begin{array}{l}\text { Clay, gray to dark-gray with oxidized zones, silty, sandy, pebbly; hard, partially } \\
\text { oxidized to unoxidized, sand zones from } 42 \text { to } 45 \text { feet and } 52 \text { to } 56 \text { feet (till) }\end{array}$ \\
\hline $105-112$ & 7 & $1,863-1,856$ & Clay, dark-gray, very silty; somewhat greasy (loess) \\
\hline $112-178$ & 66 & $1,856-1,790$ & Clay, yellowish-brown, silty, sandy, pebbly; hard, some gray till noticed with depth (till) \\
\hline $178-271$ & 93 & $1,790-1,697$ & $\begin{array}{l}\text { Clay, gray, silty, sandy, pebbly; unoxidized, gravel lens from } 265 \text { to } 267 \text { feet and } 268 \text { to } \\
271 \text { feet, rock at } 268 \text { feet (till) }\end{array}$ \\
\hline $271-400$ & 129 & $1,697-1,568$ & $\begin{array}{l}\text { Clay, gray, silty, sandy, pebbly; unoxidized, E-log signature differs from above interval, } \\
\text { rocks at } 315,381 \text { and } 400 \text { feet, gravel lens from } 319 \text { to } 322 \text { feet, } 325 \text { to } 330 \text { feet, and } \\
341 \text { to } 345 \text { feet, some oxidized till noticed (till) }\end{array}$ \\
\hline $400-433$ & 33 & $1,568-1,535$ & $\begin{array}{l}\text { Clay, yellowish-brown and gray, silty, sandy, pebbly; oxidized zones and unoxidized } \\
\text { zones, rock at } 417 \text { feet (till) }\end{array}$ \\
\hline $433-435$ & 2 & $1,535-1,533$ & Gravel, medium to coarse \\
\hline $435-472$ & 37 & $1,533-1,496$ & Clay, gray, silty, sandy, pebbly, shaley; unoxidized (till) \\
\hline $472-490$ & 18 & $1,496-1,478$ & Sand, fine to medium; partially cemented, rock at 476 feet \\
\hline $490-522$ & 32 & $1,478-1446$ & Clay, gray, shaley; hard, greasy (Pierre Shale) \\
\hline ric log & & & \\
\hline
\end{tabular}


Local number: 122N52W27BBBB R

Other identifier: R2-98-29

Site identification number: 451934097095301

Date of construction: $07-27-98$

Land surface altitude: 1,875 feet

Total depth: 477 feet

\begin{tabular}{|c|c|c|c|}
\hline Depth & $\begin{array}{l}\text { Thickness } \\
\text { (feet) }\end{array}$ & $\begin{array}{l}\text { Altitude } \\
\text { (feet) }\end{array}$ & Description \\
\hline $0-2$ & 2 & $1,875-1,873$ & Clay, black, silty (topsoil) \\
\hline $2-7$ & 5 & $1,873-1,868$ & Sand and gravel, medium sand to medium gravel; oxidized \\
\hline $7-30$ & 23 & $1,868-1,851$ & $\begin{array}{l}\text { Clay, tan, then dark-brown then yellowish-brown grading to grayish-brown, very silty; } \\
\text { soft, a few small pebbles, oxidized (loess?) }\end{array}$ \\
\hline $30-40$ & 10 & $1,851-1,841$ & Clay, gray, very silty; unoxidized (loess?) \\
\hline $40-42$ & 2 & $1,841-1,839$ & Clay, yellowish-brown, silty, sandy, pebbly; highly oxidized (till) \\
\hline $42-84$ & 42 & $1,839-1,797$ & Clay, dark-gray, very silty, sandy, pebbly; hard. unoxidized (till) \\
\hline $84-102$ & 18 & $1,797-1,779$ & Clay, gray, very silty: very soft. somewhat greasy (loess) \\
\hline $102-115$ & 13 & $1,779-1.766$ & $\begin{array}{l}\text { Clay, light-greenish-gray with some dark-brown, silty, sandy, pebbly; hard, oxidized } \\
\text { (till) }\end{array}$ \\
\hline $115-140$ & 25 & $1,766-1,741$ & $\begin{array}{l}\text { Clay, yellowish-brown grading to light-gray with brown, silty, sandy, pebbly; oxidized } \\
\text { to partially oxidized (till) }\end{array}$ \\
\hline $140-190$ & 50 & $1,741-1,691$ & Clay, gray, silty, sandy, pebbly; unoxidized (till) \\
\hline 190-194 & 4 & $1,691-1,687$ & Sand and gravel, medium to coarse sand to fine gravel \\
\hline 194-204 & 10 & $1,687-1.677$ & Clay, gray, very silty; unoxidized (lake sediment) \\
\hline 204-243 & 39 & $1,677-1,638$ & Sand and gravel, medium to coarse sand to fine gravel \\
\hline 243-256 & 13 & $1,638-1,625$ & Clay, gray, very silty; unoxidized, gravel lens from 250 feet (lake sediment?) \\
\hline $256-261$ & 5 & $1,625-1,620$ & Gravel, fine to medium \\
\hline 261-371 & 110 & $1,620-1,510$ & Clay, dark-gray, silty, sandy, pebbly, shaley; unoxidized, occasional cobbles (till) \\
\hline 371-388 & 17 & $1,510-1,493$ & Gravel, fine to medium; hard layer from 376 to 378 feet, very clayey below 378 feet \\
\hline 388-437 & 49 & $1,493-1,444$ & $\begin{array}{l}\text { Clay, gray, silty, sandy, pebbly, shaley; unoxidized, sand lens from } 402 \text { to } 404 \text { feet, rock } \\
\text { at } 390 \text { feet (till) }\end{array}$ \\
\hline $437-443$ & 6 & $1,444-1,438$ & Gravel, coarse; with cobbles \\
\hline 443-477 & 34 & $1,438-1,404$ & Clay, gray, shaley; hard, greasy (Pierre Shale) \\
\hline tric $\log$ & & & \\
\hline
\end{tabular}


Local number: 122N52W27BBBB2 R

Other identifier: R2-98-30

Site identification number: 451934097095302

Date of construction: $07-28-98$

Land surface altitude: $1,878.4$ feet

Total depth: 242 feet

\begin{tabular}{ccccc}
\hline Depth & $\begin{array}{c}\text { Thickness } \\
\text { (feet) }\end{array}$ & $\begin{array}{c}\text { Altitude } \\
\text { (feet) }\end{array}$ & Description \\
\hline $0-2$ & 2 & $1,878.4-1,876.4$ & Clay, black, silty (topsoil) \\
$2-7$ & 5 & $1,876.4-1,871.4$ & Sand and gravel, medium to coarse sand to medium gravel; highly oxidized \\
$7-16$ & 9 & $1,871.4-1,862.4$ & $\begin{array}{c}\text { Clay, tan to yellowish-brown, very silty; soft, greasy, a few small pebbles, oxidized } \\
\text { (loess?) }\end{array}$ \\
$16-22$ & 6 & $1,862.4-1,856.4$ & Clay, gray, very silty; soft, greasy, a few pebbles, unoxidized (loess?) \\
$22-36$ & 14 & $1,856.4-1,842.4$ & Clay, yellowish-brown, very silty; soft, greasy, oxidized (loess?) \\
$36-43$ & 7 & $1,842.4-1,835.4$ & Clay, yellowish-brown to brown, silty, sandy, pebbly; highly oxidized, gravel lens from \\
38 to 40 feet (till)
\end{tabular}


Local number: 123N49W24AAAA

Other identifier: R2-98-21

Site identification number: 452724096442201

Date of construction: $06-23-98$

Land surface altitude: 1,045 feet

Total depth: 102 feet

\begin{tabular}{cccl}
\hline Depth & $\begin{array}{c}\text { Thickness } \\
\text { (feet) }\end{array}$ & $\begin{array}{c}\text { Altitude } \\
\text { (feet) }\end{array}$ & \multicolumn{1}{c}{ Description } \\
\hline $0-2$ & 2 & $1,045-1,043$ & Sand, brown, coarse (roadfill) \\
$2-20$ & 18 & $1,043-1,025$ & Clay, brown, silty, sandy, pebbly; oxidized (till) \\
$20-23$ & 3 & $1,025-1,022$ & Clay, gray, silty, sandy, pebbly; unoxidized (till) \\
$23-24$ & 1 & $1,022-1,021$ & Sand, reddish-brown; oxidized \\
$24-39$ & 15 & $1,021-1,006$ & $\begin{array}{l}\text { Clay, reddish-brown, silty, very sandy, pebbly; turns grayish-pink after 32 feet (Hawk } \\
\text { Creek Till) } \\
39-42\end{array}$ \\
$42-67$ & 25 & $1,006-1,003$ & Sand and gravel, coarse sand to fine gravel \\
$67-75$ & 8 & $1,003-978$ & Clay, dark-gray, silty, sandy, pebbly; hard. unoxidized, many sand lens (till) \\
$75-102$ & 27 & $978-970$ & Clay, very light-gray, silty, sandy, pebbly; hard, gravelly, some boulders (till) \\
Electric log available & $970-943$ & Clay, gray, shaley; hard, greasy (Carlile Shale) \\
\hline
\end{tabular}


Local number: 123N50W24AAAD

Other identifier: R2-98-24

Site identification number: 452714096514501

Date of construction: 06-29-98

Land surface altitude: 1,170 feet

Total depth: 182 feet

\begin{tabular}{cccl}
\hline Depth & $\begin{array}{c}\text { Thickness } \\
\text { (feet) }\end{array}$ & $\begin{array}{c}\text { Altitude } \\
\text { (feet) }\end{array}$ & \multicolumn{1}{c}{ Description } \\
\hline $0-1$ & 1 & $1,170-1,169$ & Clay, black, silty (topsoil) \\
$1-12$ & 11 & $1,169-1,158$ & Clay, yellowish-brown, silty, sandy, pebbly; oxidized (till) \\
$12-42$ & 30 & $1,158-1,128$ & Sand and gravel, coarse sand to fine to medium gravel; some coarse gravel with depth \\
$42-47$ & 5 & $1,128-1,123$ & Clay, gray, silty, sandy, pebbly; unoxidized (till) \\
$47-50$ & 3 & $1,123-1,120$ & Gravel, coarse to very coarse; some cobbles \\
$50-102$ & 52 & $1,120-1,068$ & $\begin{array}{l}\text { Clay, dark-gray, shaley; hard, greasy, noncalcareous (Pierre Shale) } \\
102-123\end{array}$ \\
$123-138$ & 15 & $1,068-1,047$ & $\begin{array}{l}\text { Clay, black, shaley; greasy, oily (Sharon Springs Member - Pierre Shale) } \\
\text { Clay, light-gray to white, silty; hard, gritty, chalky, very calcareous (Niobrara } \\
138-182\end{array}$ \\
& 42 & $1,047-1,032$ & Formation) \\
Electric log available & $1,032-988$ & $\begin{array}{c}\text { Clay, gray, shaley; hard, greasy, noncalcareous (Blue Hill Shale Member - Carlile } \\
\text { Shale) }\end{array}$ \\
\hline
\end{tabular}


Local number: 123N51W2CCCC R

Other identifier: R2-98-33

Site identification number: 452728097011401

Date of construction: $08-19-98$

Land surface altitude: 1,500 feet

Total depth: 502 feet

\begin{tabular}{|c|c|c|c|}
\hline Depth & $\begin{array}{c}\text { Thickness } \\
\text { (feet) }\end{array}$ & $\begin{array}{c}\text { Altitude } \\
\text { (feet) }\end{array}$ & Description \\
\hline $0-1$ & 1 & $1,500-1,499$ & Clay, black. silty (topsoil) \\
\hline $1-3$ & 2 & $1,499-1.497$ & Sand and gravel; oxidized (roadfill) \\
\hline $3-6$ & 3 & $1,497-1,494$ & Clay, brown, silty, sandy \\
\hline $6-15$ & 9 & $1,494-1,485$ & Sand and gravel, coarse sand to fine gravel \\
\hline $15-70$ & 55 & $1,485-1,430$ & $\begin{array}{l}\text { Clay, gray, silty, sandy, pebbly; soft, large cuttings, sand lens from } 28 \text { to } 31 \text { feet and } 52 \\
\text { to } 54 \text { feet, unoxidized (till) }\end{array}$ \\
\hline $70-94$ & 24 & $1,430-1,406$ & Clay, gray, very silty; greasy (lake sediment) \\
\hline $94-115$ & 21 & $1,406-1,385$ & Sand and gravel, coarse sand to coarse gravel; some cobbles at base of interval \\
\hline $115-122$ & 7 & $1,385-1,378$ & Clay, gray, silty, sandy, pebbly; unoxidized (till) \\
\hline $122-134$ & 12 & $1,378-1,366$ & Sand; some clay layers \\
\hline $134-471$ & 337 & $1,366-1,029$ & $\begin{array}{l}\text { Clay, gray, silty, sandy, pebbly, shaley: rocks at } 279.284 .342,379 \text {, and } 388 \text { feet, very } \\
\text { cobbly from } 342 \text { to } 358 \text { feet, many shale inclusions, not much change in the E-log } \\
\text { signature (till) }\end{array}$ \\
\hline $471-482$ & 11 & $1,029-1,018$ & $\begin{array}{l}\text { Clay, dark-gray to black; greasy, very calcareous (Sharon Springs Member - Pierre } \\
\text { Shale?) }\end{array}$ \\
\hline $482-496$ & 14 & $1,018-1,004$ & Clay, gray, very silty; hard, very calcareous (Niobrara Formation) \\
\hline $496-502$ & 6 & $1,004-998$ & Clay, gray, shaley; hard, greasy, noncalcareous (Carlile Shale) \\
\hline Electric log & able & & \\
\hline
\end{tabular}


Local number: 123N51W19CBCB R

Other identifier: R2-99-05

Site identification number: 452507097061001

Date of construction: 06-16-99

Land surface altitude: 1,990 feet

Total depth: 792 feet

\begin{tabular}{|c|c|c|c|}
\hline Depth & $\begin{array}{l}\text { Thickness } \\
\text { (feet) }\end{array}$ & $\begin{array}{l}\text { Altitude } \\
\text { (feet) }\end{array}$ & Description \\
\hline $0-1$ & 1 & $1,990-1,989$ & Clay, black, silty (topsoil) \\
\hline $1-7$ & 6 & $1,989-1,983$ & Sand, brown, medium; oxidized \\
\hline $7-23$ & 16 & $1,983-1,967$ & Clay, yellowish-brown, silty, sandy, pebbly; oxidized (till) \\
\hline $23-27$ & 4 & $1,967-1.963$ & Gravel, coarse; with cobbles \\
\hline $27-112$ & 85 & $1,963-1.878$ & Clay, gray, silty, sandy, pebbly; hard, unoxidized (till) \\
\hline $112-194$ & 82 & $1,878-1,796$ & Clay, yellowish-brown, silty, sandy, pebbly; rock at 114 feet, oxidized (till) \\
\hline 194-302 & 108 & $1,796-1,688$ & Clay, grown and gray, silty, sandy, pebbly; granite boulder from 194 to 195 feet (till) \\
\hline $302-324$ & 22 & $1,688-1,666$ & Clay, gray and dark-brown, silty; greasy (lake clay or loess?) \\
\hline $324-376$ & 52 & $1,666-1,614$ & $\begin{array}{l}\text { Clay, yellowish-brown, silty, sandy, pebbly; rocks at } 325,343,359 \text {, and } 367 \text { feet, } \\
\text { oxidized (till) }\end{array}$ \\
\hline $376-402$ & 26 & $1,614-1,588$ & Sand and gravel, coarse sand to fine gravel; cemented, some very hard spots \\
\hline $402-456$ & 54 & $1,588-1,534$ & $\begin{array}{l}\text { Clay, dark-gray, silty, sandy, pebbly; shaley; greasy, some shale inclusions, unoxidized } \\
\text { (till) }\end{array}$ \\
\hline $456-460$ & 4 & $1,534-1,530$ & Sand; cemented, rig chatter \\
\hline $460-702$ & 42 & $1,530-1,288$ & $\begin{array}{l}\text { Clay, gray, silty, sandy, pebbly, shaley; greasy, some shale inclusions, rocks at } 533 \text { and } \\
622 \text { feet, unoxidized (till) }\end{array}$ \\
\hline $702-792$ & 90 & $1,288-1,198$ & Clay, dark-gray to black, shaley; greasy, hard, noncalcareous (Pierre Shale) \\
\hline Electric log & & & \\
\hline
\end{tabular}


Local number: 123N51W21DDDD R

Other identifier: R2-99-02

Site identification number: 452448097023001

Date of construction: 06-02-99

Land surface altitude: 1,824 feet

Total depth: 792 feet

\begin{tabular}{|c|c|c|c|}
\hline Depth & $\begin{array}{l}\text { Thickness } \\
\text { (feet) }\end{array}$ & $\begin{array}{l}\text { Aititude } \\
\text { (feet) }\end{array}$ & Description \\
\hline $0-1$ & 1 & $1,824-1,823$ & Clay, black, silty (topsoil) \\
\hline $1-13$ & 12 & $1,823-1,811$ & Clay, dark-brown, silty, sandy, pebbly; greasy, some gray 15 to 18 feet, oxidized (till) \\
\hline $13-110$ & 97 & $1,811-1,714$ & $\begin{array}{l}\text { Clay, gray, silty, sandy, pebbly; greasy, gravel lens at } 63 \text { feet, rocks } 84 \text { to } 86 \text { feet and } \\
97 \text { feet, unoxidized (till) }\end{array}$ \\
\hline $110-138$ & 28 & $1,714-1,686$ & $\begin{array}{l}\text { Sand and gravel, brown, medium sand to fine gravel; clay layer } 118 \text { to } 120 \text { feet, } \\
\text { oxidized }\end{array}$ \\
\hline $138-215$ & 77 & $1,686-1,609$ & $\begin{array}{l}\text { Clay, gray to dark-gray, silty, sandy, pebbly; hard, rocks at } 145,159 \text {, and } 169 \text { to } 171 \\
\text { feet, unoxidized (till) }\end{array}$ \\
\hline 215-229 & 14 & $1,609-1,595$ & Sand, medium to coarse; some fine gravel \\
\hline $229-336$ & 7 & $1,595-1,488$ & $\begin{array}{l}\text { Clay, light-gray then brown, silty, sandy, pebbly; rocks at } 234 \text { and } 266 \text { feet, oxidized } \\
\text { (till) }\end{array}$ \\
\hline $336-340$ & 4 & $1,488-1,484$ & Sand and gravel \\
\hline $340-769$ & 129 & $1,484-1,055$ & $\begin{array}{l}\text { Clay, gray, very silty, sandy, pebbly, shaley; very hard, some shale inclusions, rocks at } \\
\quad 614,633,657,703 \text {, and } 724 \text { feet, unoxidized (till) }\end{array}$ \\
\hline $769-780$ & 11 & $1,055-1,044$ & Gravel, coarse; with cobbles \\
\hline $780-792$ & 12 & $1,044-1,032$ & Clay, gray, silty; calcareous (Niobrara Formation) \\
\hline Electric lo & & & \\
\hline
\end{tabular}

Local number: 123N51W21DDDD2 R

Other identifier: R20-99-48

Site identification number: 452218097022902

Date of construction: $08-18-99$

Land surface altitude: $1,819.4$ feet

Total depth: 138 feet

\begin{tabular}{cccl}
\hline Depth & $\begin{array}{c}\text { Thickness } \\
\text { (feet) }\end{array}$ & $\begin{array}{c}\text { Altitude } \\
\text { (feet) }\end{array}$ & Description \\
\hline $0-1$ & 1 & $1,819.4-1,818.4$ & Clay, black. silty (topsoil) \\
$1-17$ & 16 & $1,818.4-1,802.4$ & $\begin{array}{c}\text { Clay, brown to yellowish-brown, silty, sandy, pebbly; greasy, some gray with reddish- } \\
\text { brown fracture zones from } 15 \text { to } 17 \text { feet, rock at } 17 \text { feet, oxidized (till) }\end{array}$ \\
$17-107$ & 90 & $1,802.4-1,712.4$ & $\begin{array}{c}\text { Clay, gray, silty, sandy, pebbly; greasy, gravel lens at 23 feet, rocks at } 44,73,82,83 \text { to } \\
85,86,87,95,97,100, \text { and } 107 \text { feet, unoxidized (till) }\end{array}$ \\
$107-138$ & 31 & $1,712.4-1,681.4$ & Sand and gravel, brown, medium sand to fine gravel: oxidized \\
Well screened from 118 to 138 feet; 2-inch PVC screen and casing
\end{tabular}


Local number: 123N52W3CCCC R

Other identifier: R2-99-01

Site identification number: 452727097095401

Date of construction: 04-28-99

Land surface altitude: 1,900 feet

Total depth: 507 feet

\begin{tabular}{|c|c|c|c|}
\hline Depth & $\begin{array}{l}\text { Thickness } \\
\text { (feet) }\end{array}$ & $\begin{array}{l}\text { Altitude } \\
\text { (feet) }\end{array}$ & Description \\
\hline $0-3$ & 3 & $1,900-1,897$ & Clay, black, silty (topsoil) \\
\hline $3-13$ & 10 & $1,897-1,887$ & $\begin{array}{l}\text { Clay, tan to yellowish-brown, silty, sandy, pebbly: rock at } 7 \text { feet, sand lens from } 7 \text { to } \\
9 \text { feet, oxidized (till) }\end{array}$ \\
\hline $13-46$ & 33 & $1,887-1,854$ & Clay, gray, silty, sandy, pebbly; greasy, unoxidized (till) \\
\hline $46-59$ & 13 & $1,854-1,841$ & $\begin{array}{l}\text { Sand and gravel, brown, medium; rock at } 54 \text { feet, clayey with rocks and coarse gravel } \\
54 \text { to } 59 \text { feet }\end{array}$ \\
\hline $59-64$ & 5 & $1,841-1,836$ & Clay, brown, silty; oxidized \\
\hline $64-67$ & 3 & $1,836-1,833$ & Gravel, medium to coarse \\
\hline $67-193$ & 126 & $1,833-1,707$ & Clay, brown, silty, sandy, pebbly; some gray noticed with depth, oxidized (till) \\
\hline 193-349 & 336 & $1,707-1,551$ & $\begin{array}{l}\text { Clay, brown grading to gray with depth, silty, sandy, pebbly; E-log signature differs } \\
\text { from above interval, rocks at } 283 \text { and } 338 \text { feet, oxidized (till) }\end{array}$ \\
\hline $349-356$ & 7 & $1,551-1,544$ & Sand and gravel, coarse; rock at 352 feet \\
\hline $356-404$ & 48 & $1,544-1,496$ & $\begin{array}{l}\text { Clay, dark-gray, silty, sandy, pebbly, shaley; some shale inclusions, rocks at } 372 \text { and } \\
410 \text { feet, unoxidized (till) }\end{array}$ \\
\hline $404-443$ & 39 & $1,496-1,457$ & Sand and gravel, coarse sand to fine gravel; partially cemented, rock at 435 feet \\
\hline $443-467$ & 24 & $1,457-1,433$ & Clay, gray, silty, sandy, pebbly; unoxidized (till) \\
\hline $467-498$ & 38 & $1,433-1,402$ & $\begin{array}{l}\text { Sand(?); very hard, rig chatter, E-log signature indicates possible sand, poor sample, } \\
\text { wore out the drill bit }\end{array}$ \\
\hline $498-501$ & 3 & $1,402-1,399$ & Granite boulder \\
\hline $501-507$ & 6 & $1,399-1,393$ & Clay, black, shaley; greasy, noncalcareous (Pierre Shale) \\
\hline Electric log & ble & & \\
\hline
\end{tabular}

Local number: 123N52W13AADA R

Other identifier: R2-99-03

Site identification number: 452626097061201

Date of construction: 06-02-99

Land surface altitude: 2,005 feet

Total depth: 22 feet

\begin{tabular}{cccc}
\hline Depth & $\begin{array}{c}\text { Thickness } \\
\text { (feet) }\end{array}$ & $\begin{array}{c}\text { Altitude } \\
\text { (feet) }\end{array}$ & Description \\
\hline $0-22$ & 22 & $2,005-1,983$ & Sand and gravel, reddish-brown, coarse sand to fine gravel; highly oxidized \\
\hline
\end{tabular}


Local number: 123N52W21DDDD R

Other identifier: R2-99-04

Site identification number: 452450097095501

Date of construction: 06-09-99

Land surface altitude: 2,002 feet

Total depth: 682 feet

\begin{tabular}{|c|c|c|c|}
\hline Depth & $\begin{array}{l}\text { Thickness } \\
\text { (feet) }\end{array}$ & $\begin{array}{l}\text { Altitude } \\
\text { (feet) }\end{array}$ & Description \\
\hline $0-2$ & 2 & $2,002-2,000$ & Clay, black, silty (topsoil) \\
\hline $2-4$ & 2 & $2,000-1,998$ & Sand, brown, medium; oxidized \\
\hline $4-39$ & 35 & $1,998-1,963$ & $\begin{array}{l}\text { Clay, tan to yellowish-brown, silty, sandy, pebbly; turns dark-brown then grayish-brown } \\
\text { with depth, hard, oxidized (till) }\end{array}$ \\
\hline $39-55$ & 16 & $1,963-1,947$ & Clay, gray, silty, sandy, pebbly; hard, unoxidized (till) \\
\hline $55-94$ & 39 & $1,947-1,908$ & Clay, gray, very silty; soft, layered (lake sediment) \\
\hline $94-148$ & 54 & $1,908-1,854$ & $\begin{array}{l}\text { Clay, yellowish-brown with a greenish tinge, silty, sandy, pebbly; grades to grayish- } \\
\text { brown with depth, oxidized (till) }\end{array}$ \\
\hline $148-188$ & 40 & $1,854-1,814$ & Clay, light-gray, silty, sandy, pebbly; hard (till) \\
\hline $188-217$ & 29 & $1,814-1,785$ & $\begin{array}{l}\text { Clay, dark-gray to black, very silty; greasy, some dark-brown soil-like material noticed } \\
\text { (lake sediment?) }\end{array}$ \\
\hline $217-222$ & 5 & $1,785-1,780$ & Gravel \\
\hline $222-460$ & 238 & $1,780-1,542$ & $\begin{array}{l}\text { Clay, brown and gray, silty, sandy, pebbly; sand lens at } 253,283,295,297,299 \text {, and } 332 \\
\text { feet, rocks at } 357 \text { and } 372 \text { feet, poor sample cuttings (till) }\end{array}$ \\
\hline $460-508$ & 48 & $1,542-1,494$ & $\begin{array}{l}\text { Clay, gray, silty, sandy, pebbly, shaley; greasy, some shale inclusions, poor sample } \\
\text { cuttings, unoxidized (till) }\end{array}$ \\
\hline $508-524$ & 34 & $1,494-1,478$ & Sand and gravel, coarse sand to fine gravel; cemented \\
\hline $524-574$ & 50 & $1,478-1,428$ & Clay, gray, silty, sandy, pebbly, shaley; greasy, some shale inclusions, unoxidized (till) \\
\hline $574-625$ & 51 & $1,428-1,377$ & Clay(?); very poor cuttings, drilled like clay but has questionable E-log signature (till?) \\
\hline $625-682$ & 57 & $1,377-1,320$ & Clay, dark-gray to black, shaley; greasy, hard, noncalcareous (Pierre Shale) \\
\hline Electric log & & & \\
\hline
\end{tabular}


Local number: 124N49W19BBBC

Other identifier: R2-98-22

Site identification number: 453234096514201

Date of construction: 06-24-98

Land surface altitude: 1,130 feet

Total depth: 122 feet

\begin{tabular}{|c|c|c|c|}
\hline Depth & $\begin{array}{c}\text { Thickness } \\
\text { (feet) }\end{array}$ & $\begin{array}{c}\text { Altitude } \\
\text { (feet) }\end{array}$ & Description \\
\hline $0-2$ & 2 & $1,130-1.128$ & Clay, black, silty (topsoil) \\
\hline $2-24$ & 22 & $1,128-1,106$ & $\begin{array}{l}\text { Clay, tan to yellowish-brown, silty, sandy, pebbly; oxidized. some dark-brown with } \\
\text { depth (till) }\end{array}$ \\
\hline $24-27$ & 3 & $1,106-1,103$ & Sand, brown, silty, medium to coarse \\
\hline $27-85$ & 58 & $1,103-1,045$ & $\begin{array}{l}\text { Clay, gray, silty, sandy, pebbly; unoxidized, sand lens from } 33 \text { to } 35 \text { feet, } 70 \text { to } 73 \text { feet } \\
\text { (till) }\end{array}$ \\
\hline $85-88$ & 3 & $1,045-1,042$ & Sand, gray, medium; unoxidized \\
\hline $88-98$ & 10 & $1,042-1.032$ & $\begin{array}{l}\text { Clay, gray, silty; unoxidized. very hard from } 92 \text { to } 94 \text { feet. very calcareous (Niobrara } \\
\text { Formation) }\end{array}$ \\
\hline $98-122$ & 24 & $1,032-1,008$ & Clay, gray, shaley; hard, greasy (Carlile Shale) \\
\hline Electric log & able & & \\
\hline
\end{tabular}

Local number: 124N49W21ADAD

Other identifier: R2-98-23

Site identification number: 453222096475701

Date of construction: $06-24-98$

Land surface altitude: 1,095 feet

Total depth: 122 feet

\begin{tabular}{cccc}
\hline Depth & $\begin{array}{c}\text { Thickness } \\
\text { (feet) }\end{array}$ & $\begin{array}{c}\text { Altitude } \\
\text { (feet) }\end{array}$ & Description \\
\hline $0-10$ & 10 & $1,095-1,085$ & Clay, tan to yellowish-brown, silty, sandy, pebbly; oxidized (till) \\
$10-44$ & 34 & $1,085-1,051$ & $\begin{array}{c}\text { Clay, gray, silty, sandy, pebbly; unoxidized, rock at 19 feet, sand lens at 28 feet, gravel } \\
\text { lens from 36 to } 38 \text { feet (till) }\end{array}$ \\
$44-92$ & 48 & $1,051-1,003$ & $\begin{array}{c}\text { Clay, gray, silty, sandy, pebbly; unoxidized, harder than above interval (till) } \\
\text { Clay, gray, shaley; hard, greasy, noncalcareous (Blue Hill Shale Member - Carlile } \\
\text { Shale) }\end{array}$ \\
\hline Electric log available & $1,003-973$ & \begin{tabular}{c} 
\\
\hline
\end{tabular}
\end{tabular}


Local number: 124N51W28AAAA R

Other identifier: R2-97-48

Site identification number: 453002097022701

Date of construction: $09-08-97$

Land surface altitude: 1,501 feet

Total depth: 512 feet

\begin{tabular}{cccl}
\hline Depth & $\begin{array}{c}\text { Thickness } \\
\text { (feet) }\end{array}$ & $\begin{array}{c}\text { Altitude } \\
\text { (feet) }\end{array}$ & \multicolumn{1}{c}{ Description } \\
\hline $0-19$ & 19 & $1,501-1,482$ & Clay, yellowish-brown, silty, sandy, pebbly; oxidized (till) \\
$19-24$ & 5 & $1,482-1,477$ & Clay, grayish-brown, silty, sandy, pebbly; partially oxidized (till) \\
$24-52$ & 28 & $1,477-1,449$ & Clay, gray, very silty, sandy, pebbly; soft, large cuttings, unoxidized (till) \\
$52-58$ & 6 & $1,449-1,443$ & Sand and gravel, coarse sand to coarse gravel; with cobbles \\
$58-176$ & 118 & $1,443-1,325$ & Clay, dark-gray, silty, sandy, pebbly; hard, rock at 78 feet, unoxidized (till) \\
$176-184$ & 8 & $1,325-1,317$ & $\begin{array}{l}\text { Sand and gravel, coarse sand to medium gravel } \\
184-423\end{array}$ \\
239 & $1,317-1,078$ & $\begin{array}{l}\text { Clay, gray to dark-gray, silty, sandy, pebbly; some hard zones, occasional rocks, drill rig } \\
\text { not drilling properly, unoxidized, E-log signature changes very little over the interval } \\
\text { (till) }\end{array}$ \\
$423-498$ & 75 & $1,078-1,003$ & $\begin{array}{l}\text { Sand and gravel, medium to coarse sand to fine gravel; rig chatter, drills fast, cemented } \\
\text { Clay, black, shaley; hard, greasy, calcareous (Carlile Shale) }\end{array}$ \\
$498-512$ & 14 & $1,003-989$ & \\
\hline
\end{tabular}


Local number: 124N51W30BBBB R

Other identifier: R2-99-06

Site identification number: 452958097060901

Date of construction: 07-07-99

Land surface altitude: 2,060 feet

Total depth: 847 feet

\begin{tabular}{|c|c|c|c|}
\hline Depth & $\begin{array}{l}\text { Thickness } \\
\text { (feet) }\end{array}$ & $\begin{array}{c}\text { Altitude } \\
\text { (feet) }\end{array}$ & Description \\
\hline $0-38$ & 38 & $2,060-2,022$ & Sand and gravel, brown, fine sand to fine to medium gravel; some clay layers, oxidized \\
\hline $38-70$ & 32 & $2,022-1,990$ & Clay, gray, silty, sandy, pebbly; hard, unoxidized (till) \\
\hline $70-81$ & 11 & $1,990-1,979$ & Sand and gravel \\
\hline $81-132$ & 51 & $1,979-1,928$ & Clay, brown, silty, sandy, pebbly; rocks at 87 and 88 feet, oxidized (till) \\
\hline $132-140$ & 8 & $1,928-1,920$ & Gravel, medium; much limestone, oxidized \\
\hline $140-152$ & 12 & $1,920-1,908$ & Clay, gray, silty; greasy (lake clay or loess?) \\
\hline $152-211$ & 59 & $1,908-1,849$ & Clay, grayish-brown, silty, sandy, pebbly; oxidized (till) \\
\hline $211-275$ & 64 & $1,849-1,785$ & $\begin{array}{l}\text { Clay, yellowish-brown, silty, sandy, pebbly; rock at } 224 \text { feet, highly oxidized then turns } \\
\text { grayish-brown with depth (till) }\end{array}$ \\
\hline 275-377 & 102 & $1,785-1,683$ & Clay, gray, silty, sandy, pebbly; unoxidized (till) \\
\hline $377-405$ & 28 & $1,683-1,655$ & Sand, gray, fine to medium; cemented, rig chatter \\
\hline $405-417$ & 12 & $1,655-1,643$ & Clay, gray, silty; hard, smooth \\
\hline $417-427$ & 10 & $1,643-1,633$ & Sand, gray, fine; hard. cemented, with many clay layers \\
\hline $427-722$ & 295 & $1,633-1,338$ & $\begin{array}{l}\text { Clay, gray and brown, silty, sandy, pebbly; rocks at } 440,454,459 \text {, and } 654 \text { feet, poor } \\
\text { cuttings, possibly more than one till unit (till) }\end{array}$ \\
\hline $722-730$ & 8 & $1,338-1,330$ & Gravel(?) \\
\hline $730-847$ & 117 & $1,330-1,213$ & Clay, gray, silty, sandy, pebbly; hard, unoxidized, poor cuttings (till) \\
\hline For electric & e R2-99-13 & & \\
\hline
\end{tabular}


Local number: 124N51W30BBBB2 R

Other identifier: R2-99-13

Site identification number: 453002097060902

Date of construction: $08-24-99$

Land surface altitude: 2,053.1 feet

Total depth: 422 feet

\begin{tabular}{|c|c|c|c|}
\hline Depth & $\begin{array}{l}\text { Thickness } \\
\text { (feet) }\end{array}$ & $\begin{array}{l}\text { Altitude } \\
\text { (feet) }\end{array}$ & Description \\
\hline $0-1$ & 1 & $2,053.1-2,052.1$ & Clay, tan, silty (topsoil) \\
\hline $1-6$ & 5 & $2,052.1-2,047.1$ & Sand and gravel. brown. fine sand to fine to medium gravel: rock at 6 feet. oxidized \\
\hline $6-30$ & 24 & $2,047.1-2,023.1$ & Clay, yellowish-brown, silty, sandy, pebbly: oxidized (till) \\
\hline $30-32$ & 2 & $2,023.1-2,021.1$ & Sand and gravel \\
\hline $32-40$ & 8 & $2,021.1-2,013.1$ & Clay, gray, very silty; unoxidized (lake sediment) \\
\hline $40-44$ & 4 & 2,013.1-2,009.1 & Sand, gray, fine \\
\hline $44-72$ & 28 & $2,009.1-1,981.1$ & Clay, gray, silty, sandy, pebbly; hard, rocks at 64 and 72 feet, unoxidized (till) \\
\hline $72-88$ & 16 & $1,981.1-1,965.1$ & Sand and gravel; rock at 79 feet \\
\hline $88-162$ & 74 & $1,965.1-1,891.1$ & $\begin{array}{l}\text { Clay, yellowish-brown, silty, sandy, pebbly; rocks at } 87,88 \text {, and } 92 \text { feet, grades to a } \\
\text { grayish-brown by } 120 \text { feet, oxidized (till) }\end{array}$ \\
\hline $162-199$ & 37 & $1,891.1-1,854.1$ & Clay, gray, silty, sandy, pebbly; rock at 161 feet, unoxidized (till) \\
\hline $199-275$ & 76 & $1,854.1-1,778.1$ & $\begin{array}{l}\text { Clay, yellowish-brown, silty, sandy, pebbly; rock at } 219 \text { feet, highly oxidized then turns } \\
\text { grayish-brown with depth, first few feet may have been loess (till) }\end{array}$ \\
\hline 275-374 & 99 & $1,778.1-1,679.1$ & Clay, gray, silty, sandy, pebbly; rocks at $244,246,279$, and 300 feet, unoxidized (till) \\
\hline $374-388$ & 14 & $1,679.1-1,665.1$ & Sand, grayish-brown, fine to medium; hard, cemented, rig chatter \\
\hline $388-401$ & 13 & $1,665.1-1,652.1$ & Clay, gray, silty; hard, smooth \\
\hline $401-416$ & 15 & $1,652.1-1,637.1$ & Sand, gray, fine; hard, cemented, with many clay layers \\
\hline $416-422$ & 6 & $1,637.1-1,631.1$ & Clay, gray and brown, silty, sandy, pebbly (till) \\
\hline
\end{tabular}


Local number: 124N52W9AAAC R

Other identifier: R2-99-09

Site identification number: 453234097095901

Date of construction: 08-10-99

Land surface altitude: 2,005 feet

Total depth: 807 feet

\begin{tabular}{|c|c|c|c|}
\hline Depth & $\begin{array}{l}\text { Thickness } \\
\text { (feet) }\end{array}$ & $\begin{array}{l}\text { Altitude } \\
\text { (feet) }\end{array}$ & Description \\
\hline $0-1$ & 1 & $2,005-2,004$ & Clay, black, silty (topsoil) \\
\hline $1-25$ & 24 & $2,004-1,980$ & Clay, brown, silty, sandy, pebbly; oxidized (till) \\
\hline $25-50$ & 25 & $1,980-1,955$ & Clay, grayish-brown, silty, sandy, pebbly; partially oxidized (till) \\
\hline $50-86$ & 36 & $1,955-1,919$ & Clay, gray, silty, sandy, pebbly: hard, gravel lens at 69 and 74 feet, unoxidized (till) \\
\hline $86-94$ & 8 & $1,919-1,911$ & Gravel, coarse \\
\hline $94-110$ & 16 & $1,911-1,895$ & Sand, brown, fine to medium, clayey \\
\hline $110-117$ & 7 & $1,895-1,888$ & Gravel, coarse \\
\hline $117-145$ & 28 & $1,888-1,860$ & Clay, grayish-brown to gray, silty, sandy, pebbly; unoxidized (till) \\
\hline $145-175$ & 30 & $1,860-1,830$ & $\begin{array}{l}\text { Clay, light-gray, dark-gray, brown, green and white, silty; greasy, layered, some } \\
\text { calcareous zones (lake sediment) }\end{array}$ \\
\hline $175-192$ & 17 & $1,830-1,813$ & Clay, yellowish-brown, silty, sandy, pebbly; oxidized (till) \\
\hline $192-197$ & 5 & $1,813-1,808$ & Sand and gravel, coarse sand to fine gravel; oxidized \\
\hline $197-218$ & 21 & $1,808-1,787$ & Clay, gray, silty; hard, greasy (lake sediment) \\
\hline $218-248$ & 30 & $1,787-1,757$ & Clay, yellowish-brown, silty, sandy, pebbly; oxidized (till) \\
\hline $248-280$ & 32 & $1,757-1,725$ & Clay, gray, silty, sandy, pebbly; unoxidized (till) \\
\hline $280-317$ & 37 & $1,725-1,688$ & $\begin{array}{l}\text { Clay, yellowish-brown. silty, sandy, pebbly; gravel lens from } 295 \text { to } 298 \text { feet. oxidized } \\
\text { (till) }\end{array}$ \\
\hline $317-346$ & 29 & $1,688-1,659$ & Clay, dark-gray, silty; hard, greasy (lake sediment) \\
\hline $346-348$ & 2 & $1,659-1,657$ & Gravel \\
\hline $348-472$ & 24 & $1,657-1,533$ & Clay, gray, silty, sandy, pebbly; rocks at 434,446 , and 454 feet, unoxidized (till) \\
\hline $472-484$ & 12 & $1,533-1,521$ & Sand; cemented, rig chatter \\
\hline $484-508$ & 24 & $1,521-1,497$ & Sand; cemented, with clay layers \\
\hline $508-512$ & 4 & $1,497-1,493$ & Sand and gravel \\
\hline $512-574$ & 62 & $1,493-1,431$ & Clay, gray, silty, sandy, pebbly; E-log signature change at 540 feet, unoxidized (till) \\
\hline $574-577$ & 3 & $1,431-1,428$ & Gravel. coarse \\
\hline $577-688$ & 11 & $1,428-1,317$ & Clay, gray, silty, sandy, pebbly; E-log signature change at 624 feet, unoxidized (till) \\
\hline $688-694$ & 6 & $1,317-1,311$ & Sand, cemented \\
\hline $694-807$ & 113 & $1,311-1,198$ & Clay, dark-gray to black, shaley; greasy, hard, noncalcareous (Pierre Shale) \\
\hline ectric lo & ble & & \\
\hline
\end{tabular}


Local number: 124N52W9AAAC2 R

Other identifier: R2-99-10

Site identification number: 453204097100202

Date of construction: 08-11-99

Land surface altitude: 2,003 feet

Total depth: 132 feet

\begin{tabular}{cccl}
\hline Depth & $\begin{array}{c}\text { Thickness } \\
\text { (feet) }\end{array}$ & $\begin{array}{c}\text { Altitude } \\
\text { (feet) }\end{array}$ & \\
\hline $0-1$ & 1 & $2,003-2,002$ & Clay, black, silty (topsoil) \\
$1-11$ & 10 & $2,002-1,992$ & Clay, yellowish-brown, silty, sandy, pebbly; oxidized (till) \\
$11-18$ & 7 & $1,992-1,985$ & Clay, grayish-brown, silty, sandy, pebbly; partially oxidized (till) \\
$18-20$ & 2 & $1,985-1,983$ & Clay, gray, silty, sandy, pebbly; unoxidized (till) \\
$20-24$ & 4 & $1,983-1,979$ & Clay, yellowish-brown. silty, sandy, pebbly; oxidized (till) \\
$24-87$ & 63 & $1,979-1,916$ & Clay, gray, silty, sandy, pebbly; rock at 32 feet, unoxidized (till) \\
$87-98$ & 11 & $1,916-1,905$ & Sand and gravel, coarse sand to fine to medium gravel; some coarse gravel \\
$98-102$ & 4 & $1,905-1,901$ & Clay, gray, silty \\
$102-132$ & 30 & $1,901-1,871$ & Sand and gravel, coarse sand to fine gravel; much black shale \\
Well screened from 112 to 132 feet; 2-inch PVC screen and casing \\
\hline
\end{tabular}


Local number: 124N52W29AAAA

Other identifier: R2-98-35

Site identification number: 453003097110901

Date of construction: 09-01-98

Land surface altitude: 1,965 feet

Total depth: 720 feet

\begin{tabular}{|c|c|c|c|}
\hline Depth & $\begin{array}{l}\text { Thickness } \\
\text { (feet) }\end{array}$ & $\begin{array}{l}\text { Altitude } \\
\text { (feet) }\end{array}$ & Description \\
\hline $0-1$ & 1 & $1,965-1,964$ & Clay, black, silty (topsoil) \\
\hline $1-14$ & 13 & $1,964-1.951$ & Sand and gravel, coarse sand to coarse gravel; rock at 14 feet, oxidized \\
\hline $14-26$ & 12 & $1,951-1,939$ & Clay, yellowish-brown. silty, sandy, pebbly; oxidized (till) \\
\hline $26-48$ & 22 & 1,939-1,917 & Clay, grayish-brown, silty, sandy, pebbly; partially oxidized (till) \\
\hline $48-62$ & 14 & $1,917-1,903$ & Clay, light-gray, very silty; greasy (loess) \\
\hline $62-92$ & 30 & $1,903-1,873$ & Clay, yellowish-brown, silty, sandy, pebbly; oxidized (till) \\
\hline $92-125$ & 33 & $1,873-1,840$ & Clay, grayish-brown, silty, sandy, pebbly; partially oxidized (till) \\
\hline $125-136$ & 11 & $1,840-1,829$ & Clay, gray, silty, sandy, pebbly; unoxidized (till) \\
\hline $136-146$ & 10 & $1,829-1,819$ & Clay, dark-brown, very silty; somewhat greasy, oxidized (loess) \\
\hline $146-153$ & 7 & $1,819-1,812$ & Clay, very light-gray, very silty; unoxidized (loess) \\
\hline $153-178$ & 25 & $1,812-1,787$ & Clay, yellowish-brown, silty, sandy, pebbly; oxidized (till) \\
\hline $178-229$ & 51 & $1,787-1,736$ & Clay, gray, silty, sandy, pebbly; unoxidized (till) \\
\hline $229-239$ & 10 & $1,736-1,726$ & Sand and gravel, medium sand to medium gravel; rock at 234 feet \\
\hline $239-262$ & 23 & $1,726-1,703$ & Clay, gray, silty, sandy, pebbly, shaley; unoxidized (till) \\
\hline $262-380$ & 118 & $1,703-1,585$ & $\begin{array}{l}\text { Clay, gray, silty, sandy, pebbly, shaley; E-log signature differs from above interval, } \\
\text { unoxidized (till) }\end{array}$ \\
\hline $380-450$ & 70 & $1,585-1,515$ & Clay, gray, silty, sandy, pebbly, shaley \\
\hline $450-454$ & 4 & $1,515-1,511$ & Sand, medium to coarse \\
\hline $454-597$ & 143 & $1,511-1,368$ & $\begin{array}{l}\text { Clay, gray, silty, sandy, pebbly, shaley; E-log signature differs from above interval, } \\
\text { unoxidized (till) }\end{array}$ \\
\hline $597-720$ & 123 & $1,368-1,245$ & Clay, gray, shaley; greasy, poor cuttings, unoxidized (Pierre Shale?) \\
\hline Electric log & le & & \\
\hline
\end{tabular}


Local number: 125N50W12ABCC R

Other identifier: R2-97-23

Site identification number: 453738096515401

Date of construction: 07-15-97

Land surface altitude: 1,120 feet

Total depth: 142 feet

\begin{tabular}{|c|c|c|c|}
\hline Depth & $\begin{array}{l}\text { Thickness } \\
\text { (feet) }\end{array}$ & $\begin{array}{l}\text { Altitude } \\
\text { (feet) }\end{array}$ & Description \\
\hline $0-28$ & 28 & $1,120-1,092$ & Clay, yellowish-brown, silty, sandy, pebbly; oxidized (till) \\
\hline $28-38$ & 10 & $1,092-1,082$ & Sand and gravel, coarse sand to fine gravel; some medium gravel, oxidized \\
\hline $38-41$ & 3 & $1,082-1,079$ & Clay, dark-brown, silty, sandy, pebbly; unoxidized (till) \\
\hline $41-53$ & 12 & $1,079-1,067$ & Gravel, fine to coarse \\
\hline $53-76$ & 23 & $1,067-1,044$ & Clay, gray, silty, sandy, pebbly; unoxidized (till) \\
\hline $76-81$ & 5 & $1,044-1,039$ & Clay, gray, very silty; soft, drilled fast, unoxidized (lake sediment) \\
\hline $81-92$ & 11 & $1,039-1,028$ & Clay, brown, silty, pebbly; oxidized (till) \\
\hline $92-102$ & 10 & $1,028-1,018$ & $\begin{array}{l}\text { Clay, dark-gray, silty, very sandy, pebbly; unoxidized, may be some oxidized fracture } \\
\text { zones (till) }\end{array}$ \\
\hline $102-118$ & 16 & $1,018-1,002$ & $\begin{array}{l}\text { Sand, medium to coarse; clay lens from } 113 \text { to } 116 \text { feet, some gravel from } 116 \text { to } \\
118 \text { feet }\end{array}$ \\
\hline $118-142$ & 24 & $1,002-978$ & Clay, gray, shaley; hard, greasy, noncalcareous (Carlile Shale) \\
\hline Electric log & lable & & \\
\hline
\end{tabular}

Local number: $125 \mathrm{~N} 50 \mathrm{~W} 12 \mathrm{ABCC} 2 \mathrm{R}$

Other identifier: R2-97-43

Site identification number: 453738096515402

Date of construction: $08-25-97$

Land surface altitude: $1,120.8$ feet

Total depth: 62 feet

\begin{tabular}{cccl}
\hline Depth & $\begin{array}{c}\text { Thickness } \\
\text { (feet) }\end{array}$ & $\begin{array}{c}\text { Altitude } \\
\text { (feet) }\end{array}$ & Description \\
\hline $0-25$ & 25 & $1,120.8-1,095.8$ & Clay, yellowish-brown, silty, sandy, pebbly; oxidized (till) \\
$25-62$ & 37 & $1,095.8-1,058.8$ & Sand and gravel, medium sand to medium gravel \\
Well screened from 52 to 62 feet; 2 -inch PVC casing and screen. For electric log see R2-97-23 \\
\hline
\end{tabular}


Local number: $125 \mathrm{~N} 50 \mathrm{~W} 23 \mathrm{CCCD} \mathrm{R}$

Other identifier: R2-97-22

Site identification number: 453511096534301

Date of construction: 07-14-97

Land surface altitude: 1,160 feet

Total depth: 142 feet

\begin{tabular}{|c|c|c|c|}
\hline Depth & $\begin{array}{l}\text { Thickness } \\
\text { (feet) }\end{array}$ & $\begin{array}{l}\text { Altitude } \\
\text { (feet) }\end{array}$ & Description \\
\hline $0-1$ & 1 & $1,160-1,159$ & Topsoil, black \\
\hline $1-18$ & 17 & $1,159-1,142$ & $\begin{array}{l}\text { Clay, yellowish-brown, silty, sandy, pebbly; color change to dark-brown at } 14 \text { feet, } \\
\text { oxidized (till) }\end{array}$ \\
\hline $18-100$ & 82 & $1,142-1,060$ & Clay, gray, silty, sandy, pebbly; rock at 20 feet, unoxidized (till) \\
\hline $100-112$ & 12 & $1,060-1,048$ & Sand and gravel, coarse sand to fine gravel, rock from 110 to 112 feet \\
\hline $112-117$ & 5 & $1,048-1,043$ & Clay, gray, silty; gritty, unoxidized, highly calcareous (Niobrara Formation) \\
\hline $117-142$ & 25 & $1,043-1,018$ & $\begin{array}{l}\text { Clay, gray, shaley; hard, greasy, noncalcareous, concretions at } 135 \text { and } 139 \text { feet (Carlile } \\
\text { Shale) }\end{array}$ \\
\hline Electric log & able & & \\
\hline
\end{tabular}

Local number: 125 N52W $10 B B B B R$

Other identifier: R2-97-21

Site identification number: 453751097094901

Date of construction: $07-08-97$

Land surface altitude: 1,970 feet

Total depth: 82 feet

\begin{tabular}{|c|c|c|c|}
\hline Depth & $\begin{array}{l}\text { Thickness } \\
\text { (feet) }\end{array}$ & $\begin{array}{l}\text { Altitude } \\
\text { (feet) }\end{array}$ & Description \\
\hline $0-4$ & 4 & $1,970-1,966$ & Clay, tan. silty \\
\hline $4-8$ & 4 & $1,966-1,962$ & Clay, gray, silty \\
\hline $8-10$ & 2 & $1,962-1,960$ & Sand and gravel, brown \\
\hline $10-49$ & 39 & $1,960-1,921$ & Clay, gray, silty, sandy, pebbly: rock at 14 feet, unoxidized (till) \\
\hline $49-52$ & 3 & $1,921-1,918$ & Clay, brown, silty, sandy, pebbly; many cobbles, oxidized (till) \\
\hline $52-68$ & 16 & $1,918-1,902$ & Gravel, coarse to very coarse; many cobbles from 61 to 68 feet \\
\hline $68-82$ & 14 & $1,902-1,888$ & $\begin{array}{l}\text { Clay, brown, very cobbly; with large boulders, abandoned hole because of caving gravel } \\
\text { and cobbles (till?) }\end{array}$ \\
\hline
\end{tabular}


Local number: $125 \mathrm{~N} 52 \mathrm{~W} 16 \mathrm{BABB} \mathrm{R}$

Other identifier: R2-99-07

Site identification number: 453659097104601

Date of construction: 07-21-99

Land surface altitude: 1,990 feet

Total depth: 942 feet

\begin{tabular}{|c|c|c|c|}
\hline Depth & $\begin{array}{l}\text { Thickness } \\
\text { (feet) }\end{array}$ & $\begin{array}{l}\text { Altitude } \\
\text { (feet) }\end{array}$ & Description \\
\hline $0-2$ & 2 & $1,990-1,988$ & Clay, black, silty (topsoil) \\
\hline $2-6$ & 4 & $1,988-1,984$ & Sand, brown, fine; oxidized \\
\hline $6-10$ & 4 & $1,984-1,980$ & Clay, tan, very silty; oxidized (lake sediment) \\
\hline $10-62$ & 52 & $1,980-1,928$ & Clay, gray, very silty; greasy, unoxidized (lake sediment) \\
\hline $62-222$ & 160 & $1,928-1,768$ & $\begin{array}{l}\text { Clay, gray, very silty, sandy, pebbly; soft, gravelly from } 190 \text { to } 200 \text { feet, unoxidized } \\
\text { (till) }\end{array}$ \\
\hline $222-400$ & 178 & $1,768-1,590$ & $\begin{array}{l}\text { Clay, yellowish-brown, silty, sandy, pebbly; oxidized then grades to unoxidized with } \\
\text { depth (till) }\end{array}$ \\
\hline $400-420$ & 20 & $1,590-1,570$ & Sand and gravel, coarse sand to fine gravel; cemented \\
\hline $420-522$ & 102 & $1,570-1,468$ & $\begin{array}{l}\text { Clay, grayish-brown to gray, silty, sandy, pebbly; rocks at } 471,481,485 \text {, and } 501 \text { feet, } \\
\text { gravel lens at } 490 \text { feet, hard (till) }\end{array}$ \\
\hline $522-661$ & 139 & $1,468-1,329$ & Clay, brown and gray, silty, sandy, pebbly; hard, very poor cuttings (till) \\
\hline $661-676$ & 15 & $1,329-1,314$ & Sand; very hard, cemented \\
\hline $676-847$ & 171 & $1,314-1,143$ & $\begin{array}{l}\text { Clay, brown and gray, silty, sandy, pebbly; hard, very poor cuttings, rocks at } 680,696 \text {, } \\
715,813,832,840 \text {. and } 847 \text { feet (till) }\end{array}$ \\
\hline $847-942$ & 95 & $1,143-1,048$ & $\begin{array}{l}\text { Clay, dark-gray to black. shaley; very hard, greasy, some bentonite noticed (Pierre } \\
\text { Shale) }\end{array}$ \\
\hline Electric lo & & & \\
\hline
\end{tabular}


Local number: 125N52W16BABB2 R

Other identifier: R2-99-12

Site identification number: 45365909097104502

Date of construction: $08-18-99$

Land surface altitude: $1,987.9$ feet

Total depth: 424 feet

\begin{tabular}{cccl}
\hline Depth & $\begin{array}{c}\text { Thickness } \\
\text { (feet) }\end{array}$ & $\begin{array}{c}\text { Altitude } \\
\text { (feet) }\end{array}$ & Description \\
\hline $0-2$ & 2 & $1,987.9-1,985.9$ & Clay, black, silty (topsoil) \\
$2-6$ & 4 & $1,985.9-1,981.9$ & Sand, brown, fine; oxidized \\
$6-10$ & 4 & $1,981.9-1,977.9$ & Clay, tan, very silty; oxidized (lake sediment) \\
$10-62$ & 52 & $1,977.9-1,925.9$ & Clay, gray, very silty; greasy, unoxidized (lake sediment) \\
$62-224$ & 162 & $1,925.9-1,763.9$ & $\begin{array}{c}\text { Clay, gray, very silty, sandy, pebbly; soft, sand lens from } 152 \text { to } 156 \text { feet, unoxidized } \\
\text { (till) }\end{array}$ \\
$224-405$ & 181 & $1,763.9-1,582.9$ & $\begin{array}{c}\text { Clay, yellowish-brown, silty, sandy, pebbly; oxidized then grades to unoxidized with } \\
\text { depth (till) }\end{array}$ \\
$405-419$ & 14 & $1,582.9-1,568.9$ & $\begin{array}{c}\text { Sand and gravel, coarse sand to fine gravel; cemented } \\
419-424\end{array}$ \\
\hline
\end{tabular}


Local number: 126N49W11AABA R

Other identifier: R2-97-42

Site identification number: 454303096451701

Date of construction: $08-20-97$

Land surface altitude: $1,109.7$ feet

Total depth: 262 feet

\begin{tabular}{|c|c|c|c|}
\hline Depth & $\begin{array}{c}\text { Thickness } \\
\text { (feet) }\end{array}$ & $\begin{array}{c}\text { Altitude } \\
\text { (feet) }\end{array}$ & Description \\
\hline $0-2$ & 2 & $1,109.7-1,107.7$ & Clay, black, silty (topsoil) \\
\hline $2-6$ & 4 & $1,107.7-1,103.7$ & Clay, tan, silty; oxidized (loess) \\
\hline $6-35$ & 29 & $1,103.7-1,074.7$ & $\begin{array}{l}\text { Clay, yellowish-brown, silty, sandy, pebbly; grades to dark-brown then to grayish- } \\
\text { brown, oxidized (till) }\end{array}$ \\
\hline $35-44$ & 19 & $1,074.7-1,065.7$ & Clay, gray, silty, sandy, pebbly; soft, large cuttings, unoxidized (till) \\
\hline $44-68$ & 24 & $1,065.7-1,041.7$ & Clay, gray, silty; greasy, unoxidized (lake sediment) \\
\hline $68-84$ & 16 & $1,041.7-1,025.7$ & Sand and gravel, coarse sand to fine gravel \\
\hline $84-120$ & 36 & $1,025.7-989.7$ & Clay, gray, silty, sandy, pebbly; unoxidized, rock at 120 feet (till) \\
\hline $120-199$ & 79 & $989.7-910.7$ & $\begin{array}{l}\text { Clay, gray, silty, sandy, pebbly; unoxidized, different E-log signature than above } \\
\text { interval, rock at } 164 \text { feet (till) }\end{array}$ \\
\hline $199-203$ & 4 & $910.7-906.7$ & Gravel, fine to medium \\
\hline $203-232$ & 29 & $906.7-877.7$ & Clay, gray, silty, sandy, pebbly, very gravelly; unoxidized (till) \\
\hline $232-256$ & 24 & $877.7-853.7$ & Sand and gravel, coarse sand grading to fine to medium gravel; some coarse gravel \\
\hline $256-262$ & 6 & $853.7-847.7$ & Clay, gray, silty, sandy, pebbly; unoxidized (till) \\
\hline
\end{tabular}


Local number: 126N49W11ABAA R

Other identifier: R2-97-41

Site identification number: 454305096451901

Date of construction: $08-18-19-97$

Land surface altitude: 1,111 feet

Total depth: 522 feet

\begin{tabular}{|c|c|c|c|}
\hline Depth & $\begin{array}{l}\text { Thickness } \\
\text { (feet) }\end{array}$ & $\begin{array}{l}\text { Altitude } \\
\text { (feet) }\end{array}$ & Description \\
\hline $0-2$ & 2 & $1,111-1,109$ & Clay, black, silty (topsoil) \\
\hline $2-6$ & 4 & $1,109-1,105$ & Clay, tan, silty (loess) \\
\hline $6-10$ & 4 & $1,105-1,101$ & Clay, yellowish-brown, silty, sandy, pebbly; oxidized (till) \\
\hline $10-18$ & 8 & $1,101-1.093$ & Clay, yellowish-brown, silty; greasy (lake sediment) \\
\hline $18-30$ & 12 & $1,093-1,081$ & $\begin{array}{l}\text { Clay, yellowish-brown. silty, sandy, pebbly; oxidized, grades to dark-brown by } 25 \text { feet } \\
\text { (till) }\end{array}$ \\
\hline $30-36$ & 6 & $1,081-1,075$ & Clay, grayish-brown, silty, sandy, pebbly; partially oxidized (till) \\
\hline $36-55$ & 19 & $1,075-1,056$ & Clay, gray, silty, sandy, pebbly; unoxidized, some lake sediment inclusions (till) \\
\hline $55-66$ & 11 & $1,056-1,045$ & Clay, gray, silty; soft, greasy, unoxidized (lake sediment) \\
\hline $66-84$ & 18 & $1,045-1,027$ & Sand and gravel, medium to coarse sand to fine gravel \\
\hline $84-110$ & 26 & $1,027-1,001$ & Clay, gray, silty, sandy, pebbly; unoxidized (till) \\
\hline $110-120$ & 10 & $1,001-991$ & Clay, gray, silty; soft, greasy, unoxidized (lake sediment) \\
\hline $120-136$ & 16 & $991-975$ & Sand and gravel, coarse sand to fine gravel \\
\hline $136-143$ & 7 & $975-968$ & Clay, gray, silty; soft, greasy, unoxidized (lake sediment) \\
\hline $143-148$ & 5 & $968-963$ & Sand, gray, fine to medium; unoxidized \\
\hline $148-206$ & 58 & $963-905$ & Clay, gray, silty, sandy, pebbly; unoxidized, gravel lens at 188 and 197 feet (till) \\
\hline $206-260$ & 54 & $905-851$ & Sand and gravel. medium to coarse sand to fine gravel \\
\hline $260-310$ & 50 & $851-801$ & Clay, gray, sandy, pebbly; unoxidized (till) \\
\hline $310-409$ & 99 & $801-702$ & $\begin{array}{l}\text { Clay, gray, silty, sandy, pebbly; unoxidized, different E-log signature than above } \\
\text { interval (till) }\end{array}$ \\
\hline $409-476$ & 67 & $702-635$ & Clay, gray, silty, sandy, pebbly \\
\hline $476-498$ & 22 & $635-613$ & Sand, white, fine to medium; mostly quartz, some mica (granite wash) \\
\hline $498-522$ & 24 & $613-589$ & $\begin{array}{l}\text { Clay, black with a purplish tinge, silty; very greasy, oily, noncalcareous (Graneros } \\
\text { Shale) }\end{array}$ \\
\hline Electric log & & & \\
\hline
\end{tabular}


Local number: 126N49W15ADDA R

Other identifier: R2-97-24

Site identification number: 454157096462501

Date of construction: $07-15-97$

Land surface altitude: 990 feet

Total depth: 222 feet

\begin{tabular}{|c|c|c|c|}
\hline Depth & $\begin{array}{l}\text { Thickness } \\
\text { (feet) }\end{array}$ & $\begin{array}{l}\text { Altitude } \\
\text { (feet) }\end{array}$ & Description \\
\hline $0-6$ & 6 & $990-984$ & Clay, black, silty (alluvium) \\
\hline $6-10$ & 4 & $984-980$ & Clay, brown, light-gray and black layered; some pebbles (lake sediment) \\
\hline $10-12$ & 2 & $980-978$ & Clay, black, silty (lake sediment) \\
\hline $12-19$ & 7 & $978-971$ & Clay, light-gray, silty; calcareous, some fine sand, rocks at 17 and 19 feet \\
\hline $19-39$ & 20 & $971-951$ & Sand, gray, fine; unoxidized \\
\hline $39-52$ & 13 & $951-938$ & Clay, silt and sand, gray; alternating layers (lake sediment) \\
\hline $52-118$ & 66 & $938-872$ & Clay, gray, shaley; soft, greasy, noncalcareous (Blue Hill Shale Member - Carlile Shale) \\
\hline 118-187 & 69 & $872-803$ & $\begin{array}{l}\text { Clay, dark-brownish-gray; hard, some silty zones, some greasy zones, calcareous } \\
\text { (Fairport Shale Member - Carlile Shale) }\end{array}$ \\
\hline $187-218$ & 31 & 803-772 & $\begin{array}{l}\text { Siltstone, brown; some hard cherty layers, some white chalky layers. very calcareous } \\
\text { (Greenhorn Limestone) }\end{array}$ \\
\hline $218-222$ & 4 & $772-768$ & Clay, gray; greasy (Graneros Shale) \\
\hline
\end{tabular}


Local number: 126N50W6CCCC R

Other identifier: R2-97-36

Site identification number: 454306096584301

Date of construction: $08-11-97$

Land surface altitude: 1,164 feet

Total depth: 202 feet

\begin{tabular}{cccl}
\hline Depth & $\begin{array}{c}\text { Thickness } \\
\text { (feet) }\end{array}$ & $\begin{array}{c}\text { Altitude } \\
\text { (feet) }\end{array}$ & \multicolumn{1}{c}{ Description } \\
\hline $0-3$ & 3 & $1,164-1,161$ & Clay, black (topsoil) \\
$3-32$ & 29 & $1,161-1,132$ & $\begin{array}{l}\text { Clay, yellowish-brown, silty, sandy, pebbly; covered by several feet of loess. oxidized } \\
\text { (till) }\end{array}$ \\
$32-38$ & 6 & $1,132-1,126$ & Clay, reddish-brown, silty, sandy, pebbly; oxidized (till) \\
$38-67$ & 29 & $1,126-1,097$ & Clay, gray, silty, sandy, pebbly; soft, large cuttings, unoxidized (till) \\
$67-96$ & 29 & $1,097-1,068$ & Sand and gravel, coarse sand to fine gravel; mostly shale pebbles \\
$96-104$ & 8 & $1,068-1,060$ & Clay, gray, silty, sandy, pebbly; unoxidized (till) \\
$104-165$ & 61 & $1,060-999$ & Sand, gray, fine to medium; some silt and clay layers, unoxidized \\
$165-202$ & 37 & $999-962$ & $\begin{array}{l}\text { Clay, gray, shaley; hard, greasy, noncalcareous, concretion from 187 to 188 feet (Carlile } \\
\text { Shale) }\end{array}$ \\
\hline
\end{tabular}

Local number: 126N51W27BBBB R

Other identifier: R2-97-05

Site identification number: 454030097021901

Date of construction: 06-11-97

Land surface altitude: 1,215 feet

Total depth: 62 feet

\begin{tabular}{cccl}
\hline Depth & $\begin{array}{c}\text { Thickness } \\
\text { (feet) }\end{array}$ & $\begin{array}{c}\text { Altitude } \\
\text { (feet) }\end{array}$ & \multicolumn{1}{c}{ Description } \\
\hline $0-2$ & 2 & $1,215-1,213$ & Topsoil, black \\
$2-15$ & 14 & $1,213-1,200$ & Clay, yellowish-brown, silty, sandy, pebbly; oxidized (till) \\
$15-37$ & 21 & $1,200-1,178$ & Clay, gray, silty, sandy, pebbly; unoxidized (till) \\
$37-62$ & 25 & $1,178-1,153$ & Clay, gray, shaley; hard, greasy (Pierre Shale)
\end{tabular}

Electric log available 
Local number: 126N52W1DDDD R

Other identifier: R2-97-09

Site identification number: 454305097060901

Date of construction: 06-17-97

Land surface altitude: 1,252 feet

Total depth: 82 feet

\begin{tabular}{cccl}
\hline Depth & $\begin{array}{c}\text { Thickness } \\
\text { (feet) }\end{array}$ & $\begin{array}{c}\text { Altitude } \\
\text { (feet) }\end{array}$ & Description \\
\hline $0-2$ & 2 & $1,252-1,250$ & Topsoil, black \\
$2-24$ & 22 & $1,250-1,228$ & $\begin{array}{c}\text { Clay, tan to brown, silty, sandy; turns grayish-brown at 17 feet, very shaley with depth } \\
\text { (till?) }\end{array}$ \\
$24-27$ & 3 & $1,228-1,225$ & $\begin{array}{c}\text { Sand, fine } \\
\text { Clay, tan to white, shaley; hard, greasy, mottled, turns darker brown to grayish-brown } \\
\text { with depth (weathered Pierre Shale) }\end{array}$ \\
$27-52$ & 25 & $1,225-1,200$ & $\begin{array}{c}\text { Clay, gray, shaley; hard, greasy, good cuttings (Pierre Shale) } \\
52-82\end{array}$ \\
\hline
\end{tabular}


Local number: 126N52W20CCCB R

Other identifier: R2-99-08

Site identification number: 454033097122101

Date of construction: $07-28-99$

Land surface altitude: 2,035 feet

Total depth: 747 feet

\begin{tabular}{|c|c|c|c|}
\hline Depth & $\begin{array}{l}\text { Thickness } \\
\text { (feet) }\end{array}$ & $\begin{array}{l}\text { Altitude } \\
\text { (feet) }\end{array}$ & Description \\
\hline $0-2$ & 2 & $2,035-2,033$ & Clay, brown, silty, gravelly (topsoil) \\
\hline $2-36$ & 34 & $2,033-1.999$ & $\begin{array}{l}\text { Sand and gravel, brown, medium sand grading to coarse gravel with depth; some } \\
\text { cobbles, oxidized }\end{array}$ \\
\hline $36-75$ & 34 & $1,999-1,960$ & Clay, gray, silty, sandy, pebbly; hard, unoxidized (till) \\
\hline $75-83$ & 8 & $1,960-1,952$ & Clay, $\tan$, silty; oxidized (loess) \\
\hline $83-108$ & 85 & $1,952-1,927$ & Clay, yellowish-brown, silty, sandy, pebbly; dark-brown at 96 feet, oxidized (till) \\
\hline $108-124$ & 16 & $1,927-1,911$ & Clay, dark-brown and gray, very silty; greasy, layered (lake sediment) \\
\hline $124-184$ & 60 & $1,911-1,851$ & Clay, yellowish-brown, silty, sandy, pebbly; rock at 126 feet, oxidized (till) \\
\hline $184-282$ & 98 & $1,851-1,753$ & $\begin{array}{l}\text { Clay, light-gray to gray with brown oxidation zones, silty, sandy, pebbly; partially } \\
\text { oxidized (till) }\end{array}$ \\
\hline $282-286$ & 4 & $1,753-1,749$ & Gravel \\
\hline $286-466$ & 180 & $1,749-1,569$ & Clay, gray, silty, sandy, pebbly; rock at 314 feet, unoxidized (till) \\
\hline $466-476$ & 10 & $1,569-1,559$ & Sand and gravel, coarse sand to fine gravel \\
\hline $476-627$ & 151 & $1,559-1,408$ & Clay, gray, silty, sandy, pebbly, shaley; hard, unoxidized (till) \\
\hline $627-637$ & 10 & $1,408-1,398$ & Sand; hard \\
\hline $637-720$ & 83 & $1,398-1,315$ & $\begin{array}{l}\text { Clay, gray, silty, sandy, pebbly, shaley; hard rocks at } 670,677,685,686,689,696,699 \\
\text { to } 702,703,705,707,709,710,715,717 \text {, and } 719 \text { feet, unoxidized, poor cuttings } \\
\text { (till) }\end{array}$ \\
\hline $720-747$ & 27 & $1,315-1,288$ & Clay, dark-gray, shaley; hard, greasy (Pierre Shale) \\
\hline Electric $\log$ & & & \\
\hline
\end{tabular}


Local number: 127N48W21DDDC R

Other identifier: R2-97-44

Site identification number: 454541096400501

Date of construction: $08-25-97$

Land surface altitude: 1,090 feet

Total depth: 282 feet

\begin{tabular}{|c|c|c|c|}
\hline Depth & $\begin{array}{l}\text { Thickness } \\
\text { (feet) }\end{array}$ & $\begin{array}{l}\text { Altitude } \\
\text { (feet) }\end{array}$ & Description \\
\hline $0-2$ & 2 & $1,090-1,088$ & Clay, black, silty (topsoil) \\
\hline $2-6$ & 4 & $1.088-1.084$ & Clay, tan, silty; oxidized (loess) \\
\hline $6-10$ & 4 & $1,084-1,080$ & Sand, brown, medium; dirty, oxidized \\
\hline $10-28$ & 18 & $1,080-1,062$ & Clay, yellowish-brown, silty, sandy, pebbly; oxidized (till) \\
\hline $28-60$ & 32 & $1,062-1,030$ & Sand, brown, fine to medium; oxidized \\
\hline $60-72$ & 12 & $1,030-1,018$ & Sand, gray, fine to medium; unoxidized \\
\hline $72-85$ & 13 & $1,018-1,005$ & Clay, gray, silty; greasy, unoxidized (lake sediment) \\
\hline $85-114$ & 29 & $1,005-976$ & Clay, gray, silty, sandy, pebbly; unoxidized (till) \\
\hline $114-123$ & 9 & $976-967$ & Sand and gravel, coarse sand to fine gravel; rock at 116 feet \\
\hline $123-192$ & 69 & $967-898$ & $\begin{array}{l}\text { Clay, gray, silty, sandy, pebbly; gravel lens from } 138 \text { to } 142 \text { feet, and } 148 \text { to } 150 \text { feet, } \\
\text { very gravelly clay from } 151 \text { to } 155 \text { feet. unoxidized (till) }\end{array}$ \\
\hline $192-207$ & 15 & $898-883$ & Clay, gray, very silty; hard, greasy, unoxidized (lake sediment) \\
\hline 207-226 & 19 & $883-864$ & Sand, gray, medium to coarse; rock at 220 feet \\
\hline $226-241$ & 15 & $864-849$ & Clay, gray; oily, greasy, calcareous (Fairport Shale Member - Carlile Shale) \\
\hline 241-272 & 31 & $849-818$ & $\begin{array}{l}\text { Siltstone, brown; with brown and white silty clay layers, hard, cherty, very calcareous } \\
\text { (Greenhorn Limestone) }\end{array}$ \\
\hline $272-282$ & 10 & $818-808$ & Clay, gray; hard, greasy, slightly calcareous (Graneros Shale) \\
\hline Electric log & lable & & \\
\hline
\end{tabular}


Local number: 127N48W28AAAA R

Other identifier: R2-97-45

Site identification number: 454541096400502

Date of construction: $08-26-97$

Land surface altitude: $1,090.8$ feet

Total depth: 72 feet

\begin{tabular}{cccl}
\hline Depth & $\begin{array}{c}\text { Thickness } \\
\text { (feet) }\end{array}$ & $\begin{array}{c}\text { Altitude } \\
\text { (feet) }\end{array}$ & Description \\
\hline $0-2$ & 2 & $1,090.8-1,088.8$ & Clay, black, silty (topsoil) \\
$2-8$ & 6 & $1,088.8-1,082.8$ & Clay, tan, silty; oxidized (loess) \\
$8-28$ & 20 & $1,082.8-1,062.8$ & Clay, yellowish-brown, silty, sandy, pebbly; oxidized (till) \\
$28-60$ & 22 & $1,062.8-1,030.8$ & Sand, brown, fine to medium: oxidized \\
$60-72$ & 12 & $1,030.8-1,018.8$ & Sand, gray, fine to medium; unoxidized \\
Well screened from 62 to 72 feet; 2-inch PVC casing and screen. For electric-log see R2-97-44
\end{tabular}


Local number: 127N49W21DDDC R

Other identifier: R2-97-40

Site identification number: 454541096473901

Date of construction: $08-12-97$

Land surface altitude: 1,092 feet

Total depth: 282 feet

\begin{tabular}{|c|c|c|c|}
\hline Depth & $\begin{array}{l}\text { Thickness } \\
\text { (feet) }\end{array}$ & $\begin{array}{l}\text { Altitude } \\
\text { (feet) }\end{array}$ & Description \\
\hline $0-2$ & 2 & $1,092-1,090$ & Clay, black, silty (topsoil) \\
\hline $2-26$ & 24 & $1,090-1,066$ & Clay, yellowish-brown, silty, sandy, pebbly; oxidized (till) \\
\hline $26-30$ & 4 & $1,066-1,062$ & Clay, brown, silty; oxidized (lake sediment) \\
\hline $30-35$ & 5 & $1,062-1.057$ & Clay, grayish-brown to gray, silty; hard, greasy (lake sediment) \\
\hline $35-60$ & 25 & $1.057-1,032$ & Sand and gravel. fine to very fine sand grading to coarse sand to very fine gravel \\
\hline $60-92$ & 32 & $1,032-1,000$ & Clay, gray, very silty; greasy, unoxidized, sand lens from 78 to 80 feet (lake sediment) \\
\hline $92-114$ & 22 & $1,000-978$ & Clay, gray, silty, sandy, pebbly; hard, gravelly, unoxidized (till) \\
\hline $114-145$ & 31 & $978-947$ & $\begin{array}{l}\text { Clay, gray, silty, sandy; very hard, very few pebbles, many shale inclusions, rock at } \\
138 \text { feet, unoxidized (till) }\end{array}$ \\
\hline $145-148$ & 3 & $947-944$ & Gravel, fine to medium \\
\hline $148-158$ & 10 & 944-934 & Clay, light-gray, silty, very sandy, pebbly (till) \\
\hline $158-190$ & 32 & 934-902 & $\begin{array}{l}\text { Clay, greenish-brown, silty, sandy, pebbly; rocks at } 164 \text { and } 175 \text { feet. highly oxidized } \\
\text { (till) }\end{array}$ \\
\hline $190-220$ & 30 & $902-872$ & Clay, dark-gray, silty; hard, greasy, poor cuttings (till? or lake sediment?) \\
\hline $220-270$ & 50 & $872-822$ & $\begin{array}{l}\text { Clay, brownish-black, silty; oily, greasy, chalky, calcareous (Fairport Shale Member - } \\
\text { Carlile Shale) }\end{array}$ \\
\hline $270-282$ & 12 & $822-810$ & $\begin{array}{l}\text { Siltstone, brown, hard; some brown and white clay layers, cherty, very calcareous, rig } \\
\text { chatter (Greenhorn Limestone) }\end{array}$ \\
\hline Electric log & & & \\
\hline
\end{tabular}


Local number: 127 N50W7BBBB R

Other identifier: R2-97-37

Site identification number: 454817096584601

Date of construction: $08-11-97$

Land surface altitude: 1,168 feet

Total depth: 222 feet

\begin{tabular}{|c|c|c|c|}
\hline Depth & $\begin{array}{c}\text { Thickness } \\
\text { (feet) }\end{array}$ & $\begin{array}{l}\text { Altitude } \\
\text { (feet) }\end{array}$ & Description \\
\hline $0-3$ & 3 & $1,168-1,165$ & Clay, black, silty (topsoil) \\
\hline $3-14$ & 11 & $1,165-1,154$ & Clay, yellowish-brown, silty, sandy, pebbly; oxidized (till) \\
\hline $14-18$ & 4 & $1,154-1,150$ & Clay, grayish-brown, silty, sandy, pebbly; partially oxidized (till) \\
\hline $18-82$ & 64 & $1,150-1,086$ & $\begin{array}{l}\text { Clay, gray, silty, sandy, pebbly; soft, greasy, large cuttings, sand lens from } 65 \text { to } 67 \text { feet, } \\
\text { unoxidized (till) }\end{array}$ \\
\hline $82-88$ & 6 & $1,086-1,080$ & Clay, gray, silty; greasy (lake sediment) \\
\hline $88-196$ & 108 & $1,080-1,072$ & Sand, gray, fine, silty; unoxidized \\
\hline $96-104$ & 8 & $1,072-1,064$ & Clay, gray, silty; greasy (lake sediment) \\
\hline $104-110$ & 6 & $1,064-1,058$ & Sand, gray, fine, silty; unoxidized \\
\hline $110-119$ & 9 & $1,058-1,049$ & Clay, gray, silty, sandy; unoxidized (till?) \\
\hline $119-126$ & 7 & $1,049-1,042$ & Sand and gravel, coarse sand to fine to medium gravel; rock at 125 feet \\
\hline 126-177 & 51 & $1,042-991$ & Clay, gray, silty, sandy, pebbly; hard, softer from 163 to 172 feet, unoxidized (till) \\
\hline $177-183$ & 6 & $991-985$ & Gravel, medium to coarse \\
\hline $183-222$ & 39 & $985-946$ & Clay, dark-gray, shaley; hard, greasy (Carlile Shale) \\
\hline Electric log & lable & & \\
\hline
\end{tabular}


Local number: 127N50W9AAAA R

Other identifier: R2-97-38

Site identification number: 454818096550601

Date of construction: $08-12-97$

Land surface altitude: 1,119 feet

Total depth: 162 feet

\begin{tabular}{cccc}
\hline Depth & $\begin{array}{c}\text { Thickness } \\
\text { (feet) }\end{array}$ & $\begin{array}{c}\text { Altitude } \\
\text { (feet) }\end{array}$ & Description \\
\hline $0-2$ & 2 & $1,119-1,117$ & Clay, black, silty (topsoil) \\
$2-25$ & 23 & $1,117-1,094$ & $\begin{array}{c}\text { Clay, yellowish-brown, silty, sandy, pebbly; gravel lens from 23 to 24 feet, oxidized } \\
\text { (till) }\end{array}$ \\
$25-58$ & 33 & $1,094-1,061$ & Clay, gray, silty, sandy, pebbly; unoxidized (till) \\
$58-85$ & 27 & $1,061-1,034$ & $\begin{array}{c}\text { Sand and gravel, gray, fine to medium sand grading to medium gravel, rock at } 84 \text { feet } \\
\text { Clay, gray, silty, sandy, pebbly; rocks at 98, 130, and 132 feet, gravel lens at 116 and } \\
\text { 124 feet, unoxidized (till) }\end{array}$ \\
$135-135$ & 50 & $1,034-984$ & Clay, dark-gray, shaley; hard greasy, noncalcareous (Carlile Shale) \\
\hline
\end{tabular}

Local number: 127N50W9AAAA2 R

Other identifier: R2-97-39

Site identification number: 454818096550602

Date of construction: 08-12-97

Land surface altitude: $1,119.2$ feet

Total depth: 82 feet

\begin{tabular}{cccl}
\hline Depth & $\begin{array}{c}\text { Thickness } \\
\text { (feet) }\end{array}$ & $\begin{array}{c}\text { Altitude } \\
\text { (feet) }\end{array}$ & Description \\
\hline $0-2$ & 2 & $1,119.2-1,117.2$ & Clay, black, silty (topsoil) \\
$2-25$ & 23 & $1,117.2-1,094.2$ & Clay, yellowish-brown, silty, sandy, pebbly; rock at 20 feet, oxidized (till) \\
$25-62$ & 37 & $1,094.2-1,057.2$ & Clay, gray, silty, sandy, pebbly; unoxidized (till) \\
$62-82$ & 20 & $1,057.2-1,037.2$ & Sand and gravel, coarse sand to fine gravel \\
Well screened from 72 to 82 feet; 2-inch PVC casing and screen. For electric log see R2-97-38
\end{tabular}


Local number: 127N51W9AAAA R

Other identifier: R2-97-06

Site identification number: 454820097023601

Date of construction: 06-11-97

Land surface altitude: 1,188 feet

Total depth: 242 feet

\begin{tabular}{cccl}
\hline Depth & $\begin{array}{c}\text { Thickness } \\
\text { (feet) }\end{array}$ & \multicolumn{1}{c}{$\begin{array}{c}\text { Altitude } \\
\text { (feet) }\end{array}$} & \multicolumn{1}{c}{ Description } \\
\hline $0-3$ & 3 & $1,188-1,185$ & Topsoil. black \\
$3-6$ & 3 & $1,185-1,182$ & Clay, tan, very silty (loess) \\
$6-18$ & 12 & $1,182-1,170$ & Clay, yellowish-brown, silty, sandy, pebbly; oxidized (till) \\
$18-21$ & 3 & $1,170-1,167$ & Clay, gray, silty, sandy, pebbly; unoxidized (till) \\
$21-24$ & 3 & $1,167-1,164$ & Clay, dark-brown, silty, sandy, pebbly; oxidized, rock at 23 feet (till) \\
$24-67$ & 43 & $1,164-1,121$ & $\begin{array}{l}\text { Clay, gray, silty, sandy, pebbly; large cuttings, unoxidized, gravel lens from 55 to 57 feet } \\
\text { and 58 to 59 feet (till) }\end{array}$ \\
$67-99$ & 32 & $1,121-1,089$ & $\begin{array}{l}\text { Sand and gravel, medium sand to fine gravel; some medium to coarse gravel, much } \\
\text { shale }\end{array}$ \\
$99-143$ & 44 & $1,089-1,045$ & $\begin{array}{l}\text { Clay, gray, silty, sandy, pebbly; unoxidized (till) } \\
143-177\end{array}$ \\
$177-208$ & 34 & $1,045-1,011$ & Clay, gray, very silty, sandy; some fine sand layers (lake sediment) \\
$208-242$ & 34 & $1,011-980$ & Clay, gray, silty, sandy, pebbly; unoxidized, rock at 206 feet (till) \\
Electric log available & $980-946$ & Clay, gray, shaley; greasy, noncalcareous (Carlile Shale) \\
\hline
\end{tabular}


Local number: 127N51W9AAAA2 R

Other identifier: R2-97-07

Site identification number: 454820097023602

Date of construction: 06-11-97

Land surface altitude: $1,188.7$ feet

Total depth: 102 feet

\begin{tabular}{cccl}
\hline Depth & $\begin{array}{c}\text { Thickness } \\
\text { (feet) }\end{array}$ & $\begin{array}{c}\text { Altitude } \\
\text { (feet) }\end{array}$ & Description \\
\hline $0-3$ & 3 & $1,188.7-1,185.7$ & Topsoil, black \\
$3-24$ & 21 & $1,185.7-1,164.7$ & Clay, yellowish-brown, silty, sandy, pebbly; oxidized (till) \\
$24-67$ & 43 & $1,164.7-1,121.7$ & Clay, gray, silty, sandy, pebbly; oxidized (till) \\
$67-102$ & 35 & $1,121.7-1,086.7$ & $\begin{array}{l}\text { Sand and gravel, medium sand to fine gravel; some medium to coarse gravel, much } \\
\text { shale }\end{array}$ \\
Well screened from 92 to 102 feet; 2-inch PVC screen and casing. Electric log available \\
\hline
\end{tabular}

Local number: 127N51W21DDDA R

Other identifier: R2-97-08

Site identification number: 454543097022701

Date of construction: 06-16-97

Land surface altitude: 1,155 feet

Total depth: 155 feet

\begin{tabular}{cccl}
\hline Depth & $\begin{array}{c}\text { Thickness } \\
\text { (feet) }\end{array}$ & $\begin{array}{c}\text { Altitude } \\
\text { (feet) }\end{array}$ & \multicolumn{1}{c}{ Description } \\
\hline $0-3$ & 3 & $1,155-1,152$ & Topsoil, black \\
$3-5$ & 2 & $1,152-1,150$ & Gravel and cobbles \\
$5-15$ & 10 & $1,150-1,140$ & Clay, brown, silty, sandy, pebbly; oxidized (till) \\
$15-90$ & 75 & $1,140-1,065$ & Clay, gray, silty, sandy, pebbly: sand lens from 80 to 82 feet (till) \\
$90-122$ & 32 & $1,065-1.033$ & Sand, gray, fine: unoxidzed \\
$122-135$ & 13 & $1,033-1.020$ & Clay, gray, silty; very silty to sandy from 127 to 135 feet (lake sediments) \\
$135-155$ & 20 & $1,020-1,000$ & Clay, gray; hard, greasy, noncalcareous, concretion at 147 feet (Carlile Shale) \\
\hline Electric log available & & \\
\hline
\end{tabular}


Local number: 127N52W4DCDC R

Other identifier: R2-97-19

Site identification number: 454819097102101

Date of construction: 07-07-97

Land surface altitude: 1,231 feet

Total depth: 102 feet

\begin{tabular}{cccl}
\hline Depth & $\begin{array}{c}\text { Thickness } \\
\text { (feet) }\end{array}$ & $\begin{array}{c}\text { Altitude } \\
\text { (feet) }\end{array}$ & \multicolumn{1}{c}{ Description } \\
\hline $0-5$ & 5 & $1,231-1,226$ & Clay, tan, silty; oxidized (alluvium) \\
$5-12$ & 7 & $1,226-1,219$ & Clay, yellowish-brown, silty, sandy, pebbly; oxidized (till) \\
$12-53$ & 41 & $1,219-1,178$ & Clay, gray, silty, sandy, pebbly; large cuttings, unoxidized (till) \\
$53-58$ & 5 & $1,178-1,173$ & Clay, gray, very silty; unoxidized (lake clay) \\
$58-66$ & 8 & $1,173-1,165$ & Sand and gravel, coarse sand to fine gravel \\
$66-74$ & 8 & $1,165-1,157$ & Clay, gray, silty, sandy, pebbly; unoxidized (till) \\
$74-102$ & 28 & $1,157-1,129$ & Clay, gray; hard, greasy, noncalcareous (Pierre Shale) \\
Electric log available & & \\
\hline
\end{tabular}

Local number: 127N52W25AAAA R

Other identifier: R2-97-10

Site identification number: 454538097061101

Date of construction: 06-17-97

Land surface altitude: 1,182 feet

Total depth: 182 feet

\begin{tabular}{cccl}
\hline Depth & $\begin{array}{c}\text { Thickness } \\
\text { (feet) }\end{array}$ & $\begin{array}{c}\text { Altitude } \\
\text { (feet) }\end{array}$ & \multicolumn{1}{c}{ Description } \\
\hline $0-2$ & 2 & $1,182-1,180$ & Topsoil, black \\
$2-7$ & 5 & $1,180-1,175$ & Clay, brown, very silty; oxidized \\
$7-9$ & 2 & $1,175-1,173$ & Clay, gray, very silty; unoxidized \\
$9-39$ & 30 & $1,173-1,143$ & Sand and gravel, medium sand to fine gravel \\
$39-58$ & 19 & $1,143-1,124$ & Clay, gray, silty, sandy, pebbly; unoxidized (till) \\
$58-100$ & 42 & $1,124-1,082$ & Clay, gray, shaley; hard, greasy, noncalcareous (Pierre Shale) \\
$100-151$ & 51 & $1,082-1,031$ & Clay, black, shaley; hard, gritty, oily, noncalcareous (Sharon Springs Member- Pierre \\
$151-157$ & 6 & $1,031-1,025$ & Clay, gray, silty; hard, calcareous (Niobrara Formation?) \\
$157-182$ & 25 & $1,025-1,000$ & Clay, gray, shaley; hard, greasy (Carlile Shale)
\end{tabular}

Electric log available 
Local number: 127N52W25AAAA2 R

Other identifier: R2-97-11

Site identification number: 454538097061102

Date of construction: 06-17-97

Land surface altitude: $1,182.4$ feet

Total depth: 42 feet

\begin{tabular}{cccl}
\hline Depth & $\begin{array}{c}\text { Thickness } \\
\text { (feet) }\end{array}$ & $\begin{array}{c}\text { Altitude } \\
\text { (feet) }\end{array}$ & Description \\
\hline $0-2$ & 2 & $1,182.4-1,180.4$ & Topsoil, black \\
$2-9$ & 7 & $1,180.4-1,173.4$ & Clay, brown, very silty, sandy; oxidized \\
$9-12$ & 3 & $1,173.4-1,170.4$ & Clay, gray, silty, sandy; unoxidized \\
$12-36$ & 24 & $1,170.4-1,146.4$ & Sand and gravel. fine sand grading to fine to medium gravel \\
$36-42$ & 6 & $1,146.4-1,140.4$ & Clay, gray, silty \\
Well screened from 23.1 to 33.1 feet; 2-inch PVC casing and screen. For electric log see R2-97-10
\end{tabular}


Local number: 128N48W19BCCC R

Other identifier: R2-97-30

Site identification number: 455123096434701

Date of construction: $07-28-97$

Land surface altitude: $1,078.6$ feet

Total depth: 234 feet

\begin{tabular}{ccrl}
\hline Depth & $\begin{array}{c}\text { Thickness } \\
\text { (feet) }\end{array}$ & \multicolumn{1}{c}{$\begin{array}{c}\text { Altitude } \\
\text { (feet) }\end{array}$} & \multicolumn{1}{c}{ Description } \\
\hline $0-1$ & 1 & $1,078.6-1,077.6$ & Topsoil, black, sandy \\
$1-8$ & 7 & $1,077.6-1,070.6$ & Sand, brown, fine; oxidized \\
$8-25$ & 17 & $1,070.6-1,053.6$ & Clay, brown, silty, very, sandy, pebbly; oxidized (till) \\
$25-76$ & 51 & $1,053.6-1,002.6$ & $\begin{array}{l}\text { Sand, gray, very fine to fine; grades into lake sediment with depth, some clay layers, } \\
\text { unoxidized }\end{array}$ \\
$76-102$ & 26 & $1,002.6-976.6$ & Clay, gray, very silty, sandy; greasy, unoxidized (lake sediment) \\
$102-108$ & 6 & $976.6-970.6$ & Sand, gray, fine; unoxidized \\
$108-135$ & 27 & $970.6-943.6$ & Clay, gray, silty, sandy, pebbly; unoxidized (till) \\
$135-151$ & 16 & $943.6-927.6$ & Clay, dark-gray, silty, very sandy, pebbly; harder than above interval, unoxidized rock at \\
1 144 feet (till)
\end{tabular}


Local number: 128N48W30BBBB R

Other identifier: R2-97-29

Site identification number: 455055096434801

Date of construction: $07-23-97$

Land surface altitude: 1,092 feet

Total depth: 252 feet

\begin{tabular}{|c|c|c|c|}
\hline Depth & $\begin{array}{l}\text { Thickness } \\
\text { (feet) }\end{array}$ & $\begin{array}{l}\text { Altitude } \\
\text { (feet) }\end{array}$ & Description \\
\hline $0-1$ & 1 & $1,092-1,091$ & Topsoil, black \\
\hline $1-10$ & 9 & $1,091-1,082$ & Sand, yellowish-brown, fine to medium, silty, clayey; dirty, oxidized \\
\hline $10-20$ & 10 & $1,082-1,072$ & Clay, yellowish-brown, silty, sandy, pebbly; oxidized (till) \\
\hline $20-32$ & 12 & $1,072-1,060$ & Sand, brown, fine to medium: oxidized \\
\hline $32-65$ & 33 & $1,060-1,027$ & Sand, gray, fine to medium; unoxidized \\
\hline $65-70$ & 5 & $1,027-1,022$ & Clay, gray, silty; greasy, unoxidized (lake sediment) \\
\hline $70-78$ & 8 & $1.022-1.014$ & Sand, gray, fine to medium; unoxidized \\
\hline $78-113$ & 35 & $1,014-979$ & Clay, gray. silty; greasy, unoxidized (lake sediments) \\
\hline $113-144$ & 31 & $979-948$ & Clay, gray, silty, sandy, pebbly; rock at 132 feet, unoxidized (till) \\
\hline $144-185$ & 41 & $948-907$ & $\begin{array}{l}\text { Clay, gray, silty, sandy, pebbly; harder than above interval, with a differing E-log } \\
\text { signature, unoxidized (till) }\end{array}$ \\
\hline $185-194$ & 9 & $907-898$ & Clay, gray, green, and brown, silty, sandy; alternating layers (lake sediment) \\
\hline $194-248$ & 54 & $898-844$ & Sand and gravel, coarse sand to fine gravel \\
\hline $248-252$ & 4 & $844-840$ & $\begin{array}{l}\text { Siltstone; hard, cherty, calcareous, fractured, lost all of the drilling fluid, drill stem stuck } \\
\text { in hole, abandoned hole (Greenhorn Limestone) }\end{array}$ \\
\hline
\end{tabular}


Local number: 128N49W1DDAD R

Other identifier: R2-97-31

Site identification number: 455341096434701

Date of construction: $07-29-97$

Land surface altitude: 1,072 feet

Total depth: $262 \mathrm{feet}$

\begin{tabular}{|c|c|c|c|}
\hline Depth & $\begin{array}{l}\text { Thickness } \\
\text { (feet) }\end{array}$ & $\begin{array}{l}\text { Altitude } \\
\text { (feet) }\end{array}$ & Description \\
\hline $0-4$ & 4 & $1,072-1,068$ & Clay, black, silty (topsoil and alluvium) \\
\hline $4-20$ & 16 & $1,068-1,052$ & Sand, brown, coarse; oxidized \\
\hline $20-24$ & 4 & $1,052-1,048$ & Clay, brown, silty, sandy, pebbly; oxidized (till) \\
\hline $24-54$ & 30 & $1,048-1,018$ & Sand, gray, fine, unoxidized \\
\hline $54-71$ & 17 & $1,018-1,001$ & $\begin{array}{l}\text { Silt, sand and clay, gray; very clayey from } 54 \text { to } 62 \text { feet, alternating layers (lake } \\
\text { sediment) }\end{array}$ \\
\hline $71-102$ & 31 & $1,001-970$ & Clay, gray, silty; greasy, unoxidized (lake sediment) \\
\hline $102-117$ & 15 & $970-955$ & Clay, gray, silty, sandy, pebbly; unoxidized (till) \\
\hline $117-138$ & 21 & $955-934$ & Gravel, fine to medium \\
\hline $138-204$ & 66 & $934-868$ & $\begin{array}{l}\text { Clay, gray, silty, sandy, pebbly; unoxidized, many cobbles, gravel lens from } 167 \text { to } 168 \\
\text { feet and } 170 \text { to } 172 \text { feet (till) }\end{array}$ \\
\hline $204-262$ & 58 & $868-810$ & Clay, gray, silty; greasy, some calcareous zones (Graneros Shale?) \\
\hline Electric $\log$ & ble & & \\
\hline
\end{tabular}

Local number: 128N49W1DDAD2 R

Other identifier: R2-97-32

Site identification number: 455341096435001

Date of construction: 07-29-97

Land surface altitude: $1,071.7$ feet

Total depth: 142 feet

\begin{tabular}{cccl}
\hline Depth & $\begin{array}{c}\text { Thickness } \\
\text { (feet) }\end{array}$ & $\begin{array}{c}\text { Altitude } \\
\text { (feet) }\end{array}$ & Description \\
\hline $0-4$ & 4 & $1,071.7-1,067.7$ & Clay, black, silty (topsoil and alluvium) \\
$4-35$ & 31 & $1,067.7-1,036.7$ & Sand, yellowish-brown, fine to coarse; some fine gravel, oxidized \\
$35-57$ & 22 & $1,036.7-1,014.7$ & Sand, gray, fine; some clay layers, unoxidized \\
$57-100$ & 43 & $1,014.7-971.7$ & Clay, gray, silty, sandy; greasy, unoxidized (lake sediment) \\
$100-130$ & 30 & $971.7-941.7$ & Clay, gray, silty, sandy, pebbly; sand lens at 117 feet, unoxidized (till) \\
$130-142$ & 12 & $941.7-929.7$ & Sand and gravel, coarse sand to fine to medium gravel \\
Well screened from 132 to 142 feet; 2-inch PVC casing and screen. For electric log see R2-97-31
\end{tabular}


Local number: 128N49W21DDAD R

Other identifier: R2-97-35

Site identification number: 455104096473501

Date of construction: $08-05-97$

Land surface altitude: 1,076 feet

Total depth: 322 feet

\begin{tabular}{|c|c|c|c|}
\hline Depth & $\begin{array}{l}\text { Thickness } \\
\text { (feet) }\end{array}$ & $\begin{array}{c}\text { Altitude } \\
\text { (feet) }\end{array}$ & Description \\
\hline $0-1$ & 1 & $1,076-1,075$ & Clay, black (topsoil) \\
\hline $1-16$ & 15 & $1,075-1,060$ & Clay, yellowish-brown, silty, sandy, pebbly; oxidized (till) \\
\hline $16-26$ & 10 & $1,060-1,050$ & Sand, brown, medium to coarse; oxidized \\
\hline $26-59$ & 33 & $1,050-1,017$ & Sand, gray, fine; some very silty zones, unoxidized \\
\hline $59-70$ & 11 & $1,017-1,006$ & Clay, gray, silty; greasy, unoxidized (lake sediment) \\
\hline $70-77$ & 7 & $1,006-999$ & Sand, gray, fine, silty; unoxidized \\
\hline $77-94$ & 17 & $999-982$ & Clay, gray, silty; greasy, unoxidized (lake sediment) \\
\hline $94-106$ & 12 & $982-970$ & Clay, gray, silty, sandy, pebbly; unoxidized (till) \\
\hline $106-112$ & 6 & $970-964$ & Gravel, medium to coarse; rock at 110 feet \\
\hline $112-118$ & 6 & $964-958$ & Clay, gray, silty, sandy, pebbly; unoxidized (till) \\
\hline $118-138$ & 20 & $958-938$ & Gravel, fine to coarse \\
\hline $138-160$ & 22 & $938-916$ & Sand, medium to coarse \\
\hline $160-183$ & 23 & $916-893$ & Clay, gray, silty, sandy, pebbly; unoxidized (till) \\
\hline $183-240$ & 57 & $893-836$ & Sand and gravel, coarse sand to fine gravel \\
\hline $240-291$ & 51 & 836-785 & Sand, medium to coarse; hard, cemented \\
\hline 291-294 & 3 & $785-782$ & Gravel and boulders \\
\hline 294-322 & 28 & $782-754$ & Clay, dark-gray; greasy, calcareous (Graneros Shale) \\
\hline Electric log & & & \\
\hline
\end{tabular}


Local number: 128N50W1DDDC R

Other identifier: R2-97-28

Site identification number: 455334096512001

Date of construction: $07-22-97$

Land surface altitude: 1,093 feet

Total depth: 222 feet

\begin{tabular}{|c|c|c|c|}
\hline Depth & $\begin{array}{c}\text { Thickness } \\
\text { (feet) }\end{array}$ & $\begin{array}{c}\text { Altitude } \\
\text { (feet) }\end{array}$ & Description \\
\hline $0-3$ & 3 & $1,093-1,900$ & Clay, black (topsoil) \\
\hline 3-17 & 14 & $1,090-1,076$ & Clay, tan to yellowish-brown, silty, sandy; oxidized (loess or lake sediment?) \\
\hline $17-20$ & 3 & $1,076-1,073$ & Clay, yellowish-brown, silty, sandy, pebbly; oxidized (till) \\
\hline $20-24$ & 4 & 1,073-1.069 & Clay, gray, silty, sandy, pebbly; unoxidized (till) \\
\hline 24-36 & 12 & $1,069-1,057$ & Sand, gray, fine, silty; unoxidized \\
\hline $36-64$ & 28 & $1,057-1,029$ & Silt, sand, and clay, gray; alternating layers (lake sediment) \\
\hline $64-70$ & 6 & $1,029-1,023$ & Sand, gray, fine; unoxidized \\
\hline $70-86$ & 14 & $1,023-1,007$ & Clay, gray, silty; greasy, unoxidized (lake sediment) \\
\hline $86-120$ & 34 & $1,007-973$ & $\begin{array}{l}\text { Clay, gray, silty, sandy, pebbly; rocks and gravel from } 91 \text { to } 95 \text { feet, gravel lens from } \\
108 \text { feet, unoxidized (till) }\end{array}$ \\
\hline $120-125$ & 5 & 973-968 & Sand and gravel, coarse sand to medium gravel \\
\hline $125-163$ & 38 & $968-930$ & Clay, gray, silty, sandy, pebbly; unoxidized (till) \\
\hline $163-168$ & 5 & $930-925$ & Sand and gravel, medium sand to fine gravel \\
\hline $168-180$ & 12 & $925-913$ & Clay, gray, silty; greasy, unoxidized, some sandy zones (lake sediment) \\
\hline $180-194$ & 14 & $913-899$ & Clay, gray, silty, sandy, pebbly; unoxidized (till) \\
\hline $194-198$ & 4 & $899-895$ & Sand and gravel, coarse sand to fine gravel \\
\hline $198-222$ & 24 & $895-871$ & Clay, dark-gray to brownish-black; hard, greasy, calcareous (Carlile Shale) \\
\hline Electric log & ble & & \\
\hline
\end{tabular}


Local number: 128N50W25AAAB R

Other identifier: R2-97-25

Site identification number: 455058096511801

Date of construction: $07-21-97$

Land surface altitude: 1,096 feet

Total depth: 258 feet

\begin{tabular}{cccl}
\hline Depth & $\begin{array}{c}\text { Thickness } \\
\text { (feet) }\end{array}$ & $\begin{array}{c}\text { Altitude } \\
\text { (feet) }\end{array}$ & \multicolumn{1}{c}{ Description } \\
\hline $0-1$ & 1 & $1,096-1,095$ & Clay, black (topsoil) \\
$1-10$ & 9 & $1,095-1,086$ & Clay, tan, silty (roadfill and loess) \\
$10-19$ & 9 & $1,086-1,077$ & Clay, yellowish-brown, silty, sandy, pebbly; oxidized (till) \\
$19-22$ & 3 & $1,077-1,074$ & Clay, gray, silty, sandy, pebbly; unoxidized (till) \\
$22-62$ & 40 & $1,074-1,034$ & Sand, gray, fine to medium; unoxidized clay layers from 42 to 50 feet \\
$62-83$ & 21 & $1,034-1,013$ & Clay, gray, very silty; unoxidized (lake sediment) \\
$83-92$ & 9 & $1,013-1,004$ & Sand, gray, fine to medium; unoxidized \\
$92-122$ & 30 & $1,004-974$ & Clay, gray, silty, sandy, pebbly; unoxidized, rocks at 90, 92, 105, and 122 feet, shale \\
& 88 & $974-886$ & Sravel lens at 105 feet (till) \\
$122-210$ & 12 & $886-874$ & Clay, gray, silty, sandy, pebbly; unoxidized (till) \\
$210-222$ & 26 & $874-848$ & Gravel, coarse to very coarse; with cobbles \\
$222-248$ & 8 & $848-840$ & Clay, dark-gray; hard, greasy, calcareous (Fairport Shale Member - Carlile Shale) \\
$248-256$ & 2 & $840-838$ & Siltstone, brown; very hard, cherty, very calcareous (Greenhorn Limestone) \\
$256-258$ & & & \\
\hline Electric log available & & & \\
\hline
\end{tabular}


Local number: 128N50W25AAAB2 R

Other identifier: R2-97-26

Site identification number: 455058096511802

Date of construction: $07-22-97$

Land surface altitude: $1,095.9$ feet

Total depth: 202 feet

\begin{tabular}{cccl}
\hline Depth & $\begin{array}{c}\text { Thickness } \\
\text { (feet) }\end{array}$ & $\begin{array}{c}\text { Altitude } \\
\text { (feet) }\end{array}$ & Description \\
\hline $0-17$ & 17 & $1,095.9-1,078.9$ & Clay, tan to yellowish-brown, silty (loess over oxidized till) \\
$17-26$ & 9 & $1,078.9-1.069 .9$ & Clay, gray, silty, sandy, pebbly; unoxidized (till) \\
$26-67$ & 41 & $1,069.9-1,028.9$ & Sand, gray, very fine to fine, unoxidized \\
$67-96$ & 29 & $1,028.9-999.9$ & $\begin{array}{c}\text { Clay, gray, very silty, sandy; unoxidized, gravel lens near bottom of interval (lake } \\
\text { sediment) }\end{array}$ \\
$96-130$ & 34 & $999.9-965.9$ & Clay, gray, silty, sandy, pebbly; unoxidized, gravel lens at 107 feet (till) \\
$130-202$ & 72 & $965.9-893.9$ & Sand and gravel, coarse sand to fine to medium gravel; some clay layers \\
Well screened from 190 to 200 feet; 2 -inch PVC casing and screen. For electric-log see R2-97-25. North of two wells
\end{tabular}

Local number: 128N50W25AAAB3 R

Other identifier: R2-97-27

Site identification number: 455058096511803

Date of construction: $07-22-97$

Land surface altitude: $1,095.4$ feet

Total depth: 62 feet

\begin{tabular}{cccl}
\hline Depth & $\begin{array}{c}\text { Thickness } \\
\text { (feet) }\end{array}$ & $\begin{array}{c}\text { Altitude } \\
\text { (feet) }\end{array}$ & Description \\
\hline $0-18$ & 18 & $1,095.4-1,077.4$ & Clay, yellowish-brown, silty, sandy, pebbly; oxidized (till) \\
$18-27$ & 9 & $1,077.4-1,068.4$ & Clay, gray, silty, sandy, pebbly; unoxidized (till) \\
$27-62$ & 35 & $1,068.4-1,033.4$ & Sand, gray, very fine to fine; unoxidized \\
Well screened from 52 to 62 feet; 2-inch PVC casing and screen. For electric log see R2-97-25. South of two wells \\
\hline
\end{tabular}


Local number: 128N50W28AAAA R

Other identifier: R2-97-14

Site identification number: 455054096550201

Date of construction: 06-24-97

Land surface altitude: 1,102 feet

Total depth: 342 feet

\begin{tabular}{|c|c|c|c|}
\hline Depth & $\begin{array}{l}\text { Thickness } \\
\text { (feet) }\end{array}$ & $\begin{array}{l}\text { Aititude } \\
\text { (feet) }\end{array}$ & Description \\
\hline $0-10$ & 10 & $1,102-1,092$ & Clay, yellowish-brown, silty, sandy, pebbly; oxidized (till) \\
\hline $10-17$ & 7 & $1,092-1,085$ & Clay, gray, silty, sandy, pebbly; unoxidized (till) \\
\hline $17-20$ & 3 & $1,085-1,082$ & Sand and gravel, medium sand to fine gravel \\
\hline $20-36$ & 16 & $1,082-1,066$ & Clay, gray, very silty, sandy; unoxidized \\
\hline $36-62$ & 26 & $1,066-1,040$ & Sand, gray, fine; unoxidized \\
\hline $62-114$ & 52 & $1,040-988$ & $\begin{array}{l}\text { Clay, gray, silty, very sandy, pebbly; sand lens } 75 \text { to } 80 \text { feet and } 99 \text { to } 102 \text { feet, } \\
\text { unoxidized (till) }\end{array}$ \\
\hline $114-126$ & 12 & 988-976 & Sand, gray, fine; unoxidized \\
\hline $126-202$ & 76 & $976-900$ & Clay, gray, silty, sandy, pebbly; rocks at 137 and 152 feet, unoxidized (till) \\
\hline $202-223$ & 21 & $900-879$ & Sand and gravel, medium sand to fine gravel; clay layer from 218 to 222 feet \\
\hline 223-261 & 38 & $879-841$ & Clay, dark-gray, silty; gritty, calcareous (Fairport Shale Member - Carlile Shale) \\
\hline 261-296 & 35 & $841-806$ & $\begin{array}{l}\text { Clay, brownish-gray to dark-gray, silty; some white calcareous layers, some hard } \\
\text { calcareous limestone layers, very calcareous (Greenhorn Limestone) }\end{array}$ \\
\hline 296-342 & 46 & $806-760$ & Clay, dark-gray, shaley; hard, greasy (Graneros Shale) \\
\hline \multicolumn{4}{|c|}{ Electric log available } \\
\hline
\end{tabular}

Local number: 128 N50W30BABB R

Other identifier: R2-97-13

Site identification number: 455054096582101

Date of construction: 06-23-97

Land surface altitude: 1,172 feet

Total depth: 242 feet

\begin{tabular}{|c|c|c|c|}
\hline Depth & $\begin{array}{l}\text { Thickness } \\
\text { (feet) }\end{array}$ & $\begin{array}{l}\text { Altitude } \\
\text { (feet) }\end{array}$ & Description \\
\hline $0-1$ & 1 & $1,172-1,171$ & Topsoil, black \\
\hline $1-21$ & 20 & $1,171-1,151$ & Clay, yellowish-brown, silty, sandy, pebbly; oxidized (till) \\
\hline $21-86$ & 65 & $1,151-1,086$ & Clay, gray, silty, sandy, pebbly; large cuttings, unoxidized, (till) \\
\hline $86-98$ & 12 & $1,086-1,074$ & Sand and gravel; clay layer 89 to 92 feet \\
\hline $98-193$ & 95 & $1,074-979$ & $\begin{array}{l}\text { Clay, gray, silty, sandy, pebbly; rock at } 107 \text { feet, smaller cuttings than above till interval, } \\
\text { hard, unoxidized, gravel lens } 130 \text { to } 134 \text { feet, } 147 \text { to } 151 \text { feet, } 155 \text { to } 157 \text { feet, } 164 \text { to } \\
167 \text { feet, } 187 \text { to } 190 \text { feet, and } 191 \text { to } 193 \text { feet (till) }\end{array}$ \\
\hline $193-242$ & 49 & 979-930 & Clay, dark-gray, shaley; hard, greasy (Carlile Shale) \\
\hline \multicolumn{4}{|c|}{ Electric log available } \\
\hline
\end{tabular}


Local number: 128N51W27BBBB R

Other identifier: R2-97-12

Site identification number: 455056097023101

Date of construction: 06-18-97

Land surface altitude: 1,222 feet

Total depth: 282 feet

\begin{tabular}{cccl}
\hline Depth & $\begin{array}{c}\text { Thickness } \\
\text { (feet) }\end{array}$ & $\begin{array}{c}\text { Altitude } \\
\text { (feet) }\end{array}$ & \multicolumn{1}{c}{ Description } \\
\hline $0-2$ & 2 & $1,222-1,220$ & Topsoil, black \\
$2-20$ & 18 & $1,220-1,202$ & Clay, yellowish-brown, silty, sandy, pebbly; oxidized (till) \\
$20-24$ & 4 & $1,202-1,198$ & Clay, gray, very silty; greasy (lake clay inclusion) \\
$24-112$ & 88 & $1,198-1,110$ & Clay, gray, silty, sandy, pebbly; large cuttings, unoxidized (till) \\
$112-144$ & 32 & $1,110-1,078$ & Sand and gravel, coarse sand grading to fine gravel; some coarse gravel and cobbles \\
& 28 & $1,078-1,050$ & Clay, gray, very silty; greasy (lake clay) \\
$144-172$ & 44 & $1,050-1,006$ & Sand, gray, very fine to fine; unoxidized \\
$172-216$ & 31 & $1,006-975$ & Clay, gray, silty, sandy, pebbly; some gravel lens, unoxidized (till) \\
$216-247$ & 35 & $975-940$ & Clay, dark-gray, shaley; very hard, greasy, noncalcareous (Carlile Shale) \\
$247-282$ & & & \\
\hline Electric log available & & & \\
\hline
\end{tabular}


Local number: 128N52W1DDDD R

Other identifier: R2-97-15

Site identification number: 455332097061301

Date of construction: $06-25-97$

Land surface altitude: 1,204 feet

Total depth: 262 feet

\begin{tabular}{cccl}
\hline Depth & $\begin{array}{c}\text { Thickness } \\
\text { (feet) }\end{array}$ & $\begin{array}{c}\text { Altitude } \\
\text { (feet) }\end{array}$ & \multicolumn{1}{c}{ Description } \\
\hline $0-1$ & 1 & $1,204-1,203$ & $\begin{array}{l}\text { Topsoil, black } \\
\text { Clay, yellowish-brown, silty, sandy, pebbly; turns grayish-brown at 10 feet, some } \\
\text { reddish-brown from 17 to 19 feet, oxidized (till) }\end{array}$ \\
$30-74$ & 29 & $1,203-1,174$ & $\begin{array}{l}\text { Clay, gray, silty, sandy, pebbly; large cuttings, unoxidized, fine gravel lens from 34 to } \\
\text { 37 feet (till) }\end{array}$ \\
$74-100$ & 26 & $1,174-1,130-1,104$ & $\begin{array}{l}\text { Clay, gray, very silty; unoxidized, sand content increased with depth (lake sediment) } \\
100-168\end{array}$ \\
$168-208$ & 40 & $1,104-1,036$ & $\begin{array}{l}\text { Sand, gray, fine; unoxidized } \\
\text { Clay, gray, very silty, sandy; layered, unoxidized (lake sediment) }\end{array}$ \\
$208-235$ & 27 & $996-969$ & $\begin{array}{l}\text { Clay, gray, silty, sandy, pebbly; unoxidized (till) } \\
235-262\end{array}$ \\
27 & $969-942$ & $\begin{array}{c}\text { Clay, dark-gray, shaley; hard, greasy, noncalcareous, concretion at 237 feet (Carlile } \\
\text { Shale) }\end{array}$ \\
Electric log available & & \\
\hline
\end{tabular}

Local number: 128N52W1DDDD2 R

Other identifier: R2-97-18

Site identification number: 455335097061501

Date of construction: 07-02-97

Land surface altitude: $1,204.2$ feet

Total depth: 162 feet

\begin{tabular}{cccl}
\hline Depth & $\begin{array}{c}\text { Thickness } \\
\text { (feet) }\end{array}$ & $\begin{array}{c}\text { Altitude } \\
\text { (feet) }\end{array}$ & Description \\
\hline $0-1$ & 1 & $1,204.2-1,203.2$ & Topsoil, black \\
$1-30$ & 29 & $1,203.2-1,174.2$ & $\begin{array}{c}\text { Clay, yellowish-brown, silty, sandy, pebbly; turns grayish-brown at 10 feet, some } \\
\text { reddish-brown from 17 to 19 feet, oxidized (till) }\end{array}$ \\
$30-74$ & 44 & $1,174.2-1,130.2$ & Clay, gray, silty, sandy, pebbly; large soft cuttings, unoxidized (till) \\
$74-100$ & 26 & $1,130.2-1,104.2$ & Clay, gray, very silty, unoxidized (lake sediment) \\
$100-162$ & 62 & $1,104.2-1,042.2$ & Sand, gray, fine; unoxidized \\
Well screened from 152 to 162 feet; 2-inch PVC casing and screen. For electric log see R2-97-15 \\
\hline
\end{tabular}


Local number: 128N52W3CCCB R

Other identifier: R2-97-16

Site identification number: 455337097095501

Date of construction: 06-30-97

Land surface altitude: 1,195 feet

Total depth: 242 feet

\begin{tabular}{cccc}
\hline Depth & $\begin{array}{c}\text { Thickness } \\
\text { (feet) }\end{array}$ & $\begin{array}{c}\text { Altitude } \\
\text { (feet) }\end{array}$ & Description \\
\hline $0-2$ & 2 & $1,195-1,193$ & Topsoil, black \\
$2-30$ & 28 & $1,193-1,165$ & $\begin{array}{c}\text { Clay, yellowish-brown, silty, sandy, pebbly; turns grayish-brown at 25 feet, oxidized } \\
\text { (till) }\end{array}$ \\
$30-48$ & 18 & $1,165-1,147$ & $\begin{array}{c}\text { Clay, gray, silty, sandy, pebbly; unoxidized, gravel lens from } 34 \text { to } 38 \text { feet and } 44 \text { to } 48 \\
\text { feet (till) }\end{array}$ \\
$48-70$ & 22 & $1,147-1,125$ & $\begin{array}{c}\text { Clay, gray, very silty; greasy, unoxidized (lake clay) } \\
70-112\end{array}$ \\
\hline $112-146$ & 34 & $1,125-1,083$ & Clay, gray, very silty, sandy; sand layers common, unoxidized (lake sediment) \\
$146-174$ & 28 & $1,083-1,049$ & Sand, gray, fine; unoxidized \\
$174-211$ & 37 & $1,049-1,021$ & Silt and sand; alternating layers (lake sediment) \\
$211-242$ & 31 & $984-953$ & Clay, gray, silty, sandy, pebbly; contains many gravel lens, unoxidized (till)
\end{tabular}

Electric log available

Local number: 128N52W3CCCB2 R

Other identifier: R2-97-17

Site identification number: 455337097095502

Date of construction: 07-01-97

Land surface altitude: $1,194.8$ feet

Total depth: 142 feet

\begin{tabular}{cccl}
\hline Depth & $\begin{array}{c}\text { Thickness } \\
\text { (feet) }\end{array}$ & $\begin{array}{c}\text { Altitude } \\
\text { (feet) }\end{array}$ & Description \\
\hline $0-2$ & 2 & $1,194.8-1.192 .8$ & Topsoil, black \\
$2-30$ & 28 & $1,192.8-1,164.8$ & $\begin{array}{l}\text { Clay, yellowish-brown, silty, sandy, pebbly; gravel lens from } 6 \text { to } 8 \text { feet and } 27 \text { to } \\
\text { 29 feet, oxidized (till) }\end{array}$ \\
$30-44$ & 14 & $1,164.8-1,150.8$ & Clay, gray, silty, sandy, pebbly; gravel lens from 34 to 38 feet, unoxidized (till) \\
$44-52$ & 8 & $1,150.8-1,142.8$ & Gravel, fine \\
$52-70$ & 18 & $1,142.8-1,124.8$ & Clay, gray, very silty; unoxidized (lake clay) \\
$70-112$ & 42 & $1,124.8-1,082.8$ & Clay, gray, very silty, sandy; numerous sand layers, unoxidized (lake sediment) \\
$112-142$ & 30 & $1,082.8-1,052.8$ & Sand, gray, fine; unoxidized \\
Well screened from & 132 to 142 feet; 2-inch PVC screen and casing. For electric log see R2-97-16 \\
\hline
\end{tabular}


Local number: 128N52W27BBBC R

Other identifier: R2-97-20

Site identification number: 455049097095501

Date of construction: $07-08-97$

Land surface altitude: $1,213.7$ feet

Total depth: 152 feet

\begin{tabular}{cccl}
\hline Depth & $\begin{array}{c}\text { Thickness } \\
\text { (feet) }\end{array}$ & $\begin{array}{c}\text { Altitude } \\
\text { (feet) }\end{array}$ & Description \\
\hline $0-2$ & 2 & $1,213.7-1,211.7$ & Topsoil, black \\
$2-22$ & 20 & $1,211.7-1,191.7$ & $\begin{array}{c}\text { Clay, yellowish-brown, silty, sandy, pebbly; color change to dark-brown at } 14 \text { feet, } \\
\text { oxidized (till) }\end{array}$ \\
$22-88$ & 66 & $1,191.7-1,125.7$ & Clay, gray, silty, sandy, pebbly; unoxidized (till) \\
$88-135$ & 47 & $1,125.7-1,078.7$ & $\begin{array}{l}\text { Sand and gravel, coarse sand to fine gravel; some coarse gravel, clay layers from } 96 \text { to } \\
\text { 101 feet }\end{array}$ \\
$135-152$ & 17 & $1,078.7-1,061.7$ & Clay, gray, shaley; hard greasy (Pierre Shale) \\
Well screened from 120 to 130 feet; 2-inch PVC casing and screen; 20 feet blank casing below and open to screen
\end{tabular}


Local number: 129N49W27BACC R

Other identifier: R2-97-34

Site identification number: 455557096470201

Date of construction: $08-05-97$

Land surface altitude: 1,068 feet

Total depth: 292 feet

\begin{tabular}{|c|c|c|c|}
\hline Depth & $\begin{array}{l}\text { Thickness } \\
\text { (feet) }\end{array}$ & $\begin{array}{l}\text { Altitude } \\
\text { (feet) }\end{array}$ & Description \\
\hline $0-2$ & 2 & $1,068-1,066$ & Clay, black (topsoil) \\
\hline $2-14$ & 12 & $1,066-1.054$ & $\begin{array}{l}\text { Clay, yellowish-brown, tan, and blackish-brown, very silty; some reddish-brown, with } \\
\text { concretions, layered (lake sediment) }\end{array}$ \\
\hline $14-20$ & 6 & $1,054-1,048$ & Clay, gray, very silty; greasy, unoxidized (lake sediment) \\
\hline $20-72$ & 52 & $1,048-996$ & Clay, gray, silty, sandy, pebbly; soft, large cuttings, unoxidized (till) \\
\hline $72-103$ & 31 & $996-965$ & Clay, gray, very silty, sandy, unoxidized (lake sediment) \\
\hline $103-142$ & 39 & $965-926$ & $\begin{array}{l}\text { Clay, gray, silty, very sandy, pebbly; hard, some cobbles, fine gravel lens from } 128 \text { to } \\
133 \text { feet, unoxidized (till) }\end{array}$ \\
\hline $142-148$ & 6 & $926-920$ & Gravel, coarse; with many cobbles \\
\hline $148-159$ & 11 & $920-909$ & Clay, gray; very hard, greasy, unoxidized (lake sediment) \\
\hline $159-214$ & 55 & $909-854$ & Silt grading to fine sand, gray; drills very fast, poor cuttings, unoxidized (lake sediment) \\
\hline 214-217 & 3 & $854-851$ & Chert boulder; very hard \\
\hline $217-220$ & 3 & $851-848$ & Granite boulder; hard \\
\hline $220-228$ & 8 & $848-840$ & Clay, gray, silty, sandy, pebbly; unoxidized (till) \\
\hline $228-292$ & 64 & $840-776$ & Clay, dark-gray, silty; greasy, calcareous (Graneros Shale) \\
\hline Electric log & able & & \\
\hline
\end{tabular}


Local number: 129N49W30CBBB R

Other identifier: R2-97-33

Site identification number: 455543096510701

Date of construction: $08-04-97$

Land surface altitude: 1,101 feet

Total depth: 302 feet

\begin{tabular}{|c|c|c|c|}
\hline Depth & $\begin{array}{l}\text { Thickness } \\
\text { (feet) }\end{array}$ & $\begin{array}{l}\text { Altitude } \\
\text { (feet) }\end{array}$ & Description \\
\hline $0-3$ & 3 & $1,101-1,098$ & Topsoil, black \\
\hline $3-24$ & 21 & $1,098-1,077$ & Clay, yellowish-brown, silty, sandy, pebbly; oxidized (till) \\
\hline $24-62$ & 38 & $1,077-1.039$ & Clay, gray, silty, sandy, pebbly; soft, large cuttings, unoxidized (till) \\
\hline $62-76$ & 14 & $1,039-1.025$ & Sand, gray, fine, silty; unoxidized \\
\hline $76-85$ & 9 & $1,025-1.016$ & Clay, gray, silty, sandy, pebbly; unoxidized (till) \\
\hline $85-142$ & 57 & $1,016-959$ & $\begin{array}{l}\text { Sand and gravel, coarse sand to fine gravel; grades to medium gravel with depth, some } \\
\text { coarse }\end{array}$ \\
\hline $142-193$ & 55 & $959-908$ & Clay, gray, silty, sandy, pebbly; chert boulder at 150 feet, unoxidized (till) \\
\hline 193-197 & 4 & $908-904$ & Gravel, fine to medium \\
\hline $197-219$ & 22 & $904-882$ & Clay, gray, silty, sandy, pebbly; unoxidized, rock at 198 feet (till) \\
\hline $219-225$ & 6 & $882-876$ & Gravel, fine \\
\hline $225-270$ & 45 & $876-831$ & $\begin{array}{l}\text { Clay, dark-gray to blackish-brown; hard, greasy, calcareous (Fairport Shale Member - } \\
\text { Carlile Shale) }\end{array}$ \\
\hline $270-298$ & 28 & $831-803$ & Siltstone, white and brown; hard, cherty, very calcareous (Greenhorn Limestone) \\
\hline $298-302$ & 4 & $803-799$ & Clay, gray, shaley; greasy, hard (Graneros Shale) \\
\hline Electric log & & & \\
\hline
\end{tabular}


Local number: 118N52W8AAAD R

Other identifier: R2-85-69

Site identification number: 450250097051001

Date of construction: 7-23-85

Land surface altitude: 1,795 feet

Total depth: 530 feet

\begin{tabular}{cccl}
\hline Depth & $\begin{array}{c}\text { Thickness } \\
\text { (feet) }\end{array}$ & $\begin{array}{c}\text { Altitude } \\
\text { (feet) }\end{array}$ & \\
\hline $0-1$ & 1 & $1,795-1,794$ & Topsoil, black \\
$1-56$ & 55 & $1,794-1,739$ & Clay, tan to brown, sandy, silty, pebbly (till) \\
$56-83$ & 27 & $1,739-1,712$ & Clay, gray, sandy, silty, pebbly (till) \\
$83-104$ & 21 & $1,712-1,691$ & Clay, tan, sandy, silty, pebbly (till) \\
$104-138$ & 34 & $1,691-1,657$ & Clay, tan, sandy, silty, pebbly (till) \\
$138-157$ & 19 & $1,657-1,638$ & Clay, gray, sandy, silty, pebbly (till) \\
$157-185$ & 28 & $1,638-1,610$ & Clay, gray, very sandy, silty, pebbly, (till) \\
$185-233$ & 48 & $1,610-1,562$ & Clay, gray, sandy, silty, pebbly (till) \\
$233-254$ & 21 & $1,562-1,541$ & Clay, tan, silty, sandy, pebbly; some thin sand stringers (till) \\
$254-337$ & 83 & $1,541-1,458$ & Clay, gray, silty, sandy, pebbly (till) \\
$337-341$ & 4 & $1,458-1,454$ & Sand and gravel \\
\hline $341-359$ & 18 & $1,454-1,436$ & Clay, gray, sandy, silty, pebbly (till) \\
$359-368$ & 9 & $1,436-1,427$ & Gravel, fine; coarse sand; oxidized \\
$368-413$ & 45 & $1,427-1,382$ & Clay, gray, silty, sandy, pebbly, very sandy from 395 to 404 feet (till) \\
$413-465$ & 52 & $1,382-1,330$ & Sand and gravel, gray, medium sand to fine gravel; clay stringer from 432 to 437 feet \\
& & $1,330-1,265$ & Shale, dark-gray; greasy; bentonite (Pierre Shale) \\
$465-530$ & 65 & & \\
\hline Electric log available & & & \\
\hline & & & \\
\hline
\end{tabular}


Local number: 118N52W21AAAA

Other identifier: R1-85-61

Site identification number: 450113097032401

Date of construction: 7-22-85

Land surface altitude: 1,810 feet

Total depth: 560 feet

\begin{tabular}{|c|c|c|c|}
\hline Depth & $\begin{array}{c}\text { Thickness } \\
\text { (feet) }\end{array}$ & $\begin{array}{c}\text { Altitude } \\
\text { (feet) }\end{array}$ & Description \\
\hline $0-1$ & 1 & $1,810-1.809$ & Topsoil, black \\
\hline $1-3$ & 2 & $1,809-1,807$ & Sand and gravel, brown, medium sand to fine gravel \\
\hline $3-19$ & 16 & $1,807-1,791$ & Clay, tan to brown, silty, sandy, pebbly (till) \\
\hline $19-34$ & 15 & $1,791-1,776$ & Clay, gray, very silty, sandy, pebbly (till) \\
\hline $34-47$ & 13 & $1,776-1,763$ & Clay, light-gray, very silty; calcareous (lake sediments?) \\
\hline $47-91$ & 44 & $1,763-1,719$ & Clay, brown, sandy, silty, pebbly (till) \\
\hline $91-142$ & 51 & $1,719-1,668$ & Clay, brown and gray mixed, sandy, silty, pebbly (till) \\
\hline $142-152$ & 10 & $1,668-1,658$ & Clay, gray, very sandy, silty, pebbly (till) \\
\hline $152-162$ & 10 & $1,658-1,648$ & Sand and gravel, medium sand to fine gravel; possibly oxidized \\
\hline $162-175$ & 13 & $1,648-1,635$ & Sand and gravel, medium sand to fine gravel, clayey \\
\hline $175-177$ & 2 & $1,635-1,633$ & Clay, gray, silty, sandy, pebbly (till) \\
\hline $177-181$ & 4 & $1,633-1,629$ & Sand and gravel \\
\hline $181-185$ & 4 & $1,629-1,625$ & Clay, gray, silty, sandy, pebbly (till) \\
\hline $185-191$ & 6 & $1,625-1,619$ & Sand and gravel \\
\hline $191-244$ & 53 & $1,619-1,566$ & Clay, gray, silty, sandy, pebbly (till) \\
\hline $244-276$ & 32 & $1,566-1,534$ & Clay, gray, silty, sandy, pebbly; many sand and gravel stringers (till) \\
\hline $276-278$ & 2 & $1,534-1,532$ & Sand and gravel \\
\hline $278-314$ & 36 & $1,532-1,496$ & Clay, gray, silty, sandy, pebbly; many sand and gravel stringers (till) \\
\hline 314-398 & 84 & $1,496-1,412$ & Clay, gray, silty, sandy, pebbly (till) \\
\hline $398-407$ & 9 & $1,412-1,403$ & Sand, fine to coarse; some fine gravel \\
\hline $407-461$ & 54 & $1,403-1,349$ & Clay, gray, very sandy, silty, pebbly; many sand stringers (till?) \\
\hline $461-471$ & 10 & $1,349-1,339$ & Sand, clayey \\
\hline $471-560$ & 89 & $1,339-1,250$ & Shale, dark-gray; greasy, brittle (Pierre Shale) \\
\hline
\end{tabular}

Electric log available. Clay interval from 407 to 461 feet may be a very clay rich sand (Altamont aquifer equivalent?) 
Local number: 118N53W36DDDD

Other identifier: R2-85-68

Site identification number: 445837097073502

Date of construction: 7-22-85

Land surface altitude: 1,745 feet

Total depth: 540 feet

\begin{tabular}{|c|c|c|c|}
\hline Depth & $\begin{array}{c}\text { Thickness } \\
\text { (feet) }\end{array}$ & $\begin{array}{l}\text { Altitude } \\
\text { (feet) }\end{array}$ & Description \\
\hline $0-1$ & 1 & $1,745-1,744$ & Topsoil, black \\
\hline $1-11$ & 10 & $1,744-1,734$ & Sand and gravel, tan, medium sand to fine gravel \\
\hline 11-17 & 6 & $1,734-1,728$ & Clay, tan, sandy, silty, pebbly (till) \\
\hline $17-21$ & 4 & $1,728-1,724$ & Clay, gray, sandy, silty, pebbly (till) \\
\hline $21-30$ & 9 & $1,724-1,715$ & Sand and gravel \\
\hline $30-129$ & 99 & $1,715-1,616$ & Clay, gray, very sandy, silty, pebbly; some stringers of sand (till) \\
\hline $129-139$ & 10 & $1,616-1,606$ & Sand and gravel \\
\hline $139-224$ & 85 & $1,606-1,521$ & Clay, gray, sandy, silty, pebbly (till) \\
\hline $224-244$ & 20 & $1,521-1,501$ & Clay, $\tan (?)$, sandy, silty, pebbly (till) \\
\hline $244-269$ & 25 & $1,501-1,476$ & Clay, gray, very sandy, silty, pebbly (till) \\
\hline $269-293$ & 24 & $1,476-1,452$ & Clay, gray, sandy, silty, pebbly (till) \\
\hline 293-296 & 3 & $1,452-1,449$ & Sand and gravel \\
\hline $296-332$ & 36 & $1,449-1,413$ & Clay, gray, silty, sandy, pebbly (till) \\
\hline $332-394$ & 62 & $1,413-1,351$ & Sand and gravel, clay, clayey (Altamont aquifer?) \\
\hline $394-540$ & 146 & $1,351-1,205$ & Shale, dark-gray; hard, brittle greasy (Pierre Shale) \\
\hline
\end{tabular}


Local number: 119 N52W8AAAA R

Other identifier: R1-85-62

Site identification number: 450627097110701

Date of construction: $7-23-85$

Land surface altitude: 1,818 feet

Total depth: 530 feet

\begin{tabular}{|c|c|c|c|}
\hline Depth & $\begin{array}{c}\text { Thickness } \\
\text { (feet) }\end{array}$ & $\begin{array}{l}\text { Altitude } \\
\text { (feet) }\end{array}$ & Description \\
\hline $0-1$ & 1 & $1,818-1,817$ & Topsoil, black \\
\hline $1-38$ & 37 & $1,817-1,780$ & Clay, yellow brown, sandy, silty, pebbly; soft (till) \\
\hline $38-54$ & 16 & $1,780-1,764$ & Clay, tan to brownish-gray, sandy, silty, pebbly; soft (till) \\
\hline $54-89$ & 35 & $1,764-1,729$ & $\begin{array}{l}\text { Silts, light-brown to light-gray, clayey; some fine sand; some greenish silts; upper } \\
\text { portion appears oxidized (lake sediments) }\end{array}$ \\
\hline $89-118$ & 29 & $1,729-1,700$ & Clay, tan to orangish-tan, very sandy, silty, pebbly; sand stringers(?) (till) \\
\hline $118-147$ & 29 & $1,700-1,671$ & Clay, light-gray to gray, sandy, silty, pebbly; some brownish-clays (till) \\
\hline $147-160$ & 13 & $1,671-1,658$ & Silt, medium-gray to black; greasy, noncalcareous (lake silts) \\
\hline $160-189$ & 29 & $1,658-1,629$ & Clay, yellow brown, silty, sandy, pebbly (till) \\
\hline $189-228$ & 39 & $1,629-1,590$ & Clay, gray, silty, sandy, pebbly (till) \\
\hline $228-275$ & 47 & $1,590-1,543$ & Clay, gray, silty, sandy, pebbly; hard (till) \\
\hline $275-281$ & 6 & $1,543-1,537$ & Clay, brown, silty, sandy, pebbly (till) \\
\hline $281-291$ & 10 & $1,537-1,527$ & Sand and gravel, medium sand to fine gravel; oxidized(?) \\
\hline 291-298 & 7 & $1,527-1,520$ & Clay, yellow brown, silty, sandy, pebbly (till) \\
\hline 298-358 & 60 & $1,520-1,460$ & Clay, gray, silty, sandy, pebbly; many sand and gravel stringers (till) \\
\hline $358-386$ & 28 & $1,460-1,432$ & Sand and gravel, fine sand to fine gravel; some yellow brown clay in upper portion \\
\hline $386-530$ & 144 & $1,432-1,288$ & Claystone, dark-gray to black; brittle, greasy (Pierre Shale) \\
\hline
\end{tabular}


Local number: 120 N51W9BBBC R

Other identifier: RB-86-104

Site identification number: 451140097034101

Date of construction: $10-28-86$

Land surface altitude: 1,940 feet

Total depth: $652 \mathrm{feet}$

\begin{tabular}{|c|c|c|c|}
\hline Depth & $\begin{array}{c}\text { Thickness } \\
\text { (feet) }\end{array}$ & $\begin{array}{c}\text { Altitude } \\
\text { (feet) }\end{array}$ & Description \\
\hline $0-1$ & 1 & $1,940-1,939$ & Topsoil, black \\
\hline $1-23$ & 22 & $1,939-1,917$ & Clay, yellow brown, silty, sandy, pebbly (till) \\
\hline 23-69 & 46 & $1,917-1,871$ & Clay, gray, silty, sandy, pebbly (till) \\
\hline $69-78$ & 9 & $1,871-1,862$ & Clay, dark-brown to medium gray, silty \\
\hline $78-135$ & 57 & $1,862-1,805$ & Clay, yellow brown, silty, sandy, pebbly (till) \\
\hline $135-190$ & 55 & $1,805-1,750$ & Clay, gray, silty, sandy, pebbly; sand content increases with depth (till) \\
\hline $190-286$ & 96 & $1,750-1,654$ & Clay, gray, silty, sandy, pebbly; many sand and gravel stringers (till) \\
\hline 286-306 & 20 & $1,654-1,634$ & Clay, grayish-brown. silty, sandy, pebbly (till) \\
\hline $306-396$ & 90 & $1,634-1,544$ & Clay, gray, silty, sandy, pebbly; many sand and gravel stringers (till) \\
\hline $396-408$ & 12 & $1,544-1,532$ & Sand and gravel \\
\hline $408-470$ & 62 & $1,532-1,470$ & Clay, gray, silty, sandy, pebbly, shaley (till) \\
\hline $470-486$ & 16 & $1,470-1,454$ & Sand and gravel \\
\hline $486-606$ & 120 & $1,454-1,334$ & Clay, dark-gray, silty, sandy, pebbly, shaley (till) \\
\hline $606-614$ & 8 & $1,334-1,326$ & Sand and gravel \\
\hline $614-652$ & 38 & $1,326-1,288$ & Shale, dark-gray; greasy (Pierre Shale) \\
\hline Electric $\log$ & ble & & \\
\hline
\end{tabular}


Local number: 120N51W17DDDD

Other identifier: R2-87-27

Site identification number: 451140096575301

Date of construction: $6-30-87$

Land surface altitude: 1,915 feet

Total depth: 745 feet

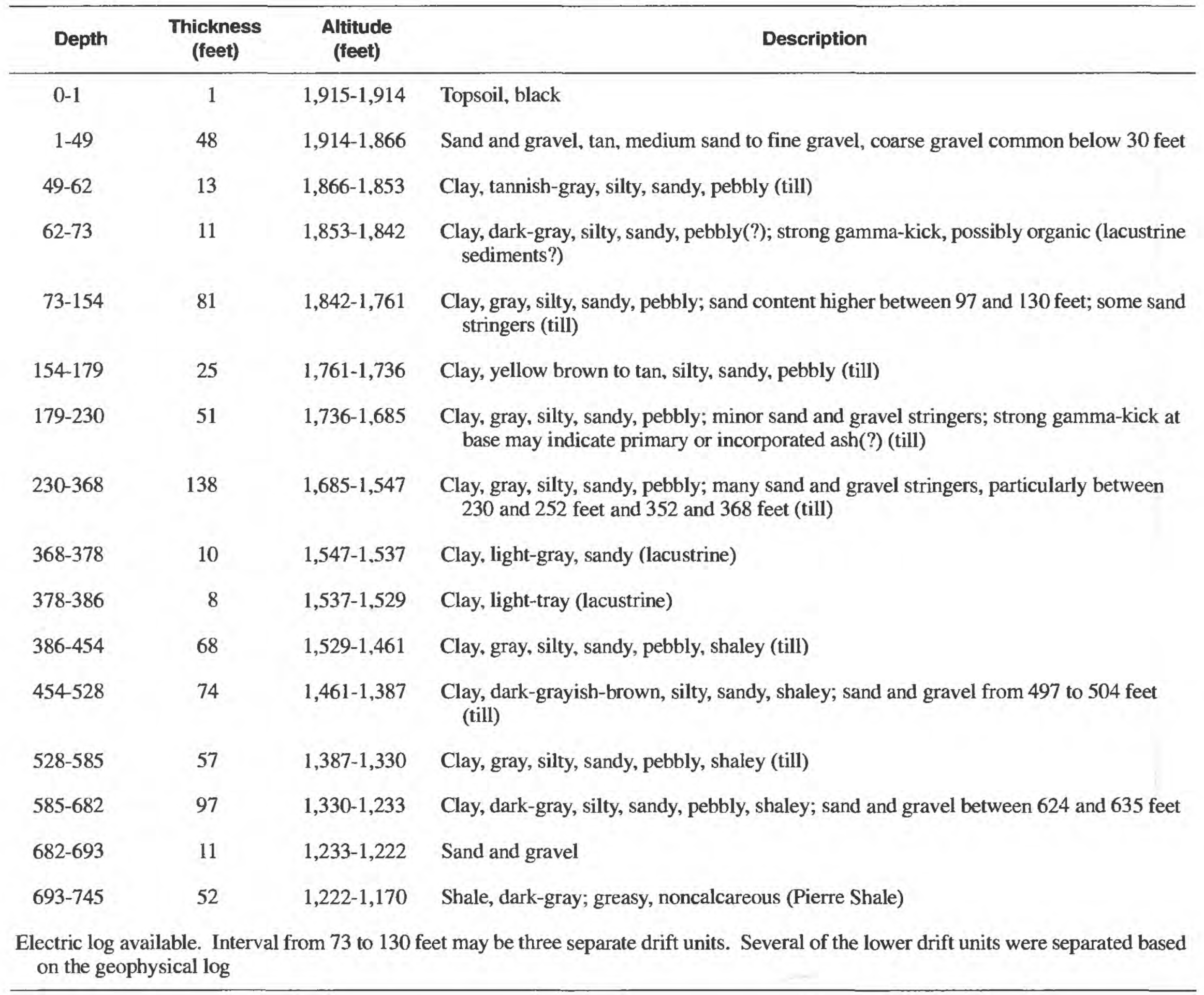


Local number: 120N52W8AAAB R

Other identifier: R2-86-107

Site identification number: 451138097111301

Date of construction: $11-4-86$

Land surface altitude: 1,850 feet

Total depth: 516 feet

\begin{tabular}{cccl}
\hline Depth & $\begin{array}{c}\text { Thickness } \\
\text { (feet) }\end{array}$ & $\begin{array}{c}\text { Altitude } \\
\text { (feet) }\end{array}$ & \\
\hline $0-2$ & 2 & $1,850-1,848$ & Topsoil, black \\
$2-31$ & 29 & $1,848-1,819$ & Clay, yellow brown to brown, very silty, sandy pebbly (till) \\
$31-57$ & 26 & $1,819-1,793$ & Clay, gray, very silty, sandy, pebbly (till) \\
$57-67$ & 10 & $1,793-1,783$ & Clay, grayish-green to olive, silty; noncalcareous (lacustrine?) \\
$67-115$ & 48 & $1,783-1,735$ & Clay, yellow brown to tan, silty, sandy, pebbly; scattered sand stringers (till) \\
$115-147$ & 32 & $1,735-1,703$ & Sand and gravel, yellow brown to gray, medium sand to fine gravel; yellow brown clay \\
& 77 & $1,703-1,626$ & Clayers, gray, silty, sandy, pebbly (till) \\
$147-224$ & 58 & $1,626-1,568$ & Sand and gravel, gray, medium to coarse sand, some fine gravel: scattered gray clay \\
$224-282$ & 11 & $1,568-1,557$ & Clayers, mostly in lower half \\
$282-293$ & 17 & $1,557-1,540$ & Clay, yellow brown, silty, sandy, pebbly; a few sand stringers (till) \\
$293-310$ & 114 & $1,540-1,426$ & Clay, gray, silty, sandy, pebbly (till) \\
$310-424$ & 58 & $1,426-1,368$ & Sand, gray \\
$424-482$ & 34 & $1,368-1,334$ & Shale, dark-gray; greasy (Pierre Shale) \\
$482-516$ & & & \\
\hline Electric log available & & & \\
\hline
\end{tabular}


Local number: 120N52W11AAAA R

Other identifier: R2-88-19

Site identification number: 451143097072501

Date of construction: 7-26-88

Land surface altitude: 1,835 feet

Total depth: 527 feet

\begin{tabular}{|c|c|c|c|}
\hline Depth & $\begin{array}{l}\text { Thickness } \\
\text { (feet) }\end{array}$ & $\begin{array}{l}\text { Altitude } \\
\text { (feet) }\end{array}$ & Description \\
\hline $0-1$ & 1 & $1,835-1,834$ & Topsoil, yellow brown, silty \\
\hline $1-27$ & 26 & $1,834-1,808$ & Sand and gravel, medium sand to medium gravel; oxidized \\
\hline $27-34$ & 7 & $1,808-1,801$ & Clay, gray, silty, sandy, pebbly (till) \\
\hline $34-39$ & 5 & $1,801-1,796$ & Sand and gravel, coarse sand and fine gravel \\
\hline $39-75$ & 36 & $1,796-1,760$ & Clay, yellow brown silty, sandy, pebbly (till) \\
\hline $75-102$ & 27 & $1,760-1,733$ & Clay, brownish-gray to gray, silty, sandy, pebbly (till) \\
\hline $102-143$ & 41 & $1,733-1,692$ & $\begin{array}{l}\text { Sand and gravel, medium sand to medium gravel; oxidized; some yellow brown till } \\
\text { stringers }\end{array}$ \\
\hline 143-161 & 18 & $1,692-1,674$ & Clay, gray, silty, sandy, pebbly (till) \\
\hline $161-181$ & 20 & $1,674-1,654$ & Gravel, medium to coarse; some sand \\
\hline $181-238$ & 57 & $1,654-1,597$ & $\begin{array}{l}\text { Clay, gray, very silty, sandy, pebbly; clayey sands from } 213 \text { to } 219 \text { feet and } 225 \text { to } \\
238 \text { feet (till?) }\end{array}$ \\
\hline 238-289 & 51 & $1,597-1,546$ & Clay, gray, silty, sandy, pebbly (till) \\
\hline 289-295 & 6 & $1,546-1,540$ & Sand and gravel \\
\hline 295-337 & 42 & $1,540-1,498$ & Clay, yellow brown to brown, silty, sandy, pebbly (till) \\
\hline $337-354$ & 17 & $1,498-1,481$ & Clay, gray, silty, sandy, pebbly; sand stringers (till) \\
\hline $354-438$ & 84 & $1,481-1,397$ & Clay, gray, silty, sandy, pebbly (till) \\
\hline $438-481$ & 43 & $1,397-1,354$ & Sand, gray, medium; some fine gravel \\
\hline $481-527$ & 46 & $1,354-1,308$ & Shale, dark-gray; greasy, noncalcareous (Pierre Shale) \\
\hline Electric $\log$ & lable & & \\
\hline
\end{tabular}


Local number: 121N47W4AAAA

Other identifier: R2-88-25

Site identification number: 451935096343201

Date of construction: $9-7-88$

Land surface altitude: 1,110 feet

Total depth: 270 feet

\begin{tabular}{|c|c|c|c|}
\hline Depth & $\begin{array}{l}\text { Thickness } \\
\text { (feet) }\end{array}$ & $\begin{array}{l}\text { Altitude } \\
\text { (feet) }\end{array}$ & Description \\
\hline $0-3$ & 3 & $1,110-1.107$ & Topsoil, black \\
\hline $3-18$ & 15 & $1,107-1,092$ & Clay, tan to light-brown, silty, sandy, pebbly (till) \\
\hline $18-43$ & 25 & $1,092-1,067$ & Clay, gray, silty, sandy, pebbly (till) \\
\hline $43-45$ & 2 & $1,067-1,065$ & Gravel, coarse \\
\hline $45-67$ & 22 & $1,065-1,043$ & Clay, gray, silty, sandy, pebbly (till) \\
\hline $67-73$ & 6 & $1,043-1,037$ & Sand and gravel \\
\hline $73-115$ & 42 & $1,037-995$ & Clay, reddish-brown, sandy, silty, pebbly (till) \\
\hline $115-120$ & 5 & $995-990$ & Sand \\
\hline $120-145$ & 25 & $990-965$ & Clay, dark-gray, silty, sandy, pebbly (till) \\
\hline $145-205$ & 60 & $965-905$ & Sand, gray, fine to medium \\
\hline $205-250$ & 45 & $905-860$ & $\begin{array}{l}\text { Siltstone, dark-brown with tan specks; calcareous (Fairport Shale Member - Carlile } \\
\text { Shale) }\end{array}$ \\
\hline $250-270$ & 20 & $860-840$ & $\begin{array}{l}\text { Siltstone, dark-brown with tan specks; calcareous; crystalline carbonate layers; } \\
\text { fractured (Greenhorn Limestone) }\end{array}$ \\
\hline
\end{tabular}


Local number: $121 \mathrm{~N} 48 \mathrm{~W} 3 \mathrm{BBCC}$

Other identifier: R2-87-20

Site identification number: 451928096404001

Date of construction: $5-28-87$

Land surface altitude: 1,150 feet

Total depth: 319 feet

\begin{tabular}{cccl}
\hline Depth & $\begin{array}{c}\text { Thickness } \\
\text { (feet) }\end{array}$ & $\begin{array}{c}\text { Aititude } \\
\text { (feet) }\end{array}$ & \multicolumn{1}{c}{ Description } \\
\hline $0-1$ & 1 & $1,150-1,149$ & Topsoil black \\
$1-12$ & 11 & $1,149-1,138$ & Clay, yellow brown to tan, silty, sandy, pebbly; sand stringers (till - New Ulm?) \\
$12-32$ & 20 & $1,138-1,118$ & Clay, yellow brown, silty, sandy, pebbly; some carbonate gravels (till - Granite Falls?) \\
$32-58$ & 26 & $1,118-1,092$ & Clay, gray, silty, sandy, pebbly; some carbonate gravels (till - Granite Falls?) \\
$58-89$ & 31 & $1,092-1,061$ & Clay, reddish-brown to reddish-gray, sandy, silty, pebbly (till - Hawk Creek?) \\
$89-124$ & 35 & $1,061-1,026$ & Clay, dark-gray, silty, sandy, pebbly (till - Whetstone?) \\
$124-261$ & 137 & $1,026-889$ & Shale, dark-gray; greasy, noncalcareous; scattered mica rich sand intervals (Blue Hill \\
& & Member - Carlile Shale) & \\
$261-318$ & 57 & $889-832$ & Shale, dark-brown with tan specks; calcareous (Fairport Shale Member - Carlile Shale) \\
$318-319$ & 1 & $832-831$ & Limestone, gray; dirty (Greenhorn Limestone) \\
Electric log available & & \\
\hline
\end{tabular}

Local number: 121N48W6AAAA

Other identifier: R2-86-83

Site identification number: 451935096430901

Date of construction: 9-11-86

Land surface altitude: 1,140 feet

Total depth: 360 feet

\begin{tabular}{cccl}
\hline Depth & $\begin{array}{c}\text { Thickness } \\
\text { (feet) }\end{array}$ & $\begin{array}{c}\text { Aititude } \\
\text { (feet) }\end{array}$ & Description \\
\hline $0-7$ & 7 & $1,140-1,133$ & Clay, yellow brown and gray, silty, sandy, pebbly (till - New Ulm or Granite Falls) \\
$7-42$ & 35 & $1,133-1,098$ & Clay, reddish-brown, sandy, silty, pebbly; many sand stringers (till - Hawk Creek) \\
$42-46$ & 4 & $1,098-1,094$ & Clay, gray, silty, sandy, pebbly (till - Whetstone) \\
$46-54$ & 8 & $1,094-1,086$ & $\begin{array}{l}\text { Clay, dark-brownish-gray, silty; greasy, noncalcareous; organic looking (Carlile Shale?) } \\
54-274\end{array}$ \\
220 & $1,086-866$ & $\begin{array}{c}\text { Shale, dark-gray; greasy, noncalcareous; scattered fine sand partings (Blue Hill Shale } \\
\text { Member - Carlile Shale) }\end{array}$ \\
$274-331$ & 57 & $866-809$ & $\begin{array}{c}\text { Shale, dark-brown with tan specks; calcareous (Fairport Shale Member - Carlile Shale) } \\
\text { Limestone, dark-brownish-gray; dirty; some dark-brown calcareous shale (Greenhorn } \\
\text { Limestone) }\end{array}$ \\
Electric log available & $809-780$ & & \\
\hline
\end{tabular}


Local number: 121N49W3BBBC

Other identifier: R1-82-47

Site identification number: 451927096480201

Date of construction: $5-18-82$

Land surface altitude: 1,195 feet

Total depth: 155 feet

\begin{tabular}{cccll}
\hline Depth & $\begin{array}{c}\text { Thickness } \\
\text { (feet) }\end{array}$ & $\begin{array}{c}\text { Altitude } \\
\text { (feet) }\end{array}$ & Description \\
\hline $0-2$ & 2 & $1,195-1,193$ & Topsoil \\
$2-13$ & 11 & $1,193-1,182$ & Clay, brown, silty, pebbly (till) \\
$13-21$ & 8 & $1,182-1,174$ & Sand, medium to coarse \\
$21-55$ & 34 & $1,174-1,140$ & Clay, gray, silty, pebbly (till) \\
$55-66$ & 11 & $1,140-1,129$ & Clay, olive, silty, pebbly (till) \\
$66-145$ & 79 & $1,129-1,050$ & Clay, gray, silty, sandy, pebbly (till) \\
$145-155$ & 10 & $1,050-1,040$ & Clay, gray (shale) \\
\hline
\end{tabular}

Local number: 121N50W1BBBB

Other identifier: R2-86-77

Site identification number: 451944096525701

Date of construction: $8-29-86$

Land surface altitude: 1,305 feet

Total depth: 500 feet

\begin{tabular}{|c|c|c|c|}
\hline Depth & $\begin{array}{l}\text { Thickness } \\
\text { (feet) }\end{array}$ & $\begin{array}{c}\text { Altitude } \\
\text { (feet) }\end{array}$ & Description \\
\hline $0-2$ & 2 & $1,305-1,303$ & Topsoil, black \\
\hline $2-17$ & 15 & $1,303-1,288$ & Clay, yellow brown to tan, silty, sandy, pebbly (till) \\
\hline $17-22$ & 5 & $1,288-1,283$ & Clay, dark-brown, silty; noncalcareous; brittle (lacustrine or shale block?) \\
\hline $22-43$ & 21 & $1,283-1,262$ & Clay, yellow brown to brown, silty, sandy, pebbly (till) \\
\hline $43-65$ & 22 & $1,262-1,240$ & Clay, gray, silty, sandy, pebbly (till) \\
\hline $65-83$ & 18 & $1,240-1,222$ & Clay, yellow brown, silty, sandy, pebbly; several sand and gravel stringers (till) \\
\hline $83-116$ & 33 & $1,222-1,189$ & Clay, gray, silty, sandy, pebbly (till) \\
\hline $116-152$ & 36 & $1,189-1,153$ & Clay, gray, silty, sandy, pebbly; sand stringers (till) \\
\hline $152-190$ & 38 & $1,153-1,115$ & Clay, gray, silty, sandy, pebbly (till) \\
\hline $190-290$ & 100 & $1,115-1,015$ & Sand and gravel, gray; scattered clay layers (Revillo aquifer?) \\
\hline $290-464$ & 174 & $1,015-841$ & $\begin{array}{l}\text { Shale, dark-blue gray; greasy, noncalcareous; scattered fine sand partings (Blue Hill } \\
\text { Shale Member - Carlile Shale) }\end{array}$ \\
\hline $464-500$ & 36 & $841-805$ & Shale, dark-brown with tan specks; calcareous (Fairport Shale Member - Carlile Shale) \\
\hline Electric log & lable & & \\
\hline
\end{tabular}


Local number: 121N50W5BBBB

Other identifier: R2-87-44

Site identification number: 451935096575101

Date of construction: 7-30-87

Land surface altitude: 1,700 feet

Total depth: 785 feet

\begin{tabular}{|c|c|c|c|}
\hline Depth & $\begin{array}{c}\text { Thickness } \\
\text { (feet) }\end{array}$ & $\begin{array}{c}\text { Altitude } \\
\text { (feet) }\end{array}$ & Description \\
\hline $0-2$ & 2 & $1,700-1,698$ & Topsoil, black \\
\hline $2-24$ & 22 & $1,698-1,676$ & Clay, yellow brown, silty, sandy, pebbly (till) \\
\hline 24-55 & 31 & $1,676-1,645$ & Clay, gray, silty, sandy, pebbly (till) \\
\hline $55-105$ & 50 & $1,645-1,595$ & Clay, gray, sandy, silty, pebbly; many sand stringers (till) \\
\hline $105-142$ & 37 & $1,595-1,558$ & Clay, gray, silty, sandy, pebbly (till) \\
\hline $142-159$ & 17 & $1,558-1,541$ & Clay, gray, silty, sandy, pebbly; many sand and gravel stringers (till?) \\
\hline $159-187$ & 28 & $1,541-1,513$ & Clay, grayish-brown, silty, sandy, pebbly (till) \\
\hline $187-214$ & 27 & $1,513-1,486$ & Clay, brownish-gray to gray, silty, sandy, pebbly (till) \\
\hline $214-301$ & 87 & $1,486-1,399$ & Clay, gray, silty, sandy, pebbly; sandier below 262 feet (till) \\
\hline $301-335$ & 34 & $1,399-1,365$ & Sand and gravel, medium and to fine gravel; scattered clay layers \\
\hline $335-419$ & 84 & $1,365-1,281$ & $\begin{array}{l}\text { Clay, gray, silty, sandy, pebbly, several sand and gravel stringers between } 410 \text { and } \\
419 \text { feet (till) }\end{array}$ \\
\hline $419-488$ & 69 & $1,281-1,212$ & Clay, gray, silty, sandy, pebbly, shaley; darker than previous interval (till) \\
\hline $488-574$ & 86 & $1,212-1,126$ & $\begin{array}{l}\text { Clay, gray, silty, sandy, pebbly, shaley; quite sandy from } 517 \text { to } 532 \text { feet and } 566 \text { to } \\
574 \text { feet (till) }\end{array}$ \\
\hline $574-661$ & 87 & $1,126-1,039$ & Clay, gray, silty, sandy, pebbly, shaley (till) \\
\hline $661-673$ & 12 & $1,039-1,027$ & Sand and gravel(?), clayey \\
\hline $673-692$ & 19 & $1,027-1,008$ & Shale, gray; greasy, noncalcareous (Carlile Shale?) \\
\hline $692-785$ & 93 & $1,008-915$ & $\begin{array}{l}\text { Shale, gray; greasy, noncalcareous; a few fine sand partings (Blue Hill Shale Member - } \\
\text { Carlile Shale) }\end{array}$ \\
\hline Electric lo & & & \\
\hline
\end{tabular}


Local number: 121N51 W14DADB

Other identifier: R2-86-85

Site identification number: 451720097002801

Date of construction: $9-18-86$

Land surface altitude: 1,990 feet

Total depth: 585 feet

\begin{tabular}{|c|c|c|c|}
\hline Depth & $\begin{array}{c}\text { Thickness } \\
\text { (feet) }\end{array}$ & $\begin{array}{l}\text { Altitude } \\
\text { (feet) }\end{array}$ & Description \\
\hline $0-1$ & 1 & $1,990-1,989$ & Topsoil, black \\
\hline $1-33$ & 32 & $1,989-1,957$ & Clay, yellow brown, sandy, silty, pebbly; many sand and gravel stringers (till) \\
\hline $33-85$ & 52 & $1,957-1,905$ & $\begin{array}{l}\text { Clay, gray, sandy, silty, pebbly; many sand and gravel stringers; sand and gravel } \\
\text { between } 42 \text { and } 49 \text { feet (till) }\end{array}$ \\
\hline $85-125$ & 40 & $1,905-1,865$ & Clay, yellow brown to tan, silty, sandy, pebbly; numerous sand and gravel stringers (till) \\
\hline $125-170$ & 45 & $1,865-1,820$ & Clay, medium-gray, silty, sandy, pebbly; many sand and gravel stringers (till) \\
\hline $170-223$ & 53 & $1,820-1,767$ & Clay, gray, silty, sandy, pebbly (till) \\
\hline $223-236$ & 13 & $1,767-1,754$ & Clay, yellow brown, silty, sandy, pebbly (till) \\
\hline $236-255$ & 19 & $1,754-1,735$ & Clay, gray, silty, sandy, pebbly; a few sand stringers (till) \\
\hline $255-269$ & 14 & $1,735-1,721$ & Clay, yellow brown, sandy, silty, pebbly (till) \\
\hline $269-301$ & 32 & $1,721-1,789$ & Clay, gray, sandy, silty, pebbly (till) \\
\hline $301-313$ & 12 & $1,789-1,777$ & Sand and gravel, medium sand to medium gravel; a few clay layers \\
\hline 313-388 & 75 & $1,777-1,602$ & $\begin{array}{l}\text { Clay, gray, silty, sandy, pebbly; may be partially oxidized from } 313 \text { to about } 340 \text { feet } \\
\text { (till) }\end{array}$ \\
\hline $388-436$ & 48 & $1,602-1,554$ & Clay, light-gray, silty, sandy, pebbly; soft, greasy; a few sand stringers (till) \\
\hline $436-493$ & 57 & $1,554-1,497$ & Clay, gray, silty, sandy, pebbly (till) \\
\hline $493-515$ & 22 & $1,497-1,475$ & Clay, olive gray, silty, sandy, pebbly; a few sand stringers (till) \\
\hline $515-562$ & 47 & $1,475-1,428$ & Clay, gray, silty, sandy, pebbly; a few sand stringers (till) \\
\hline $562-574$ & 12 & $1,428-1,416$ & Sand and gravel, clayey \\
\hline $574-585$ & 11 & $1,416-1,405$ & Shale, dark-gray; greasy, noncalcareous; inoceramus fragments (Pierre Shale) \\
\hline Electric log & & & \\
\hline
\end{tabular}


Local number: 121N51W17AAAA R

Other identifier: R2-86-103

Site identification number: 451603097034201

Date of construction: $10-22-86$

Land surface altitude: 2,030 feet

Total depth: 758 feet

\begin{tabular}{|c|c|c|c|}
\hline Depth & $\begin{array}{l}\text { Thickness } \\
\text { (feet) }\end{array}$ & $\begin{array}{l}\text { Altitude } \\
\text { (feet) }\end{array}$ & Description \\
\hline $0-1$ & 1 & $2,030-2,029$ & Topsoil, black \\
\hline $1-42$ & 41 & $2,029-1,988$ & Clay, yellow brown, silty, sandy, pebbly; minor sand and gravel (till) \\
\hline $42-100$ & 58 & $1,988-1,930$ & Clay, gray, silty, sandy, pebbly (till) \\
\hline $100-185$ & 85 & $1,930-1,845$ & Clay, gray, very silty, sandy, pebbly (till) \\
\hline $185-239$ & 54 & $1,845-1,791$ & Clay, yellow brown silty, sandy, pebbly (till) \\
\hline $239-295$ & 56 & $1,791-1,735$ & Clay, gray, silty, sandy, pebbly; a few sand and gravel stringers below 270 feet (till) \\
\hline $295-347$ & 52 & $1,735-1,683$ & Clay, gray, silty, sandy, pebbly (till) \\
\hline $347-364$ & 17 & $1,683-1,666$ & Sand and gravel; minor clay layers \\
\hline 364-399 & 35 & $1,666-1,631$ & Clay, gray, silty, sandy, pebbly (till) \\
\hline $399-423$ & 24 & $1,631-1,607$ & Sand and gravel, clayey \\
\hline $423-465$ & 42 & $1,607-1,565$ & Clay, brown, silty, sandy, pebbly; shaley; scattered sand and gravel intervals (till) \\
\hline $465-503$ & 38 & $1,565-1,527$ & Clay, gray, silty, sandy, pebbly, shaley; scattered sand and gravel intervals (till) \\
\hline $503-601$ & 98 & $1,527-1,429$ & Clay, dark-gray, silty, sandy, pebbly, shaley; scattered sand intervals (till) \\
\hline $601-701$ & 100 & $1,429-1,329$ & Clay, dark-gray, silty, sandy, pebbly, shaley (till) \\
\hline $701-739$ & 38 & $1,329-1,291$ & Sand and gravel \\
\hline $739-758$ & 19 & $1,291-1,272$ & Shale, dark-gray; greasy, noncalcareous (Pierre Shale) \\
\hline Electric $\log$ & & & \\
\hline
\end{tabular}


Local number: 121N51W35CDDD

Other identifier: R1-87-53

Site identification number: 451427097010101

Date of construction: 7-24-87

Land surface altitude: 1,955 feet

Total depth: 860 feet

\begin{tabular}{|c|c|c|c|}
\hline Depth & $\begin{array}{l}\text { Thickness } \\
\text { (feet) }\end{array}$ & $\begin{array}{c}\text { Altitude } \\
\text { (feet) }\end{array}$ & Description \\
\hline $0-1$ & 1 & $1,955-1,954$ & Topsoil, tan, sandy \\
\hline $1-26$ & 25 & $1,954-1,929$ & Sand and gravel, brown, medium sand to medium gravel: numerous clay stringers \\
\hline $26-48$ & 22 & $1,929-1,907$ & Clay, yellow brown to brown, sandy, silty, pebbly (till) \\
\hline $48-65$ & 17 & $1,907-1,890$ & Sand and gravel, lower portion clayey \\
\hline $65-113$ & 48 & $1,890-1,842$ & Clay, dark-gray, silty, sandy, pebbly (till) \\
\hline $113-117$ & 4 & $1,842-1,838$ & Sand and gravel \\
\hline $117-169$ & 52 & $1,838-1,786$ & Clay, gray, silty, sandy, pebbly (till) \\
\hline $169-212$ & 43 & $1,786-1,743$ & Clay, yellow brown to brown, silty, sandy, pebbly (till) \\
\hline $212-233$ & 21 & $1,743-1,722$ & Sand and gravel, lower portion clayey \\
\hline $233-242$ & 9 & $1,722-1,713$ & Clay, brown, silty, sandy, pebbly (till) \\
\hline $242-350$ & 108 & $1,713-1,605$ & Clay, gray, silty, sandy, pebbly (till) \\
\hline $350-378$ & 28 & $1,605-1,577$ & Sand and gravel, clayey \\
\hline $378-407$ & 29 & $1,577-1,548$ & Clay, yellow brown(?), silty, sandy, pebbly (till) \\
\hline $407-453$ & 46 & $1,548-1,502$ & Clay, gray, sandy, silty, pebbly (till) \\
\hline 453-537 & 84 & $1,502-1,418$ & Clay, gray, silty, sandy, pebbly (till) \\
\hline $537-542$ & 5 & $1,418-1,413$ & Sand \\
\hline $542-610$ & 68 & $1,413-1,345$ & Clay, gray, silty, sandy, pebbly (till) \\
\hline $610-614$ & 4 & $1,345-1,341$ & Sand and gravel, medium sand to medium gravel \\
\hline $614-680$ & 66 & $1,341-1,275$ & Clay, gray, silty, sandy, pebbly (till) \\
\hline $680-715$ & 35 & $1,275-1,240$ & Clay, brownish-gray, silty, sandy pebbly (till) \\
\hline $715-722$ & 7 & $1,240-1,233$ & Sand, brown \\
\hline $722-820$ & 98 & $1,233-1,135$ & Clay, gray, silty, sandy, pebbly (till) \\
\hline $820-860$ & 40 & $1,135-1,095$ & Shale(?) \\
\hline
\end{tabular}

Electric log available. Lithologic breaks to 551 feet were called using the geophysical log. Below 551 feet they are based on the driller's logs. Nature of the shale, Pierre Shale or Carlile Shale, is uncertain, but it is probably Carlile Shale 
Local number: 121N52W2AAAA R

Other identifier: R1-87-37

Site identification number: 451748097072501

Date of construction: 6-11-87

Land surface altitude: 1,890 feet

Total depth: 560 feet

\begin{tabular}{|c|c|c|c|}
\hline Depth & $\begin{array}{l}\text { Thickness } \\
\text { (feet) }\end{array}$ & $\begin{array}{l}\text { Aititude } \\
\text { (feet) }\end{array}$ & Description \\
\hline $0-2$ & 2 & $1,890-1,888$ & Topsoil, black, sandy \\
\hline 2-15 & 13 & $1,888-1,875$ & Gravel, brown, fine to medium; some sand \\
\hline $15-27$ & 12 & $1,875-1,863$ & Clay, yellow brown, silty, sandy, pebbly (till) \\
\hline $27-63$ & 36 & $1,863-1,827$ & Clay, gray, silty, sandy, pebbly (till) \\
\hline 63-81 & 18 & $1,827-1,809$ & Clay, olive green, silty; minor sand \\
\hline $81-94$ & 13 & $1,809-1,796$ & Clay, yellow brown, silty, sandy, pebbly (till) \\
\hline $94-144$ & 50 & $1,796-1,746$ & Clay, gray, silty, sandy, pebbly, sandy near base (till) \\
\hline $144-166$ & 22 & $1,746-1,724$ & Sand and gravel, coarse sand to medium gravel \\
\hline $166-194$ & 28 & $1,724-1,696$ & Clay, yellow brown, sandy, silty, pebbly; scattered sand stringers (till) \\
\hline $194-225$ & 31 & $1,696-1,665$ & Clay, yellow brown, silty, sandy, pebbly (till) \\
\hline $225-280$ & 55 & $1,665-1,610$ & Clay, gray, silty, sandy, pebbly; some sand stringers (till) \\
\hline $280-332$ & 52 & $1,610-1,558$ & Clay, gray, silty, sandy, pebbly (till) \\
\hline $332-345$ & 13 & $1,558-1,545$ & Clay, gray, silty, sandy, pebbly; grading to clayey sand \\
\hline 345-392 & 47 & $1,545-1,498$ & Clay, yellow brown to gray, silty, sandy, pebbly (till) \\
\hline $392-473$ & 81 & $1,498-1.417$ & $\begin{array}{l}\text { Clay, gray, silty, sandy, pebbly: sand and gravel abundant between } 423 \text { and } 435 \text { feet } \\
\text { (till) }\end{array}$ \\
\hline $473-528$ & 55 & $1,417-1,362$ & $\begin{array}{l}\text { Sand and gravel, gray, medium sand to fine gravel; several clayey zones; grades to } \\
\text { medium gravel at base }\end{array}$ \\
\hline $528-560$ & 32 & $1,362-1,330$ & Shale, dark-gray; greasy, noncalcareous (Pierre Shale) \\
\hline \multicolumn{4}{|c|}{ Electric log available } \\
\hline
\end{tabular}


Local number: 121N52W9CDDD R

Other identifier: R2-86-106

Site identification number: 451607097103001

Date of construction: $10-30-86$

Land surface altitude: 1,852 feet

Total depth: 510 feet

\begin{tabular}{|c|c|c|c|}
\hline Depth & $\begin{array}{l}\text { Thickness } \\
\text { (feet) }\end{array}$ & $\begin{array}{l}\text { Altitude } \\
\text { (feet) }\end{array}$ & Description \\
\hline $0-1$ & 1 & $1,852-1,851$ & Topsoil, black \\
\hline $1-22$ & 21 & $1,851-1,830$ & Clay, yellow brown, very silty, sandy, pebbly; soft (till) \\
\hline $22-52$ & 30 & $1,830-1,800$ & Clay, dark-brown to gray, very silty, sandy, pebbly; soft (till) \\
\hline $52-65$ & 13 & $1,800-1,787$ & Sand, gray, medium to coarse; some gravel \\
\hline $65-120$ & 55 & $1,787-1,732$ & Silt, gray, clayey; only minor sand and pebbles; some interbedded sands \\
\hline $120-127$ & 7 & $1,732-1,725$ & $\begin{array}{l}\text { Silt, black to purplish-brown to green, clayey; a strong gamma kick in this segment } \\
\text { suggests a possible ash layer }\end{array}$ \\
\hline $127-147$ & 20 & $1,725-1,705$ & Clay, yellow brown, silty, sandy, pebbly (till) \\
\hline $147-212$ & 65 & $1,705-1.640$ & Clay, gray, silty, sandy, pebbly (till) \\
\hline $212-280$ & 68 & $1,640-1,572$ & Clay, gray, sandy, silty, pebbly; a few sand and gravel stringers below 259 feet (till) \\
\hline $280-297$ & 17 & $1,572-1,555$ & Clay, yellow brown to brown, silty, sandy, pebbly (till) \\
\hline $297-349$ & 52 & $1,555-1,503$ & $\begin{array}{l}\text { Clay, gray, silty, sandy, pebbly: sandier interval or clayey sands from } 336 \text { to } 349 \text { feet } \\
\text { (till) }\end{array}$ \\
\hline $349-369$ & 20 & $1,503-1,483$ & Clay, dark-brown to gray, silty, sandy, pebbly, shaley(?) (till) \\
\hline $369-401$ & 32 & $1,483-1,451$ & Clay, dark-gray, silty, sandy, pebbly, shaley(?) (till) \\
\hline $401-434$ & 33 & $1,451-1,418$ & Clay, dark-gray, silty, sandy, pebbly, shaley(?) (till) \\
\hline $434-493$ & 59 & $1,418-1,359$ & Sand, brown to gray, medium; minor clay intervals throughout \\
\hline 493-510 & 17 & $1,359-1,342$ & Shale, dark-gray; greasy, noncalcareous (Pierre Shale) \\
\hline
\end{tabular}


Local number: 121N52W18BBBB R

Other identifier: R-65

Site identification number: 451605097133101

Date of construction: $8-12-70$

Land surface altitude: 1,873 feet

Total depth: 530 feet

\begin{tabular}{cccl}
\hline Depth & $\begin{array}{c}\text { Thickness } \\
\text { (feet) }\end{array}$ & $\begin{array}{c}\text { Altitude } \\
\text { (feet) }\end{array}$ & \multicolumn{1}{c}{ Description } \\
\hline $0-27$ & 27 & $1,873-1,846$ & Till, brown \\
$27-39$ & 12 & $1,846-1,834$ & Till gray \\
$39-63$ & 24 & $1,834-1,810$ & Silt and sand, light-gray to light-brownish bray; possibly oxidized silt or till \\
$63-65$ & 2 & $1,810-1,808$ & Gravel \\
$65-96$ & 31 & $1,808-1,777$ & Till, yellowish-brown; iron staining \\
$96-124$ & 28 & $1,777-1,749$ & Gravel; and silty layers, yellowish-brown \\
$124-128$ & 4 & $1,749-1,745$ & Clay, light-gray, silty, sandy; fat, calcareous \\
$128-245$ & 117 & $1,745-1,628$ & Till, gray to brown; rocks from 227 to 229, 231 to 233, and 244 to 245 feet; rocky and \\
& & $1,628-1,585$ & Till, light-brown \\
$245-288$ & 43 & $1,685-1,561$ & Sand and gravel; rocks \\
$288-312$ & 24 & $1,561-1,376$ & Till, brown and gray; some dark-clay in samples from 420 to 440 feet \\
$312-497$ & 185 & $1,376-1,368$ & Gravel \\
\hline $497-505$ & 8 & $1,368-1,343$ & Shale \\
$505-530$ & 25 & & \\
\hline Electric log available & & \\
\hline
\end{tabular}


Local number: 121N52W25BBBB R

Other identifier: R1-87-35

Site identification number: 451420097072301

Date of construction: 6-9-87

Land surface altitude: 1,915 feet

Total depth: 725 feet

\begin{tabular}{|c|c|c|c|}
\hline Depth & $\begin{array}{l}\text { Thickness } \\
\text { (feet) }\end{array}$ & $\begin{array}{l}\text { Altitude } \\
\text { (feet) }\end{array}$ & Description \\
\hline $0-2$ & 2 & $1,915-1,913$ & Topsoil, black \\
\hline $2-41$ & 39 & $1,913-1,874$ & Clay, yellow brown to tan, silty, sandy, pebbly (till) \\
\hline $41-87$ & 46 & $1,874-1,828$ & Clay, gray, silty, sandy, pebbly; sand and gravel from 58 to 60 feet (till) \\
\hline $87-93$ & 6 & $1,828-1,822$ & Sand and gravel \\
\hline 93-138 & 45 & $1,822-1,777$ & Clay, brown, silty, sandy, pebbly (till) \\
\hline $138-154$ & 16 & $1,777-1,761$ & Clay, gray, silty, sandy, pebbly (till) \\
\hline $154-162$ & 8 & $1,761-1,753$ & Sand and gravel, clayey \\
\hline $162-189$ & 27 & $1,753-1,726$ & Clay, gray, silty, sandy, pebbly (till) \\
\hline $189-275$ & 86 & $1,726-1,640$ & Clay, gray, sandy, silty, pebbly; many thin sand and gravel stringers (till) \\
\hline 275-306 & 31 & $1,640-1,609$ & Sand and gravel, coarse sand to medium gravel; possibly some oxidized till \\
\hline $306-358$ & 52 & $1,609-1,557$ & Clay, gray, silty, sandy, pebbly, grading to very sandy below 346 feet (till) \\
\hline $358-413$ & 55 & $1,557-1,502$ & $\begin{array}{l}\text { Clay, gray, silty, sandy, pebbly, grades to very sandy below } 399 \text { feet; there is a gamma } \\
\text { spike at the top of the interval, possibly indicating an ashy layer (till) }\end{array}$ \\
\hline $413-475$ & 62 & $1,502-1,440$ & Clay, dark-brown, silty, sandy, pebbly; a few sand stringers (till) \\
\hline $475-564$ & 89 & $1,440-1,351$ & Clay, dark-grown to gray, silty, sandy, pebbly; a few sand stringers below 535 feet (till) \\
\hline $564-696$ & 132 & $1,351-1,219$ & $\begin{array}{l}\text { Sand and gravel, gray, medium sand to fine gravel, generally clayey; possibly } \\
\text { interbedded gray till units }\end{array}$ \\
\hline $696-725$ & 29 & $1,219-1,190$ & Shale, dark-gray; greasy, noncalcareous, inoceramus fragments (Pierre Shale) \\
\hline \multicolumn{3}{|c|}{ Electric log available } & \\
\hline
\end{tabular}


Local number: 121N53W1AAAA R

Other identifier: R2-87-25

Site identification number: 451750097133501

Date of construction: $6-9-87$

Land surface altitude: 1,915 feet

Total depth: 545 feet

\begin{tabular}{|c|c|c|c|}
\hline Depth & $\begin{array}{l}\text { Thickness } \\
\text { (feet) }\end{array}$ & $\begin{array}{l}\text { Altitude } \\
\text { (feet) }\end{array}$ & Description \\
\hline $0-3$ & 3 & $1,915-1,912$ & Topsoil, black \\
\hline $3-26$ & 23 & $1,912-1,889$ & Clay, yellow brown, silty, sandy, pebbly (till) \\
\hline $26-71$ & 45 & $1,889-1,844$ & Clay, dark-yellow brown to gray, silty, sandy, pebbly \\
\hline $71-155$ & 84 & $1,844-1,760$ & $\begin{array}{l}\text { Clay, yellow brown, silty, sandy, pebbly; sand and gravel stringers common; sand } \\
\text { content increases below } 96 \text { feet (till) }\end{array}$ \\
\hline $155-185$ & 30 & $1,760-1,730$ & Clay, gray, silty, sandy, pebbly (till) \\
\hline $185-222$ & 37 & $1,730-1,693$ & Clay, tan brown, silty, sandy, pebbly (till) \\
\hline $222-309$ & 87 & $1,693-1,606$ & $\begin{array}{l}\text { Clay, brown to gray(?), sandy, silty, pebbly; numerous sand and gravel intervals; very } \\
\text { sandy below } 259 \text { feet (till) }\end{array}$ \\
\hline $309-373$ & 64 & $1,606-1,542$ & Clay, tan, silty, sandy, pebbly (till) \\
\hline $373-416$ & 43 & $1,542-1,499$ & Clay, gray, silty, sandy, pebbly (till) \\
\hline $416-449$ & 33 & $1,499-1,466$ & Sand, gray, medium; scattered clay layers \\
\hline $449-479$ & 30 & $1,466-1,436$ & Sand, gray, medium, shaley(?); some shale blocks included \\
\hline $479-545$ & 66 & $1,436-1,370$ & Shale, dark-gray; greasy, noncalcareous (Pierre Shale) \\
\hline Electric log & le. Increas & general sand & $\begin{array}{l}\text { ontent at } 96 \text { feet in the } 71 \text { - to } 155 \text {-foot interval may mark a different drift unit. Very } \\
\text { layey sand instead }\end{array}$ \\
\hline
\end{tabular}


Local number: 121N53W24DDDD R

Other identifier: R1-87-36

Site identification number: 451423097174501

Date of construction: 6-10-87

Land surface altitude: 1,855 feet

Total depth: 515 feet

\begin{tabular}{|c|c|c|c|}
\hline Depth & $\begin{array}{l}\text { Thickness } \\
\text { (feet) }\end{array}$ & $\begin{array}{l}\text { Altitude } \\
\text { (feet) }\end{array}$ & Description \\
\hline $0-2$ & 2 & $1,855-1.853$ & Topsoil, black \\
\hline $2-9$ & 7 & $1,853-1,846$ & Clay, yellow brown, sandy, silty, pebbly (till) \\
\hline $9-31$ & 22 & $1,846-1,824$ & Sand, brown to gray, medium to coarse; some gravel; clay stringers \\
\hline $31-42$ & 11 & $1,824-1,813$ & Clay, gray, silty, sandy, pebbly (till) \\
\hline $42-82$ & 40 & $1,813-1,773$ & Clay, yellow brown to tan, silty, sandy, pebbly (till) \\
\hline $82-133$ & 51 & $1,773-1,722$ & Clay, gray, silty, sandy, pebbly; possible increase in clay content at 113 feet (till) \\
\hline $133-190$ & 57 & $1,722-1,665$ & Clay, medium-gray, silty, sandy, pebbly; some oxidized clay (till) \\
\hline $190-203$ & 13 & $1,665-1,652$ & Sand and gravel, gray; interbedded clay \\
\hline $203-213$ & 10 & $1,652-1,642$ & Clay, gray, silty; minor sand \\
\hline 213-245 & 32 & $1,642-1,610$ & Clay, yellow brown, silty, sandy, pebbly; scattered sand and gravel layers (till) \\
\hline $245-294$ & 49 & $1,610-1,561$ & Clay, gray, silty, sandy, pebbly; a few sand and gravel stingers (till) \\
\hline 294-304 & 10 & $1,561-1,551$ & Sand and gravel \\
\hline $304-329$ & 25 & $1,551-1,526$ & Clay, brown(?), silty, sandy, pebbly (till) \\
\hline $329-381$ & 52 & $1,526-1,474$ & Clay, gray, silty, sandy, pebbly; scattered sand and gravel stringers (till) \\
\hline $381-420$ & 39 & $1,474-1,435$ & Clay, gray, silty, sandy, pebbly (till) \\
\hline $420-480$ & 60 & $1,435-1,375$ & Sand, gray, medium; several clay layers \\
\hline $480-515$ & 35 & $1,375-1,340$ & Shale, dark-gray; greasy, noncalcareous (Pierre Shale) \\
\hline
\end{tabular}


Local number: 122N47W35DDCD

Other identifier: R2-85-28

Site identification number: 451935096310001

Date of construction: $5-9-85$

Land surface altitude: 1,100 feet

Total depth: 200 feet

\begin{tabular}{|c|c|c|c|}
\hline Depth & $\begin{array}{l}\text { Thickness } \\
\text { (feet) }\end{array}$ & $\begin{array}{l}\text { Altltude } \\
\text { (feet) }\end{array}$ & Description \\
\hline $0-37$ & 37 & $1,100-1,063$ & Clay, brown, silty, very sandy, pebbly (till) \\
\hline $37-51$ & 14 & $1,063-1,049$ & Clay, gray, silty, very sandy, pebbly (till) \\
\hline $51-54$ & 3 & $1,049-1,046$ & Sand, coarse \\
\hline $54-59$ & 5 & $1,046-1,041$ & Clay, gray, very silty, sandy, pebbly, shaley (till) \\
\hline $59-86$ & 27 & $1,041-1,014$ & Sand, medium; to medium gravel; clay layer from 80 to 82 feet \\
\hline $86-124$ & 38 & $1,014-976$ & Clay, gray, silty, sandy, pebble; shale rich (till) \\
\hline $124-130$ & 6 & $976-970$ & Sand, coarse; to fine gravel \\
\hline $130-161$ & 31 & $970-939$ & $\begin{array}{l}\text { Clay, gray, silty, sandy, pebbly; shale rich; lake deposits, clay and fine silt from } 143 \text { to } \\
150 \text { feet (till) }\end{array}$ \\
\hline $161-182$ & 21 & $939-918$ & $\begin{array}{l}\text { Mudstone, dark-brown, tan speckles; calcareous, hard (Fairport Shale Member? - } \\
\text { Carlile Shale) }\end{array}$ \\
\hline $182-200$ & 18 & $918-900$ & $\begin{array}{l}\text { Mudstone, dark-brown, tan speckles, calcareous; crystalline carbonate fragments, soft, } \\
\text { calcareous, white clays, drill chatter (Greenhorn Limestone) }\end{array}$ \\
\hline
\end{tabular}


Local number: 122N48W35DDDD

Other identifier: R2-85-23

Site identification number: 451935096381501

Date of construction: $5-7-85$

Land surface altitude: 1,110 feet

Total depth: 290 feet

\begin{tabular}{|c|c|c|c|}
\hline Depth & $\begin{array}{l}\text { Thickness } \\
\text { (feet) }\end{array}$ & $\begin{array}{l}\text { Altitude } \\
\text { (feet) }\end{array}$ & Description \\
\hline $0-1$ & 1 & $1,110-1,109$ & Topsoil, black \\
\hline $1-18$ & 17 & $1,109-1,092$ & Clay, tan to brown, very silty, sandy, pebbly (till) \\
\hline $18-36$ & 18 & $1,092-1,074$ & Clay, gray, very silty, sandy, pebbly (till) \\
\hline $36-48$ & 12 & $1,074-1,062$ & Sand, medium to coarse; to fine travel in lower portion; upper parts look oxidized \\
\hline $48-78$ & 30 & $1,062-1,032$ & Clay, pinkish- to brownish-gray, silty, very sandy, pebbly; some sand stringers \\
\hline $78-92$ & 14 & $1,032-1,018$ & Sand, medium; to fine gravel; clay layers \\
\hline $92-118$ & 26 & $1,018-992$ & Clay, dark-gray, silty, very sandy, pebbly (till) \\
\hline $118-122$ & 4 & $992-988$ & Gravel, coarse \\
\hline $122-136$ & 14 & $988-974$ & Clay, dark-gray to black, silty, sandy, pebbly(?); might be shale \\
\hline $136-205$ & 69 & 974-905 & $\begin{array}{l}\text { Claystone, dark-brown to black; greasy, noncalcareous; fine sand partings with very soft } \\
\text { clay (Blue Hill Member? - Carlile Shale) }\end{array}$ \\
\hline $205-240$ & 35 & $905-870$ & $\begin{array}{l}\text { Mudstone, dark-brown, tan speckles; calcareous; hard (Fairport Shale Member? - } \\
\text { Carlile Shale) }\end{array}$ \\
\hline $240-290$ & 50 & $870-820$ & $\begin{array}{l}\text { Mudstone, dark-brown, tan speckles; calcareous; drill chatter; soft white calcareous } \\
\text { clays, crystalline calcite fragments (Greenhorn Limestone) }\end{array}$ \\
\hline \multicolumn{4}{|c|}{$\begin{array}{l}\text { Unit contacts in bedrock are from cuttings log, so they may both be exact. May have penetrated Graneros Shale below the Greenhorn } \\
\text { Limestone }\end{array}$} \\
\hline
\end{tabular}


Local number: $122 \mathrm{~N} 49 \mathrm{~W} 33 \mathrm{CCCB}$

Other identifier: R20-88-47

Site identification number: 451941096491701

Date of construction: 9-14-88

Land surface altitude: 1,214 feet

Total depth: 458 feet

\begin{tabular}{cccl}
\hline Depth & $\begin{array}{c}\text { Thickness } \\
\text { (feet) }\end{array}$ & $\begin{array}{c}\text { Altitude } \\
\text { (feet) }\end{array}$ & \multicolumn{1}{c}{ Description } \\
\hline $0-2$ & 2 & $1,214-1,212$ & Topsoil, black \\
$2-21$ & 19 & $1,212-1,193$ & Clay, yellow brown, silty, sandy, pebbly (till) \\
$21-107$ & 86 & $1,193-1,107$ & $\begin{array}{c}\text { Clay, gray, silty, sandy, pebbly; sand intervals from 32 to 36, } 50 \text { to } 57 \text {, and } 60 \text { to } 62 \text { feet } \\
\text { (till) }\end{array}$ \\
$107-116$ & 9 & $1,107-1,098$ & Clay, olive gray, silty, sandy, pebbly (till) \\
$116-257$ & 141 & $1,098-957$ & Sand, medium to fine, clayey to 175 feet \\
$257-362$ & 105 & $957-852$ & Clay, gray, (Blue Hill Shale Member - Carlile Shale) \\
$632-454$ & 92 & $852-760$ & Clay, dark-brown; calcareous (Fairport Shale Member - Carlile Shale) \\
$454-458$ & 4 & $760-756$ & Limestone, dark-brown (Greenhorn Limestone) \\
\hline
\end{tabular}

Local number: 122N49W35BBBB

Other identifier: R1-82-46

Site identification number: 452026096464801

Date of construction: 5-18-82

Land surface altitude: 1,169 feet

Total depth: 65 feet

\begin{tabular}{cccl}
\hline Depth & $\begin{array}{c}\text { Thickness } \\
\text { (feet) }\end{array}$ & $\begin{array}{c}\text { Altitude } \\
\text { (feet) }\end{array}$ & \multicolumn{1}{c}{ Description } \\
\hline $0-2$ & 2 & $1,169-1,167$ & Topsoil, black \\
$2-12$ & 10 & $1,167-1,157$ & Clay, brown, silty, sandy, pebbly (till) \\
$12-45$ & 33 & $1,157-1,124$ & Clay, gray, silty \\
$45-65$ & 20 & $1,124-1,104$ & Shale \\
\hline
\end{tabular}


Local number: 122N49W35DDDD

Other identifier: R2-86-80

Site identification number: 451946096453701

Date of construction: $9-9-86$

Land surface altitude: 1,165 feet

Total depth: 372 feet

\begin{tabular}{|c|c|c|c|}
\hline Depth & $\begin{array}{l}\text { Thickness } \\
\text { (feet) }\end{array}$ & $\begin{array}{l}\text { Altitude } \\
\text { (feet) }\end{array}$ & Description \\
\hline $0-2$ & 2 & $1,165-1,163$ & Topsoil, black \\
\hline $2-10$ & 8 & $1,163-1,155$ & Clay, yellow brown, silty, sandy, pebbly (till - New Ulm?) \\
\hline $10-22$ & 12 & $1,155-1,143$ & Clay, gray, silty, sandy, pebbly (till - Granite Falls? or Whetstone?) \\
\hline $22-42$ & 20 & $1,143-1,123$ & Silt, gray; calcareous; minor sand initially, increasing with depth \\
\hline $42-51$ & 9 & $1,123-1,114$ & Sand and gravel, gray \\
\hline $51-79$ & 28 & $1,114-1,086$ & Shale, dark-brownish-gray, silty; noncalcareous; organic looking (Carlile Shale?) \\
\hline 79-303 & 224 & $1,086-862$ & $\begin{array}{l}\text { Shale, dark-blue-gray; greasy, noncalcareous; some fine sand partings between } 240 \text { and } \\
280 \text { feet (Blue Hill Shale Member - Carlile Shale) }\end{array}$ \\
\hline $303-360$ & 57 & $862-805$ & $\begin{array}{l}\text { Shale, dark-brown with tan specks, silty; calcareous (Fairport Shale Member - Carlile } \\
\text { Shale) }\end{array}$ \\
\hline $360-372$ & 12 & $805-793$ & Limestone, dark-brownish-gray; dirty, some dark-brown shale (Greenhorn Limestone) \\
\hline Electric log & & & \\
\hline
\end{tabular}


Local number: 122N52W19CCCC R

Other identifier: R-70

Site identification number: 451936097133301

Date of construction: $8-20-70$

Land surface altitude: 1,870 feet

Total depth: 515 feet

\begin{tabular}{|c|c|c|c|}
\hline Depth & $\begin{array}{c}\text { Thickness } \\
\text { (feet) }\end{array}$ & $\begin{array}{c}\text { Altitude } \\
\text { (feet) }\end{array}$ & Description \\
\hline $0-14$ & 14 & $1,870-1,856$ & Sand and gravel, coarse \\
\hline $14-80$ & 66 & $1,856-1,790$ & Clay, yellowish-brown, pebbly \\
\hline 80-109 & 29 & $1,790-1,761$ & Clay, gray, pebbly \\
\hline $109-120$ & 11 & $1,761-1,750$ & Gravel and clay stringers \\
\hline $120-170$ & 50 & $1,750-1,700$ & Clay, gray, pebbly \\
\hline $170-220$ & 50 & $1,700-1,650$ & Clay; partially oxidized (till) \\
\hline $220-289$ & 69 & $1,650-1,581$ & Clay, gray, pebbly \\
\hline $289-311$ & 22 & $1,581-1,559$ & Gravel and clay stringers \\
\hline $311-336$ & 25 & $1,559-1,534$ & Clay, gray, pebbly \\
\hline $336-346$ & 10 & $1,534-1,524$ & Sand and gravel, medium to coarse \\
\hline $346-416$ & 70 & $1,524-1,454$ & Clay, gray, pebbly \\
\hline $416-437$ & 21 & $1,545-1,433$ & Sand and gravel, clayey; with clay stringers \\
\hline $437-483$ & 46 & $1,433-1,387$ & Clay, light-gray to white; some gravel (marl) \\
\hline $483-515$ & 32 & $1,387-1,355$ & Shale \\
\hline Electric log & & & \\
\hline
\end{tabular}


Local number: 122N53W16DCCD R

Other identifier: WA-6

Site identification number: 454030097174501

Date of construction: 6-66

Land surface altitude: 1,812 feet

Total depth: 445 feet

\begin{tabular}{cccl}
\hline Depth & $\begin{array}{c}\text { Thickness } \\
\text { (feet) }\end{array}$ & $\begin{array}{c}\text { Altitude } \\
\text { (feet) }\end{array}$ & \\
\hline $0-2$ & 2 & $1,812-1,810$ & Topsoil \\
$2-15$ & 13 & $1,810-1,797$ & Gravel \\
$15-47$ & 32 & $1,797-1,765$ & Clay, gray; several thin sand lenses \\
$47-80$ & 33 & $1,765-1,732$ & Gravel medium to coarse \\
$80-124$ & 44 & $1,732-1,688$ & Sand \\
$124-195$ & 71 & $1,688-1,617$ & Clay, gray; gravel stringers and 163 to 195 feet \\
$195-210$ & 15 & $1,617-1,602$ & Gravel; and clay stringers \\
$210-385$ & 175 & $1,602-1,427$ & Clay, gray; thin gravel lenses at 210 to 240 feet, rocky at 360 to 375 feet (till) \\
$385-419$ & 34 & $1,427-1,393$ & Gravel \\
\hline $419-445$ & 26 & $1,393-1,367$ & Pierre Shale
\end{tabular}

Electric log available

Local number: 123 N53W13CCCC R

Other identifier: R-71

Site identification number: 452544097144801

Date of construction: $8-20-70$

Land surface altitude: 1,874 feet

Total depth: 700 feet

\begin{tabular}{cccl}
\hline Depth & $\begin{array}{c}\text { Thickness } \\
\text { (feet) }\end{array}$ & $\begin{array}{c}\text { Altitude } \\
\text { (feet) }\end{array}$ & \multicolumn{1}{c}{ Description } \\
\hline $0-17$ & 17 & $1,874-1,857$ & Clay, yellow brown, sandy, pebbly (till) \\
$17-56$ & 39 & $1,857-1,818$ & Clay, gray, sandy, pebbly (till) \\
$56-72$ & 16 & $1,818-1,802$ & Clay, yellow brown, silty, sandy (till) \\
$72-98$ & 26 & $1,802-1,776$ & Sand, yellow brown, clayey, gravelly \\
$98-142$ & 44 & $1,776-1,732$ & Clay, dark-gray, pebbly; very hard (till) \\
$142-190$ & 48 & $1,732-1,684$ & Clay, yellow (till) \\
$190-684$ & 494 & $1,684-1,190$ & Clay, gray, silty, sandy, pebbly (till) \\
$684-700$ & 16 & $1,190-1,174$ & Shale, dark-gray; thin bentonite layers \\
Electric log available & & \\
\hline
\end{tabular}


Local number: 123N53W27CCCC R

Other identifier: R-69

Site identification number: 452357097171601

Date of construction: $8-18-70$

Land surface altitude: 1,882 feet

Total depth: 840 feet

\begin{tabular}{|c|c|c|c|}
\hline Depth & $\begin{array}{c}\text { Thickness } \\
\text { (feet) }\end{array}$ & $\begin{array}{l}\text { Altitude } \\
\text { (feet) }\end{array}$ & Description \\
\hline $0-17$ & 17 & $1,882-1.865$ & Clay, yellow, silty, pebbly, gravelly in spots \\
\hline $17-60$ & 43 & $1,865-1,822$ & Sand and gravel, medium to coarse \\
\hline $60-103$ & 43 & $1,822-1,779$ & Clay, gray, silty, pebbly \\
\hline $103-106$ & 3 & $1,779-1,776$ & Clay, yellow \\
\hline $106-123$ & 17 & $1,776-1,759$ & Clay, light-gray, silty, sandy \\
\hline $123-137$ & 14 & $1,759-1,745$ & Sand and gravel, medium to coarse; with some clay \\
\hline $137-152$ & 15 & $1,745-1,730$ & Till, yellow to gray; gravel stringers \\
\hline $152-166$ & 14 & $1,730-1,716$ & Clay, medium-gray to green; slightly oxidized, some black organic clay \\
\hline $166-185$ & 19 & $1,716-1,697$ & Clay, gray, compact \\
\hline $185-200$ & 15 & $1,697-1,682$ & Clay, gray, pebbly \\
\hline $200-207$ & 7 & $1,682-1,675$ & Sand and gravel \\
\hline $207-217$ & 10 & $1,675-1,665$ & Clay, gray \\
\hline $217-255$ & 38 & $1,665-1,627$ & Clay, yellow, gravelly; sample contains gray and yellow oxidized clay, and some gravel \\
\hline $255-360$ & 105 & $1,627-1,522$ & Clay, gray, silty, pebbly \\
\hline $360-425$ & 65 & $1,522-1,457$ & Clay, dark-gray, very sandy; with gravelly layers and rocks \\
\hline $425-620$ & 195 & $1,457-1,262$ & Clay, dark-gray, sandy, pebbly; some reworked shale present \\
\hline $620-645$ & 25 & $1,262-1,237$ & Clay, gray (lacustrine) \\
\hline $645-685$ & 40 & $1,237-1,197$ & Till(?), gray, silty, pebbly; some light-gray marl \\
\hline $685-700$ & 15 & $1,197-1,182$ & Clay, gray; lots of marl. cuttings look like shale and yellow clay \\
\hline $700-710$ & 10 & $1,182-1,172$ & Clay, medium to dark-gray, silty; some hard, dark-shale \\
\hline $710-785$ & 75 & $1,172-1,097$ & Clay, medium to light-gray, silty, marly; calcareous \\
\hline $785-840$ & 55 & $1,097-1,042$ & Shale, bluish-black; blocky, compact \\
\hline
\end{tabular}


Local number: 124N49W8ADDC R

Other identifier: RB-81-23

Site identification number: 453354096491901

Date of construction: 6-25-81

Land surface altitude: 1,022 feet

Total depth: 170 feet

\begin{tabular}{|c|c|c|c|}
\hline Depth & $\begin{array}{l}\text { Thickness } \\
\text { (feet) }\end{array}$ & $\begin{array}{l}\text { Altitude } \\
\text { (feet) }\end{array}$ & Description \\
\hline $0-2$ & 2 & $1,022-1,020$ & Clay, yellow brown, silty, sandy, pebbly (till) \\
\hline $2-20$ & 18 & $1,020-1,002$ & $\begin{array}{l}\text { Sand, brown, medium to fine, well sorted; estimate } 85-90 \% \text { of cuttings are quartz grains } \\
\text { (Veblen aquifer) }\end{array}$ \\
\hline $20-26$ & 6 & $1,002-996$ & Sand, gray, medium to fine; dominantly quartz grains (Veblen aquifer) \\
\hline $26-37$ & 11 & $996-985$ & Shale; poor sample return \\
\hline $37-50$ & 13 & 985-972 & Shale, medium-gray; noncalcareous, samples are cut into "ribbons" (Carlile Shale) \\
\hline $50-111$ & 61 & 972-911 & $\begin{array}{l}\text { Shale, medium-gray; very slightly calcareous, samples cut into "ribbons" (Carlile } \\
\text { Shale) }\end{array}$ \\
\hline 111-116 & 5 & $911-906$ & $\begin{array}{l}\text { Shale, light- to medium-brown, with orangish-brown mottling; very calcareous (Carlile } \\
\text { Shale) }\end{array}$ \\
\hline 116-145 & 29 & $906-877$ & Shale, medium-gray; noncalcareous (Carlile Shale) \\
\hline $145-148$ & 3 & $877-874$ & $\begin{array}{l}\text { Sand(?); poor return but noticed slightly harder drilling (Codell Sandstone Member - } \\
\text { Carlile Shale) }\end{array}$ \\
\hline $148-152$ & 4 & $874-870$ & Shale, medium-gray (Carlile Shale) \\
\hline $152-156$ & 4 & $870-866$ & Sand(?); poor sample return (Codell Sandstone Member - Carlile Shale) \\
\hline $156-161$ & 5 & $866-861$ & Shale, medium-gray; samples are cut into "ribbons" (Carlile Shale) \\
\hline $161-163$ & 2 & $861-859$ & Sand(?); poor sample return (Codell Sandstone Member? - Carlile Shale) \\
\hline $163-170$ & 7 & $859-852$ & Shale, medium-gray; samples are cut into "ribbons" (Carlile Shale) \\
\hline Electric log & ble & & \\
\hline
\end{tabular}


Local number: 124N50W5CADD R

Other identifier: PEEVER 8

Site identification number: 453250096565301

Date of construction: 1973

Land surface altitude: 1,185 feet

Total depth: 140 feet

\begin{tabular}{cccl}
\hline Depth & $\begin{array}{c}\text { Thickness } \\
\text { (feet) }\end{array}$ & $\begin{array}{c}\text { Altitude } \\
\text { (feet) }\end{array}$ & Description \\
\hline $0-4$ & 4 & $1,185-1,181$ & Soil, dark-gray \\
$4-13$ & 9 & $1,181-1,172$ & Clay, yellowish-brown \\
$13-126$ & 113 & $1,172-1,059$ & Clay, gray (till) \\
$126-140$ & 14 & $1,059-1,045$ & Clay, dark-gray (shale) \\
\hline
\end{tabular}

Local number: 124N50W6BBCC R

Other identifier: BARSE

Site identification number: 453318096583201

Date of construction: $12-22-76$

Land surface altitude: 1,218 feet

Total depth: 800 feet

\begin{tabular}{rccl}
\hline Depth & $\begin{array}{c}\text { Thickness } \\
\text { (feet) }\end{array}$ & $\begin{array}{c}\text { Altitude } \\
\text { (feet) }\end{array}$ & \\
\hline $0-154$ & 154 & $1,218-1,064$ & Glacial drift \\
$154-213$ & 59 & $1,064-1,005$ & Pierre Shale \\
$213-326$ & 113 & $1,005-892$ & Niobrara Formation \\
$326-331$ & 5 & $892-887$ & Codell Sandstone Member - Carlile Shale \\
$331-474$ & 143 & $887-744$ & Carlile Shale \\
$474-513$ & 39 & $744-705$ & Greenhorn Limestone \\
$513-600$ & 87 & $705-618$ & Belle Fourche Shale Member - Graneros Shale \\
$600-686$ & 86 & $618-532$ & Mowry Shale Member - Graneros Shale \\
$686-701$ & 15 & $532-517$ & Dakota Formation \\
$701-784$ & 83 & $517-434$ & Wash? \\
$784-800$ & 16 & $434-418$ & Granite Wash \\
Private well screened from 780 to 800 feet. & Yielded very little if any water. Electric log available \\
\hline
\end{tabular}


Local number: 124N53W1DDDD2 R

Other identifier: R-75

Site identification number: 453237097133802

Date of construction: $8-26-70$

Land surface altitude: 2,027 feet

Total depth: 800 feet

\begin{tabular}{|c|c|c|c|}
\hline Depth & $\begin{array}{l}\text { Thickness } \\
\text { (feet) }\end{array}$ & $\begin{array}{l}\text { Altitude } \\
\text { (feet) }\end{array}$ & Description \\
\hline $0-34$ & 34 & $2,027-1,993$ & Till, brown \\
\hline $34-37$ & 3 & $1,993-1,990$ & Till, gray \\
\hline $37-43$ & 6 & $1,990-1,984$ & Sand and gravel, medium to coarse \\
\hline $43-80$ & 37 & $1,984-1,947$ & Till, yellow \\
\hline $80-100$ & 20 & $1,947-1,927$ & Clay, grayish-brown (lacustrine) \\
\hline $100-232$ & 132 & $1,927-1,795$ & Till, yellow \\
\hline $232-264$ & 32 & $1,795-1,763$ & Till, gray \\
\hline $264-270$ & 6 & $1,763-1,757$ & Sand and gravel, clayey \\
\hline $270-290$ & 20 & $1,757-1,737$ & Till, gray \\
\hline $290-308$ & 18 & $1,737-1,719$ & Sand and gravel, well sorted \\
\hline $308-367$ & 59 & $1,719-1,660$ & Till, gray \\
\hline $367-376$ & 9 & $1,660-1,651$ & Gravel, fine, sandy \\
\hline $376-744$ & 368 & $1,651-1,283$ & Clay, gray; possibly some water laid material (till) \\
\hline $744-800$ & 56 & $1,283-1,227$ & Shale \\
\hline Electric log & lable & & \\
\hline
\end{tabular}


Local number: 124N53W33DADD2 R

Other identifier: R-72

Site identification number: 452833097172002

Date of construction: $8-24-70$

Land surface altitude: 1,929 feet

Total depth: 560 feet

\begin{tabular}{cccl}
\hline Depth & $\begin{array}{c}\text { Thickness } \\
\text { (feet) }\end{array}$ & $\begin{array}{c}\text { Altitude } \\
\text { (feet) }\end{array}$ & \multicolumn{1}{c}{ Description } \\
\hline $0-17$ & 17 & $1,929-1,912$ & Clay, yellow brown, silty; oxidized (till) \\
$17-59$ & 42 & $1,912-1,870$ & Clay, gray, silty; unoxidized (till) \\
$59-76$ & 17 & $1,870-1,853$ & Clay, yellow brown, silty, pebbly (till) \\
$76-138$ & 62 & $1,853-1,791$ & Gravel, red-brown, coarse, very sandy \\
$138-154$ & 16 & $1,791-1,775$ & Clay, yellow brown, silty, pebbly (till) \\
$154-527$ & 373 & $1,775-1,402$ & Clay, gray, sandy, very sandy at 480 to 527 feet, marly, pebbly (till) \\
$527-560$ & 33 & $1,402-1,369$ & Shale, gray \\
Electric log available & & \\
\hline
\end{tabular}


Local number: 125 N50W6BCCB R

Other identifier: RB-81-14

Site identification number: 453822096584001

Date of construction: $4-21-81$

Land surface altitude: 1,264 feet

Total depth: 215 feet

\begin{tabular}{|c|c|c|c|}
\hline Depth & $\begin{array}{l}\text { Thickness } \\
\text { (feet) }\end{array}$ & $\begin{array}{c}\text { Altitude } \\
\text { (feet) }\end{array}$ & Description \\
\hline $0-2$ & 2 & $1,264-1,262$ & Topsoil, black \\
\hline $2-26$ & 24 & $1,262-1,238$ & Clay, yellow brown, silty, pebbly (till) \\
\hline $26-29$ & 3 & $1,238-1,235$ & Clay, light-gray \\
\hline $29-43$ & 14 & $1,235-1,221$ & Clay, yellow brown, silty, sandy, pebbly (till) \\
\hline $43-60$ & 17 & $1,221-1,204$ & Clay, medium-dark-gray, silty, sandy, pebbly (till) \\
\hline $60-67$ & 7 & $1,204-1,197$ & Clay, medium-reddish-brown, silty; no sand (buried weathering horizon) \\
\hline $67-73$ & 6 & $1,197-1,191$ & Clay, medium-dark-gray, silty; non sand (loess?/lake clay?) \\
\hline $73-91$ & 18 & $1,191-1,173$ & Clay, medium-dark-gray, silty, sandy, pebbly (till) \\
\hline $91-93$ & 2 & $1,173-1,171$ & Sand, gray, fine to coarse \\
\hline 93-99 & 6 & $1,171-1,165$ & Clay, medium-dark-gray \\
\hline $99-102$ & 3 & $1,165-1,162$ & Sand, gray, fine to coarse \\
\hline $102-112$ & 10 & $1,162-1,152$ & Clay, gray, sandy, gravelly (till) \\
\hline $112-128$ & 16 & $1,152-1,136$ & Sand, gray, medium to coarse; with fine gravel \\
\hline $128-144$ & 16 & $1,139-1,120$ & Clay, medium-dark-gray, silty, sandy, pebbly (till) \\
\hline $144-156$ & 12 & $1,120-1,108$ & Gravel, gray, fine; with medium to very coarse sand \\
\hline $156-166$ & 10 & $1,108-1,098$ & Clay, gray, gravelly (till) \\
\hline $166-204$ & 38 & $1,098-1.060$ & Shale, very dark-gray (Pierre Shale) \\
\hline $204-210$ & 6 & $1,060-1,054$ & Shale, very dark-gray; rig chatters through this interval (Pierre Shale) \\
\hline $210-215$ & 5 & $1,054-1,049$ & Shale. very dark-gray (Pierre Shale) \\
\hline
\end{tabular}


Local number: 125 N50W6CCCC R

Other identifier: RB-81-15

Site identification number: 453752096584001

Date of construction: 4-21-81

Land surface altitude: 1,200 feet

Total depth: 90 feet

\begin{tabular}{cccl}
\hline Depth & $\begin{array}{c}\text { Thickness } \\
\text { (feet) }\end{array}$ & $\begin{array}{c}\text { Altitude } \\
\text { (feet) }\end{array}$ & \multicolumn{1}{c}{ Description } \\
\hline $0-3$ & 3 & $1,200-1,197$ & Topsoil, black \\
$3-20$ & 17 & $1,197-1,180$ & Sand, medium to coarse; clayey in spots \\
$20-22$ & 2 & $1,180-1,178$ & Sand, medium to coarse \\
$22-36$ & 14 & $1,178-1,164$ & Sand, gray, medium to coarse, clayey \\
$36-40$ & 4 & $1,164-1,160$ & Sand, gray, medium to coarse \\
$40-56$ & 16 & $1,160-1,144$ & Clay, medium-dark-gray; very sandy form 46 to 48 feet \\
$56-61$ & 5 & $1,144-1,139$ & Sand, gray, medium to coarse; with very fine gravel \\
$61-90$ & 29 & $1,139-1,110$ & Shale, dark-gray (Pierre Shale) \\
Well screened from 37 to 40 feet; $2-i n c h$ PVC screen and casing. Electric log available \\
\hline
\end{tabular}


Local number: 125 N50W6DCCC R

Other identifier: RB-81-16

Site identification number: 453732096580301

Date of construction: $4-22-81$

Land surface altitude: 1,230 feet

Total depth: 155 feet

\begin{tabular}{cccl}
\hline Depth & $\begin{array}{c}\text { Thickness } \\
\text { (feet) }\end{array}$ & $\begin{array}{c}\text { Altitude } \\
\text { (feet) }\end{array}$ & \multicolumn{1}{c}{ Description } \\
\hline $0-1$ & 1 & $1,230-1,229$ & Topsoil, black \\
$1-21$ & 20 & $1,229-1,209$ & Clay, yellow brown, silty, sandy, pebbly (till) \\
$21-25$ & 4 & $1,209-1,205$ & Gravel, coarse \\
$25-42$ & 17 & $1,205-1,188$ & Clay, gray, silty, sandy, pebbly (till) \\
$42-50$ & 8 & $1,188-1,180$ & Sand, gray, fine to coarse \\
$50-56$ & 6 & $1,180-1,174$ & Clay, gray, silty (loess? or lake clay?) \\
$56-64$ & 8 & $1,174-1,166$ & Sand, gray; with medium to coarse gravel \\
$64-82$ & 18 & $1,166-1,148$ & Clay, gray; very silty and sandy from 68 to 72 feet \\
$82-107$ & 25 & $1,148-1,123$ & Sand, medium to coarse; with thin clay stringers \\
$107-118$ & 11 & $1,123-1,112$ & Sand, medium to coarse; clayey \\
$118-128$ & 10 & $1,112-1,102$ & Clay, gray, silty, sandy, pebbly (till) \\
$128-134$ & 6 & $1,102-1,096$ & Sand, gray, medium to coarse; with gravel \\
$134-155$ & 21 & $1,096-1,075$ & Shale, gray (Pierre Shale) \\
Well screened from 97.4 to $102-4$ feet; 2-inch PVC screen and casing. Electric log available \\
\hline
\end{tabular}


Local number: 125 N50W8BBBA R

Other identifier: RB-81-11

Site identification number: 453751096572401

Date of construction: 4-9-81

Land surface altitude: 1,216 feet

Total depth: 140 feet

\begin{tabular}{|c|c|c|c|}
\hline Depth & $\begin{array}{l}\text { Thickness } \\
\text { (feet) }\end{array}$ & $\begin{array}{l}\text { Altitude } \\
\text { (feet) }\end{array}$ & Description \\
\hline $0-9$ & 9 & $1,216-1,207$ & Silt, black, clayey (colluvium) \\
\hline $9-17$ & 8 & $1,207-1,199$ & Clay. yellow brown, silty, sandy, pebbly (till) \\
\hline $17-43$ & 26 & $1,199-1,173$ & Clay, medium-dark-gray, silty, sandy, pebbly (till) \\
\hline $43-44$ & 1 & $1,173-1,172$ & Clay, light-gray; moderately calcareous \\
\hline $44-67$ & 23 & $1,172-1,149$ & Clay, medium-dark-gray, silty, sandy, pebbly (till) \\
\hline $67-102$ & 35 & $1,149-1,114$ & $\begin{array}{l}\text { Clay, medium-dark-gray, silty, sandy, pebbly; interbedded with very coarse sand and } \\
\text { gravel lenses (till) }\end{array}$ \\
\hline $102-114$ & 12 & $1,114-1,102$ & $\begin{array}{l}\text { Sand, gray, medium to very coarse; lots of shale grains which are very coarse sand to } \\
\text { fine gravel size }\end{array}$ \\
\hline $114-140$ & 26 & $1,102-1,076$ & Shale, dark-gray; soft on top but becoming harder with depth (Pierre Shale) \\
\hline
\end{tabular}


Local number: $125 \mathrm{~N} 50 \mathrm{~W} 8 \mathrm{CCCC} \mathrm{R}$

Other identifier: RB-81-06

Site identification number: 453700096572701

Date of construction: $4-8-81$

Land surface altitude: 1,207 feet

Total depth: 170 feet

\begin{tabular}{cccl}
\hline Depth & $\begin{array}{c}\text { Thickness } \\
\text { (feet) }\end{array}$ & $\begin{array}{c}\text { Altitude } \\
\text { (feet) }\end{array}$ & \multicolumn{1}{c}{ Description } \\
\hline $0-1$ & 1 & $1,207-1,206$ & Topsoil, brown \\
$1-6$ & 5 & $1,206-1,201$ & Sand, brown, medium to coarse \\
$6-12$ & 6 & $1,201-1,195$ & Clay, yellow brown, silty, pebbly (till) \\
$12-30$ & 18 & $1,195-1,177$ & Sand, brown, medium to very coarse \\
$30-38$ & 8 & $1,177-1,169$ & Clay, gray, very silty (lake clay) \\
$30-50$ & 12 & $1,169-1,157$ & Sand, brownish-gray, medium to very coarse; with gravel and many dark-gray 1/2-inch \\
& & & shale fragments \\
$50-58$ & 8 & $1,157-1,149$ & Clay, gray (lake clay?) \\
$58-73$ & 15 & $1,149-1,134$ & Sand, gray, medium to very coarse; with gravel and clayey from 63 to 67 feet \\
$73-100$ & 27 & $1,134-1,107$ & Clay, medium-dark-gray, silty, sandy, pebbly; soft (till) \\
$100-143$ & 43 & $1,107-1,064$ & Clay, medium-dark-gray, sandy, gravelly (till) \\
$143-170$ & 27 & $1,064-1,037$ & Shale, dark-gray; hard (Pierre Shale) \\
\hline Electric log available & & \\
\hline
\end{tabular}


Local number: 125N50W20DDDD R

Other identifier: RB-81-04

Site identification number: 453514096561401

Date of construction: 4-7-81

Land surface altitude: 1,214 feet

Total depth: 200 feet

\begin{tabular}{cccl}
\hline Depth & $\begin{array}{c}\text { Thickness } \\
\text { (feet) }\end{array}$ & $\begin{array}{c}\text { Aititude } \\
\text { (feet) }\end{array}$ & \multicolumn{1}{c}{ Description } \\
\hline $0-15$ & 15 & $1,214-1,199$ & Sand, brown, medium to very coarse \\
$15-25$ & 10 & $1,199-1,189$ & Clay, yellow brown, silty, pebbly (till) \\
$25-139$ & 114 & $1,189-1,075$ & Clay, medium-dark-gray, silty, sandy, pebbly (till) \\
$139-154$ & 15 & $1,075-1,060$ & Clay, medium-dark-gray, gravelly, sandy, (till) \\
$154-179$ & 25 & $1,060-1,035$ & Clay, medium-dark-gray, silty, sandy, pebbly (till) \\
$179-181$ & 2 & $1,035-1,033$ & $\begin{array}{l}\text { Gravel; poor sample return } \\
181-200\end{array}$ \\
& 19 & $1,033-1,014$ & $\begin{array}{l}\text { Clay, light- to medium-gray, silty; calcareous, samples turn brownish-gray upon being } \\
\text { acidized, drill rig chatters through this interval (Niobrara Formation) }\end{array}$ \\
Electric log available. E-log suggests some change occurs from 108 to 139 feet, but it was not observed in drilling
\end{tabular}

Local number: 125N51W1DBBB R

Other identifier: RB-81-19

Site identification number: 453817096591801

Date of construction: 4-22-81

Land surface altitude: 1,261 feet

Total depth: 155 feet

\begin{tabular}{cccl}
\hline Depth & $\begin{array}{c}\text { Thickness } \\
\text { (feet) }\end{array}$ & $\begin{array}{c}\text { Aititude } \\
\text { (feet) }\end{array}$ & \multicolumn{1}{c}{ Description } \\
\hline $0-1$ & 1 & $1,261-1,260$ & Topsoil, black \\
$1-42$ & 41 & $1,260-1,219$ & Clay, yellow brown, silty, sandy, pebbly (till) \\
$42-46$ & 4 & $1,219-1,215$ & Sand, brown, coarse; with very fine gravel \\
$46-58$ & 12 & $1,215-1,203$ & Clay, yellow brown, silty, sandy, pebbly (till) \\
$58-99$ & 41 & $1,203-1,162$ & Clay, medium-dark-gray, silty, sandy, pebbly (till) \\
$99-112$ & 13 & $1,162-1,149$ & Sand, gray, coarse; with cream colored rock fragments, shale grains \\
$112-120$ & 8 & $1,149-1,141$ & Gravel, gray, very coarse; 1/2- to 3/4-inch diameter, also with very coarse to coarse sand - \\
& excellent material! & & \\
$120-126$ & 6 & $1,141-1,135$ & Clay, gray; poor sample \\
$126-135$ & 9 & $1,135-1,126$ & Gravel, gray, very coarse; with very coarse sand \\
$135-155$ & 20 & $1,126-1,106$ & Shale, dark-gray (Pierre Shale) \\
Well screened from 117 to 123 feet; 2-inch PVC screen and casing. Electric log available \\
\hline
\end{tabular}


Local number: 125N51W13ABBB R

Other identifier: RB-81-09

Site identification number: 453658096591701

Date of construction: 4-9-81

Land surface altitude: 1,202 feet

Total depth: 50 feet

\begin{tabular}{cccl}
\hline Depth & $\begin{array}{c}\text { Thickness } \\
\text { (feet) }\end{array}$ & $\begin{array}{c}\text { Altitude } \\
\text { (feet) }\end{array}$ & \multicolumn{1}{c}{ Description } \\
\hline $0-9$ & 9 & $1,202-1,193$ & Sand, brown, medium to coarse \\
$9-14$ & 5 & $1,193-1,188$ & Clay, yellow brown, silty \\
$14-17$ & 3 & $1,188-1,185$ & Sand, grayish-brown, medium to coarse \\
$17-21$ & 4 & $1,185-1,181$ & Clay, gray, silty \\
$21-50$ & 29 & $1,181-1,152$ & Shale, dark-gray (Pierre Shale) \\
Electric log available & & \\
\hline
\end{tabular}

Local number: 125N51W25ABAB R

Other identifier: RB-81-01

Site identification number: 453514096591201

Date of construction: 4-6-81

Land surface altitude: 1,214 feet

Total depth: 50 feet

\begin{tabular}{cccl}
\hline Depth & $\begin{array}{c}\text { Thickness } \\
\text { (feet) }\end{array}$ & $\begin{array}{c}\text { Altitude } \\
\text { (feet) }\end{array}$ & \multicolumn{1}{c}{ Description } \\
\hline $0-2$ & 2 & $1,214-1,212$ & Topsoil, black \\
$2-7$ & 5 & $1,212-1,207$ & Clay, light-gray \\
$7-17$ & 10 & $1,207-1,197$ & Sand, brown, very coarse, poorly sorted; color becomes gray with fine gravel at the base \\
$17-20$ & 3 & $1,197-1,194$ & Clay, olive gray, very sandy (till) \\
$20-26$ & 6 & $1,194-1,188$ & Clay, medium-dark-gray, silty, sandy (till) \\
$26-50$ & 24 & $1,188-1,164$ & Shale, light-bluish-gray; color grades to dark-gray, hard \\
Electric log available & & \\
\hline
\end{tabular}


Local number: 125 N55W36CDCD

Other identifier: R-1

Site identification number: 453517097222501

Date of construction: $8-19-68$

Land surface altitude: 1,853 feet

Total depth: 900 feet

\begin{tabular}{|c|c|c|c|}
\hline Depth & $\begin{array}{l}\text { Thickness } \\
\text { (feet) }\end{array}$ & $\begin{array}{l}\text { Altitude } \\
\text { (feet) }\end{array}$ & Description \\
\hline $0-17$ & 17 & $1,853-1,836$ & Clay, pebbly, sandy, silty, $\tan$ (till) \\
\hline $17-166$ & 149 & $1,836-1,687$ & Clay, silty, very rocky, gray (till) \\
\hline $166-183$ & 17 & $1,687-1,670$ & Gravel, brownish-gray \\
\hline $183-223$ & 40 & $1,670-1,630$ & Clay, gray (till) \\
\hline $223-225$ & 2 & $1,630-1,628$ & Gravel \\
\hline $225-245$ & 20 & $1,628-1,608$ & Clay, pebbly, yellowish-brown (till) \\
\hline 245-323 & 78 & $1,608-1,530$ & Clay, pebbly, much gravel (till) \\
\hline 323-335 & 12 & $1,530-1,518$ & Gravel \\
\hline 335-395 & 60 & $1,518-1,458$ & Clay, sandy (till) \\
\hline $395-410$ & 15 & $1,458-1,443$ & Gravel \\
\hline $410-440$ & 30 & $1,443-1,413$ & Clay, gravelly, some very fine sand (till) \\
\hline $440-465$ & 25 & $1,413-1,388$ & Clay, sandy, a lot of gravel (till) \\
\hline $465-560$ & 95 & $1,388-1,293$ & Clay, sandy, dark-gray (till) \\
\hline $560-620$ & 60 & $1,293-1,233$ & Clay, gravelly (till) \\
\hline $620-677$ & 57 & $1,233-1,176$ & Clay, sandy, a lot of gravel (till) \\
\hline $677-740$ & 63 & $1,176-1,113$ & Gravel \\
\hline $740-755$ & 15 & $1,113-1,098$ & Clay, very gravelly and sandy (till) \\
\hline $755-770$ & 15 & $1,098-1,083$ & Gravel, lots of lignite \\
\hline $770-800$ & 30 & $1,083-1,053$ & Gravel \\
\hline $800-820$ & 20 & $1,053-1,033$ & Clay, some sand, light-gray \\
\hline $820-900$ & 80 & $1,033-953$ & Shale \\
\hline
\end{tabular}


Local number: 126 N49W32BBBB R

Other identifier: RB-81-25

Site identification number: 453934096495701

Date of construction: 6-30-81

Land surface altitude: 995 feet

Total depth: 230 feet

\begin{tabular}{|c|c|c|c|}
\hline Depth & $\begin{array}{l}\text { Thickness } \\
\text { (feet) }\end{array}$ & $\begin{array}{c}\text { Altitude } \\
\text { (feet) }\end{array}$ & Description \\
\hline $0-1$ & 1 & 995-994 & Topsoil, black \\
\hline $1-7$ & 6 & $994-988$ & Sand, brown; with fine gravel \\
\hline $7-24$ & 17 & 988-971 & Clay, medium-dark-gray, silty, sandy, pebbly; calcareous (till) \\
\hline $24-27$ & 3 & 971-968 & Sand, gray, medium to coarse \\
\hline $27-122$ & 95 & $968-873$ & $\begin{array}{l}\text { Shale, dark-gray; slightly calcareous, hard and cuts into "ribbons," uniform drilling } \\
\text { (Carlile Shale) }\end{array}$ \\
\hline $122-130$ & 8 & $873-865$ & Sand(?); based on electric log (Codell Sandstone Member - Carlile Shale) \\
\hline $130-185$ & 55 & $865-810$ & $\begin{array}{l}\text { Shale, dark-gray; slightly calcareous, hard and cuts into "ribbons," uniform drilling, } \\
\text { concretions(?) at } 140 \text { feet (Carlile Shale) }\end{array}$ \\
\hline 185-199 & 14 & 810-796 & $\begin{array}{l}\text { Limestone, medium-brown to dark-brownish-gray; interbedded with shale, angular } \\
\text { limestone chips are calcareous and occur in lumps of shale (Greenhorn Limestone) }\end{array}$ \\
\hline 199-222 & 23 & 796-773 & $\begin{array}{l}\text { Shale, dark-gray; interbedded with brown limestone and occasional bentonites } \\
\text { (Greenhorn Limestone) }\end{array}$ \\
\hline $222-230$ & 8 & $773-765$ & Shale, dark-gray to very dark-gray (Graneros Shale) \\
\hline
\end{tabular}

Local number: 126N51W9AAAB R

Other identifier: RB-79-18

Site identification number: 454305097023501

Date of construction: 9-19-79

Land surface altitude: 1,160 feet

Total depth: 80 feet

\begin{tabular}{cccl}
\hline Depth & $\begin{array}{c}\text { Thickness } \\
\text { (feet) }\end{array}$ & $\begin{array}{c}\text { Altitude } \\
\text { (feet) }\end{array}$ & \multicolumn{1}{c}{ Description } \\
\hline $0-1$ & 1 & $1,160-1,159$ & Topsoil, black \\
$1-14$ & 13 & $1,159-1,146$ & Clay, yellow brown (till) \\
$14-55$ & 41 & $1,146-1,105$ & Clay, gray; a few sand stringers (till) \\
$55-80$ & 25 & $1,105-1,080$ & Clay, gray, black; shell fragments, good shale cuttings (shale) \\
\hline
\end{tabular}


Local number: 126 N51W30CCCC R

Other identifier: RB-81-24

Site identification number: 453940097060701

Date of construction: 6-30-81

Land surface altitude: 1,410 feet

Total depth: 735 feet

\begin{tabular}{|c|c|c|c|}
\hline Depth & $\begin{array}{l}\text { Thickness } \\
\text { (feet) }\end{array}$ & $\begin{array}{l}\text { Altitude } \\
\text { (feet) }\end{array}$ & Description \\
\hline $0-24$ & 24 & $1,410-1,386$ & Shale, medium-dark-gray; with orangish-iron stains, bentonitic (Pierre Shale) \\
\hline 24-115 & 91 & $1,386-1,295$ & Shale, black to very dark-gray, bentonite at 85 feet (Pierre Shale) \\
\hline $115-145$ & 30 & $1,295-1,265$ & Shale, medium-dark-gray; bentonitic (Pierre Shale) \\
\hline $145-160$ & 15 & $1,265-1,250$ & Shale, medium to light-gray (Pierre Shale) \\
\hline $160-185$ & 25 & $1,250-1,225$ & Shale, medium-dark-gray (Pierre Shale) \\
\hline $185-245$ & 60 & $1,225-1,165$ & Shale, light-gray; with bentonite (Pierre Shale) \\
\hline $245-280$ & 35 & $1,165-1,130$ & $\begin{array}{l}\text { Shale, medium-gray; easier drilling than interval from } 185 \text { to } 245 \text { feet, noncalcareous } \\
\text { (Pierre Shale) }\end{array}$ \\
\hline $280-300$ & 20 & $1,130-1,110$ & Shale, medium-gray; soft, easy drilling (Pierre Shale) \\
\hline $300-350$ & 50 & $1,110-1,060$ & $\begin{array}{l}\text { Shale, medium-dark-gray; hard, cuttings almost "snap" into pieces, noncalcareous } \\
\text { (Pierre Shale) }\end{array}$ \\
\hline $350-377$ & 27 & $1,060-1,033$ & $\begin{array}{l}\text { Shale, very dark-gray; hard cuttings almost "snap" into pieces (Sharon Springs } \\
\text { Member - Pierre Shale) }\end{array}$ \\
\hline $377-397$ & 20 & $1,033-1,013$ & $\begin{array}{l}\text { Clay, medium-olive-gray; very calcareous, cuttings turn olive brown color upon } \\
\text { acidization, trace of cream colored rock chips (Niobrara Formation) }\end{array}$ \\
\hline $397-428$ & 31 & $1,013-982$ & $\begin{array}{l}\text { Shale, medium-dark-gray; with faint blue sheen; concretions, slightly calcareous } \\
\text { (Carlile Shale) }\end{array}$ \\
\hline $428-505$ & 77 & $982-905$ & Shale, medium-dark-gray; with faint blue sheen, quiet and uniform drilling \\
\hline $505-550$ & 45 & $905-860$ & Shale, dark-gray; waxy, noncalcareous (Carlile Shale) \\
\hline $550-560$ & 10 & $860-850$ & Shale, medium-dark-gray; with medium-gray calcareous fragments \\
\hline $560-580$ & 20 & $850-830$ & $\begin{array}{l}\text { Shale, medium-gray; calcareous, soft, uphole abrasion of cuttings seems to yield } \\
\text { rounded or oblate shapes (Carlile Shale) }\end{array}$ \\
\hline $580-600$ & 20 & $830-810$ & Shale, medium-dark-gray; noncalcareous (Carlile Shale) \\
\hline $600-653$ & 53 & $810-757$ & Shale, dark-gray to very dark-gray; noncalcareous (Carlile Shale) \\
\hline $653-699$ & 46 & $757-711$ & $\begin{array}{l}\text { Clay, gray; interbedded shale and limestone, rig alternately drills very hard layers and } \\
\text { then soft layers, trace of light-gray bentonite (Greenhorn Limestone) }\end{array}$ \\
\hline $699-735$ & 36 & $711-675$ & Shale, gray (Graneros Shale) \\
\hline
\end{tabular}

Electric log available to depth of 550 feet. Below 550 feet, lithology is based on cuttings sampled at 10 -foot increments and driller's log 
Local number: 126N52W16DDC R

Other identifier: LONG HOLLOW

Site identification number: 454125097102601

Date of construction: $7-2-85$

Land surface altitude: 1,730 feet

Total depth: 1,265 feet

\begin{tabular}{|c|c|c|c|}
\hline Depth & $\begin{array}{l}\text { Thickness } \\
\text { (feet) }\end{array}$ & $\begin{array}{c}\text { Altitude } \\
\text { (feet) }\end{array}$ & Description \\
\hline $0-293$ & 293 & $1,730-1,437$ & Glacial drift \\
\hline $293-635$ & 342 & $1,437-1,095$ & Pierre Shale \\
\hline $635-711$ & 76 & $1,095-1,019$ & Niobrara Formation \\
\hline $711-930$ & 219 & $1,019-800$ & Carlile Shale \\
\hline $930-972$ & 42 & $800-758$ & Greenhorn Limestone \\
\hline $972-1,176$ & 204 & $758-554$ & Graneros Shale \\
\hline $1,176-1,265$ & 89 & $554-465 ?$ & Dakota Formation \\
\hline 1,265 & & $465 ?$ & Precambrian basement (granite)? \\
\hline Electric log a & able & & \\
\hline
\end{tabular}


Local number: 126N53W10DADA R

Other identifier: R-59

Site identification number: 454228097161501

Date of construction: $8-24-71$

Land surface altitude: 1,950 feet

Total depth: 523 feet

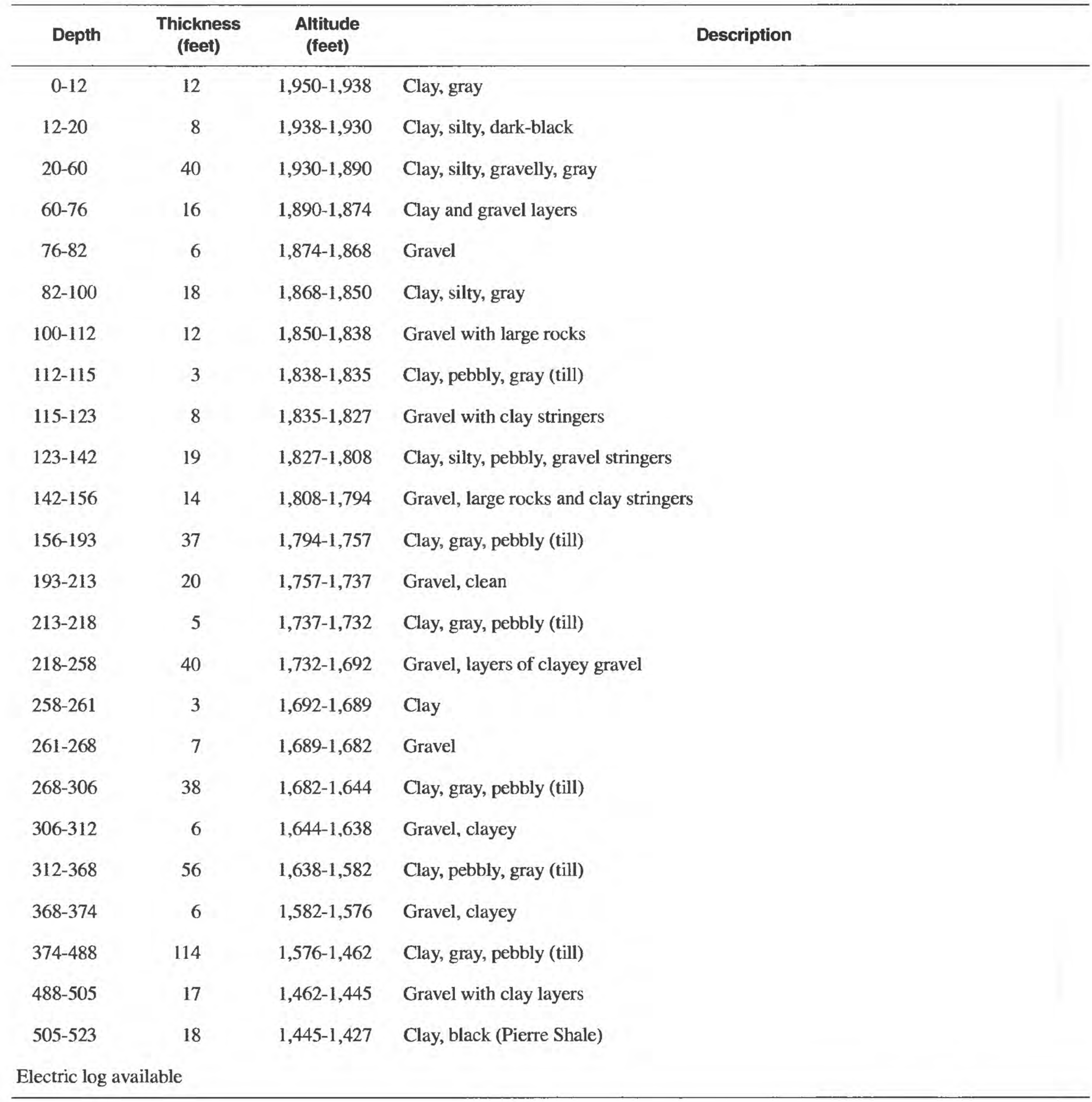


Local number: I26N54W3CBDB R

Other identifier: R-58

Site identification number: 454327097243501

Date of construction: 8-19-71

Land surface altitude: 1,862 feet

Total depth: 425 feet

\begin{tabular}{cccl}
\hline Depth & $\begin{array}{c}\text { Thickness } \\
\text { (feet) }\end{array}$ & $\begin{array}{c}\text { Altitude } \\
\text { (feet) }\end{array}$ & Description \\
\hline $0-1$ & 1 & $1,862-1,861$ & Topsoil, black \\
$1-31$ & 30 & $1,861-1,831$ & Clay, yellow brown, pebbly (till) \\
$31-80$ & 49 & $1,831-1,782$ & Clay, silty, pebbly, gray (till) \\
$80-92$ & 12 & $1,782-1,770$ & Gravel \\
$92-124$ & 32 & $1,770-1,738$ & Clay, gray \\
$124-280$ & 156 & $1,738-1,582$ & Clay, gray, pebbly (till) \\
$280-287$ & 7 & $1,582-1,575$ & Gravel \\
$287-336$ & 49 & $1,575-1,526$ & Clay, silty, gray, pebbly (till) \\
$336-342$ & 6 & $1,526-1,520$ & Gravel \\
$342-346$ & 4 & $1,520-1,516$ & Clay, gray \\
$346-376$ & 30 & $1,516-1,486$ & Sand and gravel \\
$376-393$ & 17 & $1,486-1,469$ & Clay, some sand layers \\
$393-397$ & 4 & $1,469-1,465$ & Sand, coarse \\
$397-425$ & 28 & $1,465-1,437$ & Shale. dark-gray (Pierre Shale) \\
\hline Electric log available & & \\
\hline
\end{tabular}


Local number: 126N54W25AAAA2 R

Other identifier: R-61

Site identification number: 454025097211502

Date of construction: 8-26-71

Land surface altitude: 1,845 feet

Total depth: 485 feet

\begin{tabular}{|c|c|c|c|}
\hline Depth & $\begin{array}{l}\text { Thickness } \\
\text { (feet) }\end{array}$ & $\begin{array}{c}\text { Altitude } \\
\text { (feet) }\end{array}$ & Description \\
\hline $0-8$ & 8 & $1,845-1,837$ & Clay, silty, yellowish-brown, pebbly (till) \\
\hline $8-19$ & 11 & $1,837-1,826$ & Clay, brownish-gray, pebbly (till) \\
\hline $19-23$ & 4 & $1,826-1,822$ & Gravel \\
\hline $23-95$ & 72 & $1,822-1,750$ & Clay, silty, gray, pebbly (till) \\
\hline $95-155$ & 60 & $1,750-1,690$ & Clay, gray, gravelly in spots (till), some 3 -inch to 1 -foot gravel stringers \\
\hline $155-172$ & 17 & $1,690-1,673$ & Gravel, some clay layers \\
\hline $172-215$ & 43 & $1,673-1,630$ & Clay, yellow, some gravel stringers (till) \\
\hline $215-275$ & 60 & $1,630-1,570$ & Clay, gray, pebbly, gravel stringers \\
\hline $275-292$ & 17 & $1,570-1,553$ & Gravel \\
\hline $292-338$ & 46 & $1,553-1,507$ & Clay, gray, gravelly, some gravel stringers \\
\hline $338-418$ & 80 & $1,507-1,427$ & Clay, light-gray mixed with brown, pebbly (till) \\
\hline $418-422$ & 4 & $1,427-1,423$ & Gravel \\
\hline $422-456$ & 34 & $1,423-1,389$ & Clay, light-gray an d brown, gravelly (till) \\
\hline $456-465$ & 9 & $1,389-1,380$ & Gravel \\
\hline $465-485$ & 20 & $1,380-1,360$ & Clay, dark-bluish-gray (Pierre Shale) \\
\hline Electric log & & & \\
\hline
\end{tabular}


Local number: 126N55W31CCCD

Other identifier: R-52

Site identification number: 454030097285401

Date of construction: $8-6-71$

Land surface altitude: 1,800 feet

Total depth: 785 feet

\begin{tabular}{|c|c|c|c|}
\hline Depth & $\begin{array}{c}\text { Thickness } \\
\text { (feet) }\end{array}$ & $\begin{array}{l}\text { Altitude } \\
\text { (feet) }\end{array}$ & Description \\
\hline $0-3$ & 3 & $1,800-1,797$ & Topsoil \\
\hline 3-15 & 12 & $1,797-1,785$ & Clay, yellow, pebbly (till) \\
\hline $15-25$ & 10 & $1,785-1,775$ & Clay, yellow turning gray at 25 feet (lake deposits) \\
\hline $25-28$ & 3 & $1,775-1,772$ & Clay, gray, pebbly (till) \\
\hline $28-42$ & 14 & $1,772-1,758$ & Sand, gray \\
\hline $42-63$ & 21 & $1,758-1,737$ & Clay, gray, silty, pebbly (till) \\
\hline $63-65$ & 2 & $1,737-1,735$ & Gravel \\
\hline $65-192$ & 127 & $1,735-1,608$ & Clay, gray, silty, gravelly in spots (till) \\
\hline $192-212$ & 20 & $1,608-1,588$ & Gravel, clay layer at 204 to 210 feet \\
\hline $212-239$ & 27 & $1,588-1,561$ & Clay, gray, gravelly (till) \\
\hline $239-248$ & 9 & $1,561-1,552$ & Gravel \\
\hline $248-290$ & 42 & $1,552-1,510$ & Clay, gray, gravelly (till) \\
\hline 290-308 & 18 & $1,510-1,492$ & Gravel \\
\hline 308-352 & 44 & $1,492-1,448$ & Clay, dark-gray, pebbly (till) \\
\hline $352-380$ & 28 & $1,448-1,420$ & Sand, fine to very coarse, clayey, gray \\
\hline 380-393 & 13 & $1,420-1,407$ & Clay, light-green to light-gray, light-green is clean clay, gray is pebbly \\
\hline 393-487 & 94 & $1,407-1,313$ & Clay, gray, pebbly (till) \\
\hline 487-505 & 18 & $1,313-1,295$ & Clay, gray, some very fine sand (lake deposits) \\
\hline $505-554$ & 49 & $1,295-1,246$ & Clay, dark-gray, pebbly (till) \\
\hline $554-601$ & 47 & $1,246-1,199$ & Sand, fine to very coarse; some pea size gravel, some clay \\
\hline $601-607$ & 6 & $1,199-1,193$ & Clay, dark-gray, compact, pebbly \\
\hline $607-614$ & 7 & $1,193-1,186$ & Sand \\
\hline 614-618 & 4 & $1,186-1,182$ & Clay, gray, pebbly \\
\hline $618-621$ & 3 & $1,182-1,179$ & Sand \\
\hline $621-668$ & 47 & $1,179-1,132$ & Clay, dark-gray, pebbly (till) \\
\hline $668-740$ & 72 & $1,132-1,060$ & Sand and gravel, lots of coal from 692 to 725 feet \\
\hline $740-747$ & 7 & $1,060-1,053$ & Clay, whitish-gray to light-gray, silty \\
\hline $747-785$ & 38 & $1,053-1,015$ & Clay, bluish-black, compact (Pierre Shale) \\
\hline Electric log & lable & & \\
\hline
\end{tabular}


Local number: 127N50W18AAAD R

Other identifier: SATHER

Site identification number: 454725096573301

Date of construction: 1965 ?

Land surface altitude: 1,150 feet

Total depth: 753 feet

\begin{tabular}{cccll}
\hline Depth & $\begin{array}{c}\text { Thickness } \\
\text { (feet) }\end{array}$ & $\begin{array}{c}\text { Altitude } \\
\text { (feet) }\end{array}$ & Description \\
\hline $0-85$ & 85 & $1,150-1,065$ & Clay \\
$85-99$ & 14 & $1,065-1,051$ & Poor sand \\
$99-132$ & 33 & $1,051-1,018$ & Good sand \\
$132-155$ & 23 & $1,018-995$ & Clay \\
$155-165$ & 10 & $995-985$ & Good sand \\
$165-358$ & 193 & $985-792$ & Carlile Shale \\
$358-390$ & 32 & $792-760$ & Greenhorn Limestone \\
$390-585$ & 195 & $760-565$ & Graneros Shale \\
$585-729$ & 144 & $565-421$ & Dakota Formation \\
$729-753$ & 24 & $421-397$ & Granite Wash \\
Electric log available & & \\
\hline
\end{tabular}

Local number: 127N53W1AAAA R

Other identifier: R-4

Site identification number: 454907097134401

Date of construction: 8-26-69

Land surface altitude: 1,252 feet

Total depth: 65 feet

\begin{tabular}{cccl}
\hline Depth & $\begin{array}{c}\text { Thickness } \\
\text { (feet) }\end{array}$ & $\begin{array}{c}\text { Altitude } \\
\text { (feet) }\end{array}$ & Description \\
\hline $0-3$ & 3 & $1,252-1,249$ & Topsoil, black \\
$3-12$ & 9 & $1,249-1,240$ & Clay, yellowish, some pebbles \\
$12-18$ & 6 & $1,240-1,234$ & Very large rocks (limestone?), gravel, some sand \\
$18-40$ & 22 & $1,234-1,212$ & Clay, gray, gravelly, pebbly \\
$40-65$ & 25 & $1,212-1,187$ & Shale \\
\hline
\end{tabular}


Local number: 127N54W3AAAA R

Other identifier: R-60

Site identification number: 454400097233301

Date of construction: 8-24-71

Land surface altitude: 1,847 feet

Total depth: 380 feet

\begin{tabular}{cccl}
\hline Depth & $\begin{array}{c}\text { Thickness } \\
\text { (feet) }\end{array}$ & $\begin{array}{c}\text { Altitude } \\
\text { (feet) }\end{array}$ & \\
\hline $0-7$ & 7 & $1,847-1,840$ & Clay, yellowish-brown, pebbly (till) \\
$7-11$ & 4 & $1,840-1,836$ & Clay, gray, silty, pebbly (till) \\
$11-13$ & 2 & $1,836-1,834$ & Clay, brownish-gray (lake clay?) \\
$13-18$ & 5 & $1,834-1,829$ & Clay, gray, silty, clayey (lake clay?) \\
$18-160$ & 142 & $1,829-1,687$ & Clay, gray, silty, clayey, pebbly (till) some thin gravel stringers \\
$160-164$ & 4 & $1,687-1,683$ & Clay, gray, very tough, drilled like shale, had to use pulldown \\
$164-285$ & 121 & $1,683-1,562$ & Clay, gray, gravelly in spots (till) \\
$285-295$ & 10 & $1,562-1,552$ & Clay, same as above, some brown silty clay mixed in with it \\
$295-320$ & 25 & $1,552-1,527$ & Clay, gray, pebbly (till) \\
$320-340$ & 20 & $1,527-1,507$ & Clay, gray, pebbly, gravelly in spots (till) \\
$340-364$ & 24 & $1,507-1,483$ & Clay, gray, sandy and gravelly (till) \\
$364-380$ & 16 & $1,483-1,467$ & Clay, dark-bluish-gray (shale) \\
\hline Electric log available & & \\
\hline
\end{tabular}


Local number: 127N56W36AABB

Other identifier: R-51

Site identification number: 454632097291701

Date of construction: 8-4-71

Land surface altitude: 1,840 feet

Total depth: 545 feet

\begin{tabular}{|c|c|c|c|}
\hline Depth & $\begin{array}{l}\text { Thickness } \\
\text { (feet) }\end{array}$ & $\begin{array}{l}\text { Altitude } \\
\text { (feet) }\end{array}$ & Description \\
\hline $0-5$ & 5 & $1,840-1,835$ & Sand and gravel \\
\hline $5-12$ & 7 & $1.835-1.828$ & Clay, yellowish-brown, pebbly (till) \\
\hline $12-20$ & 8 & $1,828-1.820$ & Clay, gray, pebbly (till) \\
\hline $20-32$ & 12 & $1.820-1.808$ & Clay, gray, sandy, gravelly (till) \\
\hline $32-76$ & 44 & $1,808-1,764$ & Clay, gray, pebbly (till) \\
\hline $76-81$ & 5 & $1,764-1,759$ & Gravel, some clay \\
\hline $81-140$ & 59 & $1,759-1,700$ & Clay, gray, pebbly, some thin gravel stringers (till) \\
\hline $140-148$ & 8 & $1,700-1,692$ & Sand and gravel \\
\hline $148-165$ & 17 & $1,692-1,675$ & Clay, silty, gray, pebbly (till) \\
\hline $165-172$ & 7 & $1,675-1,668$ & Sand and clay layers \\
\hline $172-185$ & 13 & $1,668-1,655$ & Clay, gray (lake deposits) \\
\hline $185-235$ & 50 & $1,655-1,605$ & Clay, silty, gray, pebbly (till) \\
\hline 235-336 & 101 & $1,605-1,504$ & Clay, gray, pebbly, some gravel layers \\
\hline $336-345$ & 9 & $1,504-1,495$ & Gravel \\
\hline $345-355$ & 10 & $1,495-1,485$ & Clay, gray, pebbly (till) \\
\hline $355-376$ & 21 & $1,485-1,464$ & Clay, greenish-brown, pebbly, hard drilling \\
\hline $376-391$ & 15 & $1,464-1,449$ & Clay, dark-gray to blackish-gray, pebbly (till) \\
\hline 391-394 & 3 & $1,449-1,446$ & Sand, coarse, some fine sand, marly \\
\hline $394-430$ & 36 & $1,446-1,410$ & Sand, fine to coarse, clay layers \\
\hline $430-475$ & 45 & $1,410-1,365$ & Clay, dark-gray, pebbly (till) \\
\hline $475-510$ & 35 & $1,365-1,330$ & Clay, gray to brown, pebbly \\
\hline $510-545$ & 35 & $1,330-1,295$ & Clay, bluish-black, compact (Pierre Shale) \\
\hline Electric log & ble & & \\
\hline
\end{tabular}


Local number: 128N53W10BBBB R

Other identifier: R-1

Site identification number: 455329097172501

Date of construction: 8-25-69

Land surface altitude: 1,250 feet

Total depth: 215 feet

\begin{tabular}{cccl}
\hline Depth & $\begin{array}{c}\text { Thickness } \\
\text { (feet) }\end{array}$ & $\begin{array}{c}\text { Altitude } \\
\text { (feet) }\end{array}$ & Description \\
\hline $0-21$ & 21 & $1,250-1,229$ & Clay, yellowish-brown \\
$21-27$ & 6 & $1,229-1,223$ & Clay, yellowish-brown, gravelly \\
$27-110$ & 83 & $1,223-1.140$ & Clay, gray, gravelly \\
$110-120$ & 10 & $1,140-1,130$ & Sand, coarse, gray \\
$120-130$ & 10 & $1,130-1,120$ & Gravel \\
$130-140$ & 10 & $1,120-1,110$ & Gravel, coarse \\
$140-160$ & 20 & $1,110-1,090$ & Gravel, fine and very coarse sand \\
$160-170$ & 10 & $1,090-1,080$ & Sand, very coarse, some gravel \\
$170-178$ & 8 & $1,080-1,072$ & Gravel \\
$178-215$ & 37 & $1,072-1,035$ & Shale \\
\hline
\end{tabular}


Local number: 128N53W12AAAA R

Other identifier: $\mathrm{R}-2$

Site identification number: 455329097134401

Date of construction: 8-26-69

Land surface altitude: 1,210 feet

Total depth: 200 feet

\begin{tabular}{cccl}
\hline Depth & $\begin{array}{c}\text { Thickness } \\
\text { (feet) }\end{array}$ & $\begin{array}{c}\text { Altitude } \\
\text { (feet) }\end{array}$ & \multicolumn{1}{c}{ Description } \\
\hline $0-28$ & 28 & $1,210-1,182$ & Clay, yellowish-brown, silty, sandy \\
$28-45$ & 17 & $1,182-1,165$ & Clay, yellowish-brown, silty, pebbly (till) \\
$45-60$ & 15 & $1,165-1,150$ & Clay, gray, very silty, slightly pebbly (till) \\
$60-75$ & 15 & $1,150-1,135$ & Clay, gray, silty, pebbly (till) \\
$75-78$ & 3 & $1,135-1,132$ & Clay, gray, shaley pebbly, gravelly \\
$78-110$ & 32 & $1,132-1,100$ & Clay, gray, silty, pebbly (till) \\
$110-120$ & 10 & $1,100-1,090$ & Sand, medium, some coarse \\
$120-130$ & 10 & $1,090-1,080$ & Sand, clayey \\
$130-140$ & 10 & $1,080-1,070$ & Sand, coarse, some very coarse \\
$140-150$ & 10 & $1,070-1,060$ & Sand, very coarse, clay layers \\
$150-168$ & 18 & $1,060-1,042$ & Sand, medium \\
$168-200$ & 32 & $1,042-1,010$ & Shale \\
Electric log available & & \\
\hline
\end{tabular}

Local number: $128 \mathrm{~N} 53 \mathrm{~W} 14 \mathrm{CCCC} \mathrm{R}$

Other identifier: R-7

Site identification number: 455148097161001

Date of construction: $9-12-70$

Land surface altitude: 1,250 feet

Total depth: 95 feet

\begin{tabular}{cccll}
\hline Depth & $\begin{array}{c}\text { Thickness } \\
\text { (feet) }\end{array}$ & $\begin{array}{c}\text { Altitude } \\
\text { (feet) }\end{array}$ & Description \\
\hline $0-1$ & 1 & $1,250-1,249$ & Topsoil \\
$1-18$ & 17 & $1,249-1,232$ & Clay, yellowish-brown, pebbly & \\
$18-78$ & 60 & $1,232-1,172$ & Clay, silty, gray, pebbly \\
$78-95$ & 17 & $1,172-1,155$ & Shale (Pierre Shale) \\
\hline
\end{tabular}


Local number: 128N53W15BAAA R

Other identifier: R-9

Site identification number: 455337097165201

Date of construction: $9-2-70$

Land surface altitude: 1,250 feet

Total depth: 200 feet

\begin{tabular}{cccl}
\hline Depth & $\begin{array}{c}\text { Thickness } \\
\text { (feet) }\end{array}$ & $\begin{array}{c}\text { Altitude } \\
\text { (feet) }\end{array}$ & \\
\hline $0-3$ & 3 & $1,250-1,247$ & Topsoil, black \\
$3-15$ & 12 & $1,247-1,235$ & Silt (lake deposits) \\
$15-120$ & 105 & $1,235-1,130$ & Clay, silty, yellowish-brown to gray \\
$120-170$ & 50 & $1,130-1,080$ & Gravel \\
$170-195$ & 25 & $1,080-1,055$ & Clay, gray, pebbly (gravel layer on top of shale) \\
$195-200$ & 5 & $1,055-1,050$ & Shale (Pierre Shale) \\
Electric log available & & \\
\hline
\end{tabular}

Local number: 128N53W15CAAA R

Other identifier: R-10

Site identification number: 455211097165201

Date of construction: $9-3-70$

Land surface altitude: 1,252 feet

Total depth: 110 feet

\begin{tabular}{cccl}
\hline Depth & $\begin{array}{c}\text { Thickness } \\
\text { (feet) }\end{array}$ & $\begin{array}{c}\text { Altitude } \\
\text { (feet) }\end{array}$ & \multicolumn{1}{c}{ Description } \\
\hline $0-26$ & 26 & $1,252-1,226$ & Clay, yellowish-brown, pebbly (till) \\
$26-58$ & 32 & $1,226-1,194$ & Clay, silty, some very fine sand, gray, some pebbles (till) \\
$58-60$ & 2 & $1,194-1,192$ & Sand and gravel \\
$60-89$ & 29 & $1,192-1,163$ & Clay, silty, some very fine sand, gray \\
$89-110$ & 21 & $1,163-1,142$ & Shale, bluish-black, compact (Pierre Shale) \\
\hline
\end{tabular}


Local number: $128 \mathrm{~N} 53 \mathrm{~W} 15 \mathrm{CBCB} \mathrm{R}$

Other identifier: R-6

Site identification number: 455204097172501

Date of construction: $9-2-70$

Land surface altitude: 1,268 feet

Total depth: 80 feet

\begin{tabular}{cccl}
\hline Depth & $\begin{array}{c}\text { Thickness } \\
\text { (feet) }\end{array}$ & $\begin{array}{c}\text { Altitude } \\
\text { (feet) }\end{array}$ & \multicolumn{1}{c}{ Description } \\
\hline $0-9$ & 9 & $1,268-1,259$ & Sand, rocks, gravel, and clay (till) \\
$9-16$ & 7 & $1,259-1,252$ & Clay, yellowish-brown, gravelly \\
$16-72$ & 56 & $1,252-1,196$ & Clay, gray, pebbly (6-inch gravel stringer at 50 feet, 2-foot gravel stringer at 60 feet) \\
$72-80$ & 8 & $1,196-1,188$ & Shale (Pierre Shale) \\
\hline
\end{tabular}

Local number: 128N53W20BBBB R

Other identifier: ATH-22

Site identification number: 455144097195501

Date of construction: 10-11-68

Land surface altitude: 1,350 feet

Total depth: 108 feet

\begin{tabular}{cccl}
\hline Depth & $\begin{array}{c}\text { Thickness } \\
\text { (feet) }\end{array}$ & $\begin{array}{c}\text { Altitude } \\
\text { (feet) }\end{array}$ & \multicolumn{1}{c}{ Description } \\
\hline $0-20$ & 20 & $1,350-1,330$ & Clay, light-tan, with light-gray streaks and some red spots (rust) \\
$20-40$ & 20 & $1,330-1,310$ & Clay, light-gray, streaked with light-tan \\
$40-95$ & 55 & $1,310-1,255$ & Clay, light-gray \\
$95-100$ & 5 & $1,255-1,250$ & Weathered shale (about 95 feet) \\
$100-108$ & 8 & $1,250-1,242$ & Shale \\
\hline
\end{tabular}


Local number: 128 N53W22CCCC R

Other identifier: ATH-20

Site identification number: 455055097172501

Date of construction: 6-25-68

Land surface altitude: 1,305 feet

Total depth: 77 feet

\begin{tabular}{cccl}
\hline Depth & $\begin{array}{c}\text { Thickness } \\
\text { (feet) }\end{array}$ & $\begin{array}{c}\text { Altitude } \\
\text { (feet) }\end{array}$ & \multicolumn{1}{c}{ Description } \\
\hline $0-2$ & 2 & $1,305-1,303$ & Topsoil \\
$2-10$ & 8 & $1,303-1,295$ & Clay, pebbly, light-brown \\
$10-12$ & 2 & $1,295-1,293$ & Clay, pebbly, yellow gray \\
$12-15$ & 3 & $1,293-1,290$ & Clay, pebbly, light-gray streaked with light-brown \\
$15-19$ & 4 & $1,290-1,286$ & Clay, pebbly, light-brown with some white streaks \\
$19-45$ & 26 & $1,286-1,260$ & Clay, light-tan with lighter and dark streaks \\
$45-55$ & 10 & $1,260-1,250$ & Clay, light-gray \\
$55-77$ & 22 & $1,250-1,228$ & Shale \\
\hline
\end{tabular}

Local number: 128N53W25AAAA R

Other identifier: R-3

Site identification number: 455052097134401

Date of construction: 8-26-69

Land surface altitude: 1,230 feet

Total depth: 110 feet

\begin{tabular}{cccl}
\hline Depth & $\begin{array}{c}\text { Thickness } \\
\text { (feet) }\end{array}$ & $\begin{array}{c}\text { Altitude } \\
\text { (feet) }\end{array}$ & \multicolumn{1}{c}{ Description } \\
\hline $0-17$ & 17 & $1,230-1,213$ & Clay, yellowish (till) \\
$17-24$ & 7 & $1,213-1,206$ & Clay, gray (till) \\
$24-72$ & 48 & $1,206-1,158$ & Clay, gray, pebbly (till) \\
$72-80$ & 8 & $1,158-1,150$ & Sand, coarse to very coarse, and gravel, gray \\
$80-92$ & 12 & $1,150-1,138$ & Gravel and very coarse sand, gray \\
$92-110$ & 18 & $1,138-1,120$ & Shale \\
Electric log available & & \\
\hline
\end{tabular}


Local number: 128N53W33DDDD R

Other identifier: ATH-21

Site identification number: 454911097173001

Date of construction: 6-25-68

Land surface altitude: 1,430 feet

Total depth: 48 feet

\begin{tabular}{rccl}
\hline Depth & $\begin{array}{c}\text { Thickness } \\
\text { (feet) }\end{array}$ & $\begin{array}{c}\text { Altitude } \\
\text { (feet) }\end{array}$ & \multicolumn{1}{c}{ Description } \\
\hline $0-10$ & 10 & $1,430-1,420$ & Sand, very coarse; gravel, very fine to medium \\
$10-15$ & 5 & $1,420-1,415$ & Sand, very coarse, silty, large cobbles; water level at 7.4 feet \\
$15-25$ & 10 & $1,415-1,405$ & Sand, coarse to very coarse, saturated, less cobbles and gravel \\
$25-30$ & 5 & $1,405-1,400$ & Sand, medium to very coarse \\
$30-43$ & 13 & $1,400-1,387$ & Clay, pebbles, gray; gravel \\
$43-48$ & 5 & $1,387-1,382$ & Shale? \\
\hline
\end{tabular}


Local number: 128N55W31CCBC R

Other identifier: R-50

Site identification number: 455102097284501

Date of construction: 8-3-71

Land surface altitude: 1,885 feet

Total depth: 470 feet

\begin{tabular}{|c|c|c|c|}
\hline Depth & $\begin{array}{c}\text { Thickness } \\
\text { (feet) }\end{array}$ & $\begin{array}{l}\text { Altitude } \\
\text { (feet) }\end{array}$ & Description \\
\hline $0-22$ & 22 & $1,885-1,863$ & Clay, yellowish-brown with some gray, pebbly (till) \\
\hline $22-67$ & 45 & $1,863-1,818$ & Clay, gray, pebbly (till) \\
\hline $67-185$ & 118 & $1,818-1,700$ & Clay, gray, sandy, gravelly in spots (till) \\
\hline $185-208$ & 23 & $1,700-1,677$ & Clay, gray, tough in spots, clayey (till) \\
\hline $208-235$ & 27 & $1,677-1,650$ & Clay, gray gravelly in spots (till) \\
\hline $235-314$ & 79 & $1,650-1,571$ & Clay, gray, pebbly (till) \\
\hline 314-386 & 72 & $1,571-1,499$ & $\begin{array}{l}\text { Clay, gray, gravelly in spots, very tough drilling (cut mud at } 327 \text { feet, drills easier now); } \\
\text { clay is very sticky, clayey, shaley at } 360 \text { feet }\end{array}$ \\
\hline $386-392$ & 6 & $1,499-1,493$ & Gravel \\
\hline $392-395$ & 3 & $1,493-1,490$ & Clay, shaley \\
\hline $395-405$ & 10 & $1,490-1,480$ & Gravel \\
\hline $405-415$ & 10 & $1,480-1,470$ & Clay, gray, sandy, pebbly, gravelly in spots \\
\hline $415-450$ & 35 & $1,470-1,435$ & $\begin{array}{l}\text { Clay, grayish-brown, tough, shaley, mud turned brown (another till?); some white marl } \\
\text { in cuttings (lake deposit?); brown clay between } 440 \text { to } 450 \text { feet }\end{array}$ \\
\hline $450-453$ & 3 & $1,435-1,432$ & Sand and gravel layer, not very good but took water \\
\hline $453-470$ & 17 & $1,432-1,415$ & Clay, bluish-black, compact (shale) \\
\hline
\end{tabular}


Local number: 128N56W1AAAA

Other identifier: R-48

Site identification number: 455606097284901

Date of construction: 8-2-71

Land surface altitude: 1,720 feet

Total depth: 260 feet

\begin{tabular}{cccl}
\hline Depth & $\begin{array}{c}\text { Thickness } \\
\text { (feet) }\end{array}$ & $\begin{array}{c}\text { Altitude } \\
\text { (feet) }\end{array}$ & \multicolumn{1}{c}{ Description } \\
\hline $0-31$ & 31 & $1,720-1,689$ & Clay, yellowish-brown, pebbly (till) \\
$31-46$ & 15 & $1,689-1,674$ & Clay, light-brownish-gray, pebbly (till) \\
$46-164$ & 118 & $1,674-1,556$ & Clay, dark-gray, pebbly (till), becomes gravelly at 98 feet \\
$164-167$ & 3 & $1,556-1,553$ & Gravel \\
$167-216$ & 49 & $1,553-1,504$ & Clay, dark-gray, very gravelly (till) \\
$216-230$ & 14 & $1,504-1,490$ & Clay, yellow brown \\
$230-260$ & 30 & $1,490-1,460$ & Clay, dark-blue gray (shale), bentonite mixed in with shale \\
\hline
\end{tabular}


Local number: 129N50W24CCD R

Other identifier: $12186 \mathrm{~A}$

Site identification number: 455755096520101

Date of construction: $09-08-88$

Land surface altitude: $1,089.52$ feet

Total depth: 215 feet

\begin{tabular}{|c|c|c|c|}
\hline Depth & $\begin{array}{l}\text { Thickness } \\
\text { (feet) }\end{array}$ & $\begin{array}{l}\text { Altitude } \\
\text { (feet) }\end{array}$ & Description \\
\hline $0-2$ & 2 & $1,089.52-1,087.52$ & Topsoil \\
\hline $2-17$ & 15 & $1,087.52-1,072.52$ & Silt, clayey to pebbly, soft, slightly plastic, oxidized; gravel from 14 to 15 feet (till) \\
\hline $17-47$ & 30 & $1,072.52-1,042.52$ & Silt, clayey to pebbly, olive-gray, soft, slightly plastic (till) \\
\hline $47-92$ & 45 & $1,042.52-997.52$ & $\begin{array}{l}\text { Sand, fine, well sorted, subrounded and rounded quartz; contains detrital lignite } \\
\text { below } 60 \text { feet }\end{array}$ \\
\hline $92-110$ & 18 & $997.52-979.52$ & Silt, clayey, soft, nonplastic \\
\hline $110-117$ & 7 & $979.52-972.52$ & Silt, clayey, soft, nonplastic; interbedded sand and gravel \\
\hline $117-123$ & 6 & $972.52-966.52$ & Gravel, fine, granular, subangular to rounded; limestone and igneous \\
\hline $123-138$ & 15 & $966.52-951.52$ & $\begin{array}{l}\text { Sand, coarse and very coarse, pebbly, subangular to rounded; interbedded clay and } \\
\text { gravel }\end{array}$ \\
\hline $138-145$ & 7 & $951.52-944.52$ & Gravel, coarse, subrounded and rounded, igneous and limestone \\
\hline $145-157$ & 12 & $944.52-932.52$ & Clay, silty to pebbly, olive-gray, firm, nonplastic (till) \\
\hline $157-177$ & 20 & $932.52-912.52$ & Clay, olive-black. firm, slightly plastic; pebbly with depth; sand from 174 to 175 feet \\
\hline $177-180$ & 3 & $912.52-909.52$ & Gravel, fine to coarse, granular \\
\hline $180-204$ & 24 & $909.52-885.52$ & Sand, fine to very coarse. granular, subrounded and rounded \\
\hline $204-215$ & 11 & $885.52-874.52$ & Shale, brownish-black, firm \\
\hline
\end{tabular}


Local number: 129N51W8CCC R

Other identifier: 13037

Site identification number: 455755097045501

Date of construction: 8-18-92

Land surface altitude: $1,196.2$ feet

Total depth: 240 feet

\begin{tabular}{|c|c|c|c|}
\hline Depth & $\begin{array}{c}\text { Thickness } \\
\text { (feet) }\end{array}$ & $\begin{array}{l}\text { Altitude } \\
\text { (feet) }\end{array}$ & Description \\
\hline $0-2$ & 2 & $1,196.2-1,194.2$ & Topsoil, black \\
\hline $2-4$ & 2 & 1,194.2-1,192.2 & Clay, light-olive gray, soft, plastic, oxidized; mottled to 10 feet (till) \\
\hline $4-23$ & 19 & $1,192.2-1,173.2$ & $\begin{array}{l}\text { Clay, silty, sandy, pebbly, light-olive-gray, slightly firm, oxidized; mottled to } 10 \text { feet } \\
\text { (till) }\end{array}$ \\
\hline $23-25$ & 2 & $1,173.2-1,171.2$ & Sand, fine and medium, oxidized \\
\hline $25-33$ & 8 & $1,171.2-1,166.2$ & Clay, silty, sandy, pebbly, light-olive-gray, slightly firm, unoxidized (till) \\
\hline $33-36$ & 3 & $1,166.2-1,160.2$ & Clay, silty, sandy, pebbly, olive-gray, oxidized \\
\hline $36-40$ & 4 & $1,160.2-1,156.2$ & Silt, clayey, light-olive-gray, oxidized \\
\hline $40-45$ & 5 & $1,156.2-1,151.2$ & Silt, clayey, olive-gray, unoxidized \\
\hline $45-77$ & 32 & 1,151.2-1,119.2 & Sand, fine, slightly pebbly, fine, well sorted, quartz, igneous, shale \\
\hline $77-81$ & 4 & $1,119.2-1,115.2$ & Silt, clayey, medium-dark-gray, soft, slightly stick, plastic \\
\hline $81-127$ & 46 & $1,115.2-1,069.2$ & Sand, fine, slightly pebbly, fine, well sorted, quartz, igneous, shale; some lignite return \\
\hline $127-136$ & 9 & $1,069.2-1,060.2$ & Silt. clayey, medium-dark-gray, soft. slightly sticky, plastic \\
\hline $136-155$ & 19 & $1,060.2-1,041.2$ & $\begin{array}{l}\text { Sand, fine and medium, pebbly, fine, medium sorted, quartz, igneous, shale; coarser } \\
\text { with depth }\end{array}$ \\
\hline $155-163$ & 8 & 1,041.2-1,033.2 & $\begin{array}{l}\text { Sand, medium and coarse, pebbly, fine to coarse, medium sorted, angular to sub- } \\
\text { rounded, igneous, carbonate, shale }\end{array}$ \\
\hline $163-189$ & 26 & $1,033.2-1,007.2$ & Silt, clayey, medium-dark-gray, soft, sticky, plastic \\
\hline $189-231$ & 42 & $1,007.2-965.2$ & $\begin{array}{l}\text { Clay, silty, sandy, pebbly, olive-gray, slightly firm: cobbles at } 189 \text { feet; firm from } 209 \text { to } \\
231 \text { feet (till) }\end{array}$ \\
\hline $231-240$ & 9 & $965.2-956.2$ & Shale, grayish-black, hard; waxy appearance \\
\hline
\end{tabular}


Local number: 129N51W19ABA R

Other identifier: 13422

Site identification number: 455701097051501

Date of construction: 9-21-94

Land surface altitude: $1,191.23$ feet

Total depth: 240 feet

\begin{tabular}{|c|c|c|c|}
\hline Depth & $\begin{array}{l}\text { Thickness } \\
\text { (feet) }\end{array}$ & $\begin{array}{l}\text { Altitude } \\
\text { (feet) }\end{array}$ & Description \\
\hline $0-2$ & 2 & $1,191.23-1,189.23$ & Topsoil. clay loam black \\
\hline $2-7$ & 5 & $1,189.23-1,184.23$ & Clay, light-olive-brown, slightly firm, slightly sticky, slightly plastic, oxidized \\
\hline $7-28$ & 21 & $1,184.23-1,163.23$ & $\begin{array}{l}\text { Clay, silty, sandy, pebbly, light-olive-brown, slightly firm, slightly sticky, slightly } \\
\text { plastic, oxidized }\end{array}$ \\
\hline $28-34$ & 6 & $1,163.23-1,157.23$ & Clay, slightly silty, light-olive-brown, soft, sticky, plastic, oxidized \\
\hline $34-53$ & 19 & $1,157.23-1,138.23$ & Clay, slightly silty, olive gray \\
\hline $53-55$ & 2 & $1,138.23-1,136.23$ & Interbedded clay, silt, and sand \\
\hline $55-65$ & 10 & $1,136.23-1,126.23$ & Sand, fine to coarse, pebbly, fine, predominantly shale, subangular to rounded \\
\hline $65-87$ & 22 & $1,126.23-1,104.23$ & Silt, clayey, sandy, olive gray; interbedded sand, silty from 74 to 75 feet \\
\hline $87-142$ & 55 & $1,104.23-1,049.23$ & $\begin{array}{l}\text { Sand, fine and medium, pebbly, fine, mixed mineralogy, subangular to rounded; } \\
\text { detrital lignite; silt, sandy, olive gray from } 103 \text { to } 108 \text { feet, sand, fine to coarse, } \\
\text { granular, pebbly, fine from } 120 \text { to } 140 \text { feet }\end{array}$ \\
\hline $142-203$ & 61 & $1,049.23-988.23$ & $\begin{array}{l}\text { Clay, silty, sandy, olive gray, soft, sticky, nonplastic; clay, slightly silty, plastic below } \\
167 \text { feet; clay, silty below } 200 \text { feet (lacustrine) }\end{array}$ \\
\hline $203-227$ & 24 & $988.23-964.23$ & $\begin{array}{l}\text { Clay, silty, sandy, pebbly, olive gray, slightly firm, slightly sticky, slightly plastic, } \\
\text { occasional cobble (till) }\end{array}$ \\
\hline $227-240$ & 13 & $964.23-951.23$ & $\begin{array}{l}\text { Clay, olive black, slightly firm, sticky, plastic, noncalcareous; waxy appearance } \\
\text { (shale) }\end{array}$ \\
\hline
\end{tabular}


Local number: 129N52W14AAA R

Other identifier: 13494

Site identification number: 455932097092401

Date of construction: 10-11-95

Land surface altitude: 1,220 feet

Total depth: 270 feet

\begin{tabular}{|c|c|c|c|}
\hline Depth & $\begin{array}{l}\text { Thickness } \\
\text { (feet) }\end{array}$ & $\begin{array}{l}\text { Altitude } \\
\text { (feet) }\end{array}$ & Description \\
\hline $0-2$ & 2 & $1,220-1,218$ & Loam, black \\
\hline $2-32$ & 30 & $1,218-1,188$ & $\begin{array}{l}\text { Clay, silty, sandy, pebbly, dusky yellow, firm slightly sticky, slightly plastic, oxidized; } \\
\text { slightly reduced, light-olive-gray with depth }\end{array}$ \\
\hline $32-52$ & 20 & $1,188-1,168$ & Clay, silty, sandy, pebbly, olive gray, firm, slightly sticky, slightly plastic, reduced \\
\hline $52-60$ & 8 & $1,168-1,160$ & Clay, very silty, olive gray, soft, slightly sticky, nonplastic \\
\hline $60-124$ & 64 & $1,160-1,096$ & Sand, very fine and fine; lignite return \\
\hline $124-218$ & 94 & $1,096-1,002$ & Clay, silty, very sandy, olive gray; lignite return \\
\hline $218-264$ & 46 & $1,002-956$ & $\begin{array}{l}\text { Clay, silty, sandy, pebbly, olive gray, firm, slightly sticky, nonplastic, rock at } 223 \text { feet } \\
\text { (till) }\end{array}$ \\
\hline $264-270$ & 6 & $956-950$ & $\begin{array}{l}\text { Clay, slightly silty, olive black, firm, sticky, plastic, noncalcareous; waxy appearance } \\
\text { (shale) }\end{array}$ \\
\hline
\end{tabular}


Local number: 129N52W19ABB R

Other identifier: 13495

Site identification number: 455658097130201

Date of construction: 10-11-95

Land surface altitude: 1,170 feet

Total depth: 220 feet

\begin{tabular}{|c|c|c|c|}
\hline Depth & $\begin{array}{l}\text { Thickness } \\
\text { (feet) }\end{array}$ & $\begin{array}{l}\text { Altitude } \\
\text { (feet) }\end{array}$ & Description \\
\hline $0-2$ & 2 & $1,170-1,168$ & Clay loam, black \\
\hline $2-12$ & 10 & $1,168-1,158$ & $\begin{array}{l}\text { Clay, slightly silty, slightly sandy, slightly pebbly, dusky yellow, firm, slightly sticky, } \\
\text { plastic, oxidized }\end{array}$ \\
\hline $12-15$ & 3 & $1,158-1,155$ & $\begin{array}{l}\text { Clay, slightly silty, slightly sandy, slightly pebbly, light-olive-gray, firm, slightly sticky, } \\
\text { plastic, slightly reduced }\end{array}$ \\
\hline $15-35$ & 20 & $1,155-1,135$ & $\begin{array}{l}\text { Clay, slightly silty, slightly sandy, slightly pebbly, olive gray, firm, sticky, plastic, } \\
\text { reduced }\end{array}$ \\
\hline $35-89$ & 54 & $1,135-1,081$ & $\begin{array}{l}\text { Clay, silty, sandy, pebbly, olive gray, slightly firm, sticky, plastic; occasional cobble; } \\
\text { interbedded sand and gravel less than } 1 \text {-foot beds, from } 47 \text { to } 56 \text { feet (till) }\end{array}$ \\
\hline $89-108$ & 19 & $1,081-1,062$ & $\begin{array}{l}\text { Sand, fine to coarse, predominantly coarse, gravel fine and medium, mixed minerology, } \\
\text { subangular and subrounded }\end{array}$ \\
\hline $108-124$ & 16 & $1,062-1,046$ & Sand, fine to coarse, predominantly coarse \\
\hline $124-132$ & 8 & $1,046-1,038$ & Clay, olive gray \\
\hline $132-140$ & 8 & $1,038-1,030$ & Clay, very sandy, olive gray \\
\hline $140-142$ & 2 & $1,030-1,028$ & Sand and gravel \\
\hline $142-202$ & 60 & $1,028-968$ & $\begin{array}{l}\text { Clay, silty, sandy, pebbly, olive gray, slightly firm; occasional cobble; interbedded sand } \\
\text { and gravel, less than 1-foot beds, from } 142 \text { to } 166 \text { feet; very sandy by } 166 \text { feet; firm } \\
\text { by } 183 \text { feet (till) }\end{array}$ \\
\hline $202-220$ & 18 & $968-950$ & $\begin{array}{l}\text { Clay, slightly silty, olive black, firm, sticky, plastic, noncalcareous; waxy appearance } \\
\text { (shale) }\end{array}$ \\
\hline \multicolumn{4}{|c|}{$\begin{array}{l}\text { Well screened from } 114 \text { to } 119 \text { feet; } 2 \text {-inch PVC screen and casing. Well flowed. less than } 1 \text { gallon per minute or about } 3.3 \text { feet above } \\
\text { land surface. Well was plugged by NDSWC on } 11-02-95\end{array}$} \\
\hline
\end{tabular}


Local number: 129N52W21CCC R

Other identifier: 12277

Site identification number: 455610097110901

Date of construction: 11-29-88

Land surface altitude: $1,221.2$ feet

Total depth: 280 feet

\begin{tabular}{|c|c|c|c|}
\hline Depth & $\begin{array}{c}\text { Thickness } \\
\text { (feet) }\end{array}$ & $\begin{array}{c}\text { Altltude } \\
\text { (feet) }\end{array}$ & Description \\
\hline $0-3$ & 3 & $1,221.2-1,218.2$ & Topsoil \\
\hline $3-16$ & 13 & $1,218.2-1,205.2$ & Clay, silty to pebbly, dark-yellowish-orange, soft, plastic, oxidized (till) \\
\hline $16-21$ & 5 & $1,205.2-1,200.2$ & Clay, slightly silty, dark-yellowish-orange, soft, plastic, oxidized (lacustrine) \\
\hline $21-41$ & 20 & $1,200.2-1,180.2$ & $\begin{array}{l}\text { Sand, fine and medium, granular, subrounded and rounded limestone, shale and } \\
\text { quartz; interbedded gravel }\end{array}$ \\
\hline $41-59$ & 18 & $1,180.2-1,162.2$ & Silt, slightly clayey, oxidized \\
\hline $59-62$ & 3 & $1,162.2-1,159.2$ & Silt, slightly clayey, olive gray \\
\hline $62-136$ & 74 & $1,159.2-1,085.2$ & $\begin{array}{l}\text { Sand, medium, subrounded and rounded, quartz and carbonate; interbedded sandy } \\
\text { clay and gravel; contains detrital lignite }\end{array}$ \\
\hline $136-186$ & 50 & $1,085.2-1,035.2$ & Clay, silty and very sandy, olive gray \\
\hline $186-189$ & 3 & $1,035.2-1,032.2$ & Gravel, rounded, shale and limestone \\
\hline $189-204$ & 15 & $1,032.2-1,017.2$ & Clay, very sandy, very pebbly, olive gray, nonplastic; interbedded gravel (till) \\
\hline $204-223$ & 19 & $1017.2-998.2$ & Clay, very silty, to pebbly, olive gray, soft, slightly plastic (till) \\
\hline $223-263$ & 40 & 998.2-958.2 & Clay, very silty, very sandy, pebbly, olive gray firm; sand from 252 to 253 feet (till) \\
\hline $263-280$ & 17 & $958.2-941.2$ & Shale, olive black, plastic \\
\hline
\end{tabular}


Local number: 129N53W4BAA

Other identifier: 13447

Site identification number: 460117097195801

Date of construction: 8-29-95

Land surface altitude: 1,163 feet

Total depth: 260 feet

\begin{tabular}{|c|c|c|c|}
\hline Depth & $\begin{array}{l}\text { Thickness } \\
\text { (feet) }\end{array}$ & $\begin{array}{l}\text { Altitude } \\
\text { (feet) }\end{array}$ & Description \\
\hline $0-29$ & 29 & $1,163-1,134$ & Clay, silty, sandy, pebbly pale yellow, gray, and brown, oxidized (till) \\
\hline $29-31$ & 2 & $1,134-1,132$ & $\begin{array}{l}\text { Sand and gravel, sand very fine to very coarse, gravel up to 1/2-inch diameter; yellow } \\
\text { stained, oxidized; angular to rounded, quartz, shield silica, carbonate, shale }\end{array}$ \\
\hline $31-36$ & 5 & $1,132-1,127$ & Sand and gravel, as above, interbedded with till as above \\
\hline $36-85$ & 49 & $1,127-1,078$ & Clay, silty, sandy, pebbly, soft, olive gray (till) \\
\hline $85-91$ & 6 & $1,078-1,072$ & Till interbedded with shale and carbonaceous sand and gravel \\
\hline $91-172$ & 81 & $1,072-991$ & $\begin{array}{l}\text { Sand }(90 \%) \text { very fine to very coarse, predominantly medium, and gravel }(10 \%) \text {, } \\
\text { predominantly very fine, up to } 1 / 4 \text {-inch diameter; occasional silty clay; clayey silt } \\
\text { layers where bit slowed; lots of quartz, shale and carbonates, shield silicates, minor } \\
\text { detrital shale, subangular to well rounded, light bit chatter; at } 142 \text { to } 144 \text { feet bit } \\
\text { slowed, silty clay/clayey silt layer }\end{array}$ \\
\hline $172-192$ & 20 & $991-971$ & $\begin{array}{l}\text { Silty, clay, slightly sandy (very fine) greenish-gray, bit slowed, soft, drilled smooth, no } \\
\text { chatter, good recovery }\end{array}$ \\
\hline $192-201$ & 9 & $971-962$ & Clay silt, as 172 - to 192 -foot interval, with sand and gravel layers \\
\hline $201-229$ & 28 & $962-934$ & $\begin{array}{l}\text { Gravel }(50-70 \%) \text { up to } 1 \text {-inch diameter, predominantly fine, and sand, very fine to very } \\
\text { coarse, predominantly coarse }(30-50 \%) \text {; lots of quartz, shield silica, carbonates, } \\
\text { shale, some occasional silty clay/clayey silty layers where bit slowed, otherwise } \\
\text { moderate bit chatter }\end{array}$ \\
\hline $229-235$ & 6 & 934-928 & $\begin{array}{l}\text { Clay, silty, brownish-black, very calcareous, does not appear to be bedrock shale, soft, } \\
\text { greasy }\end{array}$ \\
\hline $235-260$ & 25 & $928-903$ & $\begin{array}{l}\text { Clay, black, sticky, noncalcareous, does not effervesce in dilute } \mathrm{HCl} \text {; ribbon-like sample } \\
\text { returns (Carlile Shale) }\end{array}$ \\
\hline
\end{tabular}


Local number: 129N53W7BBA R

Other identifier: 9248

Site identification number: 455842097210301

Date of construction: $12-5-74$

Land surface altitude: 1,171 feet

Total depth: 220 feet

\begin{tabular}{|c|c|c|c|}
\hline Depth & $\begin{array}{c}\text { Thickness } \\
\text { (feet) }\end{array}$ & $\begin{array}{c}\text { Altitude } \\
\text { (feet) }\end{array}$ & Description \\
\hline $0-1$ & 1 & $1,171-1,170$ & Topsoil, silty loam, dusky brown \\
\hline $1-4$ & 3 & $1,170-1,167$ & Clay, silty, sandy, pebbly; grayish-orange; soft, sticky, oxidized (till) \\
\hline $4-12$ & 8 & $1,167-1,159$ & Clay, silty, sandy, pebbly; dark-yellowish-brown; firm plastic, oxidized (till) \\
\hline $12-66$ & 54 & $1,159-1,105$ & $\begin{array}{l}\text { Clay, silty, sandy, predominantly very coarse sand grains, pebbly; dark-gray; } \\
\text { moderately firm, plastic (till) }\end{array}$ \\
\hline $66-102$ & 36 & $1,105-1,069$ & Clay, silty, sandy, pebbly; olive gray; moderately firm, plastic (till) \\
\hline $102-200$ & 98 & $1,069-971$ & $\begin{array}{l}\text { Sand, medium-dark-gray, medium to very coarse, angular to round, about } 70 \% \text { quartz, } \\
10 \% \text { gneiss, } 10 \% \text { carbonates, } 8 \% \text { shale, } 2 \% \text { detrital lignite (some fragments); at } \\
\text { laminations taking some water }\end{array}$ \\
\hline $200-220$ & 20 & $971-951$ & Pierre Shale, grayish-black; silicaceous, plastic, slightly fissile, noncalcareous \\
\hline
\end{tabular}

Local number: 129N53W9AAA R

Other identifier: 9249

Site identification number: 455842097173701

Date of construction: $12-5-74$

Land surface altitude: 1,170 feet

Total depth: 240 feet

\begin{tabular}{cccc}
\hline Depth & $\begin{array}{c}\text { Thickness } \\
\text { (feet) }\end{array}$ & $\begin{array}{c}\text { Altitude } \\
\text { (feet) }\end{array}$ & Description \\
\hline $1-1$ & 1 & $1,170-1,169$ & Topsoil. silty loam, dusky brown \\
$1-6$ & 5 & $1,169-1.164$ & $\begin{array}{c}\text { Clay, silty, sandy, pebbly; grayish-orange, iron stained, moderately firm, plastic, } \\
\text { oxidized (till) }\end{array}$ \\
62 & 56 & $1,164-1,108$ & $\begin{array}{c}\text { Clay, silty, sandy, pebbly; dark-gray; moderately firm, plastic, tight; occasional gravel } \\
\text { lens, boulders (till) }\end{array}$ \\
$62-120$ & 58 & $1,108-1,050$ & $\begin{array}{c}\text { Clay, silty, sandy, pebbly; olive gray; moderately firm, plastic (till) } \\
120-180\end{array}$ \\
60 & $1,050-990$ & $\begin{array}{c}\text { Silt, slightly sandy, dark-gray; tight, moderate plasticity, thin sand lenses; occasional } \\
\text { light-gray laminae }\end{array}$ \\
$180-217$ & 37 & $990-953$ & $\begin{array}{c}\text { Sand, about 5\% gravel; fine to coarse; angular to subrounded; about 70\% quartz, } \\
10 \% \text { shale, 10\% carbonates, 10\% gneiss; silt lenses }\end{array}$ \\
$217-240$ & 23 & $953-930$ & $\begin{array}{c}\text { Pierre Shale, silicaceous, dark-gray to grayish-black; noncalcareous, plastic, tight } \\
\text { Well screened from } 198 \text { to 201 feet; 11/4-inch PVC screen and casing. Electric log available }\end{array}$ \\
\hline
\end{tabular}


Local number: 129N53W11AAA R

Other identifier: 9250

Site identification number: 455844097145701

Date of construction: 12-6-74

Land surface altitude: 1,171 feet

Total depth: 260 feet

\begin{tabular}{cccl}
\hline Depth & $\begin{array}{c}\text { Thickness } \\
\text { (feet) }\end{array}$ & $\begin{array}{c}\text { Altitude } \\
\text { (feet) }\end{array}$ & \multicolumn{1}{c}{ Description } \\
\hline $0-1$ & 1 & $1,171-1,170$ & Topsoil, silty loam, dusky yellow brown \\
$1-4$ & 3 & $1,170-1,167$ & Clay, silty, sandy, pebbly; medium-yellow-brown; soft, sticky, oxidized (till) \\
$4-26$ & 22 & $1,167-1,145$ & Clay, silty, sandy, pebbly; medium-yellow-brown; firm, plastic, oxidized (till) \\
$26-40$ & 14 & $1,145-1,131$ & Clay, silty, sandy, pebbly; dark-gray; moderately firm, plastic, gravelly (till) \\
$40-82$ & 42 & $1,131-1,089$ & Clay, silty, sandy, pebbly: olive gray, moderately firm, plastic (till) \\
$82-92$ & 10 & $1,089-1,079$ & $\begin{array}{l}\text { Sand, very fine to fine, medium-dark-gray; angular to subangular; about } 80 \% \text { quartz, } \\
10 \% \text { carbonates, 5\% gneiss, 5\% shale, trace detrital lignite; micaceous; till lenses }\end{array}$ \\
$92-174$ & 82 & $1,079-997$ & Sand, as above; clean, well sorted, little caving taking very little water (deltaic?) \\
$174-230$ & 56 & $997-941$ & Silt, olive gray, soft, poor recovery, washing out, sand-gravel lenses (deltaic?) \\
$230-260$ & 30 & $941-911$ & Pierre Shale; silicaceous; grayish-black; tight, plastic; noncalcareous \\
Well screened from 138 to 141 feet; 11/4-inch PVC screen and casing. Electric log available
\end{tabular}


Local number: 129N53W25AADA R

Other identifier: R-5

Site identification number: 455559097134401

Date of construction: $9-1-70$

Land surface altitude: 1,188 feet

Total depth: 245 feet

\begin{tabular}{cccl}
\hline Depth & $\begin{array}{c}\text { Thickness } \\
\text { (feet) }\end{array}$ & $\begin{array}{c}\text { Altitude } \\
\text { (feet) }\end{array}$ & \multicolumn{1}{c}{ Description } \\
\hline $0-11$ & 11 & $1,188-1,177$ & Clay, yellowish-brown, pebbly (till) \\
$11-40$ & 29 & $1,177-1,148$ & Sand, fine to coarse, brown \\
$40-47$ & 7 & $1,148-1,141$ & Sand, fine to coarse, light-gray \\
$47-55$ & 8 & $1,141-1,133$ & Clay, very silty, gray, with very fine sand \\
$55-75$ & 20 & $1,133-1,113$ & Sand, very fine to medium, gray \\
$75-142$ & 67 & $1,113-1,046$ & Clay, quite silty, gray, with very fine sand \\
$142-151$ & 9 & $1,046-1,037$ & Sand, medium to coarse, and gravel \\
$151-177$ & 26 & $1,037-1,011$ & Clay, very silty, gray, with very fine sand and gravel and sand stringers \\
$177-218$ & 41 & $1,011-970$ & Clay, calcareous, gray, pebbly, sandy (Niobrara Formation) \\
$218-245$ & 27 & $970-943$ & Shale, bluish-black (Carlile Shale) \\
\hline Electric log available & & \\
\hline
\end{tabular}


Local number: 129N53W27BBBB R

Other identifier: R-11

Site identification number: 455606097172501

Date of construction: $9-3-70$

Land surface altitude: 1,255 feet

Total depth: 260 feet

\begin{tabular}{|c|c|c|c|}
\hline Depth & $\begin{array}{l}\text { Thickness } \\
\text { (feet) }\end{array}$ & $\begin{array}{c}\text { Altitude } \\
\text { (feet) }\end{array}$ & Description \\
\hline $0-12$ & 12 & $1,255-1,243$ & Clay, yellowish-brown, pebbly (till) \\
\hline $12-20$ & 8 & $1,243-1,235$ & Sand, medium, some gravel, brown \\
\hline $20-30$ & 10 & $1,235-1,225$ & Sand, coarse some gravel, brown \\
\hline $30-50$ & 20 & $1,225-1,205$ & Sand, medium to coarse, brown \\
\hline $50-60$ & 10 & $1,205-1,195$ & Sand, coarse to very coarse, brown \\
\hline $60-80$ & 20 & $1,195-1.175$ & Sand, medium to coarse, brown, some very coarse from 70 to 80 feet \\
\hline $80-90$ & 10 & $1,175-1.165$ & Sand, coarse to very coarse, brown \\
\hline $90-110$ & 20 & $1,165-1,145$ & Sand. medium to coarse, brown \\
\hline $110-130$ & 20 & $1,145-1,125$ & Sand, coarse to very coarse, gray, some coal from 120 to 130 feet \\
\hline $130-140$ & 10 & $1,125-1,115$ & Sand, fine to medium, gray, some coal \\
\hline $140-150$ & 10 & $1,115-1,105$ & Sand, fine to medium, gray, some coal \\
\hline $150-160$ & 10 & $1,105-1,095$ & Sand, medium, gray, some coal \\
\hline $160-170$ & 10 & $1,095-1,085$ & Sand, medium to coarse, gray, some coal \\
\hline $170-180$ & 10 & $1,085-1,075$ & Sand, fine to medium, gray, much coal \\
\hline $180-190$ & 10 & $1,075-1,065$ & Sand, coarse, gray, some coal \\
\hline $190-203$ & 13 & $1,065-1,052$ & Sand, coarse t very coarse, gray, some coal \\
\hline $203-218$ & 15 & $1,052-1,037$ & Clay, sandy, gray, with pebbles \\
\hline $218-223$ & 5 & $1,037-1,032$ & Sand and gravel, gray \\
\hline $223-242$ & 19 & $1,032-1,013$ & Clay, gray, pebbly (till) \\
\hline $242-243$ & 1 & $1,013-1,012$ & Rocks \\
\hline $243-260$ & 17 & $1,012-995$ & Shale, bluish-black \\
\hline
\end{tabular}


Local number: 129N54W1AAA

Other identifier: 13452

Site identification number: 460118097230501

Date of construction: $9-5-95$

Land surface altitude: 1,162 feet

Total depth: 231 feet

\begin{tabular}{|c|c|c|c|}
\hline Depth & $\begin{array}{l}\text { Thickness } \\
\text { (feet) }\end{array}$ & $\begin{array}{c}\text { Altitude } \\
\text { (feet) }\end{array}$ & Description \\
\hline $0-28$ & 28 & $1,162-1,134$ & $\begin{array}{l}\text { Clay, silty, sandy, pebbly, some cobbles; pale yellow; gray brown with red yellow } \\
\text { stringers; oxidized, soft (till) }\end{array}$ \\
\hline $28-62$ & 34 & $1,134-1,100$ & Clay, as above, olive gray, unoxidized (till) \\
\hline $62-218$ & 156 & $1,100-944$ & $\begin{array}{l}\text { Sand, very fine to very coarse, predominantly medium coarse }(90 \%) \text { and gravel }(10 \%) \\
\text { up to abut } 1 / 2 \text {-inch diameter, mostly less than } 0.2 \text {-inch diameter, lots of quartz, shield } \\
\text { silica, carbonates, detrital shale and lignite, subangular to well rounded; takes water; } \\
\text { some large pebbles sized weathered niobrara shale chunks; at } 109 \text { feet, finer section, } \\
\text { (less than } 1 \% \text { very fine gravel; sand, predominantly fine to medium); At } 135 \text { to } \\
140 \text { feet, slight rig chatter numerous detrital lignite fragments; after about } 160 \text { feet, } \\
\text { finer section, mostly very fine to fine sand, good recovery; at } 180 \text { feet mixed one bag } \\
\text { bentonite }\end{array}$ \\
\hline $218-220$ & 2 & 944-942 & Silt, clayey, greenish-gray, good recovery, bit slowed, smooth \\
\hline $220-231$ & 11 & $942-931$ & Clay, black, sticky, does not effervesce in dilute $\mathrm{HCl}$, noncalcareous (Carlile Shale) \\
\hline
\end{tabular}


Local number: 129N54W3ADD R

Other identifier: 12263

Site identification number: 455914097231801

Date of construction: $10-25-82$

Land surface altitude: 1,186 feet

Total depth: 200 feet

\begin{tabular}{cccl}
\hline Depth & $\begin{array}{c}\text { Thickness } \\
\text { (feet) }\end{array}$ & $\begin{array}{c}\text { Altitude } \\
\text { (feet) }\end{array}$ & \multicolumn{1}{c}{ Description } \\
\hline $0-1$ & 1 & $1,186-1,185$ & Topsoil \\
$1-43$ & 42 & $1,185-1,143$ & Clay, yellow brown, iron stained, oxidized, silty, sandy, with pebbles (till) \\
$43-120$ & 77 & $1,143-1,066$ & Clay, olive gray, silty, sandy, with pebbles, unoxidized (till) poorly sorted \\
$120-132$ & 12 & $1,066-1,054$ & $\begin{array}{c}\text { Sand, fine to coarse, some gravel, predominantly shale, well rounded to subrounded, } \\
\text { fair sorting }\end{array}$ \\
$132-138$ & 6 & $1,054-1,048$ & $\begin{array}{l}\text { Clay, olive gray, some layers of sand and gravel } \\
138-140\end{array}$ \\
$140-156$ & 16 & $1,048-1,046$ & Sand, very fine, poor return \\
$156-188$ & 32 & $1,046-1,030$ & Silt, poor return, drills fast, olive gray \\
$188-200$ & 12 & $998-986$ & Clay, black. snaps when broken (bedrock) \\
Well screened from 125 to 128 feet; 11/4-inch PVC screen and casing
\end{tabular}


Local number: 129N54W10AAA R

Other identifier: 12264

Site identification number: 455846097231801

Date of construction: $10-26-82$

Land surface altitude: 1,186 feet

Total depth: 200 feet

\begin{tabular}{cccl}
\hline Depth & $\begin{array}{c}\text { Thickness } \\
\text { (feet) }\end{array}$ & $\begin{array}{c}\text { Altitude } \\
\text { (feet) }\end{array}$ & \multicolumn{1}{c}{ Description } \\
\hline $0-1.5$ & 1.5 & $1,186-1,184.5$ & Topsoil \\
$1.5-18$ & 16.5 & $1,184.5-1,168$ & Clay, yellow brown; iron stained, silty, sandy, pebbly, oxidized (till) \\
$18-183$ & 165 & $1,168-1003$ & $\begin{array}{c}\text { Clay, olive gray, unoxidized, silty, sandy, pebbly (till), poorly sorted sand lens at } 112 \text { to } \\
\text { 113 feet }\end{array}$ \\
$183-200$ & 17 & $1,003-986$ & Clay, black, greasy (bedrock) \\
Electric log available & & \\
\hline
\end{tabular}

Local number: 129N54W10ADD R

Other identifier: 12265

Site identification number: 455821097231801

Date of construction: $10-26-82$

Land surface altitude: 1,232 feet

Total depth: 200 feet

\begin{tabular}{|c|c|c|c|}
\hline Depth & $\begin{array}{l}\text { Thickness } \\
\text { (feet) }\end{array}$ & $\begin{array}{l}\text { Altitude } \\
\text { (feet) }\end{array}$ & Description \\
\hline $0-2$ & 2 & $1,232-1,230$ & Topsoil \\
\hline 2-13 & 11 & $1,230-1,219$ & Clay, yellow brown, oxidized, iron stained, silty, sandy, pebbly (till) \\
\hline $13-53$ & 40 & $1,219-1,179$ & Clay, olive gray, silty, sandy, pebbly (till) \\
\hline $53-69$ & 16 & $1,179-1,163$ & $\begin{array}{l}\text { Sand, medium to coarse with some pebble gravel, well rounded to subrounded, fair } \\
\text { sorting, predominantly shale with } 10 \% \text { carbonates and igneous; also a couple of clay } \\
\text { seams }\end{array}$ \\
\hline $69-94$ & 25 & $1,163-1,138$ & Till, as above \\
\hline 94-97 & 3 & $1,138-1,135$ & $\begin{array}{l}\text { Sand, drills fast, poor return, predominantly shale, composition unknown due to upper } \\
\text { sands }\end{array}$ \\
\hline $97-190$ & 93 & $1,135-1,042$ & Till, as above \\
\hline $190-200$ & 10 & $1,042-1,032$ & Clay, black, greasy, peels off like bacon (bedrock) some siltstone \\
\hline \multicolumn{4}{|c|}{$\begin{array}{l}\text { Well screen from } 58 \text { to } 63 \mathrm{feet} \text {; 11/2-inch PVC screen and casing. Well was flowing so it was plugged with grout on 11-16-82. Electric log } \\
\text { available }\end{array}$} \\
\hline
\end{tabular}


Local number: 129N54W25AAAA R

Other identifier: R-12

Site identification number: 455607097211501

Date of construction: 9-4-70

Land surface altitude: 1,260 feet

Total depth: 230 feet

\begin{tabular}{cccl}
\hline Depth & $\begin{array}{c}\text { Thickness } \\
\text { (feet) }\end{array}$ & $\begin{array}{c}\text { Altitude } \\
\text { (feet) }\end{array}$ & \multicolumn{1}{c}{ Description } \\
\hline $0-3$ & 3 & $1,260-1,257$ & Topsoil, black \\
$3-33$ & 30 & $1,257-1,227$ & Yellow brown clay, fatty \\
$33-120$ & 87 & $1,227-1,140$ & Clay, gray; 6- to 8-inch gravel stringer at 87 feet \\
$120-160$ & 40 & $1,140-1,100$ & Gravel \\
$160-173$ & 13 & $1,100-1,087$ & Silty (till), smooth cutting, gravel stringer at 173 feet \\
$173-213$ & 40 & $1,087-1,047$ & Silt; rock at 213 feet \\
$213-230$ & 17 & $1,047-1,030$ & Shale cuttings \\
\hline
\end{tabular}

Local number: 129N54W27BBBB R

Other identifier: R-13

Site identification number: 455607097245701

Date of construction: 9-8-70

Land surface altitude: 1,365 feet

Total depth: 110 feet

\begin{tabular}{cccl}
\hline Depth & $\begin{array}{c}\text { Thickness } \\
\text { (feet) }\end{array}$ & $\begin{array}{c}\text { Altitude } \\
\text { (feet) }\end{array}$ & Description \\
\hline $0-5$ & 5 & $1,365-1,360$ & Mostly gravel, some clay \\
$5-15$ & 10 & $1,360-1,350$ & Clay, yellow, very gravelly \\
$15-35$ & 20 & $1,350-1,330$ & Clay, gray, very silty \\
$35-100$ & 65 & $1,330-1,265$ & Clay, whitish-gray (drills and looks like shale) \\
$100-110$ & 10 & $1,265-1,255$ & Shale, dark-gray \\
\hline
\end{tabular}


Local number: 129N54W29CCC

Other identifier: 9244

Site identification number: 455659097291601

Date of construction: 12-3-74

Land surface altitude: 1,520 feet

Total depth: 40 feet

\begin{tabular}{cccl}
\hline Depth & $\begin{array}{c}\text { Thickness } \\
\text { (feet) }\end{array}$ & $\begin{array}{c}\text { Altitude } \\
\text { (feet) }\end{array}$ & \multicolumn{1}{c}{ Description } \\
\hline $0-4$ & 4 & $1,520-1,516$ & Clay, very silty, sandy, pebbly; moderately yellow brown; soft, sticky, oxidized (till) \\
$4-12$ & 8 & $1,516-1,508$ & Clay, silty, sandy, pebbly; dark-yellow-brown; firm, moderately plastic, oxidized (till) \\
$12-15$ & 3 & $1,508-1,505$ & Clay, silty, sandy, pebbly; dark-gray, moderately plastic (till) \\
$15-40$ & 25 & $1,505-1,480$ & $\begin{array}{l}\text { Pierre Shale; grayish-black; (brittle upper contact), tight, moderately plastic, } \\
\text { noncalcareous, bentonitic }\end{array}$ \\
\hline
\end{tabular}

Local number: 130N54W27CCC R

Other identifier: 12261

Site identification number: 460033097245701

Date of construction: 9-21-82

Land surface altitude: 1,180 feet

Total depth: 190 feet

\begin{tabular}{|c|c|c|c|}
\hline Depth & $\begin{array}{c}\text { Thickness } \\
\text { (feet) }\end{array}$ & $\begin{array}{c}\text { Altitude } \\
\text { (feet) }\end{array}$ & Description \\
\hline $0-1$ & 1 & $1,180-1,179$ & Topsoil \\
\hline $1-27$ & 26 & $1,179-1,153$ & Clay, yellow brown, oxidized, iron stains, silty, sandy, pebbly (till) \\
\hline $27-101$ & 74 & $1,153-1,079$ & Clay, olive gray, silty, sandy, pebbly (till); rock at 80 feet, put rock bit on \\
\hline $101-112$ & 11 & $1,079-1,068$ & $\begin{array}{l}\text { Sand and gravel, coarse sand to medium gravel, predominantly very coarse sand, } \\
\text { angular to rounded, predominantly rounded, predominantly quartz, some very coarse } \\
\text { detrital lignite }\end{array}$ \\
\hline $112-141$ & 29 & $1,068-1,039$ & Till, olive gray clay, slightly pebbly, cohesive and plastic \\
\hline $141-170$ & 29 & $1,039-1,010$ & $\begin{array}{l}\text { Sand, fine to medium grain, predominantly medium, predominantly rounded, } \\
\text { interbedded with sandy and/or silty clay and lenses of coarse detrital lignite; below } \\
153 \text { feet, appears to be strictly medium sand, no clay; below } 164 \text { feet, interbedded } \\
\text { clay and detrital lignite }\end{array}$ \\
\hline $170-176$ & 6 & $1,010-1,004$ & Till, brownish- to olive-gray, silty and pebbly, moderately cohesive \\
\hline $176-190$ & 14 & $1,004-990$ & Bedrock shale, dark-gray to black, interbedded with dark-gray clayey siltstone \\
\hline
\end{tabular}


Local number: 130N54W32CCC R

Other identifier: 9246

Site identification number: 455909097272801

Date of construction: $12-4-74$

Land surface altitude: 1,187 feet

Total depth: 180 feet

\begin{tabular}{|c|c|c|c|}
\hline Depth & $\begin{array}{c}\text { Thickness } \\
\text { (feet) }\end{array}$ & $\begin{array}{c}\text { Altltude } \\
\text { (feet) }\end{array}$ & Description \\
\hline $2-0$ & 2 & $1,187-1,185$ & Topsoil, silty, loam; dusky yellow brown \\
\hline $2-6$ & 4 & $1,185-1,181$ & Clay, silty, sandy, pebbly; grayish-orange, soft. sticky, oxidized (till) \\
\hline $6-23$ & 17 & $1,181-1,164$ & Clay, silty, sandy, pebbly; dark-yellow orange; firm, plastic, iron stained: oxidized (till) \\
\hline $23-70$ & 47 & $1,164-1,117$ & $\begin{array}{l}\text { Clay, very silty, sandy, few pebbles, very coarse sand grains; dark-gray; moderately soft, } \\
\text { plastic, occasional thin gravel lenses (till) }\end{array}$ \\
\hline $70-151$ & 81 & $1,117-1,036$ & Clay, silty, sandy, pebbly, olive gray; moderately firm, moderately plastic (till) \\
\hline $151-159$ & 8 & $1,036-1,028$ & Clay, silty, very sandy, pebbly; dark-gray; firm, tight, slightly friable; gravel lenses (till) \\
\hline $159-180$ & 21 & $1,028-1,007$ & Pierre Shale; gray, slightly black, plastic, tight, noncalcareous, very slightly fissile \\
\hline lectr & & & \\
\hline
\end{tabular}

Local number: 130N54W33DDD R

Other identifier: 12262

Site identification number: 455909097245901

Date of construction: 9-22-82

Land surface altitude: 1,158 feet

Total depth: 200 feet

\begin{tabular}{|c|c|c|c|}
\hline Depth & $\begin{array}{l}\text { Thickness } \\
\text { (feet) }\end{array}$ & $\begin{array}{l}\text { Altitude } \\
\text { (feet) }\end{array}$ & Descriptlon \\
\hline $0-1$ & 1 & $1,158-1,157$ & Topsoil \\
\hline $1-10$ & 9 & $1,157-1,148$ & Till, yellow brown, oxidized, silty, very pebbly; cohesive, plastic \\
\hline $10-27$ & 17 & $1,148-1,131$ & $\begin{array}{l}\text { Till, yellow brown, oxidized, slightly silty, slightly pebbly, very clayey; cohesive, } \\
\text { slightly brittle; at } 18 \text { feet, color becomes dark-brown, still oxidized }\end{array}$ \\
\hline $27-120$ & 93 & $1,131-1,038$ & $\begin{array}{l}\text { Till, olive gray, silty, pebbly, moderately cohesive, moderately plastic, below } 60 \text { feet till } \\
\text { seems softer, more pliable, same composition; at } 116 \text { to } 120 \text { feet. becomes gravelly }\end{array}$ \\
\hline $120-129$ & 9 & $1,038-1,029$ & Till, olive gray, silty, pebbly, very cohesive, tight, plastic \\
\hline $129-172$ & 43 & $1,029-986$ & $\begin{array}{l}\text { Sand and gravel, coarse sand to medium gravel, predominantly very coarse sand and } \\
\text { fine gravel, angular to rounded, predominantly subangular, equal proportions quartz, } \\
\text { shale and carbonates; below } 140 \text { feet becomes more rounded and spherical }\end{array}$ \\
\hline $172-183$ & 11 & $986-975$ & Till? no sample \\
\hline $183-200$ & 17 & $975-958$ & Bedrock shale, dark-gray to black, slightly carbonaceous. silty \\
\hline
\end{tabular}


Local number: 130N54W34DCC R

Other identifier: 12267

Site identification number: 455941097241701

Date of construction: 10-26-82

Land surface altitude: 1,155 feet

Total depth: 180 feet

\begin{tabular}{|c|c|c|c|}
\hline Depth & $\begin{array}{l}\text { Thickness } \\
\text { (feet) }\end{array}$ & $\begin{array}{l}\text { Altitude } \\
\text { (feet) }\end{array}$ & Description \\
\hline $0-1$ & 1 & $1,155-1,154$ & Topsoil \\
\hline $1-11$ & 10 & $1,154-1,144$ & Clay, yellow brown, iron stained, oxidized, silty, sandy with pebbles (till) \\
\hline $11-107$ & 96 & $1,144-1,048$ & $\begin{array}{l}\text { Clay, olive gray, unoxidized, silty, sandy with pebbles (till), layer of sand and gravel at } \\
104 \text { to } 105 \text { feet }\end{array}$ \\
\hline $107-118$ & 11 & $1,048-1,037$ & $\begin{array}{l}\text { Till with numerous layers of shale sand and gravel; drills slow then fast in 1- to 2-foot } \\
\text { increments }\end{array}$ \\
\hline $118-136$ & 18 & $1,037-1,019$ & Gray till as above, rocky \\
\hline $136-150$ & 14 & $1,019-1005$ & $\begin{array}{l}\text { Gravel, pebbly, subrounded, some medium to coarse sand, } 30 \% \text { shale, } 40 \% \text { carbonates, } \\
30 \% \text { igneous, drills choppy and fast }\end{array}$ \\
\hline $150-170$ & 20 & $1005-985$ & $\begin{array}{l}\text { Silt, olive gray, poor return, drills real smooth and fast, could be some very fine sands } \\
\text { mixed in }\end{array}$ \\
\hline $170-180$ & 10 & $985-975$ & $\begin{array}{l}\text { Clay, blackish, greasy, bedrock?, lost circulation, put soap in and gained circulation; } \\
\text { poor return? }\end{array}$ \\
\hline
\end{tabular}


Local number: 130N54W35CCC R

Other identifier: 9247

Site identification number: 455941097234001

Date of construction: 12-4-74

Land surface altitude: 1,170 feet

Total depth: 240 feet

\begin{tabular}{|c|c|c|c|}
\hline Depth & $\begin{array}{l}\text { Thickness } \\
\text { (feet) }\end{array}$ & $\begin{array}{l}\text { Altitude } \\
\text { (feet) }\end{array}$ & Description \\
\hline $0-4$ & 4 & $1,170-1,166$ & Clay, silty, sandy, pebbly; dark-yellow-orange, soft, sticky, oxidized (till) \\
\hline $4-27$ & 23 & $1,166-1,143$ & Clay, silty, sandy, pebbly; dark-yellow-brown; firm, plastic, tight, oxidized (till) \\
\hline $27-68$ & 41 & $1,143-1,102$ & $\begin{array}{l}\text { Clay, silty, sandy, pebbly; dark-gray; firm moderately plastic, tight (till); occasional thin } \\
\text { gravel lens }\end{array}$ \\
\hline $68-96$ & 28 & $1,102-1,074$ & $\begin{array}{l}\text { Clay, silty, sandy, pebbly; olive gray; moderately soft, plastic; gravel lenses; larger } \\
\text { pebbles (till) }\end{array}$ \\
\hline $96-118$ & 22 & $1,074-1,052$ & $\begin{array}{l}\text { Clay, silty, sandy, pebbly; dark-gray to grayish-black, moderately firm, plastic, } \\
\text { numerous thin gravel lenses (till) }\end{array}$ \\
\hline $118-130$ & 12 & $1,052-1,040$ & $\begin{array}{l}\text { Sand and gravel; sand medium to very coarse, angular to subrounded, about } 60 \% \\
\text { quartz, } 30 \% \text { carbonates, } 5 \% \text { shale, } 5 \% \text { gneiss; gravel, fine to coarse, angular to } \\
\text { subrounded, about } 70 \% \text { gneiss, } 10 \% \text { quartz, } 10 \% \text { shale, } 10 \% \text { carbonates, clean sorted }\end{array}$ \\
\hline $130-134$ & 4 & $1,040-1,036$ & Clay, very silty, sand, very coarse sand grains; dark-gray; soft, plastic (till) \\
\hline $134-150$ & 16 & $1,036-1,020$ & $\begin{array}{l}\text { Clay, dark-gray, noncalcareous; light-gray mottling (calcareous), tight, plastic; weak } \\
\text { laminae (lacustrine) }\end{array}$ \\
\hline $150-174$ & 24 & $1,020-996$ & Clay, as above with sand lenses \\
\hline $174-200$ & 26 & $996-970$ & $\begin{array}{l}\text { Sand and gravel, dark-gray sand, medium to very coarse, angular to round; gravel, fine } \\
\text { to medium, angular to subround, about } 60 \% \text { quartz, } 30 \% \text { shale, } 10 \% \text { carbonates, } \\
\text { igneous, and moderate lignite fragments }\end{array}$ \\
\hline $200-218$ & 18 & $970-952$ & $\begin{array}{l}\text { Clay, silty, slightly sandy, occasional coarse sand grains; grayish-black, calcareous, very } \\
\text { plastic, soft (glaciofluvial - silt/till?) }\end{array}$ \\
\hline $218-240$ & 22 & $952-930$ & Pierre Shale, grayish-black; noncalcareous, tight, plastic, stiff \\
\hline
\end{tabular}



Section B - Hydrographs 

Site number from location map: 1

Local well number: 118 N52W18BBBB2

Station identification number: 450205097073302

Other identifier: CD-77A

County: Codington, South Dakota

Aquifer: Undetermined

Altitude of land surface: 1,775 feet

Measuring point: 0.0 foot

Extremes: April 16, 1981, to November 6, 1996: Highest, -5.6 feet, August 20, 1990; lowest, 12.5 feet, June 19, 1986, July 1, 1986, July 16, 1986.

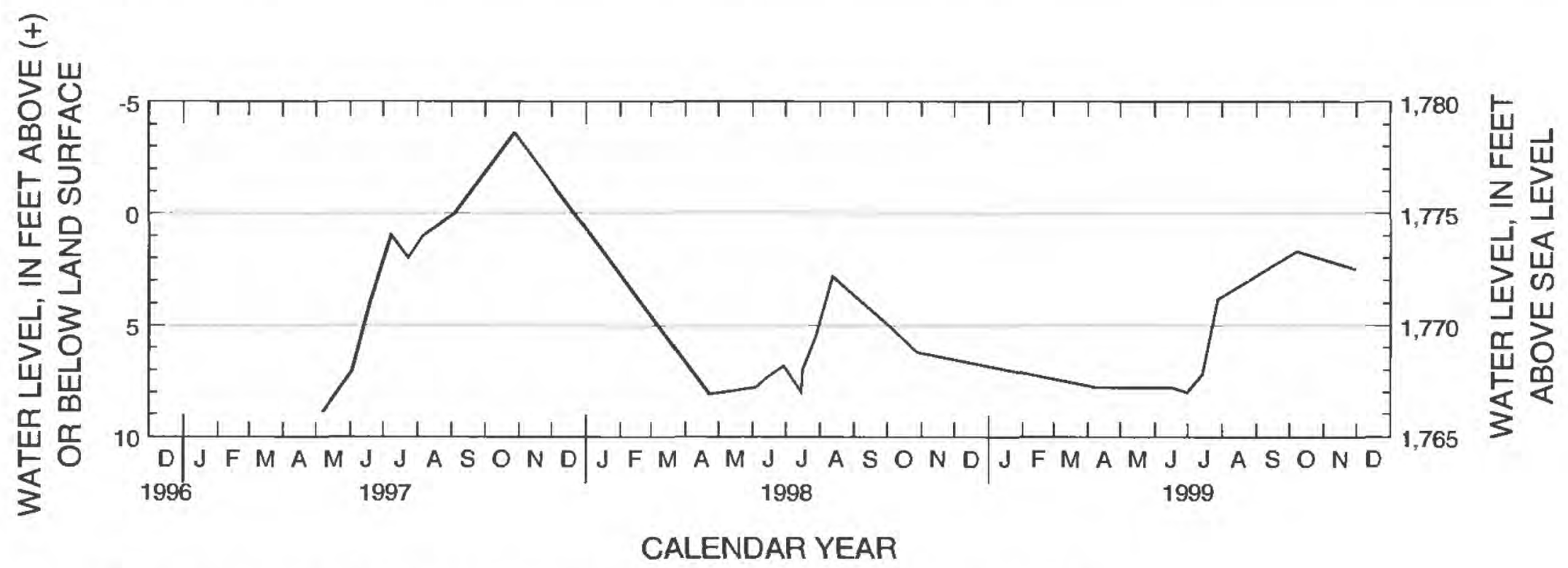

Figure B1. Hydrograph for observation well 118N52W18BBBB2 (site number 1).

Site number from location map: 2

Local well number: 118N52W30CDCD

Station identification number: 445930097070901

Other identifier: CD-76B

County: Codington, South Dakota

Aquifer: Big Sioux

Altitude of land surface: $1,753.2$ feet

Measuring point: 0.0 foot

Extremes: November 30, 1976, to October 9, 1996: Highest, 5.87 feet, March 21, 1985; lowest, 12.2 feet, July 25, 1984.

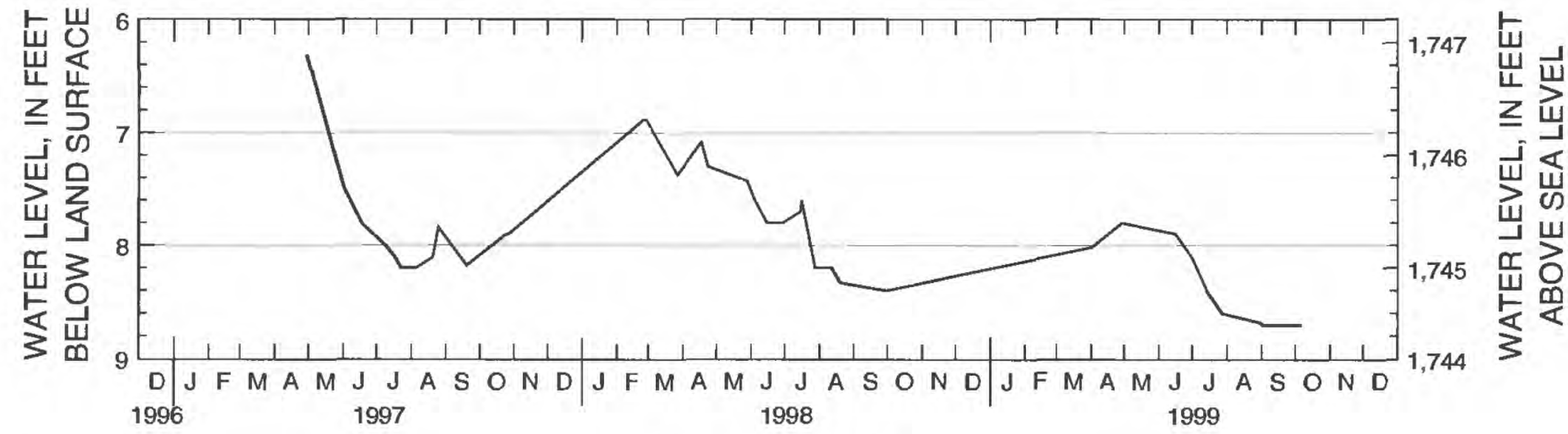

CALENDAR YEAR

Figure B2. Hydrograph for observation well 118N52W30CDCD (site number 2). 
Site number from location map: 3

Local well number: 119N52W4ADDD R

Station identification number: 450655097092301

Other identifier: CD-77B

County: Codington, South Dakota

Aquifer: Big Sioux

Altitude of land surface: 1,770 feet

Measuring point: 2.4 feet

Extremes: December 1, 1977, to October 6, 1999: Highest, 1.9 feet, July 2, 1991; lowest, 7.67 feet, December 1, 1988.

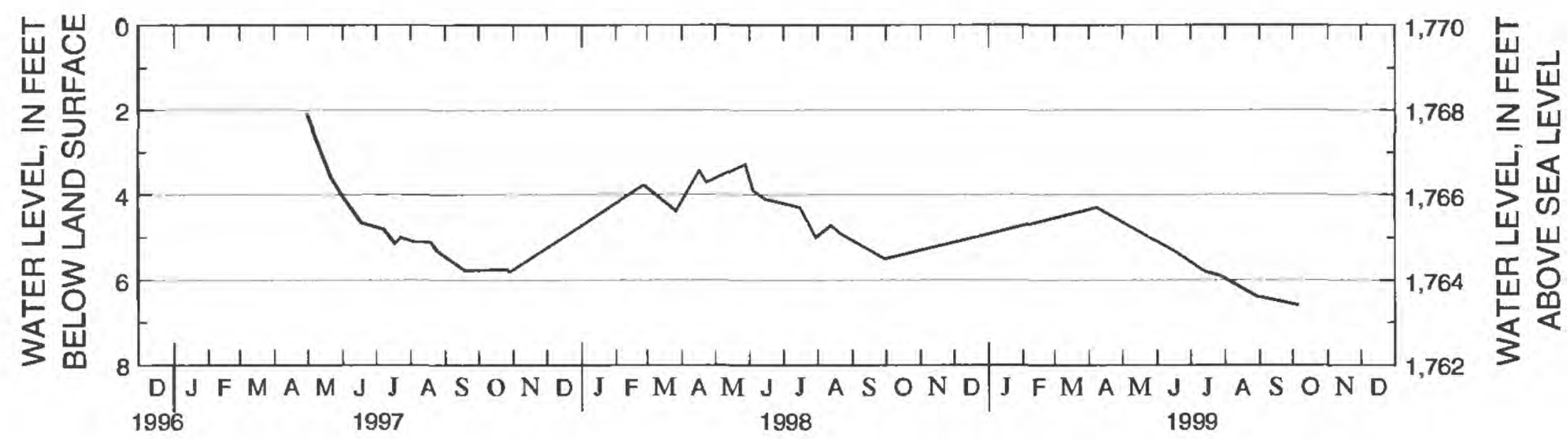

CALENDAR YEAR

Figure B3. Hydrograph for observation well 119N52W4ADDD R (site number 3).

Site number from location map: 4

Local well number: 119N52W10DDDD R

Station identification number: 450538097083901

Other identifier: CD-77C

County: Codington, South Dakota

Aquifer: Big Sioux

Altitude of land surface: 1,770 feet

Measuring point: 3.6 feet

Extremes: December 1, 1977, to October 6, 1999: Highest, 1.0 foot, June 23, 1994; lowest, 9.2 feet, December 29, 1981.

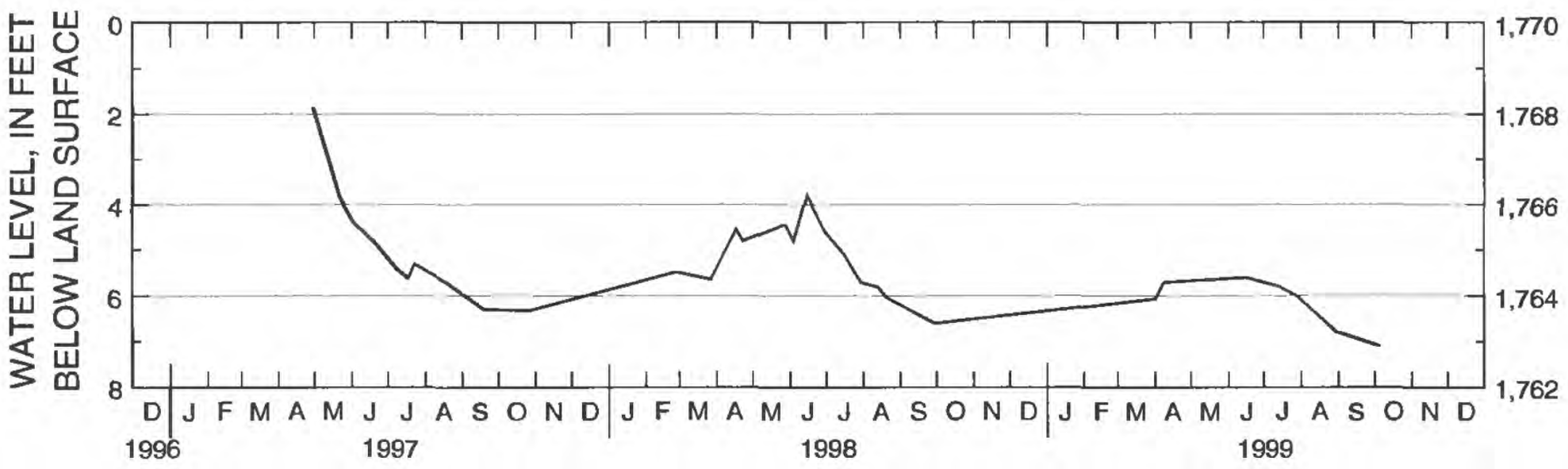

CALENDAR YEAR

Figure B4. Hydrograph for observation well 119N52W10DDDD R (site number 4). 
Site number from location map: 5

Local well number: 119N52W33DCDC R

Station identification number: 450208097101401

Other identifier: CD-60A

County: Codington, South Dakota

Aquifer: Big Sioux

Altitude of land surface: 1,745 feet

Measuring point: 1.9 feet

Extremes: June 13, 1960, to October 7, 1999: Highest, 2.80 feet, Apr. 30, 1997; lowest, 10.6 feet, November 1, 1990.



CALENDAR YEAR

Figure B5. Hydrograph for observation well 119N52W33DCDC R (site number 5).

Site number from location map: 6

Local well number: 119N53W6BBBB

Station identification number: 450904097145501

Other identifier: R2-85-111

County: Codington, South Dakota

Aquifer: Altamont

Altitude of land surface: 1,878 feet

Measuring point: 1.9 feet

Extremes: April 30, 1997, to August 31, 1999: Highest, 118.71 feet, April 1, 1999; lowest, 120.14 feet, August 25, 1997.

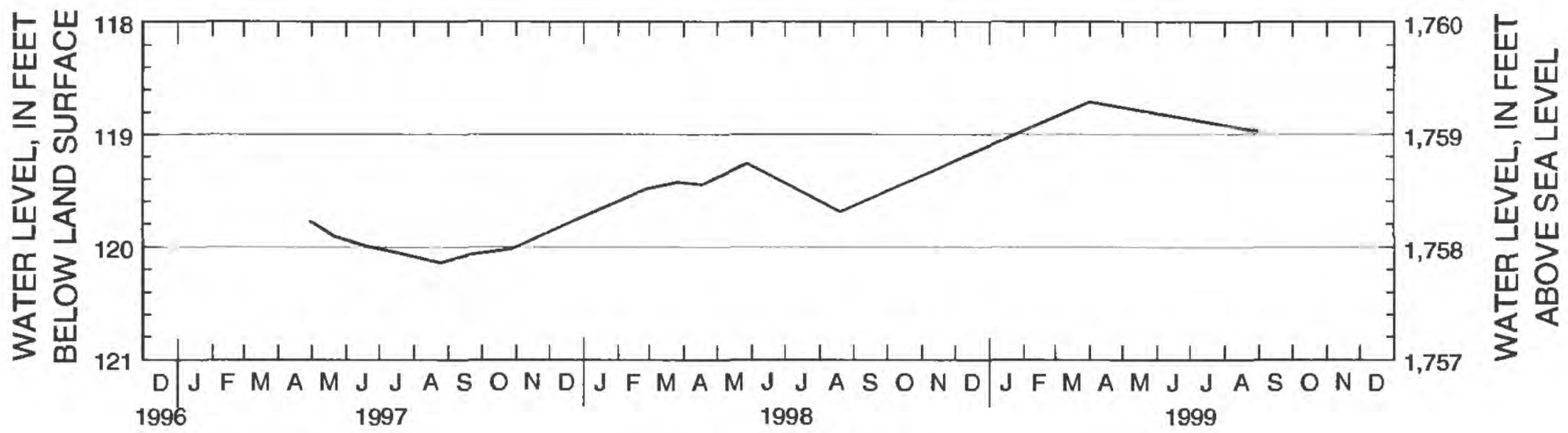

CALENDAR YEAR

Figure B6. Hydrograph for observation well 119N53W6BBBB (site number 6). 
Site number from location map: 7

Local well number: 120N51W19BBCC R

Station identification number: 450947097060901

Other identifier: GT-77A

County: Grant, South Dakota

Aquifer: Prairie Coteau

Altitude of land surface: $1,894.99$ feet

Measuring point: 2.0 feet

Extremes: July 20, 1977, to October 6, 1999: Highest, 75.5 feet, November 8, 1995; lowest, 121.5 feet, July 29, 1988.

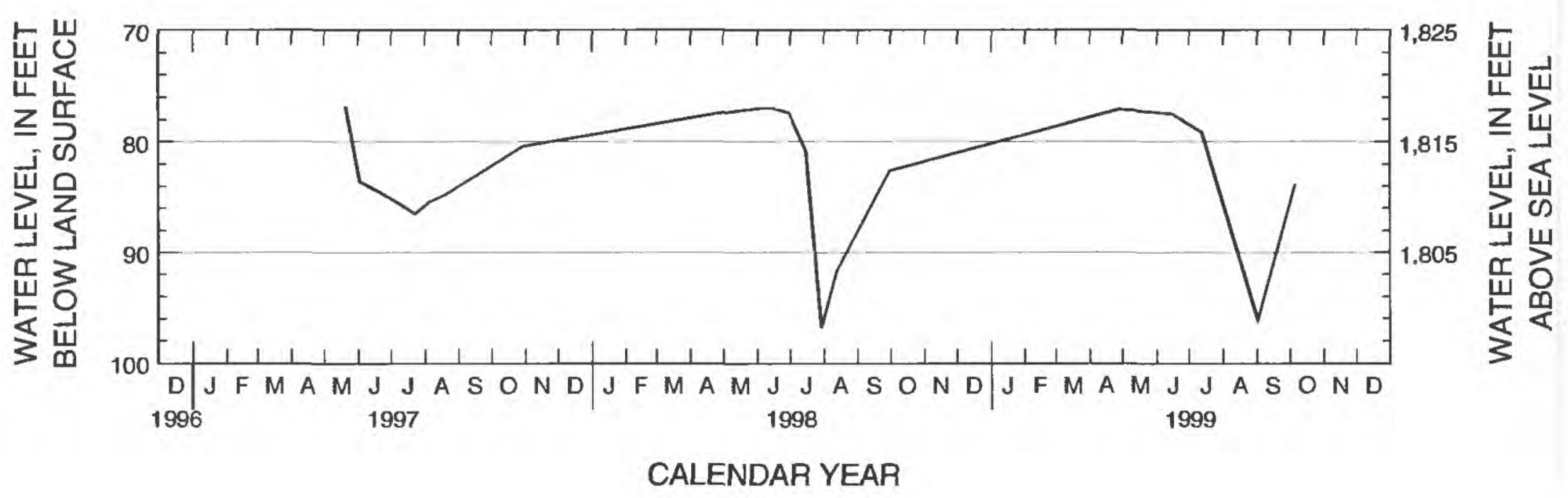

Site number from location map: 8

Local well number: 120N52W9BBBB R

Station identification number: 451143097110501

Other identifier: GT-79C

County: Grant, South Dakota

Aquifer: Prairie Coteau

Altitude of land surface: 1,834 feet

Measuring point: 1.4 feet

Extremes: October 25, 1979, to October 6, 1999: Highest, 14.5 feet, June 6. 1996; lowest, 26.2 feet, September 7, 1988.

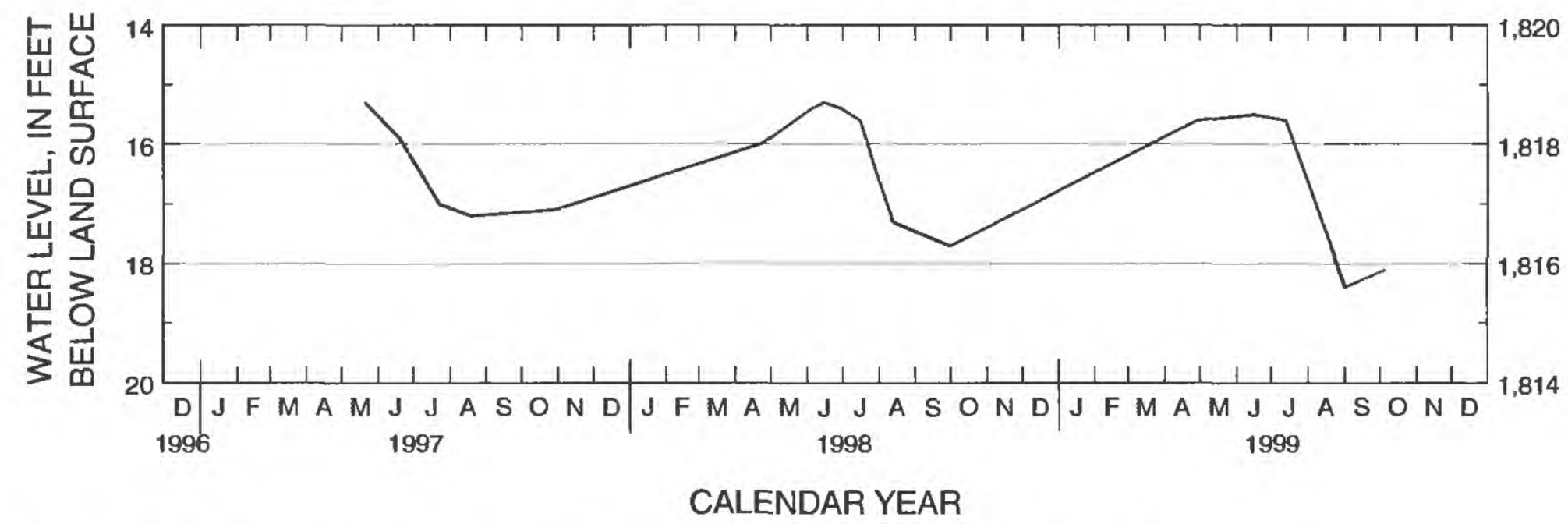

Figure B8. Hydrograph for observation well 120N52W9BBBB R (site number 8). 
Site number from location map: 9

Local well number: 120N52W12AAAB R

Station identification number: 451141097061701

Other identifier: GT-76C

County: Grant, South Dakota

Aquifer: Prairie Coteau

Altitude of land surface: $1,873.12$ feet

Measuring point: 2.1 feet

Extremes: June 17, 1977, to October 6, 1999: Highest, 50.1 feet, June 6, 1996; lowest, 97.1 feet, August 25, 1988.

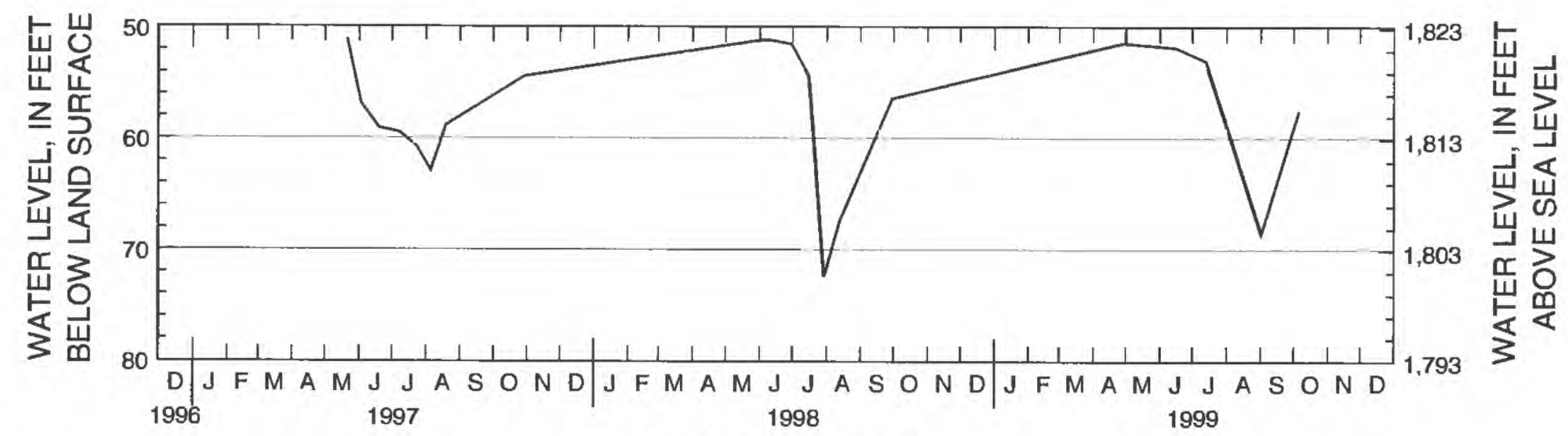

CALENDAR YEAR

Figure B9. Hydrograph for observation well 120N52W12AAAB R (site number 9).

Site number from location map: 10

Local well number: 120N52W23BBBB R

Station identification number: 450956097083401

Other identifier: GT-76B

County: Grant, South Dakota

Aquifer: Prairie Coteau

Altitude of land surface: $1,820.87$ feet

Measuring point: 3.0 feet

Extremes: June 16, 1977, to October 6, 1999: Highest, 6.3 feet, June 6, 1996; lowest, 65.0 feet, July 29, 1988.

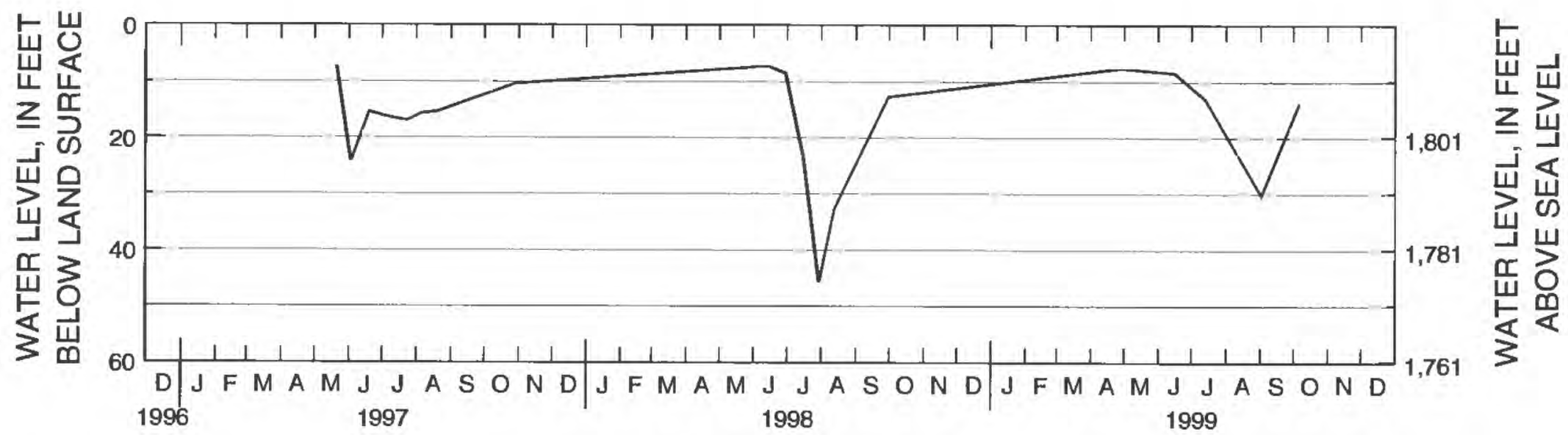

CALENDAR YEAR

Figure B10. Hydrograph for observation well 120N52W23BBBB R (site number 10). 
Site number from location map: 11

Local well number: 120N52W27CDDD R

Station identification number: 450814097091601

Other identifier: CD-76D

County: Codington, South Dakota

Aquifer: Prairie Coteau

Altitude of land surface: 1,800 feet

Measuring point: 4.6 feet

Extremes: June 16, 1976, to November 30, 1999: Highest, -4.7 feet, November 8, 1995; lowest, 52.1 feet, July 29, 1988.

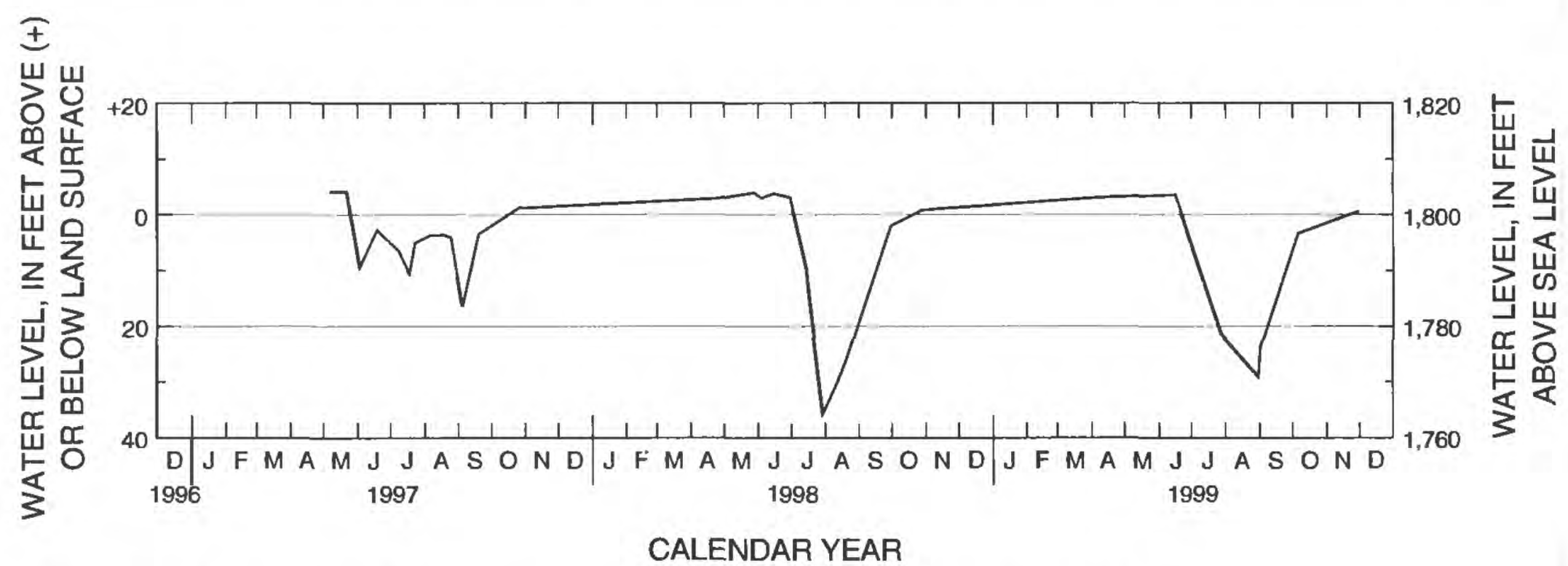

Figure B11. Hydrograph for observation well 120N52W27CDDD R (site number 11).

Site number from location map: 12

Local well number: 120N52W28DDDD R

Station identification number: 450814097095301

Other identifier: CD-56A

County: Codington, South Dakota

Aquifer: Big Sioux

Altitude of land surface: $1,781.62$ feet

Measuring point: 1.6 feet

Extremes: August 29, 1957, to October 6, 1999: Highest, 4.4 feet, July 2, 1991; lowest, 11.6 feet, December 7 , 1966.

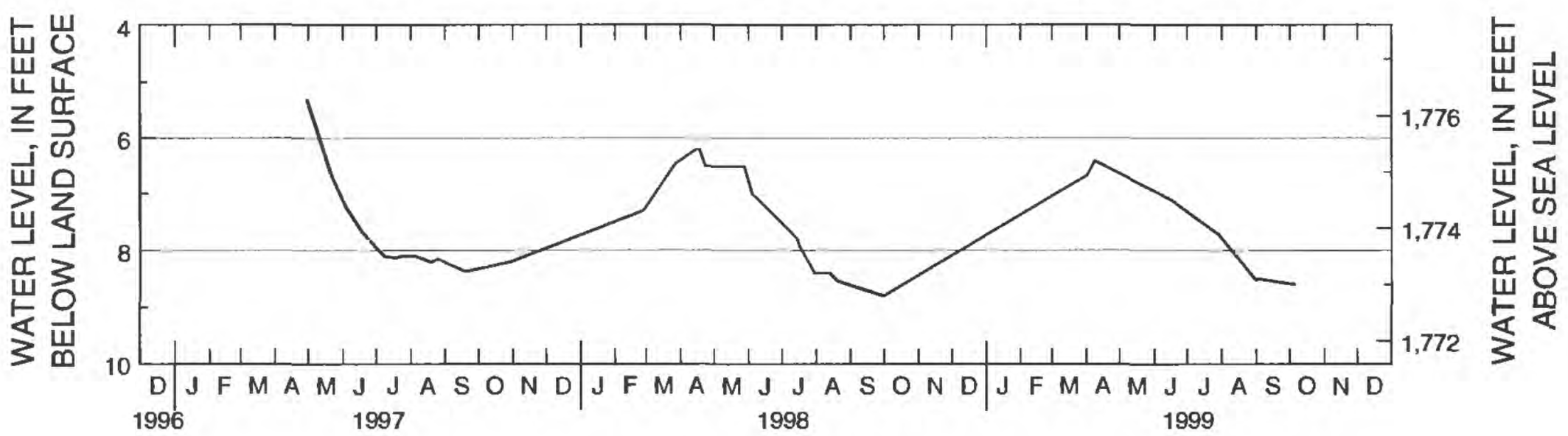

CALENDAR YEAR

Figure B12. Hydrograph for observation well 120N52W28DDDD R (site number 12). 
Site number from location map: 13

Local well number: 120N54W23DDDC

Station identification number: 451050097161601

Other identifier: DA-78H

County: Day, South Dakota

Aquifer: Prairie Coteau

Altitude of land surface: 1,830 feet

Measuring point: 3.0 feet

Extremes: October 16, 1978, to October 6, 1999: Highest, 41.7 feet, October 6, 1999; lowest, 56.2 feet, August 4, 1982, October 3, 1983.

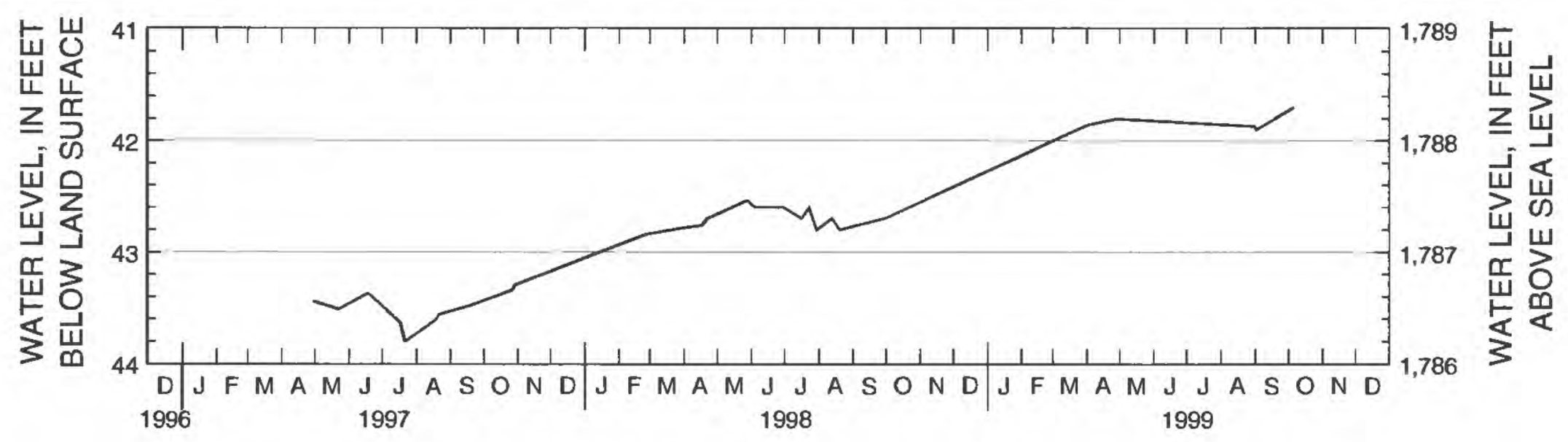

CALENDAR YEAR

Figure B13. Hydrograph for observation well 120N54W23DDDC (site number 13).

Site number from location map: 14

Local well number: 121N47W1AAAA

Station identification number: 451936096294001

Other identifier: $\mathrm{C} 0-86-03$

County: Grant, South Dakota

Aquifer: Undetermined

Altitude of land surface: 1,092 feet

Measuring point: 3.0 feet

Extremes: December 11, 1996, to August 25, 1999: Highest, 50.8 feet, June 20, 1997; lowest, 54.77 feet, July 20, 1998.

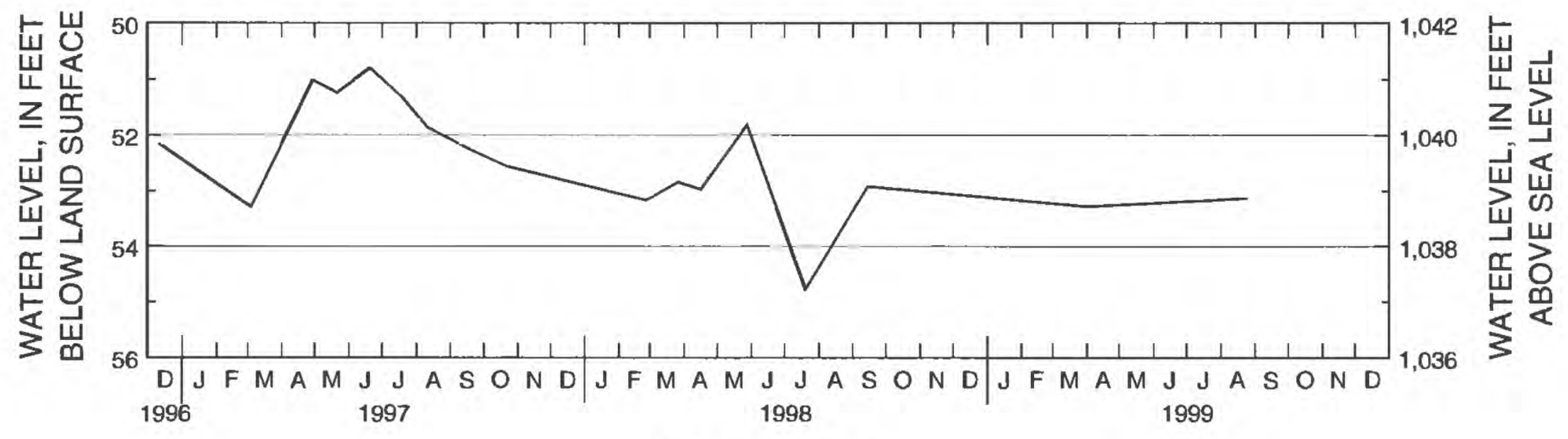

CALENDAR YEAR

Figure B14. Hydrograph for observation well 121N47W1AAAA (site number 14). 
Site number from location map: 15

Local well number: 121N47W2DDDD

Station identification number: 451844096305401

Other identifier: CO-86-05

County: Grant, South Dakota

Aquifer: Undetermined

Altitude of land surface: 1,115 feet

Measuring point: 3.0 feet

Extremes: April 29, 1997, to August 25, 1999: Highest, 45.13 feet, June 20, 1997; lowest, 48.03 feet, August 25, 1999.

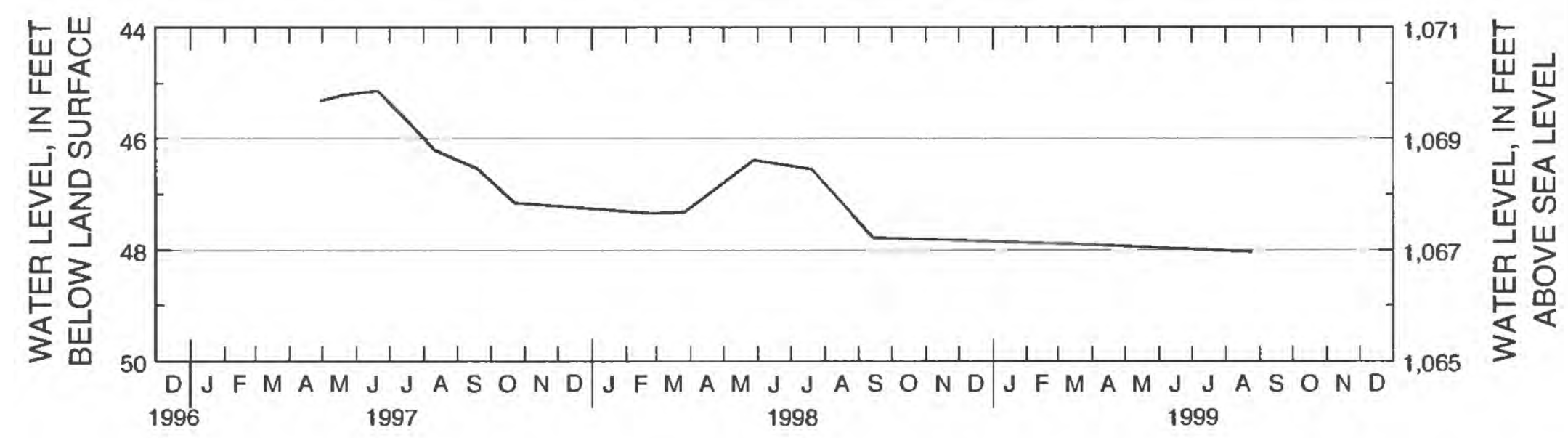

CALENDAR YEAR

Figure B15. Hydrograph for observation well 121N47W2DDDD (site number 15).

Site number from location map: 16

Local well number: 121N47W6CCCC

Station identification number: 451848096363501

Other identifier: GT-79E

County: Grant, South Dakota

Aquifer: Undetermined

Altitude of land surface: 1,104.00 feet

Measuring point: 2.8 feet

Extremes: October 25, 1979, to October 5, 1999: Highest, 19.22 feet; Apr. 29, 1997; lowest, 38.4 feet, October 4, 1983, November 8, 1983.

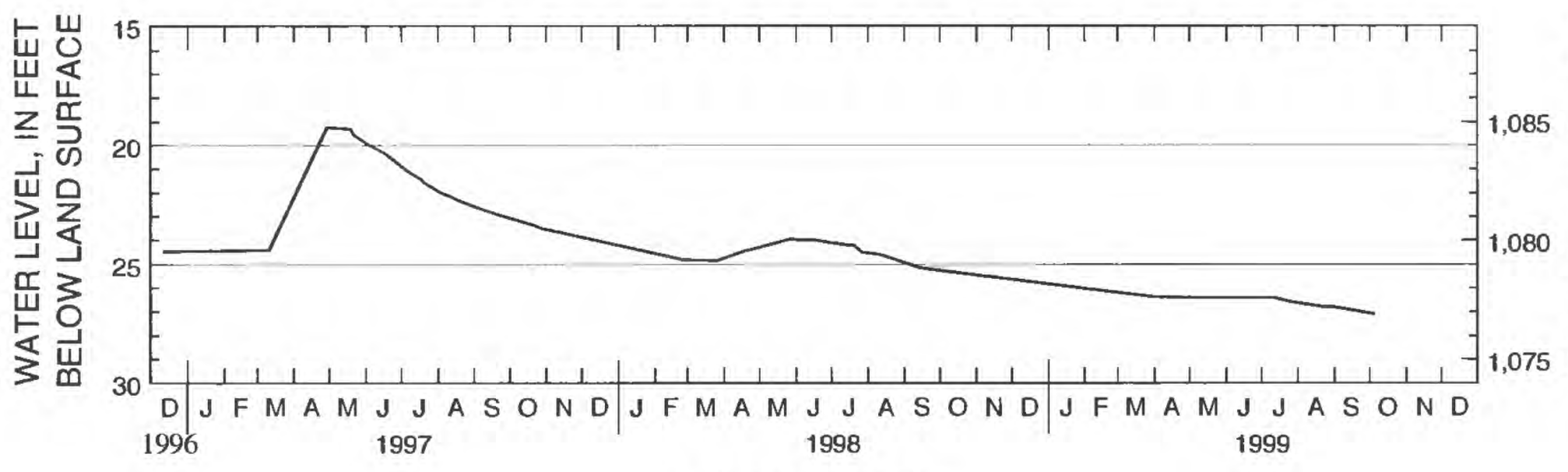

CALENDAR YEAR

Figure B16. Hydrograph for observation well 121N47W6CCCC (site number 16). 
Site number from location map: 17

Local well number: $121 \mathrm{~N} 51$ W29BBBB R

Station identification number: 451418097045501

Other identifier: GT-77E

County: Grant, South Dakota

Aquifer: Prairie Coteau

Altitude of land surface: 1,955 feet

Measuring point: 3.6 feet

Extremes: May 2, 1978, to October 6, 1999: Highest, 73.5 feet, June 6, 1996; lowest, 84.72 feet, September 26, 1988.

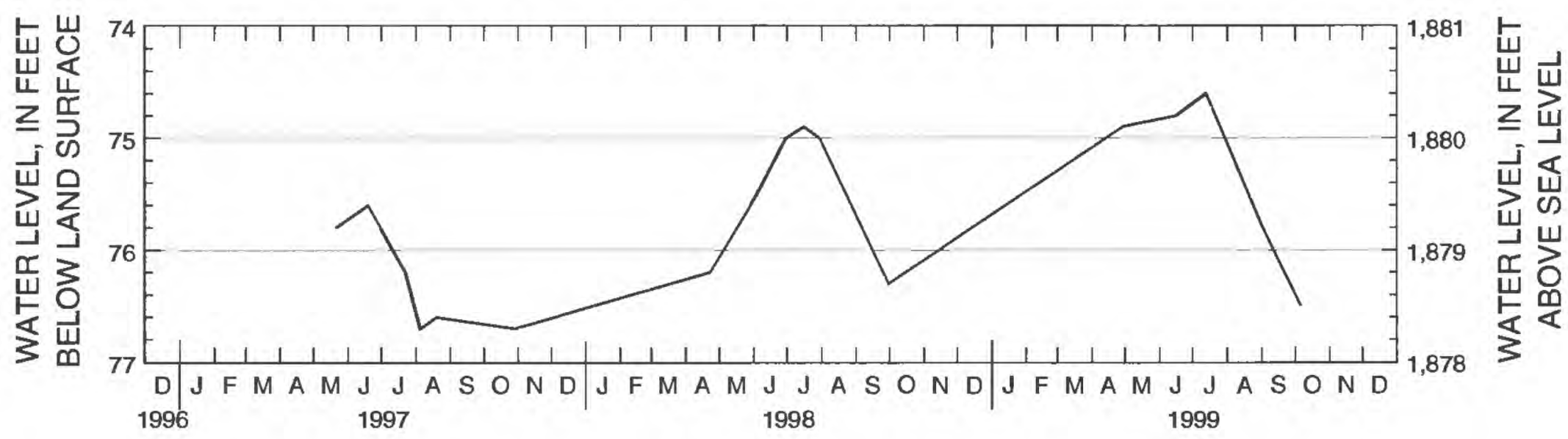

CALENDAR YEAR

Figure B17. Hydrograph for observation well 121N51W29BBBB R (site number 17).

Site number from location map: 18

Local well number: 121N52W1CBBB R

Station identification number: 451725097072101

Other identifier: GT-57A

County: Grant, South Dakota

Aquifer: Big Sioux

Altitude of land surface: 1,885 feet

Measuring point: 1.75 feet

Extremes: April 29, 1997, to October 6, 1999: Highest, 4.16 feet, April 29, 1997; lowest, 10.3 feet, October 6, 1999

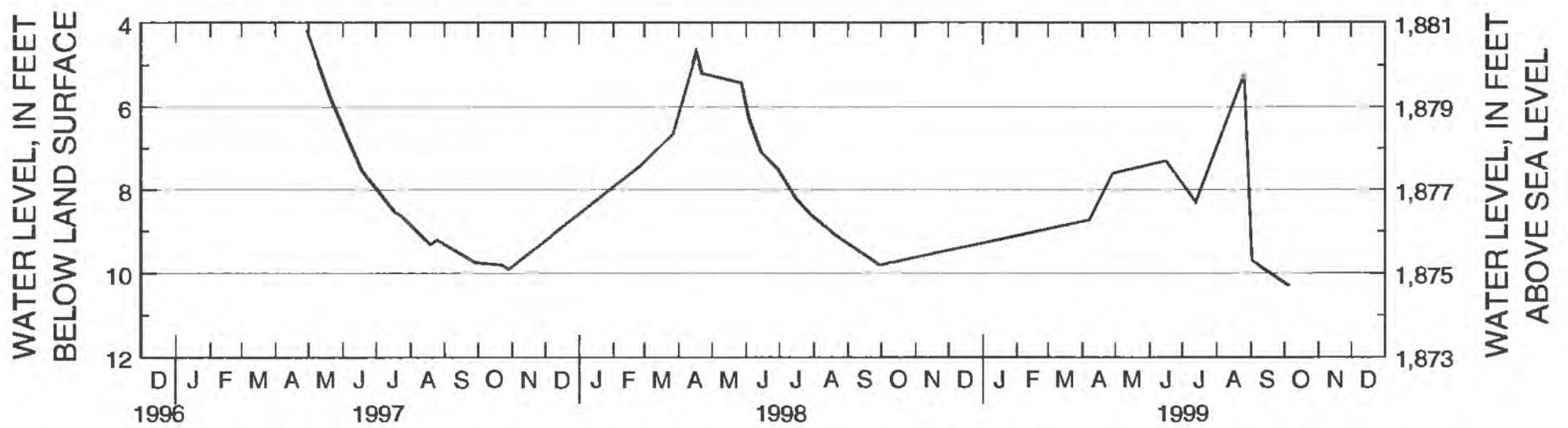

CALENDAR YEAR

Figure B18. Hydrograph for observation well 121N52W1CBBB R (site number 18). 
Site number from location map: 19

Local well number: 121N52W2ADDD R

Station identification number: 451606097072501

Other identifier: GT-82A

County: Grant, South Dakota

Aquifer: Big Sioux

Altitude of land surface: $1,881.25$ feet

Measuring point: 3.2 feet

Extremes: June 29, 1982, to October 6, 1999: Highest, 0.92 foot, April 29, 1997; lowest, 6.7 feet, July 30, 1998.

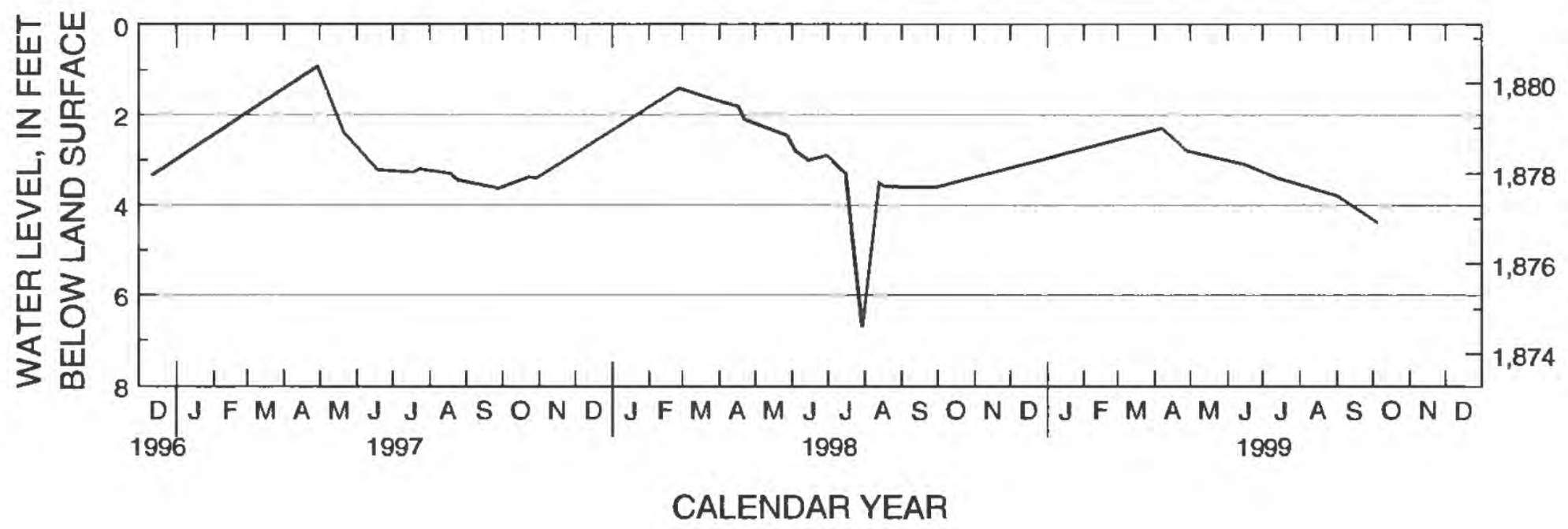

Figure B19. Hydrograph for observation well 121N52W2ADDD R (site number 19).

Site number from location map: 20

Local well number: 121N52W2BAAA R

Station identification number: 451749097080601

Other identifier: R20-84-31

County: Grant, South Dakota

Aquifer: Big Sioux

Altitude of land surface: $1,878.4$ feet

Measuring point: 3.0 feet

Extremes: April 29, 1997, to August 26, 1999: Highest, -0.49 foot, April 17, 1998; lowest, 2.07 feet, August 18, 1998.



Figure B20. Hydrograph for observation well 121N52W2BAAA R (site number 20). 
Site number from location map: 21

Local well number: 121N52W2BBBA R

Station identification number: 451750097083001

Other identifier: CO-87-66

County: Grant, South Dakota

Aquifer: Prairie Coteau

Altitude of land surface: 1,885 feet

Measuring point: 2.6 feet

Extremes: April 29, 1997, to August 26, 1999: Highest, 23.03 feet, May 28, 1998; lowest 26.37 feet, August 26, 1999.

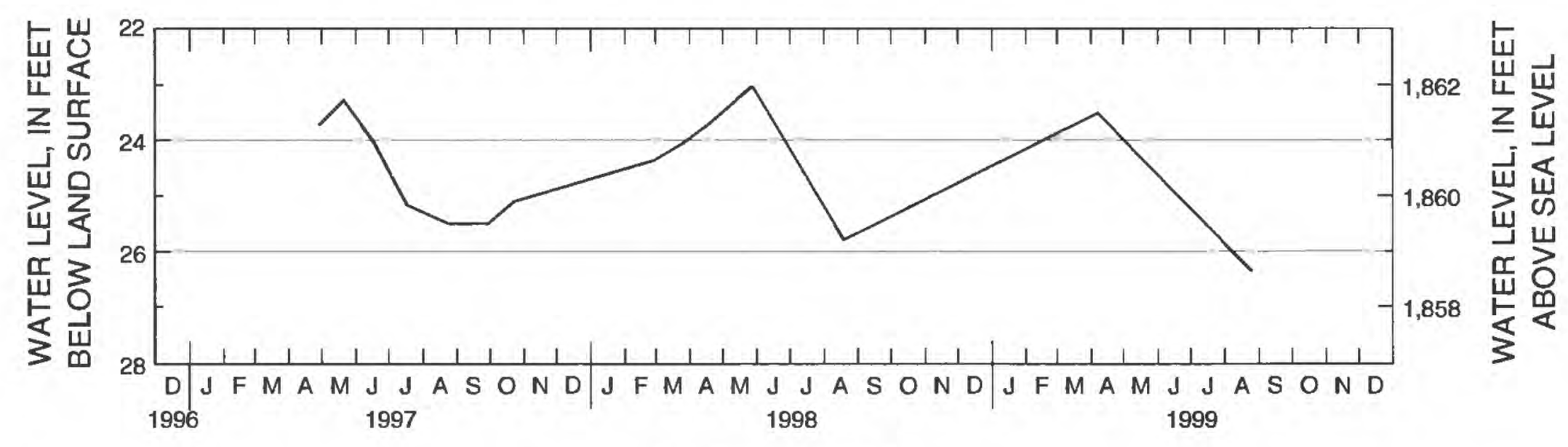

CALENDAR YEAR

Figure B21. Hydrograph for observation well 121N52W2BBBA R (site number 21).

Site number from location map: 22

Local well number: 121 N52W2CCCC R

Station identification number: 451701097083701

Other identifier: R20-84-26

County: Grant, South Dakota

Aquifer: Big Sioux

Altitude of land surface: 1,869 feet

Measuring point: 2.0 feet

Extremes: April 29, 1997, to August 26, 1999: Highest, 0.21 foot, February 26, 1998; lowest, 8.64 feet, August 26, 1999.

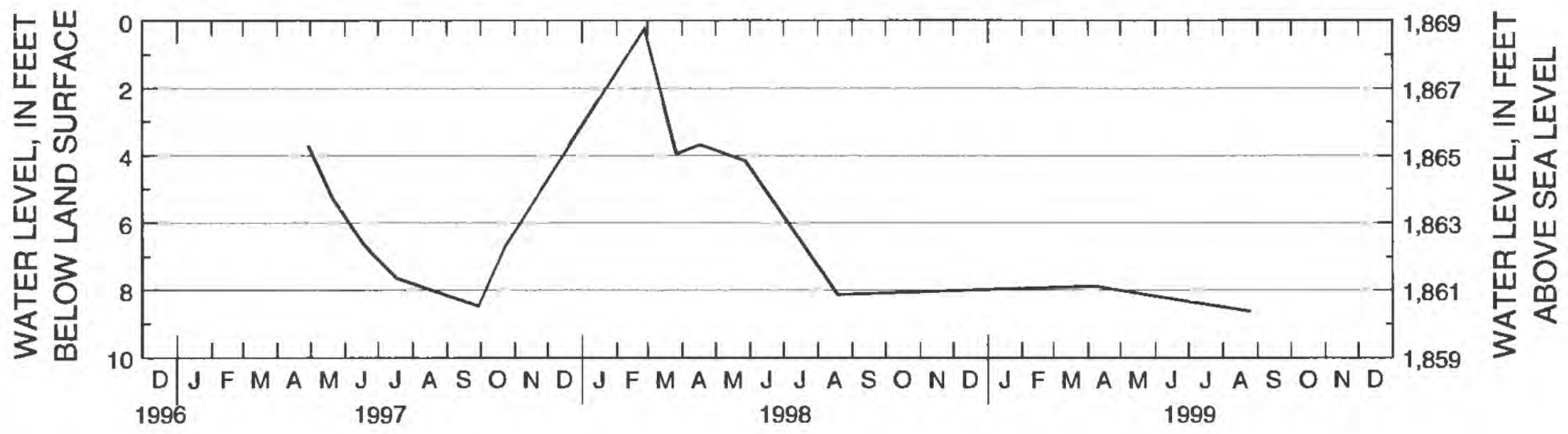

CALENDAR YEAR

Figure B22. Hydrograph for observation well 121N52W2CCCC R (site number 22). 
Site number from location map: 23

Local well number: 121N52W3CCBB R

Station identification number: 451716097095901

Other identifier: R20-84-25

County: Grant, South Dakota

Aquifer: Big Sioux

Altitude of land surface: $1,857.9$ feet

Measuring point: 3.0 feet

Extremes: April 29, 1997, to August 26, 1999: Highest, 6.74 feet, April 29, 1997; lowest, 12.8 feet, August 26, 1999.

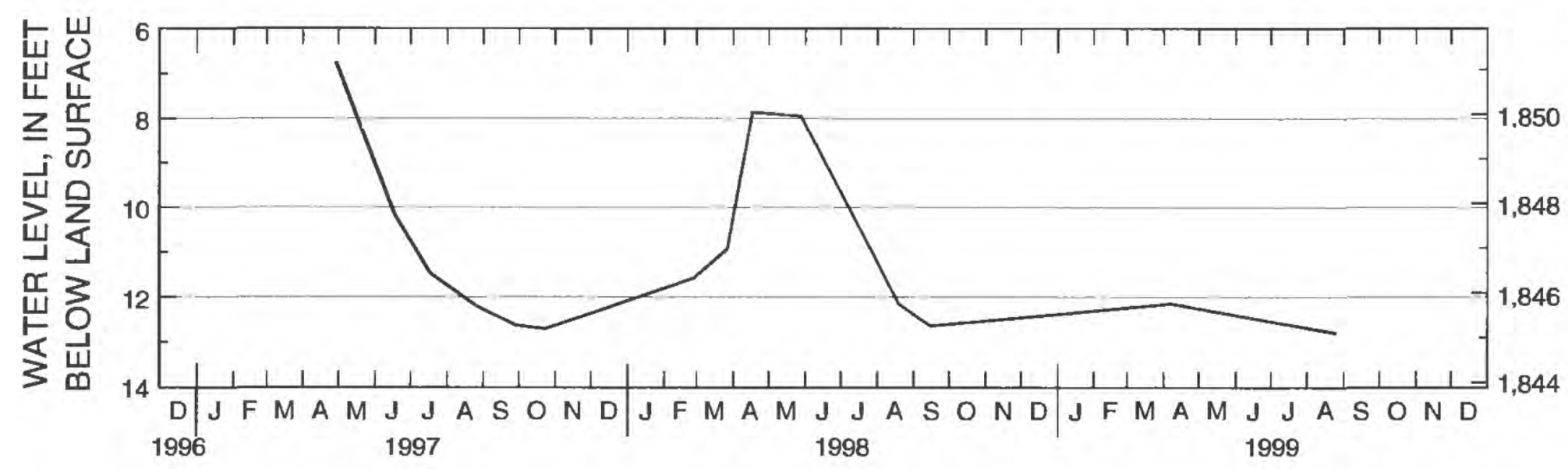

CALENDAR YEAR

Figure B23. Hydrograph for observation well 121N52W3CCBB R (site number 23).

Site number from location map: 24

Local well number: 121N52W4BBAB R

Station identification number: 451749097105501

Other identifier: R20-84-27

County: Grant, South Dakota

Aquifer: Big Sioux

Altitude of land surface: $1,843.7$ feet

Measuring point: 2.8 feet

Extremes: December 11, 1996, to August 26, 1999: Highest, 3.1 feet, April 29, 1997; lowest 13.81 feet, December 11, 1996.

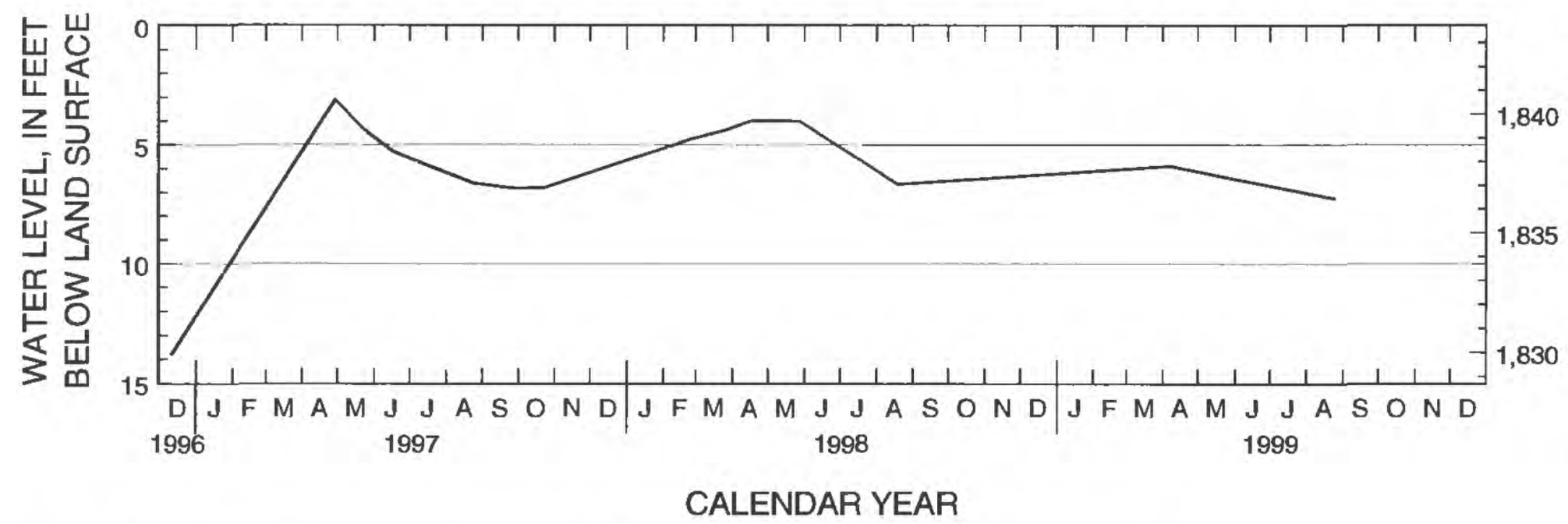

Figure B24. Hydrograph for observation well 121N52W4BBAB R (site number 24). 
Site number from location map: 25

Local well number: 121N52W8DCCC R

Station identification number: 451608097114101

Other identifier: GT-77B

County: Grant, South Dakota

Aquifer: Big Sioux

Altitude of land surface: $1,831.17$ feet

Measuring point: 2.6 feet

Extremes: December 1, 1977, to October 6, 1999: Highest, 4.7 feet, April 29, 1997; lowest, 9.8 feet, October 20, 1981.

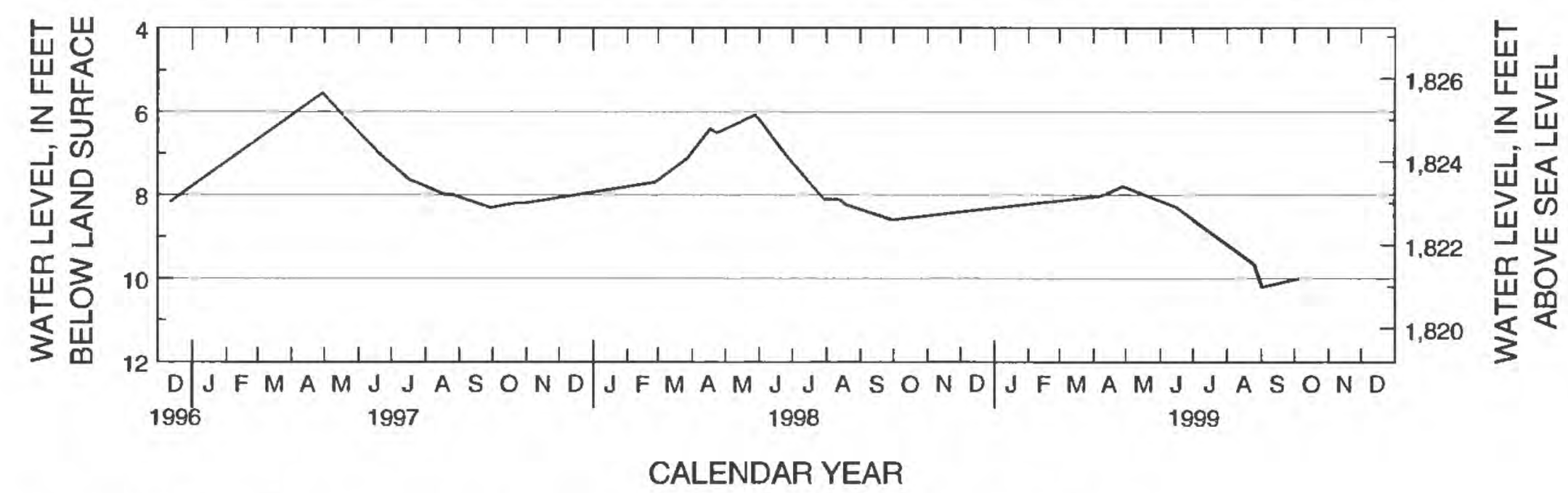

Figure B25. Hydrograph for observation well 121N52W8DCCC R (site number 25).

Site number from location map: 26

Local well number: 121N52W8DDDD R

Station identification number: 451608097110801

Other identifier: R20-84-30

County: Grant, South Dakota

Aquifer: Big Sioux

Altitude of land surface: $1,839.2$ feet

Measuring point: 3.0 feet

Extremes: April 29, 1997, to August 20, 1999: Highest, 11.39 feet, April 29, 1997; lowest 15.08 feet, August 20, 1999.
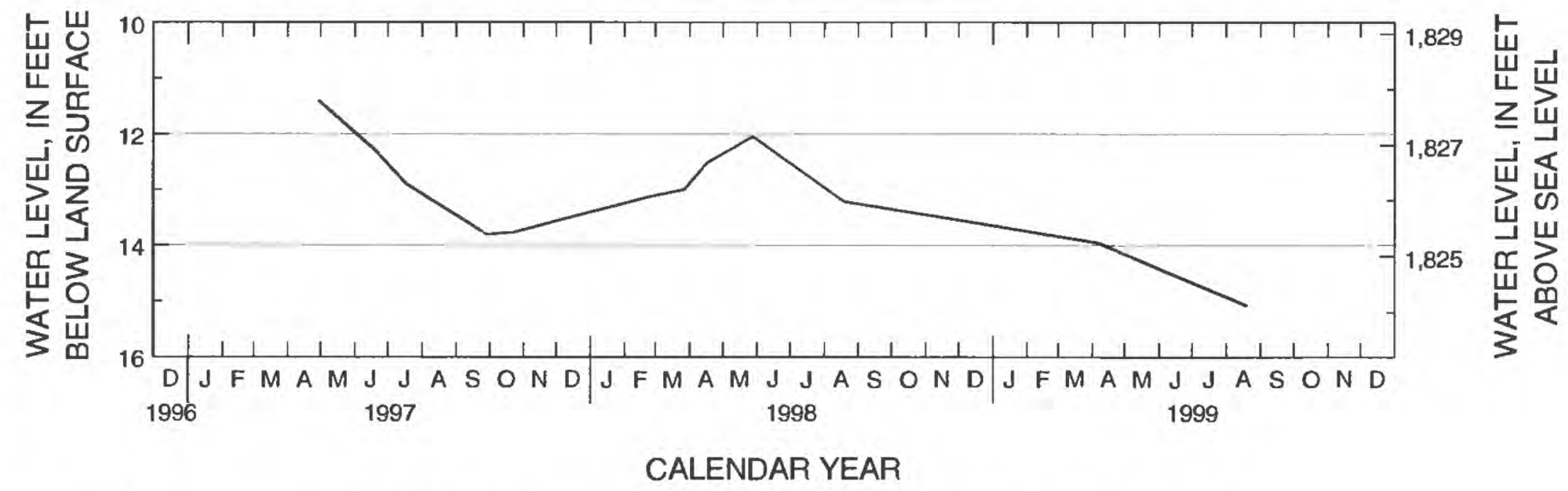

Figure B26. Hydrograph for observation well 121N52W8DDDD R (site number 26). 
Site number from location map: 27

Local well number: 121N52W13BBBA R

Station identification number: 451605097071701

Other identifier: GT-79A

County: Grant, South Dakota

Aquifer: Prairie Coteau

Altitude of land surface: $1,916.87$ feet

Measuring point: 2.2 feet

Extremes: June 9, 1982, to October 6, 1999: Highest, 66.6 feet, September 8, 1992; lowest, 85.4 feet, August 25, 1988.
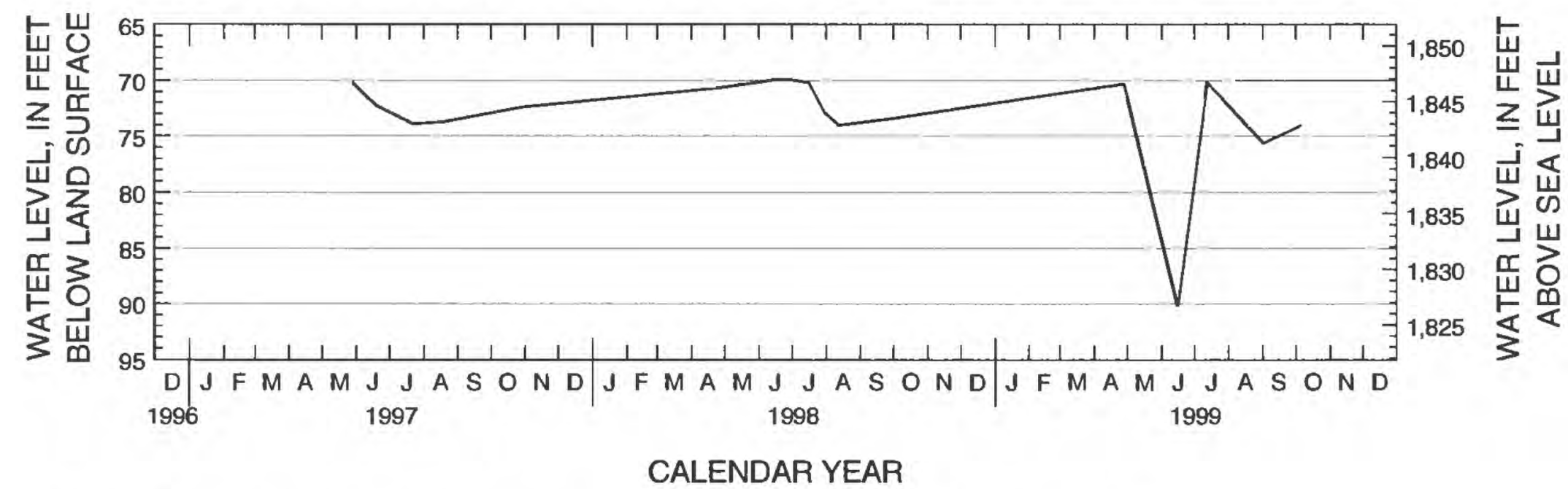

Figure B27. Hydrograph for observation well 121N52W13BBBA R (site number 27).

Site number from location map: 28

Local well number: $121 \mathrm{~N} 52 \mathrm{~W} 22 \mathrm{CCCC} \mathrm{R}$

Station identification number: 451423097095201

Other identifier: GT-77C

County: Grant, South Dakota

Aquifer: Prairie Coteau

Altitude of land surface: 1,837 feet

Measuring point: 3.0 feet

Extremes: December 1, 1977, to October 6, 1999: Highest, 2.9 feet, June 5, 10, 15, 1996; lowest, 20.8 feet, August 25, 1988.

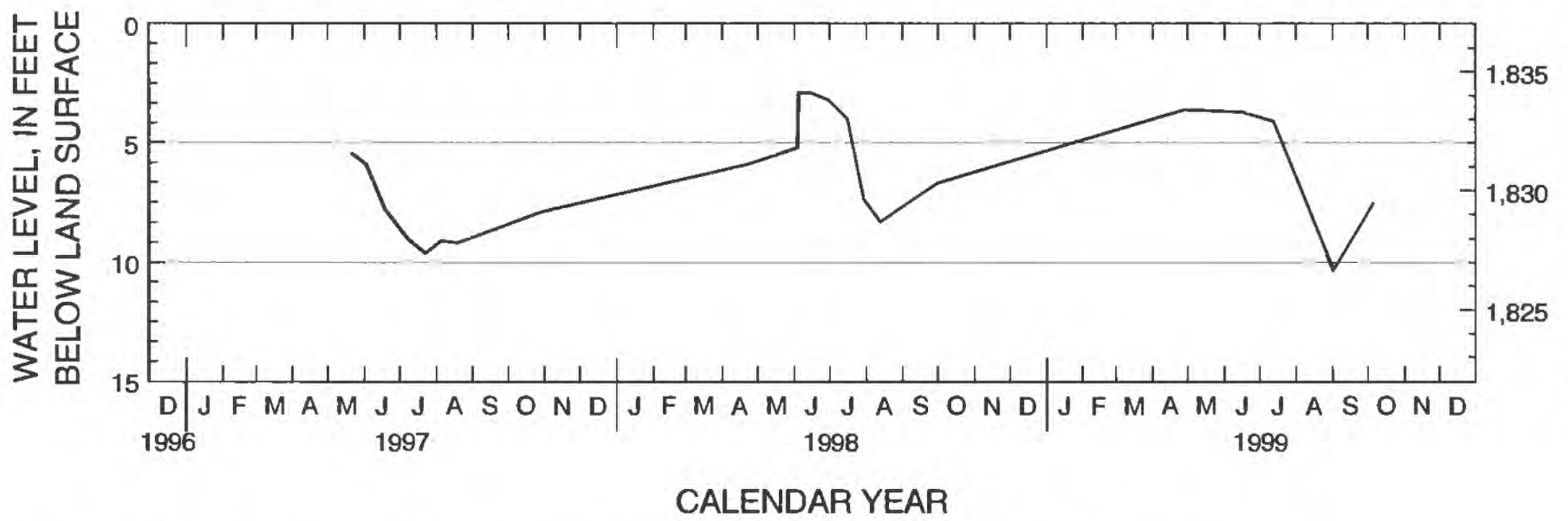

Figure B28. Hydrograph for observation well 121N52W22CCCC R (site number 28). 
Site number from location map: 29

Local well number: $121 \mathrm{~N} 52 \mathrm{~W} 25 \mathrm{CCCC} \mathrm{R}$

Station identification number: 451328097072301

Other identifier: GT-79B

County: Grant, South Dakota

Aquifer: Prairie Coteau

Altitude of land surface: 1,880 feet

Measuring point: 2.2 feet

Extremes: October 25, 1979, to October 6, 1999: Highest, 51.6 feet, June 6, 1996; lowest, 84.2 feet, July 24, 1988.

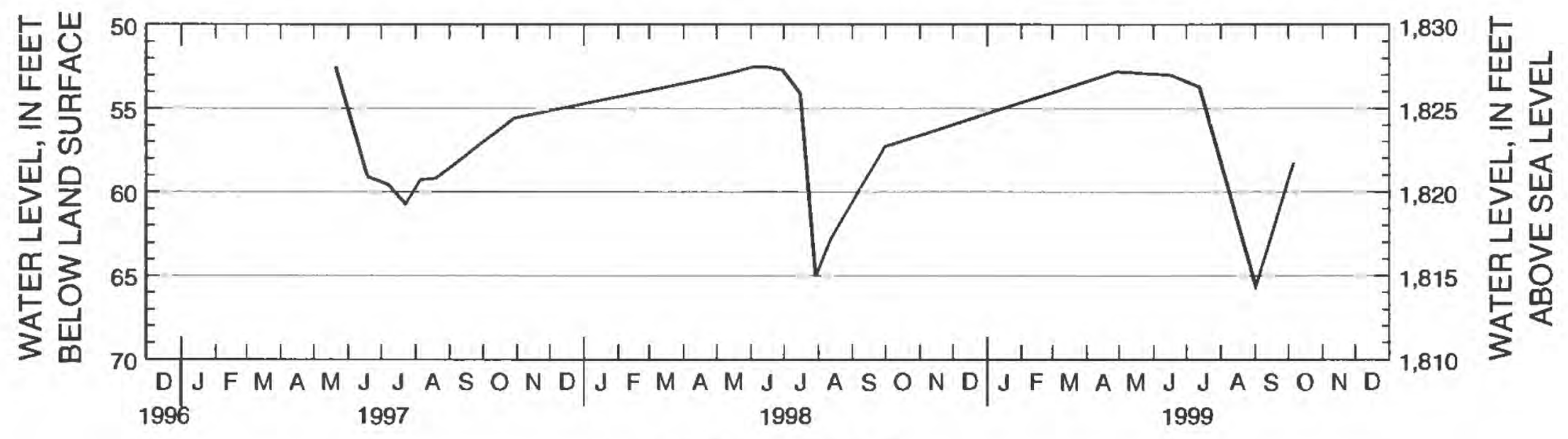

CALENDAR YEAR

Figure B29. Hydrograph for observation well 121N52W25CCCC R (site number 29).

Site number from location map: 30

Local well number: 121 N52W29CCBB R

Station identification number: 451340097121801

Other identifier: GT-77D

County: Grant, South Dakota

Aquifer: Big Sioux

Altitude of land surface: 1,860 feet

Measuring point: 1.6 feet

Extremes: December 1, 1977, to October 6, 1999: Highest, 1.4 feet, July 20, 1993; lowest, 12.1 feet, October 14, 1988.
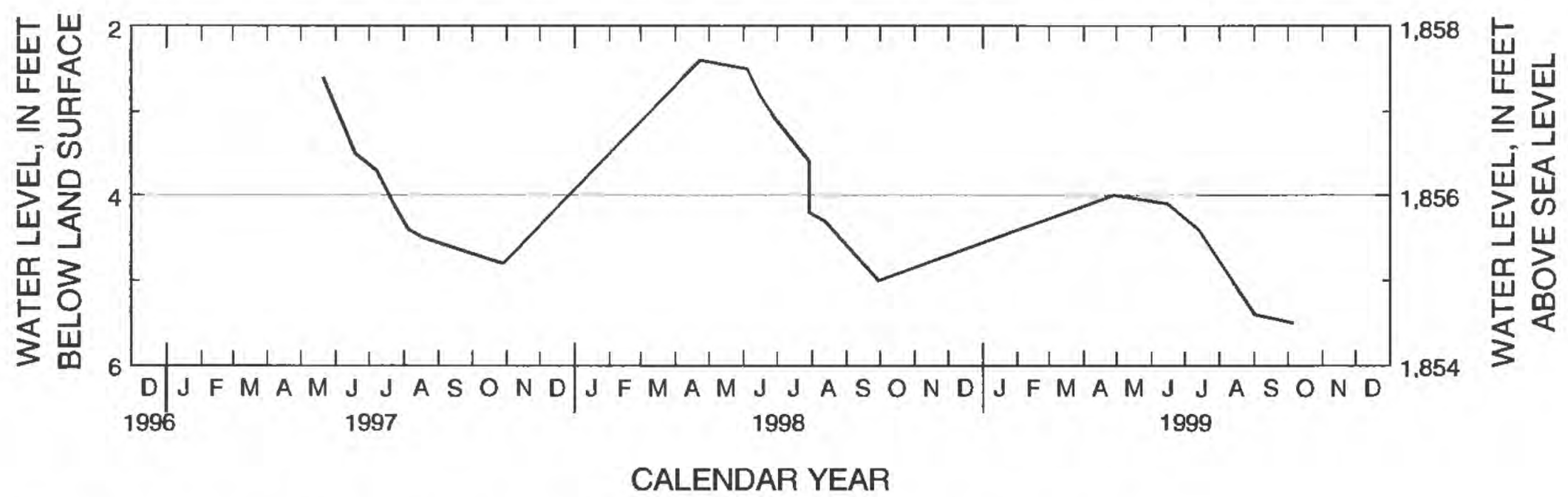

Figure B30. Hydrograph for observation well 121N52W29CCBB R (site number 30). 
Site number from location map: 31

Local well number: 121N54W34AADA

Station identification number: 451506097170401

Other identifier: DA-78E

County: Day, South Dakota

Aquifer: Prairie Coteau

Altitude of land surface: 1,803 feet

Measuring point: 3.0 feet

Extremes: September 27, 1978, to October 6, 1999: Highest, 4.5 feet, June 4. 1998; lowest, 24.7 feet, August 19, 1982.

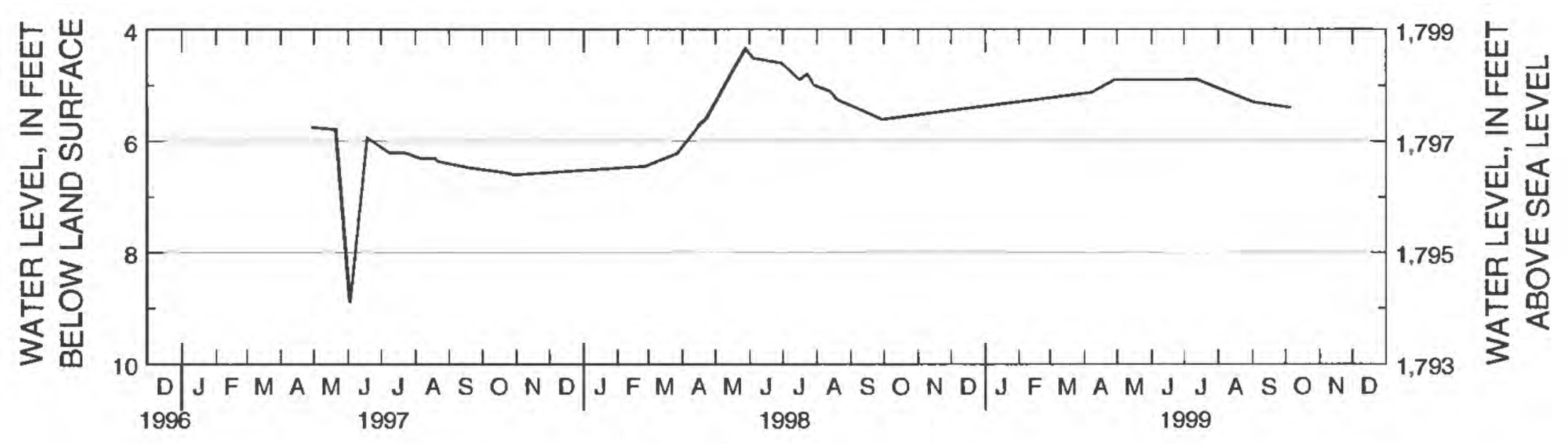

CALENDAR YEAR

Figure B31. Hydrograph for observation well 121N54W34AADA (site number 31).

Site number from location map: 32

Local well number: 122N47W35DDCD

Station identification number: 451935096310001

Other identifier: R2-85-28

County: Roberts, South Dakota

Aquifer: Wilmot

Altitude of land surface: 1,100 feet

Measuring point: 3.0 feet

Extremes: December 11, 1996, to August 25, 1999: Highest, 23.53 feet, August 25, 1999; lowest, 26.82, April 1, 1999.

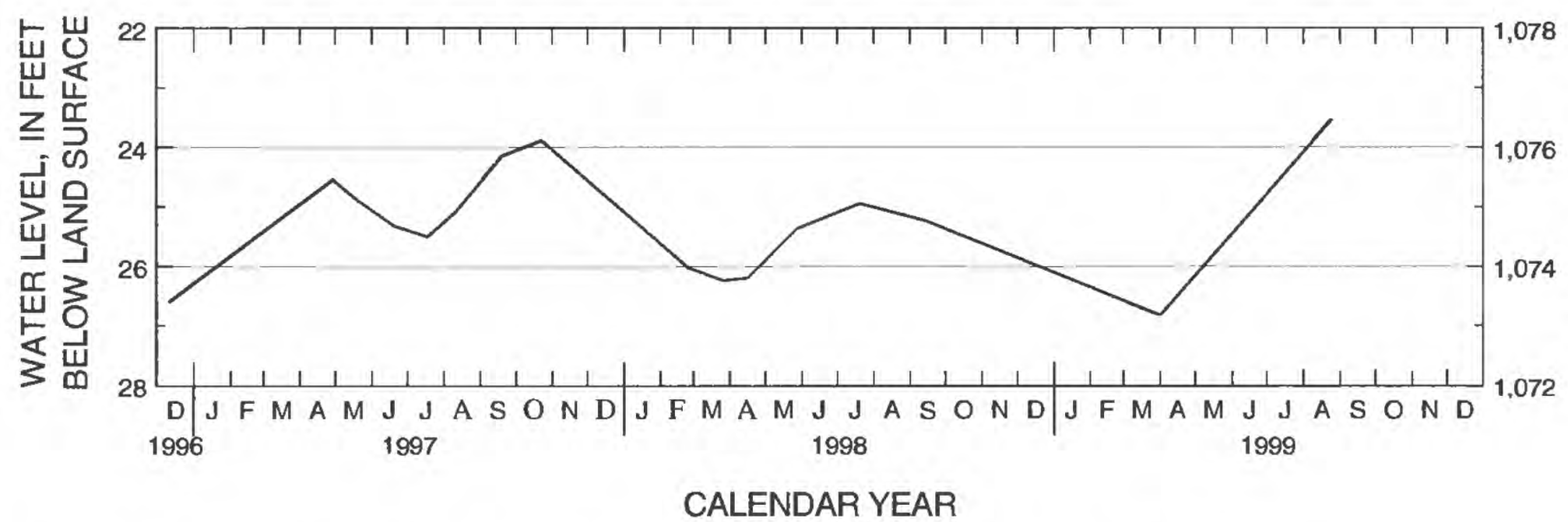

Figure B32. Hydrograph for observation well 122N47W35DDCD (site number 32). 
Site number from location map: 33

Local well number: 122N48W13DDDD2

Station identification number: 452211096365902

Other identifier: R2-98-15

County: Roberts, South Dakota

Aquifer: Undetermined

Altitude of land surface: $1,085.9$ feet

Measuring point: 2.25 feet

Extremes: April 1, 1999, to September 13, 1999: Highest, 18.96 feet, April 1, 1999; lowest, 19.89 feet,

September 13, 1999.

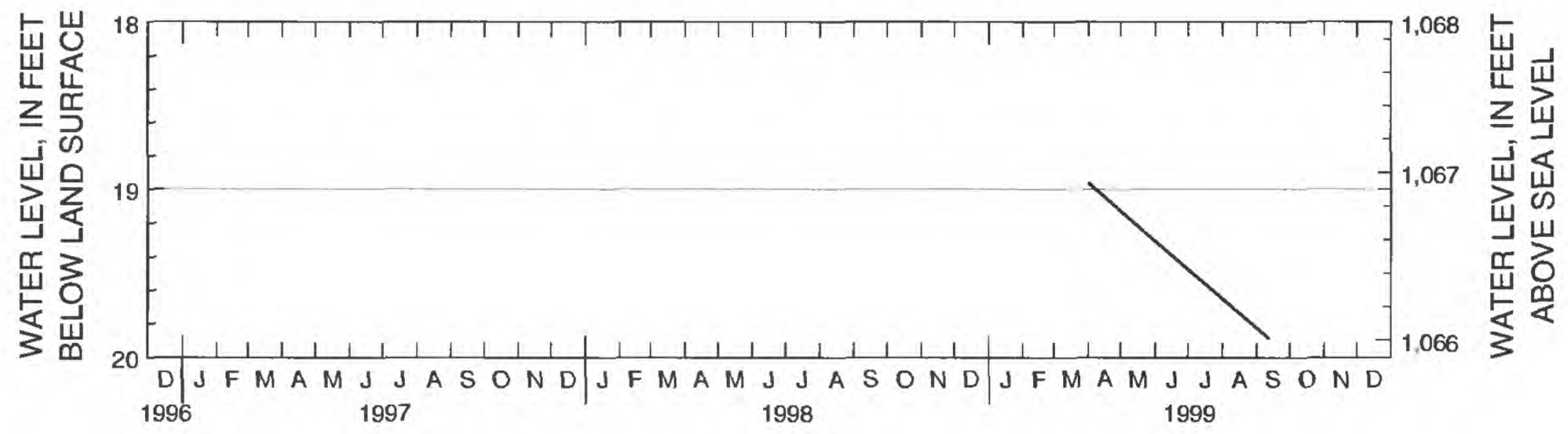

CALENDAR YEAR

Figure B33. Hydrograph for observation well 122N48W13DDDD2 R (site number 33).

Site number from location map: 34

Local well number: 122N49W7BBBB

Station identification number: 452352096513901

Other identifier: RB-77N

County: Roberts, South Dakota

Aquifer: Revillo

Altitude of land surface: 1,199.9 feet

Measuring point: 1.7 feet

Extremes: November 30, 1977, to October 5, 1999: Highest, 23.74 feet, April 29, 1997; lowest, 43.2 feet, July 28, 1988.

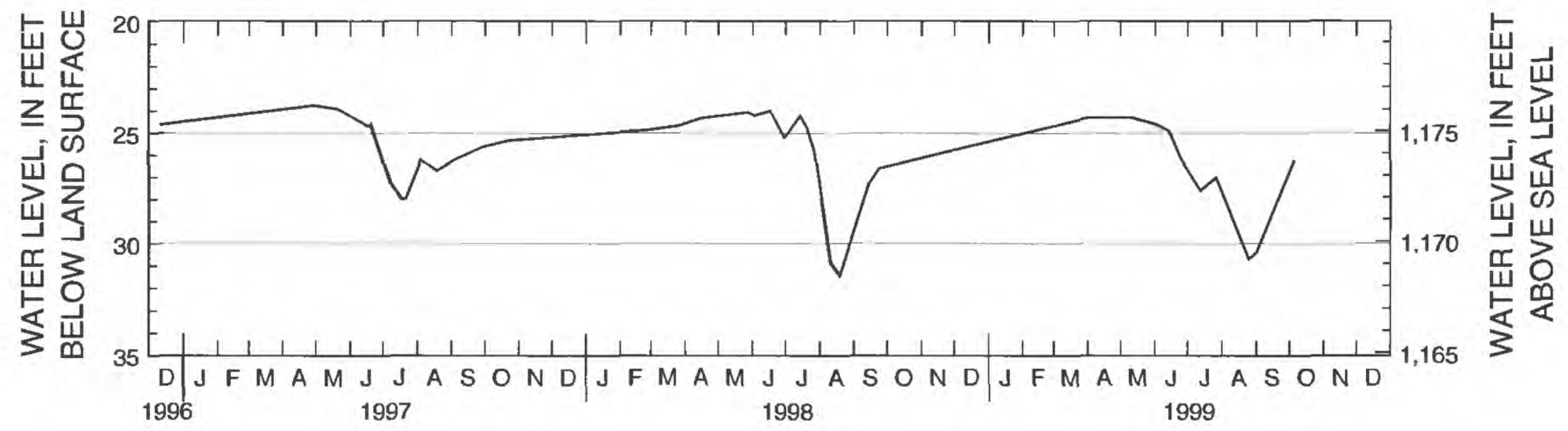

CALENDAR YEAR

Figure B34. Hydrograph for observation well 122N49W7BBBB (site number 34). 
Site number from location map: 35

Local well number: 122N49W17AAAD

Station identification number: 452257096491801

Other identifier: RB-77P

County: Roberts, South Dakota

Aquifer: Wilmot

Altitude of land surface: 1,163.9 feet

Measuring point: 2.4 feet

Extremes: November 30, 1977, to October 5, 1996: Highest, 6.7 feet, April 26, 1994; lowest, 12.1 feet, September 8, 1988.

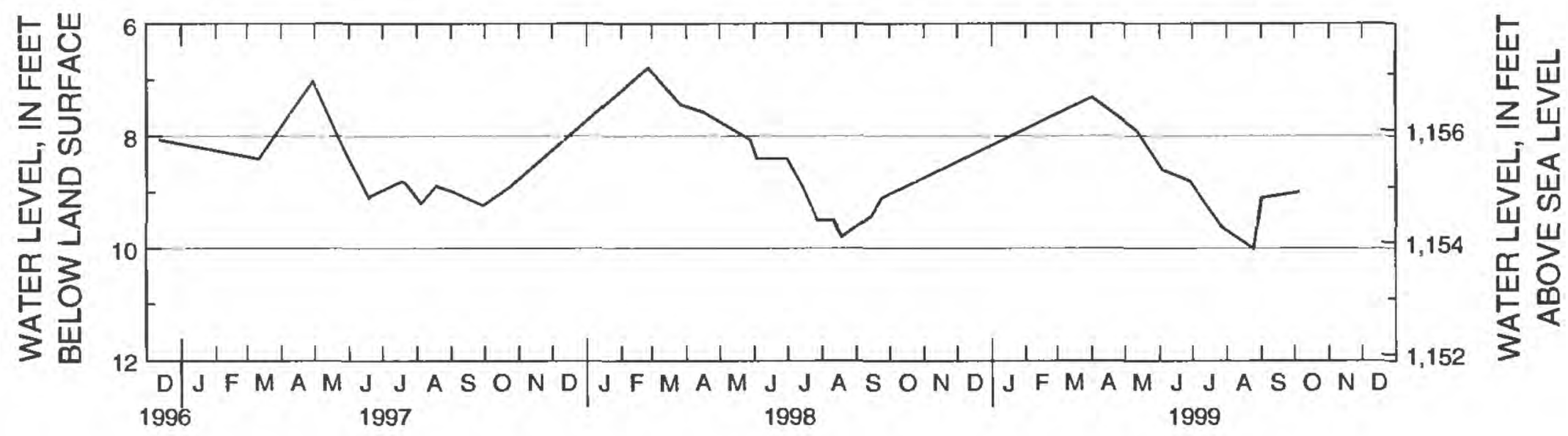

CALENDAR YEAR

Figure B35. Hydrograph for observation well 122N49W17AAAD (site number 35).

Site number from location map: 36

Local well number: 122N49W18AAAA

Station identification number: 452302096503101

Other identifier: RB-77O

County: Roberts, South Dakota

Aquifer: Wilmot

Altitude of land surface: $1,184.2$ feet

Measuring point: 1.5 feet

Extremes: November 30, 1977, to October 5, 1999: Highest, 8.16 feet, Apr. 29, 1997; lowest, 25.8 feet, July 28, 1988.

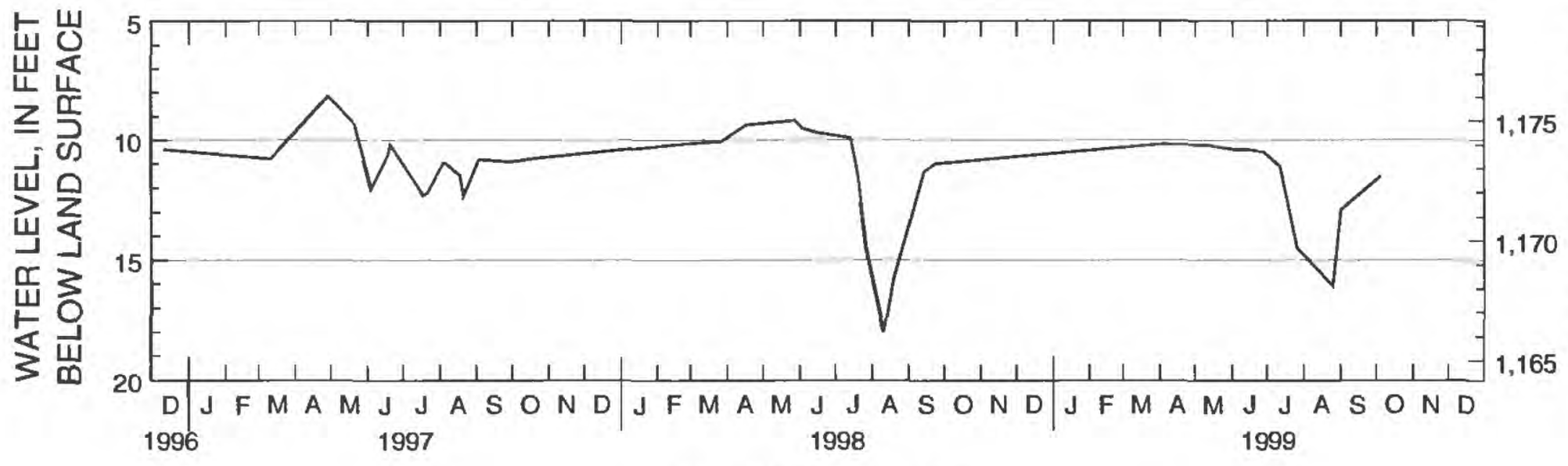

CALENDAR YEAR

Figure B36. Hydrograph for observation well 122N49W18AAAA (site number 36). 
Site number from location map: 37

Local well number: 122 N49W30BBBB

Station identification number: 452117096514201

Other identifier: RB-77Q

County: Roberts, South Dakota

Aquifer: Revillo

Altitude of land surface: $1,248.3$ feet

Measuring point: 1.0 foot

Extremes: November 30, 1977, to October 5, 1999: Highest, 42.02 feet, May 21, 1997; lowest, 49.1 feet,

August 18, 1982, September 1, 1982.
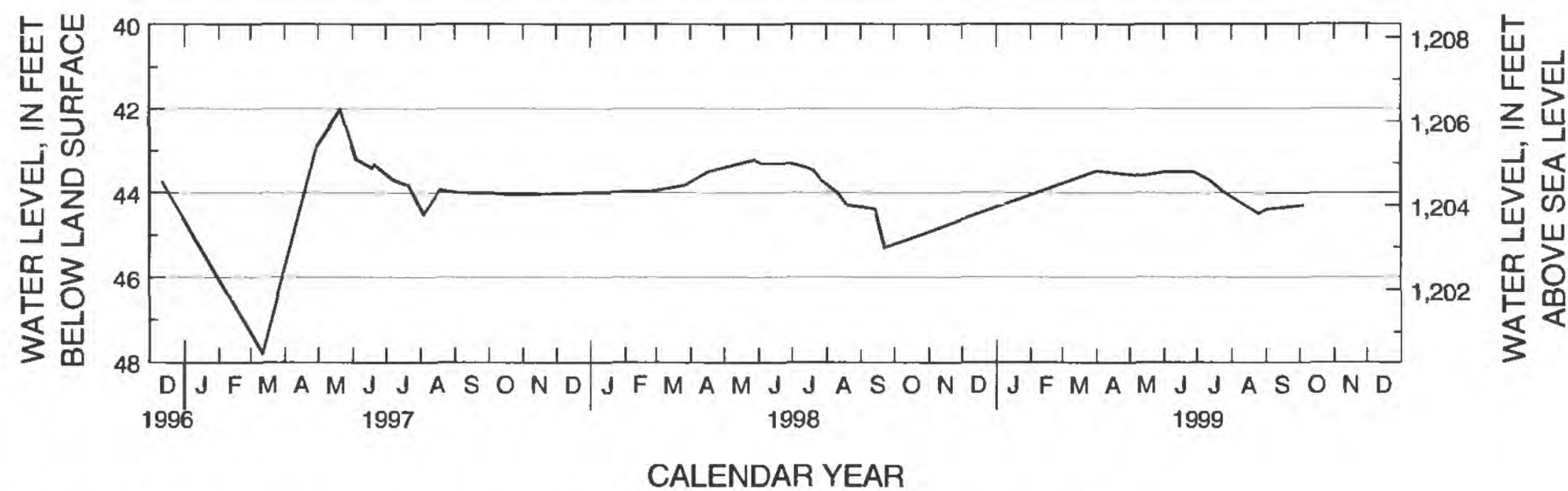

Figure B37. Hydrograph for observation well 122N49W30BBBB (site number 37).

Site number from location map: 38

Local well number: 122N50W13DDDC

Station identification number: 452210096514701

Other identifier: R2-97-46

County: Roberts, South Dakota

Aquifer: Wilmot

Altitude of land surface: 1,228 feet

Measuring point: 1.5 feet

Extremes: October 23, 1997, to August 25, 1999: Highest 19.52 feet, May 28, 1998; lowest 21.36 feet, October 23, 1997.



CALENDAR YEAR

Figure B38. Hydrograph for observation well 122N50W13DDDC (site number 38). 
Site number from location map: 39

Local well number: 122N51W4DDDD2 R

Station identification number: 452212097022702

Other identifier: R2-99-11

County: Roberts, South Dakota

Aquifer: Eden

Altitude of land surface: $1,962.2$ feet

Measuring point: 2.0 feet

Extremes: September 14, 1999, to December 12, 1999: Highest, 208.35 feet, September 14, 1999; lowest, 208.73 feet, December 8, 1999.

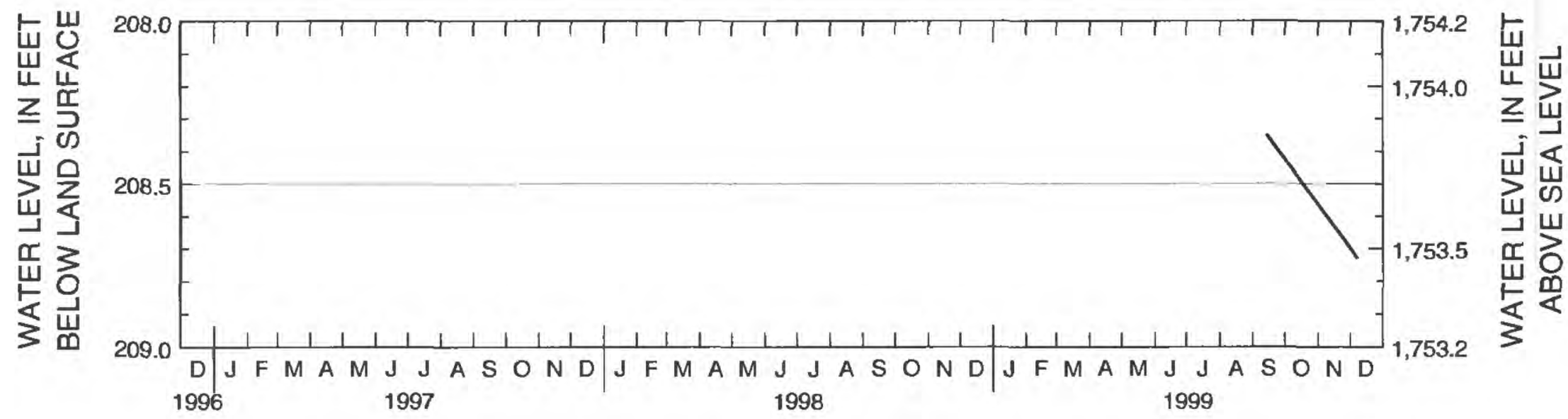

CALENDAR YEAR

Figure B39. Hydrograph for observation well 122N51W4DDDD2 R (site number 39).

Site number from location map: 40

Local well number: 122N51W27BBBB2 R

Station identification number: 451936097022602

Other identifier: R2-98-27

County: Roberts, South Dakota

Aquifer: Prairie Coteau

Altitude of land surface: 2,025.4 feet

Measuring point: 2.25 feet

Extremes: March 11, 1999, to September 14, 1999: Highest, 220.92 feet, March 11, 1999; lowest 221.01 feet, September 14, 1999.

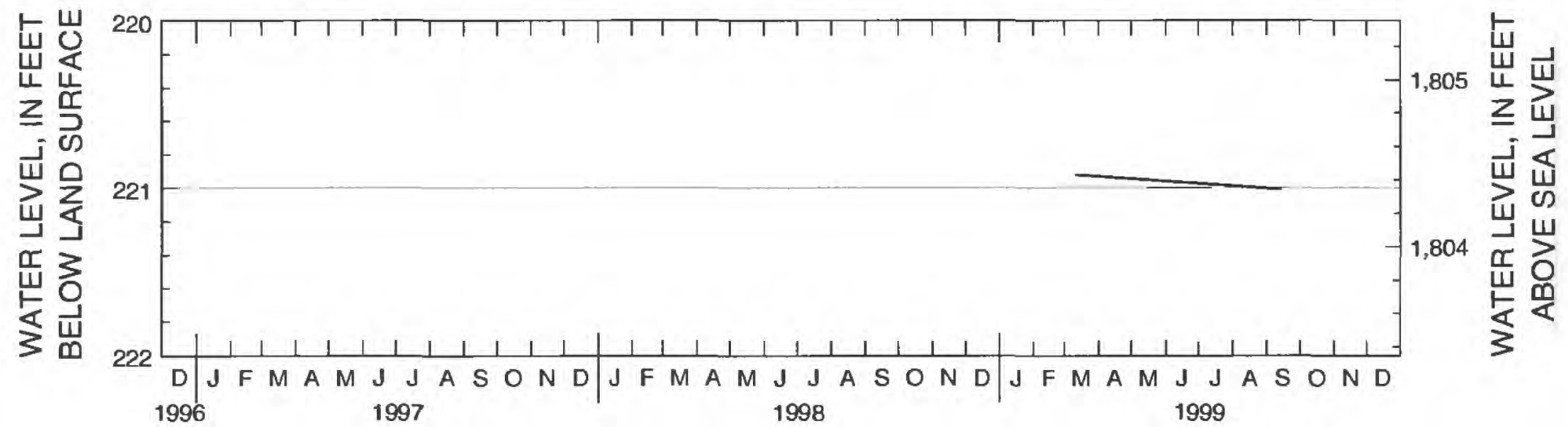

CALENDAR YEAR

Figure B40. Hydrograph for observation well 122N51W27BBBB2 R (site number 40).

Site number from location map: 41 
Local well number: 122N52W1DDCC R

Station identification number: 452213097063101

Other identifier: R2-98-31

County: Roberts, South Dakota

Aquifer: Eden

Altitude of land surface: $1,946.1$ feet

Measuring point: 3.4 feet

Extremes: March 11, 1999, to September 15, 1999: Highest, 164.67 feet, September 15, 1999; lowest, 164.7 feet, March 11, 1999.

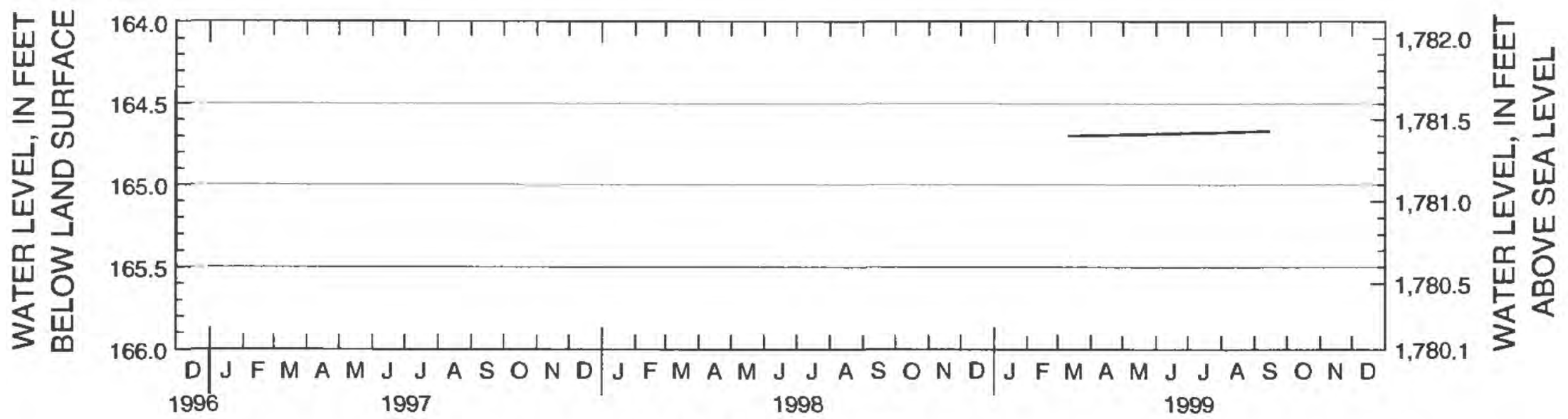

CALENDAR YEAR

Figure B41. Hydrograph for observation well 122N52W1DDCC R (site number 41).

Site number from location map: 42

Local well number: 122N52W2ABBB R

Station identification number: 452304097080201

Other identifier: CO-93-11

County: Roberts, South Dakota

Aquifer: Coteau Lakes

Altitude of land surface: 1,905 feet

Measuring point: 2.4 feet

Extremes: December 12, 1996, to August 25, 1999: Highest, -0.12 foot, March 27, 1998; lowest, 2.11 feet, December 12, 1996.

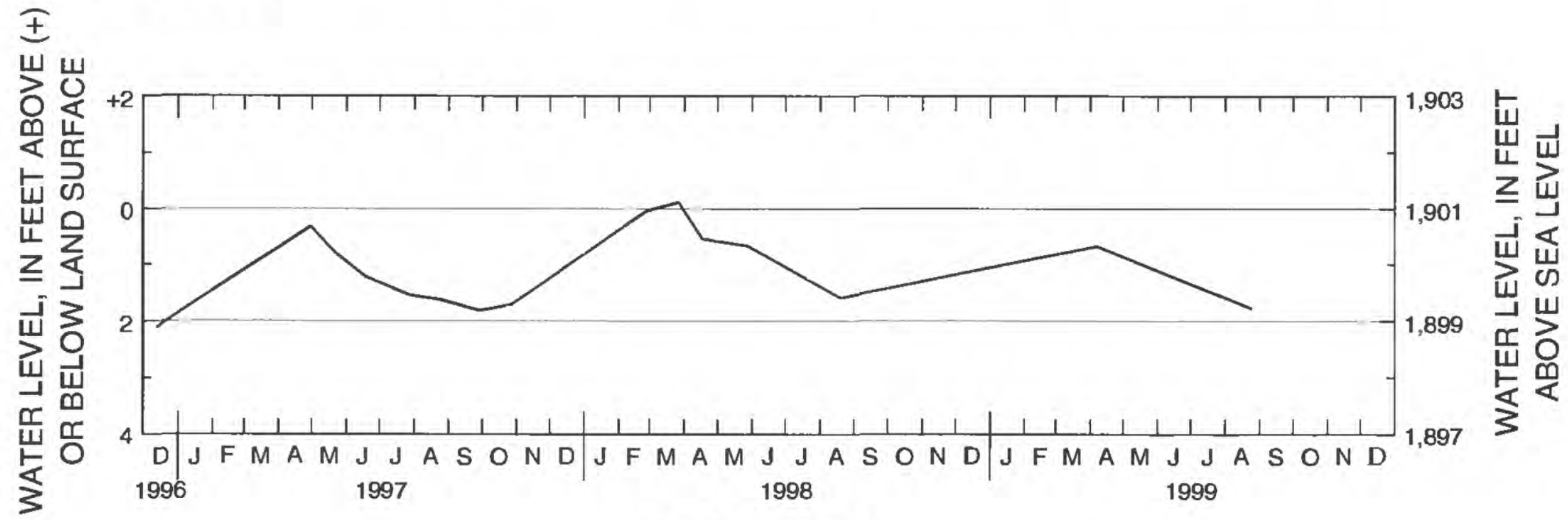

\section{CALENDAR YEAR}

Figure B42. Hydrograph for observation well 122N52W2ABBB R (site number 42). 
Site number from location map: 43

Local well number: 122N52W2BBBB R

Station identification number: 452304097083901

Other identifier: RB-77T

County: Roberts, South Dakota

Aquifer: Coteau Lakes

Altitude of land surface: $1,910.9$ feet

Measuring point: 2.1 feet

Extremes: November 30, 1977, to October 5, 1999: Highest, 10.02 feet, April 30, 1997; lowest, 23.20 feet, November 30, 1977.
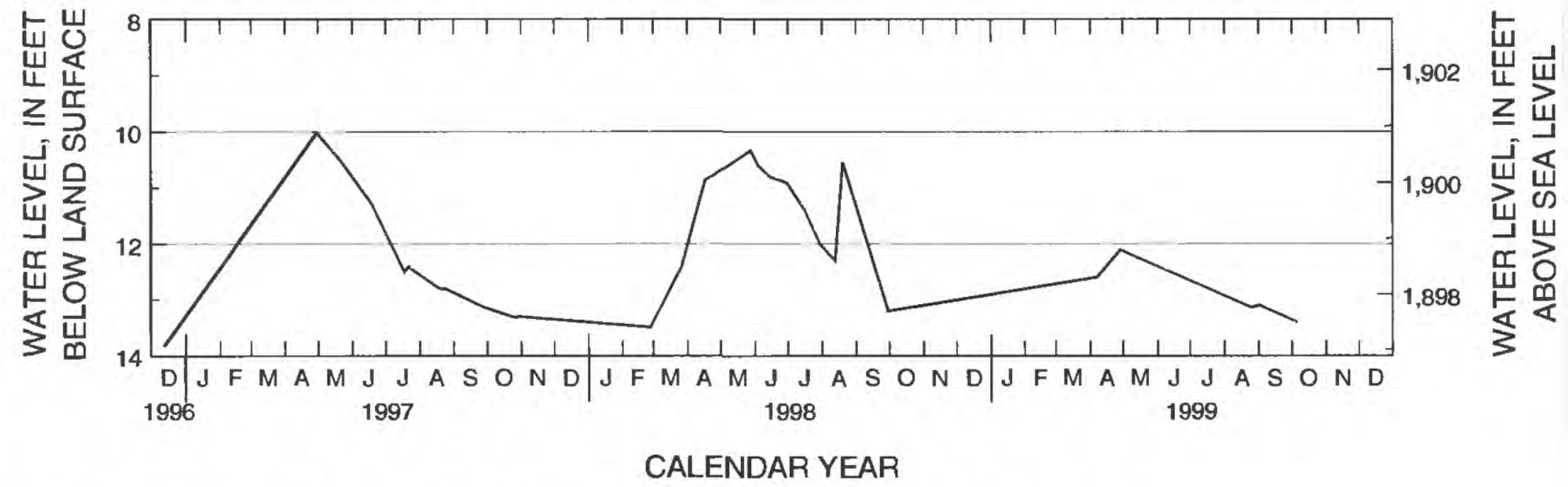

Figure B43. Hydrograph for observation well 122N52W2BBBB R (site number 43).

Site number from location map: 44

Local well number: 122N52W3ABBB R

Station identification number: 452304097091501

Other identifier: CO-93-36

County: Roberts, South Dakota

Aquifer: Coteau Lakes

Altitude of land surface: 1,908 feet

Measuring point: 2.3 feet

Extremes: December 12, 1996, to August 26, 1999: Highest, 12.0 feet, April 30, 1997; lowest 16.29 feet, February 26, 1998.

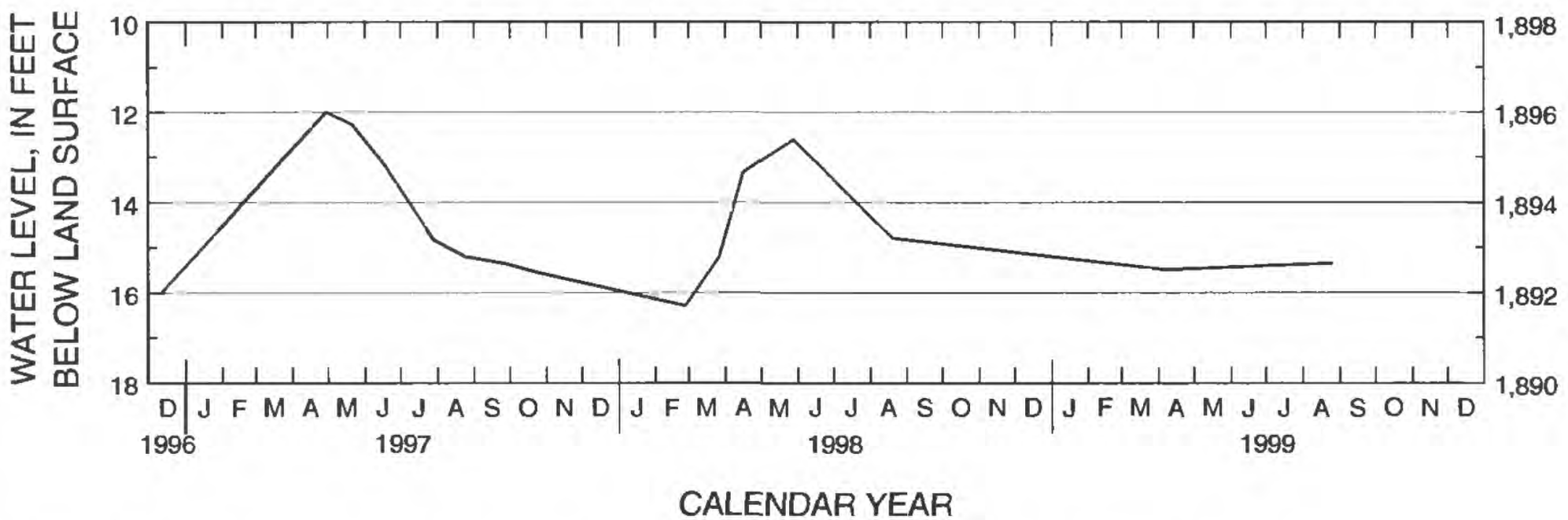

Figure B44. Hydrograph for observation well 122N52W3ABBB R (site number 44). 
Site number from location map: 45

Local well number: 122N52W3CCDD R

Station identification number: 452214097092701

Other identifier: CO-93-37

County: Roberts, South Dakota

Aquifer: Coteau Lakes

Altitude of land surface: 1,890 feet

Measuring point: 2.2 feet

Extremes: December 12, 1996, to August 26, 1999: Highest, 15.13 feet, April 30, 1997; lowest, 18.08 feet, August 26, 1999.

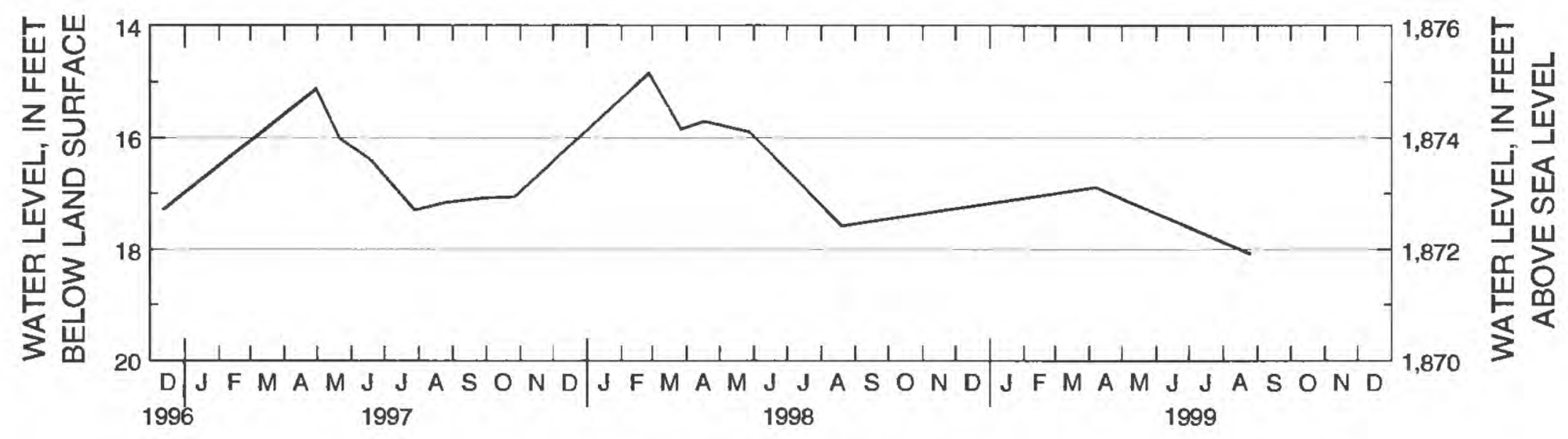

CALENDAR YEAR

Figure B45. Hydrograph for observation well 122N52W3CCDD R (site number 45).

Site number from location map: 46

Local well number: 122N52W3DAAA R

Station identification number: 452238097084001

Other identifier: CO-93-35

County: Roberts, South Dakota

Aquifer: Coteau Lakes

Altitude of land surface: 1,910 feet

Measuring point: 2.0 feet

Extremes: April 30, 1997, to August 26, 1999: Highest, 13.0 feet, April 30, 1997; lowest, 16.81 feet, February 26, 1998.

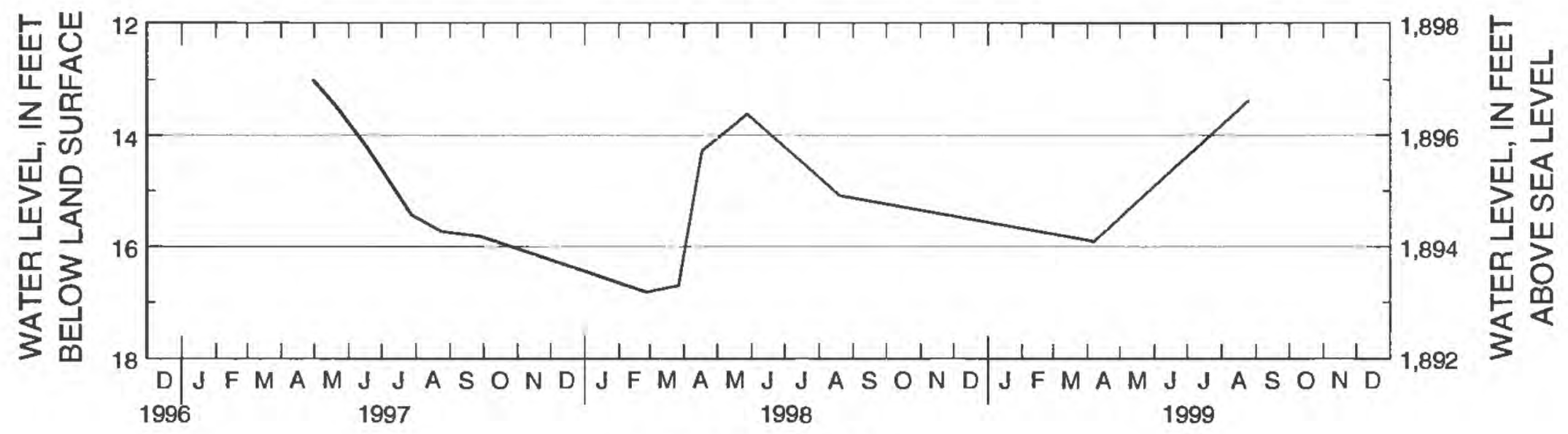

CALENDAR YEAR

Figure B46. Hydrograph for observation well 122N52W3DAAA R (site number 46). 
Site number from location map: 47

Local well number: 122N52W5CCCC R

Station identification number: 452215097121801

Other identifier: RB-77R

County: Roberts, South Dakota

Aquifer: Coteau Lakes

Altitude of land surface: $1,871.7$ feet

Measuring point: 1.5 feet

Extremes: November 30, 1977, to October 6, 1999: Highest, 7.85 feet, April 30, 1997; lowest, 16.4 feet, April 1, 1982.

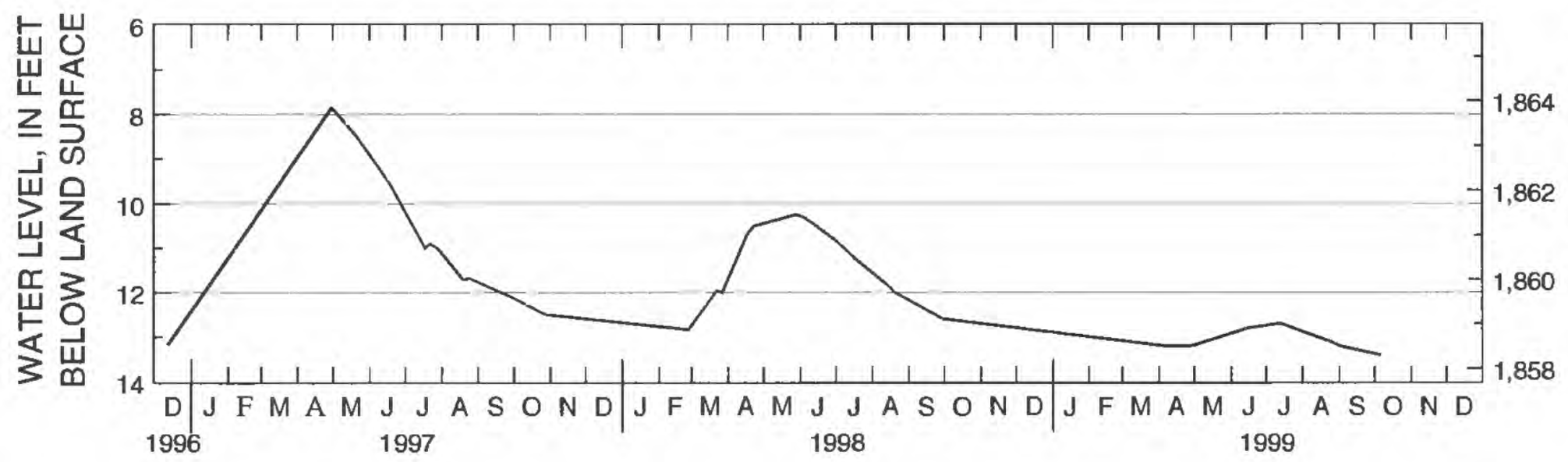

CALENDAR YEAR

Figure B47. Hydrograph for observation well 122N52W5CCCC R (site number 47).

Site number from location map: 48

Local well number: 122N52W9AAAA2 R

Station identification number: 452213097095602

Other identifier: CO-93-31

County: Roberts, South Dakota

Aquifer: Coteau Lakes

Altitude of land surface: 1,872 feet

Measuring point: 2.1 feet

Extremes: December 12, 1996, to August 26, 1999: Highest, 1.42 feet, February 26, 1998: lowest, 3.17 feet, August 26, 1999.

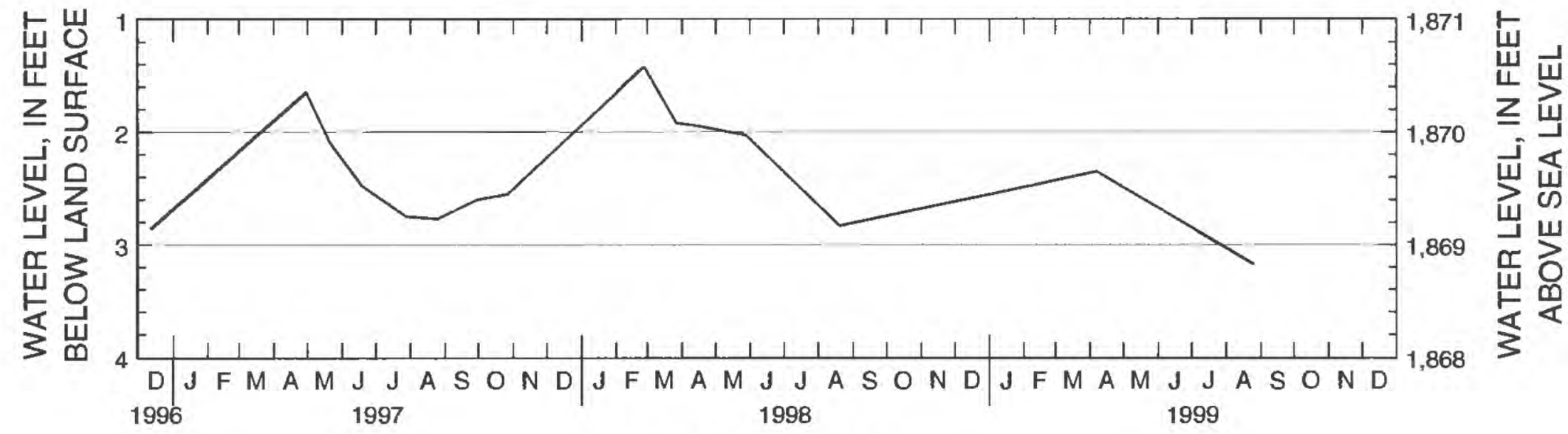

CALENDAR YEAR

Figure B48. Hydrograph for observation well 122N52W9AAAA2 R (site number 48). 
Site number from location map: 49

Local well number: 122N52W11BBBB R

Station identification number: 452212097083901

Other identifier: RB-93C

County: Roberts, South Dakota

Aquifer: Prairie Coteau

Altitude of land surface: 1,921 feet

Measuring point: 2.3 feet

Extremes: December 12, 1996, to October 6, 1999: Highest, 37.18 feet, May 21, 1997; lowest 39.82 feet, February 26, 1998.

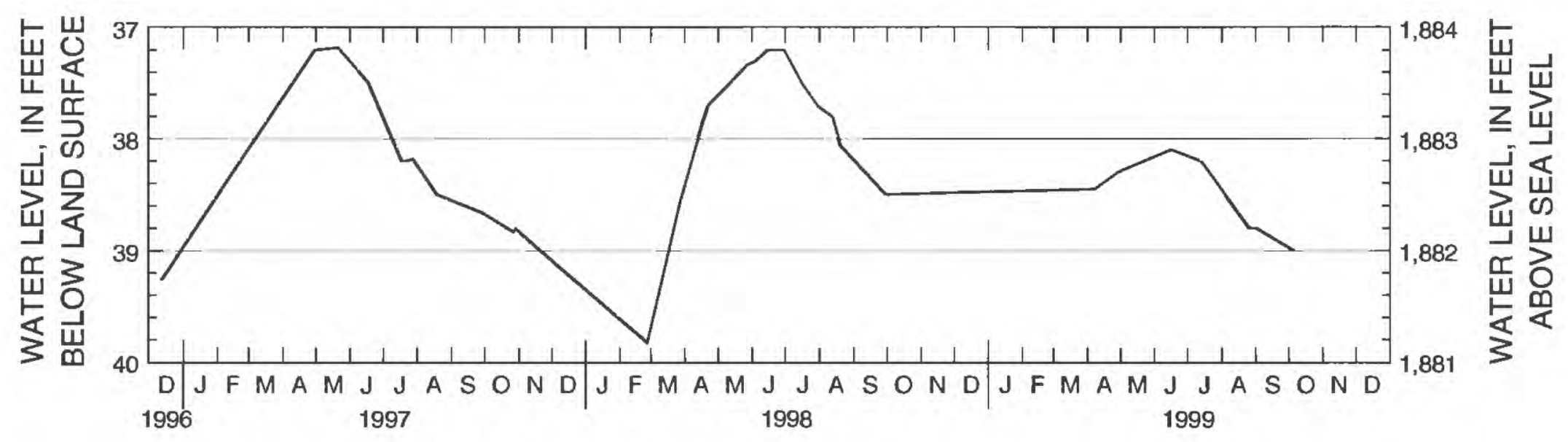

CALENDAR YEAR

Figure B49. Hydrograph for observation well 122N52W11BBBB R (site number 49).

Site number from location map: 50

Local well number: $122 \mathrm{~N} 52 \mathrm{~W} 12 \mathrm{CCCC} \mathrm{R}$

Station identification number: 452122097072301

Other identifier: RB-76B

County: Roberts, South Dakota

Aquifer: Coteau Lakes

Altitude of land surface: $1,904.9$ feet

Measuring point: 2.3 feet

Extremes: December 1, 1976, to October 9, 1996: Highest, -1.08 feet, March 26, 1998; lowest, 13.0 feet, May 7, 1991.

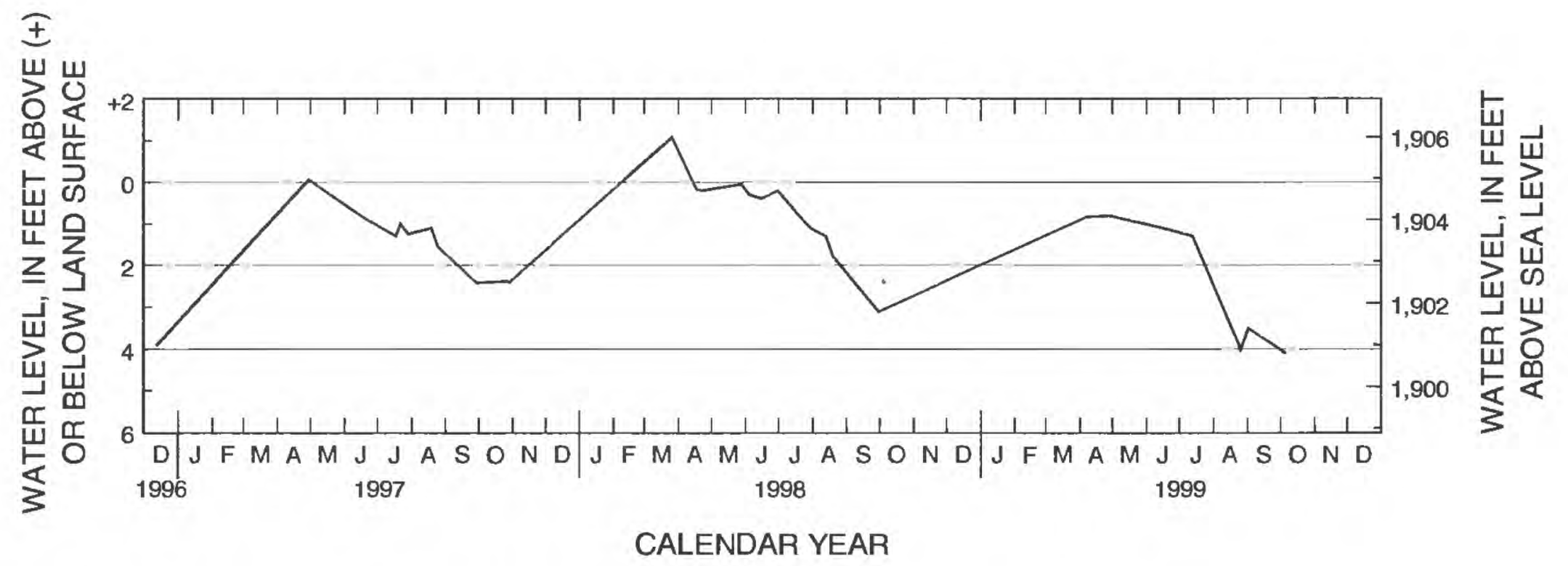

Figure B50. Hydrograph for observation well 122N52W12CCCC R (site number 50). 
Site number from location map: 51

Local well number: 122N52W14BBBB R

Station identification number: 452119097083901

Other identifier: RB-76A

County: Roberts, South Dakota

Aquifer: Coteau Lakes

Altitude of land surface: $1,897.9$ feet

Measuring point: 2.9 feet

Extremes: December 1, 1976, to October 6, 1999: Highest, 10.1 feet, October 21, 1993; lowest, 22.8 feet,

October 11, 1983, November 9, 1983.

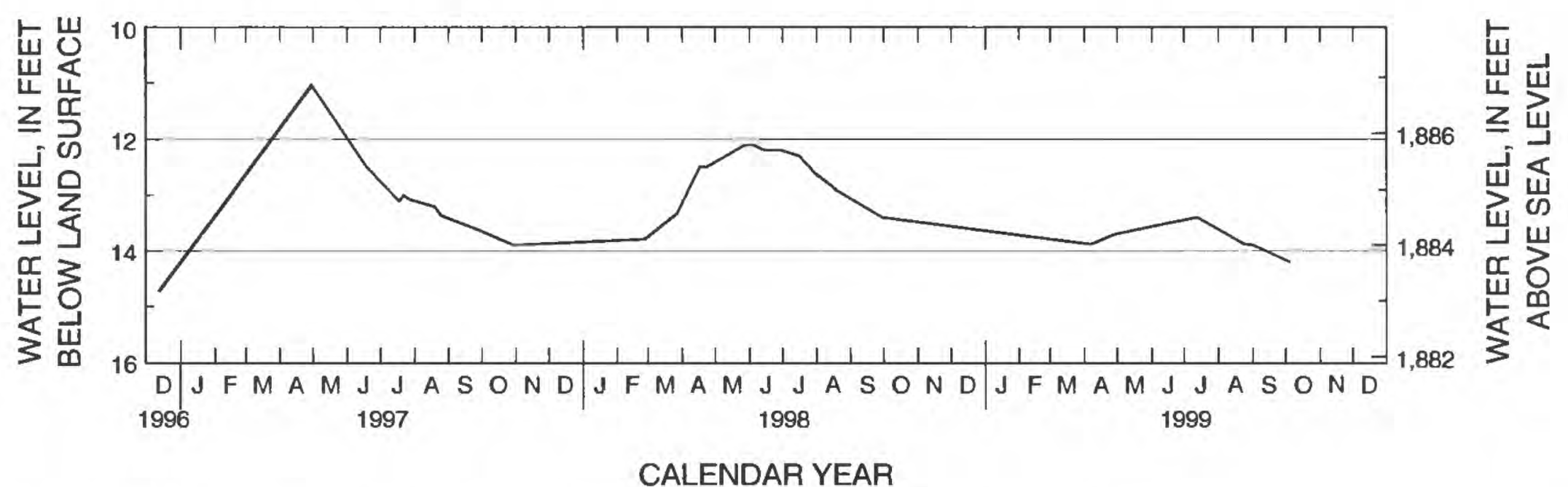

Figure B51. Hydrograph for observation well 122N52W14BBBB R (site number 51).

Site number from location map: 52

Local well number: 122N52W16BBBC R

Station identification number: 452115097110601

Other identifier: RB-82B

County: Roberts, South Dakota

Aquifer: Coteau Lakes

Altitude of land surface: $1,855.5$ feet

Measuring point: 2.7 feet

Extremes: June 29, 1982, to October 6, 1999: Highest, 12.1 feet, August 4, 1993; lowest, 14.8 feet, October 11, 1983.



CALENDAR YEAR

Figure B52. Hydrograph for observation well 122N52W16BBBC R (site number 52). 
Site number from location map: 53

Local well number: 122N52W27BBBB2 R

Station identification number: 451934097095302

Other identifier: R2-98-30

County: Roberts, South Dakota

Aquifer: Lonesome Lake

Altitude of land surface: $1,878.4$ feet

Measuring point: 2.3 feet

Extremes: March 11, 1999, to September 14, 1999: Highest, 51.14 feet, March 11, 1999; lowest, 51.54 feet, September 14, 1999.

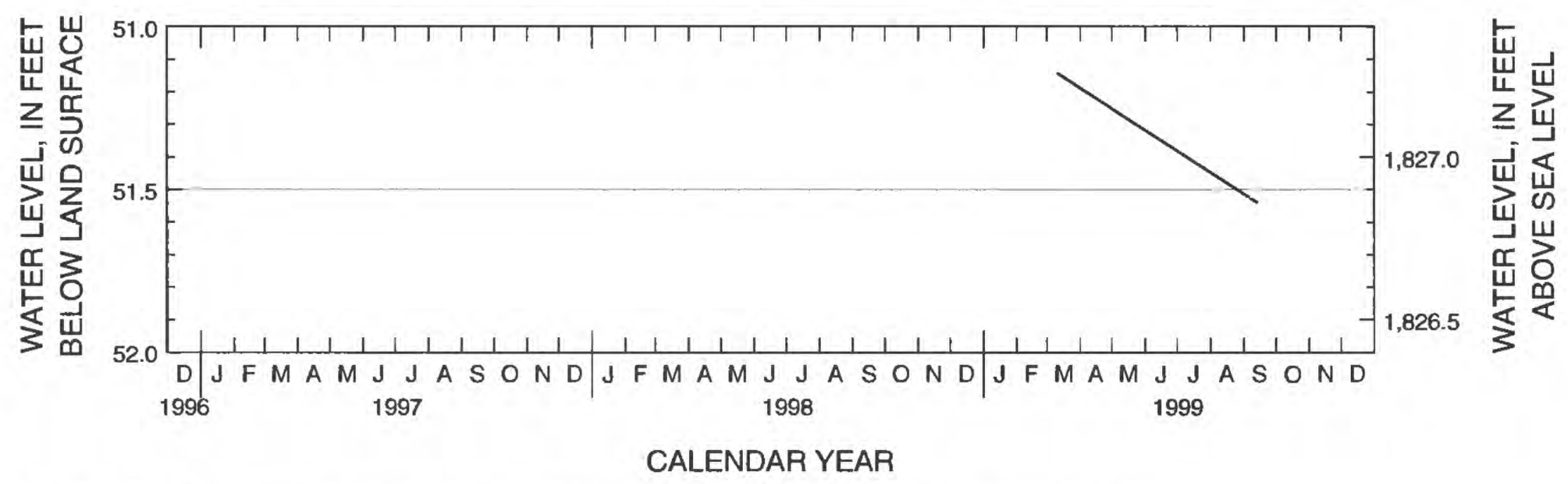

Figure B53. Hydrograph for observation well 122N52W27BBBB2 R (site number 53).

Site number from location map: 54

Local well number: 122N52W32AAAA R

Station identification number: 451841097110901

Other identifier: RB-77S

County: Roberts, South Dakota

Aquifer: Coteau Lakes

Altitude of land surface: $1,851.7$ feet

Measuring point: 1.8 feet

Extremes: May 23, 1978, to October 6, 1999: Highest, 7.6 feet, August 4, 1993; lowest, 12.0 feet, October 21, 1981.



Figure B54. Hydrograph for observation well 122N52W32AAAA R (site number 54). 
Site number from location map: 55

Local well number: $122 \mathrm{~N} 52 \mathrm{~W} 36 \mathrm{BCCB} 2 \mathrm{R}$

Station identification number: 451822097072401

Other identifier: R20-84-34

County: Roberts, South Dakota

Aquifer: Big Sioux

Altitude of land surface: 1,888 feet

Measuring point: 3.0 feet

Extremes: April 29, 1997, to August 26, 1999: Highest, 0.76 feet, March 27, 1998; lowest, 4.33 feet, August 26, 1999.

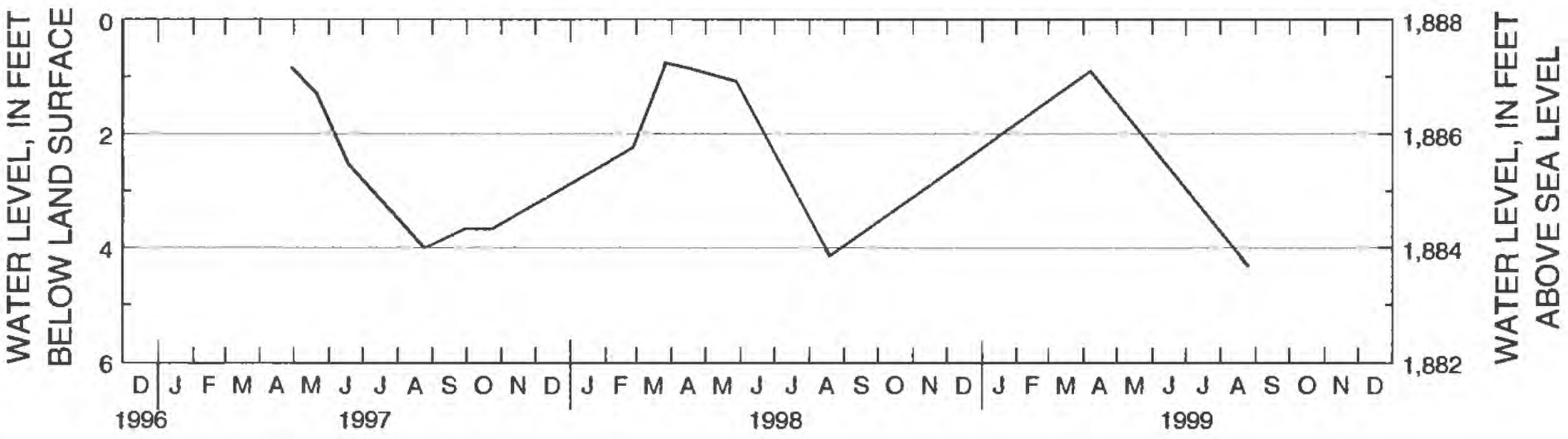

CALENDAR YEAR

Figure B55. Hydrograph for observation well 122N52W36BCCB2 R (site number 55).

Site number from location map: 56

Local well number: 122N53W11DDDD R

Station identification number: 452120097145001

Other identifier: DA-78D

County: Day, South Dakota

Aquifer: Coteau Lakes

Altitude of land surface: 1,830 feet

Measuring point: 2.8 feet

Extremes: September 27, 1978, to October 6, 1999: Highest, 0.99 foot, February 26, 1998; lowest, 10.5 feet, September 1, 1982.

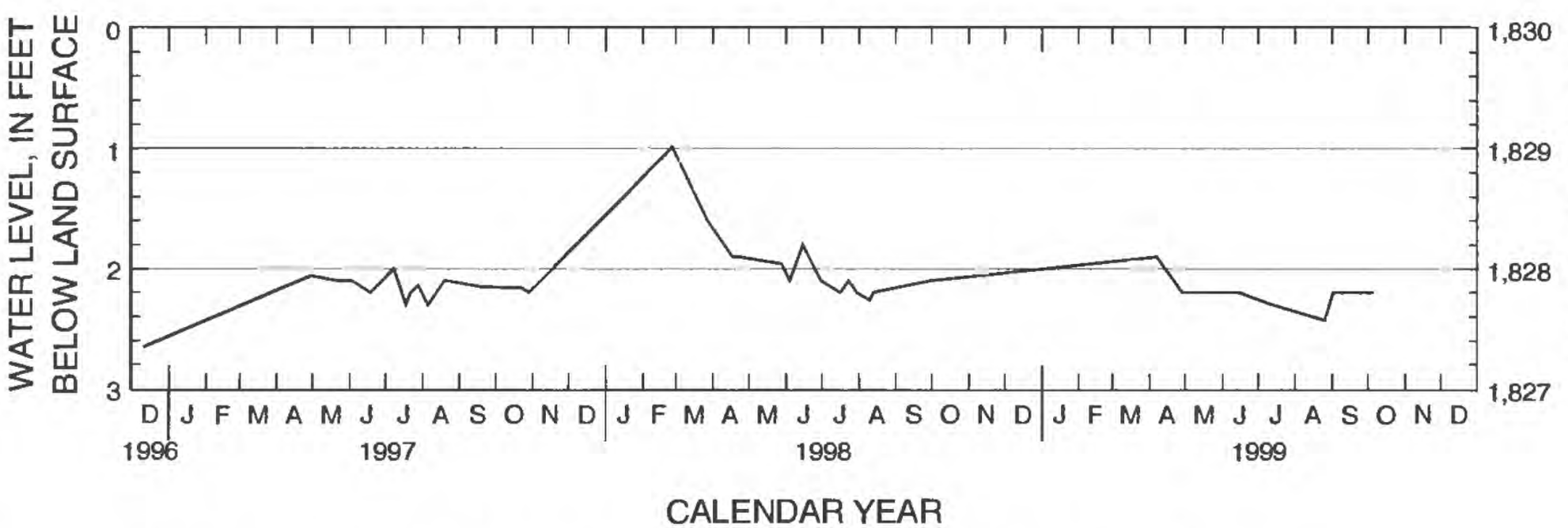

Figure B56. Hydrograph for observation well 122N53W11DDDD R (site number 56). 
Site number from location map: 57

Local well number: 123N49W24CCCC

Station identification number: 452633096453401

Other identifier: RB-81B

County: Roberts, South Dakota

Aquifer: Undetermined

Altitude of land surface: $1,148.6$ feet

Measuring point: 2.5 feet

Extremes: May 29, 1981, to October 5, 1999: Highest, 38.35 feet, June 20, 1997; lowest, 61.0 feet, November 9 , 1983.
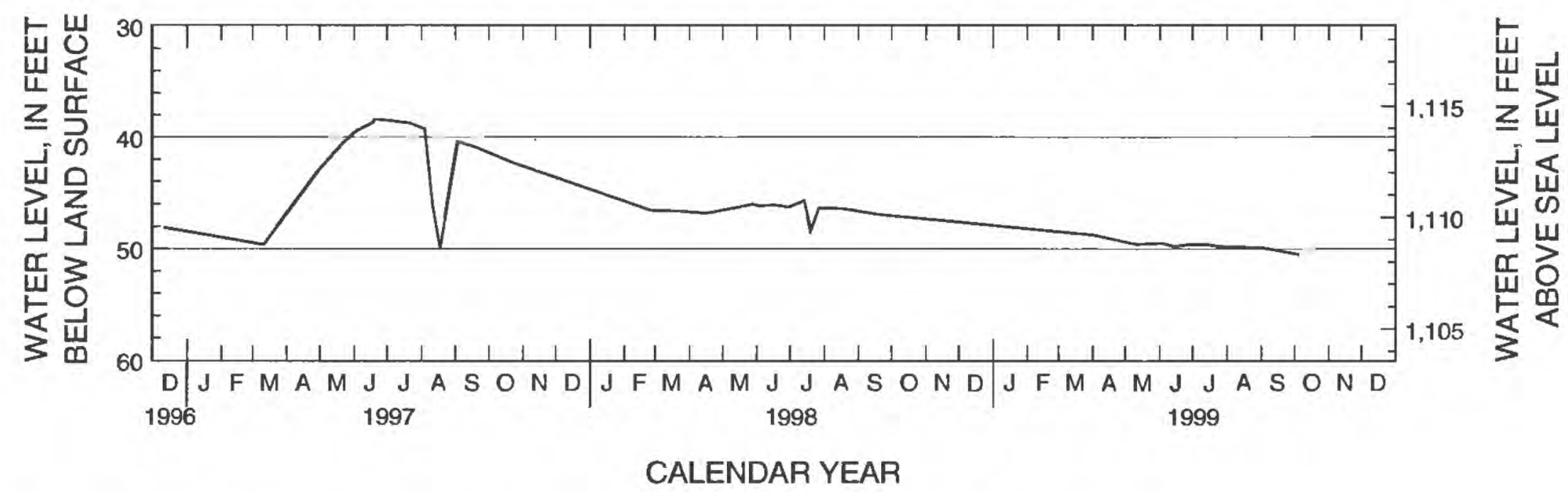

Figure B57. Hydrograph for observation well 123N49W24CCCC (site number 57).

Site number from location map: 58

Local well number: 123N50W22BBBB

Station identification number: 452722096552301

Other identifier: R2-94-66

County: Roberts, South Dakota

Aquifer: Revillo

Altitude of land surface: $1,240.3$ feet

Measuring point: 2.1 feet

Extremes: December 12, 1996, to August 25, 1999: Highest, 58.59 feet, April 29, 1997; lowest 72.95 feet, August 25, 1999.



CALENDAR YEAR

Figure B58. Hydrograph for observation well 123N50W22BBBB (site number 58). 
Site number from location map: 59

Local well number: 123N50W23AAAA

Station identification number: 452721096525701

Other identifier: RB-77L

County: Roberts, South Dakota

Aquifer: Revillo

Altitude of land surface: 1,175.2 feet

Measuring point: 3.5 feet

Extremes: November 30, 1977, to October 5, 1999: Highest, -3.5 feet, June 17, 1998; lowest, 16.0 feet, July 28, 1988.

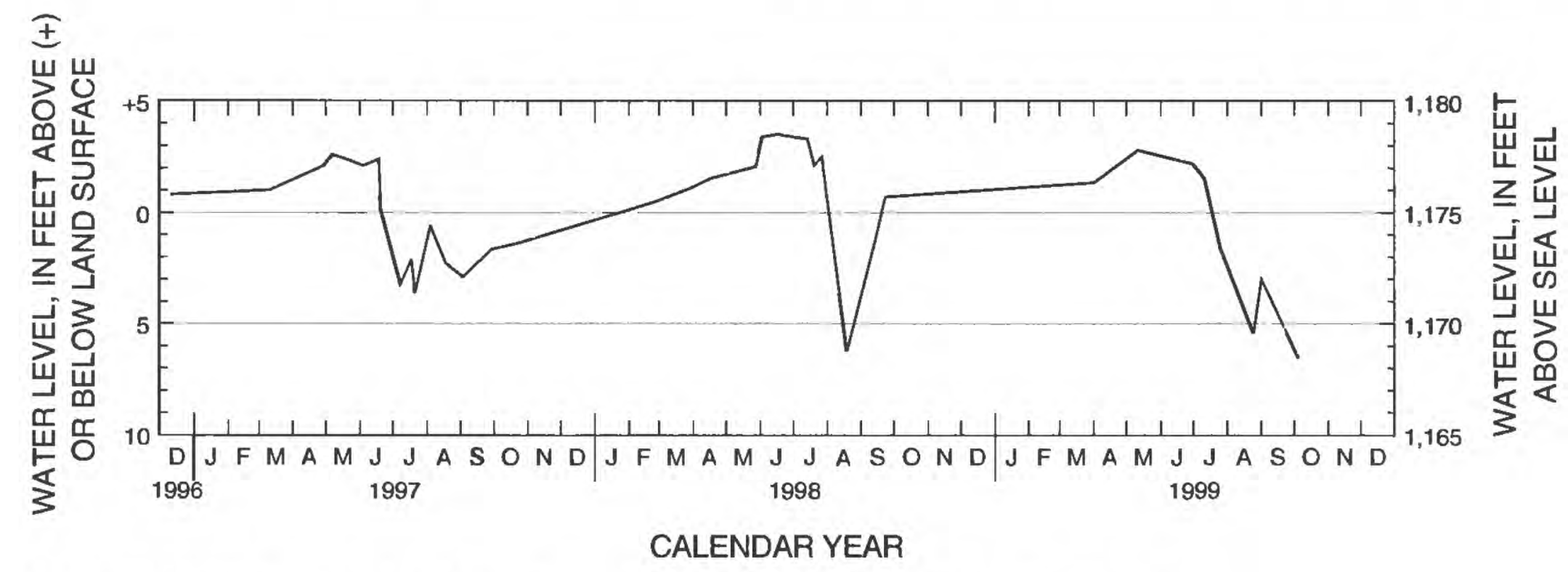

Figure B59. Hydrograph for observation well 123N50W23AAAA (site number 59).

Site number from location map: 60

Local well number: 123N50W35AAAA

Station identification number: 452537096525701

Other identifier: RB-77M

County: Roberts, South Dakota

Aquifer: Revillo

Altitude of land surface: $1,216.1$ feet

Measuring point: 2.3 feet

Extremes: November 30, 1977, to October 5, 1999: Highest, 39.91 feet, March 11, 1997; lowest, 59.1 feet, July 29, 1988.

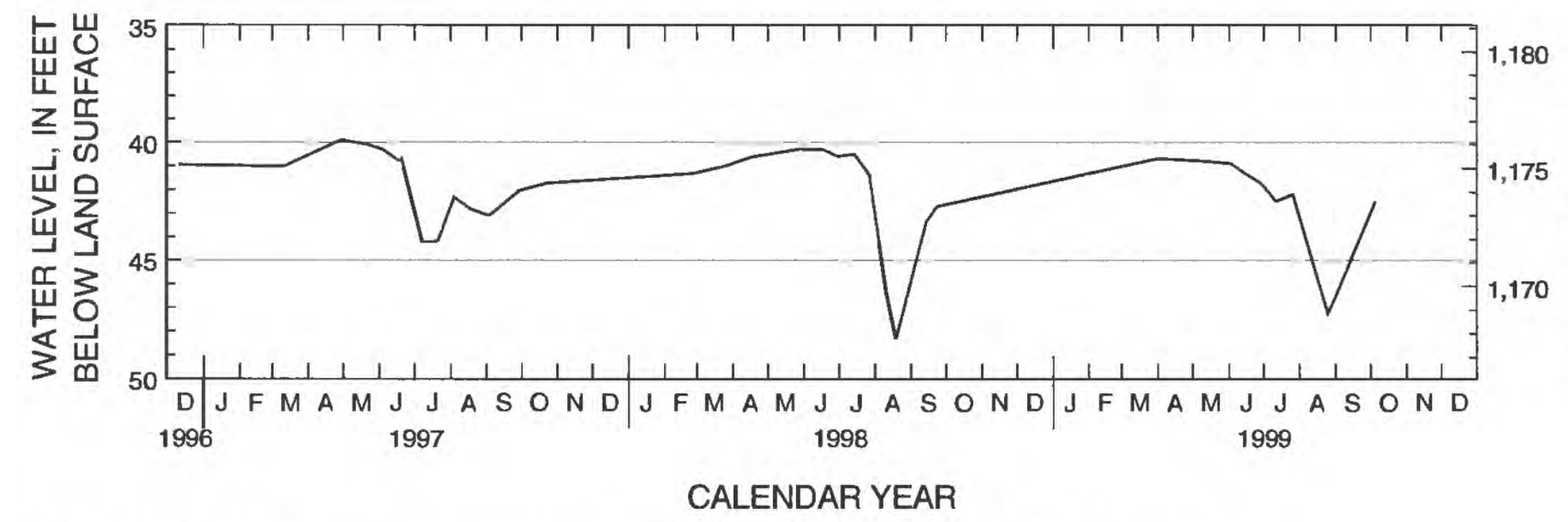

Figure B60. Hydrograph for observation well 123N50W35AAAA (site number 60). 
Site number from location map: 61

Local well number: 123N51W21DDDD2 R

Station identification number: 452218097022902

Other identifier: R20-99-48

County: Roberts, South Dakota

Aquifer: Undetermined

Altitude of land surface: $1,819.4$ feet

Measuring point: 2.0 feet

Extremes: September 14, 1999, to December 8, 1999: Highest 26.86 feet, September 14, 1999; lowest, 27.23 feet, December 8, 1999.

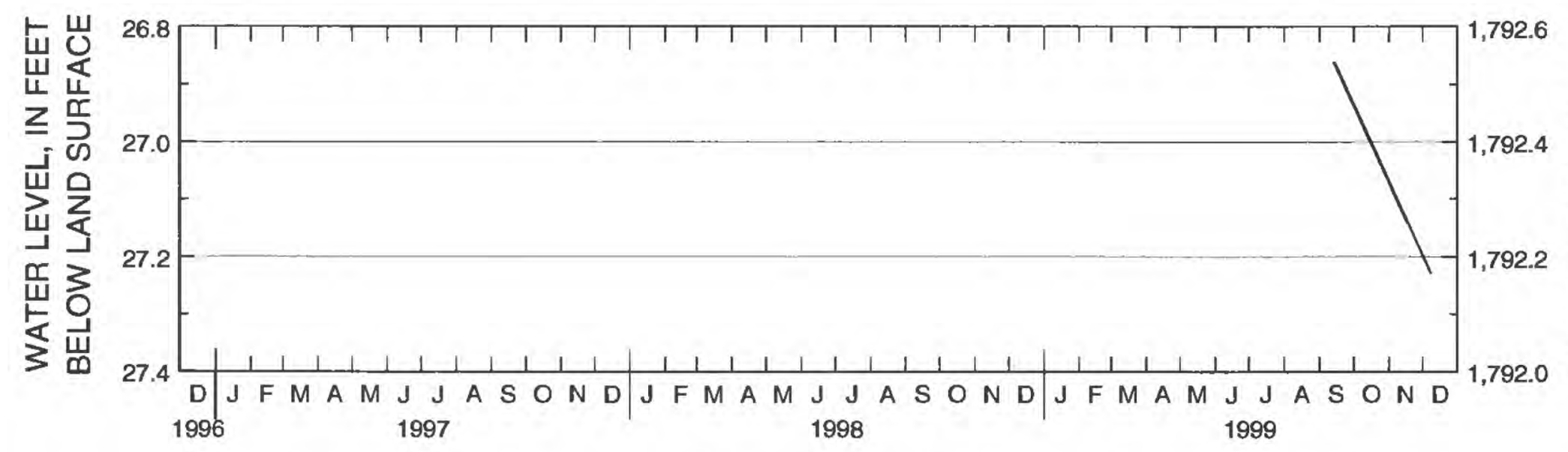

CALENDAR YEAR

Figure B61. Hydrograph for observation well 123N51W21DDDD2 R (site number 61).

Site number from location map: 62

Local well number: 123N52W6DDDA R

Station identification number: 452728097122401

Other identifier: CO-93-28

County: Roberts, South Dakota

Aquifer: Coteau Lakes

Altitude of land surface: 1,870 feet

Measuring point: 2.0 feet

Extremes: April 30, 1997, to August 26, 1999: Highest, 0.08 foot, February 26, 1998; lowest, 0.91 foot, September 18, 1997.

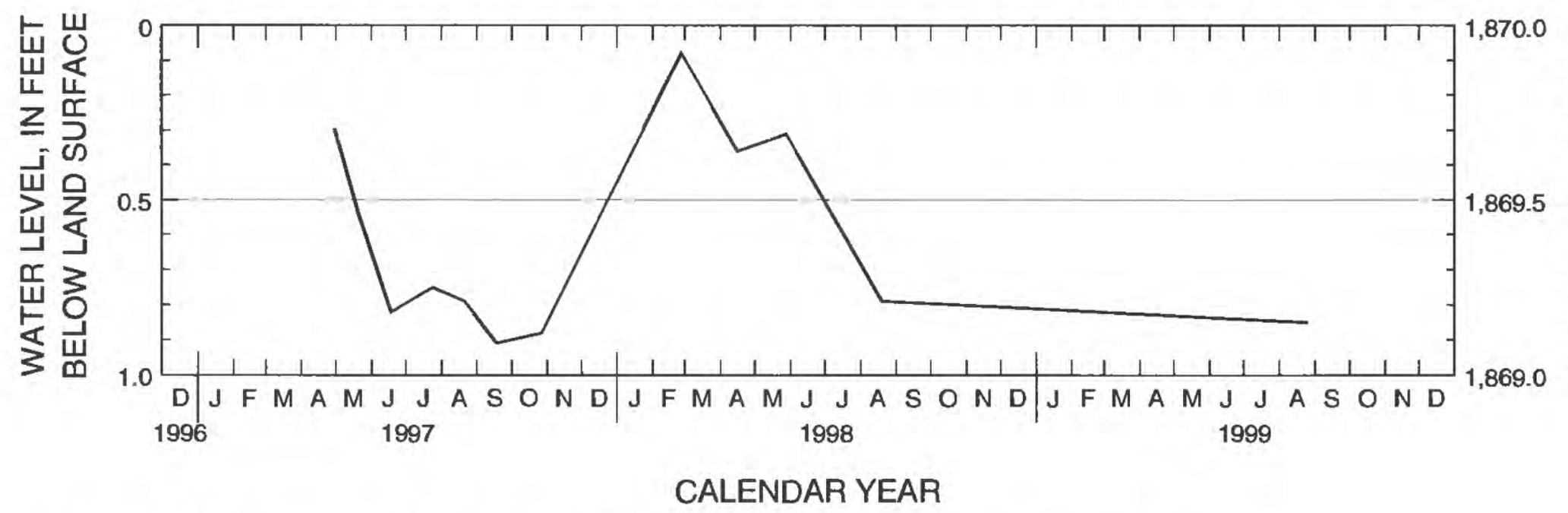

Figure B62. Hydrograph for observation well 123N52W6DDDA R (site number 62). 
Site number from location map: 63

Local well number: 123N52W8BAAA R

Station identification number: 452724097114701

Other identifier: CO-93-26

County: Roberts, South Dakota

Aquifer: Coteau Lakes

Altitude of land surface: 1,895 feet

Measuring point: 2.0 feet

Extremes: April 30, 1997, to August 26, 1999: Highest, 23.06 feet, April 30, 1997; lowest, 25.62 feet, February 26, 1998.

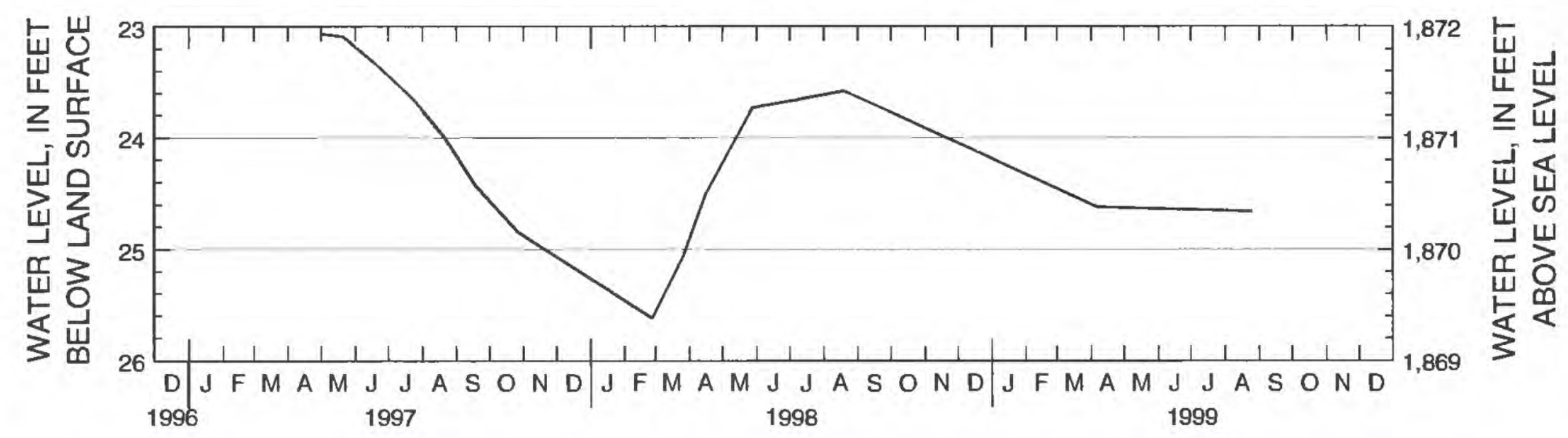

CALENDAR YEAR

Figure B63. Hydrograph for observation well 123N52W8BAAA R (site number 63).

Site number from location map: 64

Local well number: 123N52W19BABA R

Station identification number: 452540097130901

Other identifier: RB-81C

County: Roberts, South Dakota

Aquifer: Coteau Lakes

Altitude of land surface: $1,918.0$ feet

Measuring point: 2.3 feet

Extremes: August 18, 1982, to October 6, 1999: Highest, 2.5 feet, July 22, 1997; lowest, 11.1 feet, September 8, 1988.

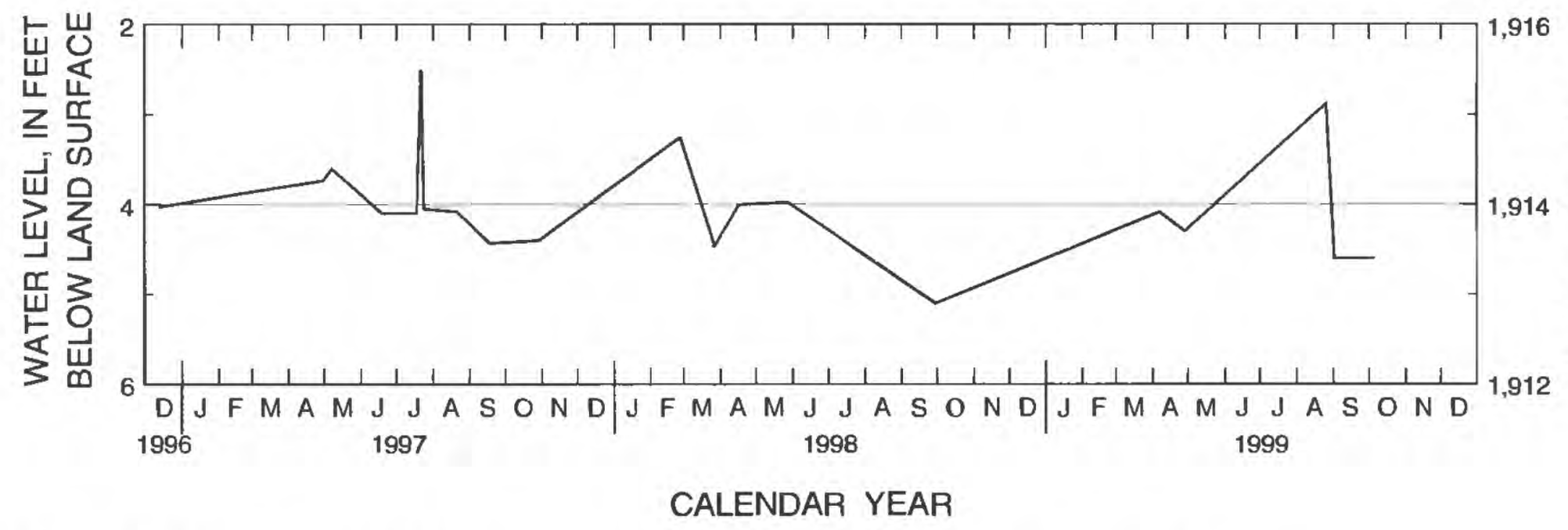

Figure B64. Hydrograph for observation well 123N52W19BABA R (site number 64). 
Site number from location map: 65

Local well number: 123N52W25CCCC R

Station identification number: 452357097072401

Other identifier: RB-81D

County: Roberts, South Dakota

Aquifer: Coteau Lakes

Altitude of land surface: $1,931.9$ feet

Measuring point: 2.5 feet

Extremes: July 20, 1982, to October 6, 1999: Highest, 5.91 feet, April 30, 1997; lowest, 19.7 feet, March 21, 1991.

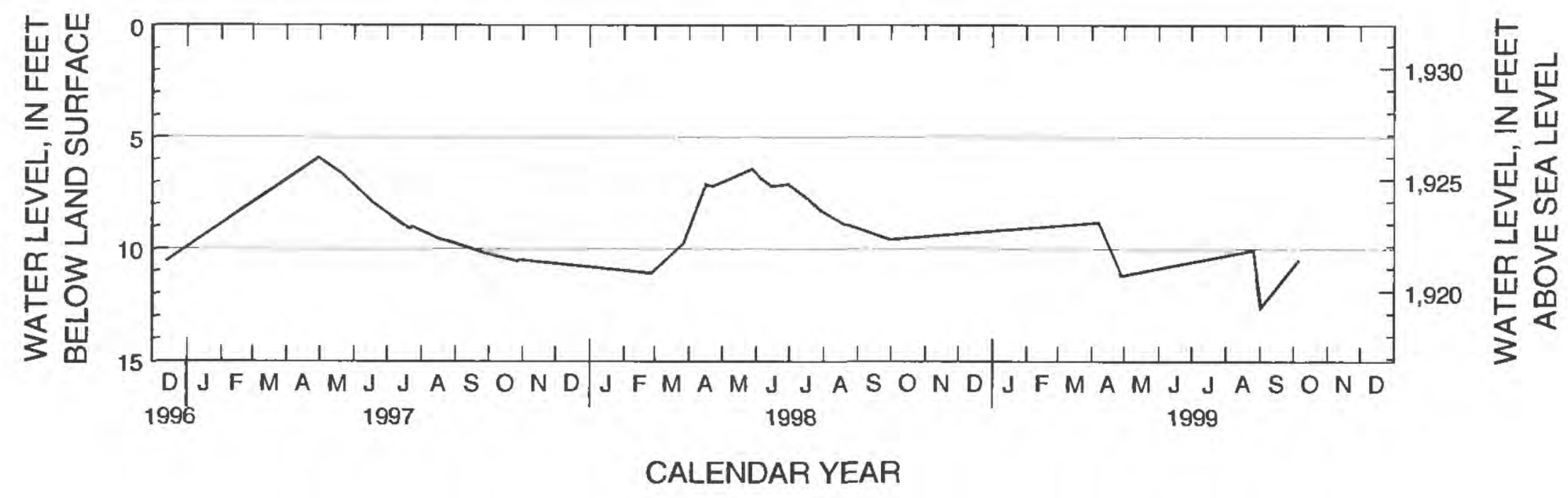

Figure B65. Hydrograph for observation well 123N52W25CCCC R (site number 65).

Site number from location map: 66

Local well number: 123 N52W35CBBB R

Station identification number: 452330097083801

Other identifier: CO-93-33

County: Roberts, South Dakota

Aquifer: Coteau Lakes

Altitude of land surface: 1,910 feet

Measuring point: 2.0 feet

Extremes: April 30, 1997, to August 26, 1999: Highest, -0.59 foot, April 30, 1997: lowest, 6.16 feet, February 26, 1998.

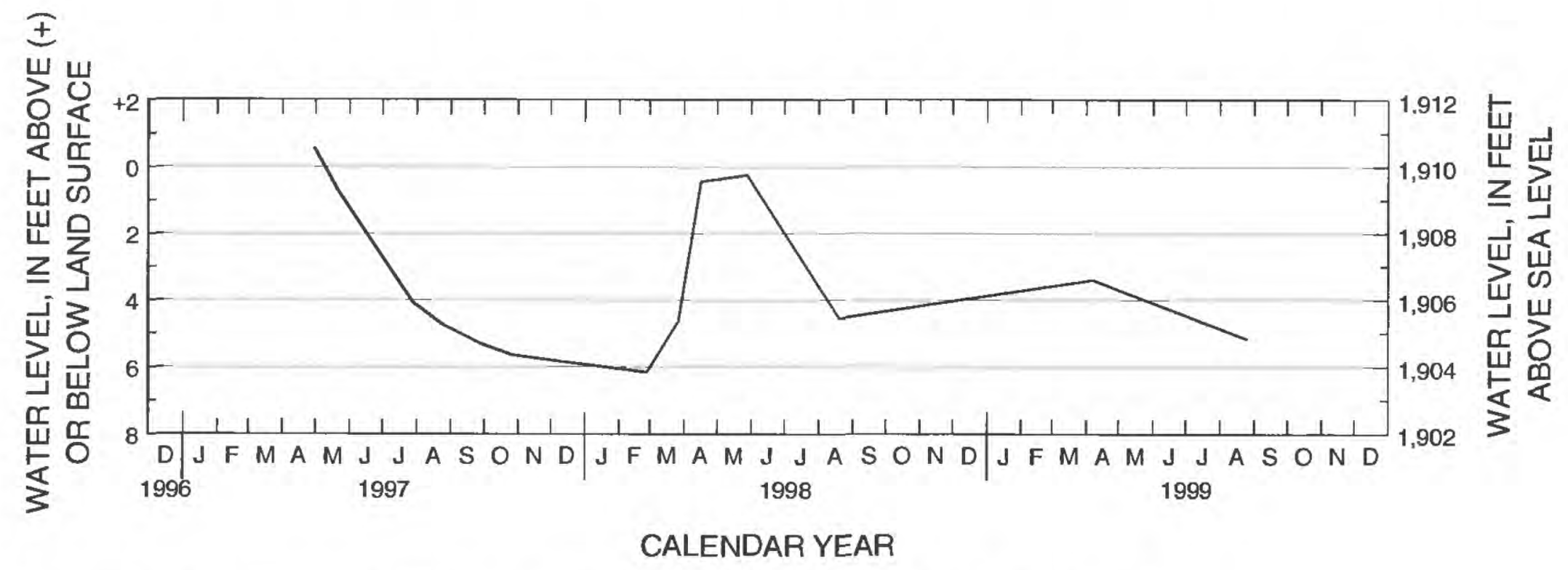

Figure B66. Hydrograph for observation well 123N52W35CBBB R (site number 66). 
Site number from location map: 67

Local well number: $123 \mathrm{~N} 52 \mathrm{~W} 35 \mathrm{CBBB} 2 \mathrm{R}$

Station identification number: 452330097083802

Other identifier: CO-93-34

County: Roberts, South Dakota

Aquifer: Coteau Lakes

Altitude of land surface: 1,910 feet

Measuring point: 2.0 feet

Extremes: April 30, 1997, to August 26, 1999: Highest, -0.95 foot, April 30, 1997; lowest, 5.83 feet, February 26, 1998.

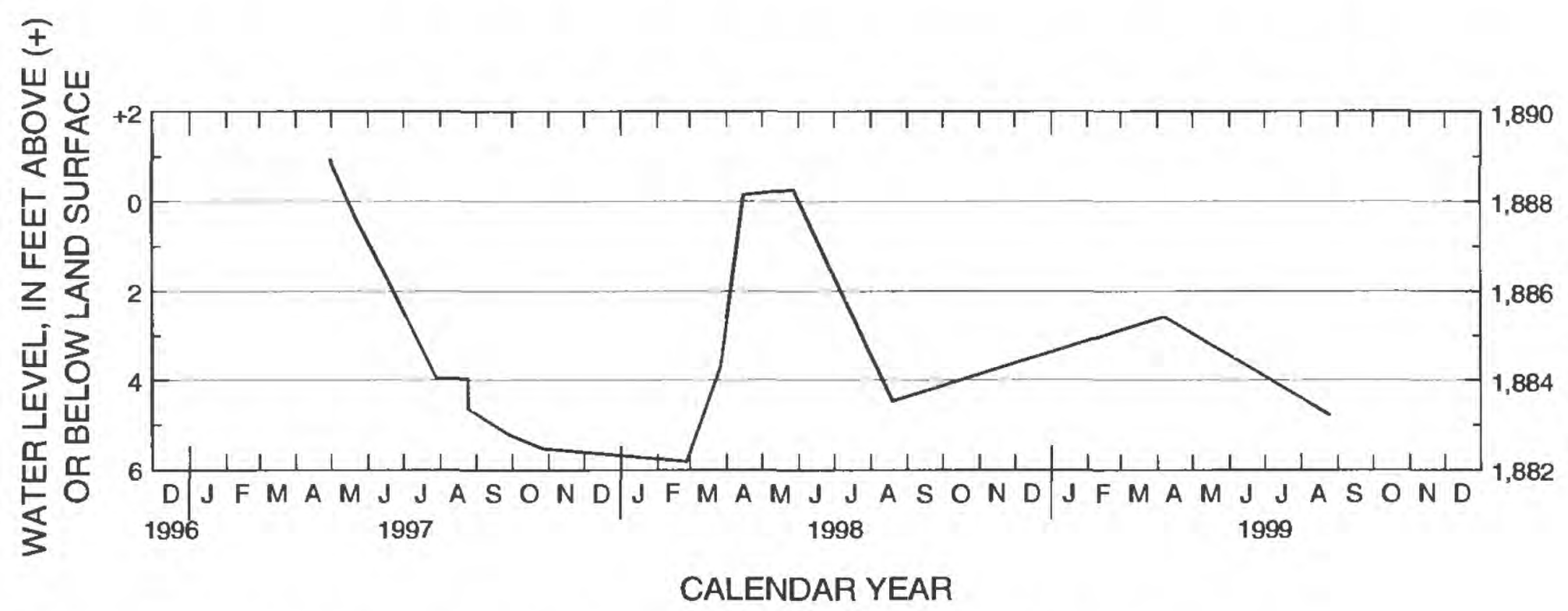

Figure B67. Hydrograph for observation well 123N52W35CBBB2 R (site number 67).

Site number from location map: 68

Local well number: 123N53W13CCCC2 R

Station identification number: 452544097144802

Other identifier: RO-7

County: Day, South Dakota

Aquifer: Prairie Coteau

Altitude of land surface: 1,874 feet

Measuring point: 1.6 feet

Extremes: April 30, 1997, to August 26, 1999: Highest, 17.72 feet, April 30, 1997; lowest, 18.8 feet, September 18, 1997, and October 27, 1997.

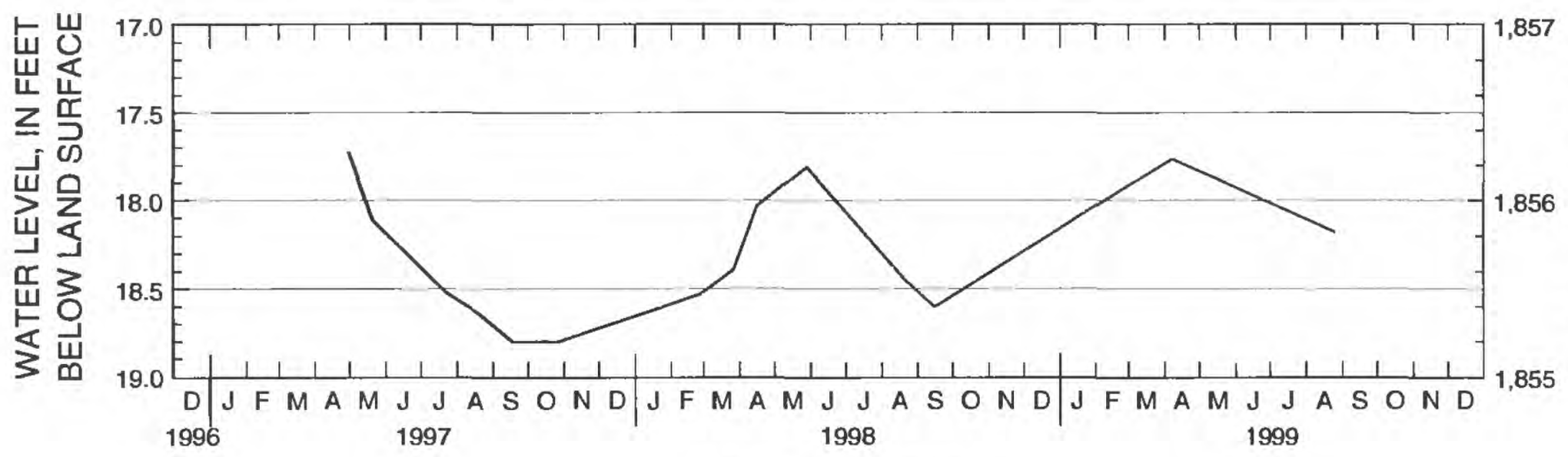

CALENDAR YEAR

Figure B68. Hydrograph for observation well 123N53W13CCCC2 R (site number 68). 
Site number from location map: 69

Local well number: 124N50W27BBBB R

Station identification number: 452959096545901

Other identifier: R2-94-54

County: Roberts, South Dakota

Aquifer: Revillo

Altitude of land surface: $1,210.1$ feet

Measuring point: 2.17 feet

Extremes: December 12, 1996, to August 25, 1999: Highest, 25.75 feet, April 29, 1997; lowest, 33.88 feet, August 19, 1998.

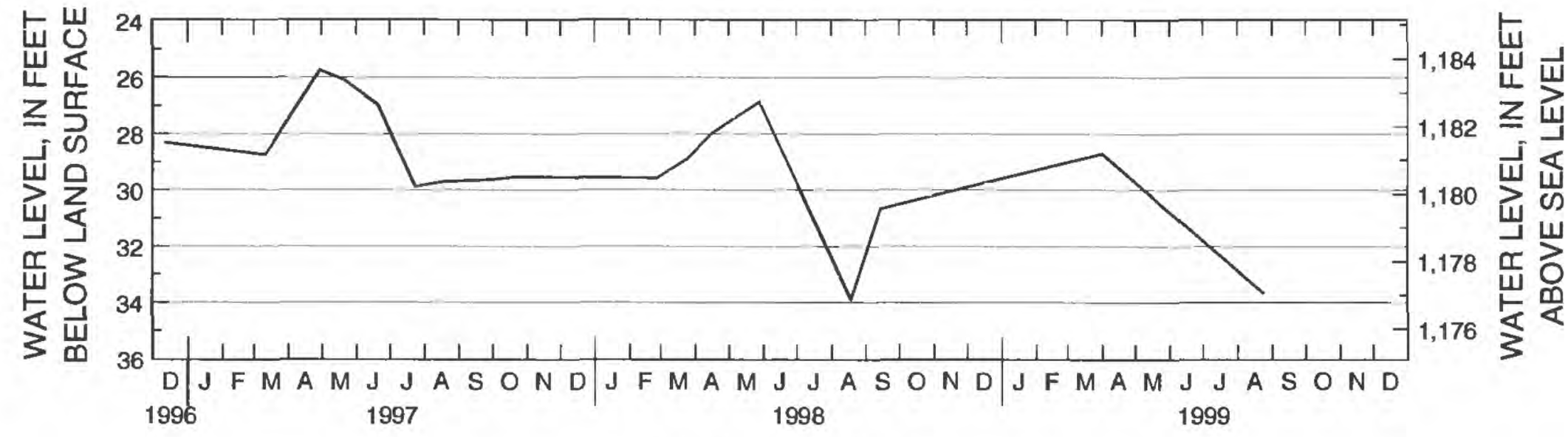

CALENDAR YEAR

Figure B69. Hydrograph for observation well 124N50W27BBBB R (site number 69).

Site number from location map: 70

Local well number: 124N51W25AAAB R

Station identification number: 453001096584801

Other identifier: R2-94-55

County: Roberts, South Dakota

Aquifer: Revillo

Altitude of land surface: 1,274 feet

Measuring point: 1.6 feet

Extremes: December 11, 1996, to August 25, 1999: Highest, 12.52 feet, April 29, 1997; lowest, 14.32 feet, February 5, 1998.

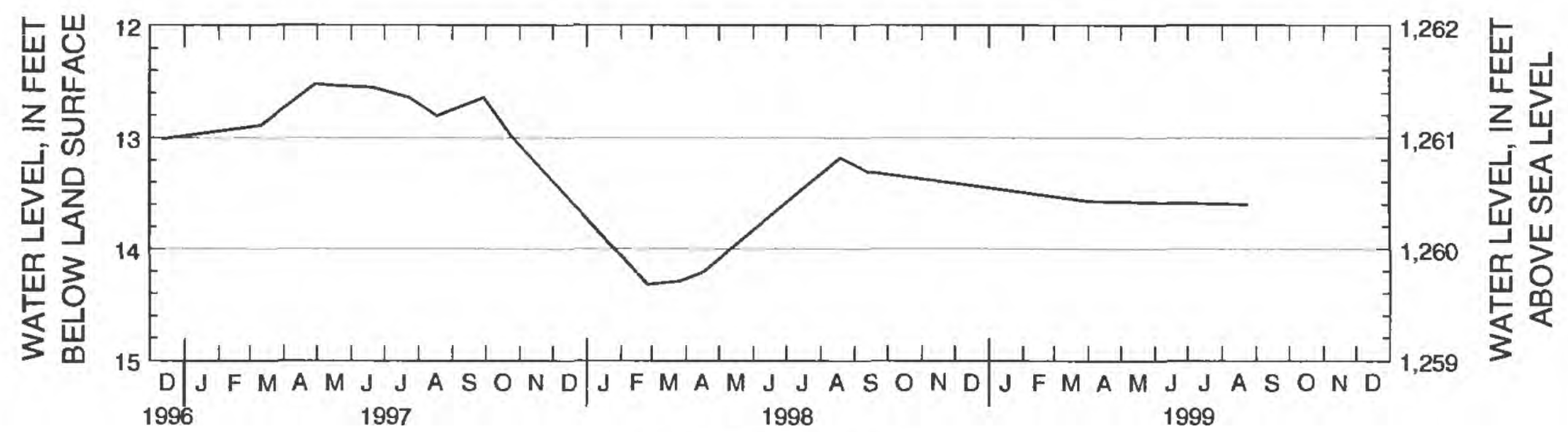

CALENDAR YEAR

Figure B70. Hydrograph for observation well 124N51W25AAAB R (site number 70). 
Site number from location map: 71

Local well number: 124N51W30BBBB2 R

Station identification number: 453002097060902

Other identifier: R2-99-13

County: Roberts, South Dakota

Aquifer: Marday

Altitude of land surface: 2,053.1 feet

Measuring point: 1.9 feet

Extremes: Single measurement of 323.44 feet on December 8, 1999.

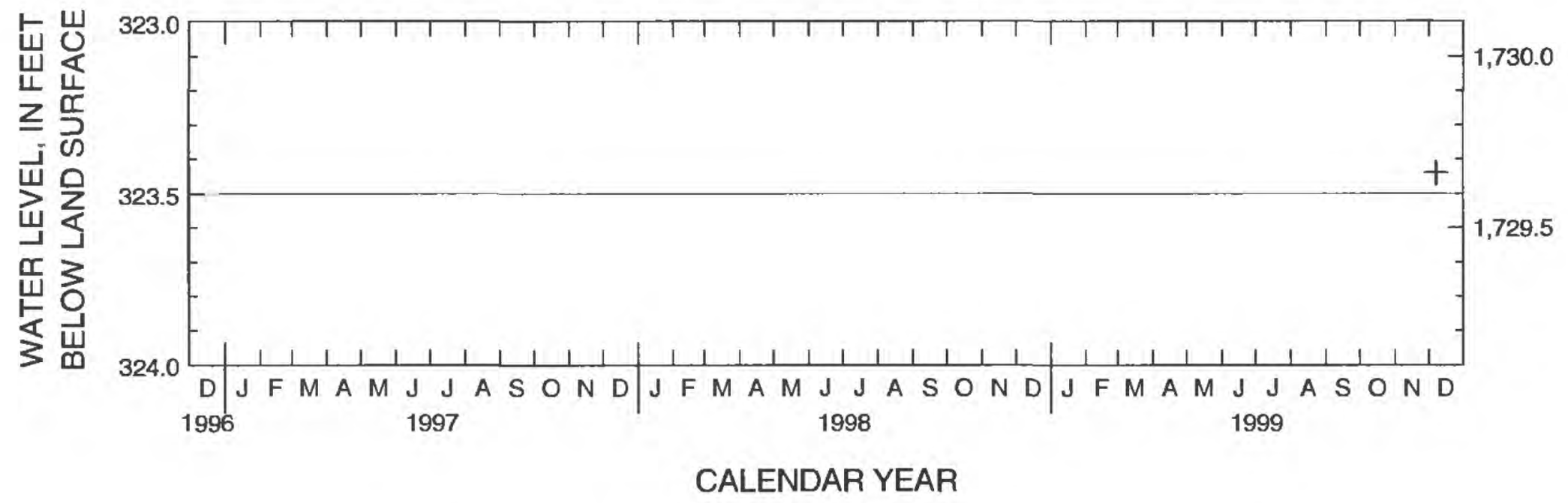

Figure B71. Hydrograph for observation well 124N51W30BBBB2 R (site number 71).

Site number from location map: 72

Local well number: 124N51W4DDDD R

Station identification number: 453240097022701

Other identifier: R2-95-01

County: Roberts, South Dakota

Aquifer: Revillo

Altitude of land surface: 1,425 feet

Measuring point: 2.5 feet

Extremes: December 11, 1996, to August 26, 1999: Highest, 156.6 feet, December 11, 1996; lowest, 161.63 feet, September 14, 1998.

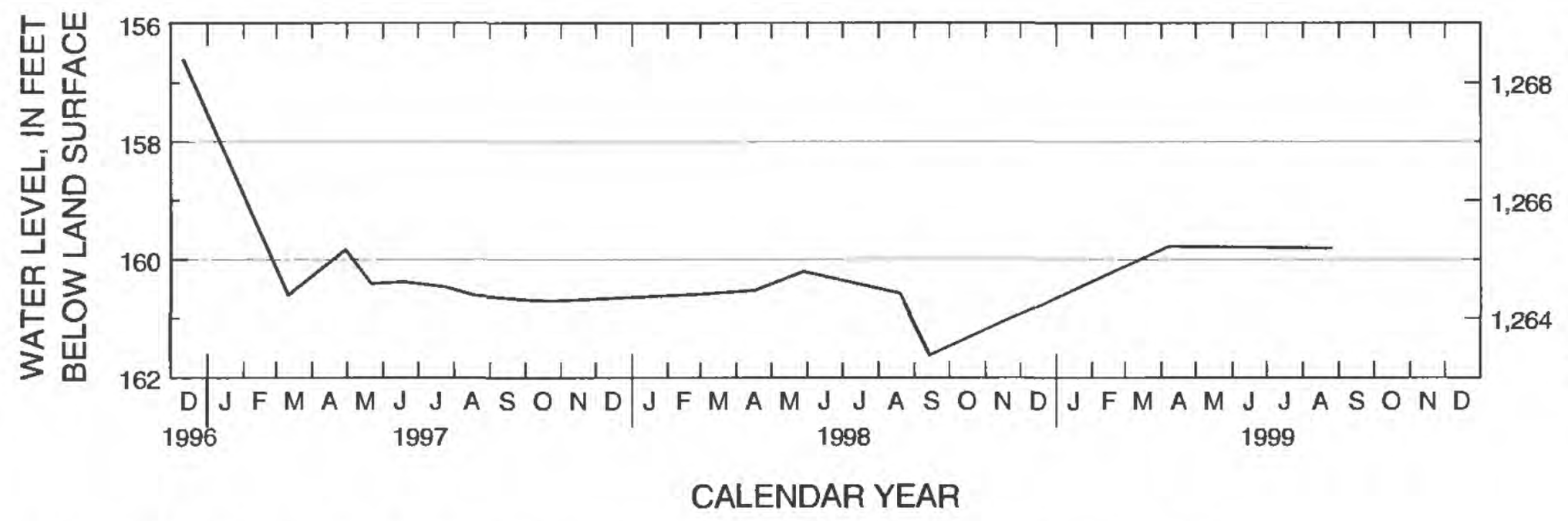

Figure B72. Hydrograph for observation well 124N51W4DDDD R (site number 72). 
Site number from location map: 73

Local well number: 124N52W9AAAC2 R

Station identification number: 453204097100202

Other identifier: R2-99-10

County: Roberts, South Dakota

Aquifer: Undetermined

Altitude of land surface: 2,003 feet

Measuring point: 1.1 feet

Extremes: September 13, 1999, to December 8, 1999: Highest, 49.48 feet, September 13, 1999; lowest, 49.57 feet,

December 13, 1999.

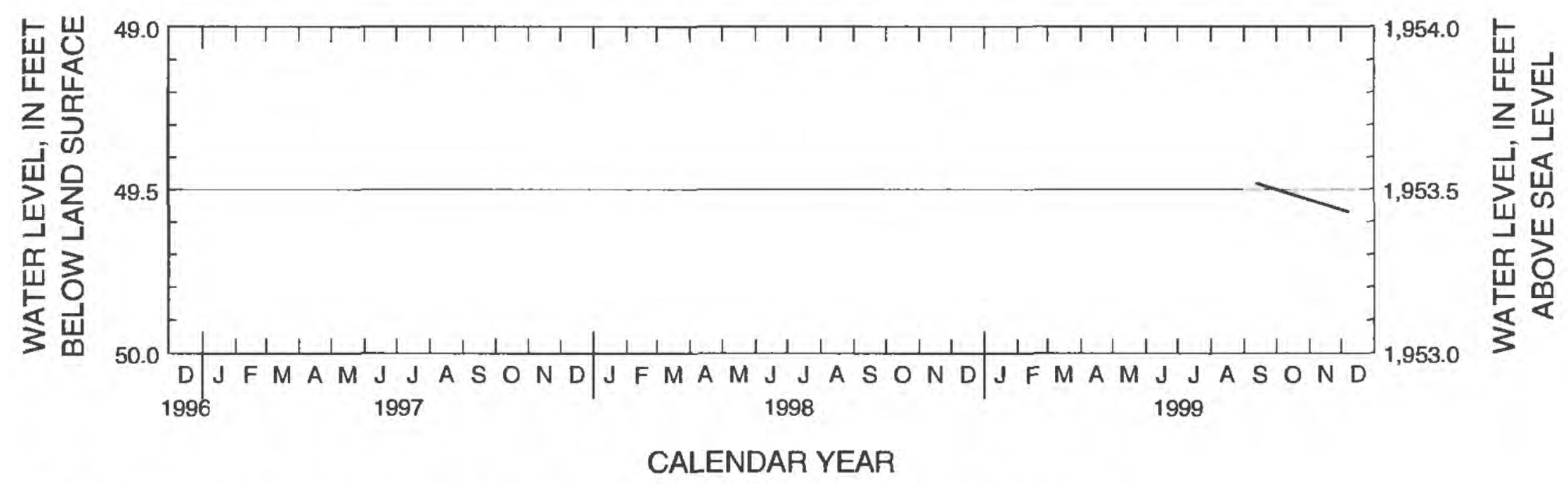

Figure B73. Hydrograph for observation well 124N52W9AAAC2 R (site number 73).

Site number from location map: 74

Local well number: 125 N50W6CBBB R

Station identification number: 453815096584001

Other identifier: R2-93-53

County: Roberts, South Dakota

Aquifer: Undetermined

Altitude of land surface: 1,225 feet

Measuring point: 1.9 feet

Extremes: April 29, 1997, to August 25, 1999: Highest, 22.07 feet, April 29, 1997; lowest, 23.82 feet, August 25, 1999.

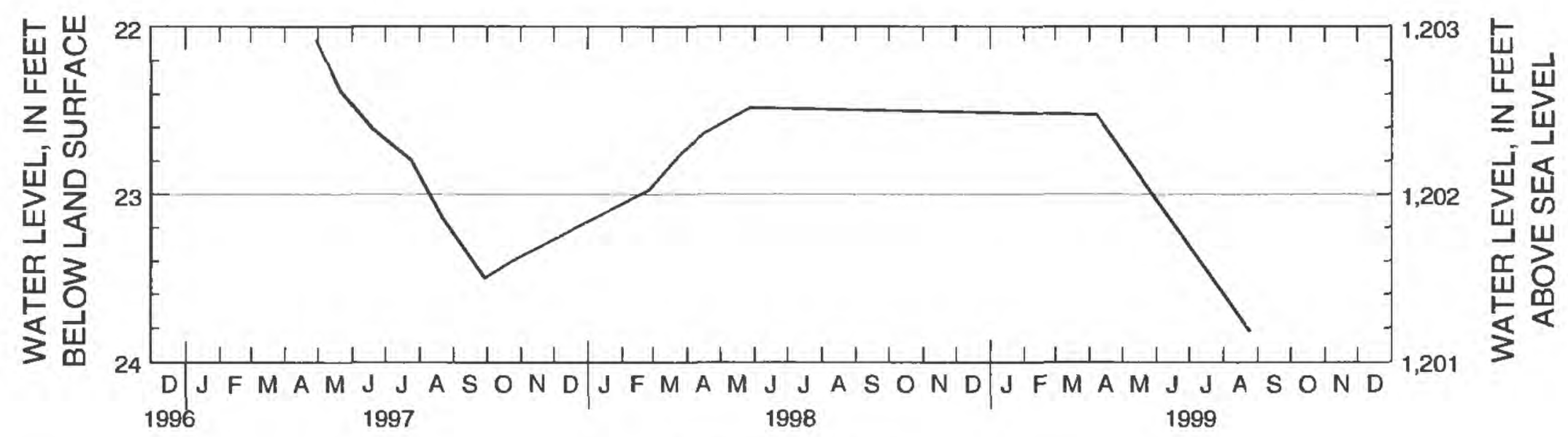

CALENDAR YEAR

Figure B74. Hydrograph for observation well 125N50W6CBBB R (site number 74). 
Site number from location map: 75

Local well number: 125 N50W6CCCC R

Station identification number: 453752096584001

Other identifier: RB-81-15

County: Roberts, South Dakota

Aquifer: Undetermined

Altitude of land surface: 1,200 feet

Measuring point: 2.5 feet

Extremes: April 29, 1997, to August 25, 1999: Highest, 4.22 feet, April 29, 1997; lowest, 7.52 feet, September 14, 1998.

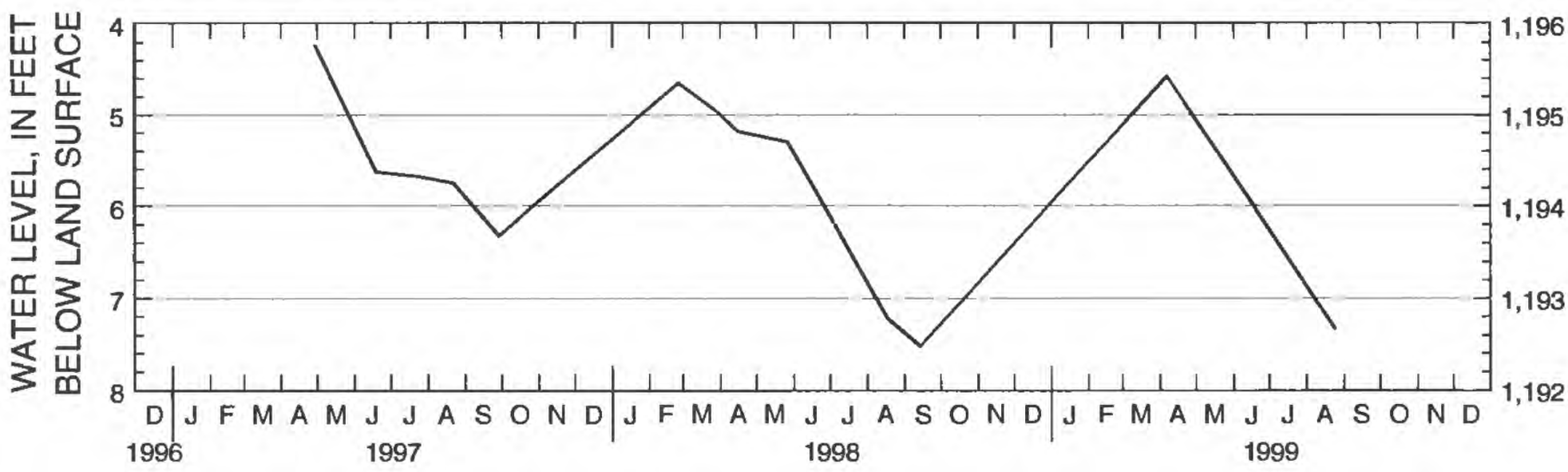

CALENDAR YEAR

Figure B75. Hydrograph for observation well 125N50W6CCCC R (site number 75).

Site number from location map: 76

Local well number: 125N50W6DCCC R

Station identification number: 453752096580301

Other identifier: RB-81-16

County: Roberts, South Dakota

Aquifer: Undetermined

Altitude of land surface: 1,230 feet

Measuring point: 1.6 feet

Extremes: April 29, 1997, to August 25, 1999: Highest, 33.76 feet, April 29, 1997; lowest, 35.44 feet, August 25, 1999.

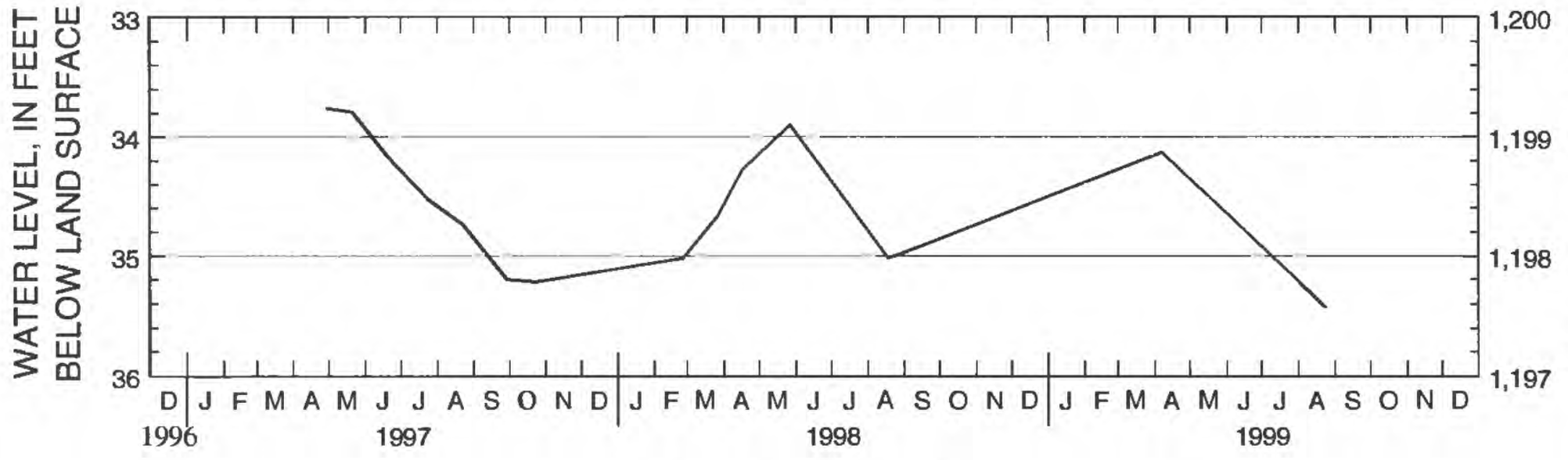

CALENDAR YEAR

Figure B76. Hydrograph for observation well 125N50W6DCCC R (site number 76). 
Site number from location map: 77

Local well number: 125N50W6DCCC2 R

Station identification number: 453752096580302

Other identifier: RB-81-17

County: Roberts, South Dakota

Aquifer: Undetermined

Altitude of land surface: 1,231 feet

Measuring point: 1.1 feet

Extremes: December 11, 1996, to October 5, 1999: Highest, 17.64 feet, May 27, 1998; lowest, 20.68 feet, March 11, 1997.



Figure B77. Hydrograph for observation well 125N50W6DCCC2 R (site number 77).

Site number from location map: 78

Local well number: 125 N50W7ADDD R

Station identification number: 453725096572801

Other identifier: R2-93-54

County: Roberts, South Dakota

Aquifer: Undetermined

Altitude of land surface: 1,205 feet

Measuring point: 2.0 feet

Extremes: April 29, 1997, to August 25, 1999: Highest, 2.92 feet, April 29, 1997; lowest, 6.04 feet, September 14, 1999.



Figure B78. Hydrograph for observation well 125N50W7ADDD R (site number 78). 
Site number from location map: 79

Local well number: 125N50W8BBBA R

Station identification number: 453751096572401

Other identifier: RB-11

County: Roberts, South Dakota

Aquifer: Undetermined

Altitude of land surface: 1,216 feet

Measuring point: 3.5 feet

Extremes: December 11, 1996, to October 5, 1999: Highest, 17.64 feet, May 27, 1998; lowest, 20.68 feet, March 11, 1997.



CALENDAR YEAR

Figure B79. Hydrograph for observation well 125N50W8BBBA R (site number 79).

Site number from location map: 80

Local well number: $125 \mathrm{~N} 50 \mathrm{~W} 8 \mathrm{CCCC} 2 \mathrm{R}$

Station identification number: 453700096572702

Other identifier: RB-7W

County: Roberts, South Dakota

Aquifer: Undetermined

Altitude of land surface: 1,207 feet

Measuring point: 2.8 feet

Extremes: December 10, 1996, to August 25. 1999: Highest, 0.25 foot, April 29, 1997; lowest. 5.98 feet, August 25, 1999.

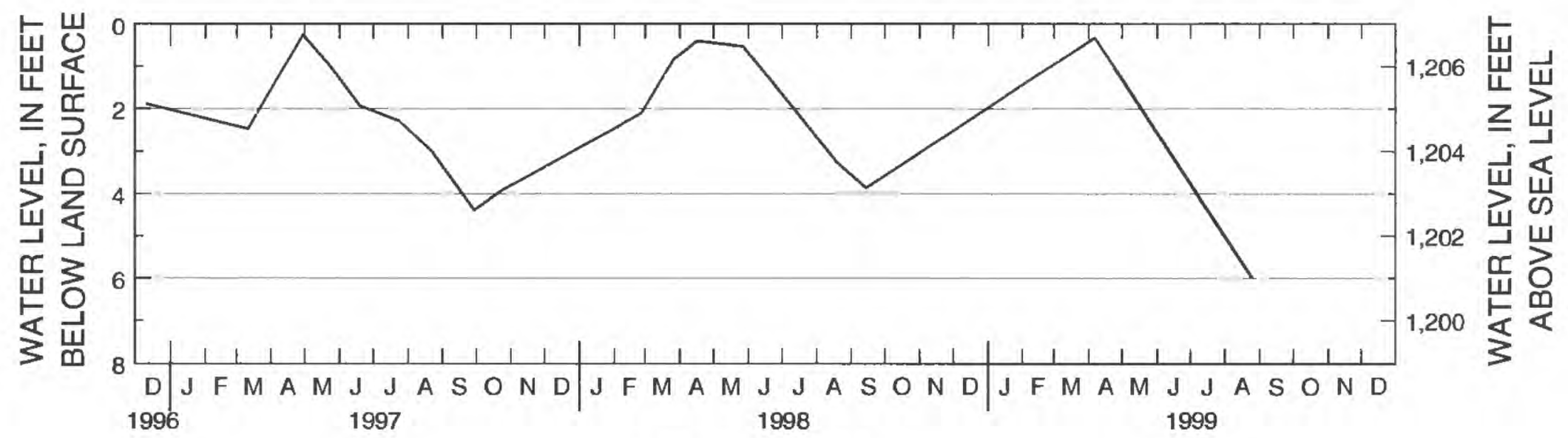

CALENDAR YEAR

Figure B80. Hydrograph for observation well 125N50W8CCCC2 R (site number 80). 
Site number from location map: 81

Local well number: $125 \mathrm{~N} 50 \mathrm{~W} 8 \mathrm{CCCC} 3 \mathrm{R}$

Station identification number: 453700096572701

Other identifier: RB-7E

County: Roberts, South Dakota

Aquifer: Undetermined

Altitude of land surface: $1,207 \mathrm{feet}$

Measuring point: 3.0 feet

Extremes: December 10, 1996, to October 5, 1999: Highest, 0.52 foot, April 29, 1997; lowest, 4.71 feet, September 29, 1997.

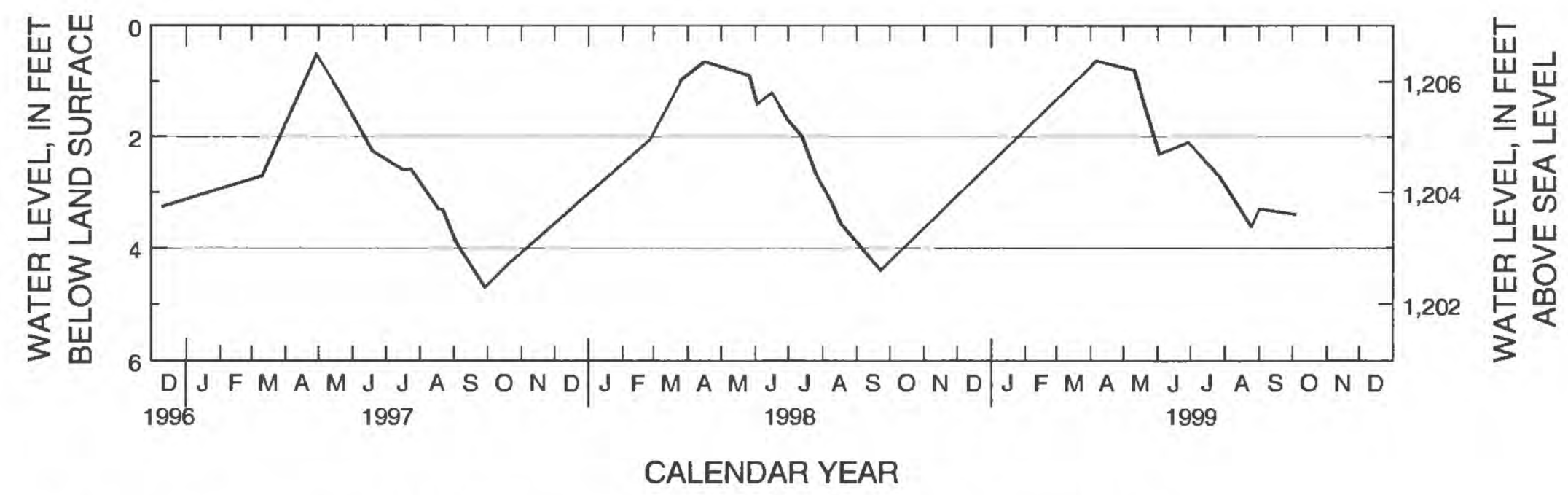

Figure B81. Hydrograph for observation well 125N50W8CCCC3 R (site number 81).

Site number from location map: 82

Local well number: $125 \mathrm{~N} 50 \mathrm{~W} 12 \mathrm{ABCC} 2 \mathrm{R}$

Station identification number: 453738096515402

Other identifier: R2-97-43

County: Roberts, South Dakota

Aquifer: Veblen

Altitude of land surface: 1,120 feet

Measuring point: 2.1 feet

Extremes: October 23, 1997, to August 25, 1999: Highest, 56.98 feet, October 23, 1997; lowest, 59.85 feet, August, 25, 1999.

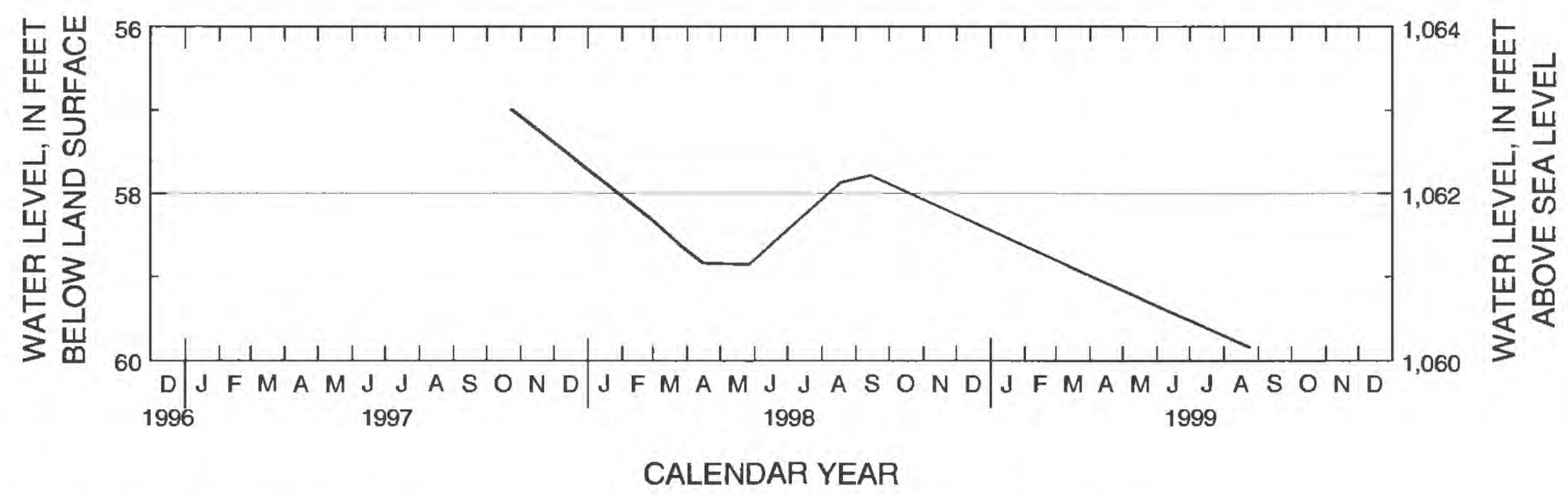

Figure B82. Hydrograph for observation well 125N50W12ABCC2 R (site number 82). 
Site number from location map: 83

Local well number: 125N50W20DDDD2 R

Station identification number: 453514096561402

Other identifier: RB-5

County: Roberts, South Dakota

Aquifer: Undetermined

Altitude of land surface: 1,215 feet

Measuring point: 3.3 feet

Extremes: December 11, 1996, to October 5, 1999: Highest, 4.96 feet, April 29, 1997; lowest, 11.94 feet, February 25, 1998.

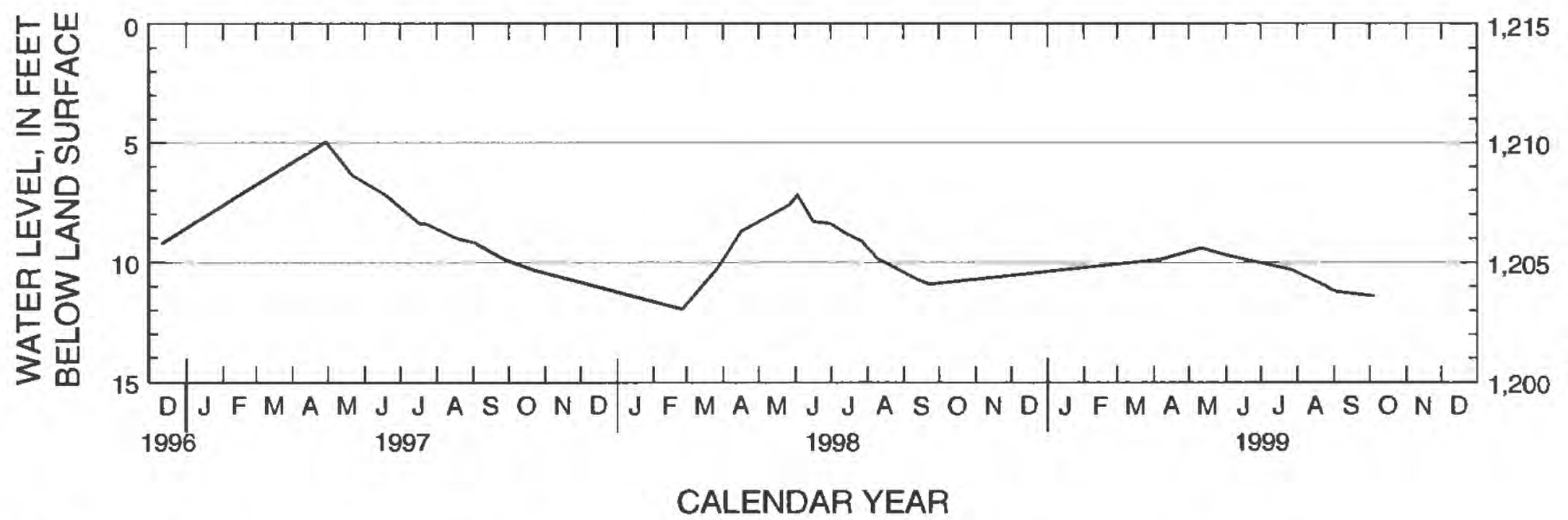

Figure B83. Hydrograph for observation well 125N50W20DDDD2 R (site number 83).

Site number from location map: 84

Local well number: 125N51W1DCCC R

Station identification number: 453752096591801

Other identifier: RB-12E

County: Roberts, South Dakota

Aquifer: Undetermined

Altitude of land surface: 1,215 feet

Measuring point: 2.3 feet

Extremes: April 29, 1997, to October 5, 1999: Highest, 19.02 feet, April 29, 1997; lowest, 20.86 feet, September 14, 1998.

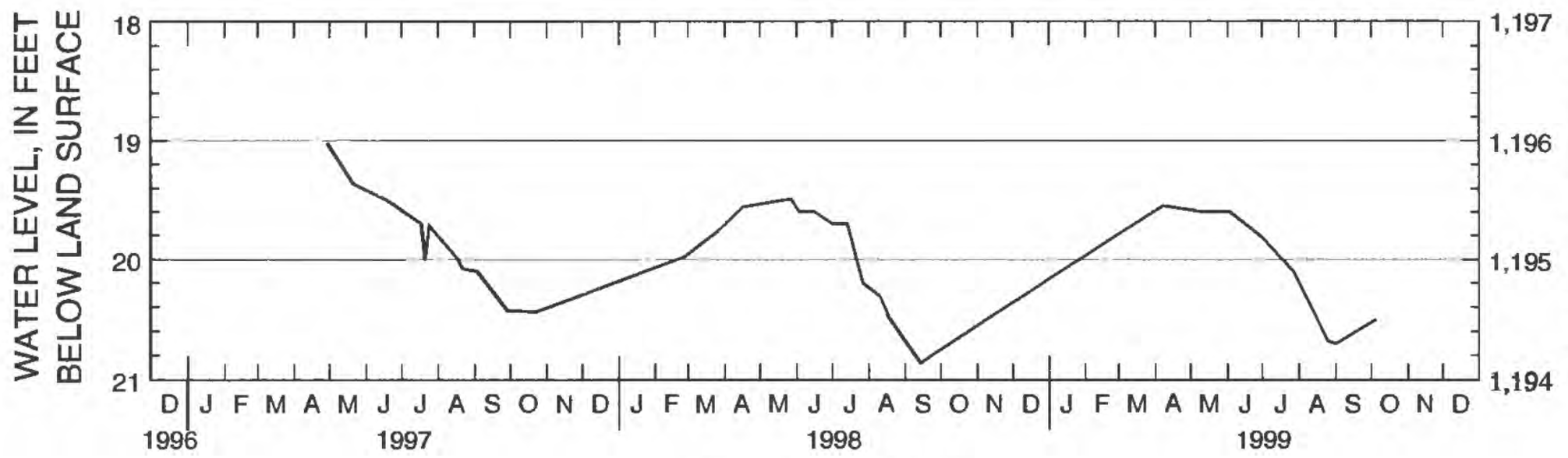

CALENDAR YEAR

Figure B84. Hydrograph for observation well 125N51W1DCCC R (site number 84). 
Site number from location map: 85

Local well number: 125N51W1DCCC2 R

Station identification number: 453752096591802

Other identifier: RB-12W

County: Roberts, South Dakota

Aquifer: Undetermined

Altitude of land surface: 1,215 feet

Measuring point: 2.3 feet

Extremes: April 29, 1997, to August 25, 1999: Highest, 18.91 feet, April 29, 1997; lowest, 21.05 feet, September 14, 1998.



Figure B85. Hydrograph for observation well 125N51W1DCCC2 R (site number 85).

Site number from location map: 86

Local well number: 125N51W13ABBB2 R

Station identification number: 453658096591702

Other identifier: RB-10

County: Roberts, South Dakota

Aquifer: Undetermined

Altitude of land surface: 1,222 feet

Measuring point: 4.3 feet

Extremes: May 27, 1997, to October 5, 1999: Highest, 19.0 feet, May 20, 1997; lowest, 21.94 feet, February 25, 1998.

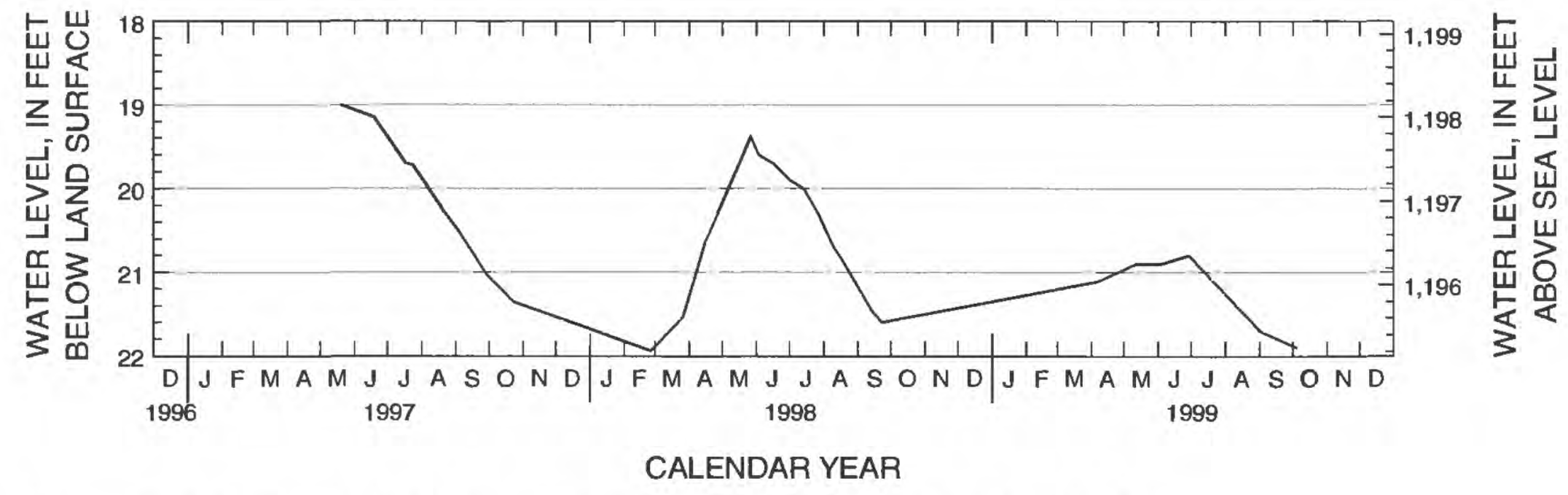

Figure B86. Hydrograph for observation well 125N51W13ABBB2 R (site number 86). 
Site number from location map: 87

Local well number: $125 \mathrm{~N} 51$ W32CCCC R

Station identification number: 453331097045301

Other identifier: R2-96-01

County: Roberts, South Dakota

Aquifer: Revillo

Altitude of land surface: $1,660.1$ feet

Measuring point: 2.0 feet

Extremes: April 29, 1997, to August 26, 1999: Highest, 396.69, August 26, 1999; lowest, 397.66 feet, October 23, 1997.



Figure B87. Hydrograph for observation well 125N51W32CCCC R (site number 87).

Site number from location map: 88

Local well number: 125N51W7BBBB2 R

Station identification number: 453751097060702

Other identifier: R2-95-05

County: Roberts, South Dakota

Aquifer: Roslyn

Altitude of land surface: 1,525 feet

Measuring point: 1.75 feet

Extremes: April 29, 1997, to August 26, 1999: Highest, 72.26 feet, August 26, 1999; lowest, 74.8 feet, April 16, 1998.

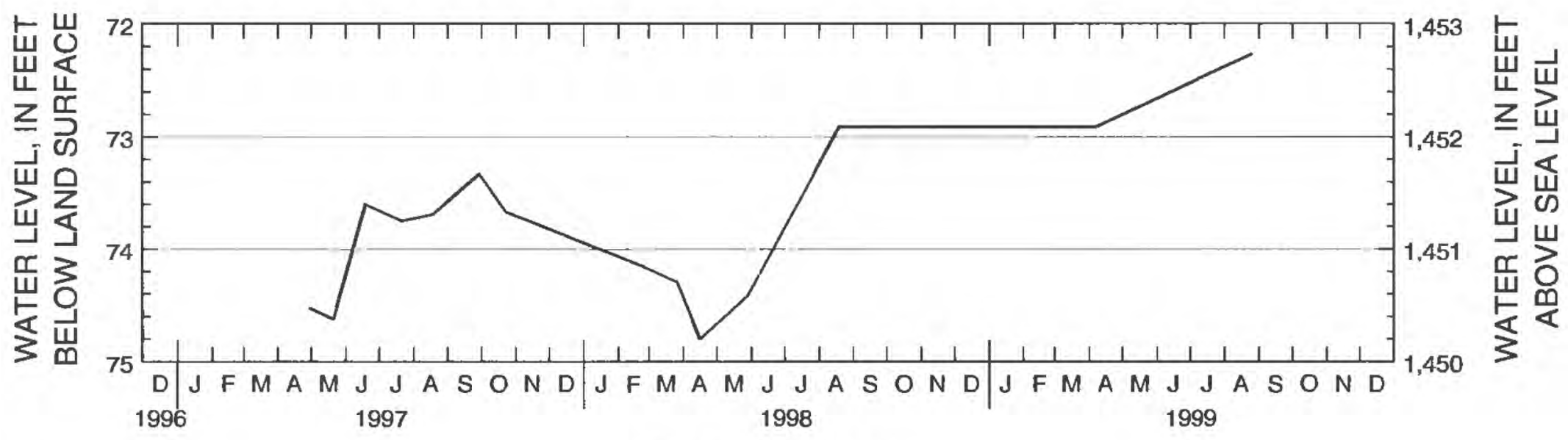

CALENDAR YEAR

Figure B88. Hydrograph for observation well 125N51W7BBBB2 R (site number 88). 
Site number from location map: 89

Local well number: 125N52W16BABB2 R

Station identification number: 453659097104502

Other identifier: R2-99-12

County: Roberts, South Dakota

Aquifer: Eden

Altitude of land surface: $1,987.9$ feet

Measuring point: 2.2 feet

Extremes: September 13, 1999, to December 8, 1999: Highest, 296.57 feet, September 13, 1999; lowest, 296.95 feet, December 8, 1999.
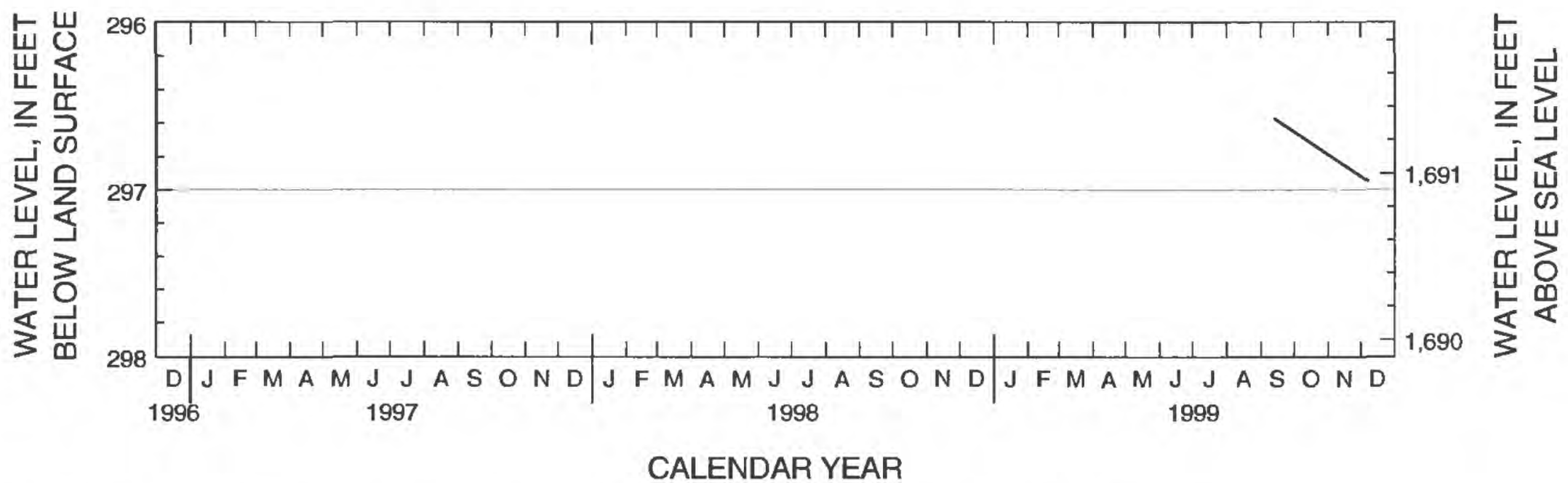

Figure B89. Hydrograph for observation well 125N52W16BABB2 R (site number 89).

Site number from location map: 90

Local well number: $125 \mathrm{~N} 52 \mathrm{~W} 23 \mathrm{CCCC} \mathrm{R}$

Station identification number: 453515097083801

Other identifier: CO-93-19

County: Roberts, South Dakota

Aquifer: Coteau Lakes

Altitude of land surface: 1,972 feet

Measuring point: 1.9 feet

Extremes: April 29, 1997, to August 26, 1999: Highest, 2.35 feet, April 7, 1999; lowest, 7.09 feet, February 25, 1998.

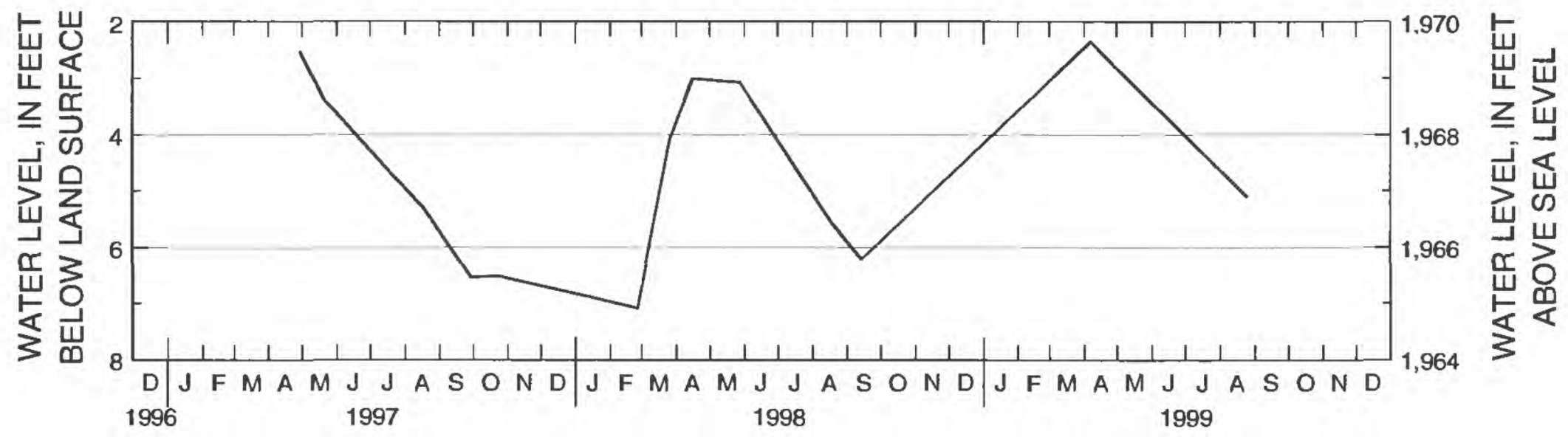

CALENDAR YEAR

Figure B90. Hydrograph for observation well 125N52W23CCCC R (site number 90). 
Site number from location map: 91

Local well number: 125N53W12CCCC R

Station identification number: 453700097144901

Other identifier: CO-93-42

County: Marshall, South Dakota

Aquifer: Coteau Lakes

Altitude of land surface: 1,875 feet

Measuring point: 2.7 feet

Extremes: March 11, 1997, to August 26, 1999: Highest, 9.43 feet, May 28, 1998; lowest, 13.52 feet, February 25, 1998.

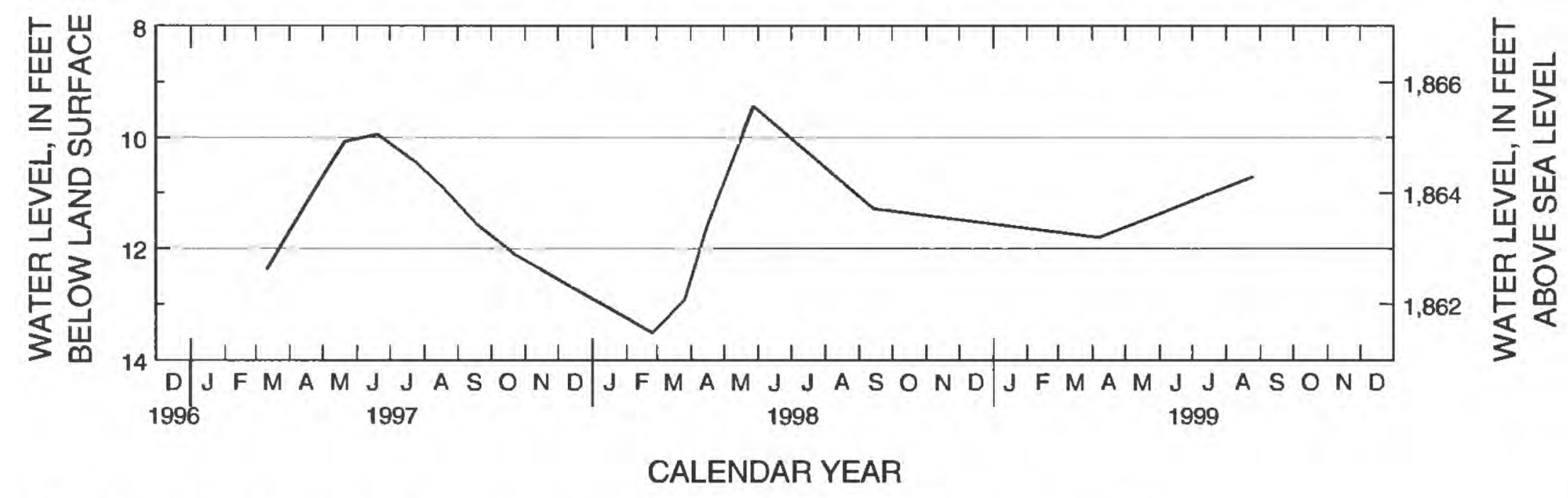

Figure B91. Hydrograph for observation well 125N53W12CCCC R (site number 91).

Site number from location map: 92

Local well number: 126N49W7BBBB2 R

Station identification number: 454304096511302

Other identifier: R2-96-56

County: Roberts, South Dakota

Aquifer: Rosholt

Altitude of land surface: 1,098.5 feet

Measuring point: 1.9 feet

Extremes: April 29, 1997, to August 25, 1999: Highest, 47.7 feet, April 6, 1999; lowest, 48.66 feet, May 20, 1997.

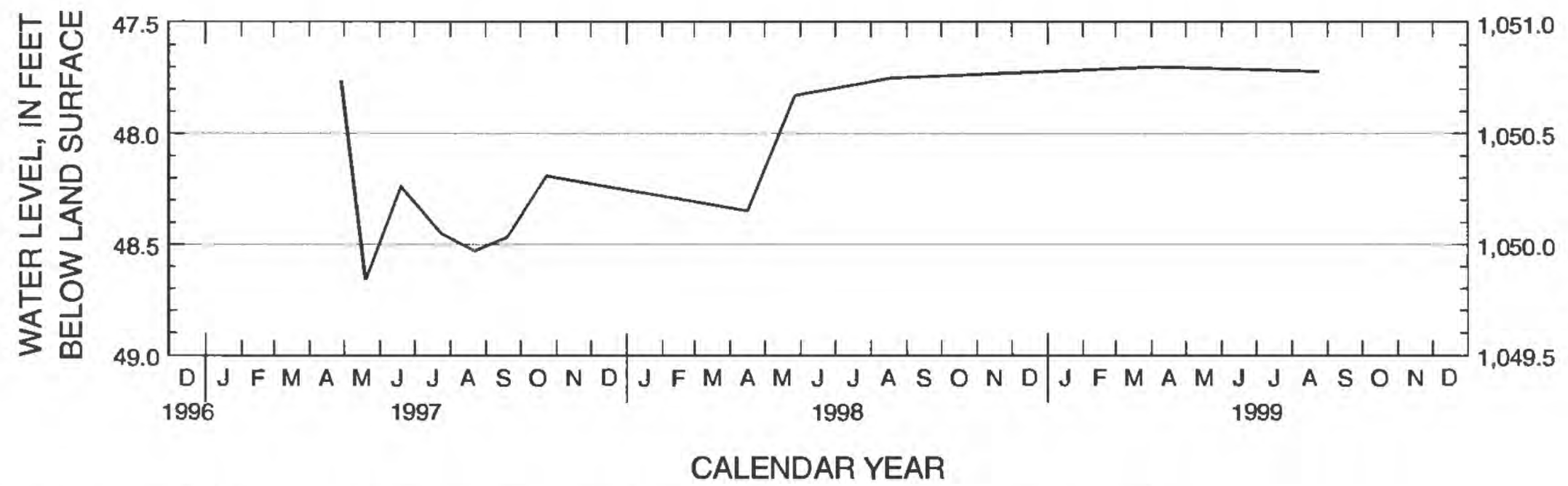

Figure B92. Hydrograph for observation well 126N49W7BBBB2 R (site number 92). 
Site number from location map: 93

Local well number: 126 N49W11AABA R

Station identification number: 454303096451701

Other identifier: R2-97-42

County: Roberts, South Dakota

Aquifer: Fairmount

Altitude of land surface: 1,111 feet

Measuring point: 2.3 feet

Extremes: October 23, 1997, to April 6, 1999: Highest. 77.92 feet, February 25, 1998; lowest, 79.53 feet,

October 23, 1997.

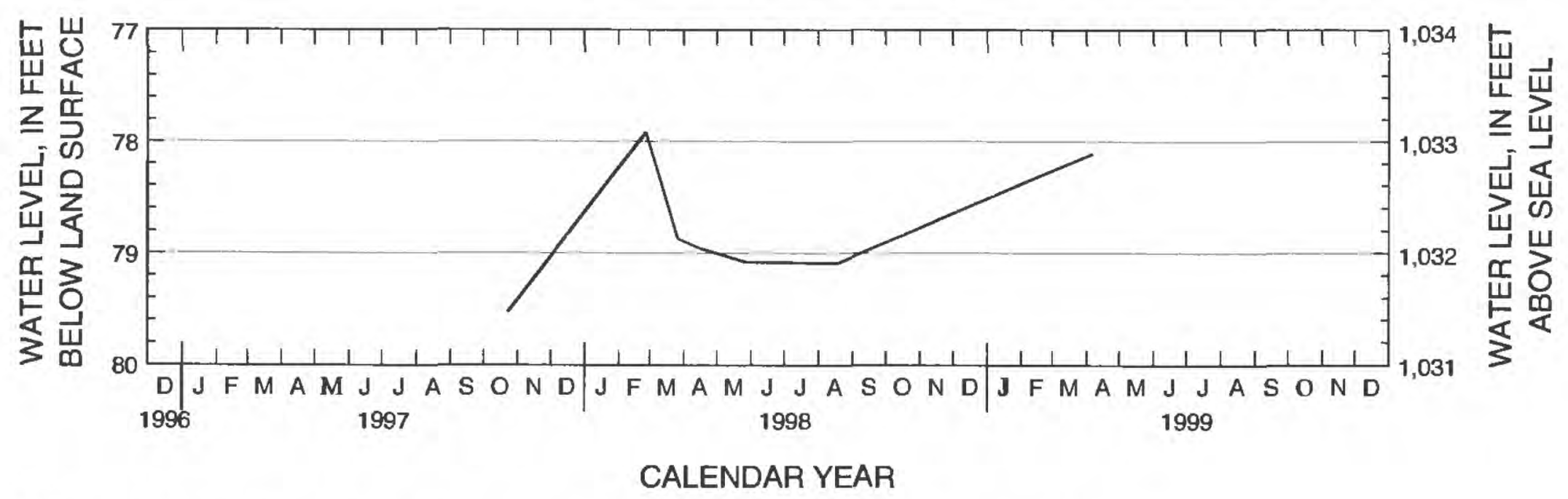

Figure B93. Hydrograph for observation well 126N49W11AABA R (site number 93).

Site number from location map: 94

Local well number: 126N50W21DDDD R

Station identification number: 454028096550001

Other identifier: R2-96-48

County: Roberts, South Dakota

Aquifer: Veblen

Altitude of land surface: $1,165.1$ feet

Measuring point: 2.2 feet

Extremes: December 10, 1996, to August 25, 1999: Highest, 65.61 feet, August 25, 1999; lowest, 68.83 feet, March 11, 1997.

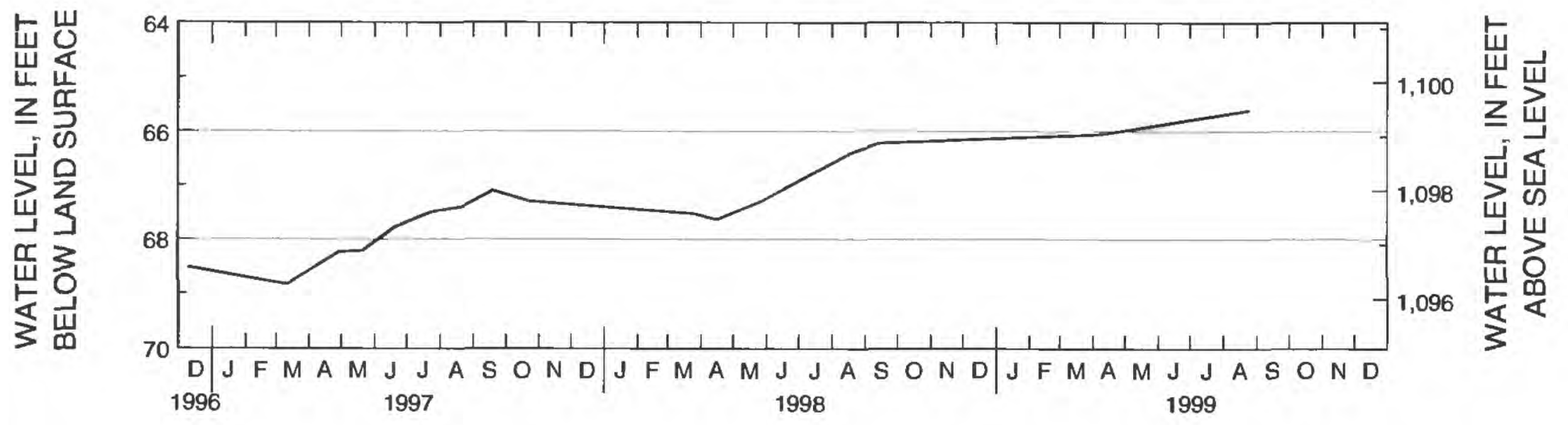

CALENDAR YEAR

Figure B94. Hydrograph for observation well 126N50W21DDDD R (site number 94). 
Site number from location map: 95

Local well number: 126 N50W32CCCB R

Station identification number: 453819096572501

Other identifier: RB-79D

County: Roberts, South Dakota

Aquifer: Veblen

Altitude of land surface: $1,164.4$ feet

Measuring point: 2.0 feet

Extremes: October 26, 1979, to October 5, 1999: Highest, 52.71 feet, April 29, 1997; lowest, 60.4 feet, September 1, 1982.



Figure B95. Hydrograph for observation well 126N50W32CCCB R (site number 95).

Site number from location map: 96

Local well number: 126N51W35AADA R

Station identification number: 453928096595601

Other identifier: RB-79C

County: Roberts, South Dakota

Aquifer: Undetermined

Altitude of land surface: $1,160.6$ feet

Measuring point: 2.5 feet

Extremes: October 26, 1979, to October 5, 1999: Highest, 2.46 feet, May 27, 1998; lowest, 14.5 feet, October 21, 1981.

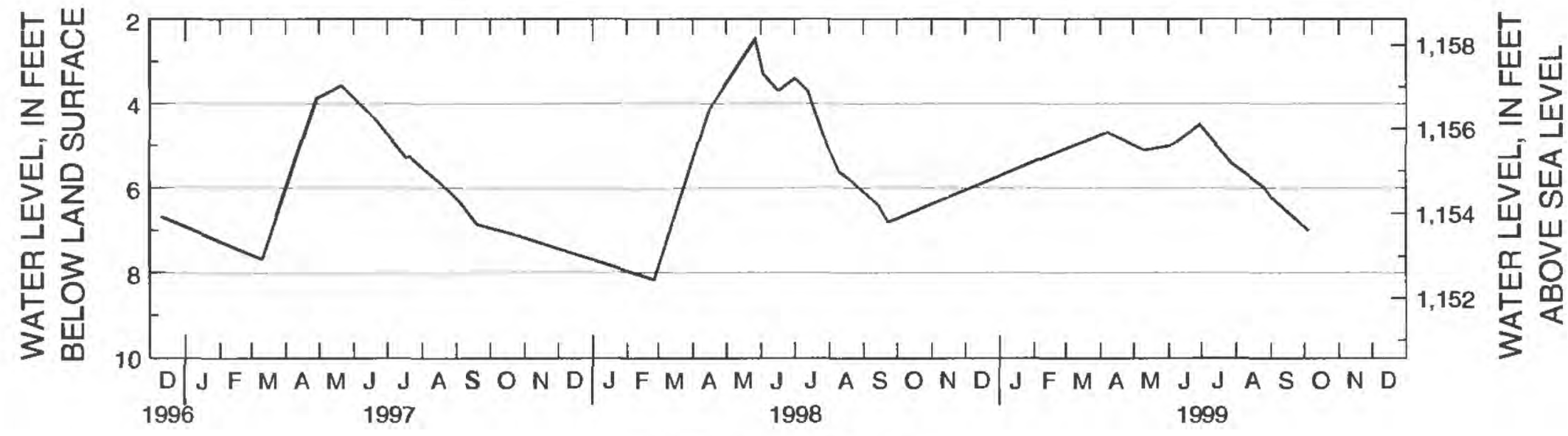

CALENDAR YEAR

Figure B96. Hydrograph for observation well 126N51W35AADA R (site number 96). 
Site number from location map: 97

Local well number: 126N51W36DCCC R

Station identification number: 453844096591801

Other identifier: RB-20

County: Roberts, South Dakota

Aquifer: Undetermined

Altitude of land surface: 1,200 feet

Measuring point: 3.1 feet

Extremes: December 11, 1996, to October 5, 1999: Highest, 5.97 feet, April 29, 1997; lowest, 7.79 feet, December 11, 1996.

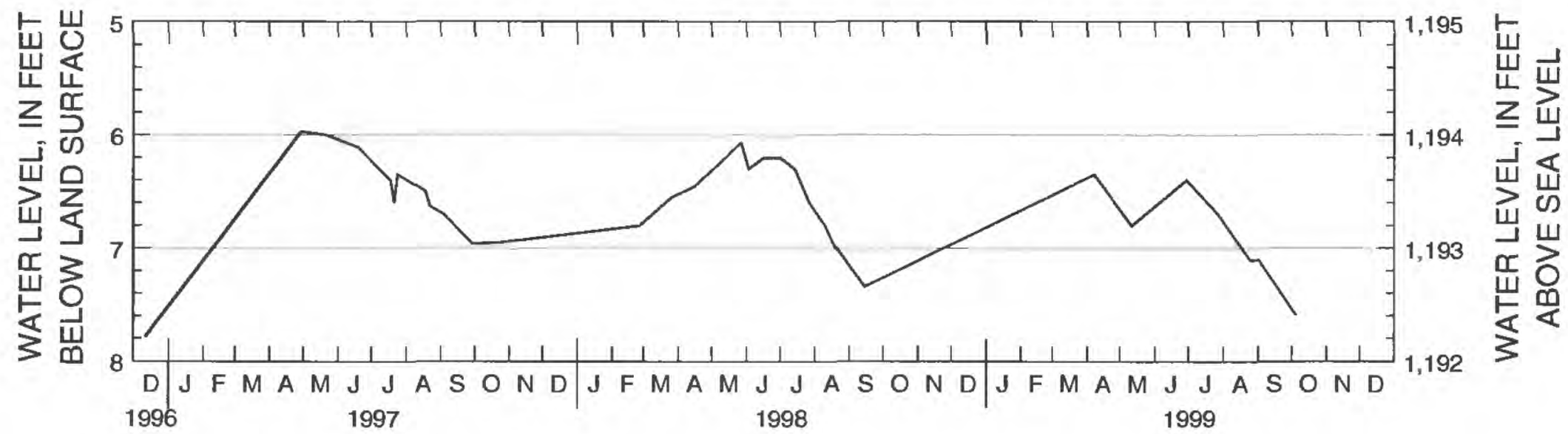

CALENDAR YEAR

Figure B97. Hydrograph for observation well 126N51W36DCCC R (site number 97).

Site number from location map: 98

Local well number: 127N48W2BBBB R

Station identification number: 454911096384601

Other identifier: RB-77F

County: Roberts, South Dakota

Aquifer: Rosholt

Altitude of land surface: 1,069 feet

Measuring point: 1.0 foot

Extremes: May 28, 1978, to October 5, 1999: Highest, 10.95 feet, April 28, 1997; lowest, 28.2 feet, July 20, 1982.

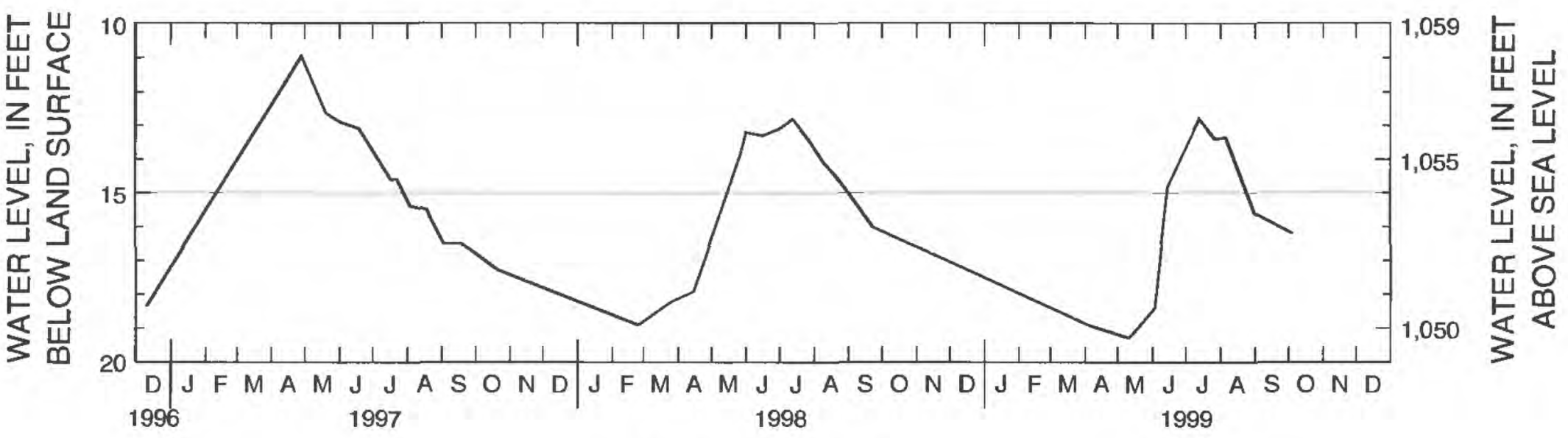

CALENDAR YEAR

Figure B98. Hydrograph for observation well 127N48W2BBBB R (site number 98). 
Site number from location map: 99

Local well number: 127N48W7BBBB R

Station identification number: 454818096434501

Other identifier: RB-77G

County: Roberts, South Dakota

Aquifer: Rosholt

Altitude of land surface: $1,079.9$ feet

Measuring point: 1.9 feet

Extremes: November 30, 1977, to October 5, 1999: Highest, 0.63 feet, April 6, 1999; lowest, 13.9 feet, October 4, 1983, November 9, 1983.
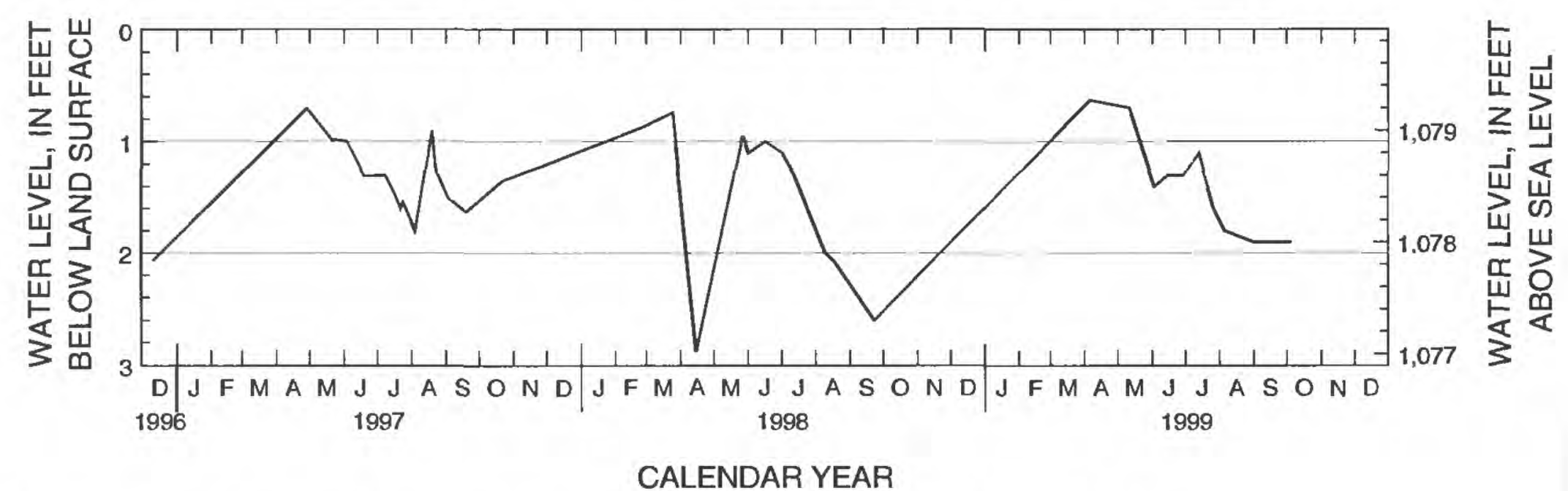

Figure B99. Hydrograph for observation well 127N48W7BBBB R (site number 99).

Site number from location map: 100

Local well number: $127 \mathrm{~N} 48 \mathrm{~W} 7 \mathrm{BBBB} 2 \mathrm{R}$

Station identification number: 454818096434502

Other identifier: $\mathrm{RB}-77 \mathrm{H}$

County: Roberts, South Dakota

Aquifer: Fairmount

Altitude of land surface: $1,079.7$ feet

Measuring point: 3.6 feet

Extremes: November 30, 1977, to October 5, 1999: Highest, 8.2 feet, July 1, 13, 1998: lowest, 20.6 feet. October 4, 1983, November 9, 1983.



Figure B100. Hydrograph for observation well 127N48W7BBBB2 R (site number 100). 
Site number from location map: 101

Local well number: 127N48W7DDBB R

Station identification number: 454737096424801

Other identifier: RB-77A

County: Roberts, South Dakota

Aquifer: Rosholt

Altitude of land surface: 1,100 feet

Measuring point: 3.0 feet

Extremes: June 6, 1977, to September 9, 1999: Highest, 12.5 feet, May 20, 1997; lowest, 32.6 feet, July 20, 1982.

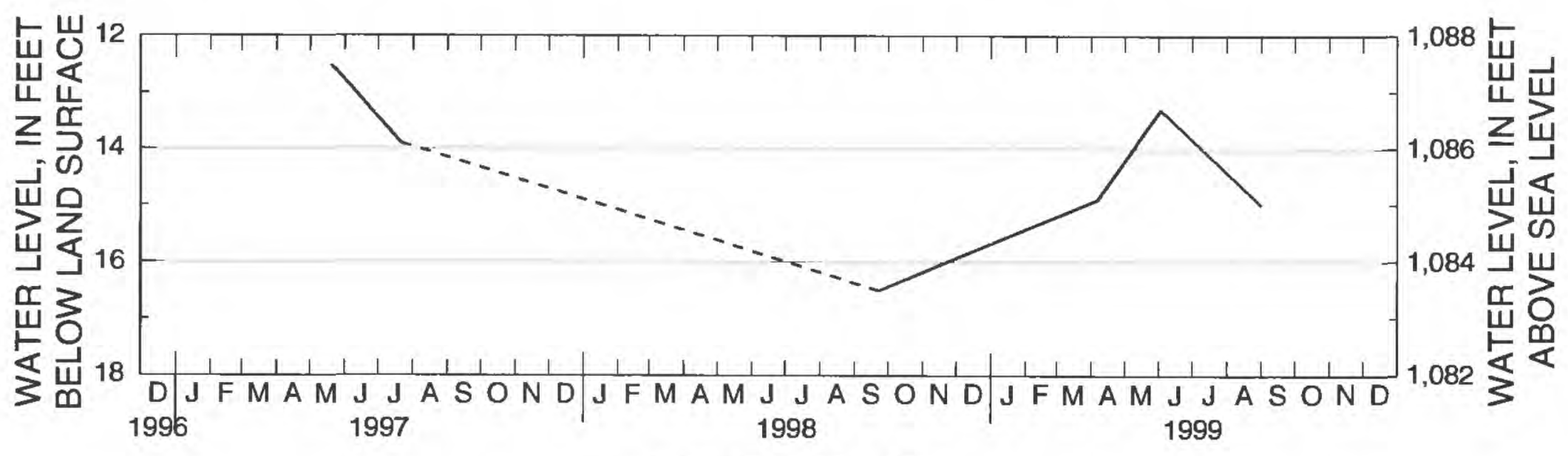

CALENDAR YEAR

Figure B101. Hydrograph for observation well 127N48W7DDBB R (site number 101).

Site number from location map: 102

Local well number: 127N48W18DDDD R

Station identification number: 454634096434501

Other identifier: RB-77B

County: Roberts, South Dakota

Aquifer: Fairmount

Altitude of land surface: $1,097.7$ feet

Measuring point: 1.3 feet

Extremes: June 6, 1977, to October 5, 1999: Highest, 32.3 feet, July 13, 1998; lowest, 44.2 feet, September 20, 1977.



Figure B102. Hydrograph for observation well 127N48W18DDDD R (site number 102). 
Site number from location map: 103

Local well number: 127N48W28AAAA R

Station identification number: 454541096400502

Other identifier: R2-97-45

County: Roberts, South Dakota

Aquifer: Rosholt

Altitude of land surface: 1,090 feet

Measuring point: 2.0 feet

Extremes: October 22, 1997, to August 25, 1999: Higher, 45.73 feet, April 6, 1999; lowest, 46.09 feet, September 14, 1998.

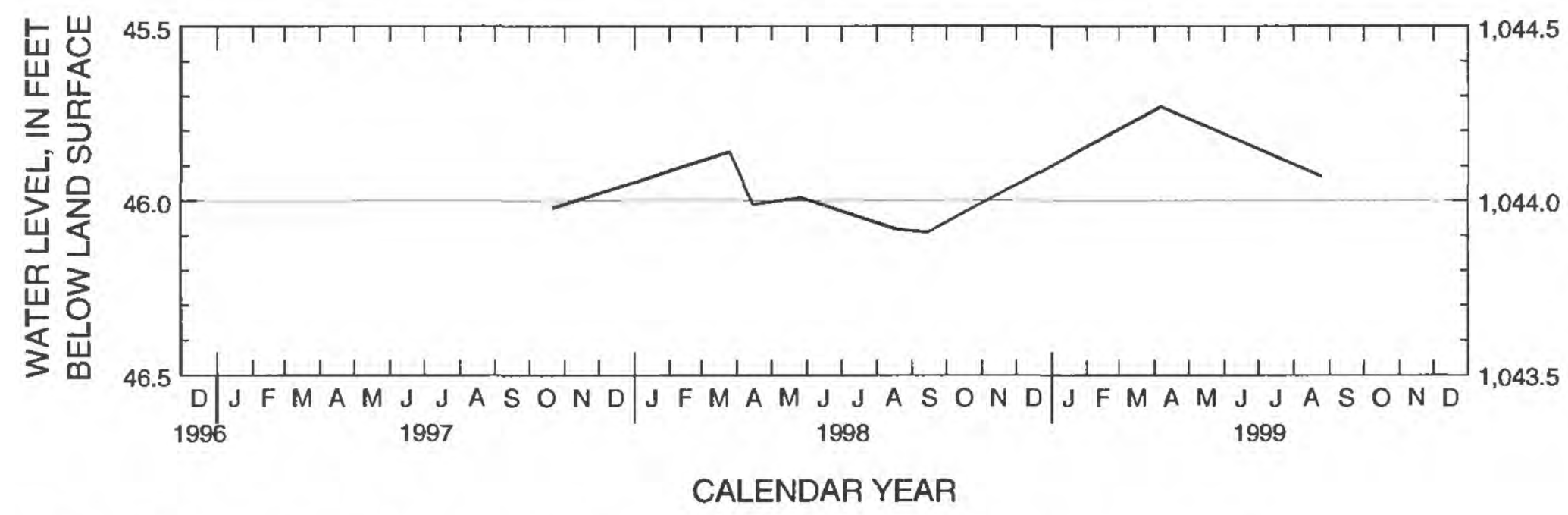

Figure B103. Hydrograph for observation well 127N48W28AAAA R (site number 103).

Site number from location map: 104

Local well number: 127 N49W10BBBB2 R

Station identification number: 454818096473002

Other identifier: R2-96-53

County: Roberts, South Dakota

Aquifer: Rosholt

Altitude of land surface: $1,089.2$ feet

Measuring point: 1.9 feet

Extremes: December 10, 1996, to August 25, 1999: Highest, 11.57 feet, May 27, 1998; lowest, 18.88 feet, August 25, 1999.

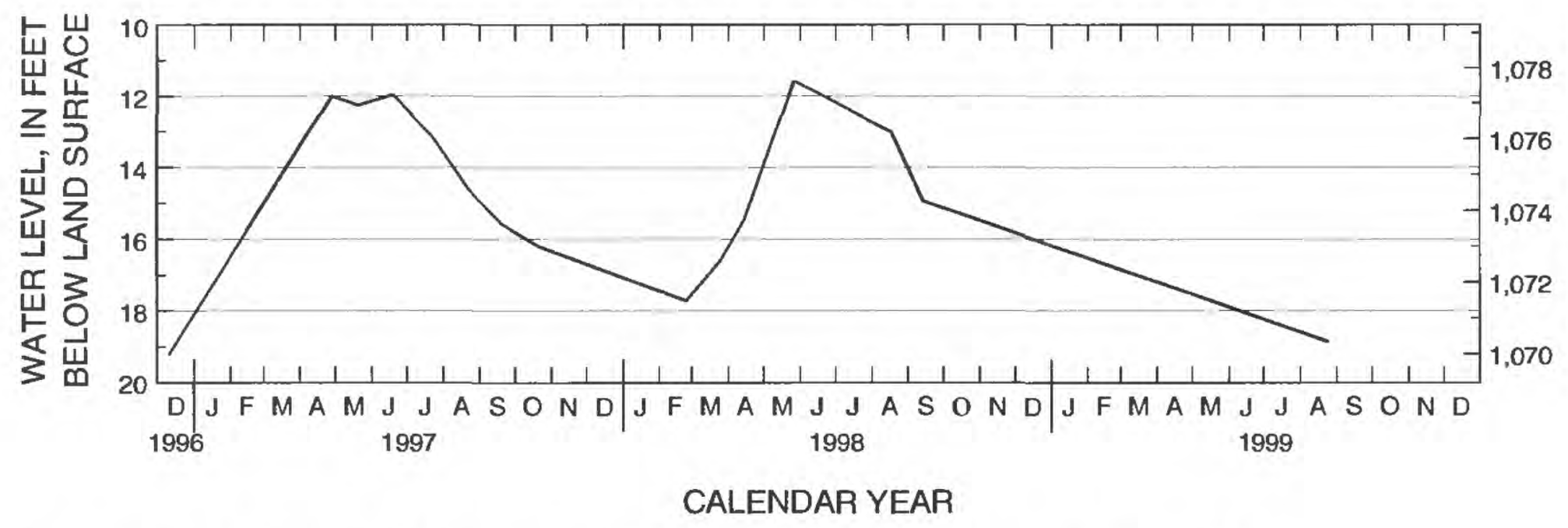

Figure B104. Hydrograph for observation well 127N49W10BBBB2 R (site number 104). 
Site number from location map: 105

Local well number: 127N49W14DAAA R

Station identification number: 454658096450301

Other identifier: RB-77I

County: Roberts, South Dakota

Aquifer: Fairmount

Altitude of land surface: $1,109.3$ feet

Measuring point: 2.2 feet

Extremes: November 30, 1977, to October 5, 1999: Highest, 36.9 feet, July 13, 1998; lowest, 51.2 feet, October 4, 1983.

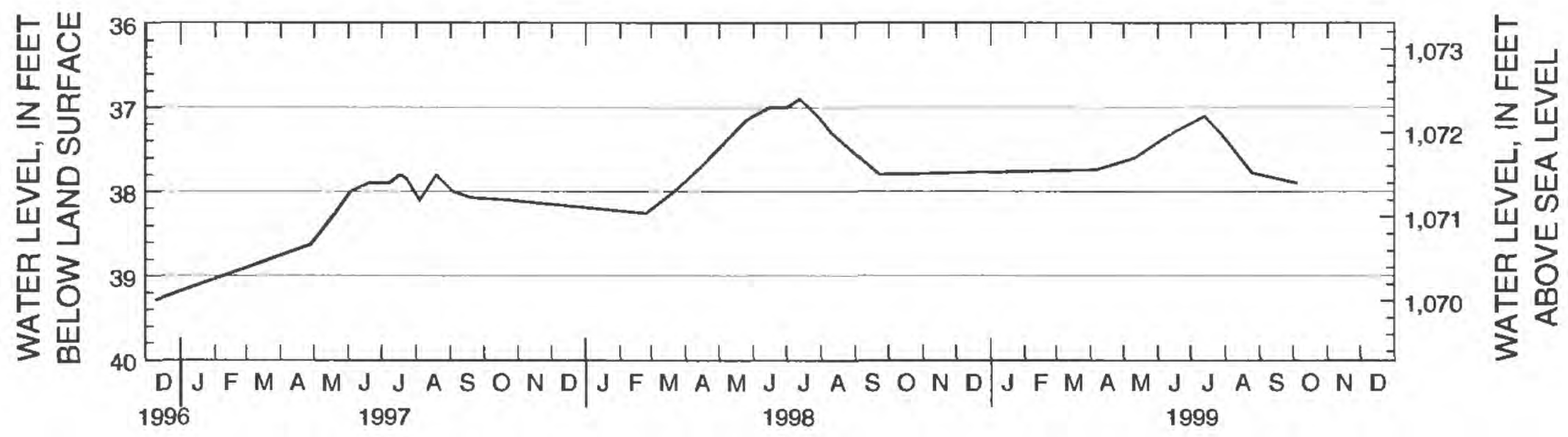

CALENDAR YEAR

Figure B105. Hydrograph for observation well 127N49W14DAAA R (site number 105).

Site number from location map: 106

Local well number: 127N49W14DAAA2 R

Station identification number: 454658096450402

Other identifier: RB-77J

County: Roberts, South Dakota

Aquifer: Rosholt

Altitude of land surface: 1,109.9 feet

Measuring point: 2.0 feet

Extremes: November 30, 1977, to October 5, 1998: Highest, 19.8 feet, June 1, 1998; lowest, 42.5 feet, October 4, 1983.

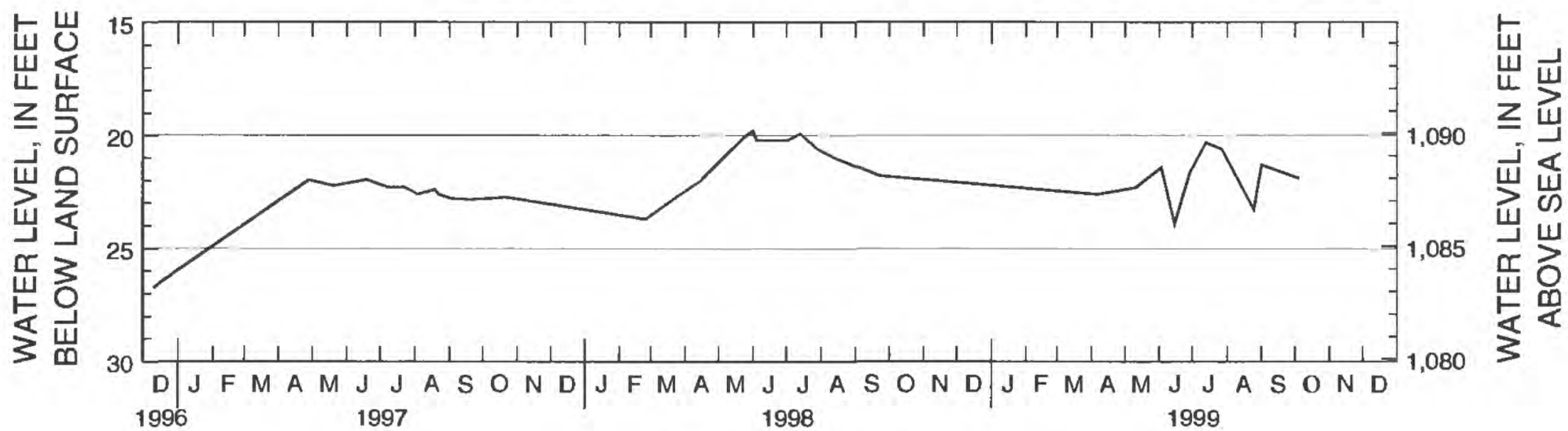

CALENDAR YEAR

Figure B106. Hydrograph for observation well 127N49W14DAAA2 R (site number 106). 
Site number from location map: 107

Local well number: 127N49W26DDDD R

Station identification number: 454449096450201

Other identifier: RB-77C

County: Roberts, South Dakota

Aquifer: Fairmount

Altitude of land surface: $1,124.5$ feet

Measuring point: 1.6 feet

Extremes: June 6, 1977, to October 5, 1999: Highest, 54.0 feet, July 13, 1998; lowest, 70.0 feet, September 20, 1977.



CALENDAR YEAR

Figure B107. Hydrograph for observation well 127N49W26DDDD R (site number 107).

Site number from location map: 108

Local well number: 127 N49W29DCDC2 R

Station identification number: 454550096491302

Other identifier: LTR-16

County: Roberts, South Dakota

Aquifer: Rosholt

Altitude of land surface: $1,111.4$ feet

Measuring point: 4.0 feet

Extremes: December 10, 1996, to August 25, 1999: Highest, 54.68 feet, August 25, 1999; lowest, 58.78 feet, December 10, 1996.

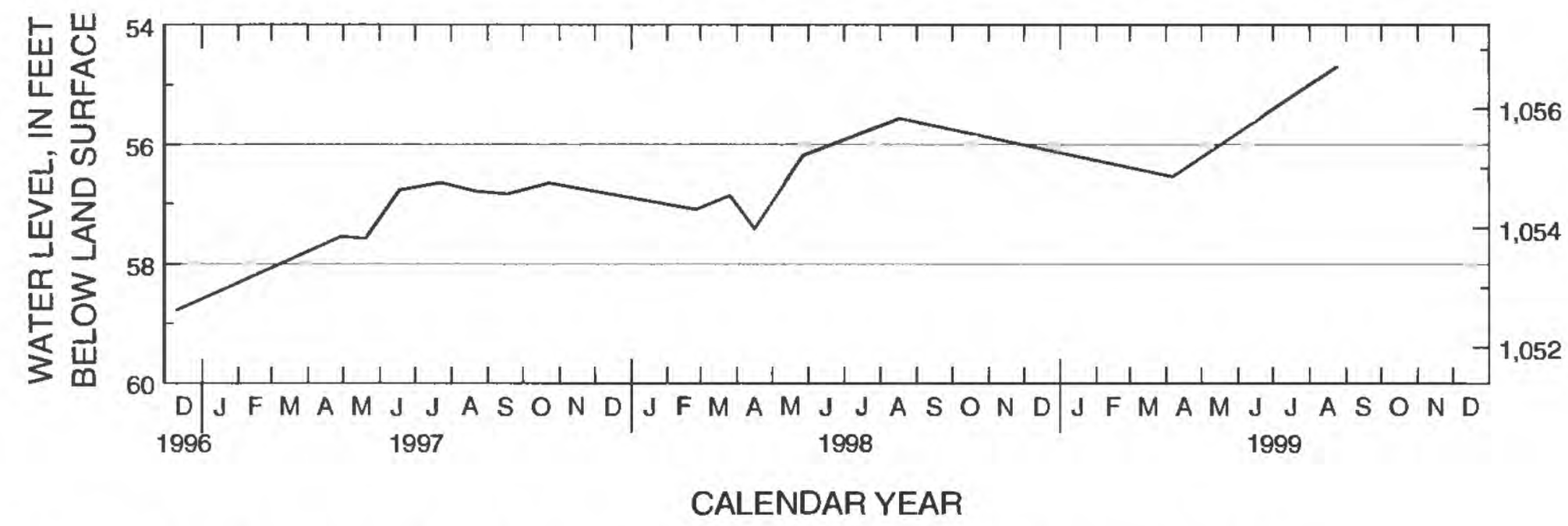

Figure B108. Hydrograph for observation well 127N49W29DCDC2 R (site number 108). 
Site number from location map: 109

Local well number: 127N49W36BBBB R

Station identification number: 454449096450101

Other identifier: RB-77K

County: Roberts, South Dakota

Aquifer: Rosholt

Altitude of land surface: $1,129.0$ feet

Measuring point: 2.8 feet

Extremes: November 30, 1977, to October 5, 1999: Highest, 56.46 feet, August 18, 1998; lowest, 77.4 feet, August 1, 1983.

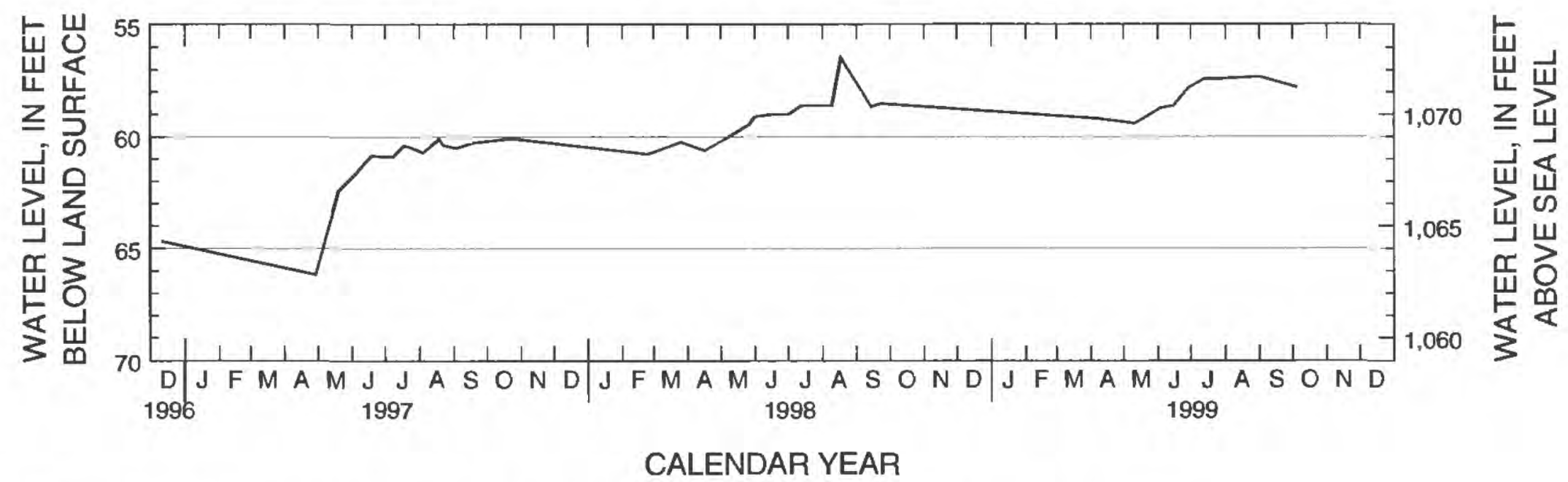

Figure B109. Hydrograph for observation well 127N49W36BBBB R (site number 109).

Site number from location map: 110

Local well number: 127 N50W9BBBB2 R

Station identification number: 454818096550602

Other identifier: R2-97-39

County: Roberts, South Dakota

Aquifer: Rosholt

Altitude of land surface: 1,120 feet

Measuring point: 2.0 feet

Extremes: October 22, 1997, to August 6, 1999: Highest, 27.9 feet, April 6, 1999; lowest. 30.3 feet, September 15. 1998.

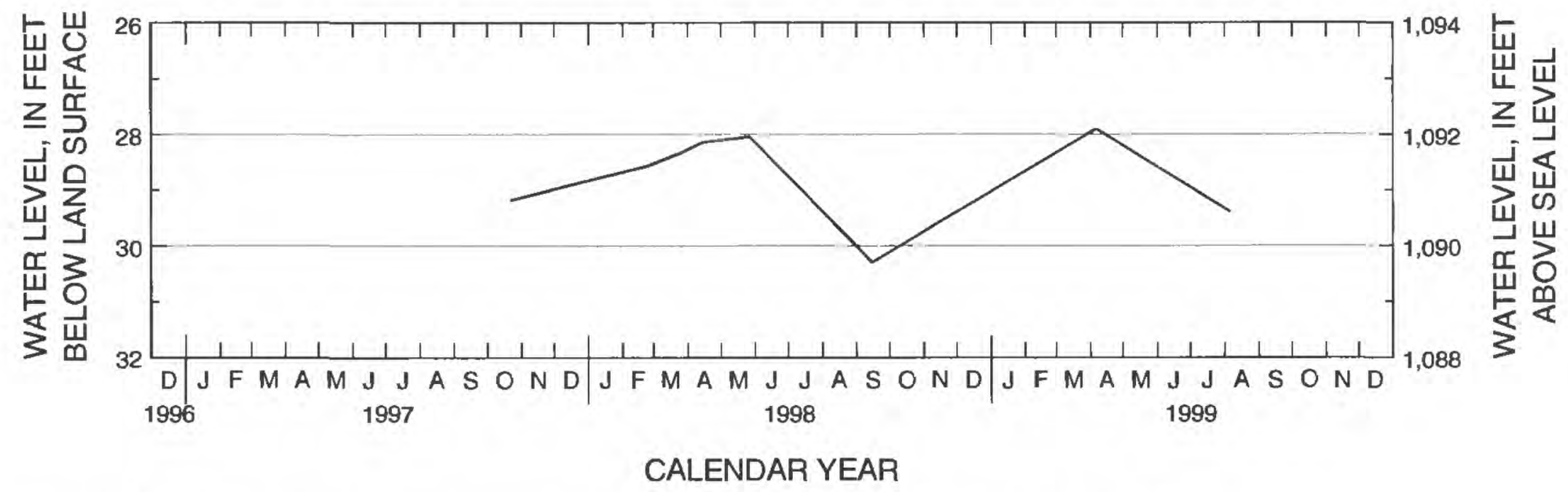

Figure B110. Hydrograph for observation well 127N50W9BBBB2 R (site number 110). 
Site number from location map: 111

Local well number: 127N50W19CCCB R

Station identification number: 454547096584301

Other identifier: LTR-2

County: Roberts, South Dakota

Aquifer: Veblen

Altitude of land surface: 1,175.4 feet

Measuring point: 2.0 feet

Extremes: February 24, 1998, to August 6, 1999: Highest, 40.09 feet, August 6, 1999; lowest, 42.45 feet, March 25, 1998.

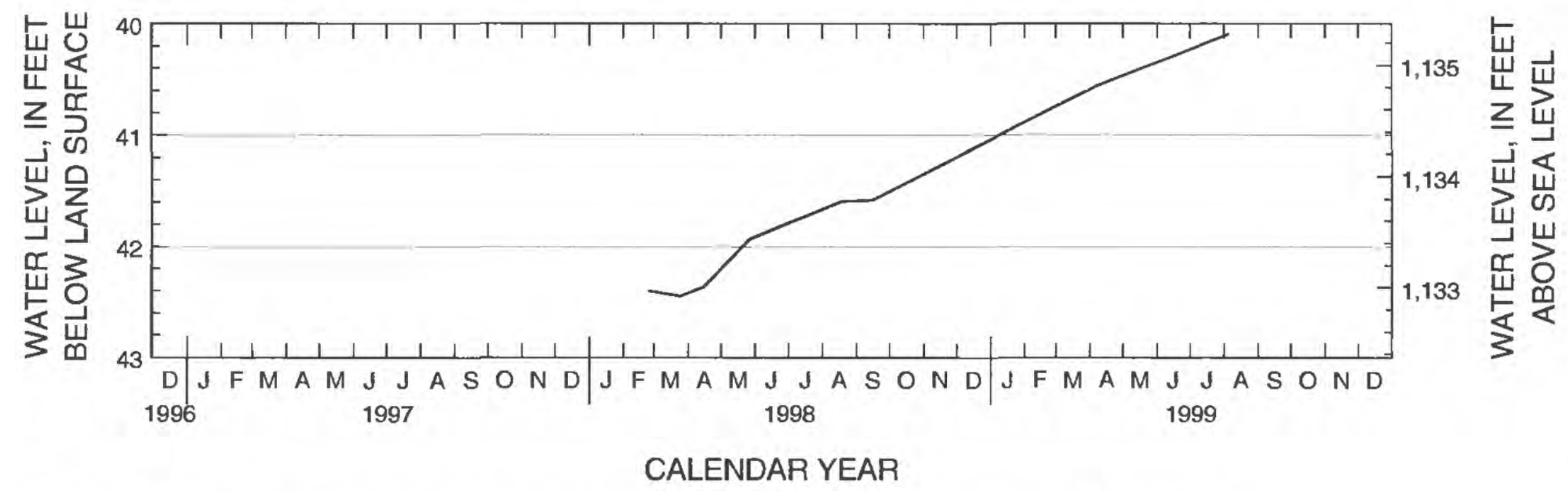

Figure B111. Hydrograph for observation well 127N50W19CCCB R (site number 111).

Site number from location map: 112

Local well number: 127N50W21DDDD R

Station identification number: 454541096550001

Other identifier: R2-96-50

County: Roberts, South Dakota

Aquifer: Veblen

Altitude of land surface: 1,139 feet

Measuring point: 2.3 feet

Extremes: April 29, 1997, to August 25, 1999: Highest, 24.63 feet, April 6, 1999; lowest, 26.69 feet, February 25, 1998.



Figure B112. Hydrograph for observation well 127N50W21DDDD R (site number 112). 
Site number from location map: 113

Local well number: 127N50W25ADAD2 R

Station identification number: 454523096511602

Other identifier: R2-96-58

County: Roberts, South Dakota

Aquifer: Rosholt

Altitude of land surface: $1,099.5$ feet

Measuring point: 1.6 feet

Extremes: April 29, 1997, to August 25, 1999: Highest, 51.48 feet, August 18, 1998; lowest, 52.23 feet, April 16, 1998.



CALENDAR YEAR

Figure B113. Hydrograph for observation well 127N50W25ADAD2 R (site number 113).

Site number from location map: 114

Local well number: 127N51W3AAAA R

Station identification number: 454910097011501

Other identifier: RB-81A

County: Roberts, South Dakota

Aquifer: Veblen

Altitude of land surface: $1,226.3$ feet

Measuring point: 1.7 feet

Extremes: May 29, 1981, to September 1, 1999: Highest, 69.4 feet, September 1, 1999; lowest, 84.8 feet, October 4, 1983.

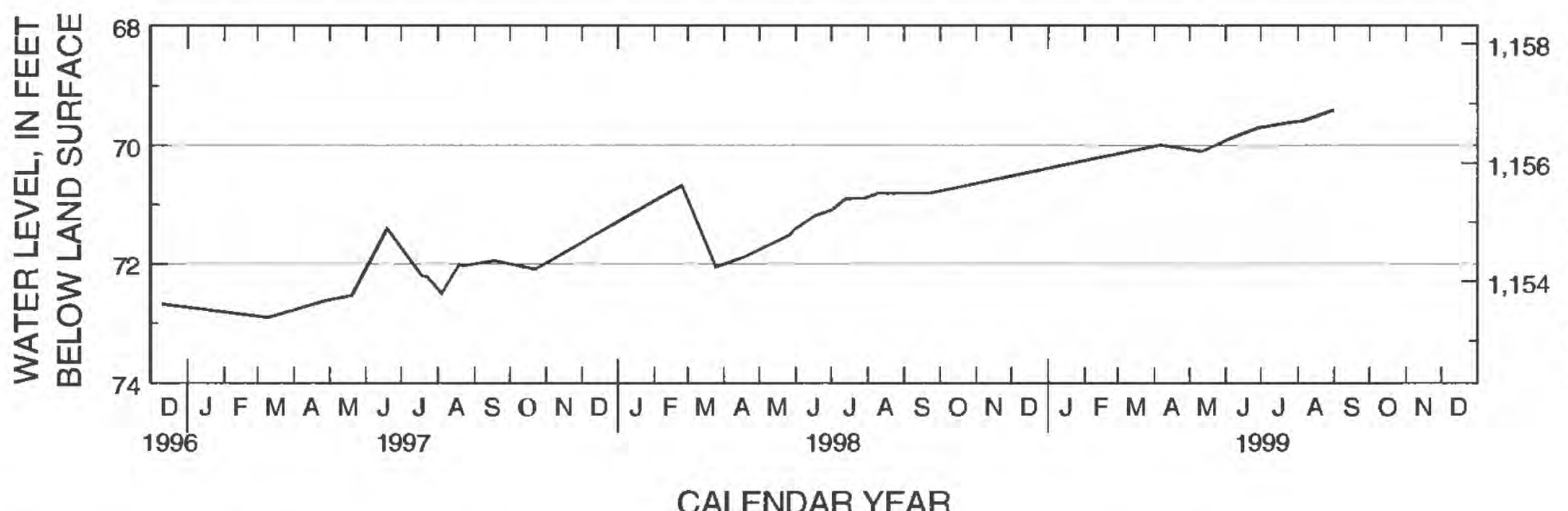

Figure B114. Hydrograph for observation well 127N51W3AAAA R (site number 114). 
Site number from location map: 115

Local well number: 127N51W9AAAA2 R

Station identification number: 454820097023602

Other identifier: R2-97-07

County: Roberts, South Dakota

Aquifer: Veblen

Altitude of land surface: 1,192 feet

Measuring point: 1.7 feet

Extremes: October 22, 1997, to August 6, 1999: Highest, 30.95 feet. August 6. 1999; lowest, 33.58 feet, February 24, 1998.

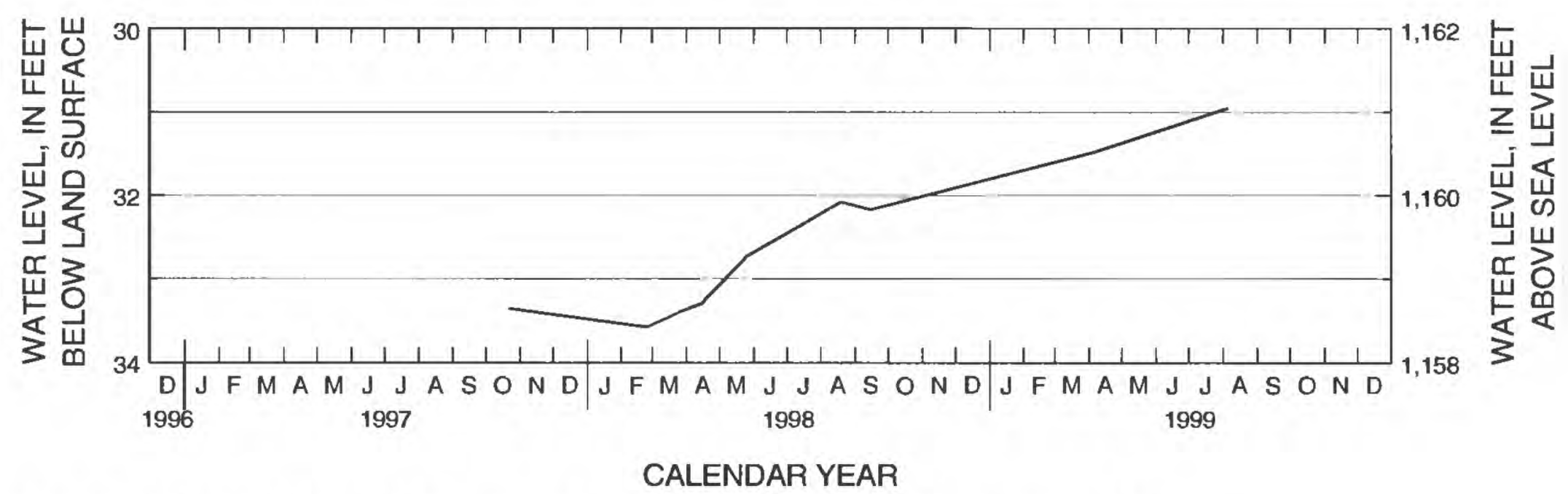

Figure B115. Hydrograph for observation well 127N51W9AAAA2 R (site number 115).

Site number from location map: 116

Local well number: 127N52W25AAAA2 R

Station identification number: 454838097061102

Other identifier: R2-97-11

County: Roberts, South Dakota

Aquifer: Undetermined

Altitude of land surface: 1,188 feet

Measuring point: 1.9 feet

Extremes: October 22, 1997, to August 6, 1999: Highest, 0.14 foot, April 6, 1999; lowest, 5.72 feet, October 22, 1997.

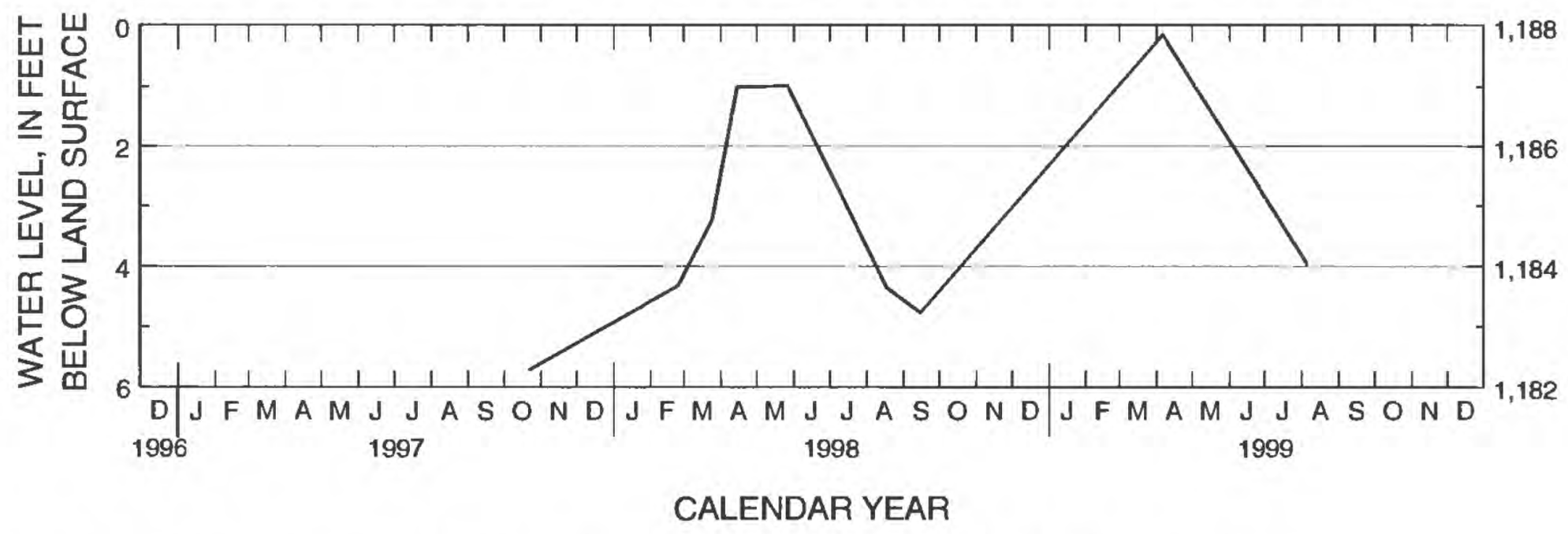

Figure B116. Hydrograph for observation well 127N52W25AAAA2 R (site number 116). 
Site number from location map: 117

Local well number: 127N52W7CCCD R

Station identification number: 454818097060401

Other identifier: R2-95-08

County: Roberts, South Dakota

Aquifer: Veblen

Altitude of land surface: 1,197 feet

Measuring point: 2.4 feet

Extremes: December 10, 1996, to August 6, 1999: Highest, 27.21 feet, August 6, 1999; lowest, 30.82 feet, March 10, 1997.

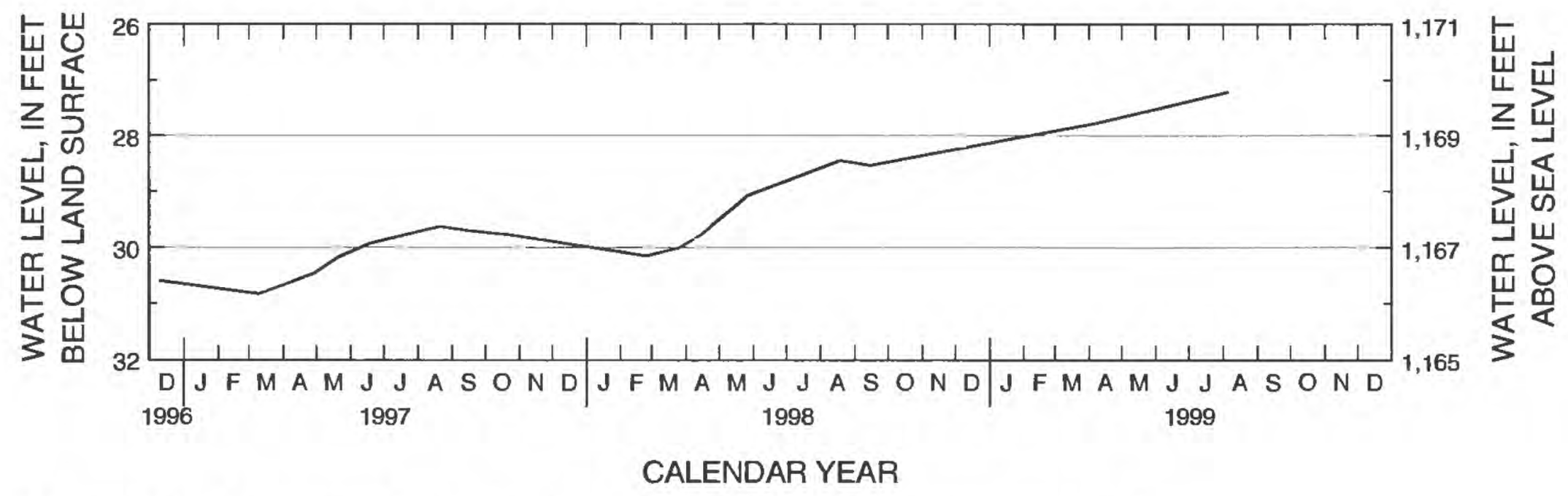

Figure B117. Hydrograph for observation well 127N52W7CCCD R (site number 117).

Site number from location map: 118

Local well number: 128 N48W1CCDC2 R

Station identification number: 455334096372102

Other identifier: R2-96-62

County: Roberts, South Dakota

Aquifer: Fairmount

Altitude of land surface: $1,021.8$ feet

Measuring point: 1.6 feet

Extremes: April 28, 1997, to August 6, 1999: Highest, 41.68 feet, April 28, 1997; lowest, 44.91 feet, July 23, 1997.

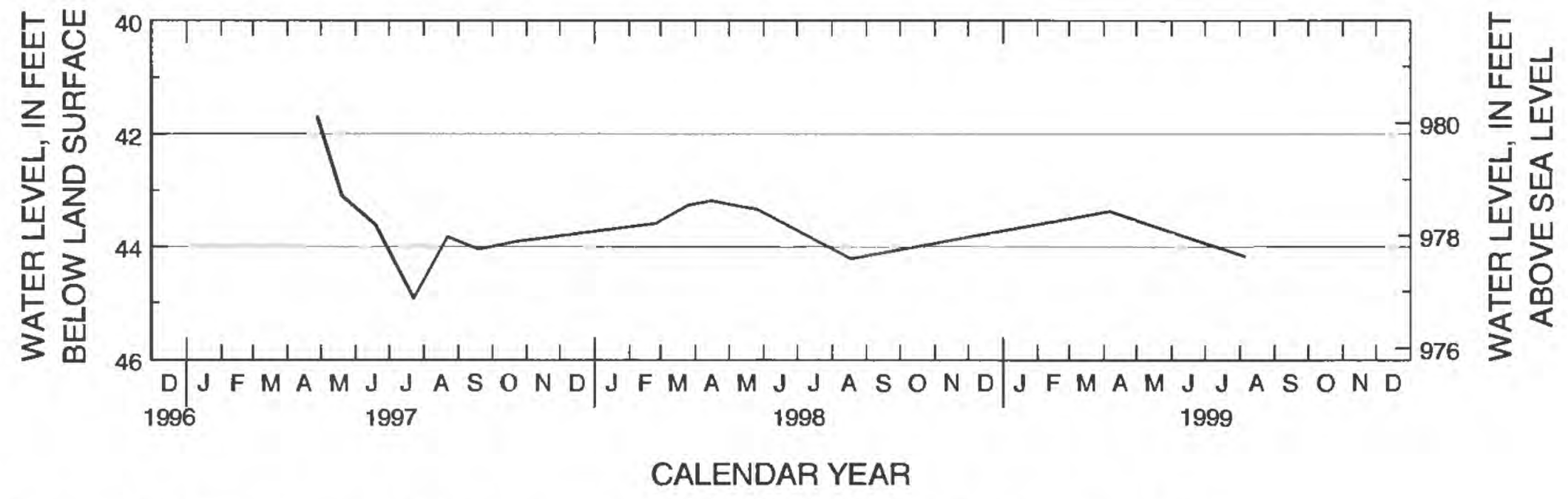

Figure B118. Hydrograph for observation well 128N48W1CCDC2 R (site number 118). 
Site number from location map: 119

Local well number: 128N48W4DDDD R

Station identification number: 455336096400301

Other identifier: R2-96-63

County: Roberts, South Dakota

Aquifer: Fairmount

Altitude of land surface: $1,046.6$ feet

Measuring point: -0.2 foot

Extremes: December 10, 1996, to April 6, 1999: Highest, 20.58 feet, December 10, 1996; lowest, 33.96 feet, March 11, 1997.

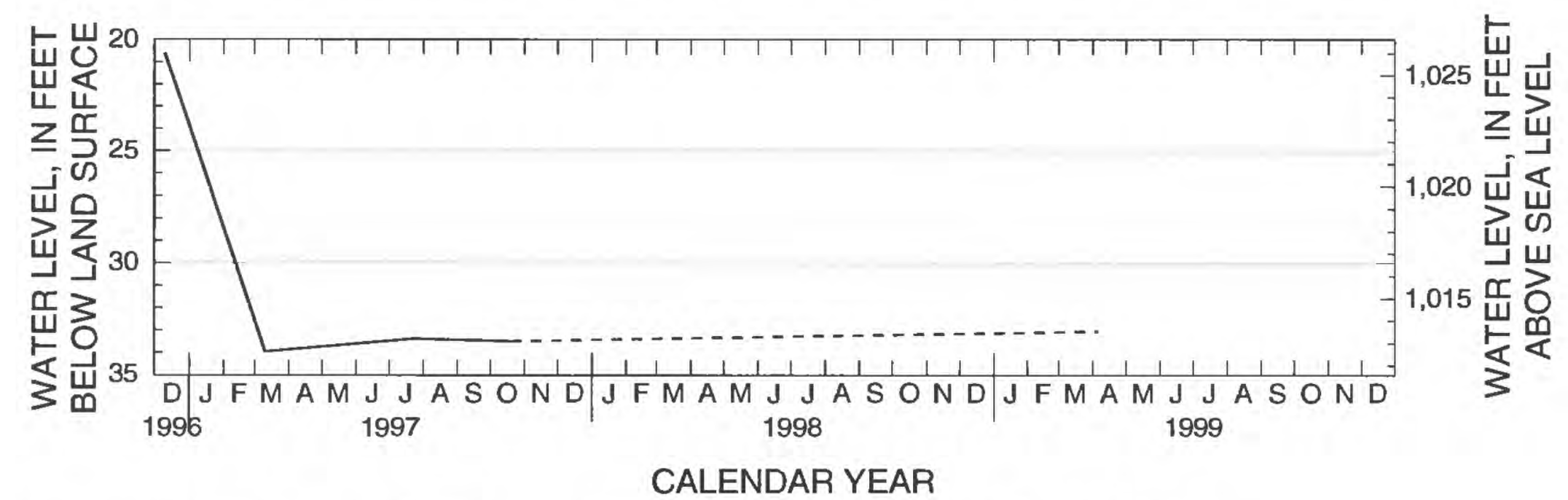

Figure B119. Hydrograph for observation well 128N48W4DDDD R (site number 119).

Site number from location map: 120

Local well number: 128 N48W6BBBB R

Station identification number: 455423096434501

Other identifier: LTR-10

County: Roberts, South Dakota

Aquifer: Fairmount

Altitude of land surface: $1,098.2$ feet

Measuring point: 2.75 feet

Extremes: April 28, 1997, to August 6, 1999: Highest, 41.33 feet, August 6, 1999; lowest, 43.85 feet, April 28, 1997.

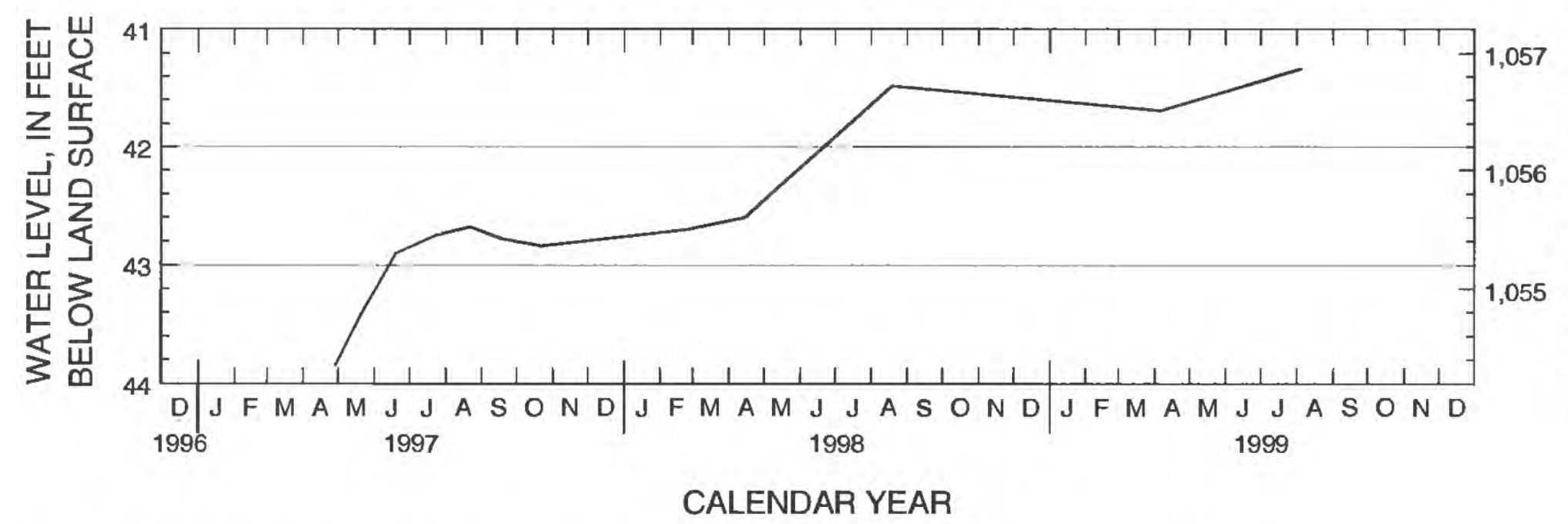

Figure B120. Hydrograph for observation well 128N48W6BBBB R (site number 120). 
Site number from location map: 121

Local well number: $128 \mathrm{~N} 48 \mathrm{~W} 19 \mathrm{BCCC} \mathrm{R}$

Station identification number: 455123096434701

Other identifier: R2-97-30

County: Roberts, South Dakota

Aquifer: Fairmount

Altitude of land surface: 1,080 feet

Measuring point: 1.7 feet

Extremes: October 22, 1997, to October 5, 1999: Highest, 8.88 feet, October 22, 1997; lowest, 23.7 feet, July 27 , 1999.



CALENDAR YEAR

Figure B121. Hydrograph for observation well 128N48W19BCCC R (site number 121).

Site number from location map: 122

Local well number: 128N48W20BBBB R

Station identification number: 455148096423101

Other identifier: RB-77D

County: Roberts, South Dakota

Aquifer: Rosholt

Altitude of land surface: $1,070.7$ feet

Measuring point: 2.1 feet

Extremes: November 30, 1977, to October 5, 1999: Highest, 1.14 feet, April 28, 1997; lowest, 18.6 feet,

October 4, 1983.

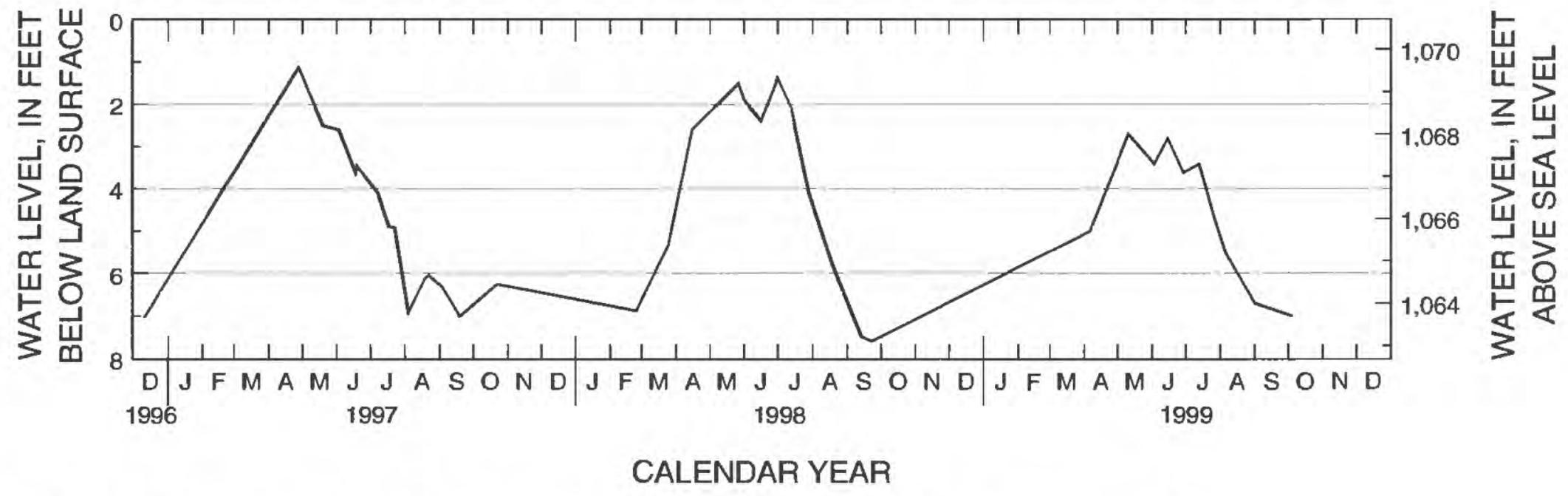

Figure B122. Hydrograph for observation well 128N48W20BBBB R (site number 122). 
Site number from location map: 123

Local well number: 128N48W29DDDD R

Station identification number: 455005096411801

Other identifier: RB-77E

County: Roberts, South Dakota

Aquifer: Rosholt

Altitude of land surface: $1,081.5$ feet

Measuring point: 1.2 feet

Extremes: May 23, 1978, to October 5, 1999: Highest, 0.33 feet, April 28, 1997; lowest, 22.6 feet, October 4, 1983.

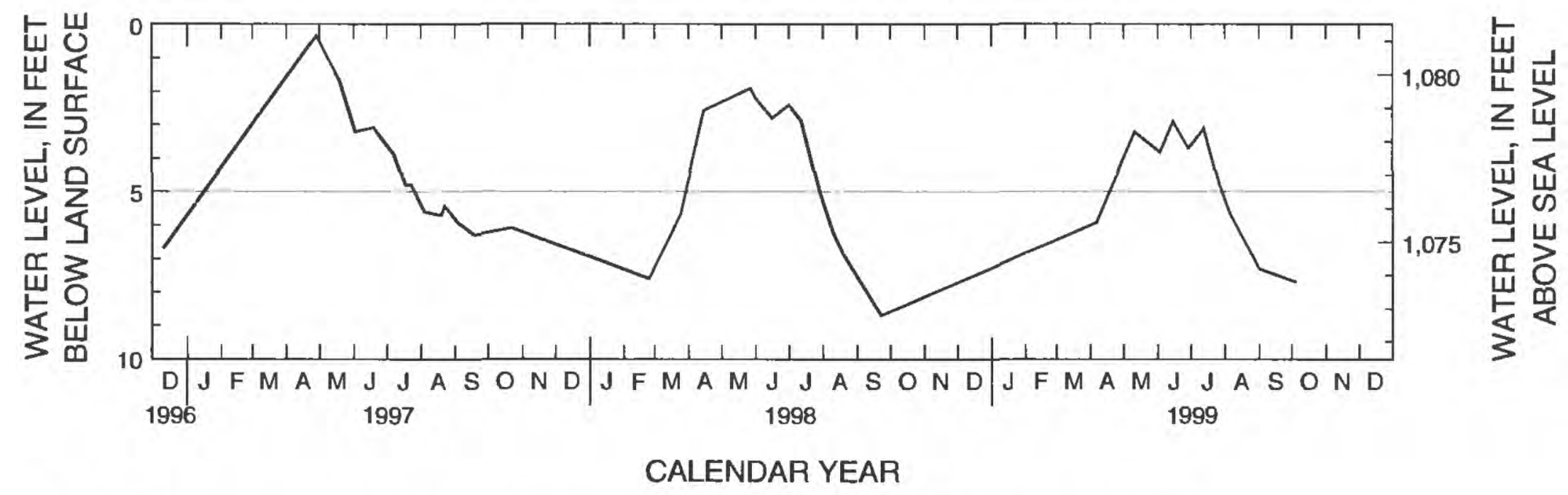

Figure B123. Hydrograph for observation well 128N48W29DDDD R (site number 123).

Site number from location map: 124

Local well number: 128N49W1DDAD2 R

Station identification number: 455341096435001

Other identifier: R2-97-32

County: Roberts, South Dakota

Aquifer: Rosholt

Altitude of land surface: 1,074 feet

Measuring point: 2.2 feet

Extremes: October 22, 1997, to August 6, 1999: Highest, 8.64 feet, August 6, 1999; lowest, 10.28 feet, February 24, 1998.



Figure B124. Hydrograph for observation well 128N49W1DDAD2 R (site number 124). 
Site number from location map: 125

Local well number: 128 N49W20BBBB R

Station identification number: 455146096500001

Other identifier: LTR-6

County: Roberts, South Dakota

Aquifer: Fairmount

Altitude of land surface: $1,082.6$ feet

Measuring point: 4.0 feet

Extremes: December 10, 1996, to August 6, 1999: Highest, 8.9 feet, April 6, 1999; lowest, 9.62 feet, December 10, 1996.

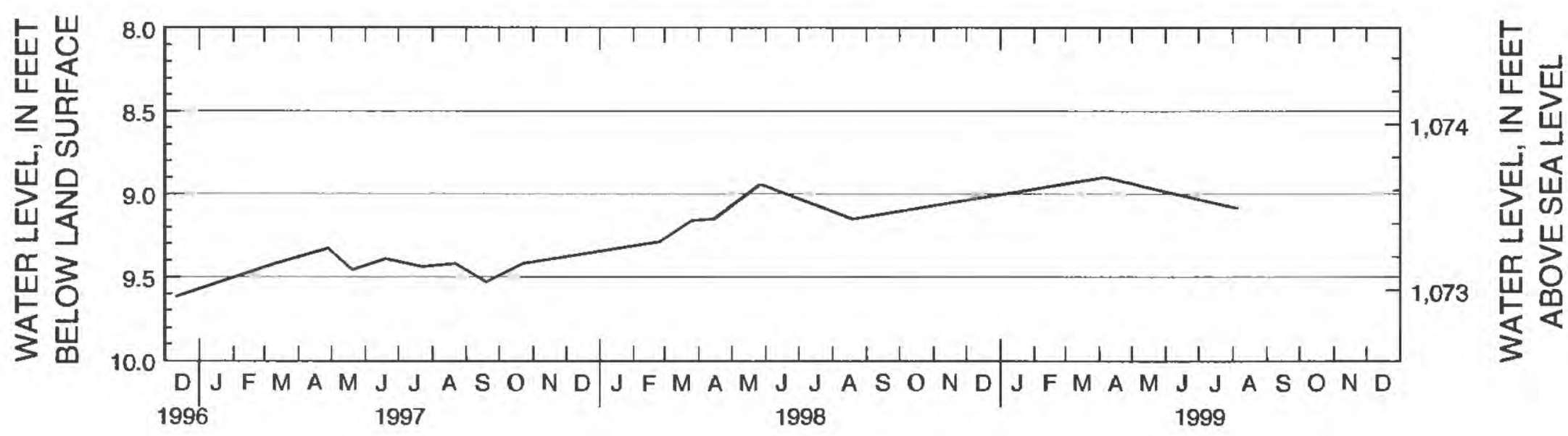

CALENDAR YEAR

Figure B125. Hydrograph for observation well 128N49W20BBBB R (site number 125).

Site number from location map: 126

Local well number: 128N49W36AAAA R

Station identification number: 455003096434701

Other identifier: LTR-21

County: Roberts, South Dakota

Aquifer: Fairmount

Altitude of land surface: $1,078.9$ feet

Measuring point: 3.9 feet

Extremes: December 10, 1996, to April 6, 1999: Highest, -1.48 feet, December 10, 1996; lowest, 1.36 feet, April 28, 1997.

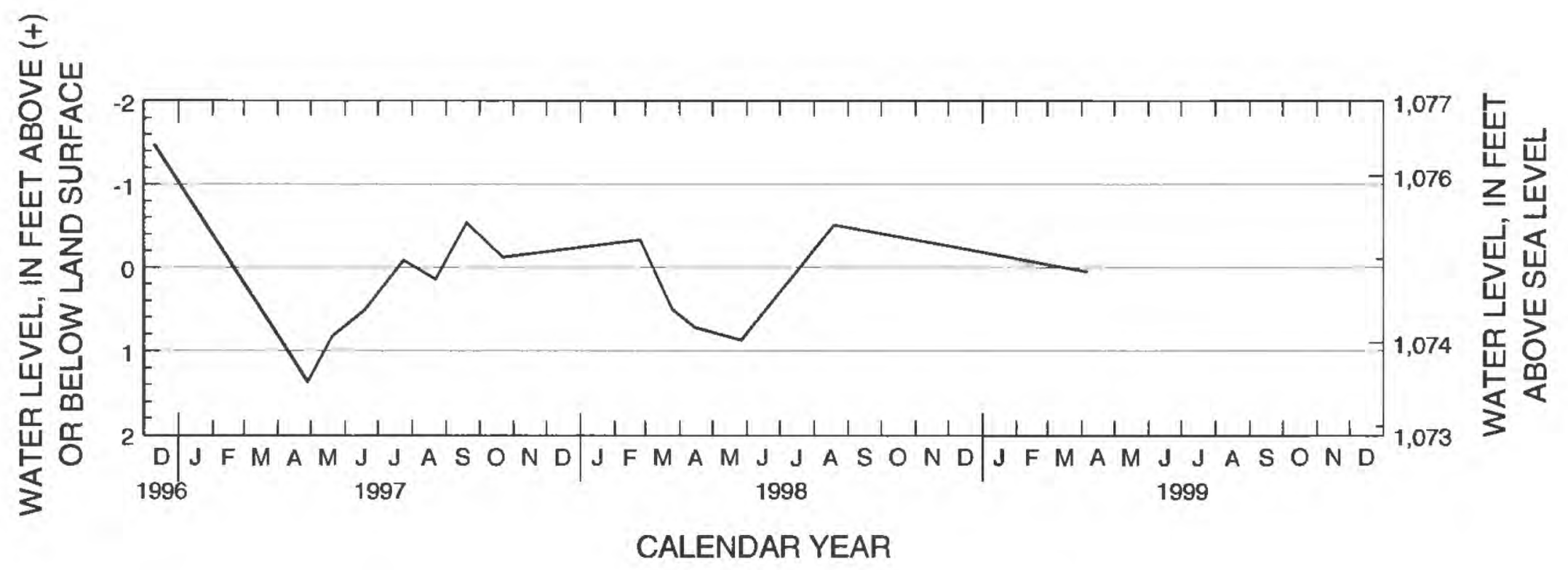

Figure B126. Hydrograph for observation well 128N49W36AAAA R (site number 126). 
Site number from location map: 127

Local well number: 128 N50W10BBBB2 R

Station identification number: 455333096550002

Other identifier: R2-95-18

County: Roberts, South Dakota

Aquifer: Rosholt

Altitude of land surface: $1,092.3$ feet

Measuring point: 3.7 feet

Extremes: December 10, 1996, to August 5, 1999: Highest, 13.96 feet, April 28, 1997; lowest, 16.53, September 15, 1998.
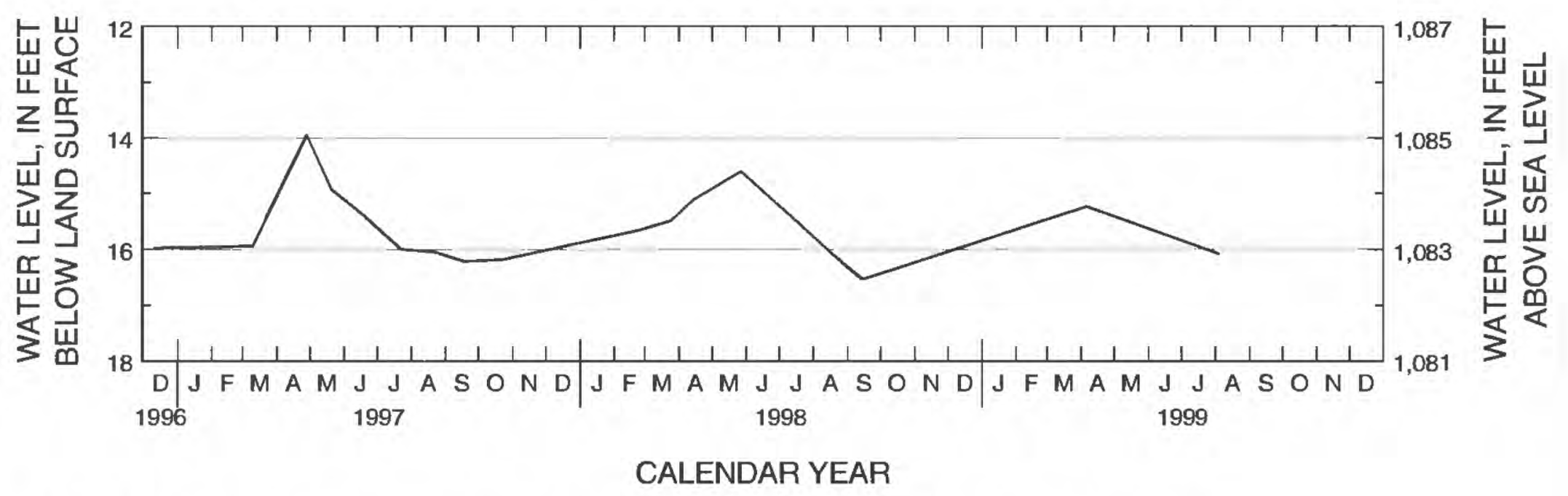

Figure B127. Hydrograph for observation well 128N50W10BBBB2 R (site number 127).

Site number from location map: 128

Local well number: 128N50W21AAAA R

Station identification number: 455148096550001

Other identifier: LTR-5

County: Roberts, South Dakota

Aquifer: Fairmount

Altitude of land surface: $1,093.7$ feet

Measuring point: 3.7 feet

Extremes: December 10, 1996, to August 5, 1999: Highest, 3.02 feet, May 27, 1998; lowest, 3.79 feet,

October 22, 1997.

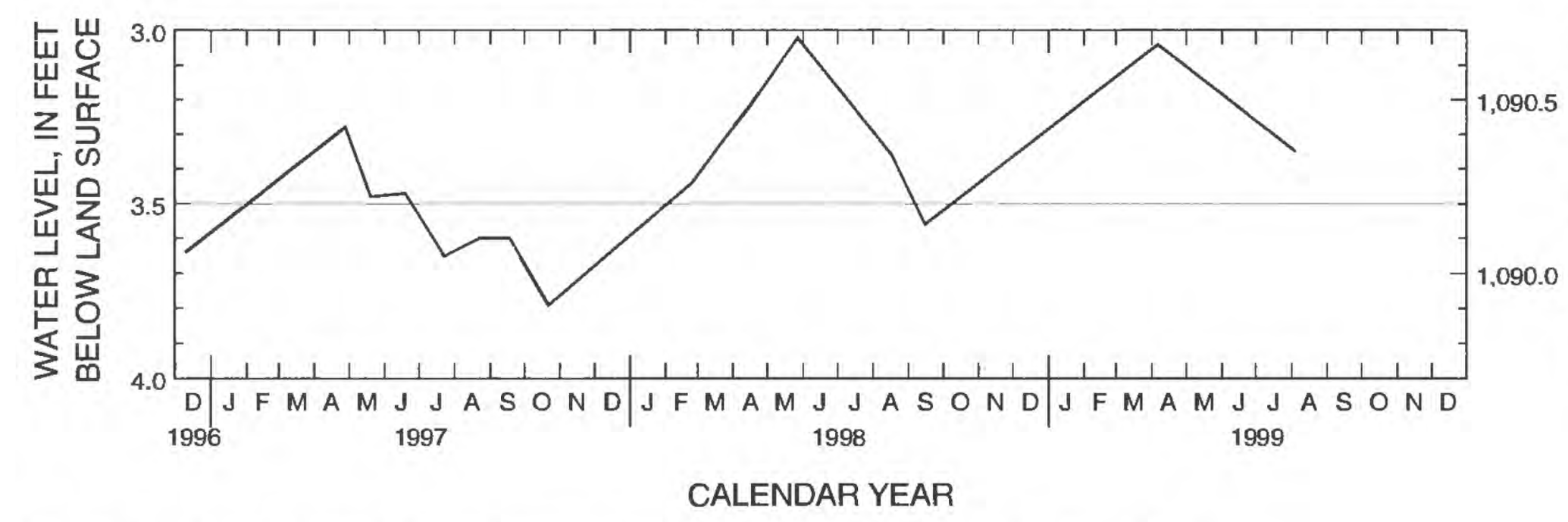

Figure B128. Hydrograph for observation well 128N50W21AAAA R (site number 128). 
Site number from location map: 129

Local well number: 128N50W21AABA R

Station identification number: 455147096551301

Other identifier: RB-79A

County: Roberts, South Dakota

Aquifer: Rosholt

Altitude of land surface: 1,094.3 feet

Measuring point: 2.5 feet

Extremes: October 26, 1979, to October 5, 1999: Highest, 0.3 feet, June 4, 1996; lowest, 6.8 feet, September 8, 1981.

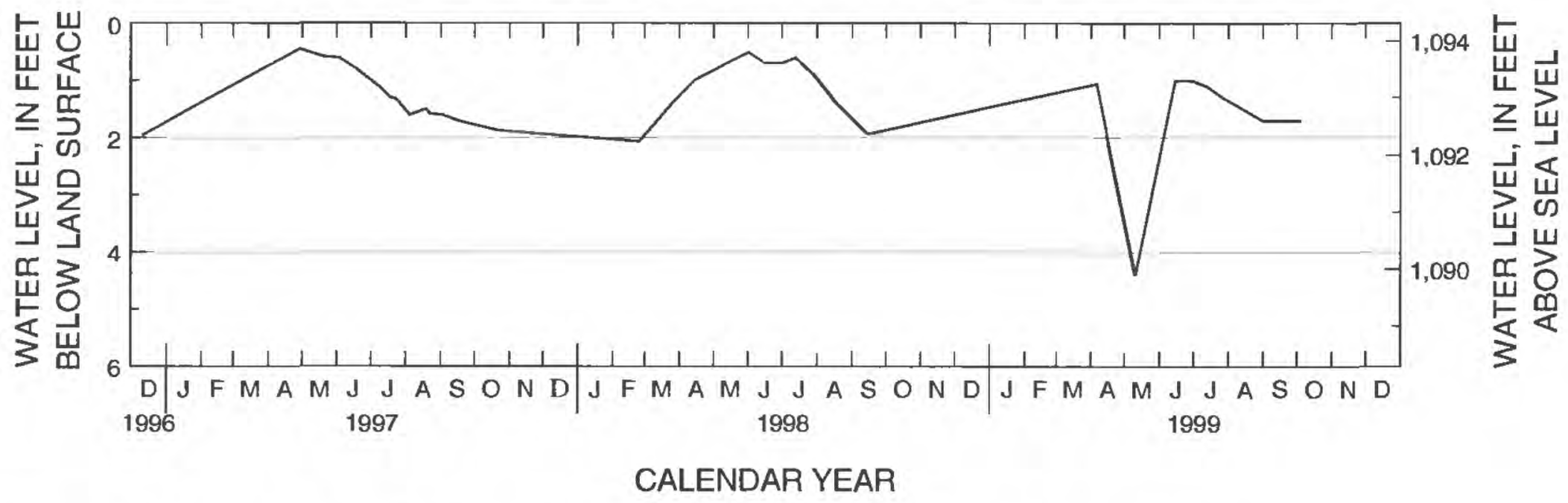

Figure B129. Hydrograph for observation well 128N50W21AABA R (site number 129).

Site number from location map: 130

Local well number: $128 \mathrm{~N} 50 \mathrm{~W} 21 \mathrm{CCCC} \mathrm{R}$

Station identification number: 455057096561501

Other identifier: RB-79B

County: Roberts, South Dakota

Aquifer: Undetermined

Altitude of land surface: $1,112.8$ feet

Measuring point: 4.25 feet

Extremes: October 26, 1979, to December 1, 1999: Highest, -4.7 feet, June 30, 1999; lowest, 4.5 feet, August 14, 1990.

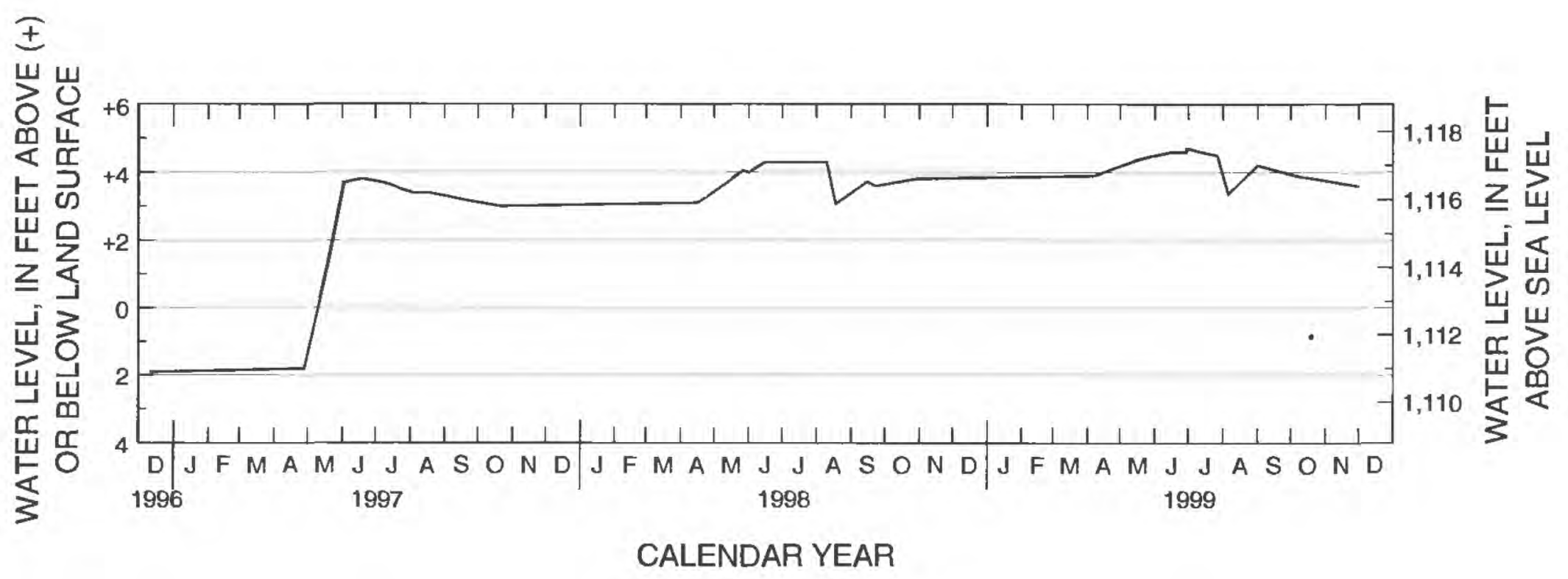

Figure B130. Hydrograph for observation well 128N50W21CCCC R (site number 130). 
Site number from location map: 131

Local well number: 128N50W25AAAB2 R

Station identification number: 455058096511802

Other identifier: R2-97-26

County: Roberts, South Dakota

Aquifer: Rosholt

Altitude of land surface: 1,095 feet

Measuring point: 1.6 feet

Extremes: October 22, 1997, to August 6, 1999: Highest, 21.78 feet, May 27, 1988 and April 6, 1999; lowest, 22.21 feet, October 22, 1997.

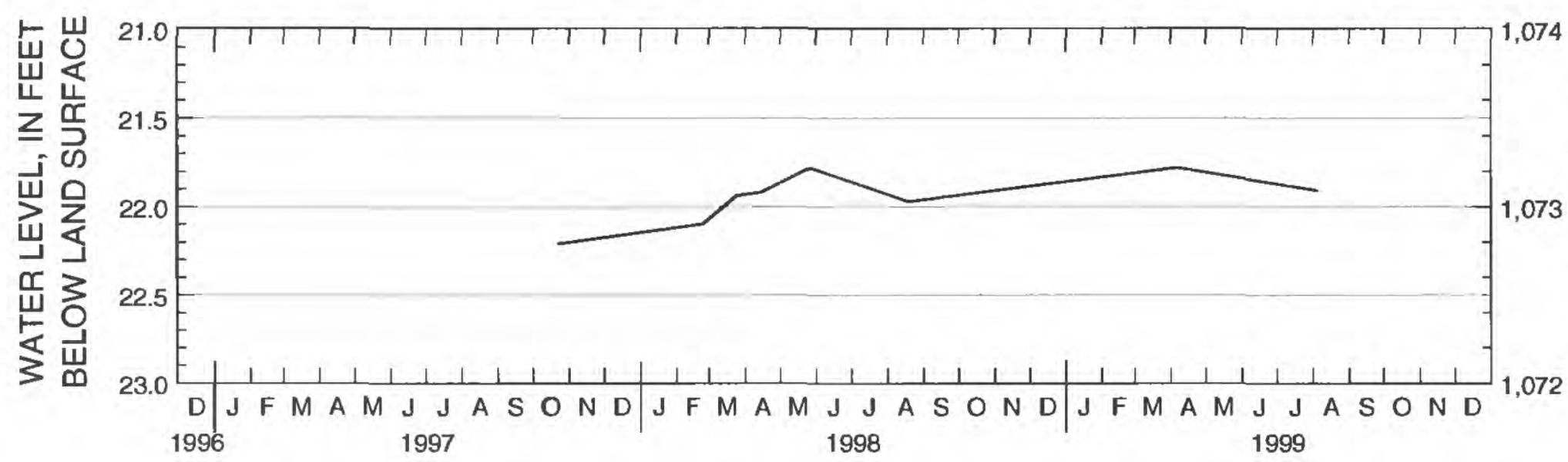

CALENDAR YEAR

Figure B131. Hydrograph for observation well 128N50W25AAAB2 R (site number 131).

Site number from location map: 132

Local well number: 128N50W25AAAB3 R

Station identification number: 455058096511803

Other identifier: R2-97-27

County: Roberts, South Dakota

Aquifer: Rosholt

Altitude of land surface: 1,095 feet

Measuring point: 2.1 feet

Extremes: October 22, 1997, to August 6, 1999: Highest, 9.69 feet, May 27, 1998; lowest, 12.08 feet, August 6, 1999.

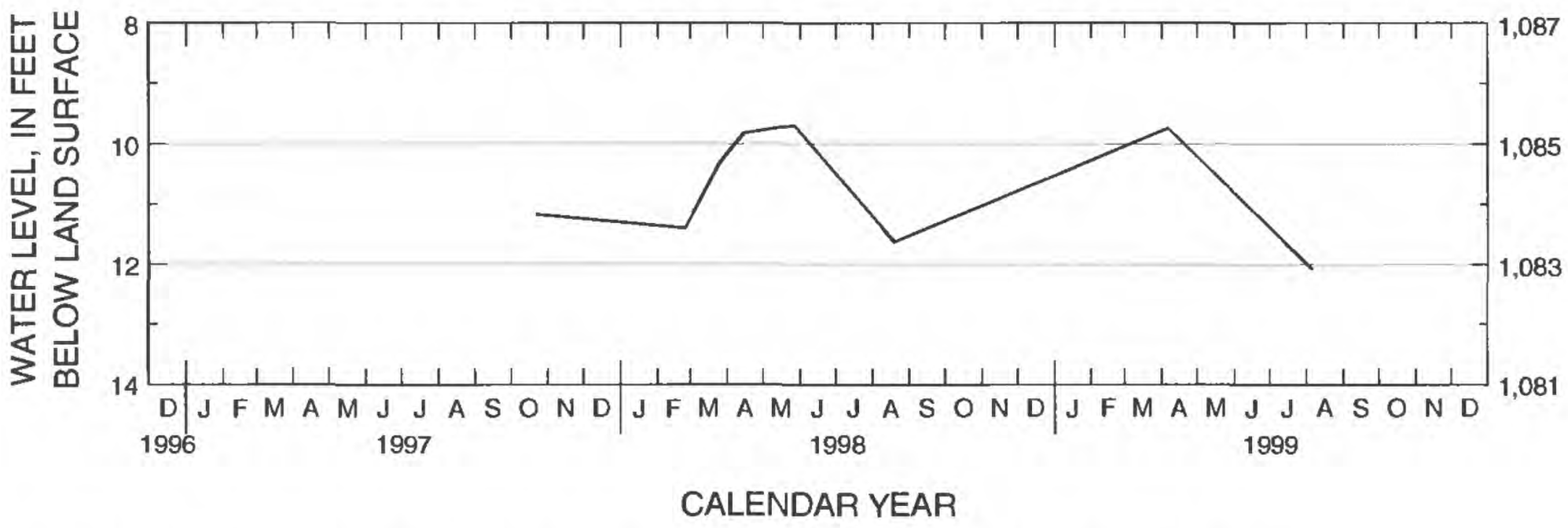

Figure B132. Hydrograph for observation well 128N50W25AAAB3 R (site number 132). 
Site number from location map: 133

Local well number: 128N51W23AABA R

Station identification number: 455148097001401

Other identifier: LTR-4

County: Roberts, South Dakota

Aquifer: Veblen

Altitude of land surface: $1,210.0$ feet

Measuring point: 2.25 feet

Extremes: December 12, 1996, to August 6, 1999: Highest, 50.23 feet, August 6, 1999; lowest, 53.64 feet,

December 10, 1996.

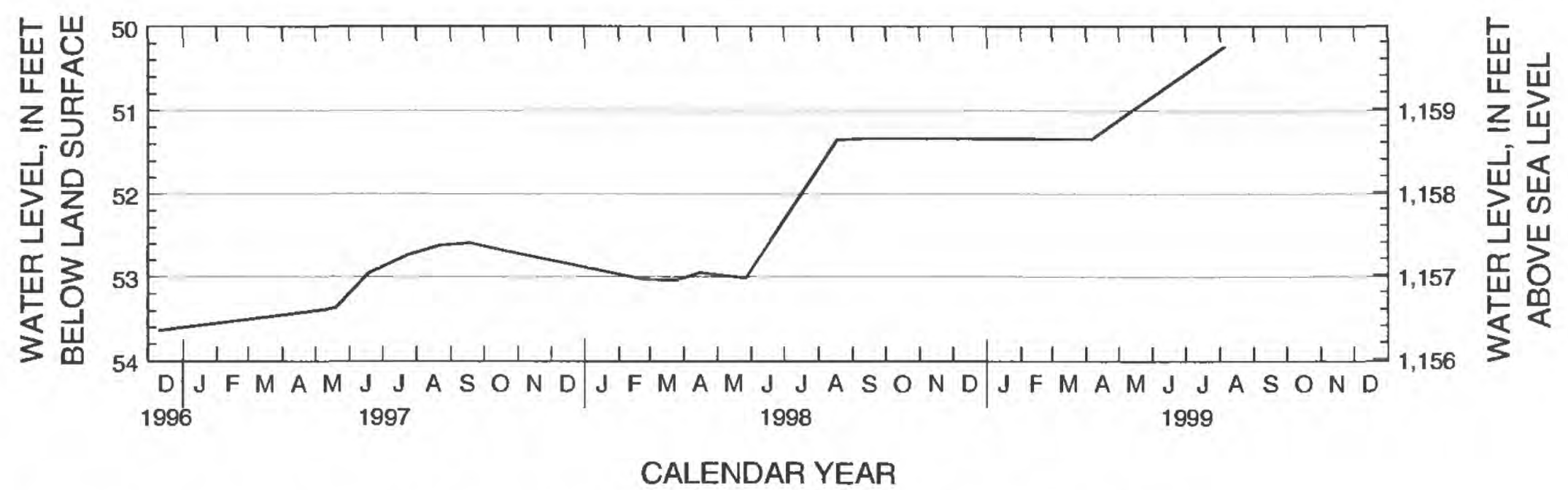

Figure B133. Hydrograph for observation well 128N51W23AABA R (site number 133).

Site number from location map: 134

Local well number: 128N51W26BBB2 R

Station identification number: 455608097010801

Other identifier: 12276B

County: Richland, North Dakota

Aquifer: Veblen

Altitude of land surface: $1,198.7$ feet

Measuring point: 2.0 feet

Extremes: March 10, 1997, to August 5, 1998: Highest, 30.24 feet, August 5, 1999; lowest, 37.39 feet, March 10, 1997.

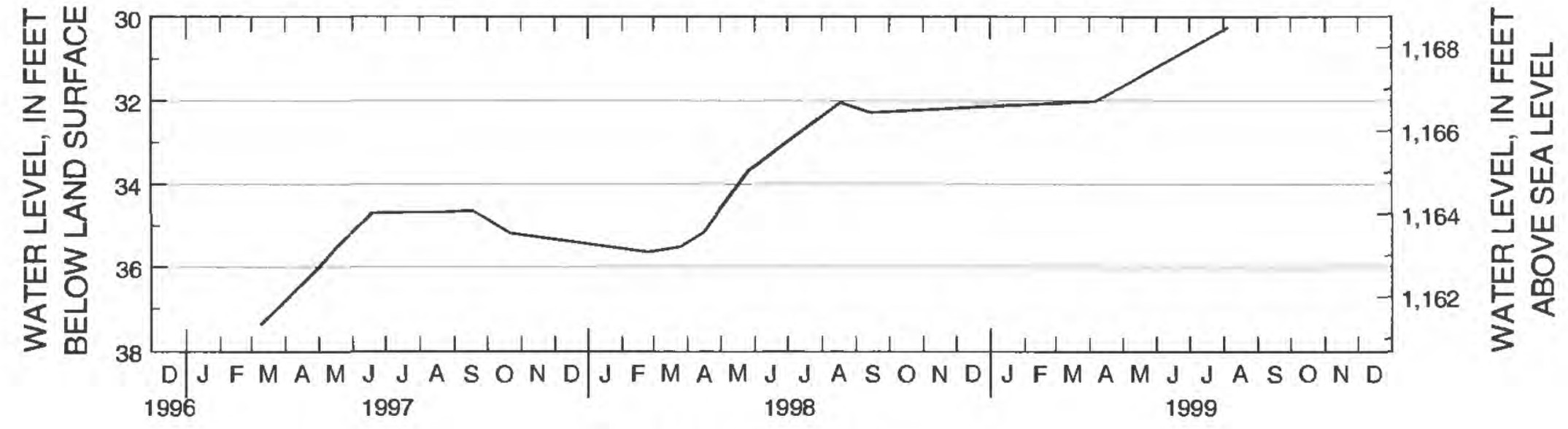

CALENDAR YEAR

Figure B134. Hydrograph for observation well 128N51W26BBBB2 R (site number 134). 
Site number from location map: 135

Local well number: 128N52W1DDDD2 R

Station identification number: 455335097061501

Other identifier: R2-97-18

County: Roberts, South Dakota

Aquifer: Veblen

Altitude of land surface: 1,205 feet

Measuring point: 2.3 feet

Extremes: October 22, 1997, to August 6, 1999: Highest, 25.43 feet, August 6, 1999; lowest, 29.84 feet, February 25 and March 25, 1998.



Figure B135. Hydrograph for observation well 128N52W1DDDD2 R (site number 135).

Site number from location map: 136

Local well number: $128 \mathrm{~N} 52 \mathrm{~W} 3 \mathrm{CCCB} 2 \mathrm{R}$

Station identification number: 455337097095502

Other identifier: R2-97-17

County: Roberts, South Dakota

Aquifer: Veblen

Altitude of land surface: 1,201 feet

Measuring point: 2.3 feet

Extremes: October 22, 1997, to August 6, 1999: Highest, 12.42 feet, August 6, 1999; lowest, 16.20 feet, August 18, 1998.

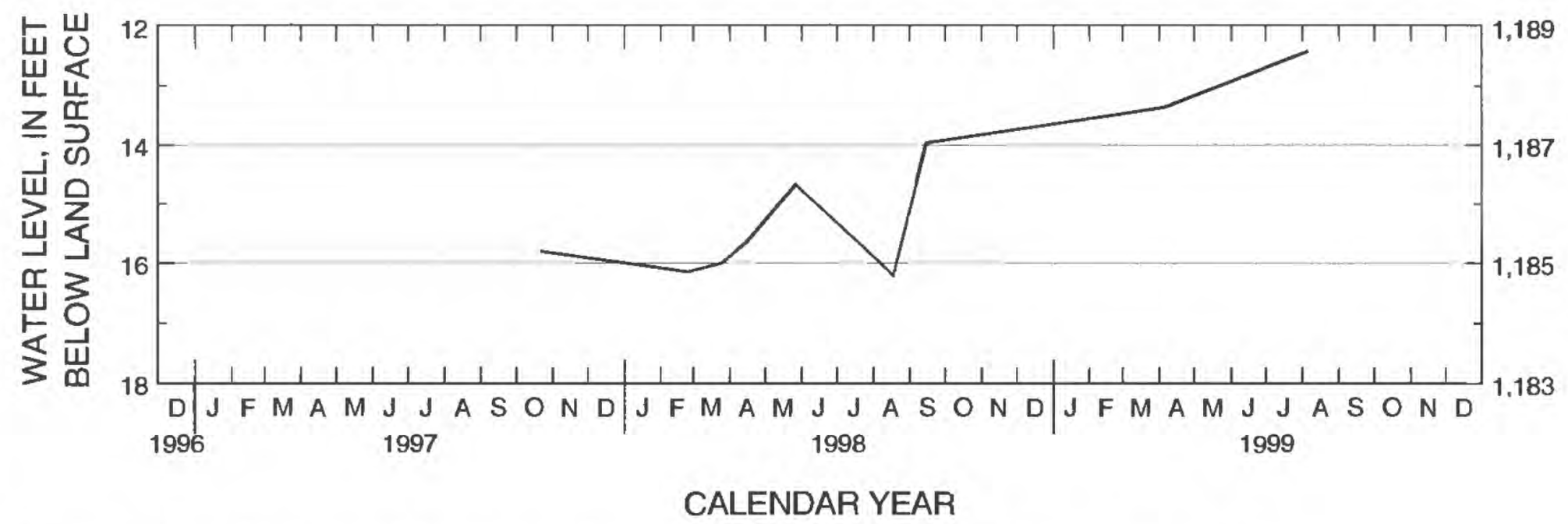

Figure B136. Hydrograph for observation well 128N52W3CCCB2 R (site number 136). 
Site number from location map: 137

Local well number: 128N52W25AAAB2 R

Station identification number: 455053097061702

Other identifier: R2-95-10

County: Roberts, South Dakota

Aquifer: Veblen

Altitude of land surface: 1,188 feet

Measuring point: 2.0 feet

Extremes: April 28, 1997, to August 6, 1999: Highest, 13.62 feet, August 6, 1999; lowest, 17.9 feet, April 28, 1997.

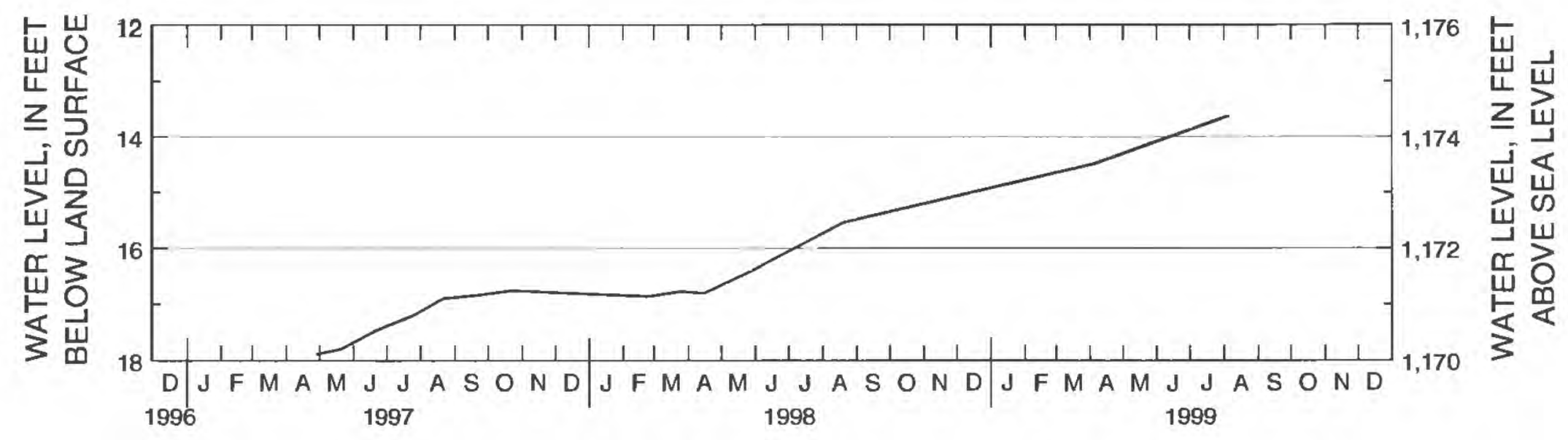

CALENDAR YEAR

Figure B137. Hydrograph for observation well 128N52W25AAAB2 R (site number 137).

Site number from location map: 138

Local well number: 128N52W25AAAB R

Station identification number: 455053097061701

Other identifier: R2-95-09

County: Roberts, South Dakota

Aquifer: Veblen

Altitude of land surface: 1,188 feet

Measuring point: 2.5 feet

Extremes: December 12, 1996, to August 6. 1999: Highest, 13.82 feet, August 6, 1999; lowest, 18.07 feet,

December 12, 1996.

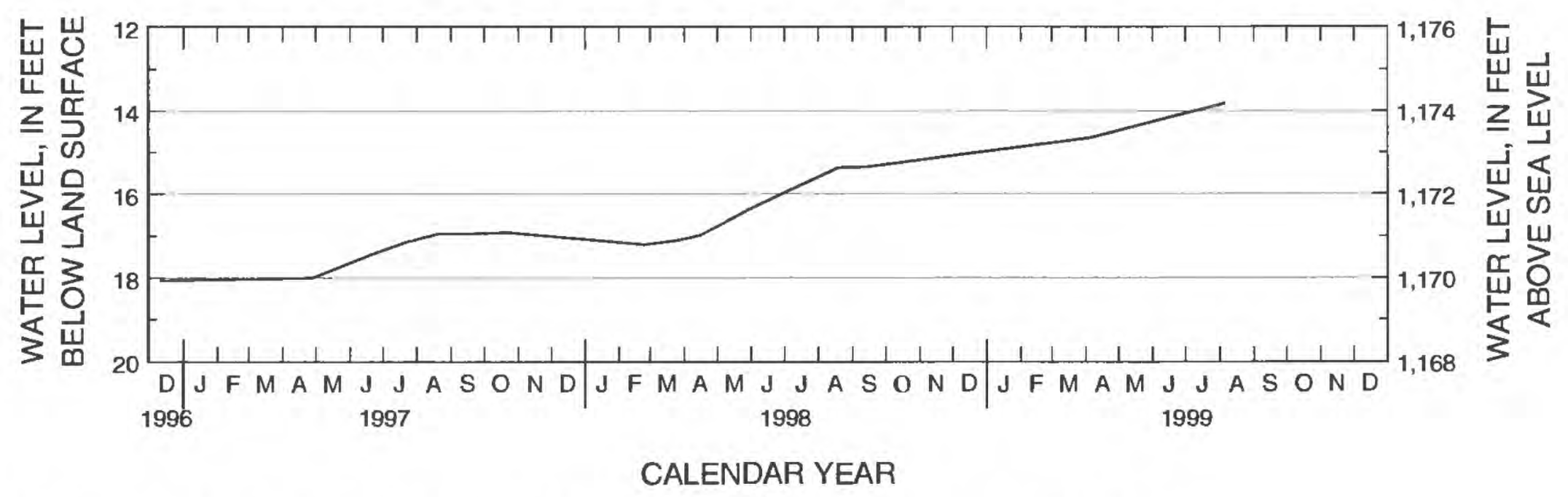

Figure B138. Hydrograph for observation well 128N52W25AAAB R (site number 138). 
Site number from location map: 139

Local well number: 128N52W27BBBC R

Station identification number: 455049097095501

Other identifier: R2-97-20

County: Roberts, South Dakota

Aquifer: Veblen

Altitude of land surface: 1,212 feet

Measuring point: 2.5 feet

Extremes: October 22, 1997, to August 6, 1999: Highest, 35.78 feet, August 6, 1999; lowest, 38.85 feet, March 25, 1998.



Figure B139. Hydrograph for observation well 128N52W27BBBC R (site number 139).

Site number from location map: 140

Local well number: 128 N53W10BBBB R

Station identification number: 455329097172501

Other identifier: ML-69A

County: Marshall, South Dakota

Aquifer: Veblen

Altitude of land surface: 1,250 feet

Measuring point: 2.3 feet

Extremes: December 4, 1969, to October 6, 1999: Highest, 59.2 feet, October 6, 1999; lowest, 75.5 feet, August 2, 1977, September 7, 1977.

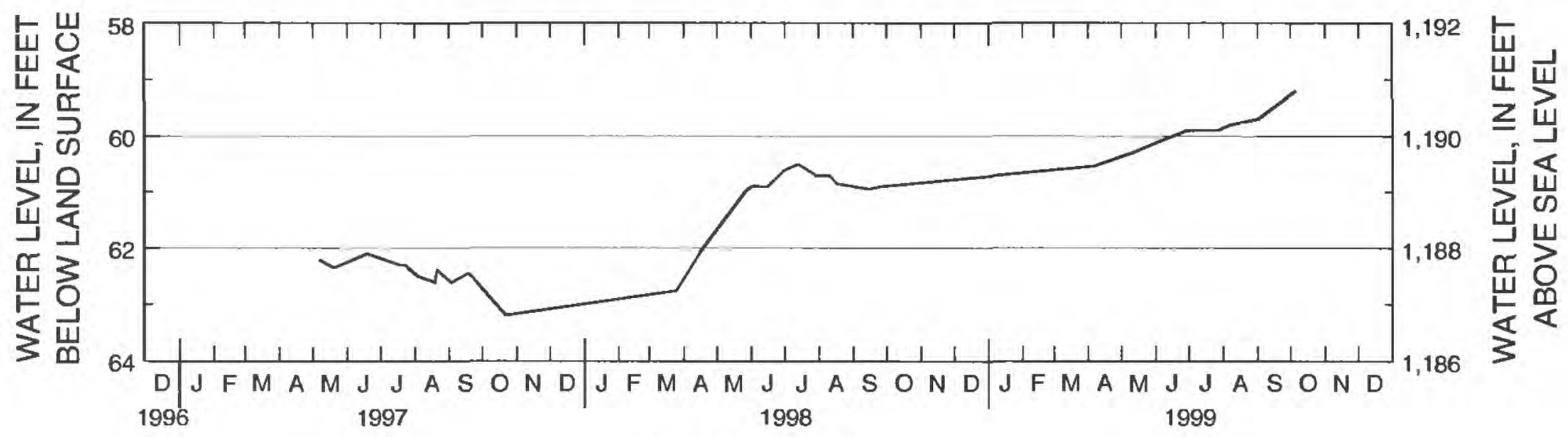

CALENDAR YEAR

Figure B140. Hydrograph for observation well 128N53W10BBBB R (site number 140). 
Site number from location map: 141

Local well number: 128 N56W3BBBB

Station identification number: 455606097322901

Other identifier: ML-70C

County: Marshall, South Dakota

Aquifer: Undetermined

Altitude of land surface: 1,352 feet

Measuring point: 0.75 foot

Extremes: June 7, 1977, to October 6, 1999: Highest, 35.49 feet, March 25, 1998; lowest, 42.9 feet, April 2, 1986.

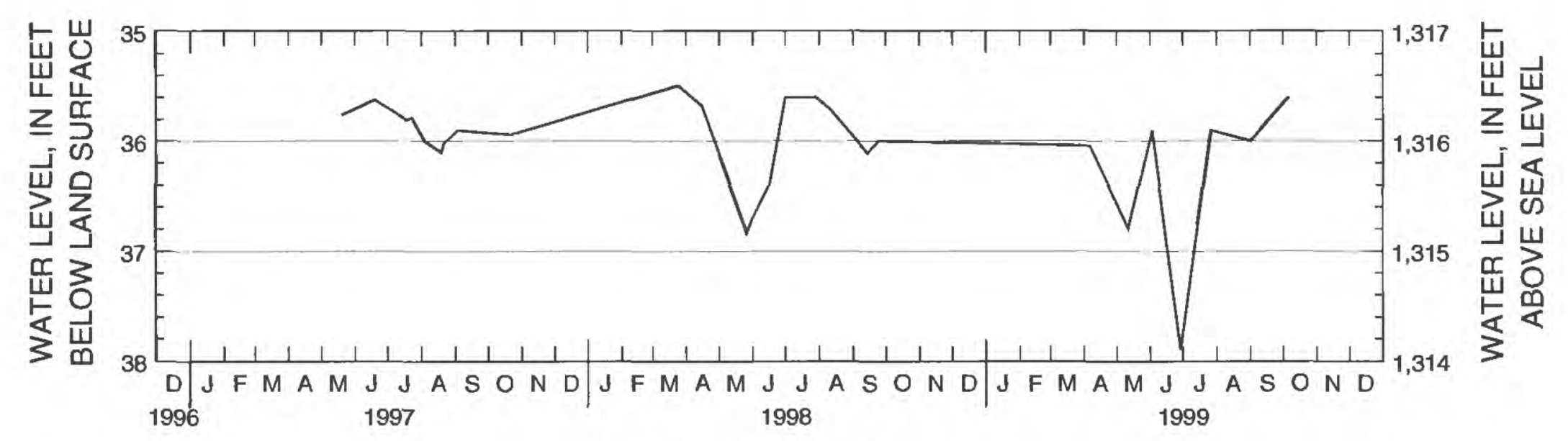

CALENDAR YEAR

Figure B141. Hydrograph for observation well 128N56W3BBBB (site number 141).

Site number from location map: 142

Local well number: 129N48W33ADDA R

Station identification number: 455457096395201

Other identifier: LTR-9

County: Roberts, South Dakota

Aquifer: Fairmount

Altitude of land surface: $1,051.7$ feet

Measuring point: 1.9 feet

Extremes: December 10, 1996, to August 6, 1999: Highest, 1.69 feet, April 6, 1999; lowest, 1.03 feet, February 24, 1998.



Figure B142. Hydrograph for observation well 129N48W33ADDA R (site number 142). 
Site number from location map: 143

Local well number: 129N50W24CCD R

Station identification number: 455611096520101

Other identifier: $12186 \mathrm{~A}$

County: Richland, North Dakota

Aquifer: Rosholt

Altitude of land surface: $1,089.5$ feet

Measuring point: 1.1 feet

Extremes: May 7, 1992, to April 6, 1999: Highest, 17.04 feet, June 6, 1996; lowest, 20.11 feet, November 10, 1992.

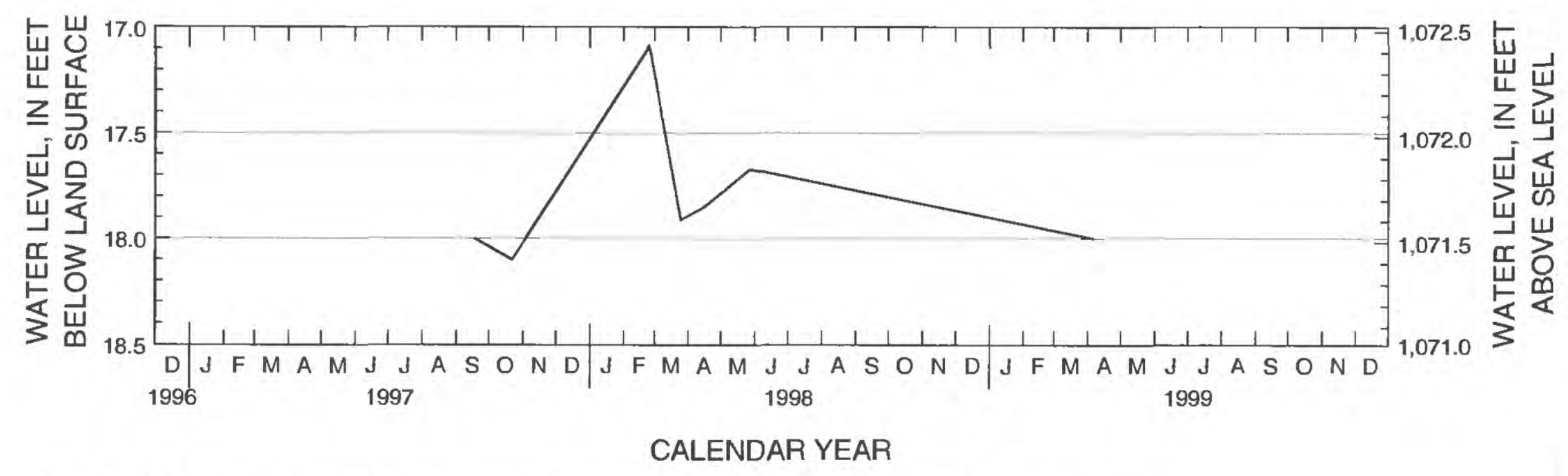

Figure B143. Hydrograph for observation well 129N50W24CCD1 R (site number 143).

Site number from location map: 144

Local well number: 129N50W24CCD2 R

Station identification number: 455611096520102

Other identifier: 12186B

County: Richland, North Dakota

Aquifer: Rosholt

Altitude of land surface: $1,089.5$ feet

Measuring point: 2.3 feet

Extremes: May 7, 1992, to April 6, 1999: Highest, 16.91 feet, May 16, 1995, lowest; 21.66 feet. October 13, 1992.

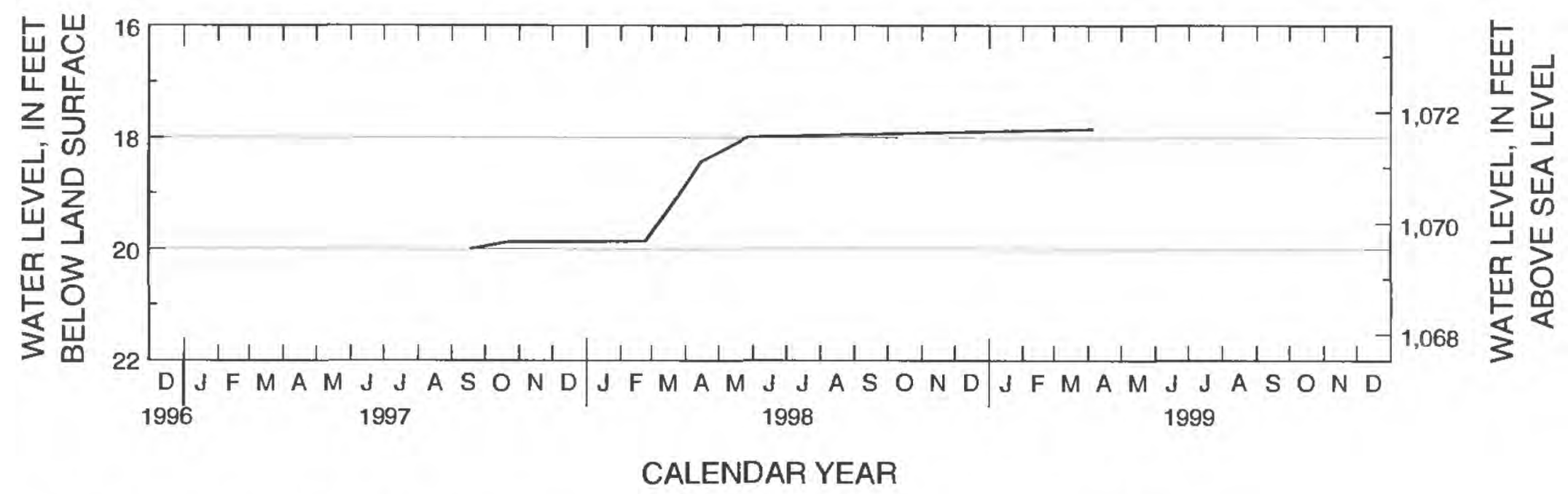

Figure B144. Hydrograph for observation well 129N50W24CCD2 R (site number 144). 
Site number from location map: 145

Local well number: 129N50W24CCD3 R

Station identification number: 455611096520103

Other identifier: $12186 \mathrm{C}$

County: Richland, North Dakota

Aquifer: Rosholt

Altitude of land surface: $1,089.5$ feet

Measuring point: 0.9 foot

Extremes: May 7, 1992, to April 6, 1999: Highest, 15.39 feet, May 16, 1995; lowest, 20.42 feet, October 13, 1992.

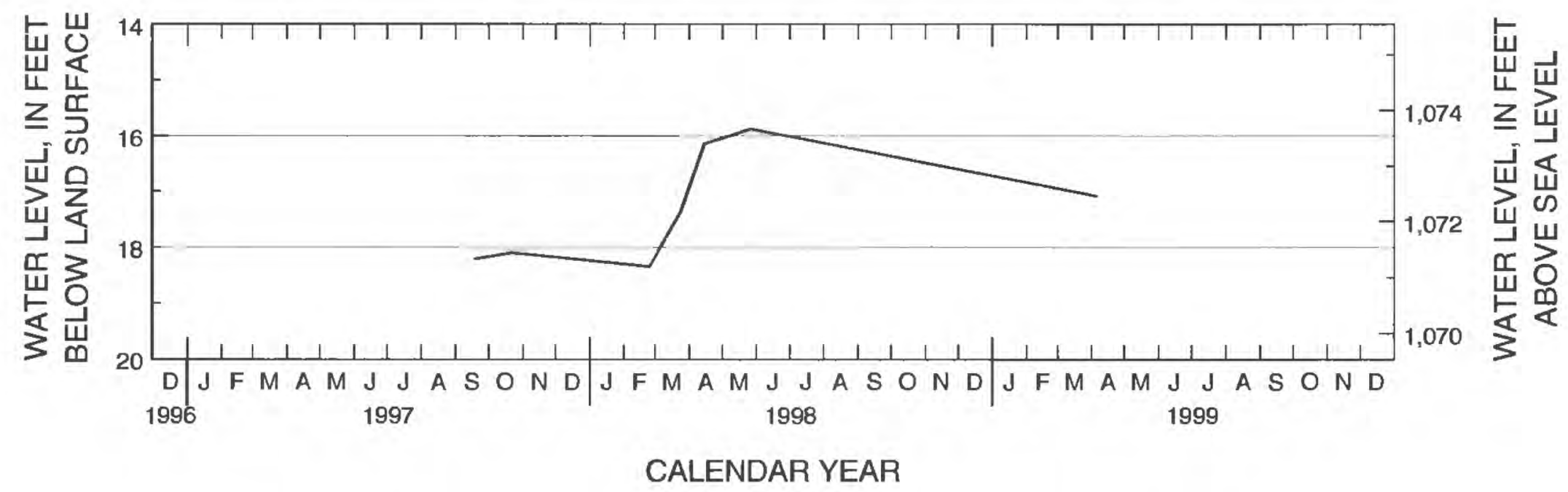

Figure B145. Hydrograph for observation well 129N50W24CCD3 R (site number 145).

Site number from location map: 146

Local well number: 129N50W27BBBB2 R

Station identification number: 455607096545002

Other identifier: R2-95-21

County: Roberts, South Dakota

Aquifer: Undetermined

Altitude of land surface: 1,106 feet

Measuring point: 2.19 feet

Extremes: December 10, 1996, to April 6, 1999: Highest, 1.05 feet, April 6, 1999; lowest, 2.14 feet, December 10, 1996.

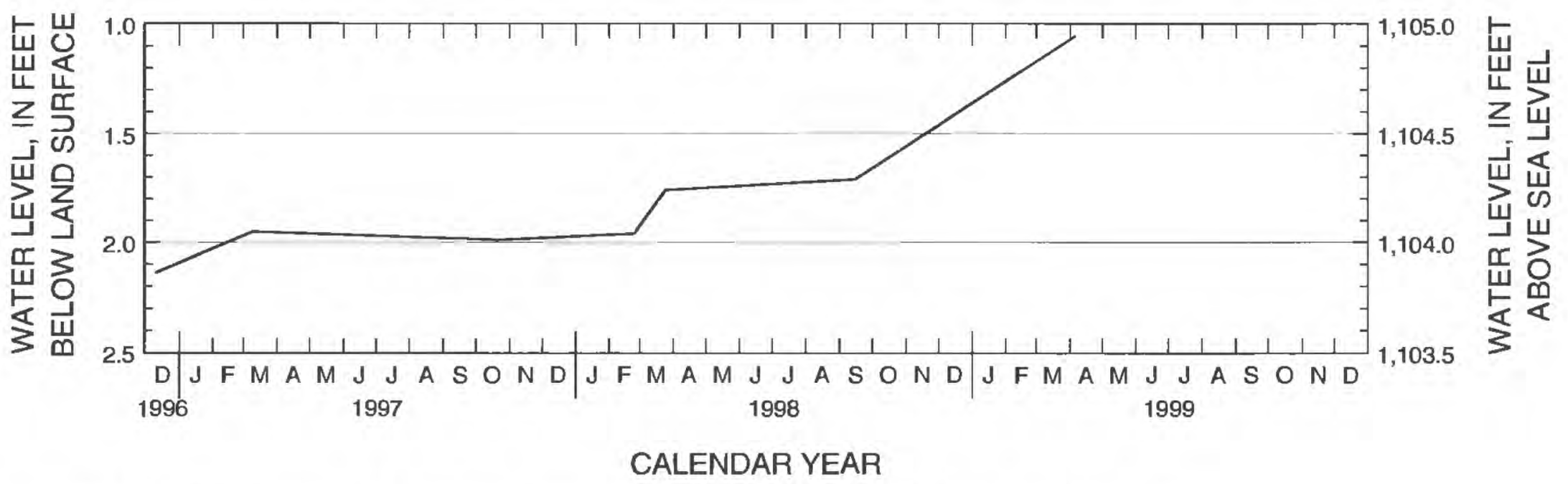

Figure B146. Hydrograph for observation well 129N50W27BBBB2 R (site number 146). 
Site number from location map: 147

Local well number: 129N51W8CCC R

Station identification number: 455755097045501

Other identifier: 13037

County: Richland, North Dakota

Aquifer: Veblen

Altitude of land surface: $1,196.2$ feet

Measuring point: 1.5 feet

Extremes: September 2, 1992, to August 5, 1999: Highest, 31.06 feet, August 5, 1999; lowest, 43.85 feet, November 10, 1992.

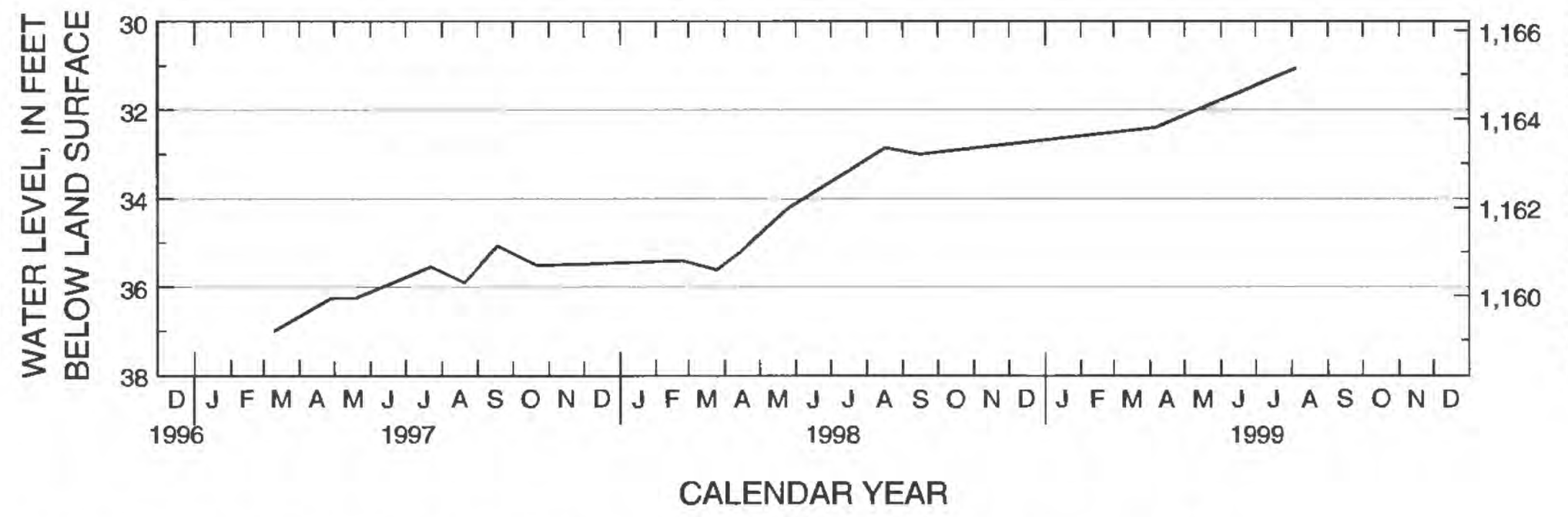

Figure B147. Hydrograph for observation well 129N51W8CCC R (site number 147).

Site number from location map: 148

Local well number: 129N51W19ABA R

Station identification number: 455701097051501

Other identifier: 13422

County: Richland, North Dakota

Aquifer: Veblen

Altitude of land surface: 1,191.2 feet

Measuring point: 1.7 feet

Extremes: October 25, 1994, to August 5, 1999: Highest, 17.65 feet, August 5, 1999; lowest, 27.36 feet,

December 14, 1994.

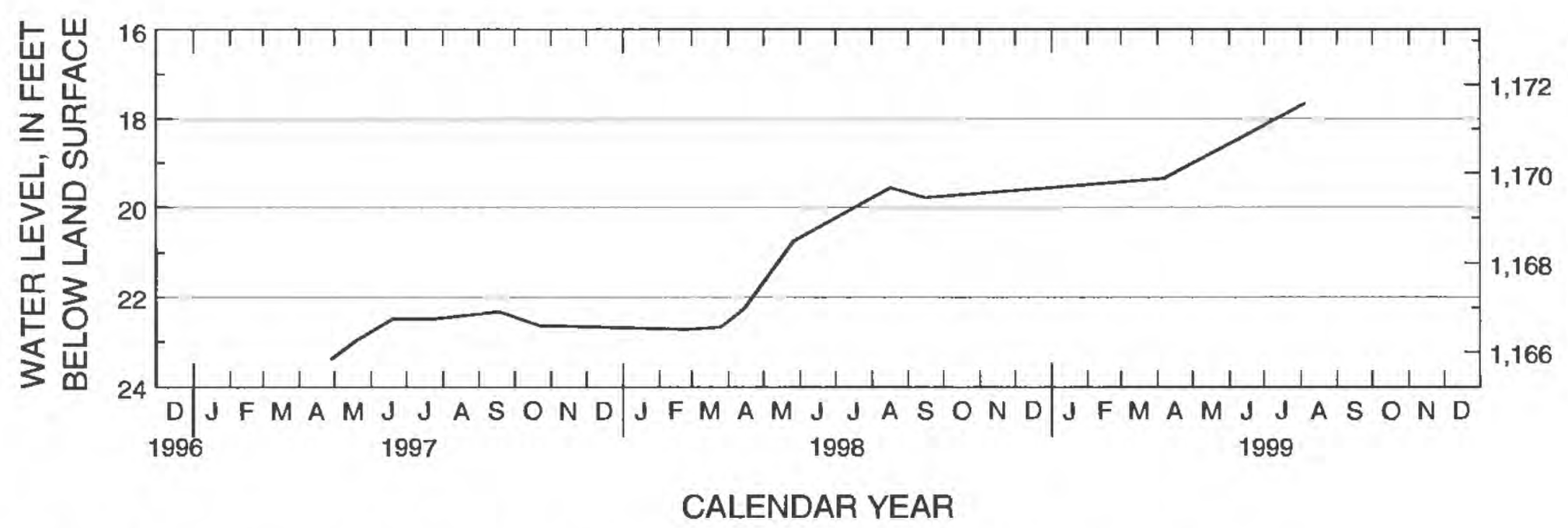

Figure B148. Hydrograph for observation well 129N51W19ABA R (site number 148). 
Site number from location map: 149

Local well number: 129N52W14AAA

Station identification number: 455932097092401

Other identifier: 13494

County: Richland, North Dakota

Aquifer: Veblen

Altitude of land surface: 1,220 feet

Measuring point: 1.6 feet

Extremes: November 21, 1995, to August 5, 1999: Highest, August 5, 1999; lowest 38.70 feet, November 21, 1995.

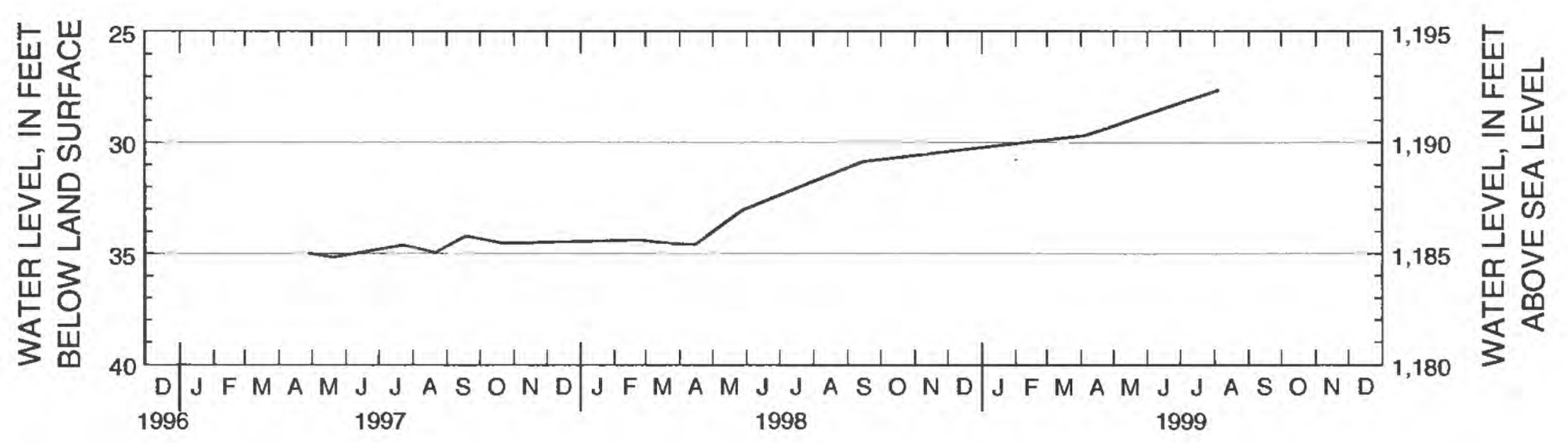

CALENDAR YEAR

Figure B149. Hydrograph for observation well 129N52W14AAA (site number 149).

Site number from location map: 150

Local well number: 129N52W21CCC R

Station identification number: 455610097110901

Other identifier: 12277

County: Richland, North Dakota

Aquifer: Veblen

Altitude of land surface: $1,221.2$ feet

Measuring point: 2.5 feet

Extremes: July 17, 1991, to August 5, 1999: Highest, 35.86 feet. August 5, 1999; lowest. 42.97 feet, April 13, 1993.

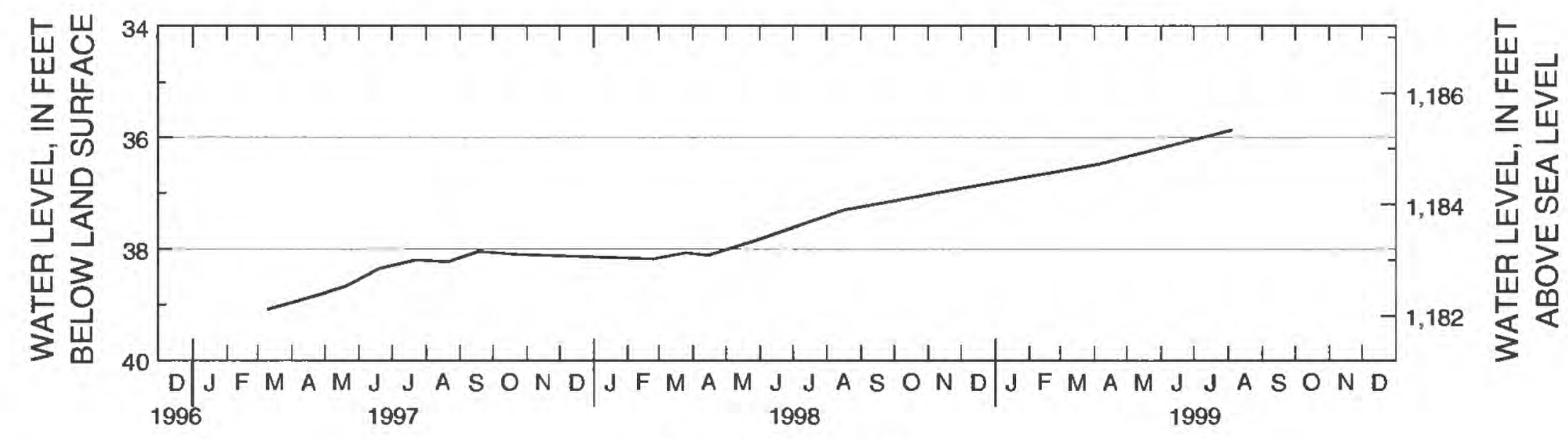

CALENDAR YEAR

Figure B150. Hydrograph for observation well 129N52W21CCC R (site number 150). 
Site number from location map: 151

Local well number: 129N52W25AAAB2 R

Station identification number: 455608097061702

Other identifier: R2-95-13

County: Roberts, South Dakota

Aquifer: Veblen

Altitude of land surface: 1,196 feet

Measuring point: 2.4 feet

Extremes: December 10, 1996, to August 5, 1999: Highest, 17.54 feet, August 5, 1999: lowest, 24.24 feet, March 10, 1997.
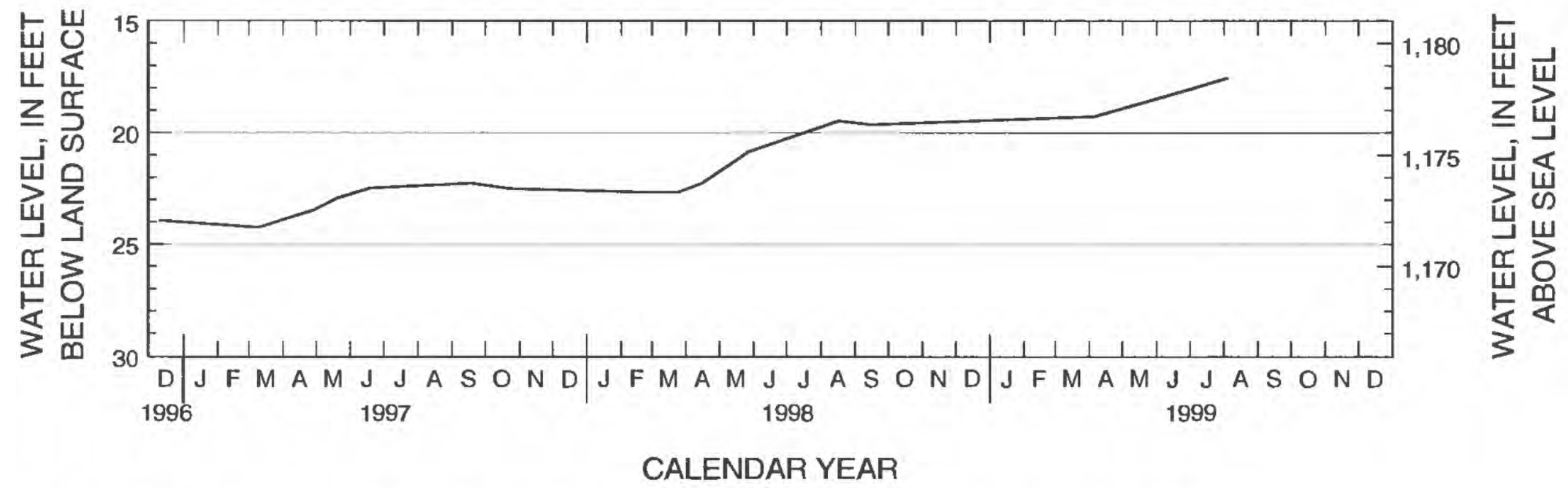

Figure B151. Hydrograph for observation well 129N52W25AAAB2 R (site number 151).

Site number from location map: 152

Local well number: 129N53W7BBA R

Station identification number: 455842097210301

Other identifier: 9248

County: Sargent, North Dakota

Aquifer: Spiritwood

Altitude of land surface: 1,171 feet

Measuring point: 1.8 feet

Extremes: March 10, 1997, to August 5, 1999: Highest, 32.54 feet, April 6, 1999; lowest, 34.46 feet, August 17, 1998.

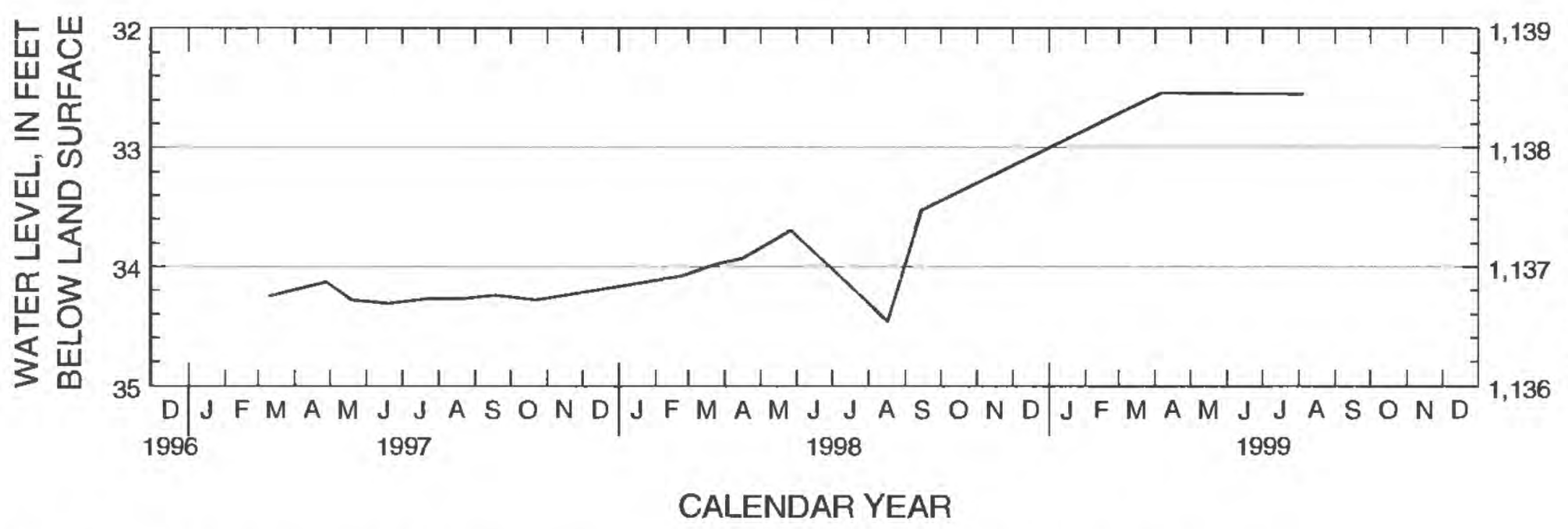

Figure B152. Hydrograph for observation well 129N53W7BBA R (site number 152). 
Site number from location map: 153

Local well number: 129N53W9AAA R

Station identification number: 455842097173701

Other identifier: 9249

County: Sargent, North Dakota

Aquifer: Spiritwood

Altitude of land surface: 1,170 feet

Measuring point: 1.2 feet

Extremes: March 10, 1997, to August 5, 1999: Highest, 29.76 feet, August 5, 1999; lowest, 31.88 feet, June 19, 1997.

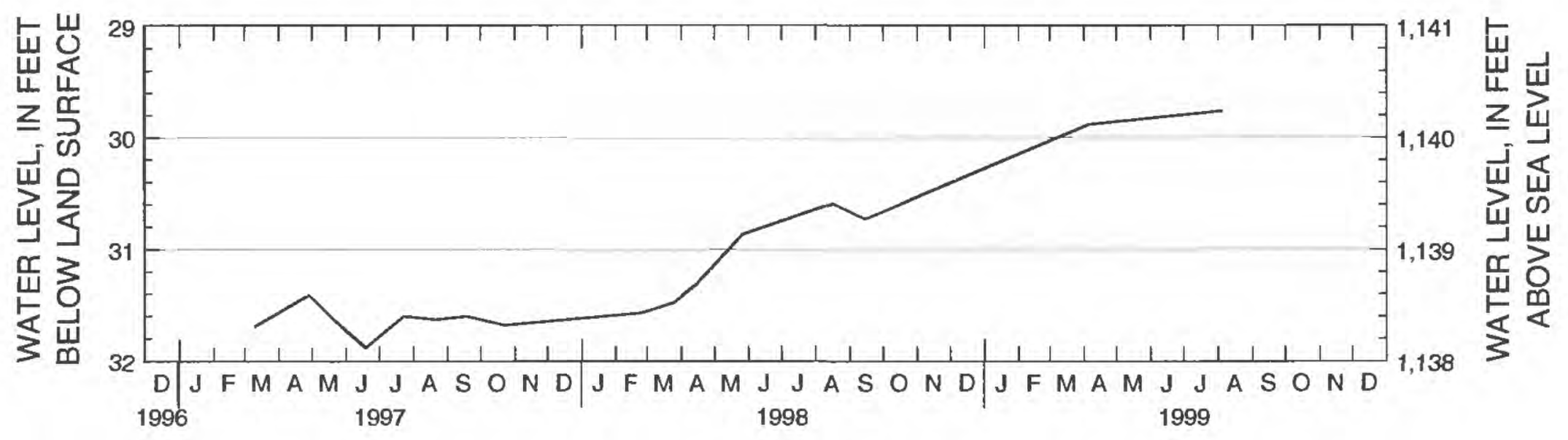

CALENDAR YEAR

Figure B153. Hydrograph for observation well 129N53W9AAA R (site number 153).

Site number from location map: 154

Local well number: 129N53W27BBBB R

Station identification number: 455606097172501

Other identifier: ML-70A

County: Marshall, South Dakota

Aquifer: Veblen

Altitude of land surface: 1,255 feet

Measuring point: 2.6 feet

Extremes: June 7, 1977, to October 6, 1999: Highest, 63.2 feet, October 6, 1999; lowest. 79.6 feet, August 19, 1983.
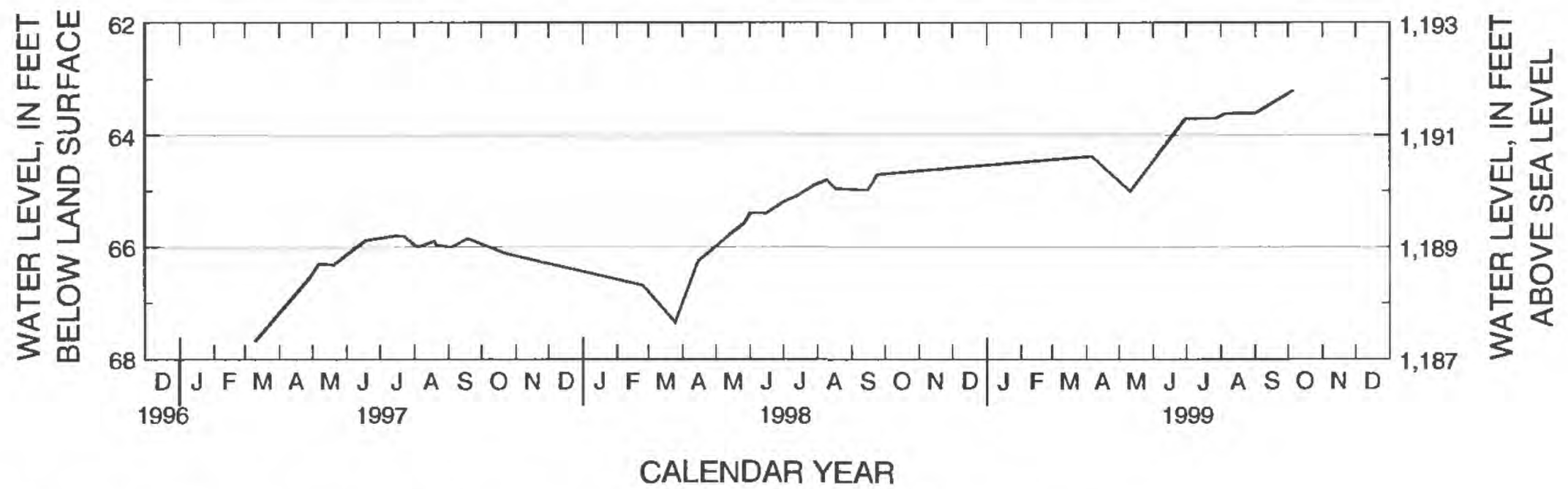

Figure B154. Hydrograph for observation well 129N53W27BBBB R (site number 154). 
Site number from location map: 155

Local well number: 129N54W1AAA

Station identification number: 460118097230501

Other identifier: 13452

County: Sargent, North Dakota

Aquifer: Spiritwood

Altitude of land surface: 1,162 feet

Measuring point: 2.1 feet

Extremes: April 28, 1997, to April 6, 1999: Highest, 13.78 feet, April 6, 1999; lowest, 15.62 feet, July 23, 1997.



Figure B155. Hydrograph for observation well 129N54W1AAA (site number 155).

Site number from location map: 156

Local well number: 129N54W1AAA2

Station identification number: 460118097230502

Other identifier: 13453

County: Sargent, North Dakota

Aquifer: Spiritwood

Altitude of land surface: 1,162 feet

Measuring point: 1.8 feet

Extremes: April 28, 1997, to April 6, 1999: Highest, 13.58 feet, April 6, 1999; lowest, 15.34 feet, October 22, 1997.



Figure B156. Hydrograph for observation well 129N54W1AAA2 (site number 156). 
Site number from location map: 157

Local well number: 129N54W3ADD R

Station identification number: 455914097231801

Other identifier: 12263

County: Sargent, North Dakota

Aquifer: Spiritwood

Altitude of land surface: 1,186 feet

Measuring point: 2.2 feet

Extremes: March 10, 1997, to August 6, 1999: Highest, 47.15 feet, April 6, 1999: lowest, 49.65 feet, April 28, 1997.

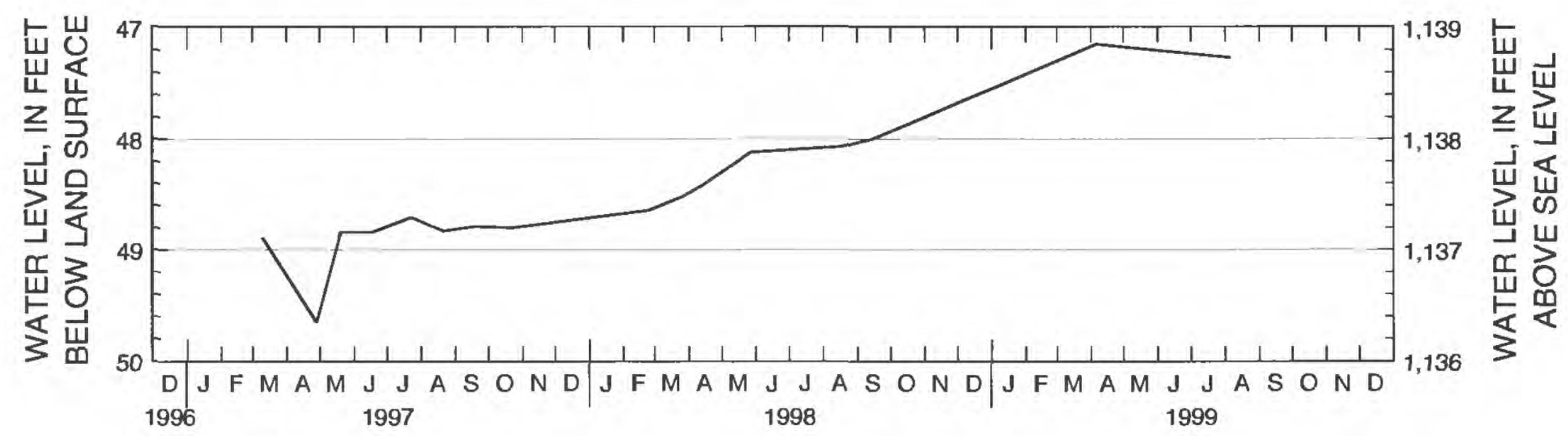

CALENDAR YEAR

Figure B157. Hydrograph for observation well 129N54W3ADD R (site number 157).

Site number from location map: 158

Local well number: 129N54W25AAAA R

Station identification number: 455607097211501

Other identifier: ML-70B

County: Marshall, South Dakota

Aquifer: Veblen

Altitude of land surface: 1,260 feet

Measuring point: 0.8 foot

Extremes: June 7, 1977, to October 6, 1999: Highest, 70.4 feet, October 6, 1999; lowest, 88.3 feet, September 7 , 1977.

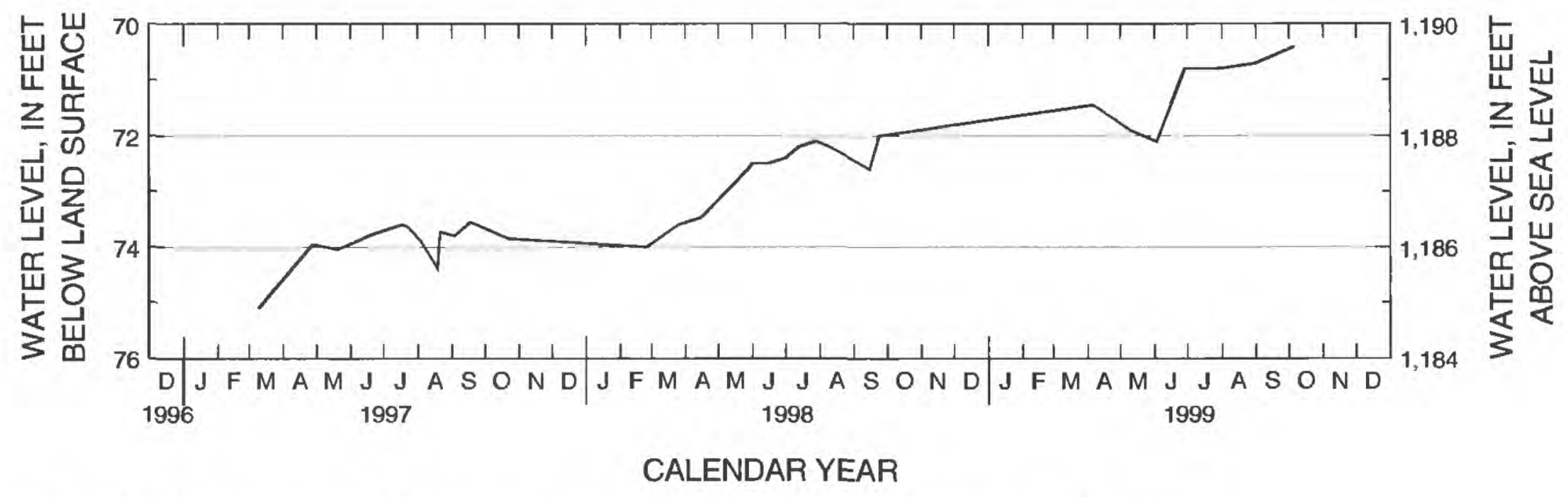

Figure B158. Hydrograph for observation well 129N54W25AAAA R (site number 158). 
Site number from location map: 159

Local well number: 130N53W4BAA

Station identification number: 460117097195801

Other identifier: 13447

County: Sargent, North Dakota

Aquifer: Spiritwood

Altitude of land surface: 1,163 feet

Measuring point: 1.5 feet

Extremes: April 28, 1997, to April 6. 1999: Highest. 25.47 feet, April 6, 1999; lowest, 27.18 feet, September 18, 1997.

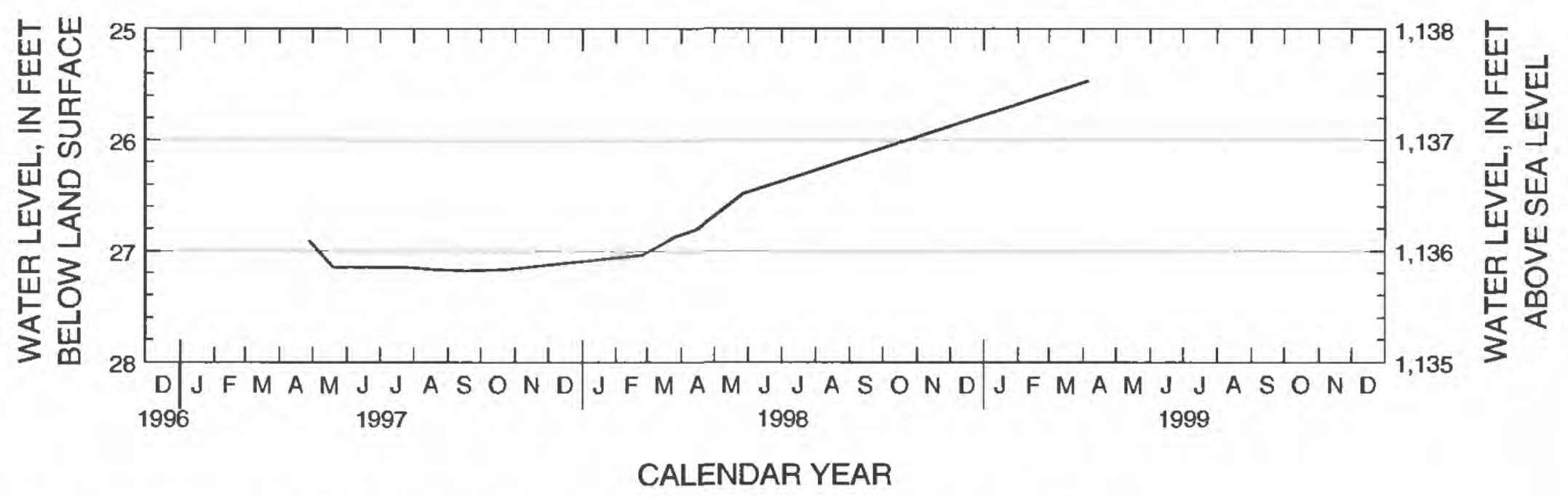

Figure B159. Hydrograph for observation well 130N53W4BAA (site number 159).

Site number from location map: 160

Local well number: 130N53W4BAA2

Station identification number: 460117097195802

Other identifier: 13448

County: Sargent, North Dakota

Aquifer: Spiritwood

Altitude of land surface: 1,163 feet

Measuring point: 1.6 feet

Extremes: April 28, 1997, to April 6, 1999: Highest, 25.06 feet, April 6, 1999; lowest, 26.78 feet, August 21, 1997.

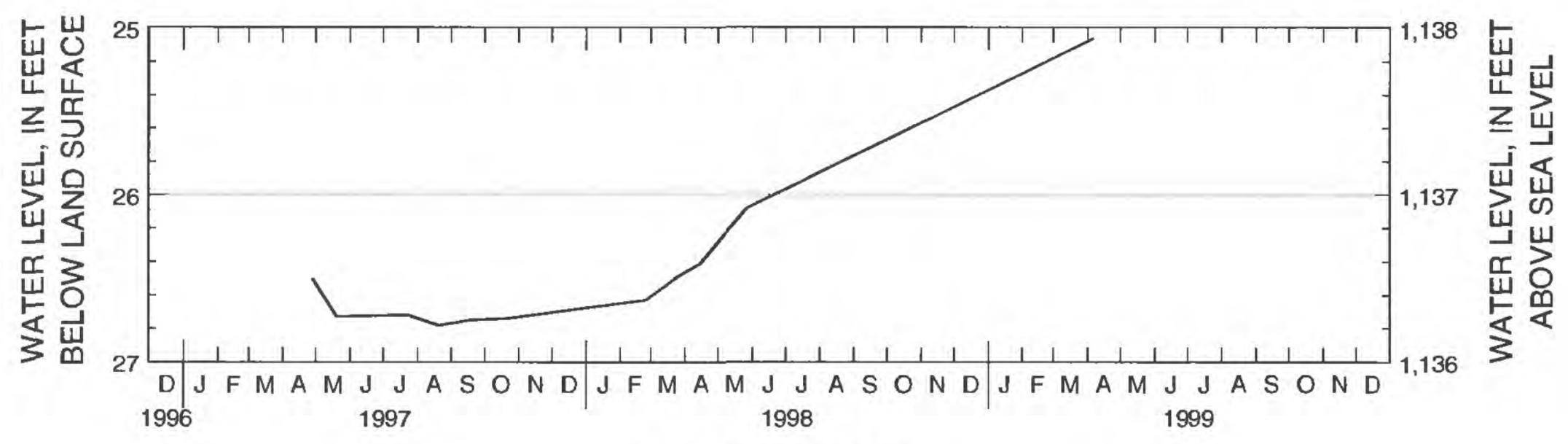

CALENDAR YEAR

Figure B160. Hydrograph for observation well 130N53W4BAA2 (site number 160). 
Site number from location map: 161

Local well number: 130N54W27CCC R

Station identification number: 460033097245701

Other identifier: 12261

County: Sargent, North Dakota

Aquifer: Spiritwood

Altitude of land surface: 1,180 feet

Measuring point: 2.1 feet

Extremes: March 10, 1997, to August 6, 1999: Highest, 34.69 feet, April 6, 1999; lowest, 36.64 feet, August 18, 1998.

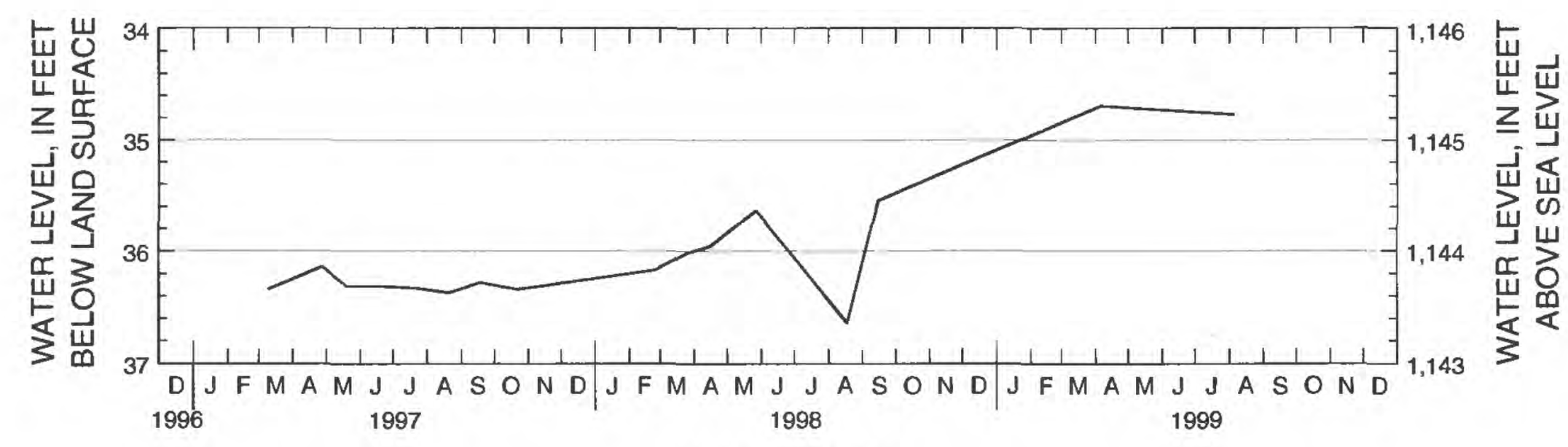

CALENDAR YEAR

Figure B161. Hydrograph for observation well 130N54W27CCC R (site number 161).

Site number from location map: 162

Local well number: 130N54W33DDD R

Station identification number: 455909097245901

Other identifier: 12262

County: Sargent, North Dakota

Aquifer: Spiritwood

Altitude of land surface: 1,158 feet

Measuring point: 2.0 feet

Extremes: March 10, 1997, to August 6, 1999: Highest, 19.86 feet, April 6, 1999; lowest, 21.54 feet, June 19, 1997.

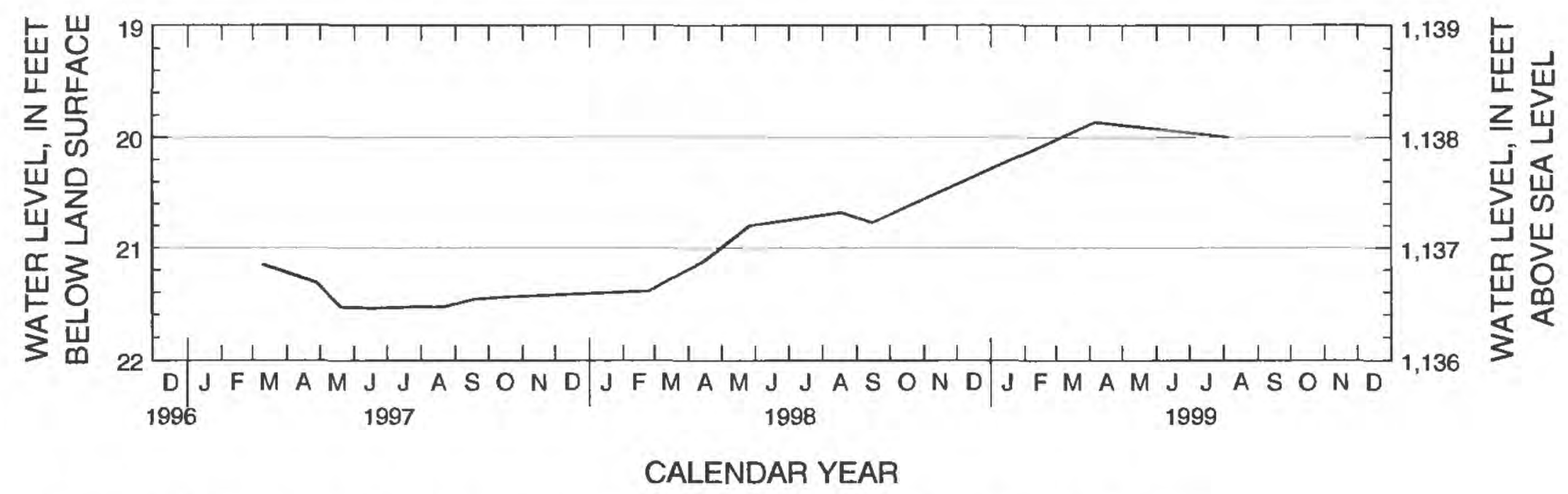

Figure B162. Hydrograph for observation well 130N54W33DDD R (site number 162). 
Site number from location map: 163

Local well number: 130N54W35CCC R

Station identification number: 455941097234001

Other identifier: 9247

County: Sargent, North Dakota

Aquifer: Spiritwood

Altitude of land surface: 1,170 feet

Measuring point: 2.4 feet

Extremes: March 10, 1997, to August 5, 1999: Highest, 43.17 feet, August 5, 1999; lowest, 50.76 feet, March 10, 1997.

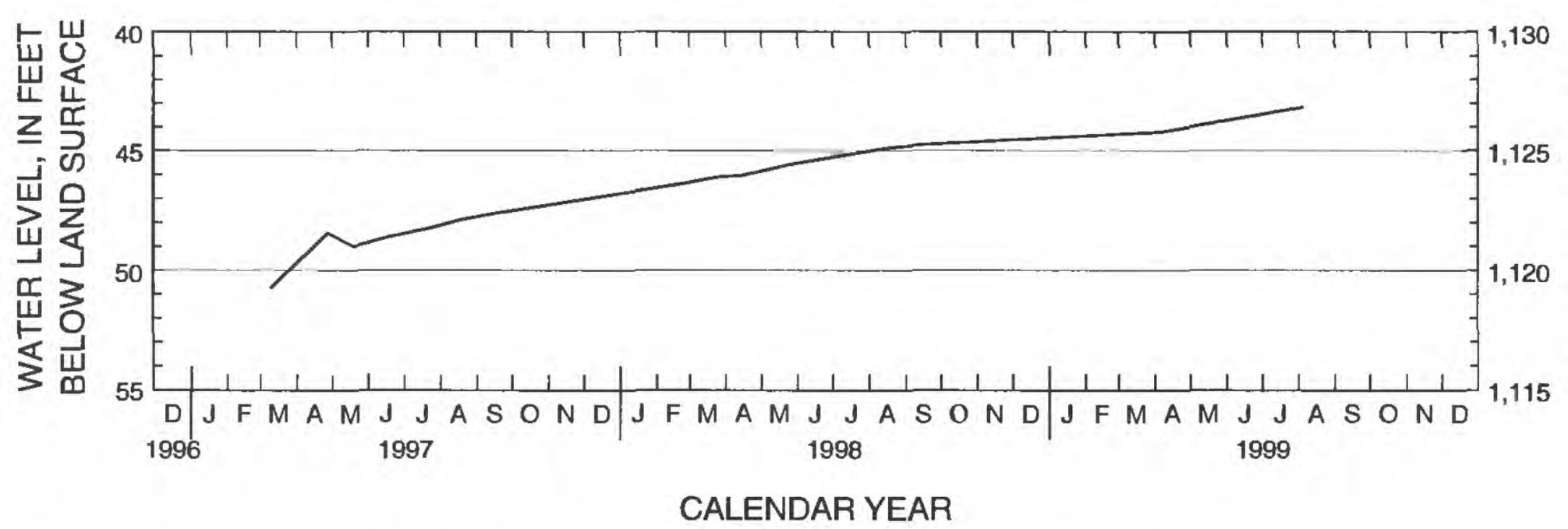

Figure B163. Hydrograph for observation well 130N54W35CCC R (site number 163). 
Section C - Annual peak-flow information

$281 \rightarrow 283$ 
Table C1. Annual peak-flow measurements for station 05050000, Bois de Sioux River near White Rock, S. Dak.

[Discharge codes: no code, peak flow or discharge is a maximum instantaneous value. Gage height codes: no code, stage or gage height is the maximum, unaffected value for the station during the year; 1 , gage height affected by backwater; 2, gage height not the maximum for the year. $\mathrm{ft}^{3} / \mathrm{s}$, cubic feet per second]

\begin{tabular}{|c|c|c|c|c|c|c|c|c|c|}
\hline $\begin{array}{l}\text { Water } \\
\text { year }\end{array}$ & Date & $\begin{array}{c}\text { Peak } \\
\text { discharge } \\
\left(\mathrm{ft}^{3} / \mathrm{s}\right)\end{array}$ & $\begin{array}{l}\text { Discharge } \\
\text { codes }\end{array}$ & $\begin{array}{c}\text { Gage } \\
\text { height } \\
\text { (feet) }\end{array}$ & $\begin{array}{l}\text { Gage-height } \\
\text { codes }\end{array}$ & $\begin{array}{l}\text { Highest } \\
\text { since }\end{array}$ & $\begin{array}{l}\text { Maximum } \\
\text { gage height } \\
\text { (feet) }\end{array}$ & Date & $\begin{array}{l}\text { Gage-height } \\
\text { codes }\end{array}$ \\
\hline 1997 & $04-20-97$ & 8,750 & & 16.90 & & & & & \\
\hline 1998 & $03-08-98$ & 1,070 & & 10.32 & 2 & & 11.81 & $03-10-98$ & 1 \\
\hline 1999 & $06-11-99$ & 737 & & 8.83 & & & & & \\
\hline
\end{tabular}

Table C2. Annual peak-flow measurements for station 05051650, La Belle Creek near Veblen, S. Dak.

[Discharge codes: no code, peak flow or discharge is a maximum instantaneous value. Gage height codes: no code, stage or gage height is the maximum. unaffected value for the station during the year; 1 , gage height affected by backwater; 2, gage height not the maximum for the year. $\mathrm{ft}^{3} / \mathrm{s}$, cubic feet per second]

\begin{tabular}{|c|c|c|c|c|c|c|c|c|c|}
\hline $\begin{array}{l}\text { Water } \\
\text { year }\end{array}$ & Date & $\begin{array}{c}\text { Peak } \\
\text { discharge } \\
\left(\mathrm{ft}^{3} / \mathrm{s}\right)\end{array}$ & $\begin{array}{l}\text { Discharge } \\
\text { codes }\end{array}$ & $\begin{array}{c}\text { Gage } \\
\text { height } \\
\text { (feet) }\end{array}$ & $\begin{array}{l}\text { Gage-height } \\
\text { codes }\end{array}$ & $\begin{array}{l}\text { Highest } \\
\text { since }\end{array}$ & $\begin{array}{l}\text { Maximum } \\
\text { gage height } \\
\text { (feet) }\end{array}$ & Date & $\begin{array}{l}\text { Gage-height } \\
\text { codes }\end{array}$ \\
\hline 1997 & 04-04-97 & 100 & & 10.60 & 1,2 & & 12.57 & $04-01-97$ & 1 \\
\hline 1998 & $05-12-98$ & 133 & & 5.41 & & & & & \\
\hline 1999 & $07-08-99$ & 97 & & 5.15 & 2 & & 5.30 & $02-26-99$ & 1 \\
\hline
\end{tabular}

Table C3. Annual peak-flow measurements for station 05289985, Big Coulee Creek near Peever, S. Dak.

[Discharge codes: no code, peak flow or discharge is a maximum instantaneous value. Gage height codes: no code, stage or gage height is the maximum, unaffected value for the station during the year; 1 , gage height affected by backwater; 2 , gage height not the maximum for the year. $\mathrm{ft}^{3} / \mathrm{s}$, cubic feet per second]

\begin{tabular}{ccccccccc}
\hline $\begin{array}{l}\text { Water } \\
\text { year }\end{array}$ & Date & $\begin{array}{c}\text { Peak } \\
\text { discharge } \\
\left(\mathbf{f t}^{3} / \mathbf{s}\right)\end{array}$ & $\begin{array}{c}\text { Discharge } \\
\text { codes }\end{array}$ & $\begin{array}{c}\text { Gage } \\
\text { height } \\
\text { (feet) }\end{array}$ & $\begin{array}{c}\text { Gage-height } \\
\text { codes }\end{array}$ & $\begin{array}{c}\text { Highest } \\
\text { since }\end{array}$ & $\begin{array}{c}\text { Maximum } \\
\text { gage height } \\
\text { (feet) }\end{array}$ & $\begin{array}{c}\text { Gage-height } \\
\text { Dodes }\end{array}$ \\
\hline 1997 & $04-05-97$ & 358 & & 7.73 & 2 & 9.43 & $03-27-97$ & 1 \\
1998 & $05-12-98$ & 614 & 7.42 & & 5.59 & $06-06-99$ & 1 \\
1999 & $10-17-98$ & 136 & 5.18 & 2 & 2 & & & \\
\hline
\end{tabular}


Table C4. Annual peak-flow measurements for station 05290000, Little Minnesota River near Peever, S. Dak.

[Discharge codes: no code, peak flow or discharge is a maximum instantaneous value. Gage height codes: no code, stage or gage height is the maximum, unaffected value for the station during the year; 1 , gage height affected by backwater; 2 , gage height not the maximum for the year. $\mathrm{ft}^{3} / \mathrm{s}$, cubic feet per second]

\begin{tabular}{|c|c|c|c|c|c|c|c|c|c|}
\hline $\begin{array}{l}\text { Water } \\
\text { year }\end{array}$ & Date & $\begin{array}{c}\text { Peak } \\
\text { discharge } \\
\left(\mathrm{ft}^{3} / \mathrm{s}\right)\end{array}$ & $\begin{array}{l}\text { Discharge } \\
\text { codes }\end{array}$ & $\begin{array}{c}\text { Gage } \\
\text { height } \\
\text { (feet) }\end{array}$ & $\begin{array}{l}\text { Gage-height } \\
\text { codes }\end{array}$ & $\begin{array}{l}\text { Highest } \\
\text { since }\end{array}$ & $\begin{array}{c}\text { Maximum } \\
\text { gage height } \\
\text { (feet) }\end{array}$ & Date & $\begin{array}{l}\text { Gage-height } \\
\text { codes }\end{array}$ \\
\hline 1997 & $03-28-97$ & 3,590 & & 14.40 & & & & & \\
\hline 1998 & $05-17-98$ & 1,340 & & 6.31 & 2 & & 6.58 & $02-29-98$ & 1 \\
\hline 1999 & $06-07-99$ & 306 & & 4.20 & & & & & \\
\hline
\end{tabular}

Table C5. Annual peak-flow measurements for station 05291000, Whetstone River near Big Stone City, S. Dak.

[Discharge codes: no code, peak flow or discharge is a maximum instantaneous value. Gage height codes: no code, stage or gage height is the maximum, unaffected value for the station during the year. $\mathrm{ft}^{3} / \mathrm{s}$, cubic feet per second]

\begin{tabular}{|c|c|c|c|c|c|c|c|c|c|}
\hline $\begin{array}{l}\text { Water } \\
\text { year }\end{array}$ & Date & $\begin{array}{c}\text { Peak } \\
\text { discharge } \\
\left(\mathrm{ft}^{3} / \mathrm{s}\right)\end{array}$ & $\begin{array}{l}\text { Discharge } \\
\text { codes }\end{array}$ & $\begin{array}{c}\text { Gage } \\
\text { height } \\
\text { (feet) }\end{array}$ & $\begin{array}{l}\text { Gage-height } \\
\text { codes }\end{array}$ & $\begin{array}{l}\text { Highest } \\
\text { since }\end{array}$ & $\begin{array}{l}\text { Maximum } \\
\text { gage height } \\
\text { (feet) }\end{array}$ & Dete & $\begin{array}{l}\text { Gage-height } \\
\text { codes }\end{array}$ \\
\hline 1997 & 04-06-97 & 7,930 & & 14.21 & & & & & \\
\hline 1998 & $03-27-98$ & 1,230 & & 7.09 & & & & & \\
\hline 1999 & $03-17-99$ & 325 & & 3.62 & & & & & \\
\hline
\end{tabular}

Table C6. Annual peak-flow measurements for station 05292000, Minnesota River at Ortonville, Minn.

[Discharge codes: no code. peak flow or discharge is a maximum instantaneous value; 7. discharge is a historic peak. Gage height codes: no code, stage or gage height is the maximum, unaffected value for the station during the year. $\mathrm{ft}^{3} / \mathrm{s}$, cubic feet per second]

\begin{tabular}{|c|c|c|c|c|c|c|c|c|c|}
\hline $\begin{array}{l}\text { Water } \\
\text { year }\end{array}$ & Date & $\begin{array}{c}\text { Peak } \\
\text { discharge } \\
\left(\mathrm{ft}^{3} / \mathrm{s}\right)\end{array}$ & $\begin{array}{l}\text { Discharge } \\
\text { codes }\end{array}$ & $\begin{array}{c}\text { Gage } \\
\text { height } \\
\text { (feet) }\end{array}$ & $\begin{array}{l}\text { Gage-height } \\
\text { codes }\end{array}$ & $\begin{array}{l}\text { Highest } \\
\text { since }\end{array}$ & $\begin{array}{c}\text { Maximum } \\
\text { gage height } \\
\text { (feet) }\end{array}$ & Date & $\begin{array}{l}\text { Gage-height } \\
\text { codes }\end{array}$ \\
\hline 1997 & $04-10-97$ & 5,070 & 7 & 12.85 & & $04-13-52$ & & & \\
\hline 1998 & 04-08-98 & 1,980 & & 8.03 & & & & & \\
\hline 1999 & $05-17-99$ & 671 & & 4.59 & & & & & \\
\hline
\end{tabular}


Table C7. Annual peak-flow measurements for station 06479215, Big Sioux River near Florence, S. Dak.

[Discharge codes: no code, peak flow or discharge is a maximum instantaneous value. Gage height codes: no code, stage or gage height is the maximum, unaffected value for the station during the year; 1 , gage height affected by backwater; 2 , gage height is not the maximum for the year. $\mathrm{ft}^{3} / \mathrm{s}$, cubic feet per second]

\begin{tabular}{ccccccccc}
\hline $\begin{array}{l}\text { Water } \\
\text { year }\end{array}$ & Date & $\begin{array}{c}\text { Peak } \\
\text { discharge } \\
\left(\mathbf{f t}^{\mathbf{3}} / \mathbf{s}\right)\end{array}$ & $\begin{array}{c}\text { Discharge } \\
\text { codes }\end{array}$ & $\begin{array}{c}\text { Gage } \\
\text { height } \\
\text { (feet) }\end{array}$ & $\begin{array}{c}\text { Gage-height } \\
\text { codes }\end{array}$ & $\begin{array}{c}\text { Highest } \\
\text { since }\end{array}$ & $\begin{array}{c}\text { Maximum } \\
\text { gage height } \\
\text { (feet) }\end{array}$ & $\begin{array}{c}\text { Gage-height } \\
\text { Dodes }\end{array}$ \\
\hline 1997 & $04-04-97$ & 2,000 & 9.32 & 2 & 9.52 & $04-02-97$ & 1 \\
1998 & $05-16-98$ & 528 & 8.12 & 2 & 8.23 & $02-26-98$ & 1 \\
1999 & $10-17-98$ & 134 & 6.55 & & & & \\
\hline
\end{tabular}

Table C8. Annual peak-flow measurements for station 06479438, Big Sioux River near Watertown, S. Dak.

[Discharge codes: no code, peak flow or discharge is a maximum instantaneous value; 7, discharge is a historic peak. Gage height codes: no code, stage or gage height is the maximum, unaffected value for the station during the year; 1 , gage height affected by backwater; 2 , gage height is not the maximum for the year. $\mathrm{ft}^{3} / \mathrm{s}$, cubic feet per second]

\begin{tabular}{ccccccccc}
\hline $\begin{array}{l}\text { Water } \\
\text { year }\end{array}$ & Date & $\begin{array}{c}\text { Peak } \\
\text { discharge } \\
\left(\mathbf{f t}^{3} / \mathbf{s}\right)\end{array}$ & $\begin{array}{c}\text { Discharge } \\
\text { codes }\end{array}$ & $\begin{array}{c}\text { Gage } \\
\text { height } \\
\text { (feet) }\end{array}$ & $\begin{array}{c}\text { Gage-height } \\
\text { codes }\end{array}$ & $\begin{array}{c}\text { Highest } \\
\text { since }\end{array}$ & $\begin{array}{c}\text { Maximum } \\
\text { gage height } \\
\text { (feet) }\end{array}$ & $\begin{array}{c}\text { Gage-height } \\
\text { Dodes }\end{array}$ \\
\hline 1997 & $04-05-97$ & 7,820 & 7 & 12.09 & & 1881 & & $02-26-98$ \\
1998 & $05-17-98$ & 1,140 & & 8.87 & 2 & & & \\
1999 & $10-17-98$ & 946 & & 8.60 & & & \\
\hline
\end{tabular}


Section D - Mean flow and summary statistics

$286 \rightarrow 289$ 
Table D1.1. Mean flow, in cubic feet per second, for station 05051650, LaBelle Creek near Veblen, S. Dak.

\begin{tabular}{llllllllllllll}
\hline $\begin{array}{c}\text { Water } \\
\text { year }\end{array}$ & \multicolumn{1}{c}{ Oct } & Nov & Dec & Jan & Feb & Mar & Apr & May & Jun & Jul & Aug & Sep & Annual \\
\hline 1988 & 0 & 0 & 0 & 0 & 0.293 & 0.932 & 0.281 & 0.091 & 0.001 & 0 & 0 & 0 & 0.133 \\
1989 & 0 & 0 & 0 & 0 & 0 & 3.13 & 5.49 & 1.18 & .115 & .020 & .281 & .028 & .854 \\
1990 & 0 & 0 & 0 & 0 & 0 & .217 & .264 & .151 & .041 & 0 & 0 & 0 & .056 \\
1991 & 0 & 0 & 0 & 0 & .078 & .361 & .669 & .495 & 3.43 & 3.07 & .389 & .105 & .718 \\
1992 & 0 & 0 & 0 & .005 & .503 & 1.79 & .858 & .132 & 3.12 & 1.83 & .072 & .241 & .710 \\
1993 & .002 & .137 & .036 & .009 & .006 & 7.03 & 12.7 & 2.50 & 6.46 & 14.1 & 7.42 & .771 & 4.29 \\
1994 & .176 & .269 & .701 & .548 & .372 & 14.3 & 13.9 & 5.84 & .206 & 1.93 & .038 & .086 & 3.21 \\
1995 & .486 & .373 & .140 & .023 & .761 & 22.4 & 11.4 & 8.67 & 2.41 & 2.78 & .455 & .235 & 4.21 \\
1996 & 2.27 & 1.16 & .154 & .105 & 3.26 & 9.86 & 1.53 & 15.1 & 2.29 & .111 & .003 & 0 \\
1997 & .146 & .084 & .240 & .326 & .258 & 1.96 & 20.9 & 3.37 & .409 & .295 & .117 & .016 & 2.33 \\
1998 & .095 & .171 & .110 & .048 & 2.41 & 7.75 & 13.6 & 11.9 & 6.25 & 3.14 & .127 & 0 \\
1999 & .842 & 2.84 & 1.81 & .303 & 1.36 & 6.59 & 5.99 & 5.53 & 1.51 & 2.74 & .417 & 7.09 & 3.08 \\
\hline
\end{tabular}

Table D1.2. Statistics on mean flow, in cubic feet per second, for station 05051650, LaBelle Creek near Veblen, S. Dak. (October 1988 through September 1999)

\begin{tabular}{|c|c|c|c|c|c|c|c|c|c|c|c|c|c|}
\hline \multirow{2}{*}{ Statistic } & \multicolumn{12}{|c|}{ Month } & \multirow{2}{*}{ Annual } \\
\hline & Oct. & Nov. & Dec. & Jan. & Feb. & Mar. & Apr. & May. & Jun. & Jul. & Aug. & Sep. & \\
\hline Number & 12 & 12 & 12 & 12 & 12 & 12 & 12 & 12 & 12 & 12 & 12 & 12 & 12 \\
\hline Maximum & 2.27 & 2.84 & 1.81 & .548 & 3.26 & 22.4 & 20.9 & 15.1 & 6.46 & 14.1 & 7.42 & 7.09 & 4.29 \\
\hline \multicolumn{14}{|l|}{ Percentile } \\
\hline 75th & .409 & .347 & .218 & .254 & 1.21 & 9.33 & 13.4 & 7.96 & 3.35 & 3.00 & .410 & .240 & 3.65 \\
\hline 50 th & .049 & .110 & .073 & .016 & .333 & 4.86 & 5.74 & 2.93 & 1.90 & 1.88 & .122 & .057 & 2.66 \\
\hline 25 th & 0 & 0 & 0 & 0 & .024 & 1.15 & .716 & .237 & .138 & .043 & .012 & 0 & .712 \\
\hline Minimum & 0 & 0 & 0 & 0 & 0 & .217 & .264 & .091 & .001 & 0 & 0 & 0 & .056 \\
\hline Mean & .33 & .42 & .27 & .11 & .78 & 6.36 & 7.30 & 4.58 & 2.19 & 2.50 & 0.78 & 0.71 & 2.20 \\
\hline $\begin{array}{l}\text { Standard } \\
\text { deviation }\end{array}$ & .66 & .83 & .53 & .18 & 1.05 & 6.67 & 6.99 & 5.02 & 2.31 & 3.88 & 2.10 & 2.02 & 1.61 \\
\hline Skewness & 2.69 & 2.70 & 2.75 & 1.66 & 1.67 & 1.42 & 0.59 & 1.07 & 0.93 & 2.79 & 3.42 & 3.39 & -0.11 \\
\hline $\begin{array}{l}\text { Coefficient } \\
\text { of varia- } \\
\text { tion }\end{array}$ & 1.98 & 1.98 & 1.97 & 1.58 & 1.36 & 1.05 & .96 & 1.10 & 1.05 & 1.55 & 2.70 & 2.83 & 0.73 \\
\hline $\begin{array}{l}\text { Percent of } \\
\text { annual } \\
\text { flow }\end{array}$ & 1.27 & 1.59 & 1.01 & 0.43 & 2.94 & 24.16 & 27.72 & 17.40 & 8.31 & 9.50 & 2.95 & 2.71 & ${ }^{1} 99.99$ \\
\hline
\end{tabular}

\footnotetext{
${ }^{1}$ Less than 100 percent due to rounding.
} 
Table D1.3. Serial correlation for 1-year lag for monthly and annual mean flows for station 05051650, LaBelle Creek near Veblen, S. Dak. (October 1988 through September 1999

\begin{tabular}{ccccccccccccc}
\hline \multicolumn{10}{c}{ Month } \\
\hline Oct. & Nov. & Dec. & Jan. & Feb. & Mar. & Apr. & May. & Jun. & Jul. & Aug. & Sep. \\
\hline 0.099 & 0.055 & 0.006 & -0.145 & 0.088 & 0.564 & 0.231 & 0.404 & -0.139 & -0.003 & -0.138 & -0.711 & 0.606 \\
\hline
\end{tabular}

Table D1.4. Correlation matrix for monthly mean flow for station 05051650, LaBelle Creek near Veblen, S. Dak. (October 1988 through September 1999)

$[-$, not computed $]$

\begin{tabular}{|c|c|c|c|c|c|c|c|c|c|c|c|c|}
\hline \multirow{2}{*}{ Month } & \multicolumn{12}{|c|}{ Month } \\
\hline & Oct. & Nov. & Dec. & Jan. & Feb. & Mar. & Apr. & May. & Jun. & Jul. & Aug. & Sep. \\
\hline Oct & 1 & 0.596 & 0.273 & 0.149 & 0.800 & 0.347 & -0.166 & 0.750 & -0.026 & -0.192 & -0.163 & 0.220 \\
\hline Nov & - & 1 & .890 & .366 & .475 & .210 & -.065 & .385 & -.053 & -.018 & -.078 & .909 \\
\hline Dec & -- & - & 1 & .646 & .195 & .205 & .151 & .181 & -.180 & -.015 & -.109 & .915 \\
\hline Jan & -- & - & -- & 1 & .057 & .286 & .539 & .211 & -.354 & -.140 & -.109 & .915 \\
\hline Feb & -- & - & -- & - & 1 & .320 & -.026 & .905 & .304 & -.166 & -.240 & .143 \\
\hline Mar & -- & - & -- & -- & - & 1 & .403 & .636 & .144 & .164 & .057 & .032 \\
\hline Apr & -- & -- & -- & - & - & -- & 1 & .275 & .184 & .294 & .251 & -.036 \\
\hline May & -- & - & -- & -- & - & - & -- & 1 & .306 & -.046 & -.131 & .041 \\
\hline Jun & - & -- & -- & -- & - & -- & - & -- & 1 & .742 & .596 & -.026 \\
\hline Jul & - & - & - & -- & -- & -- & -- & - & -- & 1 & .956 & .121 \\
\hline Aug & -- & - & -- & -- & - & -- & -- & -- & -- & -- & 1 & .047 \\
\hline Sep & -- & -- & - & - & -- & - & -- & -- & - & -- & - & 1 \\
\hline
\end{tabular}


Table D1.5. Lowest mean flow, in cubic feet per second, and ranking for the following number of consecutive days, for station 05051650, LaBelle Creek near Veblen, S. Dak.

\begin{tabular}{|c|c|c|c|c|c|c|c|c|c|c|c|c|c|c|c|c|c|c|}
\hline \multirow{3}{*}{$\begin{array}{c}\begin{array}{c}\text { Water } \\
\text { year }\end{array} \\
1988\end{array}$} & \multicolumn{18}{|c|}{ Flow, in cubic feet per second, and ranking for number of consecutive days } \\
\hline & \multicolumn{2}{|c|}{1} & \multicolumn{2}{|l|}{3} & \multicolumn{2}{|l|}{7} & \multicolumn{2}{|l|}{14} & \multicolumn{2}{|l|}{30} & \multicolumn{2}{|l|}{60} & \multicolumn{2}{|l|}{90} & \multicolumn{2}{|c|}{120} & \multicolumn{2}{|c|}{183} \\
\hline & 0 & 1 & 0 & 1 & 0 & 1 & 0 & 1 & 0 & 1 & 0 & 1. & 0 & 1 & 0 & 1 & 0.062 & 2 \\
\hline 1989 & 0 & 2 & 0 & 2 & 0 & 2 & 0 & 2 & 0 & 2 & 0 & 2 & 0 & 2 & 0 & 2 & .58 & 8 \\
\hline 1990 & 0 & 3 & 0 & 3 & 0 & 3 & 0 & 3 & 0 & 3 & 0 & 3 & 0 & 3 & 0 & 3 & .010 & 1 \\
\hline 1991 & 0 & 4 & 0 & 4 & 0 & 4 & 0 & 4 & 0 & 4 & 0 & 4 & 0 & 4 & 0 & 4 & .076 & 3 \\
\hline 1992 & 0 & 5 & 0 & 5 & 0 & 5 & 0 & 5 & 0 & 5 & 0 & 5 & 0 & 5 & 0 & 5 & .38 & 4 \\
\hline 1993 & 0 & 6 & 0 & 6 & 0 & 6 & 0 & 6 & 0 & 6 & .008 & 7 & .013 & 6 & .028 & 6 & 1.36 & 7 \\
\hline 1994 & 0 & 7 & 0 & 7 & 0 & 7 & .001 & 10 & .024 & 11 & .061 & 9 & .38 & 11 & .43 & 10 & 2.86 & 11 \\
\hline 1995 & 0 & 8 & .007 & 12 & .010 & 12 & .016 & 11 & .018 & 10 & .049 & 8 & .090 & 8 & .16 & 8 & 4.14 & 12 \\
\hline 1996 & 0 & 9 & 0 & 8 & 0 & 8 & 0 & 7 & 0 & 7 & .001 & 6 & .029 & 7 & .47 & 11 & 2.68 & 10 \\
\hline 1997 & 0 & 10 & 0 & 9 & 0 & 9 & 0 & 8 & .016 & 9 & .068 & 11 & .13 & 10 & .20 & 9 & .67 & 6 \\
\hline 1998 & 0 & 11 & 0 & 10 & 0 & 10 & 1 & 9 & 0 & 8 & .062 & 10 & .10 & 9 & .10 & 7 & 1.85 & 8 \\
\hline 1999 & 0 & 12 & 0 & 11 & .009 & 11 & .036 & 12 & .28 & 12 & .28 & 12 & .82 & 12 & 1.43 & 12 & 2.36 & 9 \\
\hline
\end{tabular}

Table D1.6. Highest mean flow, in cubic feet per second, and ranking for the following number of consecutive days, for station 05051650, LaBelle Creek near Veblen, S. Dak.

\begin{tabular}{|c|c|c|c|c|c|c|c|c|c|c|c|c|c|c|c|c|c|c|}
\hline \multirow{2}{*}{$\begin{array}{c}\text { Water } \\
\text { year }\end{array}$} & \multicolumn{18}{|c|}{ Flow, In cubic feet per second, and ranking for number of consecutive days } \\
\hline & \multicolumn{2}{|l|}{1} & \multicolumn{2}{|l|}{3} & \multicolumn{2}{|l|}{7} & \multicolumn{2}{|c|}{15} & \multicolumn{2}{|c|}{30} & \multicolumn{2}{|c|}{60} & \multicolumn{2}{|c|}{90} & \multicolumn{2}{|c|}{120} & \multicolumn{2}{|c|}{183} \\
\hline 1988 & 3.50 & 11 & 2.83 & 11 & 1.96 & 11 & 1.58 & 11 & 1.17 & 11 & 0.76 & 11 & 0.53 & 11 & 0.41 & 11 & 0.27 & 11 \\
\hline 1989 & 20.0 & 9 & 18.3 & 8 & 13.7 & 8 & 11.9 & 8 & 7.79 & 8 & 4.69 & 8 & 3.34 & 8 & 2.51 & 8 & 1.70 & 8 \\
\hline 1990 & .77 & 12 & .50 & 12 & .47 & 12 & .40 & 12 & .33 & 12 & .27 & 12 & .22 & 12 & .17 & 12 & .11 & 12 \\
\hline 1991 & 29.0 & 8 & 15.1 & 9 & 9.59 & 9 & 8.43 & 9 & 5.45 & 9 & 3.37 & 9 & 2.35 & 9 & 1.96 & 9 & 1.41 & 9 \\
\hline 1992 & 13.0 & 10 & 11.6 & 10 & 9.40 & 10 & 6.75 & 10 & 4.71 & 10 & 2.53 & 10 & 1.83 & 10 & 1.56 & 10 & 1.37 & 10 \\
\hline 1993 & 81.0 & 3 & 51.0 & 4 & 35.9 & 4 & 27.0 & 5 & 18.9 & 5 & 12.3 & 5 & 9.54 & 4 & 9.99 & 3 & 8.51 & 1 \\
\hline 1994 & 75.0 & 4 & 53.3 & 3 & 37.6 & 3 & 28.3 & 3 & 21.0 & 3 & 16.3 & 2 & 11.5 & 3 & 8.78 & 4 & 6.10 & 4 \\
\hline 1995 & 200 & 1 & 130 & 1 & 72.9 & 1 & 41.7 & 1 & 26.5 & 1 & 18.5 & 1 & 14.8 & 1 & 11.9 & 1 & 8.16 & 2 \\
\hline 1996 & 150 & 2 & 80.3 & 2 & 46.9 & 2 & 27.2 & 4 & 16.0 & 6 & 8.92 & 6 & 9.53 & 5 & 7.94 & 5 & 5.39 & 5 \\
\hline 1997 & 75.0 & 5 & 48.3 & 5 & 32.1 & 6 & 25.4 & 6 & 21.5 & 2 & 12.9 & 4 & 8.84 & 6 & 6.74 & 6 & 4.51 & 7 \\
\hline 1998 & 60.0 & 6 & 45.3 & 6 & 34.0 & 5 & 31.1 & 2 & 19.5 & 4 & 15.9 & 3 & 12.5 & 2 & 10.7 & 2 & 7.49 & 3 \\
\hline 1999 & 49.0 & 7 & 28.7 & 7 & 18.5 & 7 & 21.1 & 7 & 10.2 & 7 & 7.74 & 7 & 6.30 & 7 & 5.29 & 7 & 4.60 & 6 \\
\hline
\end{tabular}


Table D2.1. Mean flow, in cubic feet per second, for station 05289985, Big Coulee Creek near Peever, S. Dak.

\begin{tabular}{|c|c|c|c|c|c|c|c|c|c|c|c|c|c|}
\hline \multirow{2}{*}{$\begin{array}{l}\text { Water } \\
\text { year }\end{array}$} & \multicolumn{12}{|c|}{ Month } & \multirow{2}{*}{ Annual } \\
\hline & Oct & Nov & Dec & Jan & Feb & Mar & Apr & May & Jun & Jul & Aug & Sep & \\
\hline 1988 & 0 & 0 & 0 & 0 & 0.847 & 5.28 & 1.71 & 1.09 & 0.013 & 0 & 0 & 0 & 0.748 \\
\hline 1989 & 0 & 0 & 0 & 0 & 0 & 11.8 & 12.0 & 2.76 & .059 & 0 & .003 & 1.19 & 2.32 \\
\hline 1990 & .193 & .223 & 0 & 0 & 0 & 3.48 & 1.52 & 1.30 & .789 & .008 & .002 & 0 & .630 \\
\hline 1991 & .002 & .018 & .023 & 0 & 0 & 1.42 & 3.61 & 3.99 & 11.1 & 7.85 & 3.58 & 2.52 & 2.85 \\
\hline 1992 & 1.64 & .547 & .488 & .666 & 2.90 & 6.59 & 2.41 & 1.25 & 4.09 & 2.31 & .370 & 2.91 & 2.17 \\
\hline 1993 & .340 & 1.61 & .530 & .059 & .738 & 10.2 & 12.6 & 6.37 & 7.38 & 16.6 & 3.69 & .825 & 5.11 \\
\hline 1994 & 2.70 & 3.69 & 1.29 & .026 & 1.36 & 20.1 & 10.4 & 6.55 & 6.84 & 2.84 & .573 & 2.10 & 4.90 \\
\hline 1995 & 3.76 & 1.18 & .679 & .750 & 1.53 & 23.4 & 16.2 & 19.0 & 8.78 & 2.85 & 3.24 & .374 & 6.86 \\
\hline 1996 & 4.48 & 2.24 & 1.24 & .819 & 5.60 & 21.3 & 21.0 & 8.53 & 3.62 & 4.63 & 2.85 & .593 & 6.41 \\
\hline 1997 & 2.13 & .906 & .778 & .957 & 1.39 & 10.6 & 34.0 & 6.85 & 2.21 & 1.34 & .704 & .013 & 5.14 \\
\hline 1998 & .996 & 1.25 & 1.24 & .486 & 7.15 & 9.92 & 18.5 & 22.5 & 4.17 & 2.51 & .523 & .014 & 5.76 \\
\hline 1999 & 3.08 & 3.26 & 1.66 & .166 & 4.00 & 6.64 & 6.00 & 6.05 & 5.00 & 5.55 & .617 & 1.47 & 3.62 \\
\hline
\end{tabular}

Table D2.2. Statistics on mean flow, in cubic feet per second for station 05289985, Big Coulee Creek near Peever, S. Dak. (October 1988 through September 1999)

\begin{tabular}{|c|c|c|c|c|c|c|c|c|c|c|c|c|c|}
\hline \multirow{2}{*}{ Statistic } & \multicolumn{12}{|c|}{ Month } & \multirow{2}{*}{ Annual } \\
\hline & Oct. & Nov. & Dec. & Jan. & Feb. & Mar. & Apr. & May. & Jun. & Jul. & Aug. & Sep. & \\
\hline Number & 12 & 12 & 12 & 12 & 12 & 12 & 12 & 12 & 12 & 12 & 12 & 12 & 12 \\
\hline Maximum & 4.48 & 3.69 & 1.66 & .957 & 7.15 & 23.4 & 34.0 & 22.5 & 11.1 & 16.6 & 3.69 & 2.91 & 6.86 \\
\hline \multicolumn{14}{|l|}{ Percentile } \\
\hline 75th & 2.98 & 2.08 & 1.24 & .729 & 3.72 & 18.0 & 17.9 & 8.11 & 7.24 & 5.32 & 3.14 & 1.94 & 5.61 \\
\hline 50th & 1.32 & 1.04 & .604 & .112 & 1.38 & 10.1 & 11.2 & 6.21 & 4.13 & 2.67 & .595 & .709 & 4.26 \\
\hline 25 th & .050 & .069 & .006 & 0 & .184 & 5.61 & 2.71 & 1.66 & 1.14 & .341 & .095 & .013 & 2.21 \\
\hline Minimum & 0 & 0 & 0 & 0 & 0 & 1.42 & 1.52 & 1.09 & .013 & 0 & 0 & 0 & .630 \\
\hline Mean & 1.61 & 1.24 & 0.66 & 0.33 & 2.13 & 10.89 & 11.66 & 7.19 & 4.50 & 3.87 & 1.35 & 1.00 & 3.88 \\
\hline $\begin{array}{l}\text { Standard } \\
\text { deviation }\end{array}$ & 1.60 & 1.26 & 0.59 & 0.38 & 2.34 & 7.16 & 9.73 & 6.84 & 3.51 & 4.66 & 1.51 & 1.04 & 2.13 \\
\hline Skewness & .54 & .92 & .27 & .60 & 1.21 & .67 & 1.05 & 1.52 & .40 & 2.08 & .78 & .74 & -.23 \\
\hline $\begin{array}{l}\text { Coefficient } \\
\text { of varia- } \\
\text { tion }\end{array}$ & 1.00 & 1.01 & .89 & 1.16 & 1.10 & .66 & .83 & .95 & .78 & 1.20 & 1.12 & 1.04 & .55 \\
\hline $\begin{array}{l}\text { Percent of } \\
\text { annual } \\
\text { flow }\end{array}$ & 3.47 & 2.68 & 1.42 & 0.71 & 4.58 & 23.46 & 25.11 & 15.48 & 9.70 & 8.34 & 2.90 & 2.16 & ${ }^{1} 100.01$ \\
\hline
\end{tabular}

${ }^{1}$ Greater than 100 percent due to rounding. 
Table D2.3. Serial correlation for 1-year lag for monthly and annual mean flows for station 05289985, Big Coulee Creek near Peever, S. Dak. (October 1988 through September 1999)

\begin{tabular}{lllllllllllll}
\hline \multicolumn{10}{c}{ Month } \\
\hline Oct. & Nov. & Dec. & Jan. & Feb. & Mar. & Apr. & May. & Jun. & Jul. & Aug. & Sep. & Annual \\
\hline 0.572 & 0.367 & 0.703 & 0.440 & 0.207 & 0.633 & 0.510 & 0.164 & 0.192 & -0.107 & -0.178 & 0.088 & 0.675 \\
\hline
\end{tabular}

Table D2.4. Correlation matrix for monthly mean flow for station 05289985, Big Coulee Creek near Peever, S.Dak. (October 1988 through September 1999)

$[--$, not computed]

\begin{tabular}{|c|c|c|c|c|c|c|c|c|c|c|c|c|}
\hline \multirow{2}{*}{ Month } & \multicolumn{12}{|c|}{ Month } \\
\hline & Oct. & Nov. & Dec. & Jan. & Feb. & Mar. & Apr. & May. & Jun. & Jul. & Aug. & Sep. \\
\hline Oct. & 1 & 0.681 & 0.750 & 0.657 & 0.506 & 0.753 & 0.441 & 0.403 & 0.231 & -0.047 & 0.237 & 0.001 \\
\hline Nov. & -- & 1 & .888 & .077 & .422 & .518 & .193 & .251 & .298 & .263 & .084 & .164 \\
\hline Dec. & -- & -- & 1 & .383 & .742 & .474 & .400 & .501 & .215 & .135 & .025 & .022 \\
\hline Jan. & -- & -- & -- & 1 & .500 & .462 & .708 & .452 & .011 & -.169 & .157 & -.198 \\
\hline Feb. & -- & -- & -- & - & 1 & .260 & .321 & .604 & -.019 & -.031 & -.043 & -.140 \\
\hline Mar. & -- & -- & -- & -- & -- & 1 & .522 & .512 & .183 & -.034 & .287 & -.146 \\
\hline Apr. & -- & -- & -- & -- & -- & -- & 1 & .504 & -.037 & -.002 & .171 & -.443 \\
\hline May. & -- & -- & -- & -- & -- & -- & -- & 1 & .344 & .049 & .284 & -.368 \\
\hline Jun. & - & -- & -- & -- & - & - & - & - & 1 & .622 & .777 & .476 \\
\hline Jul. & -- & -- & -- & - & -- & -- & -- & - & -- & 1 & .743 & .218 \\
\hline Aug. & -- & -- & - & -- & -- & -- & -- & - & -- & -- & 1 & .116 \\
\hline Sep. & -- & -- & -- & -- & -- & -- & -- & - & - & -- & -- & 1 \\
\hline
\end{tabular}


Table D2.5. Lowest mean flow, in cubic feet per second, and ranking for the following number of consecutive days, for station 05289985, Big Coulee Creek near Peever, S. Dak.

\begin{tabular}{|c|c|c|c|c|c|c|c|c|c|c|c|c|c|c|c|c|c|c|}
\hline \multirow{3}{*}{$\begin{array}{c}\begin{array}{c}\text { Water } \\
\text { year }\end{array} \\
1988 \\
\end{array}$} & \multicolumn{18}{|c|}{ Flow, in cubic feet per second, and ranking for number of consecutive days } \\
\hline & \multicolumn{2}{|l|}{1} & \multicolumn{2}{|l|}{3} & \multicolumn{2}{|l|}{7} & \multicolumn{2}{|l|}{14} & \multicolumn{2}{|l|}{30} & \multicolumn{2}{|l|}{60} & \multicolumn{2}{|l|}{90} & \multicolumn{2}{|c|}{120} & \multicolumn{2}{|c|}{183} \\
\hline & 0 & 1 & 0 & 1 & 0 & 1 & 0 & 1 & 0 & 1 & 0 & 1 & 0 & 1 & 0 & 1 & 0.47 & 2 \\
\hline 1989 & 0 & 2 & 0 & 2 & 0 & 2 & 0 & 2 & 0 & 2 & 0 & 2 & 0 & 2 & 0 & 2 & 2.10 & 4 \\
\hline 1990 & 0 & 3 & 0 & 3 & 0 & 3 & 0 & 3 & 0 & 3 & 0 & 3 & 0 & 3 & .014 & 4 & .60 & 3 \\
\hline 1991 & 0 & 4 & 0 & 4 & 0 & 4 & 0 & 4 & 0 & 4 & 0 & 4 & .007 & 4 & .010 & 3 & .26 & 1 \\
\hline 1992 & 0 & 5 & 0 & 5 & .001 & 9 & .014 & 10 & .11 & 10 & .49 & 10 & .54 & 6 & .67 & 6 & 2.14 & 5 \\
\hline 1993 & .020 & 12 & .027 & 12 & .030 & 11 & .032 & 11 & .044 & 8 & .26 & 6 & .43 & 5 & .64 & 5 & 2.38 & 6 \\
\hline 1994 & 0 & 6 & 0 & 6 & 0 & 5 & 0 & 5 & 0 & 5 & .096 & 5 & .69 & 9 & 1.54 & 10 & 4.86 & 10 \\
\hline 1995 & 0 & 7 & 0 & 7 & 0 & 6 & 0 & 6 & .25 & 12 & .66 & 11 & .62 & 7 & .83 & 7 & 5.33 & 11 \\
\hline 1996 & 0 & 8 & 0 & 8 & 0 & 7 & 0 & 7 & .091 & 9 & .85 & 12 & 1.03 & 11 & 1.79 & 11 & 5.96 & 12 \\
\hline 1997 & .010 & 11 & .010 & 11 & .010 & 10 & .011 & 9 & .013 & 6 & .36 & 8 & .67 & 8 & .97 & 8 & 3.25 & 8 \\
\hline 1998 & 0 & 9 & 0 & 9 & 0 & 8 & 0 & 8 & .014 & 7 & .27 & 7 & .92 & 10 & 1.00 & 9 & 3.48 & 9 \\
\hline 1999 & 0 & 10 & 0 & 10 & .10 & 12 & .12 & 12 & .16 & 11 & .48 & 9 & 1.58 & 12 & 1.97 & 12 & 3.15 & 7 \\
\hline
\end{tabular}

Table D2.6. Highest mean flow, in cubic feet per second, and ranking for the following number of consecutive days, for station 05289985, Big Coulee Creek near Peever, S. Dak.

\begin{tabular}{|c|c|c|c|c|c|c|c|c|c|c|c|c|c|c|c|c|c|c|}
\hline \multirow{2}{*}{$\begin{array}{c}\text { Water } \\
\text { year }\end{array}$} & \multicolumn{18}{|c|}{ Flow, in cubic feet per second, and ranking for number of consecutive days } \\
\hline & \multicolumn{2}{|c|}{1} & \multicolumn{2}{|c|}{3} & \multicolumn{2}{|c|}{7} & \multicolumn{2}{|c|}{15} & \multicolumn{2}{|c|}{30} & \multicolumn{2}{|c|}{60} & \multicolumn{2}{|c|}{90} & \multicolumn{2}{|c|}{120} & \multicolumn{2}{|c|}{183} \\
\hline 1988 & 10.0 & 12 & 8.67 & 12 & 7.71 & 12 & 7.33 & 11 & 5.88 & 11 & 3.92 & 11 & 2.99 & 11 & 2.28 & 11 & 1.50 & 11 \\
\hline 1989 & 75.0 & 7 & 58.3 & 6 & 43.6 & 5 & 31.1 & 5 & 19.4 & 6 & 12.9 & 6 & 9.01 & 7 & 6.76 & 9 & 4.55 & 9 \\
\hline 1990 & 20.0 & 11 & 15.0 & 11 & 9.29 & 11 & 6.13 & 12 & 4.05 & 12 & 2.75 & 12 & 2.24 & 12 & 1.81 & 12 & 1.19 & 12 \\
\hline 1991 & 118 & 5 & 53.3 & 7 & 25.9 & 8 & 18.2 & 8 & 11.5 & 8 & 10.2 & 8 & 8.05 & 8 & 7.14 & 7 & 5.56 & 7 \\
\hline 1992 & 30.0 & 10 & 20.7 & 10 & 14.0 & 10 & 11.1 & 10 & 8.41 & 10 & 5.45 & 10 & 4.21 & 10 & 4.11 & 10 & 3.27 & 10 \\
\hline 1993 & 170 & 3 & 69.7 & 5 & 33.7 & 6 & 25.3 & 6 & 17.8 & 7 & 12.7 & 7 & 10.5 & 6 & 12.0 & 5 & 9.55 & 4 \\
\hline 1994 & 65.0 & 8 & 46.7 & 8 & 30.7 & 7 & 22.2 & 7 & 21.0 & 5 & 15.6 & 5 & 12.5 & 5 & 11.3 & 6 & 8.11 & 6 \\
\hline 1995 & 250 & 2 & 140 & 1 & 70.9 & 3 & 38.3 & 4 & 27.4 & 4 & 23.7 & 3 & 20.5 & 1 & 17.5 & 1 & 12.3 & 1 \\
\hline 1996 & 110 & 6 & 83.3 & 4 & 51.4 & 4 & 39.0 & 3 & 32.4 & 2 & 22.6 & 4 & 17.8 & 3 & 14.7 & 3 & 11.1 & 2 \\
\hline 1997 & 163 & 4 & 113 & 3 & 86.7 & 1 & 62.1 & 1 & 40.9 & 1 & 24.8 & 1 & 17.7 & 4 & 13.6 & 4 & 9.36 & 5 \\
\hline 1998 & 305 & 1 & 124 & 2 & 77.1 & 2 & 41.2 & 2 & 29.7 & 3 & 24.0 & 2 & 18.6 & 2 & 15.2 & 2 & 10.8 & 3 \\
\hline 1999 & 59.0 & 9 & 43.0 & 9 & 19.8 & 9 & 11.5 & 9 & 9.19 & 9 & 7.77 & 9 & 6.88 & 9 & 7.02 & 8 & 5.52 & 8 \\
\hline
\end{tabular}




\section{Section E - Water-level measurements}



Table E1. Water-level measurements for Bitter Lake in Day County

\begin{tabular}{|c|c|c|c|c|c|}
\hline Date & $\begin{array}{l}\text { Water-level } \\
\text { elevation } \\
\text { (feet) }\end{array}$ & Date & $\begin{array}{l}\text { Water-level } \\
\text { elevation } \\
\text { (feet) }\end{array}$ & Date & $\begin{array}{l}\text { Water-leve } \\
\text { elevation } \\
\text { (feet) }\end{array}$ \\
\hline $04-10-97$ & $1,786.7$ & $10-07-97$ & $1,788.4$ & 04-19-99 & $1,791.51$ \\
\hline $04-11-97$ & $1,786.7$ & $05-04-98$ & $1,790.1$ & $04-26-99$ & $1,791.53$ \\
\hline $04-12-97$ & $1,786.7$ & $05-20-98$ & 1.791 .1 & $05-05-99$ & $1,791.67$ \\
\hline $04-13-97$ & $1,787.0$ & $05-25-98$ & $1,791.3$ & $05-13-99$ & $1,791.71$ \\
\hline $04-14-97$ & $1,787.1$ & $05-26-98$ & $1,791.26$ & $06-01-99$ & $1,791.63$ \\
\hline $04-15-97$ & $1,787.2$ & $06-02-98$ & $1,791.3$ & $06-10-99$ & $1,791.91$ \\
\hline $04-16-97$ & $1,787.4$ & $06-08-98$ & $1,791.2$ & 06-14-99 & $1,791.87$ \\
\hline $04-17-97$ & $1,787.5$ & $06-12-98$ & $1,791.3$ & $06-21-99$ & $1,791.99$ \\
\hline $04-18-97$ & $1,787.6$ & $06-16-98$ & $1,791.5$ & 07-01-99 & $1,791.97$ \\
\hline $04-19-97$ & $1,787.7$ & $06-22-98$ & $1,791.5$ & $07-07-99$ & $1,791.91$ \\
\hline $04-20-97$ & $1,787.8$ & $07-06-98$ & $1,791.58$ & $07-12-99$ & 1.792 .01 \\
\hline $04-21-97$ & $1,787.9$ & $07-20-98$ & $1,791.5$ & 07-19-99 & $1,791.93$ \\
\hline $04-22-97$ & $1,788.0$ & $07-20-98$ & $1,791.3$ & $07-26-99$ & $1,791.68$ \\
\hline $04-25-97$ & $1,788.2$ & $07-27-98$ & $1,791.3$ & $08-02-99$ & $1,791.89$ \\
\hline $04-28-97$ & $1,788.2$ & 08-05-98 & $1,791.3$ & 08-09-99 & $1,792.09$ \\
\hline 05-01-97 & $1,788.5$ & 08-10-98 & 1.791 .3 & 08-16-99 & $1,791.95$ \\
\hline $05-05-97$ & $1,788.6$ & 08-21-98 & $1,791.1$ & 08-23-99 & 1.791 .81 \\
\hline 05-09-97 & $1,788.8$ & $11-13-98$ & $1,791.0$ & 09-03-99 & $1,792.11$ \\
\hline 05-13-97 & $1,788.9$ & 03-23-99 & $1,791.3$ & 09-13-99 & $1,792.05$ \\
\hline $05-14-97$ & $1,788.5$ & 04-07-99 & $1,791.47$ & $09-20-99$ & $1,792.05$ \\
\hline $05-20-97$ & $1,789.0$ & $04-12-99$ & $1,791.53$ & 09-27-99 & $1,792.11$ \\
\hline
\end{tabular}


Table E2. Water-level measurements for Blue Dog Lake in Day County

\begin{tabular}{|c|c|c|c|c|c|}
\hline Date & $\begin{array}{c}\text { Water-level } \\
\text { elevation } \\
\text { (feet) }\end{array}$ & Date & $\begin{array}{c}\text { Water-level } \\
\text { elevation } \\
\text { (feet) }\end{array}$ & Date & $\begin{array}{c}\text { Water-level } \\
\text { elevation } \\
\text { (feet) }\end{array}$ \\
\hline 04-07-97 & $1,802.3$ & $05-04-98$ & $1,802.1$ & $04-26-99$ & $1,803.61$ \\
\hline $04-10-97$ & $1,801.9$ & $05-01-98$ & $1,802.9$ & $05-05-99$ & $1,803.73$ \\
\hline $04-11-97$ & $1,802.2$ & $05-20-98$ & $1,802.87$ & 05-13-99 & $1,803.89$ \\
\hline $04-12-97$ & $1,802.1$ & $05-25-98$ & $1,802.9$ & $06-01-99$ & $1,803.83$ \\
\hline $04-13-97$ & $1,801.8$ & $05-26-98$ & $1,802.88$ & $06-10-99$ & $1,804.29$ \\
\hline $04-14-97$ & $1,801.7$ & $05-29-98$ & $1,802.9$ & 06-14-99 & $1,804.23$ \\
\hline $04-15-97$ & $1,801.5$ & $06-02-98$ & $1,802.9$ & $06-21-99$ & $1,804.21$ \\
\hline $04-16-97$ & $1,801.5$ & $06-08-98$ & $1,802.8$ & 07-01-99 & $1,804.21$ \\
\hline $04-17-97$ & $1,801.7$ & $06-15-98$ & $1,803.0$ & 07-07-99 & $1,804.19$ \\
\hline $04-18-97$ & $1,801.8$ & $06-16-98$ & $1,803.0$ & $07-12-99$ & $1,804.19$ \\
\hline $04-19-97$ & $1,802.0$ & $06-22-98$ & $1,803.0$ & 07-19-99 & $1,804.09$ \\
\hline $04-20-97$ & $1,802.1$ & $06-29-98$ & $1,803.1$ & $07-26-99$ & $1,804.06$ \\
\hline $04-21-97$ & $1,802.1$ & $07-06-98$ & $1,803.04$ & 08-02-99 & $1,803.98$ \\
\hline $04-22-97$ & $1,802.2$ & $07-20-98$ & $1,803.0$ & 08-09-99 & $1,803.92$ \\
\hline $04-25-97$ & $1,802.0$ & $07-27-98$ & $1,802.8$ & 08-16-99 & $1,803.76$ \\
\hline $04-28-97$ & $1,801.8$ & $08-05-98$ & $1,802.3$ & $08-23-99$ & $1,803.66$ \\
\hline $05-01-97$ & $1,801.6$ & $08-10-98$ & $1,802.7$ & 09-03-99 & $1,803.62$ \\
\hline $05-09-97$ & $1,801.4$ & $08-21-98$ & $1,802.5$ & $09-13-99$ & $1,803.68$ \\
\hline $05-13-97$ & $1,801.2$ & $08-25-98$ & $1,802.6$ & 09-20-99 & $1,803.56$ \\
\hline $05-16-97$ & $1,800.58$ & $08-28-98$ & $1,802.6$ & 09-27-99 & $1,803.54$ \\
\hline $05-20-97$ & $1,801.0$ & $09-04-98$ & 1.802 .5 & $10-04-99$ & $1,803.42$ \\
\hline $10-01-97$ & $1,800.5$ & $11-13-98$ & $1,802.8$ & $10-12-99$ & $1,803.36$ \\
\hline $10-07-97$ & $1,800.0$ & 04-07-99 & $1,803.55$ & $10-18-99$ & $1,803.32$ \\
\hline $03-01-98$ & $1,800.9$ & 04-12-99 & $1,803.59$ & $10-25-99$ & $1,803.3$ \\
\hline 04-01-98 & $1,802.0$ & 04-19-99 & $1,803.63$ & $11-02-99$ & 1.803 .24 \\
\hline
\end{tabular}


Table E3. Water-level measurements for Buffalo Lake North in Marshall County

\begin{tabular}{cccccc}
\hline Date & $\begin{array}{c}\text { Water-level } \\
\text { elevation } \\
\text { (feet) }\end{array}$ & Date & $\begin{array}{c}\text { Water-level } \\
\text { elevation } \\
\text { (feet) }\end{array}$ & $\begin{array}{c}\text { Water-level } \\
\text { elevation } \\
\text { (feet) }\end{array}$ \\
\hline $05-19-97$ & $1,837.7$ & $05-05-98$ & $1,836.6$ & $05-03-99$ & $1,836.4$ \\
$10-07-97$ & $1,836.5$ & $09-28-98$ & $1,834.7$ & $09-28-99$ & $1,836.0$ \\
\hline
\end{tabular}

Table E4. Water-level measurements for Buffalo Lake South in Marshall County

\begin{tabular}{cccccc}
\hline Date & $\begin{array}{c}\text { Water-level } \\
\text { elevation } \\
\text { (feet) }\end{array}$ & Date & $\begin{array}{c}\text { Water-level } \\
\text { elevation } \\
\text { (feet) }\end{array}$ & $\begin{array}{c}\text { Date } \\
\text { Water-level } \\
\text { elevation } \\
\text { (feet) }\end{array}$ \\
\hline $05-19-97$ & $1,838.6$ & $05-05-98$ & $1,838.1$ & $05-03-99$ & $1,837.6$ \\
$10-07-97$ & $1,835.3$ & $09-28-98$ & $1,835.4$ & $09-28-99$ & $1,837.0$ \\
\hline
\end{tabular}

Table E5. Water-level measurements for Clear Lake in Marshall County

\begin{tabular}{cccccc}
\hline Date & $\begin{array}{c}\text { Water-level } \\
\text { elevation } \\
\text { (feet) }\end{array}$ & Date & $\begin{array}{c}\text { Water-level } \\
\text { elevation } \\
\text { (feet) }\end{array}$ & $\begin{array}{c}\text { Water-level } \\
\text { elevation } \\
\text { (feet) }\end{array}$ \\
\hline $05-19-97$ & $1,822.9$ & $05-05-98$ & $1,822.5$ & $05-03-99$ & $1,822.3$ \\
$10-07-97$ & $1,821.5$ & $09-28-98$ & 1.821 .9 & $09-28-99$ & $1,822.0$ \\
\hline
\end{tabular}

Table E6. Water-level measurements for Clubhouse Lake in Roberts County

\begin{tabular}{ccccc}
\hline Date & $\begin{array}{c}\text { Water-level } \\
\text { elevatlon } \\
\text { (feet) }\end{array}$ & Date & $\begin{array}{c}\text { Water-level } \\
\text { elevation } \\
\text { (feet) }\end{array}$ & $\begin{array}{c}\text { Water-level } \\
\text { elevation } \\
\text { (feet) }\end{array}$ \\
\hline $05-13-97^{1}$ & 972.7 & & \\
\hline
\end{tabular}

${ }^{1}$ Water-level measurements discontinued after this date.

Table E7. Water-level measurements for Cottonwood Lake (North) in Roberts County

\begin{tabular}{cccccc}
\hline Date & $\begin{array}{c}\text { Weter-level } \\
\text { elevation } \\
\text { (feet) }\end{array}$ & Date & $\begin{array}{c}\text { Water-level } \\
\text { elevation } \\
\text { (feet) }\end{array}$ & $\begin{array}{c}\text { Water-level } \\
\text { elevation } \\
\text { (feet) }\end{array}$ \\
\hline $05-13-97$ & $1,030.6$ & $05-05-98$ & $1,030.0$ & $05-03-99$ & $1,030.0$ \\
$10-07-97$ & $1,029.6$ & $09-28-98$ & $1,029.3$ & $09-28-99$ & $1,029.7$ \\
\hline
\end{tabular}


Table E8. Water-level measurements for Cottonwood Lake in Marshall County

\begin{tabular}{cccccc}
\hline Date & $\begin{array}{c}\text { Water-level } \\
\text { elevation } \\
\text { (feet) }\end{array}$ & Date & $\begin{array}{c}\text { Water-level } \\
\text { elevation } \\
\text { (feet) }\end{array}$ & $\begin{array}{c}\text { Wate } \\
\text { elevation } \\
\text { (feet) }\end{array}$ \\
\hline $05-19-97$ & $1,827.7$ & $05-05-98$ & $1,827.3$ & $05-03-99$ & $1,827.3$ \\
$10-07-97$ & $1,826.3$ & $09-28-98$ & $1,826.5$ & $09-28-99$ & $1,827.3$ \\
\hline
\end{tabular}

Table E9. Water-level measurements for Drywood Lakes in Roberts County

[Note: North and South Drywood Lakes have coalesced into one lake]

\begin{tabular}{cccccc}
\hline Date & $\begin{array}{c}\text { Water-level } \\
\text { elevation } \\
\text { (feet) }\end{array}$ & Date & $\begin{array}{c}\text { Water-level } \\
\text { elevation } \\
\text { (feet) }\end{array}$ & $\begin{array}{c}\text { Water-level } \\
\text { elevation } \\
\text { (feet) }\end{array}$ \\
\hline $05-13-97$ & $1,948.8$ & $05-05-98$ & $1,949.1$ & $05-04-99$ & $1,950.0$ \\
$10-07-97$ & $1,947.8$ & $09-29-98$ & $1,948.7$ & $09-28-99$ & $1,949.6$ \\
\hline
\end{tabular}


Table E10. Water-level measurements for Enemy Swim Lake in Day County

\begin{tabular}{|c|c|c|c|c|c|}
\hline Date & $\begin{array}{c}\text { Water-level } \\
\text { elevation } \\
\text { (feet) }\end{array}$ & Date & $\begin{array}{c}\text { Water-level } \\
\text { elevation } \\
\text { (feet) }\end{array}$ & Date & $\begin{array}{c}\text { Water-level } \\
\text { elevation } \\
\text { (feet) }\end{array}$ \\
\hline $04-10-97$ & $1,856.0$ & $06-02-98$ & $1,854.6$ & $06-01-99$ & $1,853.92$ \\
\hline 04-11-97 & $1,856.0$ & $06-08-98$ & $1,854.4$ & 06-10-99 & $1,854.16$ \\
\hline $04-12-97$ & $1,856.1$ & $06-12-98$ & $1,854.4$ & 06-14-99 & $1,854.06$ \\
\hline $04-13-97$ & $1,856.2$ & $06-15-98$ & $1,854.4$ & 07-01-99 & $1,853.9$ \\
\hline $04-14-97$ & $1,856.2$ & $06-16-98$ & $1,854.4$ & $07-07-99$ & $1,853.59$ \\
\hline $04-15-97$ & $1,856.3$ & $06-22-98$ & $1,854.3$ & $07-12-99$ & $1,853.82$ \\
\hline $04-16-97$ & $1,856.27$ & $06-29-98$ & $1,854.2$ & 07-19-99 & $1,853.7$ \\
\hline $04-17-97$ & $1,856.2$ & $07-06-98$ & $1,854.2$ & $07-26-99$ & $1,854.03$ \\
\hline 04-18-97 & $1,856.2$ & $07-20-98$ & $1,854.0$ & 08-02-99 & $1,853.56$ \\
\hline $04-21-97$ & $1,856.1$ & $07-27-98$ & $1,853.9$ & 08-09-99 & $1,853.48$ \\
\hline $04-22-97$ & $1,856.1$ & $08-05-98$ & $1,853.7$ & $08-16-99$ & $1,853.42$ \\
\hline $04-25-97$ & $1,856.0$ & $08-10-98$ & 1.853 .6 & $08-24-99$ & $1,853.36$ \\
\hline $04-28-97$ & $1,855.8$ & $08-21-98$ & $1,853.5$ & 09-03-99 & $1,853.48$ \\
\hline $05-05-97$ & $1,855.5$ & $08-28-98$ & $1,853.6$ & 09-13-99 & $1,853.58$ \\
\hline $05-08-97$ & $1,855.4$ & 09-04-98 & $1,853.4$ & 09-20-99 & $1,853.50$ \\
\hline $05-12-97$ & $1,855.2$ & $11-13-98$ & $1,853.9$ & $09-27-99$ & $1,853.52$ \\
\hline $05-20-97$ & $1,854.9$ & 03-23-99 & $1,854.0$ & $10-04-99$ & $1,853.44$ \\
\hline $05-26-97$ & $1,854.85$ & 04-07-99 & 1.854 .14 & $10-12-99$ & $1,853.40$ \\
\hline $10-08-97$ & $1,853.3$ & $04-12-99$ & $1,854.12$ & $10-18-99$ & $1,853.38$ \\
\hline $10-29-97$ & $1,853.32$ & $04-19-99$ & $1,854.08$ & $10-25-99$ & $1,853.6$ \\
\hline $05-04-98$ & $1,854.6$ & $04-26-99$ & $1,854.02$ & $11-02-99$ & $1,853.32$ \\
\hline 05-18-98 & $1,854.85$ & $05-05-99$ & $1,854.14$ & & \\
\hline $05-26-98$ & $1,854.85$ & $05-13-99$ & $1,854.14$ & & \\
\hline
\end{tabular}


Table E11. Water-level measurements for Hillebrands Lake in Day County

\begin{tabular}{|c|c|c|c|c|c|}
\hline Date & $\begin{array}{l}\text { Water-level } \\
\text { elevation } \\
\text { (feet) }\end{array}$ & Date & $\begin{array}{l}\text { Water-level } \\
\text { elevation } \\
\text { (feet) }\end{array}$ & Date & $\begin{array}{c}\text { Water-level } \\
\text { elevation } \\
\text { (feet) }\end{array}$ \\
\hline $04-01-97$ & $1,798.3$ & $05-18-98$ & $1,802.59$ & $07-12-99$ & $1,803.61$ \\
\hline 04-10-97 & $1,797.5$ & $05-20-98$ & $1,802.6$ & $07-19-99$ & $1,803.55$ \\
\hline $05-20-97$ & $1,799.2$ & $05-26-98$ & $1,802.68$ & $07-26-99$ & $1,804.03$ \\
\hline $10-01-97$ & $1,800.08$ & $07-06-98$ & $1,802.57$ & $08-02-99$ & $1,803.97$ \\
\hline $10-08-97$ & $1,799.4$ & $11-13-98$ & 1.802 .1 & $08-09-99$ & $1,803.83$ \\
\hline $11-14-97$ & $1,799.98$ & $03-23-99$ & $1,802.7$ & $08-16-99$ & $1,803.83$ \\
\hline $04-14-98$ & $1,801.34$ & 04-07-99 & $1,802.91$ & 08-24-99 & $1,803.67$ \\
\hline $04-20-98$ & $1,801.43$ & 04-12-99 & $1,802.93$ & 09-03-99 & $1,803.75$ \\
\hline $04-27-98$ & $1,801.9$ & 04-19-99 & $1,802.93$ & 09-13-99 & $1,803.75$ \\
\hline $04-28-98$ & $1,801.9$ & $04-26-99$ & $1,802.93$ & 09-20-99 & $1,803.65$ \\
\hline $05-04-98$ & $1,801.6$ & 05-05-99 & $1,803.07$ & 09-27-99 & $1,803.65$ \\
\hline $05-04-98$ & $1,801.95$ & 05-13-99 & $1,803.21$ & $10-04-99$ & $1,803.47$ \\
\hline $05-05-98$ & $1,801.95$ & $06-01-99$ & $1,803.21$ & $10-12-99$ & $1,803.92$ \\
\hline $05-07-98$ & $1,801.94$ & $06-10-99$ & $1,803.55$ & $10-18-99$ & $1,803.37$ \\
\hline $05-11-98$ & $1,801.98$ & $06-14-99$ & $1,803.51$ & $10-25-99$ & $1,803.33$ \\
\hline $05-11-98$ & $1,802.02$ & $06-21-99$ & $1,803.53$ & $11-02-99$ & $1,803.23$ \\
\hline $05-12-98$ & $1,802.4$ & 07-01-99 & $1,803.61$ & & \\
\hline $05-15-98$ & $1,802.48$ & $07-07-99$ & $1,803.59$ & & \\
\hline
\end{tabular}

Table E12. Water-level measurements for Hurricane Lake in Roberts County

\begin{tabular}{cccccc}
\hline Date & $\begin{array}{c}\text { Water-level } \\
\text { elevation } \\
\text { (feet) }\end{array}$ & Date & $\begin{array}{c}\text { Water-level } \\
\text { elevation } \\
\text { (feet) }\end{array}$ & $\begin{array}{c}\text { Water-level } \\
\text { elevation } \\
\text { (feet) }\end{array}$ \\
\hline $05-19-97$ & $1,751.1$ & $05-05-98$ & $1,751.3$ & $05-04-99$ & $1,751.0$ \\
$10-07-97$ & $1,750.7$ & $09-29-98$ & $1,750.4$ & $09-28-99$ & $1,750.6$ \\
\hline
\end{tabular}


Table E13. Water-level measurements for Pickerel Lake in Day County

\begin{tabular}{|c|c|c|c|c|c|}
\hline Date & $\begin{array}{l}\text { Water-level } \\
\text { elevation } \\
\text { (feet) }\end{array}$ & Date & $\begin{array}{c}\text { Water-level } \\
\text { elevation } \\
\text { (feet) }\end{array}$ & Date & $\begin{array}{c}\text { Water-level } \\
\text { elevation } \\
\text { (feet) }\end{array}$ \\
\hline $04-11-97$ & $1,846.6$ & $05-26-98$ & 1.845 .89 & 06-10-99 & $1,846.08$ \\
\hline $04-12-97$ & $1,846.5$ & $06-02-98$ & $1,845.7$ & 06-21-99 & $1,845.72$ \\
\hline $04-13-97$ & $1,846.4$ & $06-12-98$ & $1,845.7$ & 07-01-99 & $1,845.66$ \\
\hline 04-14-97 & $1,846.4$ & $06-16-98$ & $1,845.7$ & 07-07-99 & $1,845.56$ \\
\hline $04-15-97$ & $1,846.4$ & $06-22-98$ & $1,845.5$ & 07-12-99 & $1,845.64$ \\
\hline $04-16-97$ & $1,846.5$ & $06-29-98$ & $1,845.5$ & 07-19-99 & $1,845.46$ \\
\hline $04-17-97$ & $1,846.5$ & $07-06-98$ & $1,845.6$ & $07-26-99$ & $1,845.38$ \\
\hline 04-18-97 & $1,846.5$ & $07-20-98$ & $1,845,3$ & 08-02-99 & $1,845.34$ \\
\hline $04-21-97$ & $1,846.5$ & $07-27-98$ & $1,845.2$ & 08-09-99 & $1,845.28$ \\
\hline $04-22-97$ & $1,846.5$ & $08-05-98$ & $1,845.0$ & $08-16-99$ & $1,845.32$ \\
\hline $04-25-97$ & $1,846.3$ & $08-10-98$ & $1,845.1$ & 08-24-99 & $1,845.26$ \\
\hline $04-28-97$ & $1,846.2$ & $08-21-98$ & $1,845.1$ & 09-03-99 & $1,845.42$ \\
\hline $05-01-97$ & $1,846.0$ & $08-28-98$ & 1.845 .2 & 09-13-99 & $1,845.54$ \\
\hline $05-05-97$ & $1,845.9$ & 09-04-98 & $1,845.0$ & 09-20-99 & 1.845 .46 \\
\hline $05-08-97$ & $1,845.9$ & 04-07-99 & $1,845.73$ & $09-27-99$ & $1,845.44$ \\
\hline $05-12-97$ & $1,845.8$ & $04-12-99$ & $1,845.68$ & $10-04-99$ & $1,845.36$ \\
\hline $05-20-97$ & $1,846.6$ & 04-19-99 & $1,845.62$ & $10-12-99$ & $1,845.36$ \\
\hline $10-08-97$ & $1,846.7$ & $04-26-99$ & $1,845.52$ & $10-18-99$ & $1,845.36$ \\
\hline $05-04-98$ & $1,846.6$ & 05-05-99 & $1,845.62$ & $10-25-99$ & $1,845.36$ \\
\hline $05-20-98$ & $1,846.00$ & $05-13-99$ & $1,845.76$ & $11-02-99$ & $1,845.38$ \\
\hline $05-25-98$ & $1,845.9$ & $06-01-99$ & $1,845.46$ & & \\
\hline
\end{tabular}

Table E14. Water-level measurements for Piyas Lake in Marshall County

\begin{tabular}{cccccc}
\hline Date & $\begin{array}{c}\text { Water-level } \\
\text { elevation } \\
\text { (feet) }\end{array}$ & Date & $\begin{array}{c}\text { Water-level } \\
\text { elevation } \\
\text { (feet) }\end{array}$ & $\begin{array}{c}\text { Water-level } \\
\text { elevation } \\
\text { (feet) }\end{array}$ \\
\hline $05-19-97$ & $1,831.5$ & $05-05-98$ & $1,831.3$ & $05-03-99$ & $1,832.4$ \\
$10-07-97$ & $1,830.9$ & $09-28-98$ & $1,831.0$ & $09-28-99$ & $1,832.6$ \\
\hline
\end{tabular}

Table E15. Water-level measurements for Red Iron Lake North in Marshall County

\begin{tabular}{cccccc}
\hline Date & $\begin{array}{c}\text { Water-level } \\
\text { elevation } \\
\text { (feet) }\end{array}$ & Date & $\begin{array}{c}\text { Water-level } \\
\text { elevation } \\
\text { (feet) }\end{array}$ & $\begin{array}{c}\text { Water-level } \\
\text { elevation } \\
\text { (feet) }\end{array}$ \\
\hline $05-19-97$ & $1,830.6$ & $05-05-98$ & $1,829.3$ & $05-03-99$ & $1,829.2$ \\
$10-07-97$ & $1,829.3$ & $09-28-98$ & $1,828.5$ & $09-28-99$ & $1,828.7$ \\
\hline
\end{tabular}


Table E16. Water-level measurements for Spring Lake in Day County

\begin{tabular}{|c|c|c|c|c|c|}
\hline Date & $\begin{array}{l}\text { Water-level } \\
\text { elevatlon } \\
\text { (feet) }\end{array}$ & Date & $\begin{array}{l}\text { Weter-level } \\
\text { elevation } \\
\text { (feet) }\end{array}$ & Date & $\begin{array}{c}\text { Water-level } \\
\text { elevation } \\
\text { (feet) }\end{array}$ \\
\hline $04-01-97$ & $1,798.22$ & $05-15-98$ & $1,802.48$ & $07-07-99$ & $1,803.59$ \\
\hline 04-10-97 & $1,796.4$ & $05-18-98$ & $1,802.59$ & $07-12-99$ & $1,803.61$ \\
\hline $05-29-97$ & $1,799.2$ & $05-20-98$ & $1,802.60$ & $07-19-99$ & $1,803.55$ \\
\hline $06-09-97$ & $1,799.6$ & $05-26-98$ & $1,802.68$ & $07-26-99$ & $1,804.03$ \\
\hline $10-01-97$ & $1,800.08$ & $07-06-98$ & $1,802.57$ & $08-02-99$ & $1,803.97$ \\
\hline $10-08-97$ & $1,799.4$ & $11-13-98$ & $1,802.1$ & 08-09-99 & 1.803 .83 \\
\hline $11-14-97$ & $1,799.98$ & $03-23-99$ & 1.802 .7 & $08-16-99$ & $1,803.83$ \\
\hline 04-14-98 & $1,801.34$ & $04-07-99$ & $1,802.91$ & 08-24-99 & $1,803.67$ \\
\hline $04-20-98$ & $1,801.43$ & $04-12-99$ & $1,802.93$ & 09-03-99 & $1,803.75$ \\
\hline $04-27-98$ & $1,801.9$ & 04-19-99 & $1,802.93$ & 09-13-99 & $1,803.75$ \\
\hline $04-28-98$ & $1,801.9$ & $04-26-99$ & $1,802.93$ & $09-20-99$ & $1,803.65$ \\
\hline $05-04-98$ & $1,801.6$ & $05-05-99$ & $1,803.07$ & $09-27-99$ & $1,803.65$ \\
\hline $05-04-98$ & $1,801.95$ & $05-13-99$ & $1,803.21$ & $10-04-99$ & $1,803.47$ \\
\hline $05-05-98$ & $1,801.95$ & 06-01-99 & $1,803.21$ & $10-12-99$ & $1,803.92$ \\
\hline $05-07-98$ & $1,801.94$ & $06-10-99$ & $1,803.55$ & $10-18-99$ & $1,803.37$ \\
\hline $05-11-98$ & $1,801.98$ & 06-14-99 & $1,803.51$ & $10-25-99$ & $1,803.33$ \\
\hline $05-11-98$ & $1,802.02$ & $06-21-99$ & $1,803.53$ & $11-02-99$ & $1,803.23$ \\
\hline $05-12-98$ & $1,802.4$ & $07-01-99$ & $1,803.61$ & & \\
\hline
\end{tabular}

Table E17. Water-level measurements for Whitestone Lake North in Roberts County

\begin{tabular}{cccccc}
\hline Date & $\begin{array}{c}\text { Water-level } \\
\text { elevation } \\
\text { (feet) }\end{array}$ & Date & $\begin{array}{c}\text { Water-level } \\
\text { elevation } \\
\text { (feet) }\end{array}$ & $\begin{array}{c}\text { Water-level } \\
\text { elevation } \\
\text { (feet) }\end{array}$ \\
\hline $05-19-97$ & $2,015.0$ & $05-05-98$ & $2,015.3$ & $05-03-99$ & $2,015.0$ \\
$10-07-97$ & $2,013.9$ & $09-29-98$ & $2,013.9$ & $09-28-99$ & $2,014.9$ \\
\hline
\end{tabular}

Table E18. Water-level measurements for Whitestone Lake South in Roberts County

\begin{tabular}{cccccc}
\hline Date & $\begin{array}{c}\text { Water-level } \\
\text { elevation } \\
\text { (feet) }\end{array}$ & Date & $\begin{array}{c}\text { Water-level } \\
\text { elevation } \\
\text { (feet) }\end{array}$ & $\begin{array}{c}\text { Date } \\
\text { Water-level } \\
\text { elevation } \\
\text { (feet) }\end{array}$ \\
\hline $05-19-97$ & $2,015.0$ & $05-05-98$ & $2,015.3$ & $05-03-99$ & $2,015.0$ \\
$10-07-97$ & $2,014.0$ & $09-29-98$ & $2,013.9$ & $09-28-99$ & $2,014.9$ \\
\hline
\end{tabular}

